Andrews University

Digital Commons @ Andrews University

Master's Theses

Graduate Research

1974

\title{
A Comparative Study of the Vocabulary of Five Basal Reading Series
}

Judith O. Young

Andrews University

Follow this and additional works at: https://digitalcommons.andrews.edu/theses

Part of the Language and Literacy Education Commons

\section{Recommended Citation}

Young, Judith O., "A Comparative Study of the Vocabulary of Five Basal Reading Series" (1974). Master's Theses. 169.

https://dx.doi.org/10.32597/theses/169

https://digitalcommons.andrews.edu/theses/169

This Thesis is brought to you for free and open access by the Graduate Research at Digital Commons @ Andrews University. It has been accepted for inclusion in Master's Theses by an authorized administrator of Digital Commons @ Andrews University. For more information, please contact repository@andrews.edu. 
Andrews Iniverity

School of Gradiate Studies

A COMPARAYYVF STUDY OF THE VOCABULARY

TW FIVE BASAL FEADTNG SERTES

A Research Project

Presented in Partial Fulfillment

of the Requirenents for the Degree

Naster of Arttz

by

Juait th 0 . Young

Angugt 1974.

Inprovel 
Chapter:

Page

1. MTRODUCHION ..................... 1

rhe Problem ....................... 1

Furpose of the study ................. 2

Definitions of Terms ................. 2

Iimitations of the Study ............. 3

II. REVIEW OF LITERATURE: AN OVERVIEW OF THE FACTORS

INCLUDED IN BASAL READERS $\ldots . . . . . . . .4$

Textbook Development ............. 4

Vocabulary ................ 5

Readability ................... 7

Summary ................... . . 9

III. RESEARCH DESIGN . . . . . . . . . . . . 11

Choice of Basal. Reading Series ........... 11

Procedures ........................ 11

Method of Analysis .................. 12

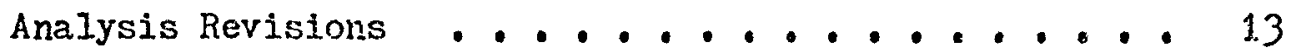

IV. FINDINGS, CONCLUSIONS, AND RECOMMENDATIONS ...... 15

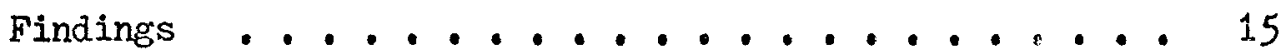

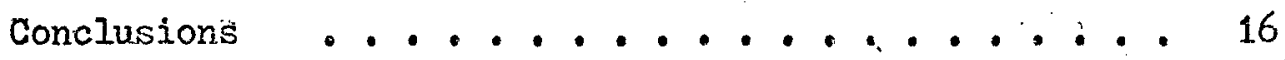

Recommendations for Further. Study . . . . . . 16

APPENDTX A

APPENDIX $B$

BIBLTOCRAPIIY 
CHAPTER I

\section{IITRODUCTION}

Much emphasis is being placed on the teaching of reading today, and yet students are still needing remedial help. The author's interest in the vocabulary comparability of basal reading textbooks began when trying to place a remedial student into a corresponding level in another basal series--there was no way to know if the vocabulary levels corresponded.

Through professional discussions with others it was found that many had faced this same problem with two types of students: 1) Those transferring to a new school from a school system using another basal series; and, 2) Those needing additional remedial help.

This study explores the possibility of obtaining a vocabulary correlation between basal reading series.

\section{The Problem}

Approximately three hundred thousand school children change schools each year between the grades of first and third. Each time these children are moved the chances are that they will have to become accustomed to a new math series, science series, spelling series, reading series, and other subject textbooks. Placement in some of these series will be done accoroing to the results of a test. However, no test exists that could test the background vocabulary a child has received in a previous reading series. 
Information in rogards to how the vocabularly at a given level in each basal series corresponds to another series at the same level is not now available. While both readers may be at what is labeled a Level 2 , who is to know if they are at the same reading level? The first series may have eleven series for up through grade 3 , while the second series may have only eight.

A second problem presented is that of the remedial reader. It is true that this child may need to repeat a step in a level, but it is harmful to the psychological state of the child to repeat this level in the same basal series. The stories, seatwork, and presentations tend to be boring the second time over and the feeling of failure is increased. To be able to place the child in another series for his remedial work would be a boon to both the child and the teacher.

\section{Purpose of the Study}

The purpose of this study is to establish a method which can be used to place a student into a new basal series by using the information from a past basal series. The needed information for the past series would be the basal series name, the level, and the page in the book the child has last successfully finished. By knowing these factors, and using them in connection with the chart given, the teacher will be able to determine at what point the student is in the new series.

\section{Definitions of Texms}

Basal Reading Series: A set of books for the teaching of reading which are a series and have a core vocabulary.

Master Series: The series which the student is moving from. Receiving Series: The sexies which the student is moving to. 
Base Word: A word not previously presented to the student which Is necessary to kngh before Griering the corresponding Receiving Series. Proper names are not included.

\section{Limitations of the study}

The study will be based only on vocabulary and where it is presented first in each series' teacher's guide. This will exclude comprehension, phonics, and other materials introduced in the series. It will also in some series exclude the words which the authors call "decodable". To be totally accurate each series would need to be completely typed into, and then analysed by, the computer.

While the study and method of placement will cover grades $K$ to 3 inclusive, it will not cover material beyond this level. Also, there are only. five basal series represented, and in these only one edition of the series. 
REVIEW OF LITERATURE:

AN OVERVIEW OF THE FACTORS INCLUDED IN BASAI READERS

\section{Textbook Development}

The first series of what is called a basal reading series appeared in America in 1790. Noah Webster, the author of these books, first published The Grammatical Institute, Part Three (Carpenter, 1963, p. 60). "Webster explained in the first part of his book that 'clear and distinct' articulation was necessary in reading, and that the pupil should notice 'the stops and make no pause where the sense required none' (Carpenter, 1963, p. 60)."

Other authors such as Caleb Bingham and Lindley Murray soon followed Webster's books. However, William McGuffery's readers, known as the "McGuffery's Eclectic Readers", were the most popular series of textbooks published in America.

The series of six books which were continually revised over the years not only taught youngsters how to read, but the more difficult volumes provided most of the literature to which three generations of Americans were exposed (Black; 1967, p. 128).

These books controlled their reader levels by controlling the length of the words in each level. But not until 1910 was comprehension taught as a part of reading.

Early in the 1960 's another step was taken. Outdated were the readers which progressed by grades. The basal series today are mainly 
on the principle of progression as quickiy as the student is ready. Therefore the basal series axe prepared by levels and numbered as such in most series.

"The textbook houses of the mid-1960's benefited from two almost concurrent boons: a population explosion and an outpouring from the Federal Treasury (Black, 1967, p. 129)."

The preparation of a basal reading series is no longer the work of one man. A linguistic consultant, a children's literature consultant, an educational consultant, advisors, editorial staff members, and artists all work together to produce a sexies.

Today the multi-colored, coordinated textbooks and workbooks are found as the core of the American reading program. "A survey of 1300 teachers sampled throughout the country indicates that 95 to 98 per cent of the primary teachers use basal readers every school day (Spache \& Spache, 1973, p. 147)." The basal reader program is also supported by a majority of reading experts, and as such is a prominent force in America today.

\section{Vocabulary}

Two important skills are included under this heading: 1) the use of meaning clues, including context; 2) instant recognition of words. To facilitate the learning of these skills the basal reader has, during the past years, had a "controlled vocabulary". This vocabulary has been arrived at independently by each publisher of the basals.

An example of this controlled vocabulary is the Free-Association Study made by Ginn and Company in 1936. This was a study aimed at graded vocabularies "involving over 20,000 children and $2,7000,000$ words of data and resulting in tentative vocabulary lists for Grades II to VIII. This list was then combined with a list of the other most important vocabulary 
studies that have been made (Enckingham \& Dolch, 1936, p. 3)." These were: "Florn's Vocabulary of Six-jear olds", "Kindergarten Onior List", "Jones", "Tidyman", "Studley-Ware", "Payne-Garrison", "Rauer", "Gates", and "Thorndike" (Buckingharn \& Jolch, 1936, p. 19).

This method used by Ginn to arrive at a graded, controlled vocabulary is typical of most curing the first half of the twentieth century. Criticism of the controlled vocabulary grew out of four unfortunate practices:

11) The emphasis in promoting readers (and in the development of them also) has been on the number of words in a series, not on the development of skills needed to identify new words.

2) Publishers competed to show how few words they used in a series. This unsound practice grew out of a sound theory: Children can best be taught to read with materials that are easy to read, other factors considered.

3) Publishers went to extremes in describing the extent of their controls.

4) The rigid application of such controls caused some authors to fall into the practice of writing inane and contrived namby-pamby selections (Harris, 1966, p. 17)."

During the past ten years the editors of the basal reader series

have moved toward a larger core vocabulary which "includes the words that most children use in their oral vocabularies and will have met in their reading after two or three years in school regardless of what kind of reading program they have been using (Johnson, Ramirez, Smith \& others, 1973, p. 33)."

At least this is the theory. However, numerous studies of the vocabularies of various basal series have, in the past, shown very little overlap. "For example, a recent survey by Selma E. Herr found only six hundred words used in all twelve series studied out of a total of approximately twelve thousand words (Spache \& Spache, 1973, p. 173)."

'In the past few years among the students who have needed remedial 
reading work, the percentage has been climbing of those who have vocabulary difficulties.

This group's exceptions to the broad developniental program must be dealt with.

"Some pupils exhibit very slow growth in sight vocabulary or in independence in word attack. Some pupils are limited in the number of new words they can deal with in a unit of time. others are so limited in language experiences (word meanings) that progress must be slow. For many of these pupils the controlled vocabulary of the basal series is essential and really basic to their needs as they gradually develop the abilities to explore other materials (Spache \& Spache, 1973, p. 175)."

\section{Readability}

Knowing where to place a child in a series is difficult and one method of placement is that of the informal reading inventory. The procedure for constructing a reading inventory is not difficult and is a real help to the teacher on the spot and pressed for time.

The first step is to select a basal reading series which your students are not familiar with. It is very important that the students are not familiar with the stories.

Step two consists of choosing twenty words from the basal vocabulaxy index of each level. Next, select a story from somewhere near the end of each level. For each story chosen, write ten comprehension questions. The questions should be divided into: two main ideas, two details, one inference, one sequence, and four vocabulaxy questions.

Each child must be tested alone. Beginning with the pre-primer level the child should read the vocabulary list upward until at least four words are missed in a given level.

"Four or more mistakes is considered a child's frustration level--the reading level that most often produces failure. 
Zero or one mistate ts the jndependent lever. Two or three mistakes, therefore, is the instrucilona? lovel-o-the level at which the ofize sholld be reading ifi sohool (Sebesta \& Wallen, 1972, yo 227)."

At this point the child should read the comprehension test for the instructional level found in vocabulary. Tris should be graded the same way. Once these two craluations are made it is best to remember one rule in grouping. Don't overload the child.

This is only one example of how to use an informal reading inventory. And there are about as many variations as there are authors. Irving Iorge at CoIumbia University developed a formula by which the difficulty of reading material could be roughly determined. Lorge was aware, however, of drawbecks of readability tests. Such elements as conceptual difficulty, organization of material, interest of material and student background. He tested students on the meaning of some passages of equal length difficulty, where vocabularies, sentence length, complexity, and the topic treated were the same. "The only apparent difference, as far as Iorge could tell, lay in organization and in clues to organization. He tested students on the meaning of these passages and found that they were better able to answer questions on passages wellorganized and bearing clues to organization (Mc Cullough, 1969, pp. 432-443)."

To know what to expect of a child in reading is difficult for the average teacher. A simple, yet usable, formula for estimating reading expectancy was suggested by Bond and Tinker (1967, p. 92):

"Reading expectancy = years in school times IQ divided by 100 , plus 1.0 "

The use of an expectancy formula such as this one may have value in helping the teacher and the pupil to set reasonable expectations of 
reading achiererent, especially for the less able pupil. Establishing reasonable expectations may go a long way towaxd relieving the excessive pressures often put on below-iverage achievers because of an unreasonable concern that they be brought up to the "norin". It may also help to challenge the more apt pupil who, with proper instruction, should perform considerably better in reading.

One final caution must be heeded. Any kind of predictive formula or expectancy formula can be terribly misunderstood or misused. Approach these with care.

\section{Summary}

Reading, like many other educational fields, can be accused of both under and: over reacting. Textbook development has moved forward in one direction, many times ignoring other areas of the same field. However, the pendulum seems to swing back and forth, much as in other areas.

Trends, redevelopment of old ideas, new philosophies--all are needed to help stimulate the professional teacher. But often this maze is confusing to the teacher and many seem to be grasping for a solid base on which to build their foundation.

The lack of consistancy fourd in the vocabuiaries of the basal reading series tends to be confusing to a reader who is being moved or is being placed in a remedial series. This confusion from a lack of background may also be seen in the readability scores. It is difficult for a child to place at a level consistent with his former work if he is faced with a seventy-five per cent new vocabulary. For this reason it would also cause one to wonder at the accuracy within a classroom when a second basal series is used as an informal test of readability. Do the two basals have similar vocabularies and styles? 
While the criticisn of a controlled vocabulary is valid, the other extreme of no basic vocabulary may be proving to be just as harmful to the children who are learning to read. No tool js going to work unless teachers use this tool with understanding and caxe. One does not get into an automobile and let it take one where it wills. No, the speed and direction are controlled by the driver. Thus with any tool used. Teachers must learn to control it and make j.t heip them. 
CHAPTER III

RESEARCH DESIGN

Choice of Basal Reading Series

The choice of the five basal series which were used in this study was taken partially from the information obtained by Rosemae Hafstrom in her research study entitled, A Survey of the Methods and Criteria Used in the Selection of Reading Textbooks, and partially from the availability of books. The five series chosen axe the following:

Series A: The Booknark Reading Program, by Harcourt Brace Jovanovich, Inc., New York, 1974.

Series B: The Houghton Mifflin Readers, by Houghton Mifflin Company, Boston, 1966.

Series C: McMillan Basal Readers, by Maclililan Company, New York, 1966.

Series D: Harper and Row Design for Reading, by Harper and Row, Publishers, Inc., New York, 1972.

Series E: Seventh-day Adventist Basal Series, Pacific Press, Mountain View, California, 1967.

\section{Procedures}

After obtaining cleararce for this research project from the department of Education, the computer center was consulted and a method of analysis was established. This method called for the use of the vocabulary lists from each basa]. series.

It was at this time that each publishing company was contacted. The reactions of the companies were varied. Some provided these lists immediately while there were two companies which refused to provide the 
vocabulary Iist.

Each participating basel series was assigned a letter which was used for ideatification throughout the study. Each series also had a number assigned for each level.

The vocabulary lists were then keypunched on to computer cards with the following information: the series identification letter, the series level number, the vocabulary word, and the page the word was first introduced into the series.

This information was then used for computer analysis as described in the section entitled "Method of Analysis".

\section{Method of Analysis}

The completed vocabulary lists were fed into the computer and a readout was given of all the words which appeared in each of the series. This readout also included an alphabetical list of the words in each series.

The second data readout was of a matrix nature showing all of the words in all of the series combined alphabetically, and on what page and level each word appeared in the series. An example of this matrix follows:

\begin{tabular}{lcccccc} 
Word List & Series: & A & B & C & D & E \\
\hline Agreed & $/$ & $/$ & $8 / 90$ & $/$ & $/$ \\
Ah & $7 / 170$ & $5 / 264$ & $/$ & $/ / 218$ \\
ahead & & $/$ & $/$ & $6 / 143$
\end{tabular}

This trix gave the information regarding just how widely the word presentation varies among the series and how often the words are presented in each series and at the same approximate levels.

The final step in the comparing of the basal series was to obtain a printout matrix of each Master Series at each level, comparing the 
words used in this Waster Serias level to boh Feceiving Series. From this printout a formula will be used to obtain the comparison percentages. The following formula was used:

MV: the vocabulary words in the Master Sexies at a given level

RV: the vocabulary words in the Receiving Series at one given level which are also in the $\mathrm{KV}$

$$
\frac{\mathrm{MN}}{\mathrm{RV}} \times 100=x \%
$$

If the $\mathrm{x} \%$ was above $75 \%$, the Master Series at that level and the Recelving Series at the given level will be considered comparable. However, when there is no directly corresponding level in a Receiving Series, the RV needed to establish $75 \%$ will be found by using page numbers in the levels. Thus Series C, Level 3, may correspond at the $75 \%$ level with Series $\mathrm{E}$, Level 5, page 123 to Level 6, page 34.

While the $75 \%$ level is a high percentage the words in both series which lie outside of this range must be dealt with. This will be done as follows: Each word in the Master Series Level which has not yet been presented in the Receiving Series is no problem, as this will be repeated. However, each word already presented in the Master Series and not in the Receiving Series will be called a Base word and in the final listing will be presented as such, and should be taught to the student before the Receiving Level is accurate.

\section{Analysis Revisions}

Owing to the impossibility of obtaining a $75 \%$ correspondence, an alternate method for comparison had to be designed when the data had begun running. The method which was used follows:

The author bégan to look for minimun and maximum levels between which all of the words for a given Receiving Series level had been 
introduced in the Haster Series. This also was at a very low percentage and a backup whal inst wascreated to showall of the words which were in the given Receiving Series level but never appeared in the entire Master Series. 


\section{Findirigs}

The data obtained was printed in tables. Because of the large " amount of data dealt with these tables are listed in Appendix A. The following explanation will explain what is contained in each table, its meaning and how it would affect a child. (This example table is the first to appear in Appendix $A_{\text {.) }}$

The back-up words for Level 1 are:

$$
\begin{aligned}
& \text { bing } \\
& \text { bug } \\
& \text { duck } \\
& \text { good } \\
& \text { in } \\
& \text { pig } \\
& \text { pond } \\
& \text { sandy } \\
& \text { turtle }
\end{aligned}
$$

There are 32 words in Level 1 of Series A. In comparing Series A with Series B, each at Level 1, there is a $34.37 \%$ match. There also is a $31.25 \%$ match (outside of Level 1) ranging from 6-159 to 2- 13.

\section{Explanation of Use of Tables}

The back-up words are words listed which appear in Series A, Leve1 1, but do not appear in Series B at all.

The percentage of words which match (appear in Level 1 of both Series $A$ and Series $B$ ) is $34.37 \%$. There is an additional $31.25 \%$ match, or percentage of words which appear in Level 1 of Series A and in 
Series B (exclusive of Ifvel i) between Leve? 6, page 159 and Level 2, page 13.

Each table gives the above information. There is one table for each series at each level as compared to each of the other four series.

\section{Conclusions}

Each publisher compiles his own core vocabulary list. This list is compiled by the individual publisher's standards. Due to this factor there is a very low comparison ratio between the different basal series vocabularies.

As a result of this stuoy, there are positive implications for the instruction of the child in decoding skills as soon as he begins his education. Structural clues, context clues, phonics, and other methods are all recormended to the instructor.

In considexation of the results of this study the student who is moved from one basal series to another and does not have sufficient decoding: skills may appear at a lower level in reading than he has achieved previously, due to yocabulary change.

An effort has been made to show the difficulties which a teacher may perceive for the student who is changing his basal series.

\section{Recommendations for Further Study}

The following list includes possibilities for further study in the area of vocabularies in basal readers. .

1. Additional information could be obtained by a study comparing a larger number of basal series.

2. A study comparing the vocabulary lists with the Dolch word lists could be made. 
3. Further information might be fourd by working with the obtained data and the Dale Chall Readability Fornula.

4. A compilation of ajl the words which appear in every basal series could be made. (Siee hppendix $B_{0}$ )

5. An alternate method of comparing the vocabularies could be to make a vocabulary list directly from the text of the book and not use the publishers' vocabulary lists. 
APPENDIX A

These tables are the comparison of each series at each level to each other serjes. The seives levels correspond to the following basal reading series:

Series A: The Bookmark Reading Program, by Harcourt Brace Jovanovich, Inc., New York, 1974.

Series B: The Houghton Mifflin Readers, by Houghton Mifflin Company, Boston, 1966.

Series C: McMillan Basal Readers, by Macliillan Company, New York, 1966.

Series D: Harper and Row Design for Reading, by Harper and Row, Publishers, Inc., New York, 1972.

Series E: Seventh-day Adventist Basal Series, Pacific Press, Mountain View, California, 1967.

\section{Explanation of Use of Tables}

The back-up words are words listed which appear in Series A, Level 1, but do not appear in Sexies B at all.

The percentage of words which match (appear in Level 1 of both Series A and Series B) is $34.37 \%$. There is an additional $31.25 \%$ match, or percentage of words which appear in Level 1 of Series A and in Series B (exclusive of Level 1) between Level 6, page 159 and Level 2, page 13. 
THE BACKMUP WORDS FOR LEVEL 1 ARE:
BING
BUE
DUCK
GOOD
IN
PIG
PEND
SANDY
TURTLE

THERE ARE 32 WOROS IN LEVEL 1 OF SERIES A. IN COMPARING SERIES A WITH SERIES B , EACH AT LEVEL, 1 THERE IS A $34.37 \%$ MATCH. THERE ALSO IS A $31.25 \%$ MATCH (OUTSIDE GF LEVEL 1 ) RANGING FROM, $6=159$ TO $2=13$. 
THE BACK $=U P$ WORDS FOR LEVEL, 2 ARE:

$$
\begin{aligned}
& \text { BOX } \\
& \text { JAN } \\
& \text { KEVIN } \\
& \text { KIDS } \\
& \text { LINDA } \\
& \text { MELVIN } \\
& \text { SHADOWS } \\
& \text { SPLASH }
\end{aligned}
$$

THERE ARE 40 WORDS IN LEVEL 2 OF SERIES A. IN COMPARING SERIES A WITH SERIES B, EACH AT LEVEL, 2 THERE IS A $10.00 \%$ MATCH. THERE ALSO IS A $62.50 \%$ MATCH (EUTSIDE OF LEVEL 2) RANGING FROM, $6=33 T \theta \quad 1=25$. 
THE BACK WUF WORDS FER LEVEL \& ARE:

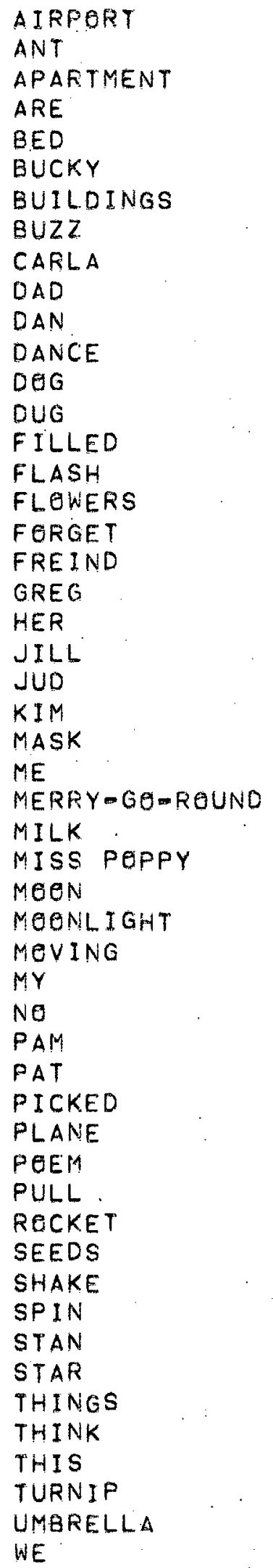

THERE ARE 131 WORDS IN LEVEL 4 EF SERIES A. IN COMPARING SERIES A WITH SERIES B, EACH AT LEVEL, 4 THERE IS A $6.11 \%$ MATCH. THERE ALSO IS A $50.38 \% \mathrm{MATCH}$ (EUTSIDE OF LEVEL 4 ) RANGING FROM. 6. 42 TO $1=136$. 
THE BACK=UP WORDS FOR LEVEL 5 ARE:

BABY
BE
BEAR
BEEHIVE
BEN
BILLY'S
BIRD
BOOK
BOW-WOW
BOZOS

BREMEN BAND

CAGE

CHEWS

CHICKEN LITTLE

CLAY

COCK-A-DEODLE-DOO

CRYING

DANNY DRAKE

DISH

DENKEY

DOOR

DUCKY LUCKY

EARS

$E G G$

FALLING

FLUUR

FEXY LOXY

FROG

GRAY

HAS

HATCH

HAVE

HEE-HAW

HEN

HENNY PENNY

HIMSELF

HOME

ITS

LAND

LIVES

LEGS

LONG

$M A D$

$M E-G W$

MILL

MISS LESTER'S

MIX

MOM

MR. GRANT

MR. MCGREGOR'S

MYSELF

NEST

QPENED

PAINTING

PENNY

PETE

PETER RABBIT

PETS

PQEM

POLICEMAN

PUIP 


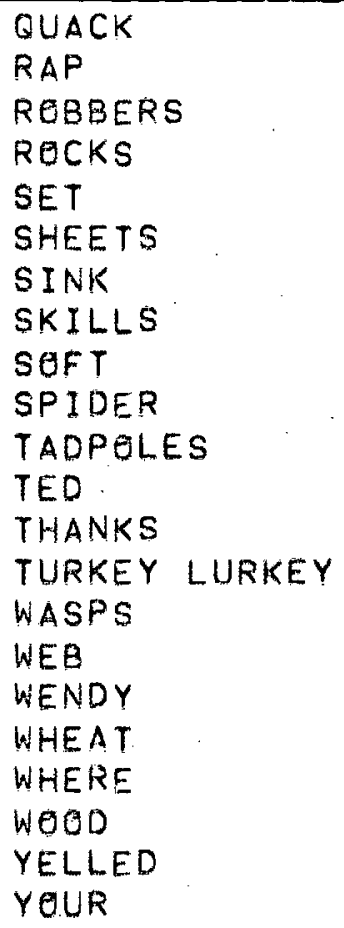

THERE ARE 162 WORDS IN LEVEL 5 OF SERIES A. IN COMPARING SERIES A WITH SERIES B ,EACH AT LEVEL, 5 THERE IS A $5.56 \%$ MATCH. THERE ALSO IS A $41.36 \%$ MATCH (OUTSIDE OF LEVEL 5 ) RANGING FROM, $6=295$ TO 1- 4 . 
THE BACK - UP WORDS FOR LEVEL 6 ARE:

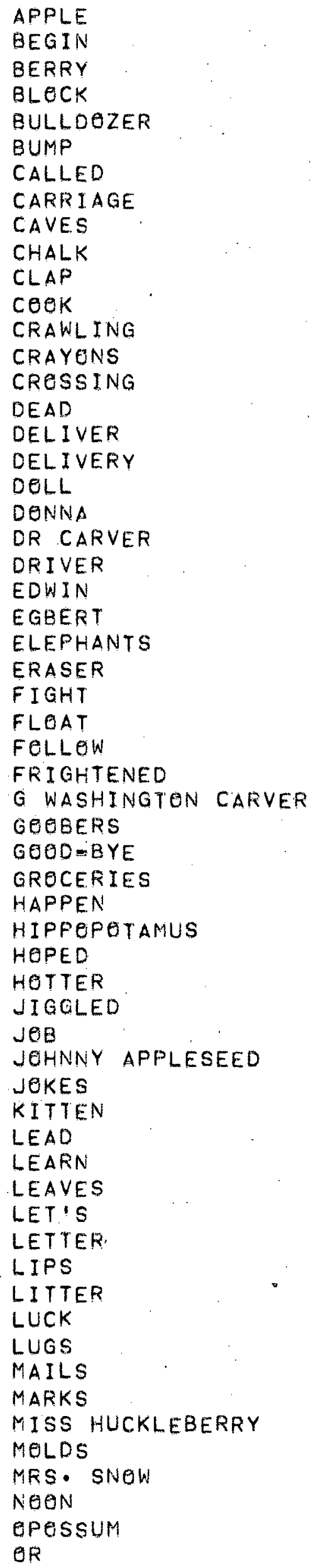




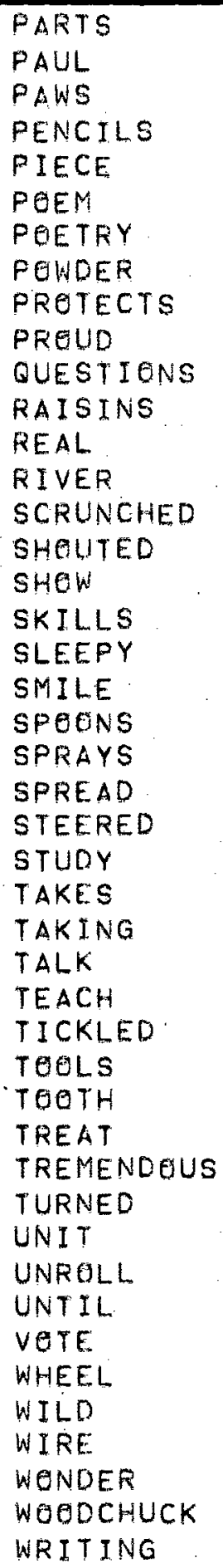

THERE ARE 210 WORDS IN LEVEL 6 OF SERIES A. IN COMPARING SERIES A WITH SERIES B , EACH AT LEVEL, 6 THERE IS A $4.29 \%$ MATCH. THERE ALSO IS A 38.57\% MATCH (EUTSIDE OF LEVEL 6) RANGING FROM, $5=272$ TO $1=139$. 
THE BACK -UP WORDS FOR LEVEL 7 ARE:

\section{$A D O B E$}

$\triangle F R A I D$

AFTERWARDS

AGAIN

AHEAD

AMERICA

ANY

$A R M$

BAGPIPES

BANANAS

BECAME

BIRDS 1

BLEW

BOAROS

$B O D Y$

BOTTLE

BOTTOM

BOUGHT

BREATHS

BUMPER

CARTONS

CHINA

CLAWS

COLLECTING

COLLECTION

CROWD

DECIDED

DINGSAURS

DRAGONS

EAGLE FEATHER

ELEVATORS

ELSA

ELVES

ENGLAND

ENVELOPES

ESCALATER

EVERY

EXIT

EXPLAIN

FAMILIES

FAMILY

FARMER

FEAR

FINALLY

FINEST

FOOT

FREEWAY

FREIGHT

FUR

GIANTS

GIVE

GLEN DEE

HANS

HARDLY

HE'S

HELPFUL

HEGAN

HOLD

HELIDAY

IT'S 
$J A P A N$

JAWS

KATHY

KILLER

KINDLY

KNOWN

LIBRARY

LOLLIPUP

LONGEST

LOVE

LUBBER

MABUNA'S

MAMMALS

MEXICO

MICHI

MIDDLE

MILLIUNS

MISERABLE

MODEL

MOUNTAINS

MUSEUM

MUSHRUSH

NAVAJO

NEAR

NEW YORK CITY

NOTICE

QVERJGYED

OXEN

PAIRS

PALE

PANCAKE

PIGGY-BACK

PLAZA

POEM

POEMS

PORRIDGE

PREUDEST

QUICKLY

RAPUNZEL

RARF.

REPOPTER

RESTAURANT

ROSF RED

ROTOR

RUIN

SCELO

SECRET

SHINE

SHINY

SHOES

SHOOK

SIGH

SIGHT

SIR CHARLES

SISTER

SKILLS

SKIRT

SMALLEST

SON

SORT

SPAIN

STARTED

STERN

STERYTELLER

SUBWAY

TFASING ROY 
TERRIBLE

THANKFUL

THEMSELVES

THEY IRE

TITO'S

TONGUE

TONIO

UNABLE

UNHAPPINESS

UNHAPPY

UNITED STATES

UNPACK

USEFUL

WALLS

WHAT' $S$

WHIRL

WHIRLY

WHISPERS

WHISTLE

WINDOWS

WI ZARD

WOKE

WERRIED

WORRY

WROTE

YESTERDAY

THERE ARE 275 WORDS IN LEVEL 7 OF SERIES A. IN COMPARING SERIES A WITH SERIES B ,EACH AT LEVEL, 7 THERE IS A .00\% MATCH.

THERE ALSO IS A $37.09 \%$ MATCH (EUTSIDE OF LEVEL. 7 ) RANGING FROM, $6-310$ TO $1=24$. 
THE BACK-UF WORDS FUR LEVEL 8 ARE:

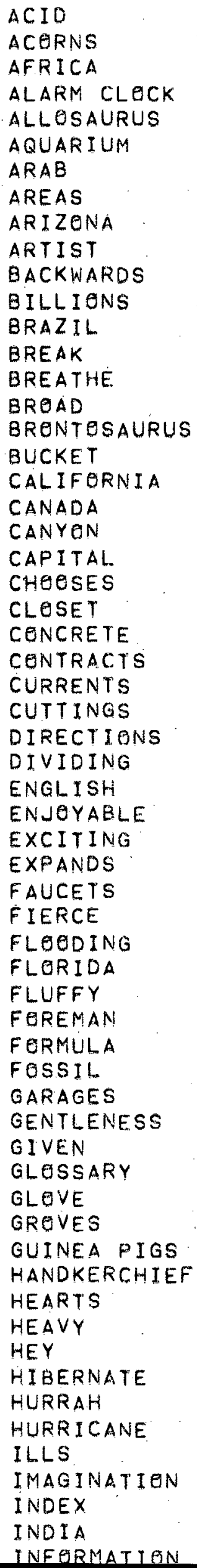


IREN

JUICE

LANGUAGES

LICHEN

MACHINE

MAGICIAN

MAGNIFYING

MESA

MESSAGES

METEOR

METEORITE

MINUTE

METIEN

MUSICIANS

MUTTERING

NAVEL

NINETY

O'CLECK

ORANGES

QRANGUTAN

OXYGEN

PADRES

PARAGRAPHS

PEPPERPOT

PERIODS

PIER

PLATES

POEM

PEETRY

POPSICLES

POURED

PRETZELS

PROBLEMS

PTERANADEN

PULLEYS

PUNCTUATION

PUPPIES

PUSHED

REALIZE

REASEN

RECOGNIZE

REDDISH

REPTILES

RESERVEIRS

RETURN

RETTEN

RQUGH

ROUSE

SHOULDER

SHEVEL

STEGESAURUS

STOMACH

STREAM

SUBJECT

TEPEE

THIRTY

THUMB

TONS

TOPIC

TUNNELS

TYRANNOSAURUS

USUALLY

VASES

VISITING

VISITERS

VAWF। 


\author{
WALLRUS \\ WARMER \\ WATERSHED \\ WE IVE \\ WEIGHED \\ WOEDEN
}

THERE ARE 199 WURDS IN LEVEL \& OF SERIES A. IN COMPARING SERIES A WITH SERIES B ,EACH AT LEVEL, 8 THERE IS A .00\% MATCH.

THERE ALSO IS A $29.65 \%$ MATCH IOUTSIDE OF LEVEL \&) RANGING FROM, $6-289$ TO $1-13 \cdot$ 
THE BACK=UP WOROS FER LEVEL 9 ARE:

ACCENTED

ACHED

$\triangle D M I R E D$

AOVENTURE

AFFECTS

AFRICAN

ALASKA

ALTHOUGH

ANCHORAGE

ANTARCTICA

$\triangle R C T I C$

ARRIVE

AUGUST

AVENUE

BOENESBOROUGH

$B$ Q

BOWSPRIT

BRITISH

BRETH

BULLETS

BUSH

BUSHONGE

BUSINESS

CAPTURE

CARNATIEN

CIBOL.A

CLIMATES

COMBINED

COMMUNICATE

CEMPUTERS

COMSAT

CONGRATULATIONS

CONGRESS

CONTENTED

CONTROL

CORAL REEFS

COURT

CUBA

CUSHION

CUSTOMER

DECEMBER

DECODE

DEPENDS

DESERTS

DETECTIVE

DIAGRAM

DIALS

DIARY

DIFF ICULT

DISAPPEINT

DOMES

DQVEKIE

DUZEN

DRAWER

ECHO

EGRET

ELECTRIC

ENJOYMENT

ESCAPE

ESKIME

ESPFCIAL Y Y 
EVENING

FABULOUS

FACTORIES

FIJI

FIJIANS

FOLK

FORTUNES

GLOBE

GLOSSARY

GRADUALLY

GRAVES

GUESTS

GUITAR

HABITAT

HAITI

HALF

HEAVEN

HEROES

HISPANIOLA

HOVER

HYDREFOIL

INTERESTED

INTERNATIONAL

ISLANDS

JUNEAU

KINGDOM

MANE

MARE

MEXICAN

MICROSCOPE

MICROWAVES

MINERALS

MINI -

MUVEMENTS

MUSTARD

NAT IVES

NEVERTHELESS

NINA

OCEANOGRAPHERS

OPERATIGN

EPPOSITE

ORDINARY

OUTNUMBERED

PATTERN

PAYMENT

PENGUIN

PINTA

PIENEERS

PLAID

POEM

POEMS

POLISHED

PREPARES

PRESIDENT

PRETENDED

PRISONER

PROPELLER

PUEBLES

PUERTO RICO

PURPOSES

RELATED

RELAY

RESCUE

ROUTE

RULER

SAN JUAN 
SAN SALVADOR

SANDWICHES

SANTA MARIA

SATELLITES

SCHWA

SCIENCE

SCIENTISTS

SEARCH

SELECTIONS

SEPTEMBER

SERGEANT

SERIQUS

SHAWNEE

SHELTER

SKIS

SPANI ARDS

STATUES

STEAL

SUBMARINES

SUFFIX

SUGAR

SURRENDER

TELSTAR

TITLE

TOLL

TEPICS

TOUCH

TREASURE

TRIAL

TREPICAL

UNDERNEATH

URGED

VOLCANO

VOYAGE

WANDERED

WHOA

WRECKED

YUGOSLAVIA

THERE ARE 203 WORDS IN LEVEL 9 OF SERIES A. IN COMPARING SERIES A WITH SERIES B ,EACH AT LEVEL, 9 THERE IS A .00\% MATCH. THERE ALSO IS A $16.75 \%$ MATCH (OUTSIDE OF LEVEL 9 ) RANGING FROM. 6-294 TO $3=225$. 
THE BACK-UP WORDS FOR LEVEL 1 ARE:

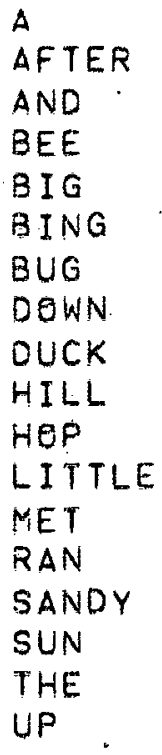

THERE ARE 33 WORDS IN LEVEL 1 OF. SERIES A. IN COMPARING SERIES A WITH SERIES C, EACH AT LEVEL, 1 THERE IS A .00\% MATCH. THERE ALSO IS A $36.36 \% \mathrm{MATCH}$ (OUTSIDE OF LEVEL 1) RANGING FROM. $7-237$ TE $4=118$. 
THE BACK-UP WORDS FOR LEVEL 2 ARE:

BILL
BOX
CAN
DID
FOR
HAT
I
IS
IT
JAN
KIDS
LOOK
LUNCH
MELVIN
NET
EN
SAID
SEE
SHADOWS
SPLASH
TE
YES

THERE ARE 39 WGRDS IN LEVEL 2 OF SERIES A. IN COMPARING SERIES A WITH SERIES C EACH AT LEVEL, 2 THERE IS A $.00 \%$ MATCH.

THERE ALSO IS A $43.59 \%$ MATCH (EUTSIDE BF LEVEL 21 RANGING FRUM; $8=217$ TO $4=60$. 
THE BACK-UP WORDS FER LEVEL 4 ARE:
A IRPERT
$A N T$
ASKED
$B O Y$
$B \cup Z Z$
CANDY
CARLA
CLEUD
CELD
COME
DAN
DANCE
DO

DUG

EAT

FAR

FILLED

FLASH

$F O G$

FORGET

FREIND

GARDEN

$G O$

GREG

JAR

$J I L L$

JUD

KIM

LIKE

MADE

MAKE

MASK

MERRY $=G O=R O U N D$

MISS POPPY

MOONLIGHT

MOTHER

MOVING

NOW.

PAM

PAT

PICKED

PLANE

PLAY

PULL

SEEDS

SHAKE

SKY

SNEW

SNOWMAN

SOMETHING

SPIN

STAN

STAR

TALL

TOY

UMBRELLA

WHAT

YOU 
WITH SERIES C , EACH AT LEVEL, 4 THERE IS A $20.00 \%$ MATCH. THERE ALSO IS A $33.08 \%$ MATCH (GUTSIDE EF LEVEL 4 ) RANGING FROM, $8=55$ TO 5-101.

THE BACK-UF WERDS FOR LEVEL 5 ARE:

BEEHIVE

BIT

BLUE

$B O W=W O W$

BOZOS

BREMEN BAND

CHEWS

CHICKEN LITTLE

CLAY

COCK - A-DEODLE-DOO

COLORS

CRYING

DANNY DRAKE

DUCKY LUCKY

EGG

FALLING

FATHER

FLOUR

FEXY LOXY

FROG

GRAY

GREEN

HAND

HEE - HAW

HELD

HEN

HENNY PENNY

HIMSELF

HOT

JEFF

KING

LAND

LAY

LESSBN

LINES

LION

LOGS

$M E=\theta W$

MILL

MISS LESTERIS

MIX

MR. GRANT

MR . MCGREGQR'S

MYSELF

PAINTING

PETE

PETER: RABBIT

PETS

PLANT

PULP

QUACK

RACE

RAP

RED

ROBBERS

RUCKS

SENT

SET

SHEETS

SHELL

SKIHIS 


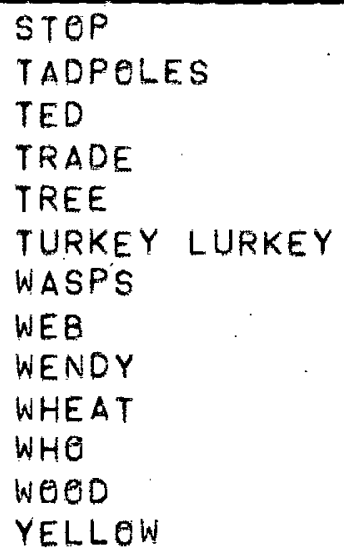

THERE ARE 163 WORDS IN LEVEL 5 OF SERIES A. IN COMPARING SERIES A WITH SERIES C . EACH AT LEVEL, 5 THERE IS A $17.79 \%$ MATCH. THERE ALSO IS A. $33.13 \%$ MATCH IOUTSIDE OF LEVEL 5 I RANGING FROM. 9-119 TO 4-129. 
THE BACK=UP WORDS FOR LEVEL 6 ARE:

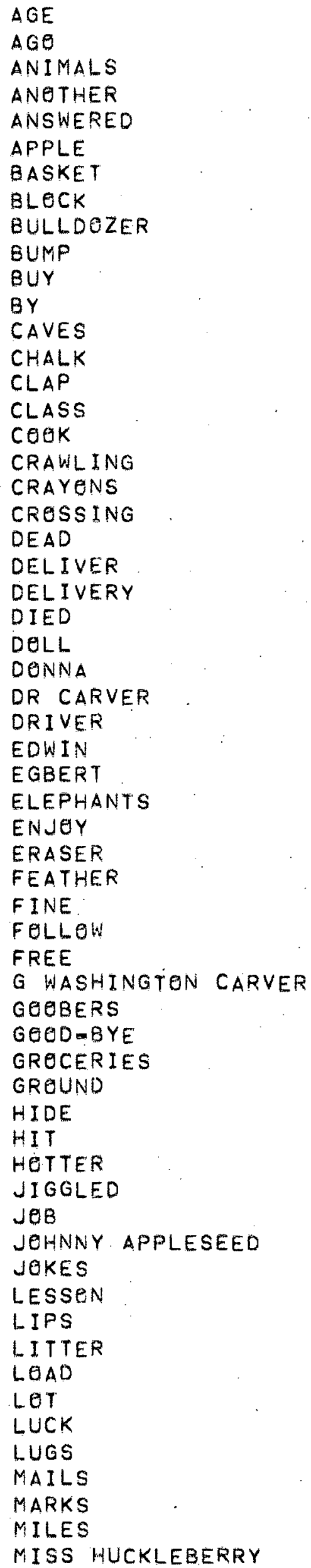




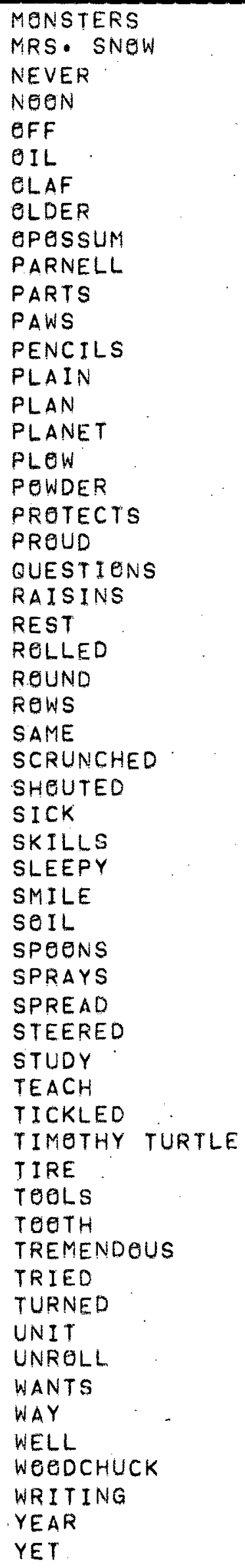

THERE ARE 210 WORDS IN LEVEL 6 OF SERIES A. IN COMPARING SERIES A WITH SERIES C , EACH AT LEVEL, 6 THERE IS A $14.29 \%$ MATCH. THERE ALSO IS A $28 \cdot 10 \%$ MATCH IUUTSIDE OF LEVEL 6) RANGING FROM. $8-235$ TO $4=146$. 
THE BACK-UP WORDS FOR LEVEL 7 ARE:

ACCIDENT

$A C H$

$A D O B E$

AFTERWARDS

AGAIN

AHEAD

$A L L O W$

ALMOST

ANY

ARM

BAGPIPES

BANANAS

BECAME

BELENG

BIRDS!

BOARDS

BQDY

BETTLE

BREATHS

BUMPER

CABUESE

CARTENS

CHINA

CLAWS

CLEVER

CERRAL

DIDN'T

DINESAURS

DRAGENS

EAGLE FEATHER

EARLY

EARN

ELEVATORS

ELSA

ELVES

ENVELEPES

ESCALATER

EXIT

FACES

FAMILIES

FAMILY

FEAR

FIELD

FREEWAY

FREIGHT

FUR

GIANTS

GLEN DEE

HAIR

HANS

HARDLY

HEAR

HEARD

HELPFUL

HERE

HOGAN

HOLD

HORNS

HERRIBLE

HERSE

ISNIT 
$I T ' S$

JACKET

JANET

KATHY

KILLER

KINDLY

LATE

LEATHER

LESSON

LIZARD

LONGEST

LUBBER

MABUNA'S

MAMMALS

MARKET

MEXICO

MICHI

MIKE

MILLIONS

MEDEL

MOUNTAINS

NAVAJO

NEAR

NEW YORK CITY

OVERJOYED

QXEN

PAIL

PAIRS

PALE

PANCAKE

PASSENGER

PIGGY-BACK

PLAZA

POEMS

PORRIDGE

PRINCE

PRINCESS

PREUDEST

RAPUNZEL

RARE

REPERTER

RESTAURANT

RQAR

RESE RED

ROTOR

RUIN

SCELD

SHINE

SHOOK

SIGH

SIR. CHARLES

SKILLS

SKIRT

SUN

SPAIN

STERN

STERYTELLER

STRAIGHT

SUBWAY

TEASING BOY

THEY I RE

TITO'S

TONGUE

TONIE

TRAFFIC

TRAILER 
UNHAPPINESS

UNHAPPY

UNITED STATES

UNPACK

USEFUL,

WALLS

WHIRL

WHIRLY

WHISPERS

WHISTLE

WIZARD

WOKE

WORRIED

YESTERDAY

THERE ARE 274 WORDS IN LEVEL 7 OF SERIES A. IN CEMPARING SERIES A WITH SERIES C , EACH AT LEVEL, 7 THERE IS A $10.95 \%$ MATCH.

THERE ALSO IS A $32.48 \%$ MATCH IOUTSIDE OF LEVEL 7 ) RANGING FROM, $9=232$ TO $4=32$. 
THE BACK=LP WORDS FQR LEVEL 8 ARE:

ACIO

ACORNS

AFRICA

ALARM CLOCK

ALLOSAURUS

ALREADY

AQUARIUM

$A R A B$

AREAS

ARIZONA

ART IST

BACKWARDS

BILLIONS

BOTHERED

BRAZIL

BREAD

BRONTESAURUS

BUCKET

CALIFURNIA

CANYEN

CAPITAL.

CHOOSES

CLOSET

CLOTHES

CLUTHESLINE

COLLAR

COMMAS

CONCRETE

CENTRACTS

CETTAGE

COVERED

CUTTINGS

DIRECTIONS

DIVIOING

ENGINE

FAUCETS

FAVORITE

FIERCE

FIGURE

FLOQDING

FLORIDA

FLUFFY

FERMULA

FESSIL

GARAGES

GENTLENESS

GIRAFFE

GIVEN

GLOSSARY

GLOVE

GROVES

GUINEA PIGS

HEARTS

HEY

HIBERNATE

HURRAH

HURR I CANE

ILLS

IMAGINATION

INDEX

INDIA 
INFORMATION

JUICE

LANGUAGES

LICHEN

LESE

MAGICIAN

MAGNIFYING

MAYGR

MESA

MESSAGES

METEOR

METEERITE

MENTH

METION

MUSICIANS

MUTTERING

NAVEL

NEIGHBER

NINETY

O.CLECK

ORANGES

ORANGUTAN

OXYGEN

PADRES

PARAGRAPHS

PEPPERPET

PERIEDS

PIER

PLATES

POPSICLES

POURED

PRETIZELS

PTERANADON

PULLEYS

PUNCTUATION

PUPPIES

PUSHED

REASEN

REDDISH

REPTILES

RESERVEIRS

RETURN

RETTEN

REUGH

ROUSE

SENTENCE

SHOULDER

SHOVEL

STEGESAURUS

SUBJECT

SYLLABLES

SYRUP

TEPEE

THIRTY

TONS

TOPIC

TYRANNOSAURUS

USUALLY

VASES

VISITING

VISITERS

VUWEL

WALRUS

WARMER

WATERSHED

WEIGHED 
THERE ARE 198 WORDS IN LEVEL 8 GF SERIES A. IN COMPARING SERIES A WITH SERIES C , EACH AT LEVEL, 8 THERE IS A $9.60 \%$ MATCH. THERE ALSO IS A $23.23 \%$ MATCH, (OUTSIDE OF LEVEL 8) RANGING FROM, $g=262$ TO $5=205$. 
THE BACK-UP WORDS FGR LEVEL 9 ARE:

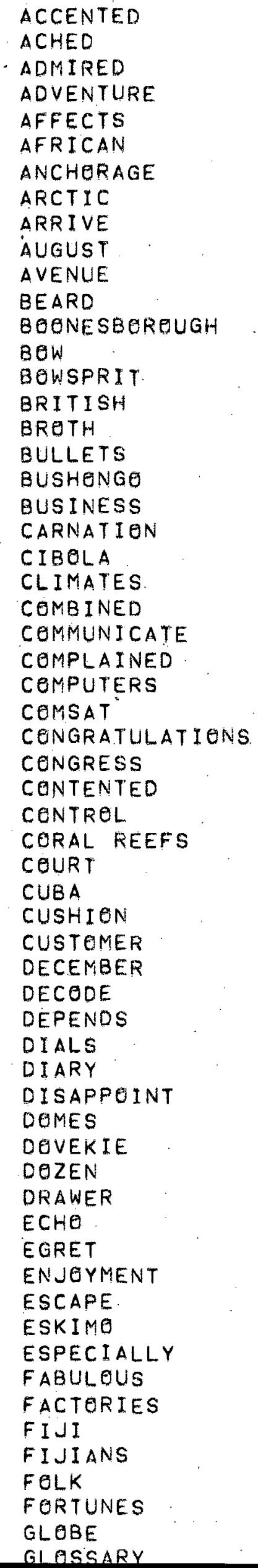


GRADUALLY

GRAVES

QUESTS

GUITAR

HABITAT

HAITI

HALF

HEAVEN

HEROES

HISPANIOLA

HOVER

HYDROFEIIL

INTERESTED

INTERNATI ONAL

ISLANDS

JUDGE

JUDGMENT.

JUNE.

JUNEAU

MANE

MARE

MEADEW

MEXICAN

MICROSCOPE

MICROWAVES

MINERALS

MINI-

MUVEMENTS

MUSTARD

NATIVES

NEVERTHELESS

NINA

OCEANOGRAPHERS

OPERATIEN

OPPESITE

ORDINARY

QUTNUMBERED

PATIENT

PATTERN

PAYMENT

PINTA

PIENEERS

PLAID

PREPARES

PRESIDENT

PRETENDED

PRISONER

PROPELLER

PUEBLES

PUERTO RICO

PURPOSES

RECE IVED

RELATED

RELAY

RESCUE

RULER

SAN JUAN

SAN SALVADOR

SANDWICHES

SANTA MARIA

SATELLITES

SCHWA

SCIENCE

SCIENTISTS

SELECTIONS

SERGEANT 
SERIOUS

SEW

SHAWNEE

SHELTER

SPANIARDS

STATUES

STEAL

SUBMARINES

SUFFIX

SUGAR

SURRENDER

TITLE

TOL!

TEPICS

TOUCH

TRIAL

TROPICAL

UNDERNEATH

URGED

VEGETABLES

VULCANO

WANDERED

WHETHER

WHOA

WON

WRECKED

YUGOSLAVIA

THERE ARE 203 WORDS IN LEVEL 9 OF SERIES A. IN COMPARING SERIES A WITH SERIES C , EACH AT LEVEL, 9 THERE IS A $9.85 \%$ MATCH. THERE ALSO IS A $12.32 \%$ MATCH IOUTSIDE OF LEVEL 9 ) RANGING FROM, $8=275$ TO $6=245$. 
THE BACK-UP WORDS FOR LEVEL 1 ARE:

\author{
BEE \\ MET. \\ PIG \\ SANDY
}

THERE ARE 33 WORDS IN LEVEL. 1 OF SERIES A. IN COMPARING SERIES A WITH SERIES D, EACH AT LEVEL, 1 THERE IS A .0O\% MATCH. THERE ALSO IS A. $72.73 \%$ MATCH IOUTSIDE OF LEVEL 1 ) RANGING FROM, $8-146$ TO $3=8$ : 
THE BACKUP WORDS FOR LEVEL 2 ARE:

$$
\begin{aligned}
& \text { BILL } \\
& \text { BOX } \\
& \text { FAST } \\
& \text { HUNGRY } \\
& \text { I } \\
& \text { JAN } \\
& \text { KEVIN } \\
& \text { KIDS } \\
& \text { LINDA } \\
& \text { MELVIN } \\
& \text { SPLASH }
\end{aligned}
$$

THERE ARE 40 WORDS IN LEVEL 2 OF SERIES A. IN COMPARING SERIES A WITH SERIES D. EACH AT LEVEL, 2 THERE IS A .00\% MATCH.

THERE ALSO IS A $70.00 \% \mathrm{MATCH}$ OUTSIDE OF LEVEL 2 ) RANGING FROM, $11-98$ TO $3=24$. 
THE BACK-UP WORDS FOR LEVEL. 4 ARE:

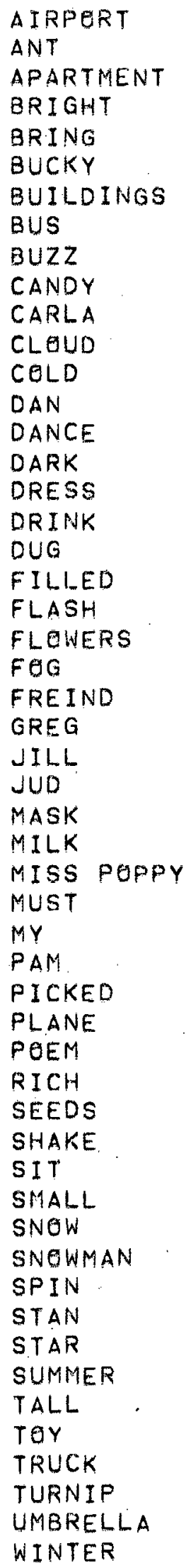

THERE ARE 130 WORDS IN LEVEL 4 OF SERIES A. IN COMPARING SERIES A WITH SERIES D, EACH. AT LEVEL, 4 THERE IS A $3.85 \%$ MATCH. THERE ALSO IS A $53.85 \%$ MATCH (OUTSIDE OF LEVEL 4 ) RANGING FROM, $11=103$ TO 3-22. 
THE BACK UP WGRDS FOR LEVEL 5 ARE:

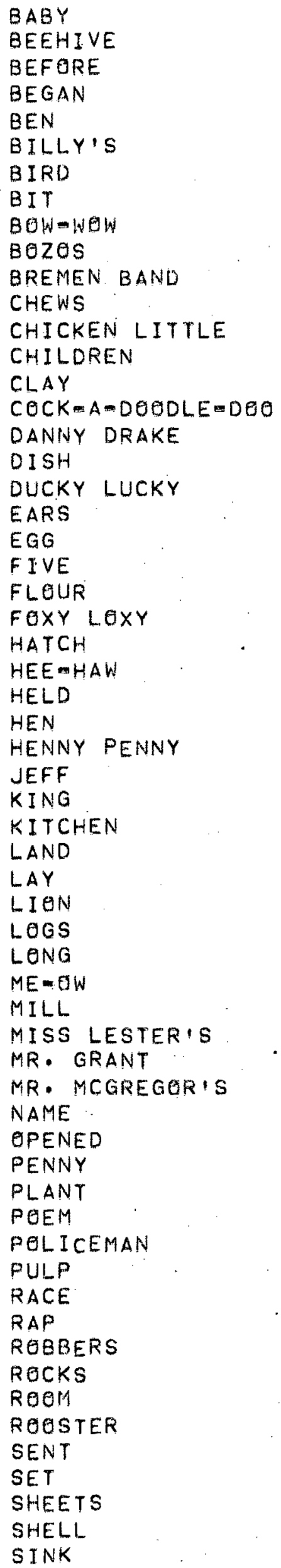


SNIFFED

SOFT

SPIDER

TADPQLES

TEACHER

TED

THANKS

TOOK

TOP

TURKEY LURKEY

WASPS

WEB

WENDY

WHEAT

WON'T

YELLED

YELLOW

THERE ARE 162 WORDS IN LEVEL 5 OF SERIES A. IN COMPARING SERIES A WITH SERIES D , EACH AT LEVEL, 5 THERE IS A $4.94 \%$ MATCH. THERE ALSE IS A $43.21 \%$ MATCH IOUTSIDE GF LEVEL 5 ) RANGING FROM, $11-104$ TO $3=.37$. 
THE BACK-UP WGRDS FER LEVEL 6 ARE:

$\triangle G E$

$A G \theta$

BALLOON

BASKET

BECAUSE

BEGIN

BERRY

BIGGER

BLOCK

BULLDEZER

BUMP

BUY

CARRIAGE

CAVES

CHALK

CLAP

CLASS

COOK

CRAWLING

CRAYENS

CRESSING

DANGER

DEAD

DEL I VER

DELIVERY

DIED

DOCTOR

DONNA

DR. CARVER

DRIVER

EOWIN

EGBERT

ELEPHANTS

ELSE

ENJOY

ERASER

FAMOUS

FEATHER

FIGHT

FLOAT

FLY

FOLLOW

FOOD

FREE

FRIGHTENED.

$G$ WASHINGTUN CARVER

GOOBERS

GOOD-BYE

GRECERIES

GROUND

GROW

HIDE

HIPPOPOTAMUS

HIT

HOTTER

JIGGLED

JOHNNY APPLESEED

JOKES

KIND

KITTEN 
LETTER

LIPS

LITTER

LOAD

LOT

MATTER

MAY

MISS HUCKLEBERRY

MOLDS

MONSTERS

MRS. SNOW

UIL.

OLAF

OLDER

OPOSSUM

QR

QVEN

PARNELL

PARTS

PAUL

PAWS

PENCILS

PLAIN

PLAN

PLANET

PEWDER

PRETECTS

PREUD

RAISINS

ROLLED

ROWS

SCRUNCHED

SEEN

SHAPE

SOIL

SPOONS

SPRAYS.

SPREAD

STEERED

STUDY

TEACH

TICKLED

TIMOTHY TURTLE

TIRE

TUOLS

TOETH

TREAT

TREMENDOUS

TRIED

TURNED

UNIT

UNRELL

UNTIL

USE

VETE

WOEDCHUCK

WOULD

WR I TING

YET

THERE ARE 210 WORDS IN LEVEL 6 OF SERIES A WITH SERIES D , EACH AT LEVEL, 6 THERE IS A

IN COMPARING SERIES A

THERE ALSO IS A $36 \cdot 19 \%$ MATCH (EUTSIDE OF LEVEL 6) RANGING FROM, $11=106$ TO $4=61$. 
THE BACK-UP WORDS FOR LEVEL 7 ARE:

ABLE

$\triangle D O B E$

$\triangle F R A I D$

AFTERWARDS

$\triangle G A I N$

ALMOST

ALSO

ALWAYS

AMERICA

ANGRY

BANANAS

BEEN

BIRDS'

BLEW

BLEW

BUARDS

BEDY

BOTTLE

BOTTOM

BOUGHT

BREAKFAST

BREATHS

BUMPER

BUSY.

CABOESE

CARTENS

CASTLE

CHANGE

CHINA

CLAWS

CLEAR

CLEVER

CLIMB

CLOSE

COLLECTING

COLLECTION

CORRAL

COURSE

DEAR

DECIDED

DINESAURS

DRAGENS

EAGLE FEATHER

EARLY

EARN

EITHER

ELEVATORS

ELSA

ELVES

ENGLAND

ENOUGH

ENVELOPES

ESCALATOR

EVEN

EVERY

EXIT

EXPLAIN

FACES

FAMILIES

FAMILY

FFAR 
FEW

FIELD

FINALLY

FLOOR

FOOT

FOREST

FREEWAY

FREIGHT

FUR

GIANTS

GLEN DEE

GREW

GRIMM

GUARDS

GUESS

HANS

HE'S

HIGH

HOGAN

HELD

HOLIDAY

HERNS

HORRIBLE

HORSE

HUNDRED

HURT

JACKET

JANET

JAWS

JUNGLE

KATHY

KILLER

LATE

LEATHER

LESSON

LIBRARI AN

LIBRARY

LIZARD

LULLIPOP

LONGEST

LOVE

LUBBER

MABUNA'S

MAMMALS

MISERABLE

MODEL

MUST

MOUNTAINS

MUSEUM

MUSHRUSH

NARREW

NAVAJO

NEAR

NEW YORK CITY

NOTICE

ONLY

OXEN

PAIL

PAIRS

PALE

PANCAKE

PASSENGER

PIGGY-BACK

PLAZA

POEM 
PERR IDGE

PRETTY

PRINCE

PRINCESS

PROMISE

PROUDEST

QUICKLY

RADIO

RAPUNZEL

RARE

REMEMBER

REPORTER

RESTAURANT

RUAR

RESE RED

ROTOR

RUIN

SCOLD

SHINE

SHINY

SHOES

SHOOK

SHORT

SHQULD

SIGH

SIGHT

SIR CHARLES

SISTER

SKILLS

SKIRT

SMALLEST

SEN

SERT

SPAIN

STERN

STURE

STORIES

STRAIGHT

SUEWAYY

SURE

SURELY

TARO

TEASING BOY

TERRIBLE

THANKFUI

THEMSELVES

THEY IRE

TITO:S

TENGUE

TENIE

TRAFFIC

TRAILER

TRAVEL

UNABLE

UNHAPPINESS

UNHAPPY

UNITED STATES

UNPACK

USEFUL

WALLS

WHAT'S

WHISPERS

WHISTLE

WIZARD

WUKE 
THERE ARE 275 WURDS IN LEVEL 7 EF SERIES A. IN COMPARING SERIES A WITH SERIES D, EACH AT LEVEL, 7 THERE IS A $2.55 \%$ MATCH. THERE ALSE IS A $17.82 \%$ MATCH IGUTSIDE OF LEVEL 7 I RANGING FROM, $11-117$ TO $4=7$. 
THE BACK=UP HORDS FUR LEVEL 8 ARE:

$\triangle C I D$

AFRICA

AGAINST

ALARM CLOCK

ALLOSAURUS

APPEARED

AQUARIUM

$A R A B$

AREAS

ARIZUNA

ARTIST

BACKWARDS

BILLIONS

BOTHERED

BRAZIL

BREAK

BREATHE

BRGAD

BRONTOSAURUS

BUCKET

CALIFORNIA

CANADA

CANYEN

CAPITAL

CARRETS

CHIEF

CHOOSES

CLOSET

CLOTHES

CLOTHESLINE

COLLAR

COMFORTABLE

COMMAS

CUNCRETE

CONTRACTS

COVERED

CURRENTS

CUTTINGS

DAUGHTER

DIRECTIONS

DIVIDING

DONE

EARTH

EASY

EIGHT

ENGINE

ENGL ISH

ENJOYABLE

EXPANDS

FAMILIAR

FAUCETS

FAVORITE

FIERCE

FIGURE

FLOODING

FLORIDA

FLUFFY

FOREMAN

FERMULA

FESSIL

FOURTH 
FRUIT

GENTLENESS

GIRAFFE

GIVEN

GLOSSARY

GLOVE

GENE

GREVES

GUINEA PIGS

HANDKERCHIEF

HEARTS

HEAVY

HEY

HIBERNATE

HUGE

HURRAH

HURR I CANE

HUSBAND

I'VE

ILLS

IMAGINATION

IMPQRTANT

INDEX

IND I A

INFORMATIEN

INSTEAD

IREN

JUICE

LANGUAGES

LICHEN

LOSE

MACHINE

MAGICIAN

MAGNIFYING

MAYOR

MESA

MESSAGES

METEER

METEGRITE

MINUTE

MONTH

MOTIEN

MUSICI ANS

MUTTERING

NAVEL

NEIGHBOR

NINETY

O'CLOCK

GCEAN

OF TEN

ORANGES

ORANGUTAN

EXYGEN

PADRES

PARAGRAPHS

PEPPERPET

PERIODS

PIER

PLATES

POEM

PQETRY

POPSICLES

POURED

PRETZELS

PROBABLY

PRARIFMS 
PTERANADEN

PULLEYS

PUNCTUATION

PUPPIES

QUIET

RATHER

REALIZE

REPTILES

RESERVOIRS

RETURN

RGTTEN

ROUGH

RQUSE

SALT

SENTENCE

SEVEN

SHOULDER

SHOVEL

SIGNAL

SIMPLE

SIMPLY

SPANISH

SPECIAL

STEGOSAURUS

STOMACH

STRANGE

STREAM

SUBJECT

SYLLABLES

SYRUP

TELEPHONE

TEPEE

THIRTY

THOUGH

TOPIC

TOWARD

TUNNELS

TWICE

TYRANNESAURUS

USUALLY

VASES

VISITING

VISITORS

VEWEL

WALRUS

WARMER

WATERSHED

WE IVE

WEAR

WEIGHED

WHOLE

WOLF

WOMEN

THERE ARE 198 WORDS IN LEVEL \& OF SERIES A.

IN COMPARING SERIES A WITH SERIES D, EACH AT LEVEL, 8 THERE IS A $2.02 \%$ MATCH.

THERE ALSE IS A $5.05 \%$ MATCH (EUTSIDE OF LEVEL 8 ) RANGING FROM, $11=102$ TO $9=72$. 
THE BACK-UP WORDS FER LEVEL 9 ARE:

ACCENTED

$\triangle C H E D$

$\triangle D M I R E D$

ADVENTURE

AFFECTS

AFRICAN

ALASKA

ALTHEUGH

ANCHERAGE

ANTARCTICA

ARCTIC

ARR IVE

AUGUST

AVENUE

BEARD

BOENESBOROUGH

BOW

BOWSPRIT

BRITISH

BRETH

BREUGHT

BULLETS

BUSH

BUSHENGO

BUSINESS

CAPTURE

CARNATION

CAUGHT

CIBOLA

CLIMATES

CLOTH

COMBINED

COMMUNICATE

COMPLAINED

COMPUTERS

COMSAT

CONGRATULATIONS

CONGRESS

CONTENTED

CONTROL

CORAL REEFS

COURAGE

COURT

CUBA

CUSHION

CUSTOMER

DECEMBER

DECODE

DELICIOUS

DEPENDS

DESERTS

DETECTIVE

DIAGRAM

DIALS

DIARY

DIFFICULT

DISAPPEINT

DOMES

DOVEKIE

DOZEN 
$\mathrm{ECHO}$

EGRET

ELECTRIC

ENJOYMENT

ESCAPE

ESKIMO

ESPECIALLY

EVENING

EXAMPLES

FACTERIES

FIJI

FIJIANS

FOLK

FORTUNES

GLOBE

GLOSSARY

GRADUALLY

GRAVES

GUESTS

GUITAR

HABITAT

HAITI

HALF

HEAVEN

HEROES

HISPANIOLA

HUVER

HYDROFOIL

IMAGINE

IMPOSSIBLE

INTERESTED

INTERNATIONAL

ISLANDS

JUDGE

JUDGMENT

JUNE

JUNEAU

KINGDOM

MANE

MARE

MEANT:

MEXICAN

MICRESCOPE

MICREWAVES

MINERALS

MINI -

MISTAKE

MEMENT

MOVEMENTS

MUSTARD

NATIVES

NEVERTHELESS

NINA

NONE

OCEANOGRAPHERS

OPERATION

OPPOSITE

QRDINARY

QUTNUMBERED

PATIENT

PATTERN

PAYMENT

PENGUIN

PINTA

PIONEERS

PLAID 
FLEASANT

POEM

PQEMS

PQLISHED

POTATO

PREPARES

PRESIDENT

PRETENDED

PRISENER

PROPELLER

PUEBLOS

PUERTO RICO

PUURPQSES

RECEIVED

RELATED

RELAY

RESCUE

REWARD

RHYMING

ROBOTS

ROUTE

RULER

SAN JUAN

SAN SALVADOR

SANDWICHES

SANTA MARIA

SATELLITES

SCENE

SCHWA

SCIENCE

SCIENTISTS

SEARCH

SELECTIONS

SEPTEMBER

SERGEANT

SERI UUS

SEVERAL

SEW

SHAWNEE

SHELTER

SKIS

SOLDIERS

SPANIARDS

SQUARE

STATUES

STEAL

SUBMARINES

SUFF IX

SUGAR

SURRENDER

TELEVISIGN

TELSTAR

TITLE

TULL

TEPICS

TOUCH

TREASURE

TRIAL

TREPICAL

UNDERNEATH

URGED

VELCANO

VGYAGE

WANDERED

WHETHER 
WHOSE

WON

WRECKED

YUGOSLAVIA

THERE ARE 203 WUROS IN LEVEL 9 EF SERIES A. IN COMPARING SERIES A WITH SERIES D ,EACH AT LEVEL, 9 THERE IS A .49\% MATCH. THERE ALSE IS A $1.48 \% \mathrm{MATCH}$ (OUTSIDE OF LEVEL 9 ) RANGING FROM, $11=57$ TO $7-81$. 
THE BACKUP WORDS FOR LEVEL 1 ARE:

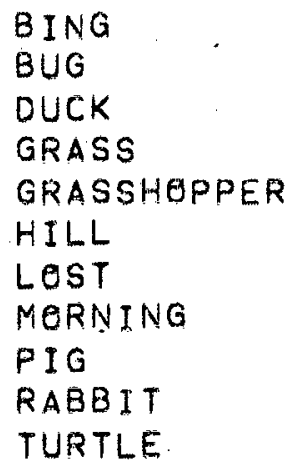

THERE ARE 33 WORDS IN LEVEL 1 OF SERIES A. IN COMPARING SERIES $A$ WITH SERIES E ,EACH AT LEVEL, 1 THERE IS A $3.03 \% \mathrm{MATCH}$. THERE ALSO IS A $60.61 \%$ MATCH (OUTSIDE OF LEVEL 1) RANGING FROM. $8=90$ TO $2-40$. 
THE BACK=UP WORDS FOR LEVEL 2 ARE:
$J A N$
JUMPED
KEVIN
KIDS
MELVIN
QVER
SAT
SHADEWS
SPLASH
VERY

THERE ARE 40 WOROS IN LEVEL 2 OF SERIES A. IN COMPARING SERIES A WITH SERIES E ,EACH AT LEVEL, 2 THERE IS A $25.00 \%$ MATCH. THERE ALSE IS A $42.50 \%$ MATCH IOUTSIDE OF LEVEL 2$)$ RANGING FROM, $6=50$ TO $3=37$. 
THE BACK-UP WORDS FOR LEVEL 4 ARE:

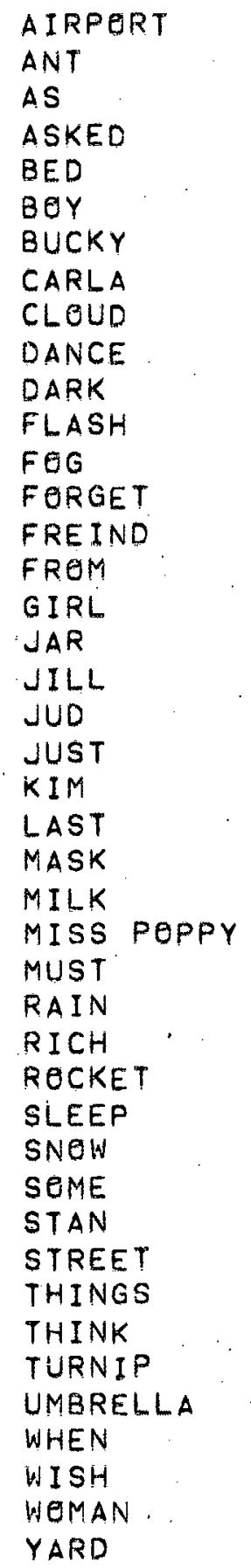

THERE ARE 130 WORDS IN LEVEL 4 OF SERIES A. IN COMPARING SERIES A WITH SERIES E , EACH AT LEVEL, 4 THERE IS A $23.85 \%$ MATCH. THERE ALSO IS A $30.00 \% \mathrm{MATCH}$ (OUTSIDE OF LEVEL 4 ) RANGING FROM, $8=192$ TO $5=168$. 
THE BACK=UP WQRDS FER LEVEL 5. ARE:

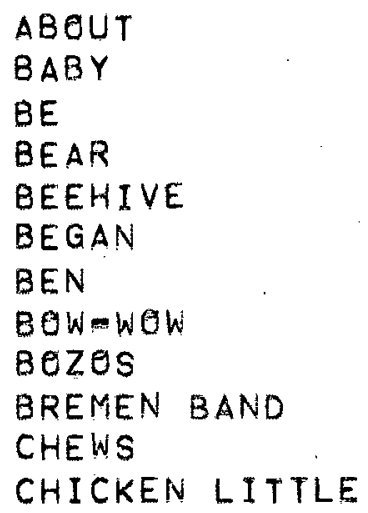

$B A B Y$

$B E$

BEAR

BEEHIVE

BEGAN

$B E N$

BOW-WOW

BEZOS

BREMEN BAND

CHEWS

CHICKEN LITTLE

CLAY

COCK-A-DEODLE-DOO

COLORS

CUT

DANNY DRAKE

DISH

DONKEY

DOER

DUCKY LUCKY

EGG

FELL

FIRST

FIVE

FLOUR

FOUR

FOXY LOXY

FROG

GRAY

GREEN

HAND

HAS

HATCH

HEE - HAW

HEN

HENNY PENNY

I'LL

I'M

ITS

JEFF

LAND

LEGS

LION

LIVES

LONG

MANY

$M E$ - OW

MILL

MISS LESTER'S

$M R$ - GRANT

MR. MCGREGOR'S

NAME

NEST

NET

NEXT

OLD

OPENED

QTHER

PAINTING

PAPEF 
PETER RABBIT

PETS

PICTURE

PLANT

PULP

RAP

RIGHT

RQBBERS

RECKS

ROOM

REOSTER

SINK

SKILLS

SPIDER

STEP

TADPQLES

TED

THANKS

TREE

TURKEY LURKEY

UNDER

WASPS

WEB

WERE

WRITE

YELLED

YELLOW

THERE ARE 163 WOROS IN LEVEL 5 OF SERIES A. IN COMPARING SERIES A WITH SERIES E EACH AT LEVEL, 5 THERE IS A $10.43 \%$ MATCH. THERE ALSO IS A $31.29 \%$ MATCH (OUTSIDE OF LEVEL. 5) RANGING FROM, 8-175 TO $4=1.07$. 
THE BACK=UP WORDS FOR LEVEL 6 ARE:

$A G E$

$A G O$

ANOTHER

ANSWERED

APPLE

BALLBON

BASKET

BEGIN

BERRY

BROWN

BULLDOZER

BUMP

BUY

CARR I AGE

CAVES

CHALK

CLAP

CLASS

CEULD

CRAWLING

CRAYENS

CRESSING

DECTER

DELL

DENNA

DR CARVER

EDWIN

EGBERT

ELEPHANTS

ENJOY

ERASER

FEATHER

FEET

FINE

FLOAT

FLY

FOLLEW

$F O 0 D$

G WAGHINGTUN CARVER

GLAD

GOQBERS

GQOD-BYE

GROCERIES

GRQUND

GROW

HAPPEN

HEAD

HIPPQPETAMUS

HOTTER

HUB

JIGGLED

JOHNNY APPLESEED

JOKES

KITTEN

LEAVES

LITTER

LQAD

LOT

LUCK

LUGS

MAILS 
MARKS

MAY

MISS HUCKLEBERRY

MELDS

MONEY

MENKEY

MONSTERS

MORE

MRS. SNOW

NIGHT

$N O O N$

UFF

OLAF

GPOSSUM

eR

PAGE

PARNELL

PARTS

PAWS

PENCILS

PIECE

PLAIN

PLAN

PLANET

PLOW

PEWDER

PROTECTS

QUESTIENS

RAISINS

READ

REUND

ROWS

SAY

SCRUNCHED

SHAPE

SHOW

SKILLS

SLEEPY

SUIL

SPBONS

SPFAYS

SPREAD

SQUIRREL

STEERED

STURY

TAIL

TAKES

TAKING

TALK

TICKLED

TIMUTHY TURTLE

TIRE

TREAT

TREMENDOUS

UNIT

UNROLL

VOTE

WAY

WELL

WHEEL

WHY

WOODCHUCK

YEAR 
WITH SERIES E , EACH AT LEVEL, 6 THERE IS A $11.43 \%$ MATCH. THERE ALSE IS A $27.62 \% \mathrm{MATCH}$ (EUTSIDE OF LEVEL 6) RANGING FROM, $8=192$ TO $3-23$.

THE BACK -UP WGRDS FOR LEVEL 7 ARE:
$\triangle \mathrm{CH}$
ADOBE
$A I R$
ALLOW
ANY
ARM
BAGPIPES
BANANAS
BEHIND
BIRDS 1
BEARDS
BOTTLE
BOTTEM
BREATHS
BRETHERS
BUMPER
BUSY
CABOESE
CALF
CARTENS
CASTLE
CHINA
CLAWS
CLEAR
CLIMB

COLLECTING

COLLECTION

CROWD

DIDN'T

DINOSAURS

DRAGENS

EAGLE FEATHER

EARLY

EARN

ELEVATORS

ELSA

ELVES

ENVELGPES

ESCALATOR

EVERY

EXIT

EXPLAIN

FACES

FAMILIES

FAMILY

FEAR

FINISH

FOOT

FREEWAY

FREIGHT

GIANTS

GIVE

GLEN DEE

HAIR

HUGAN

HOLIDAY

HORNS

HORRIBLE

HORSE

HUNDRED

INDEFD 
IT'S

JANET

JAPAN

JAWS

JUNGLE

KATHY

KILLER

KNIGHT

KNOWN

LATE

LIZARD

LOLLIPEP

LONGEST

LUBBER

MABUNA'S

MAMMALS

MARKET

MILLIONS

MISERABLE

MUUNTAINS

MUSEUM

MUSHRUSH

NAVAJO

NEAR

NEW YORK CITY

NOTICE

OFFICE

ONCE

OUR

OURSELVES

OVERJOYED

EXEN

PANCAKE

PIGQY-BACK

PLAZA

PEEMS

PERRIDGE

PROUDEST

RAPUNZEL

RARE

READY

REMEMBER

REPORTER

RESTAURANT

ROAR

ROSE RED

RETOF

RUIN

SCOLD

SHINE

SHINY

SHOES

S I GH

SIR CHARLES

SISTER

SKILLS

SKIRT

SPAIN

STERN

STQRE

STERYTELLER

SUBWAY

TEASING BEY

THOUGHT

THUUSANDS

TITO'S 
TONGUE

TENIE

TRAVEL

UNABLE

UNHAPPINESS

UNHAPPY

UNITED STATES

UNPACK

WALLS

WHICH

WHIRL

WHIRLY

WHISPERS

WHISTLE

WIND

WINDOWS

WIZARD

WOKE

WORRY

THERE ARE 275 WEROS IN LEVEL 7 OF SERIES A. IN COMPARING SERIES A WITH SERIES E EACH AT LEVEL, 7 THERE IS A $7.27 \%$ MATCH.

THERE ALSE. IS A $36.73 \% \mathrm{MATCH}$ (EUTSIDE OF LEVEL 7 ) RANGING FROM, $8=206$ TO $4=26$. 
THE BACK=UP WORDS FOR LEVEL 8 ARE:

$A C I D$

ACERNS

AFRICA

ALARM CLQCK

ALLOSAURUS

ARAB

AREAS

ARIZENA

ARTIST

BILLIONS

BGTHERED

BRAZIL

BREAD

BRENTESAURUS

CANADA

CANYEN

CAPITAL

CHOESES

COMFORTABLE

COMMAS

CENCRETE

CENTRACTS

CETTAGE

CURRENTS

CUTTINGS

DIVIDING

EARTH

ENJOYABLE

EXFANDS

FAUCETS

FAVERITE

FLEODING

FLORIDA

FEREMAN

FORMULA

FOSSIL

FRUIT

GARAGES

GENTLENESS

GIRAFFE

GLOSSARY

GLEVE

GROVES

GUINEA PIGS

HIBERNATE

HURRAH

HURR I CANE

IMAGINATION

INDEX

INDIA

INFERMATIEN

LANGUAGES

LICHEN

MACHINE

MAGICIAN

MAGNIFYING

MESA

MESSAGES

METEER

METERRITE

MONTH 
MOTION

MUSICIANS

MUTTERING

NAVEL

NINETY

NOTHING

ORANGES

BRANGUTAN

OXYGEN

PADRES

PARAGRAPHS

PEPPERPOT

PERIODS

PIER

PLATES

POPSICLES

PRETZELS

PROBLEMS

PTERANADEN

PUNCTUATIEN

PUPPIES

PUSHED

RATHER

REALIZE

RECUGNIZE

REDOISH

REPTILES

RESERVEIRS

RETURN

ROTTEN

RQUGH

RQUSE

SALT

SENTENCE

SIGNAL.

SIMPLE

SIMPLY

SPANISH

STEGOSAURUS

STOMACH

STREAM

SUBJECT

TEPEE

THIRTY

THUMB

TENS

TOPIC

TUNNELS

TYRANNOSAURUS

VASES

VISITING

VISITORS

VEWEL.

WALRUS

WARMER

WATERSHED

WE'VE

WEIGHED

WOLF

THERE ARE 199 WORDS IN LEVEL 8 EF SERIES A. IN COMPARING SERIES A WITH SERIES E EACH AT LEVEL, 8 THERE IS A $12.56 \%$ MATCH. THERE ALSE IS A $25.13 \%$ MATCH IUUTSIDE OF LEVEL \&) RANGING FROM, $7=240$ TO $4=28$. 
THE BACK - UP WORDS FOR LEVEL 9 ARE:

ACCENTED

ADMIRED

ADVENTURE

$\triangle F F E C T S$

AFRICAN

ANCHORAGE

ANTARCTICA

ARCTIC

ARRIVE

AUGUST

AVENUE

BEARD

BICYCLE

BOENESBOROUGH

BROTH

BULLETS

BUSHENGO

CAPTURE

CARNATION

CIBOLA

CLIMATES

COMBINED

COMMUNICATE

CONTENTED

CONTROL

CURAL REEFS

COURAGE

CUBA

CUSHION

CUSTEMER

DECEMBER

DECODE

DELICIOUS

DEPENDS

DESERTS

DETECTIVE

DIAGRAM

DIALS

DIARY

DIFFICULT

DISAPPEINT

DOMES

DOVEKIE

DOZEN

DRAWER

ECHO

EGRET

ENJOYMENT

ESCAPE

ESKIME

ESPECIALLY

EVENING

EXAMPLES

EXPERIMENT

FABULOUS

FACTORIES

FIJI

FIJIANS

FULK

FORTUNES

G) $9 B=$ 
GLOSSARY

GRADUALLY

GRAVES

HAITI

HALF

HEAVEN

HEROES

HISPANIOLA

HEVER

HYOROFOIL

INTERESTED

INTERNATIONAL

I SLANDS

JUDGE

JUDGMENT

JUNE

JUNEAU

KINGDOM

MANE

MARE

MEXICAN

MICROSCOPE

MICROWAVES

MINERALS

MINI

MISTAKE

MEVEMENTS

MUSTARD

NATIVES

NEVERTHELESS

NINA

OCEANGGRAPHERS

OPERATIEN

OPPOSITE

ORDINARY

OUTNUMBERED

PATTERN

PAYMENT

PENGUIN

PINTA

PIONEERS

PLAID

PREPARES

PROPELLER

PUEBLES

PUERTO RICE

PURPOSES

RECEI VED

RELATED

RELAY

REWARD

RHYMING

ROBOTS

RQUTE

SAN JUAN

SAN SALVADOR

SANDWICHES

SANTA MARIA

SATELLITES

SCENE

SCHWA

SCIENCE

SCIENTISTS

SEARCH

SELECTIONS

SFPTFMBER 
SERGEANT

SERIEUS

SHAWNEE

SHELTER

SKIS

SOLDIERS

SPANIARDS

SQUARE

STATUES

STEAL

SUBMARINES

SUFFIX

SUGAR

SURRENDER

TELEVISION

TELSTAR

TITLE

TOLL

TOPICS

TREASURE

TRIAL

UNDERNEATH

VOLCANO

VOYAGE

WHOA

WRECKED

YUGOSLAVIA

THERE ARE 203 WOROS IN LEVEL 9 OF SERIES A, IN COMPARING SERIES A WITH SERIES E EACH AT LEVEL, 9 THERE IS A $.00 \%$ MATCH. THERE ALSO IS A $18.23 \%$ MATCH (OUTSIDE OF LEVEL 9 ) RANGING FROM, $8=246$ TE $5=197$. 
THE BACK=UP WORDS FOR LEVEL 1 ARE:

BAD

BALL

BIKE

BIKES

BILL

BIRTHDAY

CAME

CAN

CAN!T

COWBEY

CEWBOYS

DET

FISH

GET

GOAT

GENE

HE

HELP

HIS

HEUSE

INTO

JUMP

JUMPED

KITES

MARY

MINE

PULLED

RIDE

RIDES

SOMEBODY

SOMETHING

STRING

TAKE

THANK

THESE

TRICKS

WANT

WANTED

WANTS

WAS

THERE ARE 139 WURDS IN LEVEL 1 OF SERIES B. IN COMPARING SERIES B WITH SERIES A EACH AT LEVEL, 1 THERE IS A $6.47 \%$ MATCH. THERE ALSE IS A $53.96 \%$ MATCH (OUTSIDE OF LEVEL 1 ) RANGING FROM, $7=238$ TO $2=52$. 
THE BACK -UP WORDS FOR LEVEL 2 ARE:

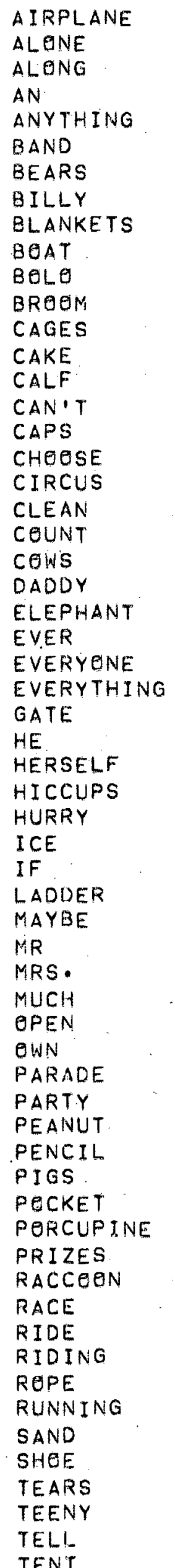


THAN

THING

VELVET

VI ULET

WHITE

WILL

WIN

WINK

WOODS

WORD

WORK

ZEBRA

THERE ARE 177 WORDS IN LEVEL 2 GF SERIES B. IN COMPARING SERIES B WITH SERIES A, EACH AT LEVEL, 2 THERE IS A $2.82 \%$ MATCH.

THERE. ALSO IS A $48.02 \%$ MATCH (OUTSIDE OF LEVEL 2 ) RANGING FROM, $8-188$ TO $1=14$. 
THE BACK-UP WOROS FOR LEVEL 3 ARE:

ACROSS

$A D D I N G$

$\triangle P A R T$

APRON

ARMS

ASK

ASKED

BABIES

BANKS

BEGINS

BESIDE

BIRDS

BOUNCED

BUNCH

BURN

CENTS

COULDNIT

CREAM

CRECODILE

CURIEUS

DEEP

DULLARS

DRANK

DREWN

ENDING

FIFTH

FLIES

FOLLOWED

FEUND

FULL.

GAMES

GELDEN

HAPPENED

HAVEN!T

HOPE

$I \cdot D$

LAMB

LANDING

LEARNED

LEFT

LEGS

LEMONADE

LISTEN

LOCK

MEN

MIGHT

MEUTH

NEED

NICKELS

OWL

PART

PIPES

PLACE

PELICE

PQP

PEST

PUPPY

PURPLE

PUSH

RAILRBAD

SAULAR 
SATURDAY

SCOOTER

SECOND

SEND

SENSE

SENT

SHALL

SILENT

SILLY

SMILEO

SONS

STANDING

STATION

STRETCH

SURPRISE

TABLE

TAKE

TAKEN

TALKING

THIRD

THROW

TIRED

TOWN

TRACTOR

TURN

TWIN

UNCLE

UNDER

VELVET'S

WASN IT

WATCHED

WE 'LL

WHEELS

WHILE

WHISPERED

WHISTLED

WINDOW

WIRES

THERE ARE 237 WORDS IN LEVEL 3 EF SERIES B. IN COMPARING SERIES B WITH SERIES A , EACH AT LEVEL, 3 THERE IS A .00\% MATCH. THERE ALSO IS A $49.79 \%$ MATCH IOUTSIDE OF LEVEL 3) RANGING FROM. $9=257$ TO $1-5$. 
THE BACK-UP WORDS FOR LEVEL 4 ARE:
APPLES
ATE
BAKERY
BAFN
BATTER
BELL
BETWEEN
BENE
BRAVE
BROKEN
BUILD
BUTTER
CARD
CARE
CAREFUL
CARPET
CHAIR

CHIMPANZEE

CHOP

CLOWN

$\mathrm{COACH}$

COEKIES

DIG

DINNER

DIRTY

DEESN'T

DOWNSTAIRS

DRAGON

EAR

EGGS

FAIRY

FEED

FEEL

FELL

FILL

FINGERS

FINISH

FLEW

FLOWER

FOOTMEN

FRESH

FRIEND

FRIGHTEN

GAY

GHOST

GINGERBREAD

GIRAFFE

GRANDFATHER

HANDLE

HASN'T

HELLO

HENS

HIND

HOLE

ISLAND

JUGGLE

KNOCKED

LAID

LARGER

LAST 
LEAVE

LIVE

LOAF

LOCKET

LOUD

LUCKY

MAGIC

MARBLE

MEAN

MERFY

MIND

MISS

MENDAY

MOVE

MUSIC

NEAT

NECK

NESTS

NOISE

NOODLE

NOSE

PAIR

PAN

PASS

PICNIC

PIECES

PINS

PITTER

POLITELY

PUFF

QUEER

QUESTIUN

QUICK

GUIET

RAGGED

REPQRT

RETURNED

RING

RQBE

RODE

ROLLED

RUFFLE.

SCISSORS

SEEMED

SELL

SHEEP

SHOUT

SHUT

SIDE

SIZE

SLEPT

SLEWLY

SMALL

SMELLED

SEMERSALLT

SPRING

START

STEP

SUCH

TEN

TEST

THURSDAY

TIDY

TIED

TEUCHEO

TOWFR 
TRUNKS

TUESDAY

TWELVE

TWICE

WAGGING

WALL

WASHED

WAVE

WEDNESDAY

WEEK

WIDE

WIFE

WINGS

WINTER

WISE

WRONG

YEU'LL

THERE ARE 207 WURDS IN LEVEL 4 EF SERIES B. IN COMPARING SERIES B WITH SERIES A , EACH AT LEVEL, 4 THERE IS A $3.38 \%$ MATCH. THERE ALSO IS A $24 \cdot 15 \%$ MATCH IOUTSIDE OF LEVEL 4 I RANGING FROM, $9=190$ TH $1=33$. 
THE BACKMUP WOROS FOR LEVEL 5 ARE:

ACTING

AFTERNEON

$\triangle G R E E$

$A H$

ALARM

AMONG

ANXIEUSLY

ARRIVED

ASHAMED

ATTENTION

BARBECUES

BARKING

BASKET

BEAMED

BEHAVED

BENT

BEYOND

BICYCLE

BLOCKS

BLOSSOMS

BOOKS

BORN

BORROW

BOTHERED

BEWED

BRANCH

BUGS

BUILT

BUNDLE

BURIED

BUS

BUTT

CASH

CERTAINLY

CHANCE

CHANGE

CHASE

CHEERFULLY

CHERRIES

CHICKEN

CHOSE

CLANG

CLIPPERS

CLOCK

CLOSE

COMPANY

COMPLETELY

CONTINUED

COOKED

COPPER

CORN

CERNER

COST

COUSIN

COWSLIPS

CRACK

CREWDED

CUP

DECIDE

DELIGHT

OFSK 
DING

DIRECTION

DISAPPEARED

DISCOVERED

DENG

DR

DREAM

DRIVING

DROP

DRY

DURING

EAGERLY

EMPTY

ENGINEER

EVENTS

EXPECTED

EXPLAINED

EXTRA

FAIR

FALL

FARTHER

FELT

FENCE

FEW

FINNALLY

FIX

FLAMES

FLASHED

FORKS

FORTH

$F O X$

FRAME

FREE

FURN I TURE

GATHERED

GIFT

GODMETHER

GRAIN

GRAPE

GRASS

GROCERY

GRQUPS

HABIT

HANG

HAPPINESS

HARBQRS

HAY

HEART

HEELS

HELD

HELICOPTER

HOUND

HOUR

INN

INSISTED

INSTEAD

INTERESTING

JELLY

LADDER

LADY

LAKES

LAMP

LEADING

LEANING

LEAPED

LEAST 
LEATHER

LEND

LENGTH

LESS

LIFE.

LIFTED

LIGHTNING

LOW

MAIN

MAJESTY

MARK

MARSH

MASTER

MATTER

MEAL

MEAT

MEET

MINUTES

MOUNTAIN

MUDDY

MULE

NAILS

NEEDLES

NEI GHBOR

NICEST

NODDED

NOTICED

ORDERED

OUGHT

PACK

PALACE

PATCH

PATH

PAY

PEACH

PEDDLERS

PEN

PERHAPS

PERSEN.

PICK

PIES

PILE

PINCH

PLENTY

POINT

PRESENT

PRINTED

QUITE

RANG

RANGER

REACH

REALIZES

REFUSES

REPLIED

RIBBONS

RICH

RID

RIPE

RIVERS

REAST

ROCKY

Re日F

- RUSHED

SAD

SAFELY

SALFS 
SALT

SAVING

SCOLDED

SCRATCH

SEAT

SHADOW

SHAKES

SHELF

SHINING

SHIPS

SHORES

SILVER

SINCE

SIR

SMART

SMOKE

SEFTLY

SERRY

SPEAKING

SPECIAL

SPEND

SPICES

SPUKE

SPREADING

SQUARE

SQUAWKS

STALL

STATE

STEEP

STICKS

STOCKINGS

STONE

STOOL

STORMY

STOVE

STRAIGHT

STRIKE

SUDDENLY

SUPPER

SUPPESE

SWEET

TEASED

TEETH

THEY:LL

THICK

THREW

TIGHT

TOES

TRACKS

TRADE

TRAIN

TRAVEL

TRIP

TRUDGED

- TRUST

UMBRELLAS

UNLESS

USUAL

VACATIEN

VISIT

VEICE

WANDER

WARM

WARN

WE 'RE

WEATHER 
WIND

WONDERFUL

WOOL

WORE

THERE ARE 369 WORDS IN LEVEL. 5. OF SERIES B. IN COMPARING SERIES B WITH SERIES A EACH AT LEVEL, 5 THERE IS A $1.90 \%$ MATCH.

THERE ALSO IS A $20.05 \%$ MATCH IOUTSIDE OF LEVEL 5) RANGING FROM, $9=242$ TO $1=33$. 
THE BACK-UP WORDS FOR LEVEL 6 ARE:

ACCOUNT

ACCUSE

$\triangle C Q R N$

ACTER

ADMITTED

$A I M$

$\triangle M A Z E D$

$\triangle M O U N T$

AMUSEMENTS

ANNGUNCER

ANNEYS

ARMY

ATTACHED

ATTRACTED

AUDIENCE

AUTUMN

AWAKE

BALCONY

BANGED

BARS

BATHFOOM"

BATTERY

BEAST

BEAT

BEGGED

BEGUN

BEND

BERRIES

BISCUIT

BITE

BLAME

BOILER

BOOMER

BOWL

BREATH

BRUSHING

BUCKSKIN

BUDS

BULLDOG

BUMBLE

BUMPED

BUSHES

BUTCHER

BUTTENS

$C A B$

CABIN

CABOOSE

CALM

CAMP

CANARIES

CANNEN

CAPE

CASE

CAST

CAUSING

CHAIN

CHALET

CHARGED

CHARMING

CHATTER 
CHEST

CHILD

CHUCKLED

CINDER

CIRCLE

CLAIM

CLAPPERS

CQAL

CELT

COMBING

CONCERNED

CONCERT

CONE

CONFESS

CONFUSED

CONTENTED

CONTENTMENT

CENTEST

CONTESTANTS

CONTRARY

$\operatorname{COOL}$

COTTAGE

CRAZY

CREATURE

CREPT

CREW

CURTAIN

CUSTOMERS

DAISY

DARING

DARKNESS

DASHING

DECALRED

DESIRE

DIFFERENCE

DIPPED

DIRECT

DISTANCE

DOUBLED

DEUBTED

DEZED

DRAW

DREW

DRIPPING

DREVE

DUCHESS

DUKE.

DUMB

ELEVEN

ELF

ENEMIES

ENGINE

ENTER

ESCAPED

EXACTLY

EXPRESS

EYEBROWS

FACTS

FAILED

FAMILIAR

FAT

FAULT

FAVORITE

FED

FELLOW

FIFRCELY 
FIFTY

FIGURE

FITS

FLEA

FLOPPED

FUELISH

FERCE

FORMS

FERT

FERTY

FORWARD

FRIDAY

FRINGE

GALLOPING

GASPED

GAZE

GEESE

GENTLEMEN

GL I TTER ING

GOBBLE

GRANT

GRINNING

GROWLING

GUNS

HARM

HATED

HEROS

HERR

HITCHED

HONEY

HOOFS

HORNETS

HUNTED

INCH

INQUIRED

INSECTS

INVISIBLE

INVITE

ITEMS

JOY

JUMBE

KEY

KICKED

KILL.

KNEES

KNITTING

LANGUAGE

LATCHED

LAZY

LED

LIST

LUCAL

LOGGERS

LOP

LOPES

LQRD

LOVED

LUMBER

LUNCH

MA'AM

MANAGED

MARRY

MIRRERS

MISCHIEF

MUNGRELS

MUSTACHE 
MYSTERY

NARRATOR

NATIENAL

NECESSARY

NEI GHBORHOOD

NEITHER

NET

NIBSLE

NICK

NIX

NUR

NOTES

NOTION

OAK

OFFER

ORIOLES

OUCH

PAID

PARENTHESES

PARENTS

PARRET

PAW

PEEKING

PERCHED

PERFECTLY

PERFORMANCE

PIANE

PITCHER

PLATFERM

PLEASURE

PLUCK

POKED

POLE

POUNDS

PRACT ICED

PRAISE

PREFIX

PREPARING

PRETEND

PROPERTY

PRETECTED

PREVED

PUNISH

PURE

PUZZLE

QUARRELING

QUARTERS

QUEEN

RASPBERRY

RATHER

RECOGNIZED

RECGRO

REMAINED

REMARKABLE

REPEATED

RIDOLES

ROSY

RUBBING

RULES

SAFETY

SAINT

SANG

SAWMILL

SCAMPERED

SCARED 
SCENLED

SCREAMED

SEARCHED

SEASONS

SEED

SELFISH

SETTLED

SHARPLY

SHEET

SHIRT

SHECKED

SHeT

SHOULDERS

SHOVELED

SHOWER

SHRUNK

SING

SINGLE

SIXTEEN

SKINKS

SKUNK

SKY

SLEEVES

SLICK

SNAILS

SNAPPED

SNIFFED

SOBS

SELD

SOLDIERS

SOLVE

SONG

SPEED

SPIED

SPILL

SPOIL

SPOT

SQUEALING

SQUIRREL

STAGE

STAKE

STARS

STATEMENT

STEALING

STEAM

STEER

STEM

STECKADES

STULEN

STREAK

STRUGGLING

STUCK

STUMP

STUPID

STUROY

SUCCESS

SUGGESTED

SULKED

SUPPLIES

SUSPECTED

SWEEP

SWEPT

SWING ING

SWITCHES

TALES 
TICKETS

TILL

TIMBER

TINKLED

TOOTHPASTE

TRAIT

TRAP

TREATED

TREMBLED

TREUSERS

TUBE

TUNE.

TURN IP-SEED

TWENTY

TWIG

UGLY

UPWARD

WAFERS

WAR

WASTE

WEAK

WEDDING

WEEP

WHEELS

WHIMPERED

WHIP

WHISKERS

WHITENESS

WICKED

WIGGLED

WIPE

WOEDSHED

WORTH

WRAPPED

WRINKLED

THERE ARE 424 WURDS IN LEVEL 6 OF SERIES B. IN COMPARING SERIES 6 WITH SERIES A, EACH AT LEVEL, 6 THERE IS A $1.89 \%$ MATCH. THERE ALSO IS A $8.25 \% \mathrm{MATCH}$ (UUTSIDE OF LEVEL 6) RANGING FROM, $g=264$ TO $1=18$. 
THE BACK $=U P$ WORDS FOR LEVEL 1 ARE:

A

AFTER

AND

ANOTHER

ASK

ASKED

BALL

BIKE

BIKES

BILL

BOY

CAN

CAN!T

COLD

COHBOY

COWBOYS

DID

DET

EAT

FIRE

GET

HE

I

IS

JEFF

KITES

LIKE

LOEK

LOOKED

MAKE

MARY

MIKE

MINE

NOT

NOW

$\mathrm{ON}$

PLAY

PLAYS

POLICEMEN

RAN

RED

RIDE

RIDES

SAID

TAKE

THE

TO

UP

US

WANT

WANTED

WANTS

WAY

WHAT

WHO

WITH

YELLOW

YES 
WITH SERIES $C$, EACH AT LEVEL, 1 THERE IS $A$

THERE ALSO IS A $53.62 \%$ MATCH IOUTSIDE OF LEVEL 1 ) RANGING FROM, $7=122$ TO $4=118$.

THE BACKMUP WERDS FER LEVEL 2 ARE:

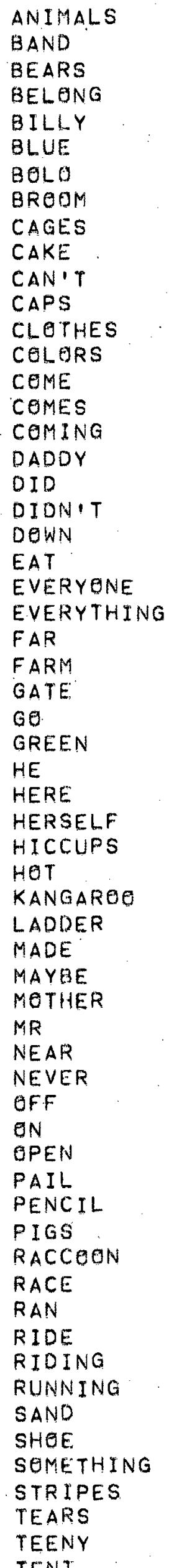


TREE

UP

VELVET

VIOLET

WILL.

WIN

WINK

YOU

ZEBRA

THERE ARE 177 WERDS IN LEVEL 2 OF SERIES B. IN COMPARING SERIES B WITH SERIES C ,EACH AT LEVEL, 2 THERE IS A .00\% MATCH. THERE ALSO IS A $53.67 \%$ MATCH (UUTSIDE OF LEVEL 2) RANGING FROM, $9=108$ TO $4-146$. 
THE BACK-UP WORDS FOR LEVEL 3 ARE:

\author{
ADDING \\ APART \\ APRON \\ $A S K$ \\ $\triangle S K E D$ \\ BANKS \\ BEGINS \\ BESIDE \\ $B I G$ \\ BIGGER \\ BLUE \\ BOY \\ BOY'S \\ BRING \\ BUNCH \\ BURN \\ BUY \\ CROCODILE \\ CURIEUS \\ DEEP \\ DIDNTT \\ DO
}

DRANK

DROWN

ENDING

FACES

FATHER

FIELD

$F \Theta R$

GAMES

GLASS

GELDEN

GREEN

HAND

HAPPENED

HAT

HAVEN 'T

HIDE

HIT

HOPE

ISN'T

IT

$J A R$

JUDGE

$\angle A M B$

LANDING

LEARNED

LIKE.

LIKES

LINES

LOOK

LEUKS

MAIL

MAKE

MAKES

MATCHES

MIGHT

NICKELS

NOW

NUMBERS OFF 


PART
PIPES
PLOW
POP
PURPLE
PUSH
RAILROAD
RED
ROUND
SAILOR
SAME
SCEOTER
SEE
SEND
SENSE
SENT
SILENT
SNOW
SONS
STANOING
STATION
STEP
STRETCH
SUITS
SUMMER
SUN
SUNSET
TAKE
TAKEN
TRACTOR
TRAFFIC
TREE
TRIEO
TURN
VELVET'S
VEWEL
WASNIT
WATCHED
WELL
WHAT
WHEELS
WHISTLED
WINDOW
WIRES
YEAR
YELLOW
YES

THERE ARE $237^{\circ}$ WORDS IN LEVEL 3 GF SERIES $B$. IN COMPARING SERIES $B$ - WITH SERIES C , EACH AT LEVEL, 3 THERE IS A .00\% MATCH. THERE ALSO IS A $51.90 \%$ MATCH (OUTSIDE OF LEVEL 3) RANGING.FROM. $9=266$ TU $4=111$. 
THE BACK-UP WORDS FOR LEVEL 4 ARE:

ANSWERED

APPLES

ATE

BAKERY

BATTER

BEARD

BELL

BUILD

BUTTER

CARD

CARPET

CHIMPANZEE

CHOP

CLEVER

CLOWN

CEACH

COVERED

$D I G$

DEESN T

DEWNSTAIRS

DRAGEN

EAR

FEED

FEEL

FINE

FINGERS

FINISH

FRIGHTEN

GARDEN.

GAY

GHEST

GINGERBREAD

GIRAFFE

GRANDFATHER

GROUND

HAIR

HASN'T

HENS

HILL

HIND

HELE

HURDY = GURDY

ISLAND

JUKES

JUGGLE

KNOCKED

LARGER

LIZARD

LOAF

LECKET

LUCKY

MARBLE

MAYOR

MERRY

MENDAY

NEAT

NECK

NESTS

NUODLE

NOSE

PAIR 


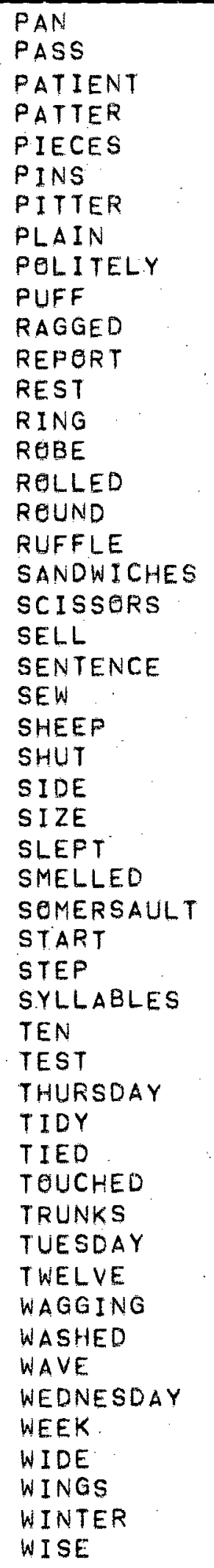

THERE ARE 207 WORDS IN LEVEL 4 OF SERIES B. IN COMPARING SERIES B WITH SERIES C , EACH AT LEVEL, 4 THERE IS A $3.38 \%$ MATCH. THERE ALSE IS A $39.13 \% \mathrm{MATCH}$ (OUTSIDE OF LEVEL 4 ) RANGING FROM. $9=175$ TO $5=185$. 
THE BACK UP WORDS FGR LEVEL 5 ARE:

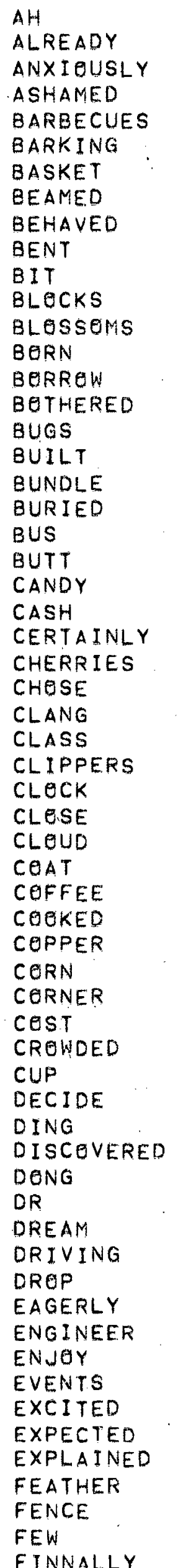


$F \cup G$

FURKS

FORTH

FRAME

FREE

FURN I TURE

GIFT

GODMOTHER

GRAIN

GRAPE

GROCERY

GROUPS

HAB IT

HANG

HARBERS

HAY

HORNS

HOUNO

HOUR

INN

INSISTED

INSTEAD

INTERESTING

JELLY

$J \theta B$

KING

LADDER

LADY

LAKES

LAMP

LAYY

LEADING

LEANING

LEAPED

LEAST

LEATHER

LEND

LENGTH

LESS

LIFE

LOSE

LUST

LOT

MAIN

MAJESTY

MARSH

MASTER

MEAL

MEAT

MEET

MINUTES

MONTH

MUDDY

MULE

NAILS

NEEDLES

NEIGHBER

NICEST

NODDED

NETICED

OIL

QRDERED

QUGHT

PATCH

PATH

PAY 
PEACH

PEDDLERS

PEN

PERSEN

PICK

PIES

PILE

PINCH

PLAN

POINT

POUF

POWER

PRESENT

PRINCE

PRINTED

RAISING

RANCHERS

RANG

RANGER

REACH

REALIIZES

RECEIVED

REFUSES

RIBBONS

RICH

RID

RIPE

RIVERS

ROCKY

ROOSTER

$S A D$

SAFELY

SALES

SALT

SAVING

SCOLDED

SCRATCH

SHAKES

SHEOK

SHEP

SHERES

SICK

SILVER

SINCE

SIR

SEFTLY

SPEAKING

SPECIAL

SPEND

SPICES

SPEKE

SPREADING

STATE

STEEP

STICKS

STECKINGS

STEOL

STERMY

STOVE

STRAIGHT

SWEET

TALL

TAP

TASTED

TEASED 
TQES

TRUDGED

TRUST

UMBRELLAS

UNLESS

VEGETABLES

WHETHER

WICK

WILDLY

WOKE

WOLF

WOEL

WOULDN'T

YET

THERE ARE 370 WORDS IN LEVEL 5 OF SERIES B. IN COMPARING SERIES B WITH SERIES C , EACH AT LEVEL, 5 THERE IS A $3.78 \%$ MATCH. THERE ALSO IS A $32.43 \%$ MATCH IOUTSIDE OF LEVEL 5) RANGING FROM, $9=222$ TO $4-126$. 
THE BACK-UP WORDS FOR LEVEL 6 ARE:

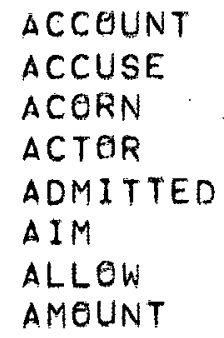


CONCERNED

CONCERT

CONE

CONFESS

CONFUSED

CONTENTED

CENTENTMENT

CONTEST

CONTESTANTS

CONTRARY

COOL

COTTAGE

CRAZY

CREATURE

CREPT

CREW

CUSTOMERS

DAISY

DARING

DARKNESS

OASHING

DECALRED

DESIRE

DIED

DIFFERENCE

DIPPED

DIRECT

DEUBLED

DUUBTED

DEZED

DRAW

DREW

DRIPPING

DROVE

DUCHESS

DUKE.

DUMB

ENGINE

ENTER

ESCAPED

EXPRESS

EYEBREWS

FACTS

FAILED

FAT

FAULT

FAVORITE

FED

FELLEW

FIGURE

FITS

FLEA

FLQPPED

FGRMS

FERT

FORTY

FERWARD

FRE I GHT

FRIDAY

FRINGE

GALLOPING

GAZE

GEESE

GENTLEMEN

GLITTERING GOBBLE 
GRANT

GR INN ING

GROWLING

GUNS

HARM

HERDS

HERF

HITCHED

HOOFS

HORNETS

HUMAN

HUMMING

HUNTED

HYACINTH

IMPATIENTLY

INCH

INQUIRED

INSECTS

ITEMS

JACKET

JAIL

$J A M$

JUY

JUMBO

JUNGLE

KICKED

KILL

KNEES

KNITTING

LATCHED

LED

LESSON

LETTUCE

LECAL

LGGGERS

LOP

LOPES

LURD

LUMBER

LUNCH

MAIAM

MARKET

MEADOW

MELT

MESSENGER

MET

MIKE

MIRRERS

MISCHIEF

MONGRELS

NARRATER

NATIUNAL

NECESSARY

NEITHER

NET

$N Q R$

NOTES

NOTIEN

BAK

OFFER

ORIOLES

EUCH

PARENTHESES

PARENTS

PARROT

PASSFNGFR 
PASTURE

PAUSED

PAW

PERCHED

PERFECTLY

PERFERMANCE

PITCHER

PLANT

PLATFGRM

PLEASURE

PLUCK

POKED

PULE

POUNDS

PRACTICED

PRAISE

PREFIX

PREPARING

PRETEND

PROPERTY

PRETECTED

PROVED

PUNISH

PURE

PUZZLE

QUARRELING

QUARTERS

QUEEN

RAGE

RAPIDLY

RASPBERRY

RATHER

RECOGNIZED

RECORD

REMAINED

REMARKABLE

REPEATED

RIDDLES

REAR

ROBBED

ROOT

RESY

ROWS

RUBBING

RULES

SAFETY

SAINT

SAWMILL

SCAMPERED

SCOWLED

SCREAMED

SEARCHED

SEASONS

SEED

SETTLED

SHARPLY

SHEFT

SHEI.L

SHIRT

SHOCKED

SHOULDERS

SHOVELED

SHOWER

SHRUNK

SILL

SING 
SINGLE

SIXTEEN

SKINKS

SLICK

SNAILS

SNAPPED

SOBS

SOIL

SOLO

SOLDIERS

SOLVE

SONG

SPEED

SQUEALING

STARS

STATEMENT

STREAK

STRUGGLING

STUCK

STUMP

STUFID

STURDY

SUCCESS

SUGGESTED

SULKED

SUPPLIES

SUSPECTED

SWEEP

SWINGING

SWITCHES

TALES

TILL

TIMBER

TINKLED

TOETHPASTE

TRAIT

TRAP

TREATED

TREMBLED

TRQUSERS

TUBE

TUNE

TURNIP =SEED

TWIG

UPWARD

WAFERS

WAR

WASTE

WEDDING

WEEP

WHEELS

WHIMPERED

WHIP

WHISKERS

WHITENESS

WICKED

WI GGLED

WIPE

WOODSHED

WRAPPED

THERE ARE 424 WORDS IN LEVEL 6 OF SERIES B: IN COMPARING SERIES B WITH SERIES C , EACH AT LEVEL, 6 THERE IS A 2.36\% MATCH.

THERE ALSO IS A $17.22 \%$ MATCH (OUTSIDE OF LEVEL 6) RANGING FROM, 
THE BACK - UP WORDS FOR LEVEL 1 ARE:

BIKE
BIKES
BILL
COLO
COWBOY
COWBOYS
CRIED
DOT
FAST
GAVE
GOAT
GONE
HIGH
JEFF
KITES
MIKE
MINE
POLICEMEN
SOMEBODY
STRING
TRICKS

THERE ARE 139 WORDS IN LEVEL 1. OF SERIES B. IN COMPARING SERIES B WITH SERIES D ,EACH AT LEVEL, 1 THERE IS A . 00\% MATCH. THERE ALSE IS A $80.58 \%$ MATCH (OUTSIDE OF LEVEL 1 ) RANGING FROM. 11* 73 TO 3- 32. 
THE BACK=UP WORDS FOR LEVEL 2 ARE:

AIRPLANE

ALENE

ALENG

BAND

BECAUSE

BILLY

BLANKETS

BLUE

BOAT

BELO

BROOM

CAPS

CHEOSE

CIRCUS

CLEAN

CLOTHES

COWS

ELEPHANT

FIVE

FOOD

HICCUPS

HORSE

HOT

HURRY

LADDER

MUST

NEAR

QPEN

ER

ORANGE

ESTRICH

OTHER

EUR

PAIL

PAINT

PAL

PARADE

PEANUT

PENCIL

PIGS

PERCUPINE

PRIZES

RACCOEN

RACE

RQPE

SAND

SEEN

SEVEN

SHOE

SHOULD

TEENY

TENT

THAN

TINY

TEY

TRUCK

TRUE

USE

VELVET

VIOLET WIN 
WINK

WOULD

ZEBRA

THERE ARE 177 WORDS IN LEVEL 2 OF SERIES B. IN COMPARING SERIES B WITH SERIES D ,EACH AT LEVEL, 2 THERE IS A .00\% MATCH. THERE ALSO IS A $57.06 \%$ MATCH IOUTSIDE OF LEVEL 2) RANGING FROM, $11=110$ TO. $3=37$. 
THE BACK -UP WORDS FOR LEVEL 3 ARE:

ADDING
AFRAID
AFTER
AIR
ALMOST
ALWAYS
ANGRY
APART
APRON
ARMS

BABIES

BALLEON

BANKS

BEEN

BEGINS

BOARD

BOTH

BOTTEM

BOUNCED

$B O Y$

BOY:S

BRIDGE

BRING

BUNCH

BURN

BUY

CHIEF

CHILDREN

CITY

CREAM

CRECEDILE

CURIEUS

CUT

DELLARS

DRANK

DRESS

DRINK

DREWN

ENDING

ENOUGH

EVEN

FACES

FIELD

FIFTH

FLIES

FEUNO

FOURTH

FULL

GLASS

GELDEN

HAVEN'T

HIDE

HIT

HEPE

HURT

I'D

JUDGE

KIND

LAND ING

LOCK MAIL 
MAKE

MAKES

MATCHES

MIGHT

MEUTH

NAME

NEED

NENE

ONLY

OVEN

PART

PIPES

PLACE

PQP

RAILROAD

REMEMBER

SAILER

SAME

SCENE

SCHEOL

SCEOTER

SEND

SENSE

SENT

SHALL

SHORT

SNOW

SENS

STANDING

STIRE

STORIES

STERY

STRANGE

STRETCH

SUGAR

SUNSET

SURE

SURPRISE

TABLE

TELEPHENE

THIRD

THROW

TOOK

TOP

TOWN

TRACTER

TRAFFIC

TREE

TWIN

VELVETIS

VEWEL

WATCHED

WE! LL

WEAR

WELL

WHILE

WHISTLED

WINDOW

WIRES

THERE ARE 237 WORDS IN LEVEL 3 OF SERIES B. WITH SERIES ' $D$, EACH AT LEVEL, 3 THERE IS A -42\% MATCH. IN COMPARING SERIES $B$ THERE ALSO IS A $40.51 \%$ MATCH (OUTSIDE QF LEVEL 3 ) RANGING FROM, $11=105$ TO $4=32$. 
THE BACK-UP WORDS FER LEVEL 4 ARE:
$A L S O$
APPLES
ATE
BAKERY
BATTER
BEARD
BELL
BLOW
BONE
BRAVE

BREAKFAST

BROKEN

BUILD

BUTTER

CASTLE

CHIMPANZEE

CHOP

CLEVER

CLIMB

CLOTH

CLOWN

COACH

COEKIES

COVERED

DANCE

DEAR

DOWNGTAIRS

DRAGON

EAR

EGGS

FILL

FINGERS

FINISH

FOOTMEN

FRESH.

FRIEND

FRIGHTEN

GAY

GHEST

GINGERBREAD

GIRAFFE

HANDLE

HASN T T

HENS

HIND

HUNGRY

HURDY = GURDY

I'VE

ISLAND

JOKES

JUGGLE

KNOCKED

LAID

LARGEF

LIZARD

LUAF

LUCKET

LUUD

LUCKY

MARBLE 
MEAN

MIND

MENDAY

MEST

NARREW

NEAT

NECK

NESTS

NOISE

NOEDLE

EFTEN

$O H$

PAIR

PAN

PASS

PATIENT

PATTER

PET

PICNIC

PINS

PITTER

PLAIN

POLITELY

PRETTY

PRINCESS

PROMISE

PUFF

QUEER

QUESTION

QUICK

QUIET

REPQRT

REST

RING

RUBE

RODE

RELLED

ROUND

RUFFLE

SANDWICHES

SCISSORS

SELL

SENTENCE

SEW

SHAPE

SIT

SIZE

SLEPT

SLEWLY

SMALL

SMELLED

SOMERSAULT

SYLLABLES

TEACHER

THURSDAY

TIDY

TIED

TOUCHED

TOWER

TRETTED

TRUNKS

TUESDAY

TWELVE

TWICE

UNHAPPY

WASHED 
WAVE

WEDNESDAY

WEEK

WHELE

WIDE

WIFE

WINGS

WINTER

THERE ARE 207 WERDS IN LEVEL 4 OF SERIES B. IN COMPARING SERIES B - WITH SERIES D ,EACH AT LEVEL, 4 THERE IS A .00\% MATCH.

THERE ALSE IS A $27.54 \% \mathrm{MATCH}$ (OUTSIDE OF LEVEL 4 ) RANGING FROM, $11=109$ TO $7=96$. 
THE BACK UP WORDS FOR LEVEL 5 ARE:

ABLE

$A C T I N G$

AGAINST

$\triangle G E$

AGREE

AH

ALARM

AMUNG

ANXIEUSLY

APPEARED

$\triangle R R I V E D$

ASHAMED

AT TENTION

BARBECUES

BASKET

BEAMED

BEHAVED

BENT

BEYEND

BICYCLE

BIT

BLECKS

BLOSSOMS

BRIGHT

BREUGHT

BUGS

BUILT

BUNDLE

BURIED

BUS

BUSY

BUTT

CANDY

CASH

CERTAINLY

CHANCE

CHANGE

CHASE

CHEERFULLY

CHERRIES

CHICKEN

CHESE

CLANG

CLASS

CLEAR

CLIPPERS

CLUCK

CLOSE

CLOUD

COAT

COFFEE

COMFORTABLE

COMPANY

COMPLETELY

CONT INUED

COUKEO

COPPER

CORN

CERNER

COST

COURSE 
CEUSIN

COWSLIPS

CRACK

CUP

DANGER

DAUGHTER

DECIDE

DELIGHT

DESK

DING

DIRECTION

DISAPPEARED

DISCOVERED

DECTOR

DONG

DR

DREAM

DURING

EAGERLY

EARLY

EARTH

EASY

ENG INEER

ENJEY

ESPECIALLY

EVENTS

EXAMPLES

EXCEPT

EXCITED

EXCLAIMED

EXPECTED

EXPLAINED

EXTRA

FAIR

FALL

FARTHER

FEATHER

FELT

FENCE

FEW

FINNALLY

F IX

FLAMES

FLASHED

FOG

FORKS

FERTH

FRUIT

FURNI TURE

GIFT

GEDMOTHER

GRAIN

GRAPE

GRASS

GROCERY

GRUUPS

HAB I T

HALF

HANG

HAPPINESS

HARBORS

HAY

HEART

HEAVY

HEELS

$H F \mid D$ 
HEL I CQPTER

HERNS

HOUND

HUUR

HUNDRED

HUNG

IMAG INE

IMMEDIATELY

IMPQRTANT

INDEED

INN

INSISTED

INSTEAD

INTEREST ING

JELLY

KING

KITCHEN

LADDER

LADY

LAKES

LAMP

LAY

LEADING

LEANING

LEAPED

LEAST

LEATHER

LEND

LENGTH

LESS

LIFE

LIFTED

LIGHTNING

LESE

LOST

LOT

LOW

MAIN

MAJESTY

MARSH

MEAT

MEET

MIDDLE

MIDNIGHT

MILES

MINUTES

MISTAKE

MONTH

MUDDY

MULE

NAILS

NEEDLES

NEIGHBBR

NICEST

NODDED

NOTICED

OCEAN

OIL

ORDERED

QUGHT

OURSELVES

PACK

PALACE

PATCH

PATH

PAY 
PEACH

PEDDLERS

PEN

PERHAPS

PERSON

PICK

PIES

PILE

PINCH

PLAN

PLENTY

POINT

POTATO

PQUR

PQWER

PRESENT

PRINCE

PRINTED

PREBABLY

PROBLEM

PREUDLY

QUITE

RAISING

RANCHERS

RANG

RANGER

REACH

REALIZES

RECEIVED

REFUSES

REPLIED

RIBBENS

RICH

RID

RIPE

RIVERS

ROAST

ROCKY

ROOF

ROESTER

RUSHED

SALES

SALT.

SAVING

SCELDED

SCRATCH

SERIUUS

SERVANTS

SEVERAL

SHADEW

SHAKES

SHELF

SHINING

SHIPS

SHOOK

SHOP

SHORES

SIGHT

SILVER

SINCE

SIR

SISTER

SMOKE

SURRY

SPEAKING SPECIAL 


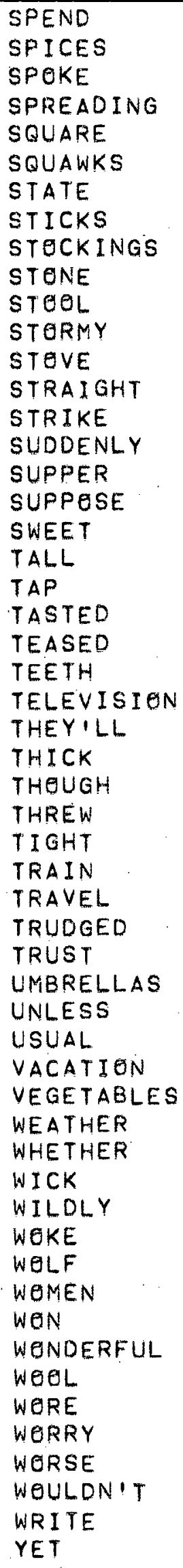

THERE ARE 369 WERDS IN LEVEL 5 GF SERIES B. IN COMPARING SERIES B WITH SERIES D, EACH AT LEVEL, 5 THERE IS A .00\% MATCH. THERE ALSE IS A $10.57 \% \mathrm{MATCH}$ (OUTSIDE OF LEVEL 5) RANGING FROM, 11-109 TO 3- 6 . 
THE BACK-UP WERDS FOR LEVEL 6 ARE:

ACCOUNT

ACCUSE

$\triangle C O R N$

ADMITTED

AIM

ALLOW

AMAZED

AMOUNT

AMUSEMENTS

ANNGUNCER

ANNEYS

ARMY

ATTACHED

ATTRACTED

AUDIENCE

AUTUMN

AWAKE

BALCONY

BANGED

BARS

BATHROOM

BATTERY

BEAST

BEAT

BEE

BERRIES

BISCUIT

BITE

BOILER

BOOMER

BREAK

BREATH

BRUSHING

BUCKSKIN

BUDS

BULLDEG

BUMBLE

BUMPED

BUSHES

BUSINESS

BUTCHER

BUTTONS

$C A B$

CABIN

CABUESE

CALM

CAMP

CANARIES

CANNON

CAPE

CARRETS

CASE

CAST

CAUSING

CHAIN.

CHALET

CHARGED

CHARMING

CHATTER

CHECK 
CHILD

CHUCKLED

CINDER

CIRCLE

CLAIM

CLAPPERS

CUAL

COLT

CEMBING

COMMAS

COMMEN

COMPARED

COMPETE

COMPLAINED

CONCERNED

CONCERT

CONE

CONFESS

CONFUSED

CONTENTED

CONTENTMENT

CONTEST

CONTESTANTS

CONTRARY

COGL

COTTAGE

CQURAGE

CRAZY

CREATURE

CREPT

CREW

CURTAIN

CUSTOMERS

DAISY

DARING

DELICIOUS

DESIRE

DIED

DIFFERENCE

DIPPED

DIRECT

DISTANCE

DUUBLED

DEUBTED

DEZED

DRAW

DREW

DRIPPING

DREVE

DUCHESS

DUKE

DUMB

EIGHT

ELEVEN

ELF

ENEMIES

ENGINE

ENTER

ESCAPED

EXACTLY

EXPERIMENT

EXPRESS

EYEBROWS

FACTS

FAILED 
FAMOUS

FAT

FIERCELY

FIFTY

FIGURE

FITS

FLEA

FLOPPED

FOOLISH

FERCE

FEREST

FERMS

FURT

FORTY

FORWARD

FRE IGHT

FRIDAY

FRINGE

GALLEPING

GASPED

GAZE

GEESE

GENTLEHEN

GLITTERING

GOBBLE

GRANT

GREW

GRINN ING

GROWLING

GUNS

HARM

- HERDS

HERR

HITCHED

HENEY

HEOFS

HORNETS

HUGE

HUMAN

HUMMING

HUNTED

HUSBAND

HYACINTH

IMPATIENTLY

IMPOSSIBLE

INCH

INVISIBLE

INVITE

I TEMS

JACKET

JAIL

JAM

JOY

JUMBE

JUNGLE

KNEES

KNITTING

LANGUAGE

LATCHED

LED

LESSON

LETTUCE

$L I O N$

LIST

LEAD 
LGGGERS

$\angle Q P$

LOPES

LQRD

LUVEO

LUMBER

LUNCH

MA'AM

MANAGED

MARRY

MEANT

MELT

MESSENGER

MET

MIKE

MIRRERS.

MISCHIEF

MOMENT

MONGRELS

MUSTACHE

NIBBLE

NICK

NIX

NER

NOTES

NETION

OAK

QFFER

ORIOLES

EUCH

PAID

PARENTHESES.

PARENTS

PASSENGER

PASTURE

PAUSED

PAW

PEEKING

PERCHED

PERFECTLY

PERFERMANCE

PIANO

PITCHER

PLANT

PLATFERM

PLEASANT

PLEASURE

PLUCK

PRACTICED

PRAISE

PREF IX

PREPARING

PRETEND

PROPERTY

PRETECTED

PROVED

PUNISH

PURE

PUZZLE

QUARRELING

QUARTERS

QUEEN

RADIO

RAGE

RAPIDLY

RASPBERRY 
RATHER

RECOGNIZED

RECQRD

REMAINED

REMARKABLE

REPEATED

REWARD

ROAR

REBBED

ROOT

ROSY

ReWS

RUBB ING

RULES

SAFETY

SAINT

SANG

SAWMILL

SCEWLED

SCREAMED

SEARCHED

SEASENS

SEED

SELFISH

SETTLED

SHARPLY

SHEET

SHELL

SHIFT

SHECKED

SHOT

SHOULDERS

SHOVELED

SHOWER

SHRUNK

SIGNAL

SILL

SIMPLE

SING

SINGLE

SIXTEEN

SKINKS

SKUNK

SKY

SLEEVES

SLICK

SNAILS

SNAPPED

SNIFFED

SOBS

SOIL

SELD

SOLDIERS

SELVE

SPEED

SPIED

SPILL

SPEIL

SPOT

SQUEALING

SQUIRREL

STAGE

STAKE

STARS

STATEMENT

STFALING 
STEAM

STEER

STEM

STOCKADES

STOLEN

STREAK

STRUGGLING

STUCK

STUMP

STUPID

STURDY

SUCCESS

SUGQESTED

SULKED

SUPPLIES

SUSPECTED

SWEEP

SWEPT

SWINGING

SWITCHES

THIEF

TICKETS

TILL

TIMBER

TINKLED

TOOTHPASTE

TRAIT

TRAP

TREATED

TREMBLED

TROUSERS

TUBE

TURN I P - SEED

TWIG

UGLY

WAFERS

WAR

WASTE

WEAK

WEDD ING

WEEP

WHIMPERED

WHIP

WHISKERS

WHITENESS

WHOSE

WICKED

WI GGLED

WIPE

WOODSHED

THERE ARE 424 WOROS IN LEVEL 6 OF SERIES B. IN COMPARING SERIES $B$ WITH SERIES D, EACH AT LEVEL, 6 THERE IS A . OO\% MATCH.

THERE ALSO IS A $6.37 \%$ MATCH (OUTSIDE OF LEVEL 6) RANGING FROM, 11-115 TG $7-81$. 
THE BACK - UP WORDS FOR LEVEL 1 ARE:

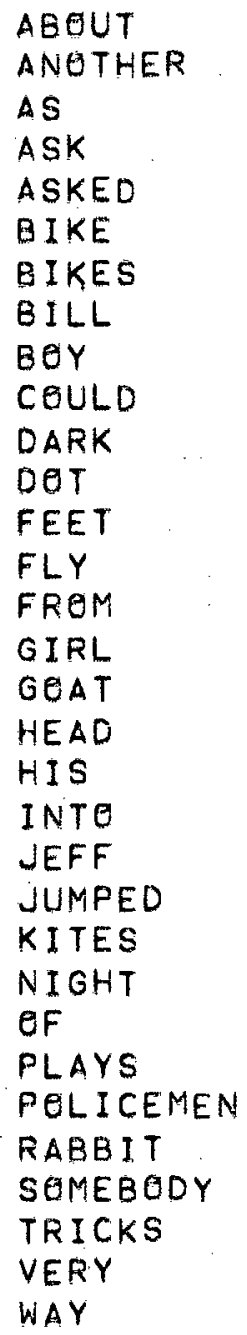

THERE ARE 139 WORDS IN LEVEL 1 OF SERIES B, IN COMPARING SERIES B WITH SERIES E ,EACH AT LEVEL, 1 THERE IS A $4.32 \%$ MATCH. THERE ALSO IS A $65.47 \% \mathrm{MATCH}$ (OUTSIDE OF LEVEL 1 ) RANGING FROM, $8=15 \cdot T 6 \quad 2=28$. 
THE BACK-UP WORDS FOR LEVEL 2 ARE:

$\triangle G A I N$

AIRPLANE

ANYTHING

BAND

BLANKETS

BEAT

$B O L O$

BROOM

BROWN

CAGES

CAKE

CALF

CAPS

CHEOSE

CIRCUS

COLERS

DIDN'T

EVERYONE

EVERYTHING

FIRST

FIVE

FOOD

FOUR

HICCUPS

HURRY

I'LL

I'M

ICE

JUST

LONG

MADE

MANY

MONEY

MERE

MR

MRS •

MUCH

MUST

NEXT

EFF

UPEN

$O R$

URANGE

OSTRICH

OTHER

OUR

OVER

PAINT

PAL

PARTY

PENCIL

PICTURE

FECKET

PORCUPINE

PRIZES

RACCEON

RUNNING

SAND

SAT

$S A Y$

SHOE 
SOME

STOP

STREET

STRIPES

TENT

THAN

THING

THINK

TOY

TREE

VELVET

VIOLET

WERE

WHEN

WHICH

WHY

WINK

WISH

WISHES

WOODS

ZEBRA

THERE ARE 177 WORDS IN LEVEL 2 OF SERIES B. IN COMPARING SERIES B WITH SERIES E, EACH AT LEVEL, 2 THERE IS A $3.95 \%$ MATCH.

THERE ALSO IS A $4.4 .63 \% \mathrm{MATCH}$ IUUTSIDE OF LEVEL 2 ! RANGING FROM, $8=178$ TO $3=41$. 
THE BACK=UP WERDS FOR LEVEL 3 ARE:

$\triangle D D I N G$

$A I R$

ASK

$\triangle S K E D$

BALLOON

BANKS

BEGINS

BESIDE

BIRDS

BOUNCED

BOY

BOY'S

BRIDGE

BRETHERS

BUNCH

BURN

BUY

CRECODILE

CUT

DIDNIT

DEGR

DRANK

DREWN

EARN

ENDING

FACES

FIFTH

FOLLOWED

FEUND

GAMES

GLAD

GELDEN

GREEN

HAND

HOPE

JAR

JUDGE

LAMB

LANDING

LEFT'

LEGS

LEMONADE

LOCK

MAIL

MEN

MORNING .

NAME

NICKELS

NETHING

NUMBERS

OFF

OFFICE

ELD

ONCE

OWL

PIPES

PLEW

PEST

PUPPY

PURPLE

PUSH 


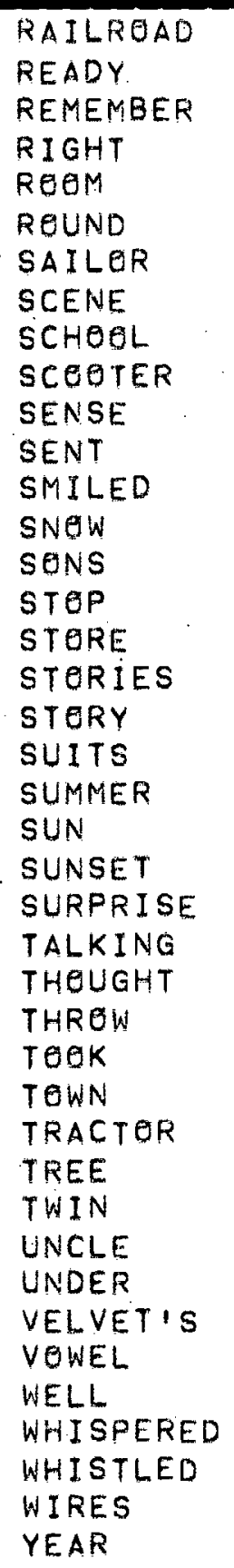

THERE ARE 237 WORDS IN LEVEL 3 OF SERIES B. IN COMPARING SERIES B WITH SERIES E EACH AT LEVEL, 3 THERE IS A $5.91 \%$ MATCH. THERE ALSO IS A $48.95 \%$ MATCH IOUTSIDE OF LEVEL 3) RANGING FROM, $8-149$ TO $4=138$. 
THE BACK-UP WORDS FUR LEVEL 4 ARE:

APPLES

ATE

BAKERY

BARN

BATTER

BEARD

BENE

CARD

CARPET

CASTLE

CHAIR

CHIMPANZEE

CHOP

CLIMB

COACH

COEKIES

DANCE

DINNER

EAR

EGGS

FAIRY

FEED

FEEL

FELL

FILL

FINE

FRIGHTEN

GARDEN

GAY

GHEST

GROUND

GREW

HAIR

HILL

HIND

HURDY = GURDY

JOKES

JUGGLE

KNOCKED

LAST

LATE

LIVE

LIZARD

LOAF

LBCKET

MARBLE

MERRY

MISS

MENDAY

MEVE

NESTS

NOODLE

PAPER

PASS

PATTER

PICNIC

PINS

PITTER

PLAIN

QUESTIEN

RAGGED 
READ

REPORT

REBE

ROUND

RUFFLE

SCISSORS

SENTENCE

SHAPE

SMELLED

SOMERSAULT

SPRING

STEP

TAIL

THURSDAY

TIDY

TIED

TOUCHED

TOWER

TUESDAY

WASHED

WAVE

WEDNESDAY

WEEK

WINGS

WINTER

WOMAN

THERE ARE 207 WORDS IN LEVEL 4 OF SERIES B. IN COMPARING SERIES B WITH SERIES E , EACH AT LEVEL, 4 THERE IS A $4.35 \%$ MATCH. THERE ALSE IS A $49.28 \%$ MATCH (OUTSIDE OF LEVEL 4 ) RANGING FRBM, $8=206$ TO $5=40$. 
THE BACK UP WORDS FOR LEVEL 5 ARE:

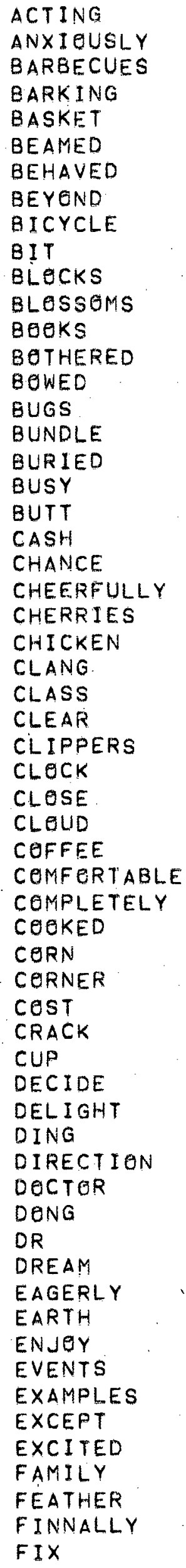


FERKS

FRAME

FREE

FRUIT

GATHERED.

GQDMETHER

GRAPE

GRASS

GRECERY

GREUPS

HABIT

HALF

HARBERS

HAY

HEART

HORNS

HOUND

HOUR

HUNDRED

INDEED

INN

JELLY

LAKES

LAMP

LEADING

LEANING

LEAPED

LEND

LENGTH

LESS

MARSH

MEET

MINUTES

MISTAKE

MENTH

NEEDLES

NICEST

QRDERED

PEACH

PEDDLERS

PEN

PIES

PILE

PINCH

PLAN

POINT

PUUR

PRESENT

RAIN

RAIS ING

RANCHERS

RANG

RANGER

REALIZES

RECEIVED

REFUSES

RIBBENS

RICH

RIPE

RIVERS

ROAST

ROEF

RUOSTER

RUSHED

SALES

SAIT 


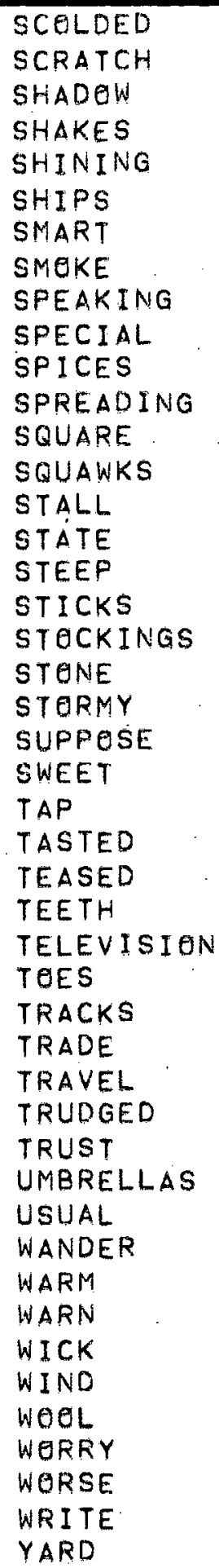

THERE ARE 370 WORDS IN LEVEL 5 OF SERIES B. IN COMPARING SERIES B WITH SERIES E , EACH AT LEVEL, 5 THERE IS A $5.95 \%$ MATCH. THERE ALSO IS A $42.43 \%$ MATCH IEUTSIDE OF LEVEL 5 I RANGING FROM, $8-254$ TO $4=48$. 
THE, BACK -UP WORDS FOR LEVEL G ARE:

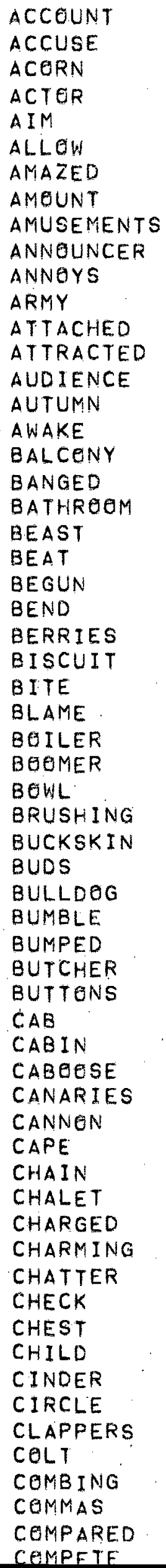


COMPLAINED

CENCERNED

CONCERT

CONE

CONFESS

CONFUSED

CONTENTED

CENTENTMENT

CONTEST

CONTESTANTS

CENTRARY

COOL

COTTAGE

COURAGE

CRAZY

CREATURE

CREPT

CREW

CURTAIN

CUSTOMERS

DAISY

DARING

DARKNESS

DASHING

DECALRED

DELICIOUS

DESIRE

DUUBLED

DOUBTED

DOZED

DRIPPING.

DUCHESS

DUKE

ESCAPED

EXPERIMENT

EXPRESS

EYEBROWS

FACTS

FAILED

FITS

FLEA

FLEPPED

FERCE

FORMS

FORT

FREIGHT

FRINGE.

GAZE

GENTLEMEN

GLITTERING

GRANT

GREWLING

GUNS

HERDS

HERR

HITCHED

HGOFS

HORNETS

HUMAN

HUMMING

HUNTED

HYACINTH

INCH

INQUIRED

INSECTS

INVISIBIF 
INVITE

ITEMS

JUMBG

JUNGLE

KILL

KNEES

KNITTING

LATCHED

LAZY

LETTUCE

LION

$\angle Q A D$

LOCAL

LOGGERS

LQP

LOPES

LORD

LOVED

LUMBER

MA'AM

MANAGED

MARKET

MELT

MESSENGER

MET

MIRRERS

MISCHIEF

MYSTERY

NARRATOR

NATIENAL

NECESSARY

NET

NIBBLE

NICK

NIX

NOR

NETES

NETIEN

ERIOLES

OUCH

PAGE

PAID

PARENTHESES

PARENTS

PARRET

PEEKING

PERCHED

PITCHER

PLANT

PLATFORM

PLEASURE

PLUCK

POKED

PELE

POUNDS

PRACTICED

PRAISE

PREFIX

PREPARING

PRETEND

PREPERTY

PROTECTED

PUNISH

PURE

PUZZLE

QUARRELING 
QUARTERS

QUEEN

RAGE

RAPIOLY

RASPBERRY

RATHER

RECOGNIZED

RECERD

REWARD

RIDOLES

ROAR

ROBBED

ROWS

RULES

SAFETY

SAINT

SANG

SAWMILL

SCAMPERED

SCARED

SCEWLED

SEARCHED

SEASONS

SELF ISH

SETTLED

SHARPLY

SHEET

SHOCKED

SHOULDERS

SHOVELED

SHOWER

SHRUNK

SIGNAL

SILL

SIMPLE

SIXTEEN

SKINKS

SKUNK

SKY

SLEEVES

SLICK

SEBS

SOIL

SOLD

SOLDIERS

SUL.VE

SPEED

SPIED

SPILL

SPOIL

SQUIRREL

STAGE

STAKE

STARS

STEALING

STEAM

STREAK

STRUGGLING

STUMP

STUPID

STURDY

SUCCESS

SULKED

SUPPLIES

SUSPECTED

SWFEP 
SWEPT

SWING ING

SWITCHES

TALES

THIEF

TICKETS

TIMBER

TINKLED

TOBTHPASTE

TRAIT

TRAP

TREATED

TURNIP $=$ SEED

TWIG

WAFERS

WAR

WASTE

WEAK

WEDDING

WEEP

WHIMPERED

WHITENESS

WI GGLED

WIPE

WOODSHED

WRAPPED

WRINKLED

THERE ARE 424 WORDS IN LEVEL 6 OF SERIES B. IN COMPARING SERIES B WITH SERIES E ,EACH AT LEVEL, 6 THERE IS A $7.31 \%$ MATCH. THERE ALSE IS A 20.99\% MATCH (OUTSIDE GF LEVEL 6) RANGING FROM, $8-247$ TO $4=14 \cdot$ 
THE BACK-UP WOROS FOR LEVEL 4 ARE:
ASKS
AT
$B A D$
BAKE
BOLO!S
BOUNCE
BOYS
BUSMAN
CALLS
CAPPY
CAPPY'S
CATS
DAYS
DOGS
EATS
FALL
FLEW
GETS
GEAT
GUATS
HELLE
HELP
HIS
HUUSE
HURRY
IF
JUMP
JUMPED
JUMPS
KNOWS
LIGHTS
LOOKED
MIKE'S
MR .
PAINT
PEANUT
PEANUTS
PET
PICNIC
PICNICS
POCKET
RUNS
SEES
SITS
TELLS
THANK
THEY
TREES
TRICK
TRICKS
TRIES
WALL.
WANTED
ZERE

THERE ARE 146 WORDS IN LEVEL 4 GF SERIES C. IN COMPARING SERIES C WITH SERIES A , EACH AT LEVEL, 4 THERE IS A $15.75 \%$ MATCH. THERE ALSE IS A $34.93 \% \mathrm{MATCH}$ IOUTSIDE OF LEVEL 4 I RANGING FROM. $7=169$ TO $1-30$. 
THE BACK $=U P$ WORDS FOR LEVEL 5 ARE:

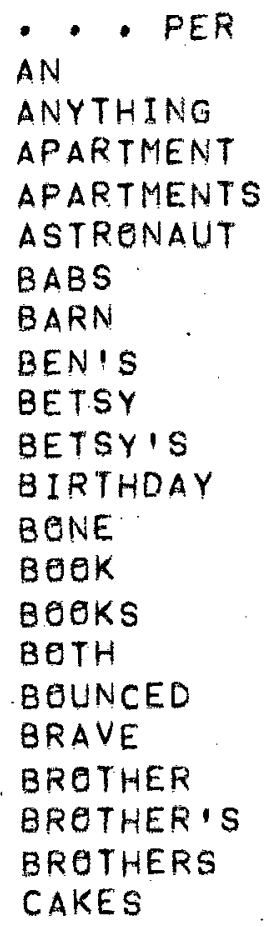


MAKE-BELIEVE

MEN

MEOW

METHER'S

MOTHERS

MOUNTAIN

NAMES

NEWS

NEXT

NOISE

CRBIT

QRB I TED

ORBITS

OTHER

ETHERS

OWN

PACK

PACKAGE

PACKS

PATCHES

PETER

PLAYED

PLAYING

PULE

POLICEMAN

POOL

PRIZE

PRIZES

PUPPY

PUPPY'S

QUARREL

ROCKETS

ROPE

SAYS

SCARED

SCHOOL

SCREEN

SEAT

SHOWED

SNAPPER

TALKING

TEDDY

TEDDY'S

TERRY

TERRY'S

THIEF

THING

THINGS

TOM

TOMMY

TOMMY'S

TWIN

TWINS

WALKED

WALKING

WEATHER

WEI GH

WUOF

WGRKED

WORKING

WORKS

WRITES

YELL

YELLED

YELLING 
THERE ARE 250 WORDS IN LEVEL 5 OF SERIES C. IN COMPARING SERIES C WITH SERIES A, EACH AT LEVEL, 5 THERE IS A $8.00 \%$ MATCH,

THERE ALSO IS A $24.80 \%$ MATCH (OUTSIDE OF LEVEL 5 ) RANGING FROM, $8=226$ TO $1=5$. 
THE BACKMUP WOROS FOR LEVEL 6 ARE:

ACRESS

ADDRESS

ALIVE

ANDREA

ANDY

ANGRY

ANIMAL

ANSINER

ARMY

BACON

BANK

BARK

BEAT

BEAUTIFUL

BEND

BENJAMIN

BETTY

BEBBY

BOUIE

BOTTLES

EOWED

BOWL

BREAD

BUTCH

CANDLE

CARS

CELLAR .

CHAIR

CHERRY

CHET

CHICKEN

CHOOSE

CIRCUS

CLEAN

COMING

COEKBEOK

COUSINS

CEVER

CRACK

CREAM

CRIED

DANNY

DAVID

DAWSEN

DINNER

DIRTY

DECK

DELLARS

OR.

DREOP

DROPPED

OREPPING

DRY

DUCKLING

EGGS

ELECTRICITY

EYEGLASSES

EYES

FAIRY

FALLEN

FFEL 
FILL

FIRES

FIX

FERGOTTEN

FOUND

FRANK

FRANKLIN

FUNNY

GATES

GAVE

GETTING

GNOMES

GUES

GUNE

GUODNESS

GRANDER

GRANDMA

GRIND

GROWN

HA

HANGING

HAPPINESS

HIGHNESS

HUBBY

HOLES

HONORABLE

HOUND -PUP

ICE

JENNY

KAKAI

KEPT

KEY

KINDNESS

LANDLORD

LATER

LEFT

LISTEN

LOCK

LEVELY

MAGIC

MARIA

MISS

MRS •

MUCH

NAVY

NEDDIE

NEED

NEST

NINETY =FIVE

OLDEST

OUTSIDE

QVEN

PANTS

PAPER

PATRICK

PEDAL

PEEP

PENNIES

PENNY

PETTED

PINT'

PITCH

PLACE

PLAYGROUND

PUSH 
QUITE

RAMBLING

READY - MIX

REAL

ROBERT

RUBBED

SACK

SADLY

SALT

SATURDAY

SAUSAGE

SEEMED

SHAW

SHOUT

SILK

SILLY

SIXTY=NINE

SKATES

SKATING

SOUP

SPACE

SPARK

SPET

SPRING

STONE

STOPPED

STORE

STORIES

STRIKE

STRING

SUE

SURPRISE

SUSAN

SWANS

SWIM

SWUNG

TABLE

TAIL

TAKEN

TAKING

TEMPERATURE

THREW

THROW

TIED

TIGER

TIMMY

TOWN

TRIP

TURNS

TWO=WHEELER

UGLY

VINEGAR

WAGGED

WAGGER

WAGQN

WAIT

WASH

WAWA

WOBBLY

WOEDCUTTER

WOODS

WURE

YOURSELF 
WITH SERIES A ,EACH AT LEVEL, 6 THERE IS A $6.69 \%$ MATCH.

THERE ALSO IS A $26.75 \% \mathrm{MATCH}$ (BUTSIDE OF LEVEL 6) RANGING FROM, $9=264$ TO $2=13$.

THE BACKMUP WORDS FUR LEVEL 7 ARE:

ABEY

$A C T$ ING

ADDRESSED

ADVENTURES

AFTERNOEN

ALICE

ANNACONDA

ANYBEDY

ARMS

AWAKE

AWFUL

BASKETS

BATHE

BATHS

BEACHES

BEAR

BEGINNING

BEHIND

BETWEEN

BEYOND

BLIINKED

BLINKY

BLIZZARD

BLOWHOLE

BLUEBERRIES

BRANCHES

BREATH

BREATHE

BUBBLES

BUCKSKIN

CARPENTER

CARRI AGE

CATTLE

CAUGHT

CENTS

CERTAIN

CHANCE

CHASE

CIRCLE

CITY

COILED

CONTESTS

COOKIES

CQPY

CORNER

CRAWLED

CREAKING

CRESS

CURLED

CURLY $Y$ EARED

DIRECTIUN

DISCOVER

DISTANCE

DIZZY

DOLPHIN

DOUGH

DRAINBGARD

EATEN

ELEVEN

EVERYBODY

EXPLARF 
FACE

FAUCET

FAVURITES

FIELDS

FIFTH

FLUTTER

FOLLOWED

FEOD

FORCE

FULL

GRANNY

GRINNED

GRQUP

HANDLE

HE! D

HE ILL

HELPLESS

$H I-Y I-Y I I P P I-Y I$

HIDDEN

HENEY

HOEP

HOPED

HOTEL.

HOURS

HUNT ING

HURRIED

JUE

JOHN

JUHNNY

JOINED

JUNATHAN

KENNELS

LANGFORD

LANGUAGE

LARGE

LAZY

LEANED

LEAPED

LIFEGUARD

LIFTED

LILLY

LEUD

LOUDSPEAKER

LOW

LYING

MAILED

MANAGED

MARK

MEAN

MILD

MIND

MINDY

MOUSE

MEUTH

MOVE

MULLAH

NEI GHBORS

NIBBLE

NOBODY

NOISY

OVERLOOKING

OWL

OWLGLASS

PAID

PARTY

PASSED 
PAW

PEEKING

PLANTS

PLAYFUL

PLENTY

POINTY-EARED

POPPY

POST

POTTER

POURING

QUACKING

RAFT

RAINIEST

RANCH

REACHED

RECEIVE

REPEAT

RETURNED

ROCK

RODE

RODEO

ROGER

ROOF

ROUNDISH

RUSTLE

RUSTLERS

SANG

SCAMPER

SETH

SHAG

SHALL

SHAPE

SHELF

SHINING

SHET

SKIED

SKIING

SLOWLY

SMOKE

SNAKE

SNOBPY

SUMEBODY

SOMETHINGNESS

SPATTERED

SPLASHED

SPEUTS

SQUANTE

SQUEEZE

SQUIRREL

STARED

STEPS

STICKING

STRETCHED

STROKE

SUCH

SUDDENLY

SUNRISE

SUPPER

SUPPESE

SWIRLED

SWIRL ING

TAPES

TEASING

TEETH

THESE

THFY'L 
THIRO

THESE

THOUGH

THOUGHTLESS

TIRED

TONGUES

TRACKS

TRAINED

TRAP

TROJAN

TRQIL

TRUE

TRULY

TRUTH

TUMBLING

UH

UNSEE-ABLE

UNTIED

VISIBLE

VISIT

VUICE

WAGGLES

WARM

WATCHED

WATCHING

WAVED

WE'LL

WE!RE

WEEK

WHIRLED

WHISPERED

WORRYING

WRONE

YEU'LL

YEU'RE

YUU'VE

THERE ARE 348 WORDS IN LEVEL 7 OF SERIES 6. IN COMPARING SERIES C WITH SERIES A EACH AT LEVEL, 7 THERE IS A $8.05 \%$ MATCH.

THERE ALSO IS A $17.24 \%$ MATCH (BUTSIDE SF LEVEL 7 ) RANGING FROM. 9-265 TH $1=53$. 
THE BACK = UP WERDS FOR LEVEL 8. ARE:

$\triangle B B O T T$

AGREED

$\triangle M O N G$

ANGIE

$A R R I V E D$

ARROWS

ASHORE

ATTENTION

AWE

BABIES

BANANA

BANDANNA

BARREL

BEEZUS

BEGGAR

BEL INDY

BOARD

BREKEN

BRUSHES

BUNG

BUSTLING

CANNEN

CAPTAIN

CARDBEARD

CHILD

CHIMNEY

CHRISTMAS

CHUCKLED

CHUTE

CIDER

CLAIMS

CLANCY

CLIFF

CLIPPITY

CLOTHING

CONDITION

COEP

COTTAGES

COUNCILOR

COUNTRIES

COUNTS

COURSE

CRATES

CREAKY

CRISPY

CROP

CROWD

CURIEUSLY

CURRENTS

CURVE

DADGER

DEAL

DELIVERING

DENMARK

DIFFERENCE

DINING

DIRECT

DISAPPEARED

DISAPPOINTED

DISLIKE 
DRAGGING

DRIED

DRIVEN

EASIER

EASILY

ELEPHANT

ENERMOUS

ENTERS

ENTRY

ESTHER

EXAMINING

EXCEPT

EXERCISE

EXPLODE

EXTRA

FARTHER

FEATHERS

FIFTEEN-MAN

FIFTY

FIGURED

FLIERS

FLIES

FGNDER

FGOLISH

FORTH

FORWARD

FOUNDRY

FOUNTAINS

FREEZING

FRESH

FRITZL

FREWN

FRUIT

FURTHER

FURTURE

GENEVIEVE

GENTLE

GERALDINE

GEWNS

GRASP

GUES

GUESS

HANDFULS

HAPPILY

HARNESS

HATED

HAUS

HEART

HEAVENS

HEAVIER

HELPLESSLY

HENERY'S

HENRI

HERRING

HES I TATED

HEPEFUL

HOPING

HUGGINS

HUI

HULLA

HUSTLING

I D

IMMEDIATELY

IMPAT IENT

INSECTS

INVENTED 
INVENTION

ISABEL

JACKSON

$J A G Q E D$

JAN IE

JENNIFER

JQYFULLY

JUDGES

JUICIER

JUICY

JUL I ANA

LAID

LECLERC

LIESI

LUADING

LEFT

LOLLIPEP

MACLEOD

MAIDSERVENTS

MAMMY

MARRIEO

MARRY

MARSHES

MASTS

MEECHAM

MELTED

MIRRER

MODELS

MOTGR

MOVEMENT

MUSTACHE

MYSTERY

OFFERED

ORVILLE

PAINFUL

PALACE

PAPPY

PARADE

PARENTS

PASSAGE

PASTURE

PATIENTLY

PEERED

PEGGY

PENGUIN

PERHAPS

PESTERING

PIDGEON

PIERRE

PILOT

PLANKTEN

PLEWED

PEISEN

POISONEUS

PORCUPINE

PRAISED

PRESENTS

PRQGRAM

PROMISE

PROVE

PUTTER

PUZZLED

QUARTER

QUEER

QUESTION

QUTMBY 
RAISED

REARED

RECORD

REFRIGERATOR

REFUSED

REINS

REPAIR

REPLIED

RIKKI

ROMONA

ROYAL

SAILURS

SAKES

SANDWICH

SANTA CLAUS

SARAH

SAUCEPAN

SCAR ING

SCRATCHING

SCREAMING

SERVICE

SEVENTEEN

SHAFTS

SHARED

SHE ILL

SHERIFF

SHUTTLE.

SIGHED

SILENTLY

SINES

SIRREE

SKEIN

SMOOTH

SNARLING

SOW

SPARKLING

SPECIAL

SPIDER

SPIED

SPINDLE

SPINNERETS

SPITZ

SPLIT

SPLUTTERING

SPRANG

SPREAD

SPREUT

SQUARE

SQUEAKY

SQUINTING

STARING

STEALING

STREDE

STRENG

STRUGGLED

STUDIED

STUDYING

SUCCEEDED

SUCK

SUPPORTS

SURFACE

SWELLED

TARANTULA

TEARING

TEBOGGAN

TRETTED 
TUGGED

TUNNELS

UMLEADING

UNABLE

UNTIE

USELESS

USUAL

VACATION

VEGETABLE

VEICLE

WACOLED

WANDER

WEAK

WHALES

WHARF

WHIRLWIND

WILBUR

WILLINGLY

WISDOM

WORRIES

WURSE

WRIGHT

WR I TTEN

YEDER

THERE ARE 357 WORDS IN LEVEL 8 OF SERIES C. IN COMPARING SERIES C WITH SERIES A ,EACH AT LEVEL, \& THERE IS A $1.20 \%$ MATCH.

THERE ALSE IS A $12.04 \%$ MATCH (UUTSIDE GF LEVEL 8 ) RANGING FROM, $9=265$ TE $2=9$. 
THE BACK -UP WORDS FOR LEVEL 9 ARE:

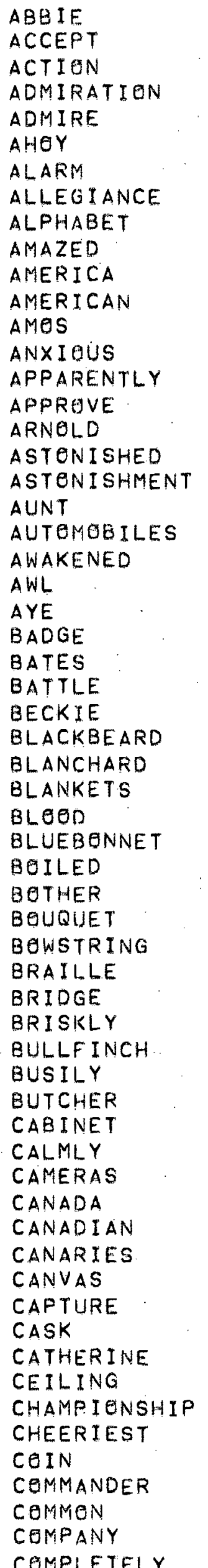


COMPLEX

COMPUTE

COMPUTER

CONT INUED

CORRECT

COSTUME

COUNCIL

COVE

CRACKLING

CRAMMED

CREDIT

CROWNED

CRUEL

CUPBOARD

CURTAIN

DEFEATED

DEFEND

DELICIOUS

DELIGHT

DEMANDING

DENNIS

DEPEND

DESCRIBED

DESERTS

DESERVE

DESPAIR

DETECTIVE

DIAGRAM

DI AMEND

DIFFICULT

DIGNITY

DIVIDED

DUEDLE

DRAWERS

DROUGHT.

DUNES

DUNN

DURING

EAGER

EAGLE

EASEL

ELECTRICAL.

EMBLEM

ENEMY

ENTRANCE

EUREPE

EXACTLY

EXAMPLES

EXCHANGE

EXCITEMENT

EXCLAIMED

EXPECTION

EXPENSIVE

EXPERIMENTAL

FANCY

FASTENED

FAVOR

FIERCELY

FIFE

FIFT IETH

FLAVER

FLUSHED

FOLKS

FOREIGN

FEUGHT 
FRECKLES

FREEOOM

FRITZ

GALE

GASPED

GATHERED

GAZED

GESSLER

GIANT

GIGGLE

GLADDENED

GLANCED

GLERY

GUBLINS

GRATEFUL

GROANED

GROUCH

GRUMPY

GUARDS

GUNPEWDER

HARBER

HAWAII

HEARTIES

HONOR

HUE

INVITE

INWARD

I RENE

IVERSON'S

JERQME

JEWELS

JUHANN

KILDEE

LABORATERY

LARS

LATIN

LEGEND

LENGTH

LEVEL

LIBERTY

LIEUTENANT

LOOSE

LOOSENED

LOUIS

MALLARD

MARIE

MARYGOLD

MATTRESSES

MAYNARD

MECHANICAL

MEMORIAL

MEMORY

MENTION

MERCHANT

MESSAGES

METAL

MICREPHONE

MICREWAVE

MIDAS

MILITARY

MINNY

MOLTING

MOOSE

MUFFLED

MUSEUM

MUSIC 
MUSICAL

MUTTERED

MYSTERIOUS

NATI GN

NEIGHBORHOOD

NOVEMBER

OARS

EBEY

OCRACEKE

OLIVE

ONION

OUTSMARTED

PACKET

PANEL

PARIS

PARSLEY

PAULA

PEABEDY

PEDDLER

PENELQPE

PERFECT

PERMISSION

PIANO

PILLEW

PIRATES

PLEDGE

PLUNGE

POLICE

POLISH

PESITION

PUSSESSIUN

POSSIBLE

POSSIBLY

PRACTICE

PRAYER

PRECIUUS

PREPARE

PRETENDED

PREFESSOR

PROPER

PROTECT

PUMPKINS

PUPILS

RACCEONS

RACKET

REAPPEARED

REPRESENT

RESPECT

RETREAT

RIEHL

REAST

REBET

ROSE-PETALS

RUSHED

RYAN

SALUTE

SATELLITE

SATISFIED

SAUCER

SCARCELY

SCARY

SCATTERED

SCITUATE

SCERED

SCRUBBED

SCURRIER 
SEASEN

SECTIUNS

SEIZE

SELFISH

SENSE

SENSER

SEPTEMBER

SERVE

SEWING

SHADEW

SHELTERED

SHOLDERS

SHOVED

SHUFFLING

SILVERSPOT

SIMON

SIMPLE

SKIRTS

SLANTED

SNICKER

SNIFFED

SELVING

SPIRITS

SPOILED

SPRIG

SPRINKLE

STAMPED

STAR-SPANGLED

STARTLED

STATUTE

STILLNESS

STIRRING

STOLEN

STOMACH

STRENGTH

SUBJECTS

SUCCESS

SUCCESSFULLY

SUIT

SUPERMAN

SUPERTEOL

SWENSEN

SWEPT

SWISS

SWIYZERLAND

SWERD

SYMBELS

SYNCEM

TANGLED

TASTIEST

TAXI

TEAMMATES

TENNIS

TEPEES

TEXAS

THIRTEEN

THOUGHTFULLY

THOUSAND - POUND

TICKETS

TEOTING

TETING

TOUCHING

TOWER

TROUTT

TWANG

TWIT 
TYRANT

UMPIRE

UNCLE

UNEARTHED

UNHAPPILY

UNIFORM

UNITED

USABLE

VALLEY

VALUE

VENTURE

VIEW

WARD

WARDEN

WARRIOR

WARSHIPS

WATERMELON

WEALTH

WELCOME

WESTERN

WHOO-00-00

WHOOOO=OO

WHESE

WIDOW

WILLIAM

WORTH

WORTHY

WRINKLED

YANKEE

YOURSELVES

THERE ARE 394 WORDS IN LEVEL 9 OF SERIES C. IN COMPARING SERIES C WITH SERIES A EACH AT LEVEL, 9 THERE IS A $3.30 \%$ MATCH.

THERE ALSO IS A $3.81 \%$ MATCH IOUTSIDE OF LEVEL 9) RANGING FROM,

$8=216$ TO $7=180$. 
THE BACK $=$ UP WORDS FOR LEVEL 4 ARE:
ASKS
AT
BABY
$B A C K$
$B A D$
BAKE
BILLYIS
BIRD
BIRDS
BELO'S
BOUNCE
BOYS
BUSMAN
CAGE
CALL
CALLS
CAPPY
CAPPY'S
CATS
DOG
DOGS
DENTT
EATS
FIGHT
GETS
HAS
HAVE
IN
LIGHTS
MAY
ME
MIKE'S
MOON
MR.
MY
NE
PICNICS
ROCKET
RUNS
SEES
SHOW
SHOWS
SITS
SUCKET
TAKES
TALK
TALKS
THANKS
THAT
THAT IS
TEO
TREES
TRICK
TRICKS
TRIES
WE
WHEEL
ZERO 
WITH SERIES $B$,EACH AT LEVEL, 4 THERE IS A $4.11 \%$ MATCH.

THERE ALSO IS A $42.47 \%$ MATCH (OUTSIDE EF LEVEL 4 ) RANGING FROM, 5-232 TO $1-130$.

THE BACK $=U P$ WERDS FUR LEVEL 5 ARE:

- - PER

APARTMENT

APARTMENTS

$\triangle R E$

ASTRENAUT

BABS

$B E$

BED

BEDS

BEN

BEN!S

BLAST

BLAST-EFF

BLEW

BEATS

BRETHER

BROTHER'S

BROTHERS

CAKES

CALF

CALLED

CAME

COW

CRY

DOING

ELLEN

ELLEN'S

FATHER'S

FATHERS

FIND

FLOAT

FLEATED

FLOATING

HAP

HAP'S

HELIUM

HELPS

HER

HOLIDAYS

HOME

JACK

JANE

JIM

JONES

JUMP ING

KITTEN

KITTENS

KNEW

LINDA

LOOKING

LOVE

LOVED

LUCY

LUCY'S

MAD

MAKE = BELIEVE

MOTHER'S

MOTHERS

MOUNTAIN

ORBIT 
ORBITS

PACKAGE

PACKS

PATCHES

PETER

PETS

PIG

PLAYED

PLAYING

POLICEMAN

POOL

PRIZE

PRIZES

RECKETS

SAYS

SCREEN

SHOWED

SISTERS

SLEEP

SNAPPER

STAR

TEDDY

TEDDY'S

TERRY

TERRY'S

TRYING

TV

WEIGH

WHITES!

WeOF

WRITES

YELL

YELLED

YELLING

ZIP

THERE ARE 250 WORDS IN LEVEL 5 OF SERIES C. IN COMPARING SERIES C WITH SERIES 8 , EACH AT LEVEL, 5 THERE IS A $4.80 \%$ MATCH. THERE ALSO IS A $35.60 \%$ MATCH (OUTSIDE OF LEVEL 5 ) RANGING FROM, 6-179 TO $1-149$. 
THE BACK=UP WORDS FOR LEVEL 6 ARE:

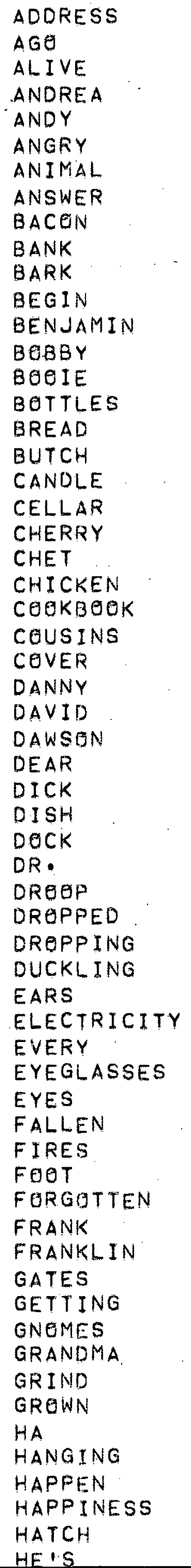


HEAD

HIGHNESS

HOBBY

HOLES

HUNERABLE

HOUND - PUP

ITS

JAPAN

JENNY

KAKAI

KINDNESS

KNOWN

LANDLORD

LATER

LEARN

LIBRARIAN

LIBRARY

LOVELY

MARIA

NAVY

NEDDIE

NEED

NEST

NINETY - F IVE

OLDEST

OPENED

QUTSIDE

OVEN

PANTS

PAPER

PATRICK

PEDAL

PEEP

PENNIES

PENNY

PETTED

PINT

PITCH

PLAYGREUND

PGPCBRN

PUPPED

POPPER

QUICKLY

RAMBLING

READY = MIX

REAL

REALLY

ROBERT

RUBBED

SACK

SADLY

SECRET

SHAW

SHINY

SHIPS

SHISH

SILK

SINK

SIXTY -NINE

SKATES

SKATING

SNOWSTORM

SOFT

SEUP

SPACE

SPARK 
STARTED

STEPPED

STORE

STORIES

SUE

SUGAR

SWIM

SWUNG

TEMPERATURE

TURNS

TWE-WHEELER

UGLY

VINEGAR

VUTE

WAGGED

WAGGER

WAGON

WAIT

WASH

WAWA

WHAT'S

WINDOWS

WIRE

WEBBLY

WOEDCUTTER

WGEDS

WRETE

YQURSELF

THERE ARE 314 WURDS IN LEVEL 6 OF SERIES C. IN COMPARING SERIES C WITH SERIES B , EACH AT LEVEL, 6 THERE IS A $3.18 \%$ MATCH. THERE ALSO IS A $41.08 \%$ MATCH (OUTSIDE OF LEVEL. 6) RANGING FROM, $5=266$ TE $1=23$. 
THE BACK-UP WORDS FOR LEVEL 7 ARE:

$A B B Y$

ADDRESSED

ADVENTURES

ALICE

ANNACENDA

ANYEODY

AWFUL

BASKETS

BATHE

BATHS

BEACHES

BEAR

BEGINNING

BERRY

BLINKED

BLINKY

BLIZZARD

BLOWHOLE

BLUEBERRIES

BRANCHES

BUBBLES

BUCKSKIN

BUSH

BUSHES

CARPENTER

CARRIAGE

CATTLE

CAUGHT

CEILED

CENTESTS

COPY

CRAVILED

CREAKING

CROSS

CURLED

CURLY - EARED

DISCOVER

DENKEY

DQUGH

DRAINBEARD

DRIVE

DROWNING

EATEN

EDGE

EVERYBGDY

EXCITING

EXPLAIN

EXPLORE

FACE

FAUCET

FAVORITES

FIELDS

FINEST.

FLUWERS

FLUTTER

FRIGHTENING

GRANNY

GRINNED

GREUP

HE' $O$ 
HELPLESS

$H I=Y I-Y I I P P I=Y I$

HIDDEN

HOGP

HOPED

HOTEL

HOURS

HUNT ING

HURRIED

I RON

IRONWOOD

JACKDAW

$J U E$

JOHN

JOHNNY

JOINED

JONATHAN

KENNELS

LANGFERD

LANGUAGE

LARGE

LEANED

LEAVES

LIFEGUARD

LIFTED

LILLY

LYING

MAILED

MILD

MILK

MINO

MINOY

MEUSE

MOUTH

MEVE

MULLAH

NEIGHBORS

NEBEDY

NOISY

OVERLOOKING

OWL

OWLGLASS

PASSED

PLAYFUL

PQEM

POINTED

POINTY-EARED

PEND

PEPPY

POTTER

PEURING

PREUD

QUACKING

RAFT

RAINIEST

RANCH

REACHEO

RECEIVE

RIVER

RECK

REUNDISH

RUSTLE

RUSTLERS

SCAMPER

SCIENTISTS

SEARCH 
$S E E-A B L E$

SETH

SHAG

SKIED

SKIING

SKIS

SKUNK

SLID

SLOWLY

SNAKE

SNEEPY

SOMETHINGNESS

SORT

SOUND

SPATTERED

SPLASHED

SPEUTS

SQUANTE

SQUEEZE

SQUIRREL

STARED

STEPS

STICKING

STREAM

STRETCHED

STROKE

SUNRISE

SWIRLED

SWIRLING

TAPES

TEASING

TERRIBLE

THANKFUL

THOUGHTLESS

THUMB

TUNGUES

TRAINED

TREAT

TREJAN

TRQIL

TUMBLING

TURTLE

TWIRLING

UH

UNSEE-ABLE

UNTJED

VISIBLE

VISIT

VEICE

WAGGLES

WHIRLED

WILD

WONDER

WONDERFUL

THERE ARE 348 WORDS IN LEVEL 7 BF SERIES $C$. IN COMPARING SERIES C WITH SERIES B , EACH AT LEVEL, 7. THERE IS A .0O\% MATCH. THERE ALSO IS A $38.22 \%$ MATCH IBUTSIDE OF LEVEL 7 ) RANGING FROM, 6-306 TO $1-120$. 
THE BACK-UP WORDS FOR LEVEL 8 ARE:

ABBOTT

AGREED

ALTHOUGH

ANGIE

ANTARCTICA

AWE

BABIES

BANANA

BANDANNA

BARREL

BEEZUS

BEGGAR

BELINDY

BRUSHES

BUILDINGS

BUNG

BUSTLING

CAPTAIN

CARDBOARD

CARRIED

CHECKERS

CHEERFULLY

CHIMNEY

CHRISTMAS

CHUCKLED

CHUTE

CIDER

CLAIMS

CLANCY

CLIFF

CLIPPITY

CLOTHING

CENDITIEN

CEOP

COTTAGES

COUNCILOR

COUNTRIES

COUNTS

CRATES

CREAKY

CRISPY

CREP

CROWD

CURIOUSLY

CURRENTS

CURVE

DADGER

DEAL

DECIDED

DEL IVER ING

DENMARK

DINING

DRAGGING

DRIED

ORIVEN

EASIER

EASILY

ENGLAND

ENERMEUS

ENTERS

ENTRY 
ESTHER

EVENING

EXAMINING

EXERCISE

EXPANDS

EXPLODE

FEATHERS

FIF TEEN = MAN

FIGURED

FINALLY

FLIERS

FLIES

FENDER

FQREMAN

FEUNDRY

FOUNTAINS

FREEZING

FRITZL

FREWN

FRUIT

FURTHER

FURTURE

GENEVIEVE

GENTLE

GERALDINE

GEWNS

GRASP

GUES

GUESS

HANDFULS

HAPPILY

HARNESS

HEAVENS

HEAVIER

HELPLESSLY

HENERY'S

HENRI

HERRING

HESITATED

HIFPEPETAMUS

HOLIDAY

HOLLY

HOLMES

HOPEFUL

HOPING

HUGGINS

HUI

HULLA

HUSTL ING

INVENTED

INVENTION

ISABEL

JACKSON

JAGGED

JANIE

JENNIFER

JOYFULLY

JUDGES

JUICIER

JUICY

JUL I ANA

KEVIN

KINGDOM

KINNDLI

LEAD 


\section{LIESI}

LOADING

LOFT

LQLLIPOP

MACHINE

MACLEOD

MAIDSERVENTS

MAMMY

MARRIED

MARRY

MARSHES

MASTS

MEECHAM

MELTED

MINUTE

MIRRER

MODELS

MOM

MEMENT

MOTUR

MOVEMENT

NOTICE

NOTICING

OFFERED

ORVILLE

PAINFUL

PAPPY

PARADE

PASSAGE

PATIENTLY

PAUL.

PEERED

PEGGY

PENGUIN.

PESTERING

PIDGEON

PIERRE

PILUT

PIONEERS

PLANKTEN

PLEWED

POISEN

POISENOUS

PRAISED

PRESENTS

PREBLEMS

PROGRAM

PROMISE

PREVE

PUTTER

PUZZLED

QUARTER

QUIMBY

RAISED

REARED

REFRIGERATOR

REFUSED

REINS

REPAIR

RIKKI

ROMENA

ROUTE

ROYAL

SAILURS

SAKES

SANDWICH 
SANTA CLAUS

SARAH

SAUCEPAN

SCARING

SCRATCHING

SCREAMING

SERVICE

SEVENTEEN

SHAFTS

SHARED

SHE'LL

SHERIFF

SHOES

SHUTTLE

SIGHED

SILENTLY

SINES

SIRREE

SKEIN

SMEOTH

SNARLING

SOW

SPARKLING

SPECIAL

SPIDER

SPIED

SPINDLE

SPINNERETS

SPITZ

SPLIT

SPLUTTERING

SPRANG

SPREAD

SPROUT

SQUARE

SQUEAKY

SQUINTING

STARING

STRODE

STRONG

STRUGGLED

STUDIED

STUDYING

SUCCEEDED

SUCK

SUPPERTS

SURFACE

SWELLED

TARANTULA

TEAR ING

THEMSELVES

THOUSANDS

THREAD

TOBGGGAN

TUGGED

TUNNELS

TURNIP

TURNEUT

TWISTED

UMLOADING

UNABLE

UNTIE

USELESS

USUAL

VACATION

VEGE TABLE 


VEICLE
VISITORS
WADDLED
WE V VE
WEAK
WHALES
WHARF
WHIRLWIND
WILBUR
WILLINGLY
WISDEM
WORRIES
WRIGHT
WRITTEN
YODER

THERE ARE 358 WORDS IN LEVEL 8 OF SERIES C. IN COMPARING SERIES C WITH SERIES $B$, EACH AT LEVEL, 8 THERE IS A .00\% MATCH. THERE ALSO IS A $19.27 \%$ MATCH IOUTSIDE OF LEVEL \&! RANGING FROM, $6=286$ TO $2-61$. 
THE BACK-UP WORDS FQR LEVEL 9 ARE:

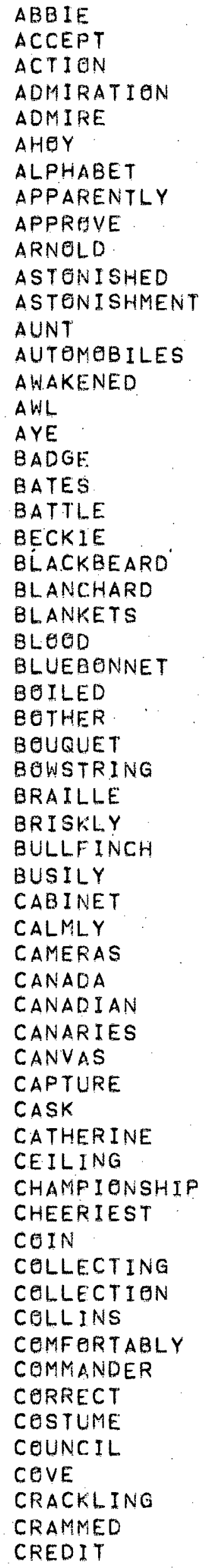


CRUEL

CUPBEARD

CURTAIN

DEFEATED

DEFEND

DELICIOUS

DELIGHT

DEMANDING

DENNIS

DEPEND

DESCRIBED

DESERTS

DESERVE

DESPAIR

DETECTIVE

DIAGRAM

DIAMOND

DIFFICULT

DIGNITY

DIVIDED

DQODLE

DRAWERS

DREUGHT

DUNES

DUNN

DURING

EAGER

EAGLE

EASEL

ELECTRIC

ELECTRICAL

EMBLEM

ENEMY

ENGLISH

ENTRANCE

EXCHANGE

EXCITEMENT

EXCLAIMED

EXPECTION

EXPENSIVE

EXPERIMENTAL

- FANCY

FASTENED

FAVER

FLAVER

FLUSHED

FOLKS

FUREIGN

FUUGHT

FRANCE

FRECKLES

FREEDOM

FRITZ

GALE

GAZED

GESSLER

GI ANT

GIGGLE

GLADDENED

GLANCED

GLERY

GEBLINS

GRATEFUL

GROANED

GROUCH

GRUMPY 
GUARDS

GUNPOWDER

HANDKERCHIEF

HARBBR

HAWAII

HEARTIES

HONOR

HUE

INFERMATION

INNING

INTELLIGENCE

INTEND

INTRODUCED

IVERSON'S

JEROME

JEWELS

JUHANN

KILDEE

LABERATORY

LARS

LATIN

LEGEND

LEVEL

LIBERTY

LIEUTENANT

LOQSE

LOOSENED

LOUIS

MALLARD

MARIE

MARYGOLD

MATTRESSES

MAYNARD

MECHANICAL

MEMURTAL

MEMORY

MENTION

MERCHANT

MESSAGES

METAL

MICREPHONE

MICRUWAVE

MIDAS

MILITARY

MINNY

MISERABLE

MUFFLED

MUSEUH

MUSIC

MUSICAL

MUTTERED

MYSTERI OUS

NATIQN

NOVEMBER

OARS

OBEY

OCRACOKE

OLIVE

ONION

OPPOSITE

OUTSMARTED

PACKET'

PANEL

PARIS

PARSLEY

PaULA 
PEABODY

PEDDLER

PENELOPE

PERFECT

PERMISSION

PILLOW

PIRATES

PLEDGE

PLUNGE

PELISH

POSITJEN

POSSESSION

PESSIELE

POSSIBLY

PRACTICE

PRAYER

PRECIEUS

PREPARE

PRETENDED

PROFESSOR

PROPER

PROTECT

PUMPKINS

PUPILS

RACCOONS

RACKET

REALIZE.

REAPPEARED

RECUGNIZE

RECOVERED

RELAYED

REPRESENT

RESPECT

RETREAT

RIEHL

RQBOT

ROSE $=P E T A L S$

SATELLITE

SATISFIED

SAUCER

SCARCELY

SCARY

SCATTERED

SCITUATE

SCORED

SCRUBBED

SCURRIED

SEASEN

SECTIENS

SEIZE

SELFISH

SERVE

SEWING

SHADEW

SHEL TERED

SHOLDERS

SHOVED

SHUFFLING

SILVERSPOT

SIMON

SIMPLE

SKIRTS

SLANTEO

SNICKER

SNIFFED

SOLVING 
SPIRITS

SPOILED

SPRIG

SPR INKLE

STAMPED

STAR = SPANGLED

STARTLED

STATUTE

STI ILLNESS

STIRRING

SUBJECTS

SUCCESS

SUCCESSFULLY

SUIT

SUPERMAN

SUPERTOUL

SWENSON

SWEPT

SWISS

SWITZERLAND

SWERD

SYMBOLS

SYNCEM

TANGLED

TASTIEST

TAXI

TEAMMATES

TELSTAR

TENNIS

TEPEES

TEXAS

THIRTEEN

THEUGHTFULLY

THOUSAND-POUND

TOETING

TOTING

TUUCHING

TREASURE

TROUT

TWANG

TWIT

TYRANT

UMPIRE

UNCLE

UNEARTHED

UNHAPPILY

UNIFORM

UNI TED

USABLE

VALLEY

VALUE

VENTURE

VIEW

VOYAGE

WARD

WARDEN

WARRIER

WARSHIPS

WATERMELON

WEALTH

WELCOME

WESTERN

WHOO-00=00

WHOOOO=0O

WHESE

WIDOW 
WILLIAM

YANKEE

YOURSELVES

THERE ARE 394 WORDS IN LEVEL 9 OF SERIES C. IN CUMPARING SERIES C WITH SERIES 8 ,EACH AT LEVEL, 9 THERE IS A .00\% MATCH. THERE ALSO IS A $9.39 \%$ MATCH IOUTSIDE OF LEVEL 9 ) RANGING FROM, $6=270$ TO $3=65$. 
THE BACK=UP WORDS FOR LEVEL 4 ARE:
BAKE
BILLYIS
BIRD
BOAT
BELO.S
BOUNCE
BUS
BUSMAN
BUT
CAPPY
CAPPY'S
CATS
CHILDREN
DOCTOR
EATS
FARTHER
FIGHT
GOAT
GOATS
MIKE!S
MR.
MUST
$M Y$
PEANUT
PEANUTS
PET
PICNIC
PICNICS
RUNS
SHOWS
SIT
SITS
SOCKET
TALKS
TRICK
TRICKS
TRIES
WON'T
ZERO

THERE ARE 146 WURDS IN LEVEL 4 OF SERIES C. IN COMPARING SERIES C WITH SERIES D, EACH AT LEVEL, 4 THERE IS A $6.16 \%$ MATCH. THERE ALSO IS A $61.64 \% \mathrm{MATCH}$ (QUTSIDE OF LEVEL 4) RANGING FROM, $11=99$ TO $3-37$, 
THE BACK - UP WORDS FOR LEVEL 5 ARE:

- - per

AIR

AIRPLANE

AIRPLANES

APARTMENT

APARTMENTS

ARE

BABS

BALLEON

BEN'S

BETSY

BETSY'S

BLAST

BLAST-OFF

BLEW

BOATS

BONE

BOOK

BeUKS

BOTH

BOUNCED

BRAVE

BRIGHT

BRETHER'S

BROTHERS

CAKES

CALF

CLOSE

CeW

Cows

DRESS

EARTH

ELLEN

ELLEN'S

FAIR

FELT

FIVE

FLQAT

FLOATED

FLOATING

GAS

HAP

HAP!S

HIGH

HOLIDAYS

HOME

HORSE

HORSES

HURT

JACK

JANE

$J I M$

JENES

JUMPING

KIND

KITTEN

KITTENS

KNEW

LETTER

LETTERS

LINDA 


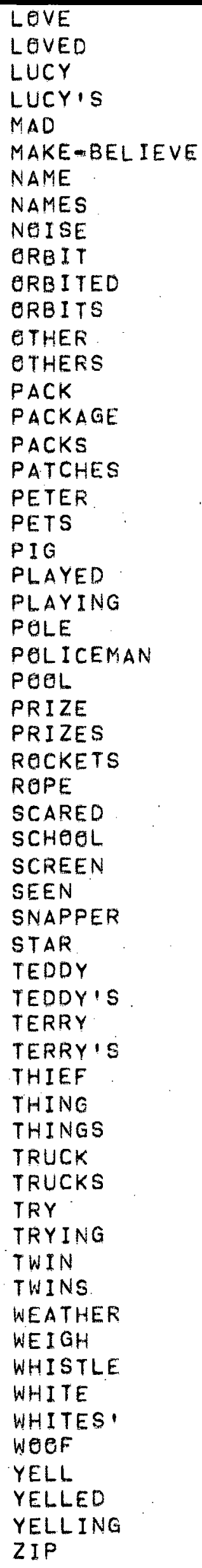

THERE ARE 250 WORDS IN LEVEL 5 OF SERIES C. IN COMFARING SERIES C WITH SERIES D, EACH AT LEVEL, 5 THERE IS A $2.40 \%$ MATCH. THERE ALSE IS A $40.40 \% \mathrm{MATCH}$ (OUTSIDE OF LEVEL 5 ) RANGING FROM. 11 - 99 TO $3-37$. 
THE BACK -UP WOROS FOR LEVEL 6 ARE:

ABLE
ADDRESS
AFRAID

ALIVE

ALMOST

ALONE

ALWAYS

ANDREA

ANDY

ANGRY

ANIMAL

ARMY

BACON

BANK

BEAT

BEAUTIFUL

BECAUSE

BEEN

BEGIN

BEND

BENJAMIN

BLOW

BOBBY

BEUIE

BOTTLES

BOWED

BOWL

BREAD

BUTCH

CANDLE

CAREFUL

CELLAR

CHERRY

CHET

CHICKEN

CHOOSE

CIRCUS

CLEAN

CEOKBOOK

COUSINS

COVER

CRACK

CREAM

CRIED

DANCE

OANNY

DAWSON

DEAR

DICK

DINNER

DIRTY

DISH

DECK

DOLLARS

DONE

DR.

DREQP

DUCKLING

EARS

EGQS

EIGHT 
ELECTRICITY

EVEN

EXPERIMENT

EYEGLASSES

EYES

FAIRY

FALLEN

FAST

FILL

FINISH

FIRES

FIX

FLOOR

FLY

FOOT

FOUND

FRANK

FRANKLIN

GAVE

GNOMES

GENE

GEODNESS

GRANDER

GRANDMA

GREW

GRIND

GREW

HA

HANG ING

HAPPEN

HAPPINESS

HATCH

HE!S

HEAD

HELD

HIGHNESS

HOBBY

HUNORABLE

HOUND PUP

HUNDRED

HUNGRY

I'LL

$I \cdot M$

ICE

IMPORTANT

JENNY

'KAKAI

KEPT

KEY

KINDNESS

KITCHEN

KITE

KNOCK

LANDLERD

LIBRARI AN

LIBRARY

LIGHTNING

LOCK

LEVELY

MARIA

MEST

NAVY

NEDDIE

NEED

NEST

NINETY-FIVE 
ONLY

OPENED

PANTS

PATRICK

PEDAL

PEEP

PENNIES

PENNY

PETTED

PINT

PITCH

PLACE

PLAYGROUND

QUICKLY

QUITE

RAMBLING

READY-MIX

REAL

REALLY

RICH

RICHARD

ROBERT

RUBBED

SACK

SADLY

SALT

SEVEN

SHAW

SHINY

SHIPS

SHISH

SHOP.

SHOULD

SHOUT

SIGHT

SILK

SILLY

SINK

SIXTY-NINE

SMALLEST

SMART

SMELL

SMILED

SNOWSTORM

SEFT

SQUP

SPACE

SPARK

STURE

STORIES

STRAIGHT

STRIKE

STRING

SUE

SUGAR

SUMMER

SURE

SUSAN

SWANS

SWUNG

TABLE

TAIL

TAKEN

TAKING

TEACHER 


TEMPERATURE
THREW
THROW
TIED
TIGER
TIMMY
TUP
TURNS
TWICE
TWO-WHEELER
UGLY
UNHAPPY
UNTIL
USE
VINEGAR
VETE
WAGGED
WAGGER
WAGON
WAIT
WASH
WAWA
WEAR
WHAT'S
WOBBLY
WOEDCUTTER
WGRE
WEULD
WRETE

THERE ARE 315. WORDS IN LEVEL 6 OF SERIES $C$. . IN COMPARING SERIES C WITH SERIES D, EACH AT LEVEL, 6 THERE IS A .32\% MATCH. THERE ALSO IS A $22.54 \%$ MATCH (OUTSIDE OF LEVEL 6) RANGING FREM. $11-117$ TO $3=4$. 
THE BACK-UP WOROS FOR LEVEL 7 ARE:

$\triangle B B Y$

$\triangle C T$ ING

ADDRESSED

ADVENTURES

ALICE

$A L S O$

ANNACENDA

ANYBEDY

ARMS

AWFUL

BASKETS

BATHE

BATHS

BEACHES

BEAR

BEGINNING

BEHIND

BERRY

BLINKY

BLIZZARD

BLEWHELE

BLUEBERRIES

BOTTOM

BOUGHT

BRANCHES

BREAK

BREAKFAST

BREATH

BREATHE

BREUGHT

BUBBLES

BUCKSKIN

BUSH

BUSHES

BUSY

CARPENTER

CARRIAGE

CARRY

CATTLE

CAUGHT

CENTS

CERTAIN

CHANCE

CHASE

CIRCLE.

CITY

CLEAR .

CLIMB

COILED.

CUNTESTS

COOKIES

COPY

CORNER

CURLEO

CURLY $=E A R E D$

DIRECTIEN

DISCUVER

DISTANCE

DIZZY

DOLPHIN

DEUGH 
DRAINBEARD

DRINK

DRIVE

DROWNING

EASY

ESCAPES

EVERYEODY

EXPLORE

FACE

FAUCET

FAVORITES

FEW

FLOWERS

FLUTTER

FEOD

FURCE

FOREST

FOURTH

FRIGHTENING

FULL

GRANNY

GRINNED

GROUP

HANDLE

HE!D

HE 'LL

HEAVY

HELPLESS

$H I-Y I-Y I I P P I=Y I$

HIDDEN

HENEY

HOOP

HOPEO

HOTEL

HOURS

HUGE

HUGGED

HUMMINGBIRD

HUNTING

HURRIED

HUSBAND

I V VE

IDEA

IMPOSSIBLE

INSTEAD

INTEREST

INVISIBLE

IRON

IRENWOOD

JACKDAW

$J O E$

JEHN

JOHNNY

JUINED

JONATHAN

KENNELS

LANGFERD

LANGUAGE

LARGE

LEANED

LEAPED

LIFEGUARD

LIFTED

LILLY

LIEN

LEUD 
LOUDSF'EAKER

LOW

LYING

MAILED

MANAGED

MATTER

MEAN

MIDDLE

MILD

MILK

MIND

MINDY

MULLAH

NEIGHBORS

NIBBLE

NEBODY

NOISY

GFTEN

OVERLGOKING

OWL

OWLGLASS

PAID

PAW

PEEKING

PLANTS

PLAYFUL.

PLENTY

POEM

PUINTED

PUINTY EARED

POPPY

PEST

POURING

PRETTY

PREUD

QUACKING

QUIET

RAFT

RAINIEST

RANCH

REACHED

RECEIVE

REMEMBER

REPEAT

REAN

ROARED

ROCK

REDE

REDEE

ROGER

REOF

ROUNDISH

RUSTLE

RUSTLERS

SANG

SCAMPER

SCIENTISTS

SEARCH

SEE-ABLE

SETH

SHAG

SHALL

SHAPE

SHELF

SHINING

SHERT 
SHOT

SKIING

SKIS

SKUNK

SLIO

SLQWLY

SMEKE

SNAKE

SNEEPY

SOMEBOOY

SOMETHINGNESS

SERT

SOUND

SPATTERED

SPLASHED

SPEUTS

SQUANTE

SQUEEZE

SQUIREEL

STICKING

STRANGE

STREAM

STRETCHED

STROKE

SUPPER

SUPPESE

SWIRLED

SWIRLING

TAPES

TEASING

TEETH

TERR IBLE

THANKFUL.

THESE

THEY,LL

THIRD

TONGUES

TRAINED

TRAP

TREAT

TREJAN

TRQIL

TRUE

TRULY

TRUTH

TUMBLING

TWIRLING

UH

UNSEE - ABLE

UNTIED

VISIBLE

VISIT

VUICE

WAGGLES

WATCHED

WATCHING

WE? LL

WE'RE

WEEK

WHIRLED

WILD

WOLF

WOMAN

WORRY

WORRY ING 
THE BACK=UP WORDS FOR LEVEL 8 ARE:

ABGETT

AGAINST

$\triangle G R E E D$

ALTHOUGH

AMONG

ANGIE

ANTARCTICA

APPEARED

ARR I VED

ARROWS

ASHORE

ATTENTION

AWE

BABIES

BANANA

BANDANNA

BARREL

BEEZUS

BEGGAR

BELINDY

BOARD

BROKEN

BRUSHES

BUILDINGS

BUNG

BUSTLING

CANNEN

CAPTAIN

CARDBEARD

CASTLE

CHANGE

CHECKERS

CHEERFULLY

CHILD

CHIMNEY

CHRISTMAS

CHUCKLED

CHUTE

CIDER

CLAIMS

CLANCY

CLIFF

CLIPPITY

CLETH

CLOTHING

COMFURTABLE

CENDITION

COOP

COTTAGES

COUNCILOR

COUNTS

COURSE

CRATES

CREAKY

CRISPY

CREP

CURIQUSLY

CURRENTS

CURVE

DADGER 
DANGEROUS

DANGL ING

DAUGHTER

DEAL

DECIDED

DELIVERING

DENMARK

DIFFERENCE

DINING

DIRECT

DISAPPEARED

DISAPPOINTED

DISLIKE

DISPLACE

DEZEN

DRAGGING

DRIED

DRIVEN

EASIER

EASILY

ELEPHANT

ENGLAND

ENORMOUS

ENTERS

ENTRY

ESTHER

EVENING

EXAMINING

EXCEPT

EXERCISE

EXPANDS

EXPLEDE

EXTRA

FAMOUS

FARTHER

FIFTEEN-MAN

FIFTY

FIGURED

FINALLY

FLIERS

FLIES

FONDER

FOOLISH

FUREMAN

FURTH

FORWARD

FEUNDRY

FOUNTAINS

FREEZING

FRESH

FRITZL

FROWN

FRUIT

FURTHER

FURTURE

GENEVIEVE

GENTLE

GERALDINE

GOWNS

GRASP

GUES

GUESS

HALF

HANDFULS

HEAVENS 


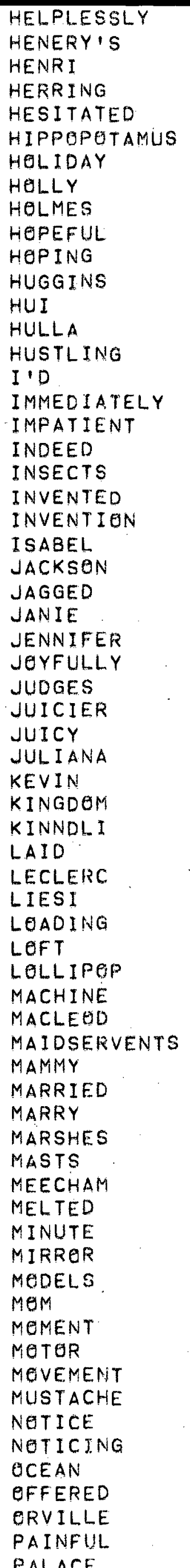




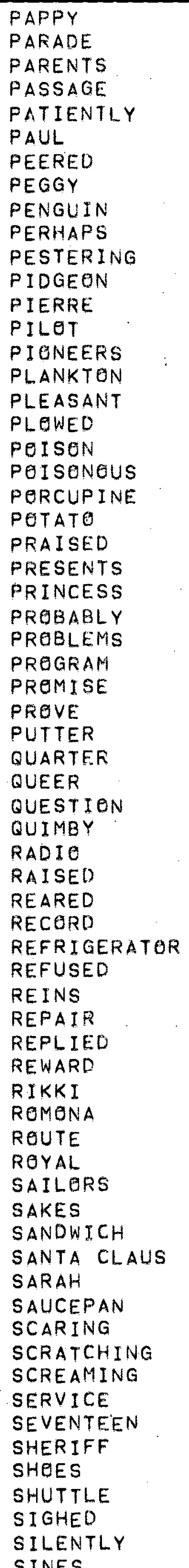


SIRREE

SKEIN

SMOOTH

SNARLING

SULDIERS

SELEMN

SORRY

SOW

SPECIAL

SPIDER

SPIED

SPINDLE

SPINNERETS

SPITZ

SPLIT

SPLUTTERING

SPRANG

SPREAD

SPRQUT

SQUARE

SQUEAKY

SQUINTING

STEALING

STRODE

STRONG

STRUGGLED

STUDIEO

STUDYING

SUCCEEDED

SUCK

SUPPERTS

SURFACE

SWELLED

TARANTULA

TEARING

TELEVISION

THEMSELVES .

THEUSANDS

THREAD

TINY

TOBOGGAN

TRAVEL

TROTTED

TUGGED

TUNNELS

TURN I P

TURNOUT

TWISTED

UMLOADING

UNABLE

UNTIE

USELESS

USUAL

VACATION

VEGETABLE

- VEICLE

VISITERS

WADDLED

WANDER

WE'VE

WEAK

WHALES

WHARF

WHIRLWIND

WHOLE

WILBUR 
WISDEM

WOMEN

WORRIES

WORSE

WRIGHT

YODER

THERE ARE 357 WURDS IN LEVEL 8 GF SERIES C. IN COMPARING SERIES C WITH SERIES D, EACH AT LEVEL, 8 THERE IS A $.56 \%$ MATCH.

THERE ALSE IS A $5.04 \%$ MATCH (EUTSIDE OF LEVEL 8). RANGING FROM, $11=97$ TO $9=67$. 
THE BACK=UP WORDS FGR LEVEL 9 ARE:

$A B B I E$

ACCEPT

$\triangle C T I O N$

ADMIRATION

ADMIRE

AHOY

ALARM

ALASKA

$\triangle L L E G I A N C E$

ALPHABET

AMAZED

AMERICA

AMERICAN

AMOS

ANXI UUS

APPARENTLY

APPROVE

$\triangle R N O L D$

$A \cup N T$

AUTGMOBILES

AWAKENED

AWL

AYE

BADGE

BATES

BATTLE

BECKIE

BLACKBEARD

BLANCHARD

BLANKETS

BLGOD

BLUEBONNET

BOILED

BOTHER

BOUQUET

BOWSTRING

BRAILLEE

BRIDGE

BRISKLY

BULLFINCH

BUSILY

BUTCHER

CABINET

CALMLY

CAMERAS

CANADA

CANADIAN

CANARIES

CANVAS

CAPTURE

CARROTS

CASK

CATHERINE

CEILING

CHAMPIONSHIP

CHEERIEST

CHIEF

CLATTERED

COIN

COLLECTING 
COLLINS

COMFERTABLY

COMMANDER

COMMON

COMPANY

COMPLETELY

COMPLEX

COMPUTE

COMPUTER

CONTINUED

CBRRECT

COSTUME

COUNCIL

COURAGE

COVE

CRACKLING

CRAMMED

CREDIT

CROWNED

CRUEL

CUPBEARD

CURTAIN

DEFEATED

DEFEND

DELICIOUS

DELIGHT

DEMANDING

DENNIS

DEPEND

DESCR I BED

DESERTS

DESERVE

DESPAIR

DETECTIVE

DIAGRAM

DI AMEND

DIFFICULT

DIGNITY

DIVIDED

DOEDLE

DRAWERS

DREUGHT

DUNES

DUNN

DURING

EAGER

EAGLE

EASEL

ELECTRIC

ELECTRICAL

EMBLEM

ENEMY

ENGL ISH

ENTRANCE

ESPECIALLY

EURAPE

EXACTLY

EXAMPLES

EXCHANGE

EXCITEMENT

EXCLAIMED

EXPECTION

EXPENSIVE

EXPERIMENTAL

FAMILIAR

FANCY 
FAVUR

FIERCELY

FIFE

FIFTIETH

FLAVER

FLUSHED

FOLKS

FOREIGN

FOUGHT

FRANCE

FRECKLES

FREEDOM

FRITZ

GALE

GASPED

GIANT

GIGGLE

GLADDENED

GLANCED

GLURY

GQBLINS

GRATEFUL

GREANED

GRQUCH

GRUMPY

GUARDS

GUNPOWDER

HANDKERCHIEF

HARBQR

HAWAI I

HEARTIES

HEL ICGPTER

HELIPORT

HISTERY

HONOR

HUE

IMAGINE

INFORMATION

INNING

INTELLIGENCE

INTEND

INTRODUCED

INVITE

INWARD

IRENE

IVERSON'S

JEROME

JEWELS

JUHANN

KILDEE

LABORATORY

LARS

LATIN

LEGEND

LENGTH

LEVEL

LIBERTY

LIEUTENANT

LOOSE

LOOSENED

LEUIS

MALLARD

MARIE

MARYGQLD

MATTRESSES

MAYNARD 
MEANT

MECHANICAL

MEMORIAL

MEMORY

MENTION

MERCHANT

MESSAGES

METAL

MICROPHONE

MICROWAVE

MIDAS

MILITARY

MINNY

MISERABLE

MISTAKE

MOLTING

MEOSE

MUFFLED

MUSEUM

MUSIC

MUSICAL

MUTTERED

MYSTERIOUS

NATIEN

NEI GHBORHOOD

NOVEMBER

EARS

QBEY

ECRACOKE

OLIVE

ENION

EPPOSITE

QURSELVES

OUTSMARTED

PACKET

PANEL

PARIS

PARSLEY

PAULA

PEABODY

PEDDLER

PENELOPE

PERFECT

PERMISSION

PIANE

PILLOW

PIRATES

PLEDGE

PLUNGE

POLICE

PULISH

PESITION

POSSESSION

POSSIBLE

POSSIBLY

PRACTICE

PRAYER

PRECIUUS

PREPARE

PRETENDED

PREFESSER

PROPER

PROTECT

PUMPKINS

PUPILS

RACCEONS 
RACKET

RATHER

REALIZF

REAPPEARED

RECGGNIZE

RECOVERED

RELAYED

REPRESENT

RESPECT

RETREAT

RIEHL

ROAST

ROBOT

ROSE-PETALS

RUSHED

RYAN

SALUTE

SATELLITE

SATISFIED

SAUCER

SCARCELY

SCARY

SCATTERED

SCITUATE

SCERED

SCRUBBED

SCURRIED

SEASEN

SECTIONS

SEIZE

SELFISH

SENSE

SENSOR

SEPTEMBER

SERIOUS

SERVE

SEVERAL

SEWING

SHADEW

SHELTERED

SHELDERS

SHOVED

SHUFFLING

SILVERSPGT

SIMON

SIMPLE

SKIRTS

SLANTED

SNICKER

SNIFFED

SULVING

SPIRITS

SPOILED

SPRIG

SPRINKLE

STAMPEO

STAR - SPANGLED

STARTLED

STATUTE

STILLNESS

STIRRING

STOLEN

STOMACH

STRENGTH

SUBJECTS

SUCCFSS 
SUCCESSFULLY

\section{SUIT}

SUPERMAN

SUPERTEEL

SWENSEN

SWEPT

SWISS

SWITZERLAND

SWORD

SYMBELS

SYNCEM

TANGLED

TASTIEST

TAXI

TEAMMATES

TELSTAR

TENNIS

TEPEES

TEXAS

THIRTEEN

THEUGHTFULLY

THOUSAND-POUND

TICKETS

TOOTING

TOTING

TOUCHING

TOWER

TREASURE

TREUT

TWANG

TWIT

TYRANT

UMPIRE

UNCLE

UNEARTHED

UNHAPPILY

UNIFORM

UNITED

USABLE

VALLEY

VALUE

VENTURE

VIEW

VOYAGE

WARD

WARDEN

WARR I QR

WARSHIPS

WATERMELEN

WEALTW

WELCOME

WESTERN

WHOO-OO-OO

WHOOOO-00

WHOSE

WIDOW

WILLIAM

YANKEE

YOURSELVES

THERE ARE 394 WORDS IN LEVEL 9 OF SERIES $C$. WITH SERIES D, EACH AT LEVEL, 9 THERE IS A IN COMPARING SERIES C . 25\% MATCH. THERE ALSO IS A $1.27 \%$ MATCH IEUTSIDE OF LEVEL 9 ) RANGING FROM, $11-115$ TO $11=22$. 
THE BACKMUP WORDS FOR LEVEL 4 ARE:
ABOUT
AGAIN
$\triangle S K S$
$B A B Y$
BACK
BIRD
BIRDS
BUAT
BOLOIS
BOUNCE
BROWN
BROWN'S
BUSMAN
CALL
CALLS
CAPPY
CAPPY'S
CATS
DAYS
DECTER
EATS
FIRST
FROM
GEAT
GOATS
HAS
HIS
HURRY
JUMPED
JUMPS
JUST
KNOWS
LIGHTS
LOST
MAY
MR •
MUST
NIGHT
OR
PAINT
PICNIC
PICNICS
POCKET
RIGHT
ROCKET
SAY
SEES
SHOW
SHOWS
SECKET
STORY
TAKES
TALK
TALKS
TELL
TELLS
TREES
WHEEL
ZERO 
THERE ARE 146 WOROS IN LEVEL WITH SERIES E, EACH AT LEVEL,

4 OF SERIES $C$

IN COMPARING SERIES $C$ THERE ALSO IS A $13.01 \% \mathrm{MATCH}$ (EUTSIDE EF LEVEL 4 ) RANGING FROM, $8=117$ TO $\quad S=136$. 
THE BACK-UP WEROS FOR LEVEL 5 ARE:

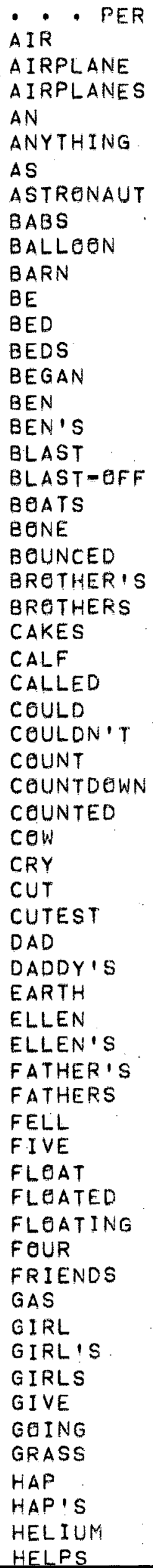


INTO

JACK

$J I M$

- JUNES

JUMPING

KITTEN

KITTENS

KNEW

LAST

LETTERS

LIKED

LIVE

LIVED

LIVES

LOOKING

LUCY'S

MAKE-BELIEVE

MANY

MEN

MEOW

MORE

MURNING

MOTHER'S

MOTHERS

NAME

NAMES

NEWS

NEXT

OLD

ERBIT

ORB ITED

ORBITS

OTHER

QTHERS

OVER

PACK

PACKAGE

PACKS

PATCHES

PETER

PETS

PIG

PLAYING

POLICEMAN

PUPPY'S

RABBIT

RAIN

READY

RECKETS

SAT

SAYS

SCARED

SCREEN

SHOWED

SISTERS

SLEEP

SNAPPER

STREET

STREETS

TALKED

TALKING

TEDDY

TEDDY'S

TERRY

TERRY'S 
THING

THINGS

TOMMY'S

TOEK

TRUCKS

TWIN

TWINS

VERY

WEIGH

WERE

WHEN

WHISTLE

WIND

WOOF

WORKS

WRITE

ZIP

THERE ARE 250 WORDS IN LEVEL 5 GF SERIES C. IN COMPARING SERIES C WITH SERIES E , EACH AT LEVEL, 5 THERE IS A $13.20 \%$ MATCH. THERE ALSO IS A $20.00 \%$ MATCH (OUTSIDE OF LEVEL 5 ) RANGING FROM, $8=192$ TO $4-15$. 
THE BACK =UP WORDS FOR LEVEL 6 ARE:

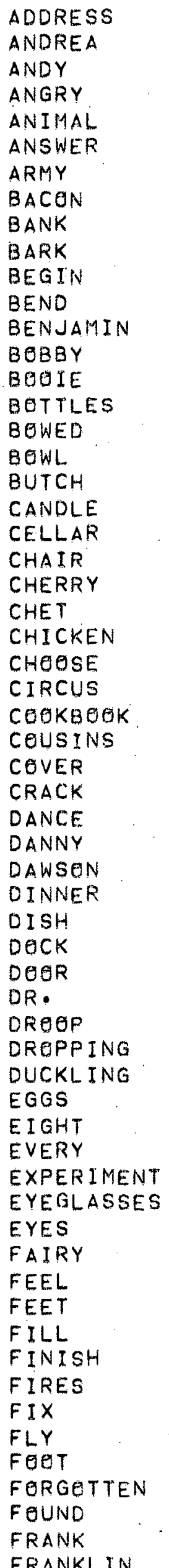


GATES

GLAD

GNOMES

GRANDER

GRANDMA

GRIND

GROW

GROWN

HA

HANGING

HAPPEN

HATCH

HIGHNESS

HEBBY

HELES

HOUND-PUP

HUNDRED

I'LL

$I \cdot M$

ICE

ITS

JENNY

KAKAI

KINDNESS

KNOWN

LANDLERD

LEFT

LIBRARIAN

LIBRARY

LECK

LONG

MARIA

MISS

MONEY

MONKEY

MRS.

MUCH

NAVY

NEDDIE

NEED

NEST

NINETY-FIVE

OLDEST

ONCE

OPENED

OUR

PAGE

PANTS

PAPER

PATRICK

PINT

PITCH

PLAYGROUND

PEPCERN

PUPPED

POPPER

RAMBLING

READ

READY-MIX

REAL

RICH

RICHARD

ROBERT

ROOM

SALT

SHAW 


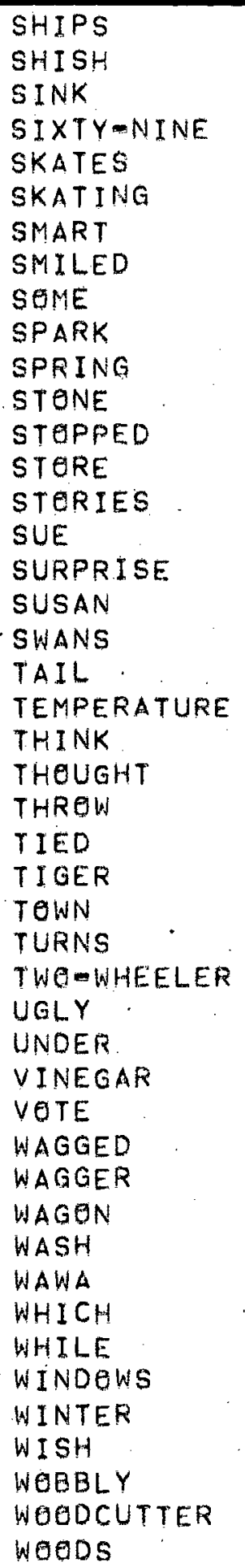

THERE ARE 315 WERDS IN LEVEL 6 OF SERIES C. IN COMPARING SERIES C WITH SERIES E ,EACH AT LEVEL, 6 THERE IS A $15.24 \%$ MATCH. THERE ALSO IS A $27.30 \%$ MATCH (OUTSIDE OF LEVEL 6) RANGING FROM, $8=191$ TO. $3=31$. 
THE BACK-UP WORDS FER LEVEL 7 ARE:

$A B B Y$

$\triangle C T I N G$

ADDRESSED

ALICE

ANNACONDA

ANYBODY

AWAKE

BASKETS

BATHE

BATHS

BEACHES

BEAR

BEHIND

BERRY

BEYOND

BLINKED

BLINKY

BLIZZARD

BLEWHOLE

BUCKSKIN

BUSY

CARPENTER

CARRIAGE

CLEAR

CLIMB

COILED

CENTESTS

COOKIES

COPY

CRAWLED

CREAKING

CURLED

CURLY $=E A R E D$

DARK

DIRECTION

DISCEVER

DIZZY

DOLPHIN

DONKEY

DEUGH

DRAINBOARD

DROWNING

ESCAPES

EXPLAIN

EXPLERE

FAMILY

FAUCET

FIELDS

FIFTH

FLUTTER

FOLLOWED

FOQD

FERCE

GRQUP

HE'D

HE'LL

HELPLESS

$H I=Y I=Y I I P P I=Y I$

HIDDEN

HEOP 
HURRIED

JUE

JUHN

JUHNNY

JONATHAN

KENNELS

LANGFERD

LANGUAGE

LAZY

LEAPED

LEAVES

LEGS

LIFEGUARD

LIFTEO

LILLY

LIEN

LOUDSPEAKER

MAILED

MANAGED

MARK

MILD

MILK

NEIGHBORS

NIBELE

NEBCDY

NETHING

NETHINGNESS

OVERLOOKING

UWL

OWLGLASS

PARTY

PASSED

PEEKING

PICTURE

PIECE

PLAYFUL

POINTED

POINTY $=E A R E D$

POPPY

POST

POTTER

PEURING

QUACKING

RAFT

RAINIEST

REACHED

RECEIVE

REMEMEER

REPEAT

REQF

REUNDISH

RUSTLE

RUSTLERS

SANG

SCAMPER

SCIENTISTS

SEARCH

SEE-ABLE

SETH

SHAG

SHINING

SKIING

SKIS

SKUNK

SMOKE

SNAKF 
THE BACK-UP WORDS FGR LEVEL 8 ARE:

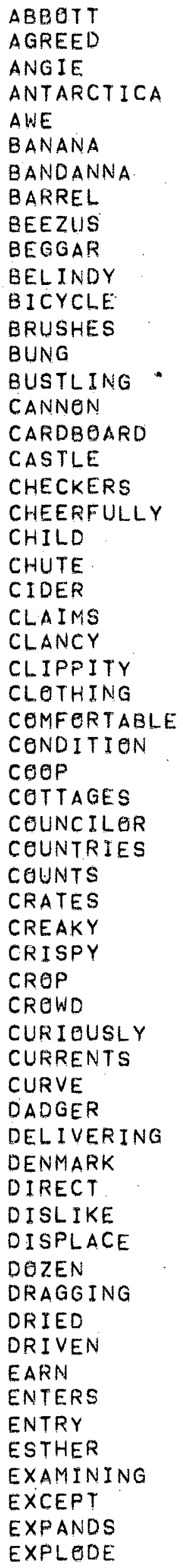


FIFTEEN=MAN

FIFTY

FIGURED

FLIERS

FLIES

FONDER

FOREMAN

FOUNDRY

FOUNTAINS

FREEZING

FRESH

FRITZL

FREWN

FRUIT

FURTHER

FURTURE

GENEVIEVE

GENTLE

GERALDINE

GOWNS

GRASP

GUES

HALF.

HANDFULS

HAPPILY

HARNESS

HAUS

HEART

HEAVENS

HEAVIER

HELPLESSLY

HENERY'S

HENRI

HERRING

HES I TATED

HIPPOPOTAMUS

HOLIDAY

HELLY

HOLMES

HOPEFUL

HOP ING

HUGGINS

HUI

HULLA

HUSTLING

I MPAT IENT

INDEED

INSECTS

JACKSON

JAGGED

JANIE

JENNIFER

JUYFULLY

JUDGES

JUICIER

JUICY

JUL I ANA

KEVIN

KINGDOM

KINNDLI

LECLERC

LIESI

LOADING

LOLLIPOP

MACHINE

MACLEBO 
MAIDSERVENTS

MAMMY

MARRIED

MARSHES

MASTS

MEECHAM

MELTED

MIRRER

MODELS

MUTOR

MOVEMENT

MUSTACHE

MYSTERY

NOTICE

NOTICING

OFFERED

ORVILLE

PAINFUL

PAPPY

PARADE

PASSAGE:

PATIENTLY

PEGGY

PENGUIN

PESTERING

PIDGEON

PIERRE

PLANKTON

POISON

POISONOUS

PERCUPINE

PRAISED

PROBLEMS

PROGRAM

PROMISE

PROVE

PUTTER

QUARTER

QUEER

QUESTIEN

QUIMBY

RAISED

REARED

REINS

REPAIR

REWARD

RIKKI

ROMONA

REUTE

SAILORS

SAKES

SANDWICH

SANTA CLAUS

SAUCEPAN

SCARING

SCRATCHING

SCREAMING

SERVICE

SEVENTEEN

SHAFTS

SHARED

SHE'LL

SHERIFF

SHOES

SHUTTLE

SINES 
THE BACK-LP WORDS FOR LEVEL 9 ARE:

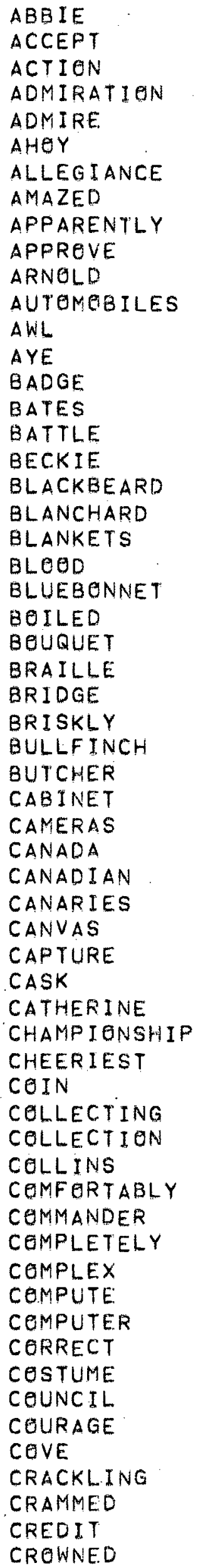


CURTAIN

DEFEATED

DEFEND

DELICIOUS

DELIGHT

DEMANDING

DENNIS

DEPEND

DESCRI BED

DESERTS

DESERVE

DESPAIR

DETECTIVE

DIAGRAM

DIAMEND

DIFFICULT

DIGNITY

DOEDLE

DRAWERS

DROUGHT

DUNES

DUNN

DURING

EAGLE

EASEL

ENEMY

ENTRANCE

EXAMPLES

EXCHANGE

EXPECTIEN

EXPENSIVE

EXPERIMENTAL

FAVOR

FLAVER

FULKS

FERE I GN

FOUGHT'

FRANCE

FRECKLES

FREEDEM

FRITZ

GALE

GATHERED

GESSLER

GLADDENED

GLANCED

GLORY

GEBLINS

GRATEFUL

GREANED

GROUCH

GRUMPY

GUARDS

GUNPEWDER

HEARTIES

HONER

HUE

INFERMATIEN

INNING

INTELLIGENCE

INTEND

INTREDUCED

INVITE

INWARD

IRENE

IVERSON'S 
JEWELS

JOHANN

KILDEE

LABORATORY

LARS

LATIN

LEGEND

LENGTH

LEVEL

LIBERTY

LIEUTENANT

LGESENED

MALLARD

MARIE.

MARYGOLD

MATTRESSES

MAYNARD

MECHANICAL

MEMORIAL

MEMORY

MENTION

MERCHANT .

MESSAGES

MICROPHONE

MICREHAVE

MIDAS

MILITARY

MINNY

MISERABLE

MISTAKE

MOLTING

MEESE

MUFFLED

MUSEUM

MUSIC

MUSICAL

MUTTERED

MYSTERIOUS

NATION

NOVEMBER

OCRACOKE

OFFICE

OLIVE

ONIEN

EPPOSITE

QURSELVES

OUTSMARTED

PACKET

PANEL

PARSLEY

PAULA

PEABQDY

PEDDLER

PENELOPE

PERMISSION

PIANE

PIRATES

PLEDGE

PLUNGE

POSSESSION

PESSIBLE

PESSIBLY

PRAYER

PROFESSOR

PROPER

PRETFCT 
PUPILS

RACKET

RATHER

REALIZE

REAPPEARED

RECOGNIZE

RECOVERED

RELAYED

REPRESENT

RESPECT

RETREAT

RIEHL

REAST

ROBOT

RESE-PE.TALS

RUSHED

RYAN

SALUTE

SATELLITE

SATISFIED

SAUCER

SCARCELY

SCITUATE

SCERED

SCURRIED

SEASON

SECTIONS

SEIZE

SELFISH

SENSE

SENSOR

SEPTEMBER

SERIOUS

SERVE

SEWING

SHADOW

SHELDERS

SHUFFLING

SILVERSPOT

SIMON

SIMPLE

SKIRTS

SLANTED

SNICKER

SOLVING

SPRINKLE

STAR-SPANGLED

STATUTE

STILLNESS

STIRRING

STELEN

STOMACH

STRENGTH

SUB JECTS

SUCCESS

SUCCESSFULLY

SUPERMAN

SUPERTOOL

SWENSON

SWEPT

SWISS

SWITZERLAND

SWORD

SYMBELS

SYNCEM

TANGLED 
TASTIEST

TAXI

TEAMMATES

TELSTAR

TENNIS

TEPEES

THIRTEEN

THOUGHTFULLY

THOUSAND - POUND

TICKETS

TOQTING

TETING

TOUCHING

TOWER

TREASURE

TROUT

TWANG

TWIT

TYRANT

UMPIRE

UNCLE

UNEARTHED

UNHAPPILY

UNIFERM

UNITED

USABLE

VIEW

VGYAGE

WARD

WARDEN

WARRIOR

WARSHIPS

HATERMELON

WEALTH

WELCOME

WESTERN

$M H O O-00-00$

WHOOOO-OO

WHOSE

WIDOW

YANKEE

YOURSELVES

THERE ARE 394 WORDS IN LEVEL

9 OF. SERIES C. IN CEMPARING SERIES C WITH SERIES E, EACH AT LEVEL, 9 THERE IS A . OO\% MATCH,

THERE ALSO IS A $17.77 \%$ MATCH (GUTSIDE OF LEVEL 9). RANGING FROM, $8-240$ TO $5-226$. 
THE BACK -UP WORDS FOR LEVEL 3 ARE:
DADDY
DAVID
JUMP
MARK
PATTY
RIDE
TINA

THERE ARE 15 WORDS IN LEVEL 3 GF SERIES D. IN COMPARING SERIES D WITH SERIES A , EACH AT LEVEL, 3 THERE IS A . OO\% MATCH. THERE ALSO IS A $46.67 \%$ MATCH IOLTSIDE OF LEVEL 3) RANGING FROM, $7=18$ TO 1 - 11 . 
THE BACK-UP WORDS FER LEVEL 4 ARE:
ADAM
GET
JUMPS
PEDRE
SAYS
WANT
WANTS
WITH

THERE ARE 33 WOROS IN LEVEL 4 OF SERIES D. IIN COMPARING SERIES D WITH SERIES A EACH AT LEVEL, 4 THERE IS A 18.18\% MATCH.

THERE ALSO IS A 51.52\%. MATCH (UUTSIDE OF LEVEL 4 ) RANGING FROM, $7=50$ TO $1-5$. 
THE BACK-UP WQRDS FGR LEVEL 5 ARE:
BROTHER
DAVIDIS
GEING
HE
METHER'S
ENES
RIDES
SEES
SECKS
STEPS
THANK
THAT
TINA'S
WALKS
WILL
YOURS

THERE ARE 48 WORDS IN LEVEL 5 OF SERIES D. IN COMPARING SERIES D WITH SERIES A. EACH AT LEVEL, 5 THERE IS A $16.67 \%$ MATCH.

THERE ALSE IS A $37.50 \%$ MATCH (OUTSIDE OF LEVEL 5 ) RANGING FROM, $7=10$ TO 1 - 25 . 
THE BACKMUP WORDS FOR LEVEL 6 ARE:

IRITING
SOMEONE
TOWN

THERE ARE 18 WERDS IN LEVEL 6 OF SERIES D. IN COMPARING SERIES D WITH SERIES A , EACH AT LEVEL, 6 THERE IS A $5.56 \%$ MATCH.

THERE ALSO IS A $72.22 \%$ MATCH (GUTSIDE OF LEVEL 6 ) RANGING FROM. $5=149$ TO $1-5$. 
THE BACK=UP WURDS FOR LEVEL 7 ARE:

ANDY

BAD

BALL

BASEBALL

BEARS

BIRTHDAY

BIRTHDAYS

CAKE

CALF

CAME

CARD

CARLOS

CATCH

COAT

COLT

DIME

FASTER

FEEL

FLYING

GAME

GAMES

GIVES

GEMEZ.

GRANDMETHER

GRASS

HAMBURGER

HAMBURGERS

HELP

ICE CREAM

JOHN

JOHN'S

KITE

KNOWS

$\angle A M B$

MARY

MAYS

MISS

MOTHERS

$M R$

MRS .

NEWS

NICKEL

NICKELS

NIGHT

O.K.

PARTY

PATTY'S

PICKLE

PICKLES

FICTURE

PICTURES

PIGEON

PIGEONS

PLAYS

PUPPET

PUPPETS

PUPPY

RAINCEAT

SCRAPBOEK

SHADEW

SHADOWS 
SHEEP

SISTER'S

SISTERS

SKATE

SKATES

SNAKE

SNAKES

SOCK

SQUIRRELS

SURPRISES

SWEATER

SWIM

$T=S H I R T$

$T=S H I R T S$

TAIL

TELL

THAT'S

TING-AMLING

UNCLE

WATERS

WHITE

WHO

YOURSELF

THERE ARE 161 WURDS IN LEVEL 7 OF SERIES D. IN COMPARING SERIES D WITH SERIES A , EACH AT LEVEL, 7 THERE IS A $4.35 \%$ MATCH. THERE ALSO IS A 32.30\% MATCH IOUTSIDE OF LEVEL 7 ) RANGING FROM, $9=173$ TB $1=5$. 
THE BACK-UP WORDS FOR LEVEL 8 ARE:

ACORN
ACERNS
ACRESS
ADAM!S
ALIKE
ALENG
AN

ANIMAL

ANIMALS

ANETHER

ANSWER

ANSWERS

ASLEEP

ASTRENAUT

BACKWARD

BARGAIN

BARK

BARN

BLUE-EYED

BOLD

$B B O K$

BOOKS

BORN

BRANCH

BREWN-EYED

CALL

CALLED

CALLS

CANNOT

$C A P$

CAR

CARS

CATBIRD

CHASE

CHITTER = CHATTER

CLOVER

COLTS

COMPASS

CQVER

CRESS

CRY

DEORWAY

DRY

END

EVERYONE

EYE

EYES

FARMS

FEELING

FENCE

FIND

FLEW

FOLLOWEO

FUUR-LEAF

FEX

FRIEND

FRIENDS

FROGS

FREM

FRENT

GATE 
GETS

GQOD-BY

HANDS

HAPPENEO

HARK

HERSELF

HIS

HOLE

HEUSE

INSIDE

INTO

KEEN

KIM'S

LANDED

LARK

LAUGH

LAUGHED

LEFT

LEG

LIKED

LISTEN

LEOKED

MASTER

MAYBE

MENKEYS

MUCH

MUD

NEWBERN

NEXT

NEXT-BEST'

NONSENSE

NOSE

OLDTEWN

ONE-YEAR-OLD

OUTDEERS

OWL

GWN

PAINT

PAINTS

PAJAMAS

PATTED

PEEPED

PLAYTIME

POCKET.

POKED

PONY

RIBBEN

RIDDLE

RIDDLES

RUNN ING

SAFE

SATURDAY

SCAMPERED

SEEING

SHARK

SHIRT

SINGS

SKIP

SLOW

SLOWPOKE

SLOWPOKES

SMART

SEFTLY

SELD

SOMETIME

SEMETIMES 
SPACE

SPARK

SPARKS

SPELL

SPELLER

SPELLS

SPRING

SPRINGTIME

START

STARTED

STARTS

STICK

SUIT

SUITS

TALKEO

TALKING

TEASE

THAN

THREE = YEAR - OLD

TONIGHT

TRIP

TUMBLING

TURN

$T W O-Y E A R=\theta L D$

UPON

WALKED

WANTED

WARM

WHIZZED

WISE

WISH

WI THOUT

WOODLAND

WOODS

WORKOUT

WORKS

YEARS

THERE ARE 281 WUROS IN LEVEL 8 OF SERIES D.. IN COMPARING SERIES D WITH SERIES A EACH AT LEVEL, 8 THERE IS A $1.07 \%$ MATCH. THERE ALSO IS A $32.03 \%$ MATCH (BUTSIDE OF LEVEL 8) RANGING FROM, $7=170$ TO $1-30$. 
THE BACK = UP WORDS FOR LEVEL 9 ARE:

ACTOR
ACTORS
AFTERNGON

ANYONE

ANYWHERE

ASK

ASKED

ASKS

BACKYARD

$B A D$

BARKING

BAT

BEING

BESIDE

BIRDS

BLAME

BLAMED

BROWN'S

BUDDIES

BUDDY

CABIN

CABINS

CAKE

CALLING

CAME

CAN'T

CAR

CARE

CHAIR

CHASED

COMING

COULDN'T

COUNT

COUNTING

DADDY'S

DADS

DAVE

DETECTIVES

DRIVES

DRIVING

EVER

EVERY

EVERYTHING

EVERYWHERE

FALL'

FALLING

FAULT

FELLOW.

FFEUND

FINDING

FINDS

FOREVER

FORGIVE

FERGOT

$F E X$

FRIENDLY

FRENT-PAGE

FUNNY

GANG

GARAGE 


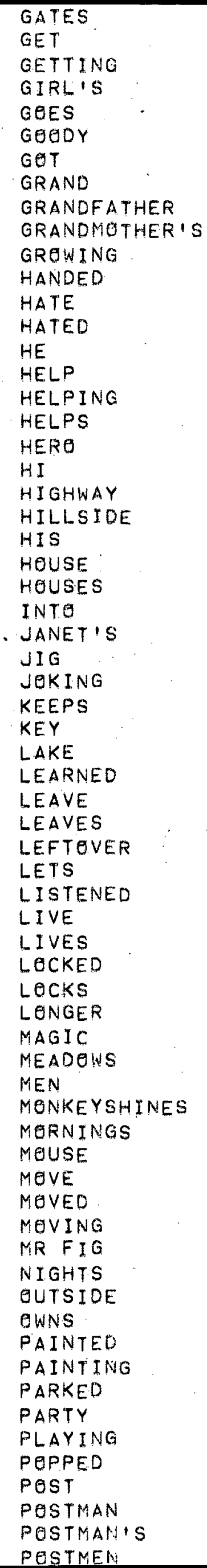


PULLED

PULLING

RAINED

REDS

REDWOOD

RIDE

ROUNDS

RUSTY

RUSTY'S

SAD

SAYING

SEEMED

SHOUT

SHOUTING

SHOWING

SING

SKATER

SOMEHOW

SONG

SEON

SPQOKIER

SPOEKY

STAND

STANDS

STARTING

STATION

STAYED

STAYS

STEP

STEPS

STILL

STOPPED

STREETS

SUNDAY

SURPRISE

SURPRISED

SURPRISING

SWAM

TAKE

TAKES

TELL

TELLING

TELLS

THAT

THEY

THINKING

TIRED-QUT

TODAY

TOGETHER

TRACK

TRACKS

TREES

WAGGED

WAGG ING

WAGON

WAIT

WAITED

WAITING

WALKER

WALKING

WASN I T

WELLS

WHISPER

WHISPERED

WHISPERING

WILL 
WITCH

WITH

WONDERING

WORKED

WORKING

WRONG

THERE ARE 262 WURDS IN LEVEL 9 OF SERIES D. IN COMPARING SERIES D WITH SERIES A, EACH AT LEVEL, 9 THERE IS A $.76 \%$ MATCH.

THERE ALSO IS A $14.50 \%$ MATCH IOUTSIDE OF LEVEL 9 ) RANGING FROM, $8-206$ TO $2=51$. 
THE BACK=UP WORDS FOR LEVEL 11 ARE:

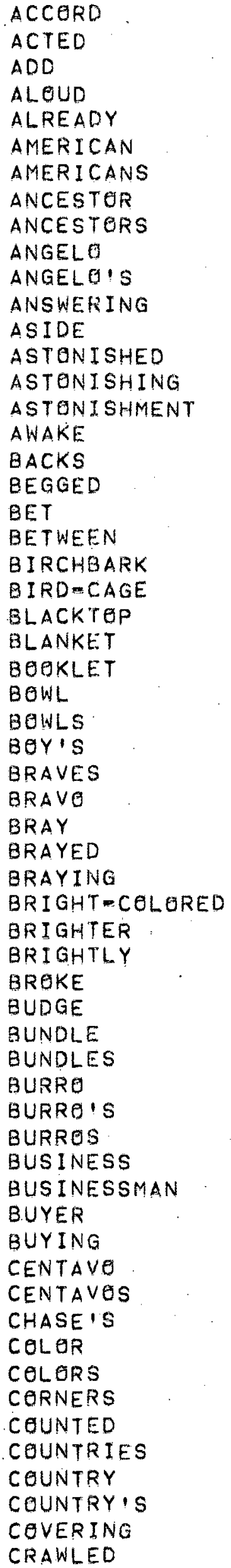


CROWD

CREWDED

CROWDS

DANCING

DARKNESS

DAY'S

DAYDREAM

DEEP

DESCENDANT

DESCENDANTS

DISAPPOINTMENT

DISTANCE

DE-NETHING

DROP

DROPPED

DUCKED

DUSTY

EACH

EATING

ELSE'S

EXCITED

EXCITING

EXCLA IMED

FEED

FEEDING

FEET

FELL

FIELDS

FINISHED

FIREWOED

FELDED

FELLOWING

FURGQTTEN

FORWARD

GOODS

GRANOFATHER'S

GRANDFATHERS

GREAT $=$ GRANDFATHERS

GREAT $=$ GREAT $=$ GRANDFAT

GREATEST

GREENF I ELD

GREWN

GROWS

GUESS

HANDSOME

HANDWORK

HAPPENING

HAPPILY

HARD - EARNED

HAROWORKING

HARNESS

HARNESSEO

HAS

HAT

HELLE

HIDDEN

HILLS

HILLTEP

HOMEWARO

INDIAN

INDI ANS

ITALY

KNOWING

KNEWINGLY

KOBO

LAMBS 
PUSHERS

PUSHING

PUZZLED

RAGGED

RAMON

RAMON'S

READING

REINS

RESTED

RETOLD

RETURNED

RIDING

RUBBING

SAFELY

SANDINO

SANDINO'S

SCHOULROOM

SCREECHED

SCREECHING

SEAT

SECOND

SERAFINA

SERAFINA'S

SERAPE

SERAPES

SHADE

SHADEWY

SHAFTS

SHEPHERD

SIDES

SIDEWAYS

SLEEPIER

SLIPPED

SLOWED

SOMEWHAT

SOUNER

SPARKLING

SPQT

SPOTS

SPRINGFIELD

STALL

STARE

STARED

STARING

STEEP

STRAPS

STRIPES

STUBBORN

SUCH

SUNNY

SWIMS

TALE

TALES

TEARS

TEMPERAMENTAL

TEN

TIED

TIRES

TOES

TRADING

TREE-SHADED

TREETOPS

TRUE.

TRY

TRYING

TUNE 
TUNES

TV

TWENTY

TWENTY - QNE

TWE-WHEELED

UNDERSTAND

UNDERSTANDS

UNWILLINGLY

UPTURNED

UPWARD

VOICES

WALL

WATCH

WATERIS

WAVED

WAYSIDE

WEAVER

WELL-MADE

WHITE-HAIRED

WHIZZING

WILLINGLY

WINTERS

WINTERS I

WISEST

WISHING

WORKMAN

WERLD

WGRTH

WRITTEN

2I PEPPI'S

THERE. ARE 399 WORDS IN LEVEL 11 OF SERIES D. IN COMPARING SERIES D WITH SERIES A, EACH AT LEVEL, 11 THERE IS A $.00 \%$ MATCH.

THERE ALSE IS A $14.54 \%$ MATCH (OUTSIDE OF LEVEL 11 ) RANGING FROM, $g=157$ TO $2=34$. 
THE BACK-UP WORDS FQR LEVEL 3 ARE:
DAVID
JANET
PATTY
TINA

THERE ARE 15 WURDS IN LEVEL 3 EF SERIES D. IN COMPARING SERIES D WITH SERIES B , EACH AT LEVEL, 3 THERE IS A $6.67 \%$ MATCH.

THERE ALSO IS A $60.00 \%$. MATCH IOUTSIDE OF LEVEL 3) RANGING FROM, $5=66$ TO $1=83$. 
THE BACK-UP WORDS FOR LEVEL 4 ARE:
ADAM
HAVE
IN
JUMPS
ME
MY
PEDRE
SAYS
THIS
WE

THERE ARE 34 WORDS IN LEVEL 4 OF SERIES D. IN COMPARING SERIES D WITH SERIES B ,EACH AT LEVEL, 4 THERE IS A .00\% MATCH. THERE ALSE IS A $67.65 \%$ MATCH IOUTSIDE OF LEVEL 4.) RANGING FROM, 3- 4 TO $1=12$. 
THE BACK-UP WORDS FOR LEVEL 5 ARE:
ARE
QE
BRETHER
DAVIDIS
GOING
HOME
LOOKING
LOOKS
MARKS
MAY
MOTHER!S
NO
ONES
SEES
SECKS
THAT
TINA'S
TOE
WALKS
YEUR
YOURS

THERE ARE 48 WEROS IN LEVEL 5 EF. SERIES D. IN COMPARING SERIES D WITH SERIES $B$, EACH AT LEVEL, 5 THERE IS A $2.08 \%$ MATCH. THERE ALSO IS A 50.00\% MATCH IOUTSIDE OF LEVEL 5 I RANGING FROM, $3=53 \mathrm{TO} 1-11$. 
THE BACK -UP WORDS FOR LEVEL 6 ARE:

IRITING
GOQD
HER
SOMEENE

THERE ARE 18 WORDS IN LEVEL 6 OF. SERIES D. IN COMPARING SERIES D WITH SERIES B , EACH AT LEVEL, 6 THERE IS A .0O\% MATCH. THERE ALSO IS A $72.22 \%$ MATCH (OUTSIDE OF LEVEL 6) RANGING FROM, 3-170 TO $1-139$. 
THE BACK=UP WORDS FOR LEVEL 7 ARE:

ANDY

BASEBALL

BEAR

BED

BOYS

CARLOS

DIME

DUCK

DUCKS

EARRINGS

FASTER

FREG

GAME

GAMES

GRANDMOTHER

HAMBURGER

HAMBURGERS

ICE CREAM

IT'S

JUHN

JUHN'S

KIM

KITE

MAYS

MEADEW

MOTHERS

U.K.

PATTYIS

PETE

PICKLE

PICKLES

PICTURE

PICTURES

PIGEON

PIGEENS

POND

PULL

PUPPET

PUPPETS

PUPPY

RAINCOAT

SCRAPBOEK

SISTER'S

SISTERS

SKATE

SKATES

SNAKE

SNAKES

SECK

SOMEDAY

STAYING

STEVE

SURPRISES

SWEATER

SWIM

$T$-SHIRT

T-SHIRTS

TAIL

TALK

THAT'S

TING $A=1$ ING 
THERE ARE 161 WORDS IN LEVEL 7. OF SERIES D. IN COMPARING SERIES D WITH SERIES B , EACH AT LEVEL, 7 THERE IS A . OO\% MATCH. THERE ALSE IS A $47.20 \%$ MATCH (EUTSIDE OF LEVEL 7 ) RANGING FROM. $6-256$ TO $1=4$. 
THE BACK-UP WORDS FUR. LEVEL \& ARE:

ADAM'S

$A G O$

AHEAD.

ALIKE

ANIMAL

ANIMALS

ANY

ANYTHING

ANYWAY

APPLE

ASLEEP

ASTRONAUT

BACKWARD

BARGAIN

BARK

BARN

$B L U E=E Y E D$

BELD

BOOK

BOOKS

$B R O W N=E Y E D$

CALL

CALLED

CALLG

CANNOT

CAP

CAR

CATBIRD

CHITTER - CHATTER

CLOVER

COLTS

COMPASS

CUVER

CROSS

CRY

DEORWAY

END

EYE

FIND

FQRGET

FEUR-LEAF

GETS

$G O O D-B Y$

HANDS

HIMSELF

ITS

ITSELF

KEEN

KIM'S

LANDED

LARK

LAUGH

LAUGHED

LEARN

LIKED

LUCK

MAD

MADE

MENKEYS

MEON

NEWBORN 
NEXT

NEXT - BEST

NONSENSE

OLDTGWN

ONE-YEAR-OLD

OUTDOORS

PATTED

PEEPED

PLAYTIME

PONY

QUACK

RIBBON

RIDDLE

RIDDLES

ROCKET

SAFE

SEEING

SHARK

SHEUTED

SHOW

SHOWED

SINGS

SKIP

SLOW

SLOWPOKE

SLOWPOKES

SMART

SOMETIME

SOMETIMES

SPACE

SPARK

SPARKS

SPELI.

SPELLER

SPELLS

STARTED

STARTS

STICK

SUIT

SUITS

TALKED

TALKING

TEASE

THREE $=$ YEAR $=Q L D$

TON I GHT

TUMBLING

TURTLE

$T W O-Y E A R=O L D$

UPON

WALKED

WHIZZED

WI THOUT

WONDER

WOOD

WOEDLAND

WOODS

WORKOUT

WORKS

YEARS

THERE ARE 281 WORDS IN LEVEL 8 OF SERIES D. IN COMPARING SERIES D WITH SERIES $B$, EACH AT LEVEL, 8 THERE IS A .00\% MATCH, THERE ALSO IS A $45.20 \%$ MATCH (UUTSIDE OF LEVEL 8 ) RANGING FROM, 6-256 TO 1-149. 
THE BACK -UP WOROS FGR LEVEL 9 ARE:

ANYONE

ANYWHERE

BAT

BECAME

BEING

BUDDIES

BUDOY

CALLING

CAR

CHASED

DAVE

DETECTIVE

DETECTIVES

DQG

DQGHQUSE

DQGS

DOING

DRIVE

DRIVES

ORIVING

EVERYWHERE

FFOUND

FINDING

FINDS

FOREVER

FORGIVE

FORGET

FRIENDLY

FRONT=PAGE

GANG

GARAGE

GARAGES

GATES

GOODY

GOT

GRANO

GRANDFATHER

GRANOMOTHER'S

GROWING

HANDED

HAPPEN

HARDLY

HATE

HATED

HERE

$\mathrm{HI}$

HIGHWAY

HILLSIDE

JANET'S

JIG

JOKING

KEEPS

KNOWN

LAKE

LETS

LISTENED

LOCKS

LONGER

MEADOWS

MEM 
MOENLIGHT

MORNINGS

MEUSE

NIGHTS

NGON

NOENTIME

NOT $=T O O-B I G$

OUTSIDE

OWNS

PAINTED

FAINTING

PARKED

PAT

PLAYING

POPPED

PULLING

REOS

REDWEOD

ROUNDS

RUSTY

RUSTY'S

SAD

SAYING

SECRET

SKATER

SLEEP

SMILE

SMILED

$S O=S O$

SOMEHOW

SPEOKIER

SFGOKY

STAND

STANDS

STARTING

STAYED

STAYS

STEPPED

STREETS

SUNDAY

TAKES

THAT

THINKING

TIRED-BUT

TODAY

TRACK

TRACKS

TREES

WAGGED

WAGGING

WAGON

WAIT

WAITED

WAITING

WALKER

WALKING

WELLS

WHERE

WHISPER

WHISPERED

WHISPER ING

WITCH

WITH

WONDERING

WORKED

WERK ING 
THERE ARE 262 WORDS IN LEVEL 9 OF SERIES D. IN COMPARING SERIES D WITH SERIES B , EACH AT LEVEL, 9 THERE IS A . O0\% MATCH.

THERE ALSQ IS A $29 \cdot 39 \%$ MATCH IOUTSIDE OF LEVEL 9 ) RANGING FROM, $6=285$ TO $1-123$. 
THE BACK-UP WORDS FOR LEVEL 11 ARE:

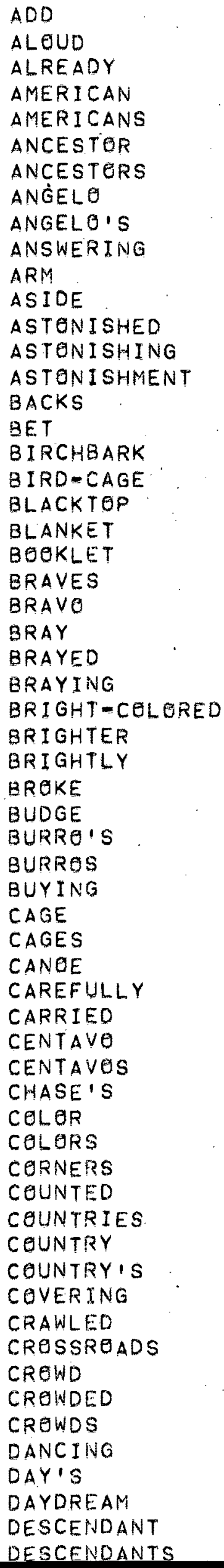


DISAPPOINTMENT

DO-NOTHING

DOLL

DONKEY

DONKEYS

DRIVER'S

DUSTY

EACH

EATING

ELSE'S

EXPLAIN

EXPLAINED

FARMER

FARMERS

FASTEN

FASTENED

FATHER

FATHER'S

FEATHERS

FIELDS

FIREWOOD

FOLDED

FOLLOWING

FORGOTTEN

GATHER

GATHERED

GBODS

GRANDFATHER'S

GRANDFATHERS

GRAY

GREAT $\approx$ GRANDFATHERS

GREAT $\approx$ GREAT $=$ GRANDFAT

GREATEST

GREENFIELD

GROWN

GROWS

GUESS

HANDSGME

HANDWURK

HAPPENING

HAPPILY

HARD -EARNED

HARDWORKING

HARNESS

HARNESSED

HAS

HAT

HIDDEN

HILLS

HILLTEP

HOLES

HOMEWARD

HOPED

HOPPED

ITALY

JAPAN

JAR

JARS

JINGLED

KINDLY

KNOWING

KNOWINGLY

KOBO

LAMBS

LATER

LAUGHJNG 
LAUGHS

LAZIEF

LAZY

LEAD

LEADER

LEADS

LET'S

LIVELY

LONG $=L O S T$

LEOKEUT

LOUDLY

LOVED

LOVINGLY

MAMA

MAN'S

MANY-COLERED

MAP

MAPS

MARCO

MARCO'S

MARKER

MARKET

MERR ILY

MERRY

MERRY =GO-ROUND

MEXICO

MIRRER

MIRROS

MISSES

MIST

MISTS

MIX

MIXED

NEEDS

NEWLY

NIGHTTIME

NURTH

OATS

OLDER

OLDEST

ONLOOKERS

BNTE

OWNER

OWN ING

PAGES

PAPA

PASSED

PEEPING

PIECE

PIECES

PLACED

POINTED

POINTING

PQNIES

PUOR

POTTER

POTTERS

POTTERY

PREUD

PROUDLY

RAMEN

RAMEN'S

READING

REAL

RESTED

RETELD 
RETURNED

RIVER

SANDINE

SANDINEIS

SCHOELREOM

SCREECHED

SCREECHING

SERAF INA

SERAFINA IS

SERAPE

SERAPES

SHADE

SHADOWY

SHAFTS

SHEPHERD

SIDES

SIDEWAYS

SIGN

SLEEPIER

SLIPPED

SLOWED

SEMEWHAT

SEONER

SPETS

SPRINGFIELO

STARE

STARED

STARING

STORYTELLER

STERYTELLER'S

STRAP

STRAPS

STUBBORN

SUNNY

SWIMS

TALE

TALES

TEMPERAMENTAL

TEN

TIRES

TRADING

TREE-SHADED

TREETOPS

TRIPS

TROT

TUNES

TV

UNDERSTAND

UNDERSTANDS

UNWILLINGLY

UPTURNED

UPWARD

VEICES

WATCH

WATER!S

WAVED

WAYSIDE

WEAVER

WELL-MADE

WHEEL

WHENEVER

WHITE-HA IRED

WHIZZING

WILLINGLY

WINDEWS

WINTERS 
WINTERS :

WISEST

WISHING

WERKMAN

WORLD

WRITTEN

ZI PEPPI'S

THERE ARE 399 WORDS IN LEVEL 11 GF SERIES D. IN COMPARING SERIES D WITH SERIES B , EACH AT LEVEL, 11 THERE IS A . O0\% MATCH.

THERE ALSO IS A $18.80 \%$ MATCH IOUTSIDE OF LEVEL 11 ) RANGING FROM, $6=236$ TO $1=160$. 
THE BACKUP WORDS FUR LEVEL 3 ARE:

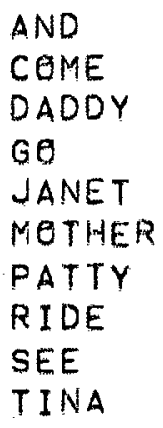

THERE ARE 15 WORDS IN LEVEL 3 OF SERIES D. IN COMPARING SERIES D WITH SERIES C , EACH AT LEVEL, 3 THERE IS A. .00\% MATCH. THERE ALSO IS A $26.67 \% \mathrm{MATCH}$ (EUTSIDE OF LEVEL 3) RANGING FROM, 7-109 TO 4- 36 . 
THE BACK - UP WORDS FER LEVEL 4 ARE:
$A$
ADAM
CAN
CAN'T
CQMES
FUR
GET
HERE
I
Is
IT
NOT
UN
PEDRE
SOMETHING
THE
TO
WANT
WANTS
WITH
You

THERE ARE 33 WORDS IN LEVEL 4 OF SERIES D. IN COMPARING SERIES D WITH SERIES C , EACH AT LEVEL, 4 THERE IS A $30.30 \%$ MATCH. THERE ALSE IS A $6.06 \%$ MATCH (OUTSIDE OF LEVEL 4 ) RANGING FROM, $5=100$ TO $5=25$. 
THE BACK-UP WGRDS FOR LEVEL 5 ARE:
BIG
BLUE
DAVID'S
DID.
De
$H E$
LIKE
LIKES
LITTLE
LOOK
LGGKS
MAKE
MAKES
MARKS
NOW
QNES
PLAY
RIDES
SAID
SOCKS
STeP
STOPS
TINA'S
WALKS
WHAT
WILL
YES

THERE ARE 48 WORDS IN LEVEL 5 OF SERIES D. IN COMPARING SERIES D WITH SERIES C EACH AT LEVEL, 5 THERE IS A $16.67 \%$ MATCH. THERE ALSE IS A $25.00 \%$ MATCH (OUTSIDE OF LEVEL 5 ) RANGING FROM, $6=15 T \theta 4=129$. 
THE BACK=UP WORDS FGR LEVEL 6 ARE:

IRITING

DOWN

GREEN

RED

SOMEONE

UP

THERE ARE

17 WURDS IN LEVEL G OF SERIES D.

IN COMPARING SERIES D WITH SERIES C , EACH AT LEVEL, 6 THERE IS A $11.76 \%$ MATCH.

THERE ALSO IS A $52.94 \%$ MATCH (OUTSIDE OF LEVEL 6) RANGING FROM, $5=82 T O 4=88$. 
THE BACK $=$ UP KORDS. FOR LEVEL 7 ARE:

BALL

BASEBALL

BEARS

CARD

CARLOS

CEAT

COLT

DIME

DUCK

DUCKS

EARRINGS

EAT

FASTER

FROG

GAME

GAMES

GRANDMETHER

HAIR

HAMBURGER

HAMBURGERS

HOP

ICE CREAM

ISN' T

IT'S

JOHN'S

KIM

LAMB

MARY

MAYS

MEADOW

$M R$

NICKEL

NICKELS

NIGHT

Q.K.

PATTY'S

PETE

PICKLE

PICKLES

PICTURES

PIGEON

PIGEENS

PLAYS

PUI.L

PUPPET

PUPPETS

PUPPY

RAINCEAT

RAN

REST

SCRAPBOOK

SISTER'S

SISTERS

SKATE

SKATES

SKY

SNAKES

SOCK

SOMEDAY

SQUIRRELS SUN 
SUN-YEE

$T-S H I R T$

TOSHIRTS

TAIL

TING $=A-L I N G$

TREE

WATERS

WHO

THERE ARE 162 WORDS IN LEVEL 7 OF SERIES D. WITH SERIES $C$, EACH AT LEVEL, 7 THERE IS A

IN COMPARING SERIES D THERE ALSO IS A $40.74 \%$ MATCH. IOUTSIDE OF LEVEL 7 ) RANGING FROM, $9=266$ TO $4=189$. 
THE BACK-UP WORDS FOR LEVEL 8 ARE:

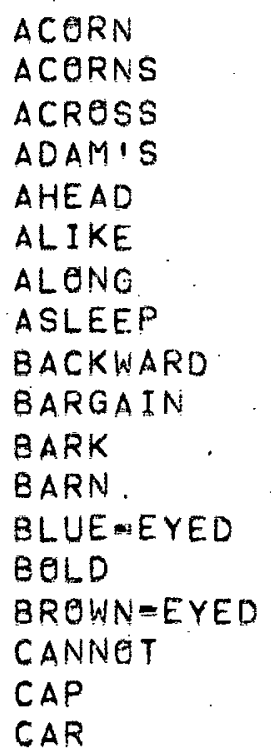


MOEN

NEWBGRN

NOSE

OLDTOWN

CNE-YEAR - OLD

OUTDOORS

PATTED

PEEPED

PLAYTIME

POKED

PENY

QUACK

RIBBON

RIDDLE

RIDOLES

ROUND

RUNNING

SAFE

SAME

SCAMPERED

SEE ING

SHARK

SHIRT

SHOUTED

SHUT

SIDE

SING

SINGS

SKIP

SLOW

SLOWFGKE

SLOWPEKES

SOFTLY

SOLD

SOMET IME

SOMETIMES

SPARKS

SPELL

SPELLER

SPELLS

STARTS

STICK

TEASE

THAN

$T H R E E-Y E A R=O L D$

TONIGHT

TRADE

TURN

TWG-YEAR-OLD

WAY

WELL

WENT

WERE

WHIZZED

WISE

WISH

WITHOUT

WeED

WEEDLAND

WOEDS

WERKEUT

WORKS

YEAR

YEARS 
THERE ARE 281 WORDS IN LEVEL

WITH SERIES $C$, EACH AT LEVEL,

IN COMPAR ING SERIES D

THERE ALSO IS A $40.57 \%$ MATCH IOUTSIDE OF LEVEL

$g=271$ TO $4-172$.

8) RANGING FROM, 
THE BACK=UP WORDS FOR LEVEL 9 ARE:

ACTOR

ACTORS

ANYONE

ANYWHERE

ASK

ASKED

ASKS

BACKYARD

BARKING

BAT

BECAME

BEING

BELONG

BELONGED

BELONGS

BESIDE

BLAME

BLAMED

BUDDIES

BUDDY

CABIN

CABINS

CALLING

CAN'T

CAR

CHASED

COUNTING

CRYING

CUTTING

DAD

DADDY'S

DADS

DAVE

DETECTIVES

DRIVES

DRIVING

EVERYTHING

EVERYWHERE

FALLING

FAULT

FELLOW

FFEUND

FINDING

FINDS

FUREVER

FERGIVE

FORGOT

FRIENDLY

FRONT-PAGE

GANG

GARAGE

GARAGES

GATES

GET

GEEDY

GET

GRAND

GRANDFATHER

GRANOMUTHER'S

GROWING

HANDED 
HARDLY

HATE

HATED

HE

HERO

HI

HIGHWAY

HILLSIDE

JANET'S

$J I G$

JUKING

KEEPS

L.AKE

LEARNED

LEAVE

LEAVES

LEFTEVER

LETS

LISTENED

LIVE

LOCKED

LOCKS

LONGER

LUNCH

MEADOWS

MENKEYSHINES

MOENLIGHT

MURNINGS

NEVER

NIGHTS

NEON

NOONTIME

NOT-TOE-BIG

NOTHING

UWNS

PAINTED

PAINTING

PARKED

PAT

PULLING

REDS

REDW66D

RIDE

ROUNDS

RUSTY

RUSTY'S

SAD

SAYING

SING

SKATER

SMILE

SMILED

SO-SO

SOMEHEW

SENG

SUQN

SPEOKIER

SPEOKY

STAND

STANDS

STARTING

STATION

STAYED

STAYS

STEP

STEPS 
STILL

SUNDAY

THINKING

TIREC-OUT

TODAY

TOGETHER

TRACK

TRACKS

WAGG ING

WALKER

WALKING

WASN'T

WELLS

WHISPER

WHI SPERED

WHISPERING

WILL

WITCH

WITH

WONDERING

THERE ARE 262 WORDS IN LEVEL 9 OF SERIES D. IN COMPARING SERIES D WITH SERIES C , EACH AT LEVEL, 9 THERE IS A $.76 \%$ MATCH.

THERE ALSE IS A 30.92\% MATCH (EUTSIDE OF LEVEL 9) RANGING FROM, $8=245$ TO $4=51$. 


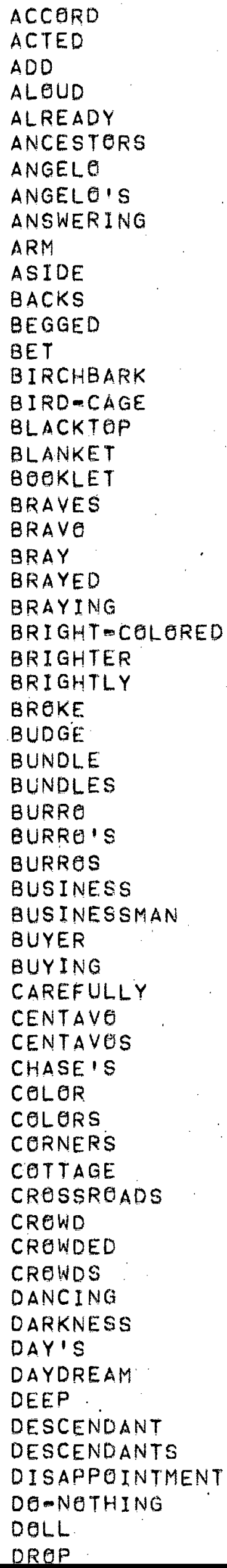


DRSPPED

DUCKED

DUSTY

EACH

EATING

ELSE'S

EXCITED

FINE

FINEST

FINISHED

FIREWOUD

FOLDED

FOLLOWING

GARDEN

GATHER

GATHERED

GLADLY

GOATSKIN

GOODS

GRANDFATHER'S

GRANDFATHERS

GRAY

GREAT - GRANDFATHERS

GREAT $=$ GREAT $=$ GRANDFAT

GREATEST

GREENFIELD

HANDSOME

HANDWERK

HAPPENING

HAPPILY

HARD-EARNED

HARDWORKING

HARNESS

HARNESSED

HAS

HAT

HILLS

HILLTOP

HEMEWARD

HOPPED

ITALY

JINGLED

KINOLY

KNOWING

KNOWINGLY

KeBO

LAMBS

LAUGHING

LAUGHS

LAZIER

LAZY

LEAD

LEADER

LEADS

LIVELY

LONG-LOST

LOOKEUT

LOUDLY

LOVINGLY

MAMA

MAN!S

MANY = COLORED

MAP

MAPS

MARCE

MARCG'S 
MARKER

MARKET

MERRILY

MERRY

MERRY - GO - ROUND

MEXICO

MILES

MIST

MISTS

MIX

MIXEO

NEEDS

NEWLY

NIGHTTIME

NERTH

GATS

OLDER

OLDEST

ONLOOKERS

ONTO

ORANGE

ORANGE - COLORED

$O U T=B F=T H E-W A Y$

OWNER

OWNING

PAGES

PAPA

PARRET

PEEPING

PLATFERM

PUSHED

PUSHER

PUSHERS

PUSHING

PUZZLED

RAGGED

RAMON

RAMON'S

READING

REASON

RESTED

RETULD

RETURNED

RUBBING

SAFELY

SANDINO

SANDINO'S

SCHOOLROOM

SCREECHED

SCREECHING

SERAF INA

SERAFINA IS

SERAPE

SERAPES

SHADE

SHADEWY

SHAFTS

SHEPHERO

SHOOK

SHEP

SHEPS

SICK

SIDES

SIDEWAYS

SIGN

SLEEPIER 


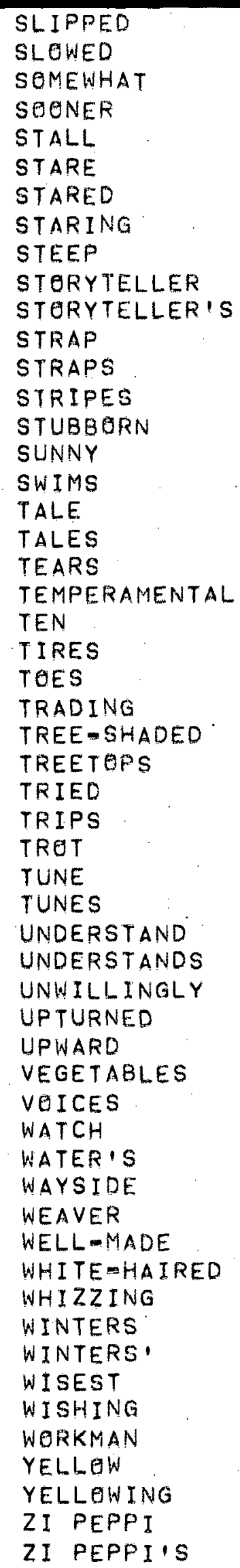

THERE ARE 399 WERDS IN LEVEL 11 OF SERIES D. IN COMPARING SERIES D WITH SERIES C, EACH AT LEVEL, 11 THERE IS A .00\% MATCH. THERE ALSO IS A 22.31\% MATCH (EUTSIDE OF LEVEL 11 ) RANGING FROM, $9=196$ TO $4-158$.

THERE ARE 15 WORDS IN LEVEL 3 OF SERIES D. IN COMPARING SERIES D WITH SERIES E, EACH AT LEVEL, 3 THERE IS A $40.00 \%$ MATCH. THERE ALSO IS A $20.00 \% \mathrm{MATCH}$ (UUTSIDE OF LEVEL 3) RANGING FROM, 
THE BACK-UP WOROS FOR LEVEL. 4 ARE:

JUMPS

PEDRO

SAYS

$\begin{array}{ll}\text { THERE ARE } & 33 \text { WORDS IN LEVEL } 4 \text { OF SERIES } 0 . \text { IN COMPARING SERIES D } \\ \text { WITH SERIES F, EACH AT LEVEL, } 4 \text { THERE IS A } 45.45 \% \text { MATCH. }\end{array}$ THERE ALSO IS A $6.06 \%$ MATCH (OUTSIDE OF LEVEL 4 ) RANGING FROM, $3=44$ TO $3=23$. 
THE BACK - UP WORDS FOR LEVEL 5 ARE:

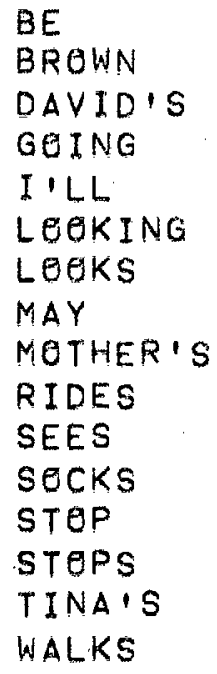

THERE ARE

48 WORDS IN LEVEL 5 OF SERIES D. WITH SERIES THERE ALSO I 4-107 TO 3-58.

IN CEMPARING SERIES D 4.17\% MATCH. 
THE BACK=UP WORDS FOR LEVEL 6 ARE:

IRITING

FLY

GREEN

JUST

MORNING

OLD

TOWN

THERE ARE 18 WORDS IN LEVEL 6 GF SERIES D. IN COMPARING SERIES.D WITH SERIES E EACH AT. LEVEL, 6 THERE IS A .00\% MATCH.

THERE ALSO IS A $55.56 \% \mathrm{MATCH}$ (OUTSIDE OF LEVEL 6) RANGING FROM, $5=162$ TO $4=37$. 
THE BACK-UP WEROS FOR LEVEL 7 ARE:

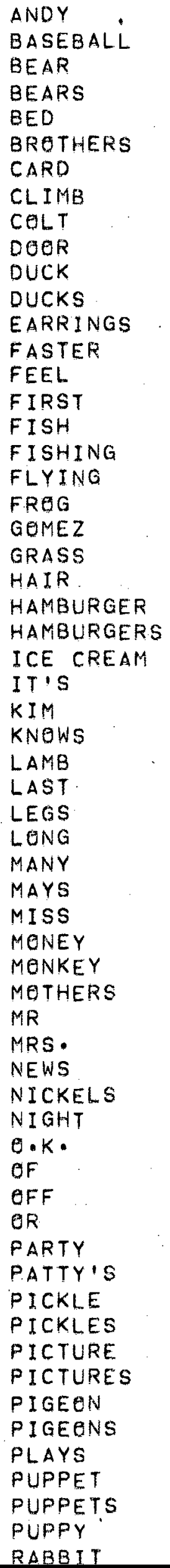


RABBITS

RAIN

RAINCEAT

RIGHT

SAT

SAY

SCRAPEOEK

SHADOW

SHADOWS

SISTER'S

SISTERS

SKATE

SKATES

SKY

SNAKE

SNAKES

SOCK

SUME

SOMEDAY

SQUIRREL

SQUIRRELS

SURPRISES

SWEATER

$T=S H I R T$

T-SHIRTS

TAIL

TALK

TELL

$T I N G=A-L I N G$

TREE

UNCLE

WATERS

WHY

THERE ARE 162 WORDS IN LEVEL 7 EF SERIES D. IN COMPARING SERIES D
WITH SERIES E, EACH AT LEVEL, 7 THERE IS A $1.23 \%$ MATCH. THERE ALSO IS A $31.48 \% \mathrm{MATCH}$ IOUTSIDE EF LEVEL 7 ) RANGING FROM. $8-238$ TO $4-58$. 
THE BACK - UP WOROS FOR LEVEL \& ARE:

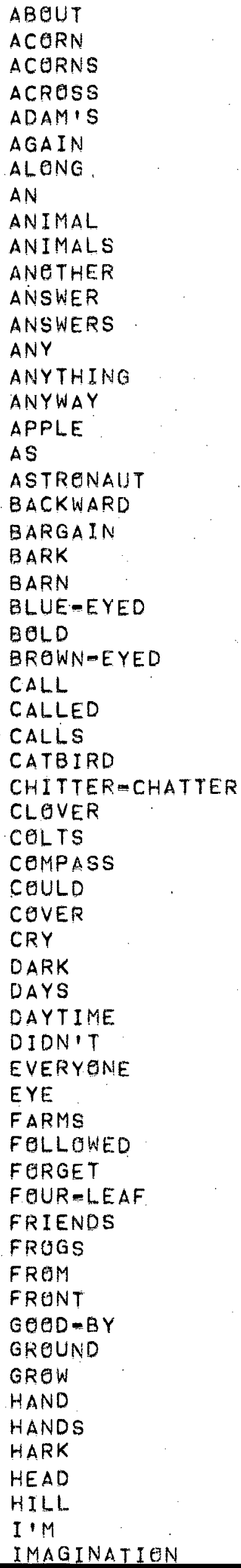


INTO

ITS

ITSELF

KEEN

KIM!S

LARK

LAUGH

LAUGHED

LEFT

LEG

LIKED

LOST

LUCK

MADE

MONKEYS

NEWBERN

NEXT

NEXT-BEST

NONSENSE

OLDTEWN

ONCE

ONE $=$ YEAR-BLD

OUR

OVER

OWL

OWN

PAGE

PAINT

PAINTS

PAJAMAS

PAPER

PAPERS

PEEPED

PLAYTIME

POCKET

PQKED

READ

READY

RIDDLE

RIDDLES

RECKET

ROBM

REUND

RUNN ING

SCAMPERED

SHARK

SHOW

SHOWED

SINGS

SKIP

SLOW

SLOWPOKE

SLUWPOKES

SMART

SOMETIMES

SPARK

SPELL

SPELLER

SPELLS

SPRING

SPRINGTIME

STARTS

STICK

TALKING

TEASE

THAN 
THING

THINGS

THINK

THINKS

THREE - YEAR - ULD

TONIGHT

TUMBLING

TURTLE

$T W O-Y E A R=O L D$

UNDER

WALKED

WARM

WAY

WELL

WHEN

WHIZZED

WINO

WEODLANO

WOEDS

WBRKEUT

WERKS

WRITE

YEAR

YEARS

THERE ARE 281 WORDS IN LEVEL \& OF SERIES D. IN COMPARING SERIES D WITH SERIES E , EACH AT LEVEL, 8 THERE IS A $2.85 \%$ MATCH. THERE ALSO IS A $37.01 \%$ MATCH (OUTSIDE OF LEVEL B) RANGING FROM. $7=228 \cdot$ TO. $3=20$. 


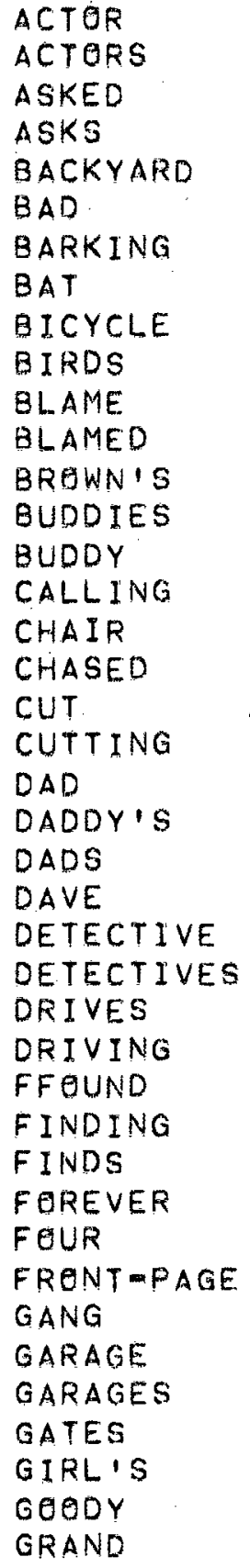




\section{LETS}

LIVE

LIVES

MEADEWS

MEN

MONKEYSHINES

MOONLIGHT

MORNINGS

MOVE

MEVED

MOVING

MR FIG

NIGHTS

NOGN

NOONTIME

NET-TOO-BIG

NETHING

OFFICE

OTHER

OWNS

PAINTED

PAINTING

PARKED

PARTY

PLAYING

POST

PESTMAN

PESTMAN'S

POSTMEN

PULLING

RUESTIUN

QUESTIENS

RAINED

REDS

REDWEOD

REUNDS

SKATER

SLEEP

SEMEHOW

SPOOKIER

SPOEKY

STANDS

STARTING

STAYS

STEP

STEPS

STEPPED

STREETS

SUNDAY

SURPRISE

SURPRISED

SURPR ISING

SWAM

TAKES

TELL

TELLING

TELLS

THINKING

TIRED - OUT

TEDAY

WAGGED

WAGGING

WAGEN

WALKER

WALKING

WELLS 
WHISPER

WHI ISPEREO

WHISPERING

WI TCH

WURKED

WORKING

YARD

YARDS

THERE ARE 262 WERDS IN LEVEL 9 OF SERIES D. WITH SERIES E EACH AT LEVEL, 9 THERE IS A

IN COMPARING SERIES D THERE ALSO IS A $35.88 \%$ MATCH (EUTSIDE OF LEVEL . OO\% MATCH.

9) RANGING FROM, 8-207 TO $4=11$. 
THE. BACK = UP WOROS FOR LEVEL 11 ARE:

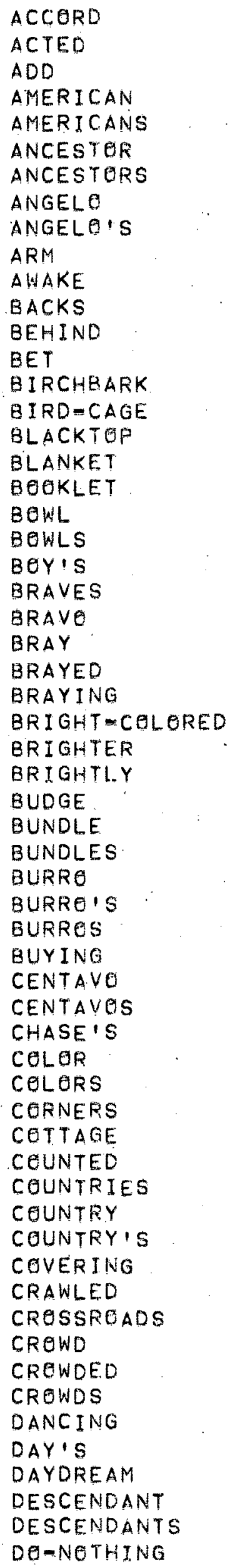


DONKEY

DONKEYS

ORIVER'S

DUCKED

DUSTY

EACH

EARN

EARNED

EXPLAIN

EXPLAINED

FAMILY

FEED

FEEDING

FEET

FELL

FIELDS

FINE

FINEST

FIREWOED

FOLDED

FOLLOWING

FORGETTEN

GARDEN

GLADLY

GOATSKIN

GOODS

GRANDFATHER'S

GRANDFATHERS

GRAY

GREAT = GRANDF ATHERS

GREAT -GREAT-GRANDFAT

GREATEST

GREENFIELD

GREWN

GROWS

HANDSOME

HANDWORK

HAPPENING

HAPPILY

HARD-EARNED

HARDWORKING

HARNESS

HARNESSED

HAS

HIDDEN

HILLS

HILLTOP

HOMEWARD

INDEED

INDI AN

INDI ANS

I TALY

JINGLED

LAMBS

LAZY

LEAD

LEADER

LEADS

LIVELY

LONG - LOST

LOOKOUT

LQUDLY

LOVEO

LOVINGLY

MAMA

MAN'S 
MANY=COLBRED

MAP

MAPS

MARCO

MARCO'S

MARKER

MARKET

MERRILY

MERRY

$M E R R Y=G O=R O U N D$

MIRRER

MIRRES

MISSES

MISTS

NEEDS

NIGHTTIME

OATS

OUT-OF-THE-WAY

PAPA

PARRET

PEEPING

PIECE

PIECES

PLATFORM

PEINTED

PEINTING

POTTER

POTTERS

POTTERY

RAGGED

RAMEN

RAMON'S

READING

REINS

RESTED

RETOLD

-RETURNED

SANDINO

SANDINO'S

SCHOOLROOM

SCREECHED

SCREECHING

SERAFINA

SERAFINA:S

SERAPE

SERAPES

SHEPHERD

SIDES

SLEEPIER

SLIPPED

SOONER

STALL

STARE

STARED

STARING

STEEP

STORYTELLER

STERYTELLER'S

STRAP

STRAPS

STRIPES

SUNNY

SWIMS

TALE

TALES

TEMPERAMENTAL 
THQUGHT

TIED

TIRES

TUES

TRADING

TREE - SHADED

TREETOPS

TRIPS

TROT

TUNES

UPTURNED

UPWARD

VILLAGERS

VOICES

WATER'S

WAVED

WAYSIDE

WEAVER

WELL-MADE

WHEEL

WHICH

WHITE-HA IRED

WHIZZING

WILLINGLY

WINDOWS

WINTERS

WINTERS I

WISEST

WISHING

WOMAN

WOMAN'S

WERKMAN

YELLOWING

ZI PEPPI

THERE ARE 399 WORDS IN LEVEL 11 OF SERIES D. IN COMPARING SERIES D WITH SERIES E EACH AT LEVEL, 11 THERE IS A .00\% MATCH. THERE ALSO IS A $27.82 \%$ MATCH (OUTSIDE EF. LEVEL 11 ) RANGING FROM, $8=217$ T' $4-89$. 
THE BACK-UP WURDS FOR LEVEL 1 ARE:

$$
\begin{aligned}
& \text { DICK } \\
& \text { GET } \\
& \text { HELP } \\
& \text { JANE } \\
& \text { JUMP } \\
& \text { PUFF } \\
& \text { SALLY } \\
& \text { SPOY } \\
& \text { TIM }
\end{aligned}
$$

THERE ARE. 21 WORDS IN LEVEL 1 OF SERIES E. IN COMPARING SERIES E WITH SERIES A EACH AT LEVEL, 1 THERE IS A $4.76 \%$ MATCH. THERE ALSO IS A $52.38 \% \mathrm{MATCH}$ (OUTSIDE OF LEVEL 1) RANGING FROM, $7=238$ Tै $2-30$. 
THE BACK-UP WORDS FOR LEVEL 2 ARE:
BALL
DICK
DID
FIND
GET
HELP
JANE
JESUS
JUMP
PUFF
SALLY
SPET
THAT
THE
TIM
WANT
WITH

THERE ARE 45 WORDS IN LEVEL 2 OF SERIES E. IN COMPARING SERIES E WITH SERIES A IEACH. AT LEVEL, 2 THERE IS A $20.00 \%$ MATCH. THERE ALSE IS A $40.00 \%$ MATCH (BUTSIDE OF LEVEL 2) RANGING FROM, $7=238$ TO $1=39$. 
THE BACK-UP WORDS FOR LEVEL 3 ARE:
BALL.
CAR
CARS
FIND
GETS
HELP
HOUSE
JANE
JESUS
JUMP
PUFF
RIDE.
SPOT
THANK
THAT
THE
TIM
WANT
WANTS
WILL.
WITH

THERE ARE 85 WGRDS IN LEVEL 3 GF SERIES E. IN COMPARTNG SERIES E WITH SERIES A EACH AT LEVEL, 3 THERE IS A .00\% MATCH. THERE ALSO IS A $58.82 \%$ MATCH. IOUTSIDE OF LEVEL 3 I RANGING FROM. $7=238$. TO $1-5$. 
THE BACK=UP WBRDS FER LEVEL 4 ARE:
BALL
BARKED
BIBLE
BILLY
BILLY'S
BIRTHDAY
BEOTS
BOYS
CAKE
CHICKENS
COAT
CoWs
DICK
DICK'S
DIO
DUCKS
FIND
FRIENO
FUNNY
GAME
GET
GIRLS

GRANDFATHER

GRANDMOTHER

HELP

HENS

HER

HERE

HOUSE

JANE

JESUS

JUMP

MAYBE

PET

PETE

PETE'S

PQNY

PUFF

RABBITS

READS

RIDE

SABBATH

SAID

SALLY

SALLY'S

SPOT

SUSAN

TAILS

TAKE

THANK

THAT

THE

TIM

TIME

TOM

TRAIN

WANT

WANTED

WHITE

WHO

WILL 
THERE ARE 186 WORDS IN LEVEL 4 OF SERIES E. IN COMPARING SERIES E WITH SERIES A EACH AT LEVEL, 4 THERE IS A $17.74 \%$ MATCH.

THERE ALSO IS A $41.40 \%$ MATCH (OUTSIDE OFF LEVEL 4 ) RANGING FROM, $8=49$ TO 1 - 30 . 
THE BACK-UP WORDS FGR LEVEL 5 ARE:

ACRESS

ALIVE

ANN

ANYUNE

ASIDE

$\triangle S K I N G$

$\triangle S L E E P$

BEARS

BEAUTIFUL

BECAME

BECAUSE

BEDREOM

BEE

BEING

BELL

BELONG

BELONGED

BESIDES

BEST

BETTER

BEATMAN

$B O B$

$B Q R G$

BUTH

BUILD

BUILDINGS

CAMP

CATCHING

CHEESE

CLEAN

CLEANED

CLEANING

CLIMBED

CLIMBING

CLYDE

CORNF IELO

CEULDN'T

CEUNTRY

DAD'S

DEEP

OIG

OIME

DO IT Y YOURSELF

DOING

DEN

DOBRBELL

DOQRWAY

DEWNTOWN

DRYER

END

EVER

EVERYBODY

FALL

FALLING

FENCE

FIREPLACE

FISH

FLASHED

FLASHLIGHT

FOOTBALL

FARD 
FULL

GAVE.

GET = TEGETHER

GETTING

GOD'S

GENE

GRASSY

GREENHOUSE

HANDING

HARRIS

HAYDEN

HEIS

HEADING

HEAR

HEARD

HEARING

HEINZ

HENRY'S

HEREAFTER

HERSELF

HERSHEY

HILLSIDES

HOOK.

HOQKED

HOWEVER

HURRIED

HURT

IF

INSIDE

JUHN

JOLLY

KITE

KNOWING

KODA

KRAFT

LARGE

LAUGHING

LETTERING

LEW

LISTEN

LISTENED

LISTENING.

LIVED

LIVELONG

LUNG $=$ TAILED

LONGING

LOTS

LOUGHBOROUGH

MACHINES

MAKING

MARRIETT

MILTEN

MISSION

MEVED

MUCH

NAMED

NEWSPAPER

NICE

NIGHTEIRDS

NOJSE

EUTDEORS

QUTSIDE

OWN

OWNED

PADDY

PAINTER 
PAPERS

PAST

PATH

PAY

PAYDAY

PAYING

PERHAPS

PICK

PICKED

PICKING

PILED

PILES

PLANTED

PLANTING

PLANTS

PLAYED

PLAYHOUSE

PLAYTHINGS

PLEASANT

PLEASEO

POTATOES

PRAYING

QUICK

RACCOBNS

RAKE

RANG

RING

RINGING

$R \in A D$

RELL

ROLLED

ROLLING

$S A D$

SAYING

SECONO

SEED

SEEING

SEEN

SEESAW

SEND

SHEEP

SHOUT

SHOUTED

SHOUTING

SHOVEL

SHOWING

SIDE

SIDEWALK

SIGN

SILVA

SLIDES

SLOWED

SMALL

SMEKED

SUMEONE.

SOMEWHAT

SOMEWHERE

SON

SERRY

SPRINGTIME

STAND

STANDING

START

STARTED

STATIEN

STAY 
STAYED

STAYING

STOREHOUSE.

SUDDENLY

TEN

THAT'S

THERE'S

THEREBY

THIRO

THOSE

TIE

TITHE

TI THED

TOMMY

TONY

TOELHOUSE

TOOLS

TREETEPS

TRICK

TRIED

TRIP

TURN

TURNED

TV

UNDERCLOTHES

UNDERSTAND

UPQN

VISITED

WANT ING

WATCH

WATCHED

WATCHING

WATER

$W H-E E-E$

WHATEVER

WHEAT

WHEELED

WHEELS

WHENEVER

WHO'S

WILL.ING

WINDOW

WISE

WORKMEN

YQU'LL

YOURSELF

THERE ARE 370 WOROS IN LEEVL 5 OF SERIES E. IN COMPARING SERIES E WITH SERIES A EACH AT LEVEL, 5 THERE IS A $3.51 \%$ MATCH. THERE ALSE IS A $18.92 \%$ MATCH (OUTSIDE OF LEVEL 5) RANGING FROM, $9=37$ TU $1=5$. 
THE BACK=UP WURDS FOR LEVEL 6 ARE:

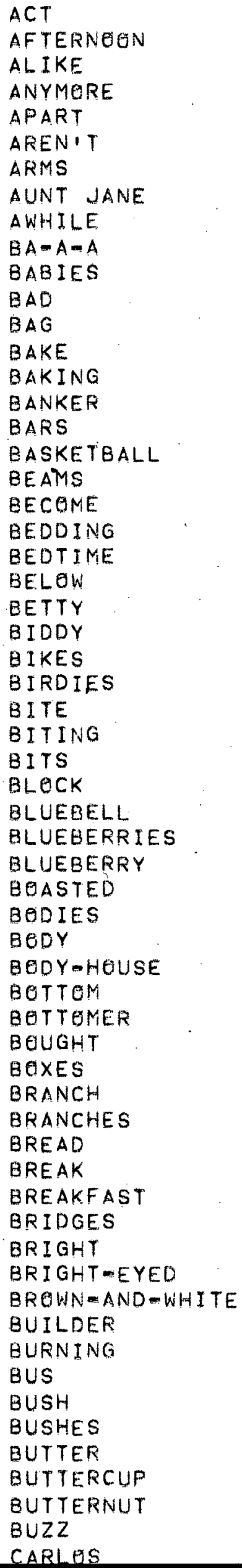


CERTAINLY

CHANCED

CHANGE

CHASE

CHASING

CHIN

CLEANER

CLECK

CLOSE

CLESER

CEAX

CEOL

COUNT

CREAM

CROSS

CREWDED

CREWFEOT

CRYING

CUPBOARD

CUPCAKES

CURLS

CURLY

CURLY - HAIREO

DADDY.

DASHED

DAVID

DAYTIME

DIRT

DIRTY

DECTOR-FATHER

DECTERS

DOESN'T

DOWNSTAIRS

DRAW

DRESS=UP

DRESSES

DRINK

DRIVE

DRIVER

DRIVING

DRQP

DREPPED

EARTHMEVERS

EARTHY

EASIER

EASILY

EATEN

EGG-HOUSES

EMPTY

EMPTY-HEADED

ENGINE

EXCITED

EXCITING

EYE-HULES

FACE

FAIR

FARAWAY

FEELING

FELT

FEW

FIREBELL

FIREHOUSE

FIREMAN'S

FIREMEN

FISHES

FIXED 
$F L A G$

FLEW

FLIES

FLOUR

FLYER

FOLLOWING

FOOL

FOOLISH

FOOTSTEPS

FORGETTING

FURGIVE

FORGOT

FORTH

FRIDAY

FRIENDLY

FRIGHTENED

FRUITS

FUNNIEST

FUNNY-LOOKING

GALLOPING

GARAGE

GARZA

GASTRIC

GATE

GAY'S

GEQRGE

GOBBLE

GOES

GOOD - LUCK

GOBD-TO-EAT

GRAB

GRABBED

GRAYLEGS

GREAT

GREATHOUSE

GRIN

GRINNED

GRINNING

HADN'T

HAPPENED

HASNIT

HATBOX

HAVEN'T

HAVING

HE'LL

HEALTH

HEALTHIER

HEALTHIEST

HEALTHY

HEALTHY=LOOKING

HEARTS

HEAVIER

HEAVY

HELD

HERE'S

HI

HIGHER

HIGHWAY

HILLER

HILLYY

HITTING

HONEY

HOPING

HOPPED

HOPPING

HOSE 
HOSPITAL

HOUSEKEEPER

HOUSETEPS

HOW'S

HUNT

I 10

ICE

INTERESTING

ITSELF

JAY

JERRY

JIMMY

JEHNNY

KATYDIDS

KEEPER

KEPT

KICKED

KILLS

KITTY

KNECK

LADDER

LAKE

LANDED

LAN I

LARGER

LATER

LEAP

LEAST

LEAVING

LED

LETTING

LIFE

LIFTED

LIONS

LIVING

LONGER

LONGHOUSE

LEUD

LUUDER

LQUDEST

LOVE

LOW

LEWER

LUCKY

LUNCHREOM

LUNCHT IME

$M=M=M$

MALEC

MARCHERS

MARY

MEAN

MEAT

MEET ING

MERRIEST

MERRY $=G E=R O U N D$

MELLY

MOUTH

NECK

NEWEST

NEWSBOY

NO-WHEELED

NED

NODDED

NOISEMAKER

NOISEMAKING

NOISIEST 
NOISY

NOSE

NOTE

NUMBER

NUTMEAT

GAK

BBEY

OFFER

OFTEN

$\mathrm{OH}-\mathrm{H} \rightarrow \mathrm{H}$

OIL

OIL-COVERED

OILY

OLDER

ONE $\cong$ WHEELED

ONO

ONTO

OUGHT

OWNER

PABLQ

PALYTIME

PAN

PARADE

PART

PARTIES

PATTED

PAW

PEANUT

PECKING

PETERSON'S

PETTED

PFT $-F T$

PIE

PIGS

PINK

PIPER

PLAY = IN-THE = SUN

PLENTY

POINTING

POISONED

POLICE

PENIES

PEOL

$P \bigoplus O R$

$P Q P$

PQPPED

PORCH

PQSTURE

POTATE

POTTED

$P R=R=R$

PRESENTS

PRETENDING

PRINCE

PUMPKINS

PUP

PUTTING

QUARREL

QUEER

QUICKLY

QUIET

QUINN

QUIT

RACE

RACING

RAGS 
RAINY

RAINY=DAY

RAKER'S

RAKING

RATS

RATTLE

REACH

REAL

REMEMBERED

REPLIED

REPLY

RETURNED

RIDING

ROARED

ROCK

RECKY

RODE

ROPE

ROSY

ROW

RUBBED

RUBBING

- RUBS

RUNNY

RUSHING

SAM

SANTES

SATAN

SAVE

SAVING.

SCARECROW

SCARY

SCHOELCHILDREN

SCHOQLTEACHER

SCREAMED

SECOND=FLGOR

SEEMED

SHIRT

SHOULDN'T

SHUT

SID

SIDEWAYS

SINCE

SING

SITTING

SLOW-MEVING

SLOWLY

SLY

SLYMAN

SMALLER

SMALLEST

SMARTEST

SMARTY

SMILE

SMOKING

SN IFF-SNIFF

SNIFFED

SNIFFY

SOAPBOX

SOMEDAY

SENNY

SORE

SOUND

SPECKLE

SPOKE

SPETTED 
SQUEAKY

SQUEALING

STAIRS

STAIRWAYS

STAR

STARRY

STEPPED

STEVE

STOMACH-CLOCKS

STOMACHS

STOOD

STORYBOOK

SUDDEN

SUNNY $=D A Y$

SUPPER

SUPPERTIME

SUPPESED

SURE

SURELY

SWIFTLY

SWIM

SWIMMING

TABLE

TAKEN

TAKING

TALL

TALLER

TALLEST

TASTE

TEACH

TEACHER

TEETH

TEMPLES

THERE ' LL

THESE

THEY ILL

THEY IRE

THREE - WHEELED

THROAT

THROWING

TIMMY

TIRED

TRACK

TRAINER

TRETTED

TREUBLE

TRUNKS

TWE-WHEELED

UGLY

UNCLE BOB

UNDERHANDED

UPPER

UPSET

UPSTAIRS

UPTEWN

USEFUL

VALENTINES

WADDLES

WALKERS

WARMED

WASN'T

WATCHES

WATCHMAN

WAVED

WAVING

WE'D 


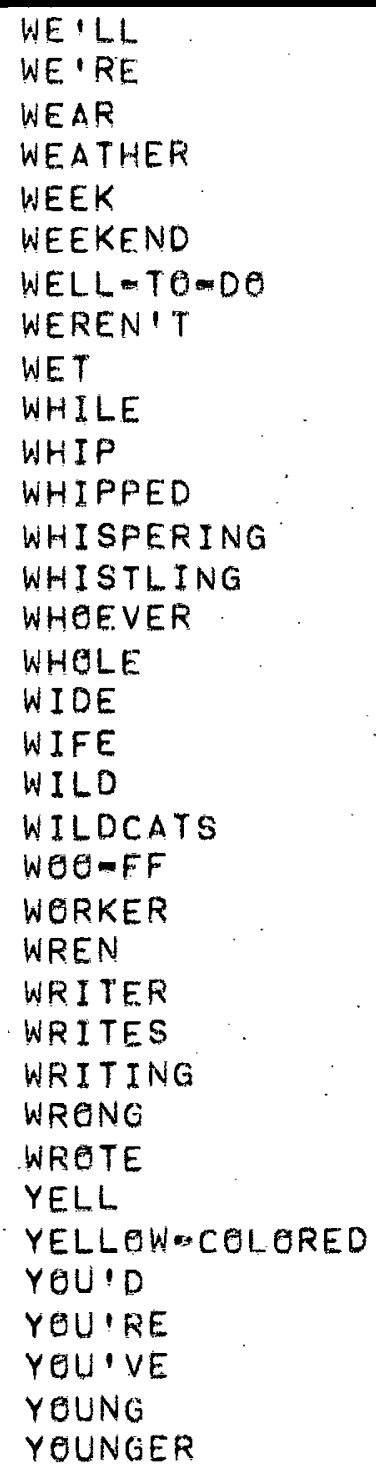

THERE ARE 609 WERDS IN LEVEL 6 OF SERIES E. IN COMPARING SERIES E WITH SERIES A EACH AT LEVEL, 6 THERE IS A $2.30 \%$ MATCH. THERE ALSO IS A $10.67 \%$ MATCH (EUTSIDE OF LEVEL 6) RANGING FROM, $9=157$ TO $1-6$. 
THE BACKMUP WORDS FOR LEVEL 7 ARE:

$\triangle E R I A L$

AGREE

AIMED

AIRPLANES

ALABAMA

ALARM

ALLERTON

ALVIN'S

AMATEUR

AMEN

AMERICA

AMY

ANKLE

ANYBEDY'D

APPREACHING

ARRENS

ASHORE

AT TACKED

AWFUL

BABCECK

BALANCE

BARTH

BASE

BATTED

BATTERY

BEACH

BEAT

BEAUTY

BEGGED

BEGINNING

BETSY

BETWEEN

BLADES

BLAZED

- BLEW

BLOWN

BONNIE

BOWSTRING

BR.ADFERD

BRASS

BRAVE

BRIGHTEN

BRIGHTLY

BROKE

BROKEN.

BUILT

BULB

BUNDLES

BUSILY

BUTTEN

CABIN

CALM

CALMLY

CANDLES

CAPTAIN

CARDS

CAROLERS

CARVER

CASE

CHARLES

CHECK 
CHEERFUL

CHICAGO

CHIEF

CHIMNEY

CHOSE

CHOSEN

CHRISTMAS

CHURN

CIRCLE

CLEARING

CLIP

CLOSELY

CLOSET

CLOTHESPIN

CLOWN

COCOA

CEDE

COMMON

COMPANY

CONNECT

CONSTANCE

COPPER

CRAMER

CRASH

CREEK

CROPS

CRUELTY

CUPFUL

CURB

DANGEROUS

DAPHNE

DARKNESS

DAYDREAMING

DECK

DEERSKIN

DEFROST

DELIVER

DIAL

DIPLOMACY

DIRECTIONS

DIRECTER

DISAPPEARED

DISHES

DOUBT

DEUBTFULLY

DRAWN

DR IF TING

ELDER

ELECTRIC

ELECTRICITY

ENGINEER

ENVELOPE

EVENLY

EXACTLY

EXPECTED

EXPLAINED

EXPLORING

FAINT

FALLEN

FANCY

FARTHER

FASTENED

FEAST

FIERCELY

FINGERS

FINISHED 
FLAT

FLOBDED

FOEL I SHLY

FERT

FRESH

FRESHLY

FRIGHT

GARDENIA

GATHER

GEESE

GILES

GINGEREREAD

GLOOMILY

GLOOMY

GOESE

GOVERNOR

GRAIN

GREATLY

GROWN-UPS

GRUNTED

HALL

HANDFUL.

HANDLE

HANS

HARBOR

HEAVILY

HILDE'S

HISTORY

He

HOBBLE

HONK

HOPKINS

HORN

HOURS

HOWL

HEZAK

HUFFMAN

HUNGRILY

INGRID

INVENTEO

INVENTIONS

INVENTOR

INVITED

JERK

JUINED

JUKE

KATALIN

KATRINA

KEY

KILPATRICK

KNIFE

KNEB

LANES

LANGUAGE

LANTERN

LEONARD

LEVERS

LIST

LOCKED

LOGS

LOOSE

LOESELY

LOOSEN

LOVELY

LUCKILY

MAGNET 
MAILBGX

MASSACHUSETTS

MEDICINE

MELTS

MESSAGE

METAL

MILKMEN

MILLION

MESTLY

MOTIONS

METERBOAT

MOUSE

MUDDY

MUSIC

NANCY 10

NEARLY

NEMETH

NEWLY

NICKEL

NINE

NUISILY

NOTICED

EK

OLD FFASIONED

ORDERS

OTTO

PAPPAS

PARENTS

PARROT'S

PARTLY

PASSED

PASTURE

PEDAL

PERFECT

PERFECTLY

PERFUME

PIECES

PILGRIM

PILUT

PIENEERS

PITCHING

PLANNING

PLATE

PLENTIFUL

PLOWED

PLUNGED

PLYMOUTH

POL ITELY

POSITIEN

PUSSIBLE

POSSIBLY

POWER

PRACTICE

PRAIRIE

PREPARED

PREVENTION

PROJECT

PROMISE

PROUDLY

PURPLE

PUZZLED

QUIETLY

QUITE

RAISE

RECREATION REFI 
REFR IGERATOR

RELEASE

RID

REASTED

ROGERS

ROEFED

RUG

SADLY

SAFE

SAFELY

SAILING

SALE

SALTY

SAMOSET

SATURDAY

SCATTERED

SCOLDING

SCRUBBED

SEAT

SECRET

SEMAPHORE

SENT

SHE'D

SHIP

SHOIE

SHEOT

SHORES

SHERTLY

SHOT

SHOVED

SHULTZ

SILENT

SILENTLY

SILVER

SIMPSON

SKIP.PED

SLINGER

SMELL

SNAPPED

SNOWFLAKES

SNEWSTORM

SECIETY

SEFTLY.

SOS

SQUP

SPACE

SPEAK

SPECIAL

SPEND

STANDISH

STEADILY

STEADY

STEEL

STEER

STEPHEN

STEW

STOVE

STRA I GHTENING

STRANGELY

STRIPS

STRUCK

STUEY

STUMBLED

SWAN

SWOUPED

TAPE 
TAUGHT

TAXICABS

TEAMS

TELESCOPE

TENSIEN

TERRIBLY

TEST

THANKFUL

THATCH

THEY'D

THIRSTY

THUNDER

TICK

TIGHT

TEE

TOOT

TRANSOM

TRAPPED

TRUE

TRULY

TUBE

TUNE

TURKEYS

TURNIPS

UNBEL IEVINGLY

UNCAOKED

UNCQVERED

UNDERSTEOD

UNEASILY

UNEXPECTED

UNLOCK

UNSAFE

UNSETTLED

UNTIE

UNWILLINGLY

USELESS

VACATIEN

VACUUM

VAN VLIET

VIOLIN

VISITOR

VOICE

WAKED

WAKENED

WALKIE-TALKIE

WARNED

WATCHFULLY

WE'D

WEBER

WEIGHT

WELCOMES

WHIRL ING

WIDELY

WILDLY

WILLIAM

WILSON'S

WOLVES

WONDERFULLY

WORRIEDLY

WORSE

WORSHIP

WOUND 
THERE ALSE IS A $6.52 \%$ MATCH (EUTSIDE OF LEVEL 7) RANGING FRGM. $9=264$ TO $4=26$.

THE BACK -UP WORDS FOR LEVEL 8 ARE:

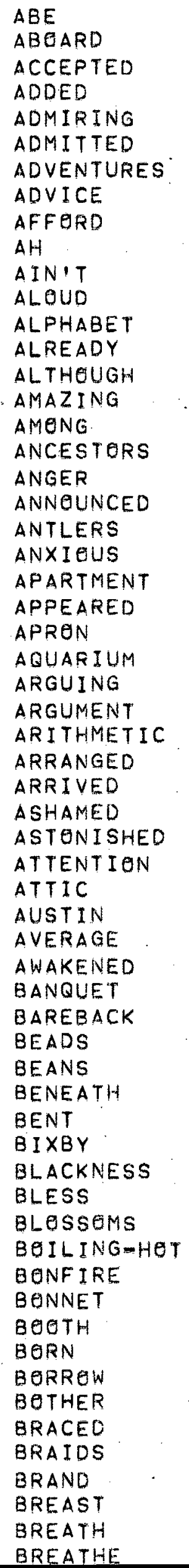


BREATHLESSLY

BREEDS

BRIDLE

BRIEF

ERUSH

BUBBLES

BUCK

BUD

BUNK

BURST

CAPTURED

CARTWRIGHT

CATTLE

CAUTIOUSLY

CEILING

CELEBRATED

CELERY

CENTEPE:D

CENTS

CHAIRBOUND

CHANTED

CHARLEY

CHARLIE

CHATTED

CHEEKS

CHEROKEE

CHINATOWN

CHINESE

CHEKED

CHOPS

CHORES

CHUCKLED

CLAIM

CLAPFED

CLASSES

CLIFF

CLETH

CLOUDY

CLUBS

COAL

COCONUT

COLLAR

COMFEFT

COMMITTEE

CONFUCIOUS

CONSIDER

CENTEST

CONTINUED

CENVENIENT

CEPYCAT

CUNNING

CUNNINGHAM

CURIOUS

CUSTOMERS

D.C.

DALE

DAMP

DANCES

DANDER

DARED

DECLAFED

DECERATIONS

DECOY

DELIGHTED

DENMARK

DFNNIS 
DESERT

DESERVES

DESK

DESPAIR

DESTREY

DETOUR

DEW

DIDOS

DIFFERENCE

DIFF I CULTY

DINING

DIEN

DIPPED

DISAPPEINTED

DISAPPOINTMENT

DISCOURAGED

DISCOVERED

DISEASE.

DISSATISFIED

DISTANCE

DISTANT

DITCH

DIVED

DIVIDED

DEE

DELLARS

DRAGON

DRAIN

DREW

DROVE

DROWNED

DULL

DUMB

DUST

EAGER

EAGERNESS

EARNED

EARTHQUAKE

ELEVEN

EMPTINESS

ENITH

ENJOYED

ENORMOUS

ENTER

ENVY

ERRAND

EXAMINE

EXCEPT

EXCITEMENT

EXCUSE

EXERCISE

EXPERIMENTS

EXTRA

FACT

FAILURE

FAITHFUL

FAMILIAR

FAULT

FAVORITES

FEARFULLY

FEARLESS

FELLOW

FERNWGOD

FEVER

FIFTEEN

FIFTY 
INTRODUCING

INVESTMENT

ISABELLA

I SLAND

JEALEUS

JEAN

JEROME

JEHNSTON

JUSEPH

JOY

JUDG ING

KALLER

KANGAROO

KAYAK

KETTLE

KIMMY

KIRSTEN

LACEUR

$\angle A D$

LADY

LAID

LAME

LANKY

LARSEN

LATHERED

LAUGHTER

LAWN

LEAFY

LEAKING

LEDGE

LEI

LESS

LEVEE

LI

LICK

LID

LIE

LIN

LINCOLN

LGANED

LOFT

LUHMANN

LONELY

LONESOME

LQRD'S

LUSE

LUTTIE

LOUELLA

LOUIS

LUMP

LYING

MA

MAGIC

MAILE'S

MAIN

MAMMA

MANAGE

MANALUK'S

MAPLE

MARKED

MARR I AGE

MARRY

MASTER

MATE

MATHILDA

MATOAKA 
MAYOR

MEADOW

MEAL

MEANT

MEASURED

MEASUREMENT

MEEKLY

MEISTER

MEMBERS

MENTIUN

MID

MINE

MIRACLE

MISCHIEF

MISSISSIPPI

MISSUURI

MIST

MISTAKEN

MITE

MIX

MOM

MEMENT

MONTHS

MEON

MOUNTAIN

MOWER

MUMPS

MURMUR

MUTTERED

NATURALLY

NEAT

NEEDLE

NEGRE

NEI THER

NETS

NURSE

OARS

OBLIGE

OFF ICER

OKLAHOMA

OUTFIT

OUTWARD

OVERAGE

BWE

$P A$

PACKED

PADDLE

PAID

PAIN

PAIRS

PALACE

PALE

PANTING

PARDEN

PARIS

PAUSED

PEACE

PEACHES

PEBBLES

PECULIAR

PEERED

PEQNY

PERSEN

PIGEON

PILLEW

PINES 
PING

PITY

PLAINLY

PBCAHONTAS

PUSTMASTER

POUND

POURED

POWHATAN

PREACHER

PRECIOUS

PRESIDENT

PROTECTED

PROTESTED

PROVED

PUDDLE

PUNCH

QUESTI INED

QUETATION

RADISH

RAM

RANCH

REALIZED

REALTIONS

REAR

REASON

REBECCA

REBUILT

RECKON

RECORD

REFUSED

RELIEVED

REMAINED

REMINDED

REPEATED

RESCUE

RESERVATION

REVOLVING

REWARDS

RIBBEN

RICE

RICHES

RINSE

RISE

ROD

ROLFE

RQET

ROYAL

RUFE

RUFFLING

RUSTY

SACK

SADDLE

SADNESS

SAN FRANCISCO'S

SANK

SARAH

SATISFIED

SCAMP

SCRAPE

SCUFFLED

SEA

SEARCHING

SEASENED

SEAWEED

SELL

SEOUGYAH'S 
SERVANTS

SHARP

SHAVED

SHELTERED

SHETLAND

SHINY

SHIVER

SHENE

SHOP

SHOWER

SHR IEKED

SHYLY

SICK

SICKNESS

SIGHED

SILENCE

SILK

SINGLE

SI GUX

SIR

SIZE

SLID

SLIP

SMITH

SMEOTH

SNAILS

SNATCHED

SNUG

SEARED

SUBBING

SOLES

SONG

SOO-PUNG

SQRT

SOUTH

SPANKED

SPARED

SPARKLING

SPARKS

SPEECH

SPEECHLESS

SPELLING

SPENT

SPILLED

SPIN

SPINE

SPIRITS

SPROUTS

SPUTTERED

SQUISH

STAG

STARED

STARTLED

STATEMENT

STATES

STATUE

STEAMING

STERNLY

STIFFLY

STOLEN

STOOL

STRAY

STREAKS

STRETCH

STRIKE

STUBRERN 
STUFF

STUPID

SUCCEEDED

SUFFER

SUGGESTED

SWALLOWED

SWIRLING

SWITCHING

SWUNG

SYLLABLES

SYRUP

TAG

TAMEST

TASK

TEARS

TENDERLY

TENTS

TERR IF IC

TERROR

TEXAS

THEE

THICK

THOMAS

THOUGH

THUNDERBOLT

TIDE

TILL

TIPP ING

TOASTY

TONGUES

TOSSED

TEWERING

TRAVELWAYS

TREMBLED

TRIBE

TRIM

TREPICAL

TROUGH

TROUSERS

TRUSTED

TRUTH

TUBS

TUTU

TWELVE

TWENTY

TWICE

TWINKLED

TWISTED

TWE-TONED

UNCOMFORTABLE

UNITED

UNLESS

UPWARD

URGED

VALLEY

VICIEUS

VIRGINIA

VIRUS

WAGGING

WAILED

WAIST

WALL

WARRIURS

WASHINGTON 


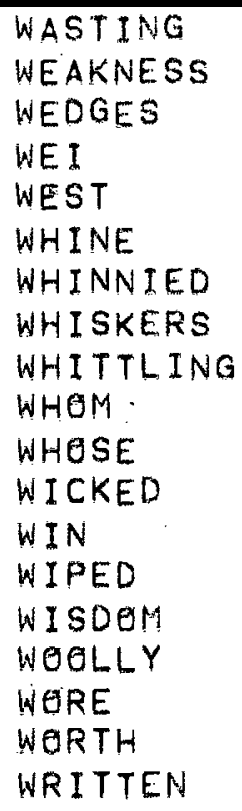

THERE ARE 697 WORDS IN LEVEL 8 OF SERIES E. IN COMPARING SERIES E WITH SERIES A , EACH AT LEVEL, 8 THERE IS A $1.58 \%$ MATCH. THERE ALSO IS A $5.31 \%$ MATCH (OUTSIDE OF LEVEL 8 ) RANGING FROM; $S=279$ TO $1=31$. 
THE BACK=UP WORDS FOR LEVEL 1 ARE:

$$
\begin{aligned}
& \text { DICK } \\
& \text { JANE } \\
& \text { ME } \\
& \text { PAM } \\
& \text { PENNY } \\
& \text { SALLY } \\
& \text { TIM }
\end{aligned}
$$

THERE ARE 21 WORDS IN LEVEL 1 OF SERIES E. IN COMPARING SERIES E WITH SERIES B , EACH AT LEVEL, 1 THERE IS A $33.33 \%$ MATCH. THERE ALSE IS A $33.33 \% \mathrm{MATCH}$ (UUTSIDE UF LEVEL 1 ) RANGING FROM, $6=37$ TO $2=6$. 
THE BACK $=U P$ WORDS FOR LEVEL 2 ARE:

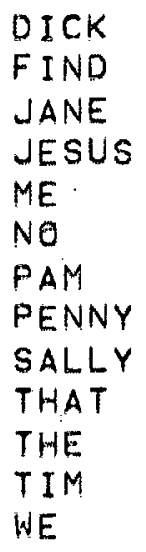

THERE ARE 44 WORDS IN LEVEL 2 OF SERIES E. IN COMPARING SERIES E WITH SERIES B, EACH AT LEVEL, 2 THERE IS A $15.91 \%$ MATCH. THERE ALSO IS A $54.55 \%$ MATCH (BUTSIDE OF LEVEL 2) RANGING FROM, $6=37$ TO 1- 12: 
THE BACK-UP WORDS FOR LEVEL 3 ARE:

$$
\begin{aligned}
& \text { BOOK } \\
& \text { CAR } \\
& \text { DICK } \\
& \text { DEG } \\
& \text { DOGS } \\
& \text { FIND } \\
& \text { HAVE } \\
& \text { IN } \\
& \text { JANE } \\
& \text { JESUS } \\
& \text { MY } \\
& \text { NE } \\
& \text { PENNY } \\
& \text { SALLY } \\
& \text { THIS } \\
& \text { TIM } \\
& \text { WE }
\end{aligned}
$$

THERE ARE 85 WORDS IN LEVEL 3 OF SERIES E. IN COMPARING SERIES E WITH SERIES B ,EACH AT LEVEL, 3 THERE IS A $15.29 \%$ MATCH. THERE ALSO IS A $50.59 \%$ MATCH (OUTSIDE IF LEVEL 3) RANGING FROM, $6=37$ TO $1=12$. 
THE BACK=UP WORDS FOR LEVEL 4 ARE:

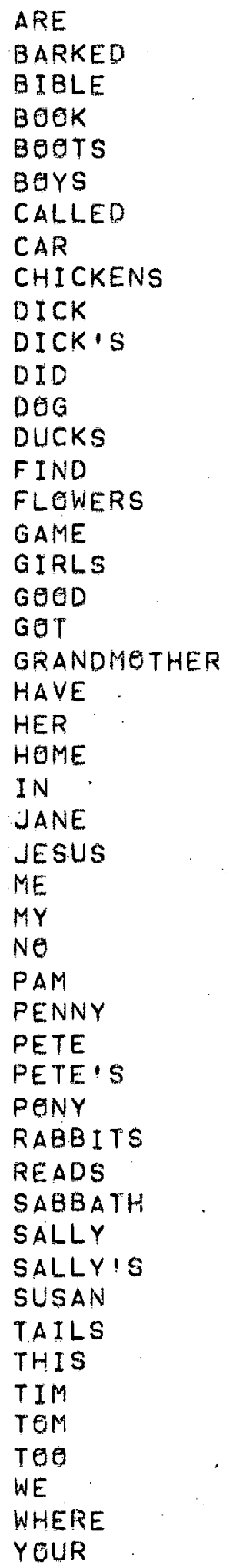

THERE ARE 186 WORDS IN LEVEL 4 OF SERIES E. IN COMPARING SERIES E WITH SERIES B , EACH AT LEVEL, 4 THERE IS A $5.38 \%$ MATCH. THERE ALSE IS A $62.90 \%$ MATCH (OUTSIDE OF LEVEL 4) RANGING FREM, $6=37$ TO $1=12$. 


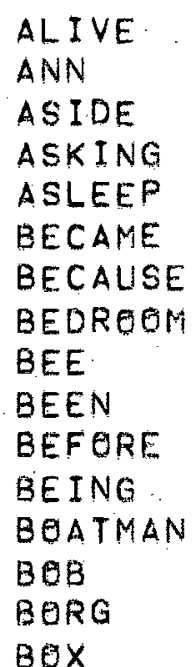

BOXMAKING

BRICK

CANNOT

CAP

CATCHING

CHEESE

CLIMBING

CLYDE

CORNFIELD

DAD'S

DEING

DON

DOORBELL

DOERINAY

DOWNTOWN

DRYER

EARS

END

FALLING

FARMER

FARMHEUSE

FIREPLACE

FLASHLIGHT

FEOTBALL

$F G R D$

GET - TUGETHER

GETTING

GOD'S

GRASSY

GREENHOUSE

HANDING

HARRIS

HAYDEN

HE'S

HEADING

HEAR

HEARD

HEARING

HEINZ

HELPFUL

HENRY'S

HEREAFTER

HILLSIDES

HIMSELF HoOK 
HEOKED

HOWEVER

HURRIED

INSIDE

JOHN

JELLY

KITE

KNOWING

$K B D A$

KRAFT

LARGE

LALGHING

LEARN

LEARNED

LEARNING

LINDA

LINES

LIVED

LIVELONG

LONG-TAILED

LONGING

LOTS

LOUGHBORQUGH

MACHINES

MAKING

MARRIOTT

MILTEN

MINUTE

MISSIEN

MOVED

NAMED

NEWSPAPER

NICE

NIGHTBIRDS

O. CLECK

OUTDOERS

OUTSIDE.

PAINTER

PAPERS

PAST

PAYDAY

PAYING

PICKED

PICKING

PILED

PILES

PLANTED

PLANTING

PLANTS

PLAYED

PLAYHOUSE

PLAYTHINGS

PLEASANT

PLEASED

POND

POTATEES

PRAYING

PULL

PULLED

QUACK

RACCUONS

RAKE

ROLL

ROLLED

RQLLING

SAYING 
SEESAW

SILVA

SLIDES

SLEWED

SMALL

SMOKED

SNOWMAN

SNOWMEN

SGAP

SOFT

SOMEONE

SOMEWIHAT

SOMEWHERE

SON

SPRINGTIME

STAND

STANDING

STOREHUUSE

TARO

THAT'S

THERE'S

THEREBY

TIE

TI THE

TITHED

TOOLHOUSE

TOOLS

TOP

TREETOPS

TRICK

UNDERCLOTHES

UNDERSTAND

UPON

VISITED

WANT ING

WATCH

WATCHED

WATCHING

WATER

WENDY

$W H=E E-E$

WHATEVER

WHEAT

WHEELED

WHEELS

WHENEVER

WHO'S

WILLING

YESHI

YEURSELF

THERE ARE 370 WORDS IN LEVEL 5 OF SERIES E. WITH SERIES B EACH AT LEVEL,. 5 THERE IS A

IN COMPARING SERIES E

THERE ALSE IS A $27.84 \%$ MATCH IOUTSIDE GF LEVEL 5 ) RANGING FROM, $6-276$ TO $1=120$. 
THE BACK -UP WORDS FGR LEVEL 6 ARE:

$A C T$

$A G O$

AHEAD

ALIKE

ANYMORE

AREN I T

ARMS

AUNT JANE

AWHILE

$B A=A=A$

BABIES

$B A D$

$B A G$

BAKE

BAKING

BANKER

BASKETBALL

BEAMS

SECOME

BEDDING

BEDTIME

BELOW

BETTY

BIDDY

BIROIES

BITING

BITS

BLECK

BLUEBELL

BLUEBERRIES

BLUEBERRY

BOASTED

BODIES

BEDY

BEDY=HOUSE

BOXES

BRIDGES

BRIGHT

BRIGHT-EYED

BROWN-AND-WHITE

BUILDER

BURNING

BUS

BUSH

BUSHES

$B \cup Z Z$

CAGE

CART

CHANCED

CHANGE

CLEANER

COOK

CRACKING

CROSS

CREWDED

CROWFEOT

CRYING

CUPBEARD.

CUPCAKES

CURLS

CUIRIY 


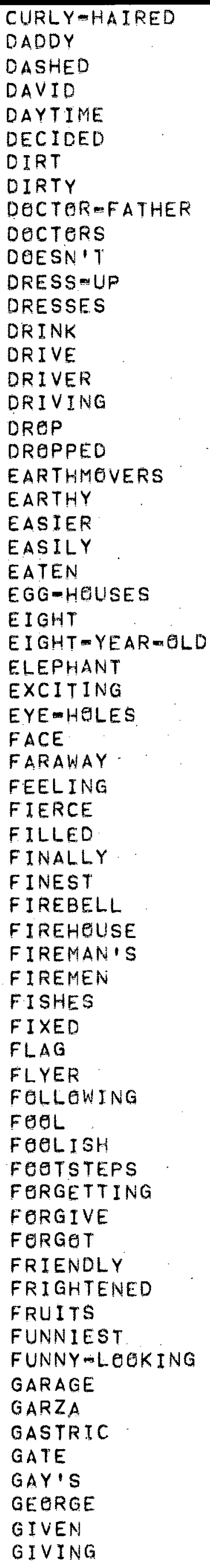


GLASSES

GLOVES

GBED-LUCK

GOED-TO-EAT

GRAB

GRABBED

GRAYLEGS

GREAT

GREATHOUSE

GRIN

GRINNED

GRINNING

HADN T

HANDKERCHIEF

HANDY

HANG

HAPPENED

HARDLY

HATBEX

HEALTH

HEALTHIER

HEALTHIEST

HEALTHY

HEALTHY=LEEKING

HEARTS

HEAVIER

HEAVY

HELD

HERE'S

HI

HIGHEP

HIGHWAY

HILLER

HILLY

HITTING

HOLD

HOPED

HOPING

HEPPED

HEPPING

HOSE

HOSPITAL

HUUSEKEEPER

HOUSETEPS

HOW:S

HUGE

HUMMINGB IRD

HUNT

IRON

ITSELF

JAY

JERRY

JIMMY

JEHNNY

JUICE

KATYOIDS

KEEPER

KEPT

KILLS

KITTY

KNOCK

LAKE

LANDEO

LANI

LARGER

LATER 
LEAP

LEAVING

LED

LETTING

LIONS

LIVING

LONGER

LONGHOUSE

LUNCHREEM

LUNCHTIME

$M=M=M$

MALEC

MARCHERS

MEETING

MERRIEST

MERRY - GB-REUND

MOLLY

MEVING

MYSELF

NEAREST

NEWEST

NEWSBEY

NO-WHEELED

NOD

NODDED

NOISEMAKER

NUISEMAKING

NOISIEST

NOISY

NOSE

NOTE

NUMBER

NUTMEAT

OAK

OBEY

$\mathrm{OH}-\mathrm{H}-\mathrm{H}$.

OIL

OIL $\rightarrow$ CEVERED

OILY

ELDER

ENE - WHEELED

ENO

ONTE

OWNER

PABLO

PALYTIME

PAN

PATTED

PAUL

PETTED

PFT $\rightarrow F T$

PIE

PINK

PIPER

$P L A Y=I N-T H E=S U N$

POINTING

POISONED

PONIES

PEOL

PEOR

$P Q P$

PEPPED.

PORCH

POSTURE

PETATE

POTTED 
$P R-R=R$

PRESENTS

PRETENDING

PUMPKINS

PUP

PUTTING

QUARREL

QUICKLY

QUIET

QUINN

QUIT

RAINY

RAINY ODAY

RAKER'S

RAKING

RATS

RATTLE

REACH

REAL

REMEMBERED

RIVER

ROARED

RECK

ROCKY

REDE

REW

RUBBED

RUBB ING

RUBS

RUNNY

RUSHING

SAM

SANDY

SANTES

SATAN

SAVE

SAVING

SCARECROW

SCARY

SCHOQLCHILDREN

SCHOOLTEACHER

SECEND-FLOUR

SET

SHAKE

SHAKING

SHALL

SHOLLDN'T

SID

SIDEWAYS

SITTING

SLOW-MEVING

SLOWLY

SLY

SLYMAN

SMALLER

SMALLEST

SMARTEST

SMARTY

SMILE

SMOKING

SNIFF-SN IFF

SNIFFED

SNIFFY

SEAPBOX

SOMEDAY

SENNY 
SORE

SPECKLE

SPOTTED

SQUEAKY

SQUEALING

STAIRS

STAIRWAYS

STAR

STARRY

STEPPED

STEVE

STOMACH-CLOCKS

STOMACHS

STEOD

STERYBEOK

STRINGER

SUDDEN

SUNNY $=D A Y$

SWIM

SWIMMING

TALLER

TALLEST

TASTE

TEACH

TEACHER

TEMPLES

TERRIBLE

THERE'LL

THESE

THROAT

THROWING

TIMMY

TOETH

TOUCH

TRACK

TRAINER

TWO - WHEELED

UGLY

UNCLE BOB

UNDERHANDED

UNHAPPY

UPPER

UPSET

UPSTAIRS

UPTOWN

USEFUL

VALENTINES

VEGETABLES

$\checkmark$ INE

WADDLE-WADDLE

WADDLES

WALKERS

WARMED

WATCHES

WATCHMAN

WAVED

WAVING

WE!D

WE ILL

WE'RE

WEAR

WEATHER

WEREN IT

WET

WHAT'S

WHIPPED 
WHI SPER ING

WHISTLING

WHEEVER

WHOLE

WILD

WILDCATS

WOO-FF

WOEDEN

WORKER

WREN

WR ITER

WRITES

WRITING

WRONG

WRETE

YELL

YELLOW-COLORED

YESTERDAY

YET

YOU'D

TMERE ARE 609 WORDS IN. LEVEL 6 OF SERIES E. IN COMPARING SERIES E WITH SERIES B. EACH AT LEVEL, 6 THERE IS A $3.28 \%$ MATCH. THERE ALSE IS A $20.03 \%$ MATCH (QUTSIDE OF LEVEL 6) RANGING FROM, $5=275$ TO $1-155$. 
THE BACK UP WORDS FOR LEVEL 7 ARE:

ACCIDENT

$\triangle E R I A L$.

AIMED

AIRPLANES

ALABAMA

ALARM

ALLERTEN

ALVIN'S

AMATEUR

AMEN

AMERICA

AMY

ANKLE

ANYBEDY'D

APPREACHING

ARROWS

ASHORE

ATTACKED

AWFUL.

BABCACK

BALANCE

BARTH

BASE

BATTED

BEAUTY

BLADES

BLAZED

BLEW

BLOWN

BONNIE

BOW

BUWSTRING

BRADFORD

BRASS

BRAVE

BRIGHTEN

BRIGHTLY

BROKE

BROKEN

BUCKET

BUSILY

BUTTEN

CAPTAIN

CARDS

CAROLERS

CARVER

CASE

CHARLES

CHIMNEY

CLEARING

CLIP

CLOSELY

CLOSET

CLOTHESPIN

COCOA

CEDE

CONNECT

CONSTANCE

CRAMER

CRASH

CREFK 
CROPS

CRUELTY

CUPFUL

CURB

DAN

DANA

DANGER

DANGERQUS

DAPHNE

DARKNESS

DAYDREAMING

DECK

DEERSKIN

DEFROST

DELIVER

DIAL

DIPLOMACY

DIRECTIONS

DIRECTER

DISAPPEARED

DISHES

DEUBT

DOUBTFULLY

ORAWN

DRIF TING

DUG

EDDIE

EDGE

ENVELOPE

EVENLY

FALLEN

FANCY

FASTENED

FEAST

FINISHED

FLAT

FLOODED

FOULISHLY

GARDENIA

GATHER

GILES

GINGERBREAD

GLOOMILY

GLOOMY

GOUSE

GOVERNOR

GRAIN

GREATLY

GROWN=UPS

GRUNTED

GUESTS

HALL

HANOFUL.

HANDLE

HANS

HARBOR

HEAVILY

HILDE!S

HISTORY

HO

HOBBLE

HONK

HOPKINS

HORN

HOURS

HOWL 
HOZAK

HUFFMAN

HUNGRILY

INVENTIONS

INVENTER

INVITED

JERK

$J O B$

JOINED

JUKE

KATALIN

KATRINA

KILPATRICK

KINDLY

KNIFE

KNOB

LANES

LANGUAGE

LANTERN

LEAD

LEANED

LEQNARD

LEVERS

LIBRARY

LOCKED

LOGS

LOESE

LOESELY

LOESEN

LOVELY

LUCKILY

MAGNET

MAILBQX

MASSACHUSETTS

MEDICINE

MELTS

MESSAGE

METAL

MODEL

MOSTLY

METIENS

METORBOAT

MEUSE

NANCY'D

NEARLY

NEMETH

NEWLY

NICKEL

NINE

NGISILY

OK

ELD-FASHIENED

QRDERS

OTTO

PAPPAS

PARROT'S

PARTLY

PASSED

PEDAL

PERFECT

PERFECTLY

PERFUME

PILGRIM

PILOT

PIONEERS

PITCHING 
PLANNING

PLATE

PLENTIFUL

PLOWED

PLUNGED

PLYMOUTH

PEEM

POSITIGN

PUSSIBLE

POSSIBLY

PREPARED

PREVENTIGN

PROJECT

PROMISE

PREUD

PROUDLY

PULLEYS

PUZZLED

QUIETLY

QUITE

R.AISE

RECREATION

REEL

REFRIGERATOR

RELEASE

ROASTED

REGERS

ROEFED

RUG

RULER

SADLY

SAFE

SAFELY

SAILING

SALE

SALTY

SAMOSET

SCIENCE.

SCELDING

SCRUBBED

SECRET

SEMAPHORE

SETTLERS

SEVENTH=DAY ADVENTIS

SEVERAL

SHE'D

SHIP

SHOIE

SHOOT

SHORTLY

SHET

SHOVED

SHULTZ

SIMPSON

SKIPPED

SLINGER

SMELL

SNEWFLAKES

SNEWSTERM

SOCIETY

SEFTLY

SeS

SQUP

SPACE

SPEAK 
SPEND

SPREAD

SQUANTE

STAMPED

STANDISH

STEADILY

STEADY

STEEL

STEPHEN

STEW

STRA I GHTENING

STRANGELY

STRIPS

STRUCK

STUEY

STUMBLED

SUIT

SUPERMARKET

SWAN

SWEGPED

TAPE

TAUGHT

TAXICAES

TEAMS

TELESCOPE

TENSION

TERRIBLY

TEST

THANKFUL

THATCH

THEY D

THIRSTY

THUNDER

TICK

TOE

TEOT

TRANSBM

TRAPPED

UNEELIEVINGLY

UNCEUKED

UNCEVERED

UNDEFSTOOD

UNEASILY

UNEXPECTED

UNLECK

UNSAFE

UNSETTLED

UNTIE

UNWILLINGLY

USELESS

VACATION

VACUUM

VAN VLIET

VIULIN

VISITER

VOICE

WAKED

WAKENEO

WALKIE $=$ TALKIE

WARNED

WATCHFULLY

WE! D

WEBER

WEIGHT

WELCOMES

WHIRLING 
WIDELY

WILLIAM

WILSEN'S

WIRE

WOLVES

WONDERFULLY

WERRIED

WORR IEDLY

WORSE

WERSHIP

WOUND

THERE ARE 445 WORDS IN LEVEL 7 OF SERIES E. IN COMPARING SERIES E WITH SERIES B ,EACH AT LEVEL, 7 THERE IS A .0O\% MATCH.

THERE: ALSE IS A $16.40 \% \mathrm{MATCH}$ (OUTSIDE OF LEVEL 7 ) RANGING FROM, $6=293$ TO $2=155$. 
THE BACK-UP WOROS FUR LEVEL 8 ARE:

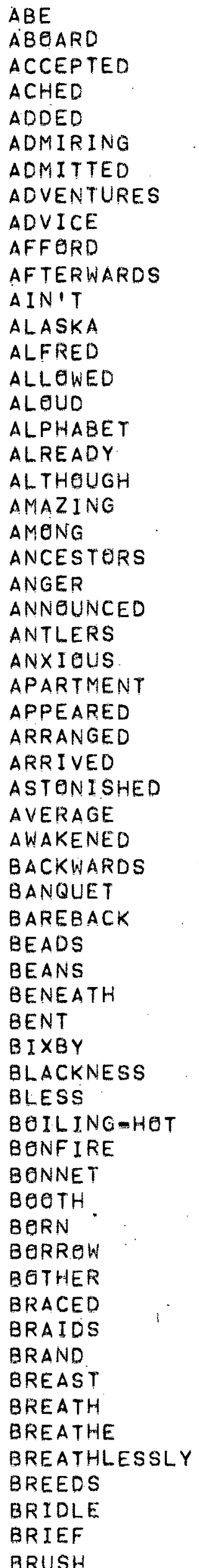


BUBBLES

BUCK

BUD

BUNK

BURST

CALIFURNIA

CAPTURED

CARTURIGHT

CATTLE

CAUTIOUSLY

CEILING

CELEBRATED

CELERY

CENTERED

CENTS

CHAIRBEUND

CHANTED

CHARLEY

CHARLIE

CHATTED

CHEEKS

CHEROKEE

CHINATOWN

CHINESE

CHOKED

CHOPS

CHORES

CHUCKLED

CLAPPED

CLASSES

CLIFF

CLEUDY

CLUBS

COCONUT

COLLAR

COMFORT

COMMITTEE

CONFUCIOUS

CONSIDER

CONVENIENT

COPYCAT

CORRAL

COSTS

COSTUMES

CETTEN

COUPLE

CRAWFORD

CRAWL

CRIPPLED

CUNNING

CUNN I NGHAM

CURIUUS

$D \cdot C$.

DALE

DAMP

DANCES

DANDER

DARED

DEAD

DEAL

DEBBIE

DEBT

DECEIVE

DECLARED

DECORATIONS DECOY 
DELIGHTED

DENMARK

DENNIS

DESERT

DESERVES

DESK

DESPAIF

DESTREY

DETOUR

DEW

DIDOS

DIFFERENCE

DIFFICULTY

DINING

DION

DIPPED

DISAPPOINTED

DISAPPOINTMENT

DISCQURAGED

DISCOVERED

DISEASE

DISSATISFIED

DISTANCE

DISTANT

DITCH

DIVED

DIVIDED

DEE

DELLARS

DREWINED

DULL

DUMB

DUST

EAGER

EAGERNESS

EARNED

EARTHQUAKE

EMPT INESS

ENGLAND

ENGLISH

ENITH

ENJOYED

ENERMEUS

ENTER

ENVY

ERRAND

ESPECIALLY

EXAMINE

EXCITEMENT

EXCUSE

EXERCISE

EXPERIMENTS

FACT

FAILURE

FAITHFUL

FAVERITES

FEARFULLY

FEARLESS

FERNWOOD

FEVER

FIFTEEN

FIRMLY

FLIGHT

FLOATING

FLOCK

FLUFFY 
FLUSHED

FEOLISHNESS

FQRENEON

FQRTUNATELY

FERTY

FORWARD

FOURTEEN

FREEZE

FREST

FROWNED

FUR

FURNI TURE

GAZED

GENTLE

GERTRUDE

GIANT

GIFT

GIGGLE

GLANCED

GLARE

GELD

GOODNESS

GOSPEL

GOURDFUL

GRACEFUL

GRANNY

GRATEFULLY

GROWL

GRUMBLED

GUN

GUPPIES

HALFWAY

HAMILTON

HAMMER

HARE

HARM

HASTILY

HEAVED

HEIGHT

HERD

HEY

HID

HEE

HOGBACK

HOLIDAYS

HOLLOW

HONORABLE

HENORS

HOTEL

HUG

HUNDREOS

HUT

ICEBERG

ILLS

IMAGINE

IMMED I ATELY

IMPATIENTLY

IMPOLITELY

INCHES

INTREDUCING

INVESTMENT

ISABELLA

ISLAND

JEROME

JOHNSTON

JESEPH 
KALLER

KANGAREO

KAYAK

KETTLE

KIMMY

KIRSTEN

LACOUR

LAD

LAME

LANKY

LARSEN

LATHERED

LAUGHTER

LAWN

LEAFY

LEAKING

LEDGE

LEI

LEVEE

LI

LICK

LID

LIE

LIN

LINCELN

LIPS

LOANED

LOFT

LOHMANN

LONELY

LONESOME

LORD'S

LOSE

LOTTIE

LQUELLA

LOUIS

LUMP

LYING

MA

MAD

MAGIC

MAILE'S

MAIN

MAMMA

MANAGE

MANALUK'S

MAPLE

MARKED

MARRIAGE

MARRY

MATE

MATHILDA

MATOAKA

MAYOR

MEADOW

MEAL

MEMBERS

MENTION

MEXICO

$M I D$

MIRACLE

MISSISSIPPI

MISSOURI

MIST 
MISTAKEN

MITE

MIX

MOM

MOMENT

MONTHS

MOON

MOWER

MUMPS

MURMUR

MUTTERED

NATURALLY

NEEDLE

NEGRO

NETS

NOODLES

NORTH

NURSE

EARS

OBL IGE

BFF ICER

OKLAHGMA

EUTFIT

OUTWARD

OVERAGE

OWE

PA

PACKEO

PADDLE

PAID

PAIN

PAIRS

PARDEN

PARIS

PEACE

PEACHES

PEBELES

PECULIAR

PEERED

PEONY

PIGEON

PILLOW

PINES

PING

PITY

PLAINLY

POCAHONTAS

PEEM

PORTER

POSTMASTER

PUUND

PQURED

POWHATAN

PREACHER

PRECIUUS

PRESICENT

PUDDLE

PUNCH

QUESTIENED

GUOTATIEN

RADISH:

RAM

RANCH

REALIZED

REALTIONS RFAR 
REASEN

REBECCA

REBUILT

RECKEN

RECERD

REFUSED

RELIEVED

REMAINED

REMINDED

REPEATED

RESCUE

RESERVATION

REVELVING

REWAFDS

RIBBEN

RICE

RICHES

RINSE

RISE

ROD

RELFE

ROYAL

RUFE

RUFFLING

RUSTY

SACK

SADDLE

SADNESS

SAN FRANCISCO'S

SANK

SARAH

SATISFIED

SCAMP

SCRAPE

SCUFFLED

SEA

SEARCHING

SEASEMED

SEAWEED

SEQUOYAH'S

SHARP

SHAVED

SHEET

SHELF

SHELL

SHELTERED

SHETLAND

SHINY

SHIVEP

SHONE

SHOULDER

SILENCE

SILK

SLID

SLIP

SMITH

SMESTH

SNAILS

SNATCHED

SNUG

SEARED

SOBBING

SOLES

SOB-PUNG

SORT

SOUTH 
SPANKED

SPARED

SPARKLING

SPARKS

SPEECH

SPEECHLESS

SPELLING

SPENT

SPILLED

SPIN

SPINE

SPIRITS

SPROUTS

SPUTTERED

SQUISH

STAG

STARED

STARTLED

STEAMING

STERNLY

STIFFLY.

STRAY

STREAKS

STUBBERN

STUCK

STUDY

STUFF

STUPID

SUCCEEDED

SUFFER

SWALLOWED

SWIRL ING

SWITCHING

SWUNG

SYLLABLES

SYRUP

TAG

TAMEST

TASK

TENDERLY

TENTS

TERRIF IC

TERROR

TEXAS

THEE

THEMSELVES

THOMAS

THOUSANDS

THREAD

THREA TENED

THREW

THUMBS

THUNDERBOLT

TIDE

TIPPING

TEASTY

TONGUES

TOSSED

TOWERING

TRAILER

TRAVELWAYS

TROPICAL

TREUGH

TRQUSERS

TRUSTED 


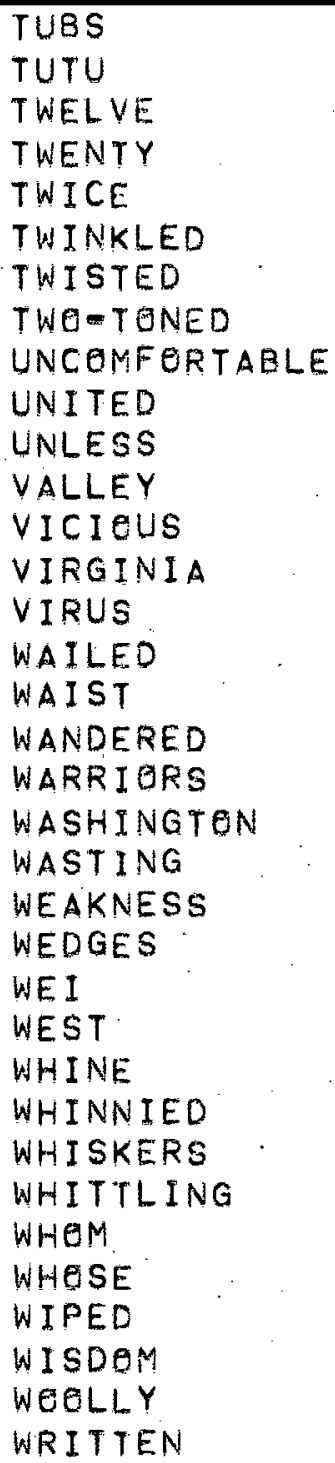

THERE ARE 697 WORDS IN LEVEL. \& EF SERIES E. IN COMPARING SERIES E WITH SERIES 8 , EACH AT LEVEL, 8 THERE IS A .00\% MATCH. THERE ALSO IS A $12.77 \%$ MATCH IEUTSIDE OF LEVEL 8 ) RANGING FROM, $6=310 \mathrm{TE} \quad 1=14$. 
THE BACK-LP WORDS FUR LEVEL 1 ARE:
COME
DEWN
GET
GQ
HERE
LUEK
MIKE
PAM
PUFF
SALLY
TIM

THERE ARE 21 WERDS IN LEVEL. 1 OF SERIES E. IN COMPARING SERIES E WITH SERIES C , EACH AT LEVEL, 1 THERE IS A .0O\% MATCH.

THERE ALSE IS A $47.62 \%$ MATCH IEUTSIDE OF LEVEL 1 ) RANGING FROM. $6=119$ TO $4=36$. 
THE BACK - UP WORDS FOR LEVEL 2 ARE:
AND
BALL
CAN
COME
FATHER
GET
$G \theta$
HERE
I
IS
JESUS
LEEK
MIKE
METHER
NET
PAM
PLAY
PUFF
SAID
SALLY
SEE
THE
TIM
TO
WANT
WITH
YeU

44 WORDS IN LEVEL 2 OF SERIES E.

IN COMPARING SERIES E WITH SERIES C , EACH AT LEVEL, 2 THERE IS A . OQ\% MATCH.

THERE ALSE IS A $34.09 \%$ MATCH (EUTSIDE OF LEVEL 2) RANGING FROM, $6=119$ TE $4=11$. 
THE BACK=UP WORDS FOR LEVEL. 3 ARE:

A
AND
BALL
BIG
CAN
CAR
COME
COMES
DOWN
FATHER
FOR
GET
GETS
GO
HERE
I
IS
IT
JESUS
LIKE
LIKES
LITTLE
LOOK
MIKE
MOTHER
NOT
NOW
PLAY
PUFF
RED
RIDE
SAID
SALLY
SEE
SOMETHING
THE
TIM
TO
UP
US
WANT
WANTS
WHAT
WHO
WILL
WITH
YOU

THERE ARE 84 WORDS IN LEVEL 3 OF SERIES E. IN COMPARING SERIES E WITH SERIES C , EACH AT LEVEL, 3 THERE IS A .00\% MATCH. THERE ALSO IS A $39.29 \%$ MATCH (OUTSIDE OF LEVEL 3) RANGING FREM. $8=24$ TO $4=11$. 
THE BACK-UP WORDS FOR LEVEL 4 ARE:

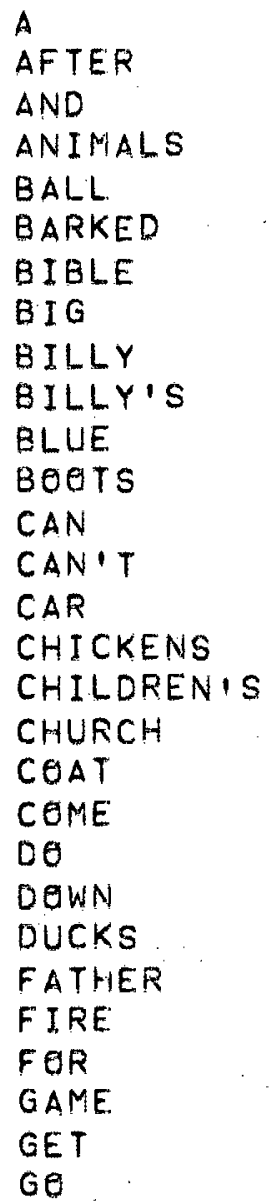

GRANDFATHER

GRANDMOTHER

HAT

HE

HENS

HER

HERE.

HEP

I

IS

IT

JESUS

LIKE

LITTLE

LOOK

LOOKED

MAKE

MAYBE

MIKE

MIKE'S

MOTHER

NOT

NOW

$O N$

PAM

PETE.

PETE'S

PLAY

PONY

PUFF

RABBITS 
READS

RED

RIDE

SABBATH

SAID

SALLY

SALLYIS

SEE

SOMETHING

TAILS

TAKE

THE

TIM

TIME

TO

TRAIN

UP

US

WANT

WANTED

WHAT

WHO

WILL.

WITH

YELLOW

YES

YOU

THERE ARE 186 WORDS IN LEVEL 4 OF SERIES E. IN COMPARING SERIES E WITH SERIES C EACH AT LEVEL, 4 THERE IS A $27.96 \%$ MATCH. THERE ALSO IS A $22.58 \%$ MATCH (OUTSIDE OF LEVEL 4) RANGING FROM, $8-24$ TH $5=129$. 
THE BACK -UP WORDS FOR LEVEL 5 ARE:

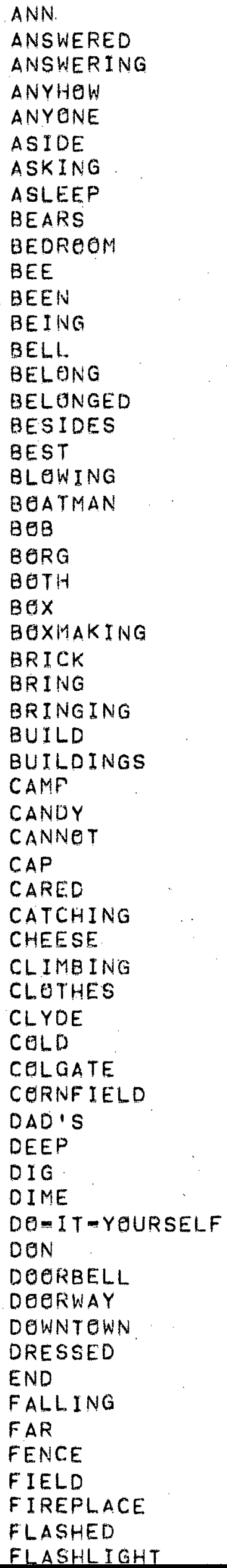


FOOTBALL

FERD

GET - TOGETHER

GOD'S

GRASSY

GREENHUUSE

HANDING

HARR IS

HAYDEN

HEADING

HEAR

HEARD

HEARING

HEINZ

HELPFUL

HENRY'S

HEREAFTER

HERSELF

HERSHEY

HIDE

HIGH

HILLSIDES

HIMSELF

HOOK

HOOKED

HOT

HOWEVER

INSIDE

ISN'T

JOLLYY

KNOWING

$K O D A$

KRAFT

LAUGHING

LETTER ING

LEW

LINES

LIVELONG

LONG - TA ILED

LONGING

LOTS

LOUGHBOROUGH

LUNCH

MACHINES

MAKING

MARRIGTT

MET

MILTEN

MISSIEN

MOVED

NAMED

NEAR

NEARBY

NEARING

NEED

NEEDED

NEIGHBOR

NEIGHBORHOOD

NEVER

NEWSPAPER

NICE

NIGHTBIRDS

- ' CLOCK

OUTDOORS

OUTSIDE

OWNED 


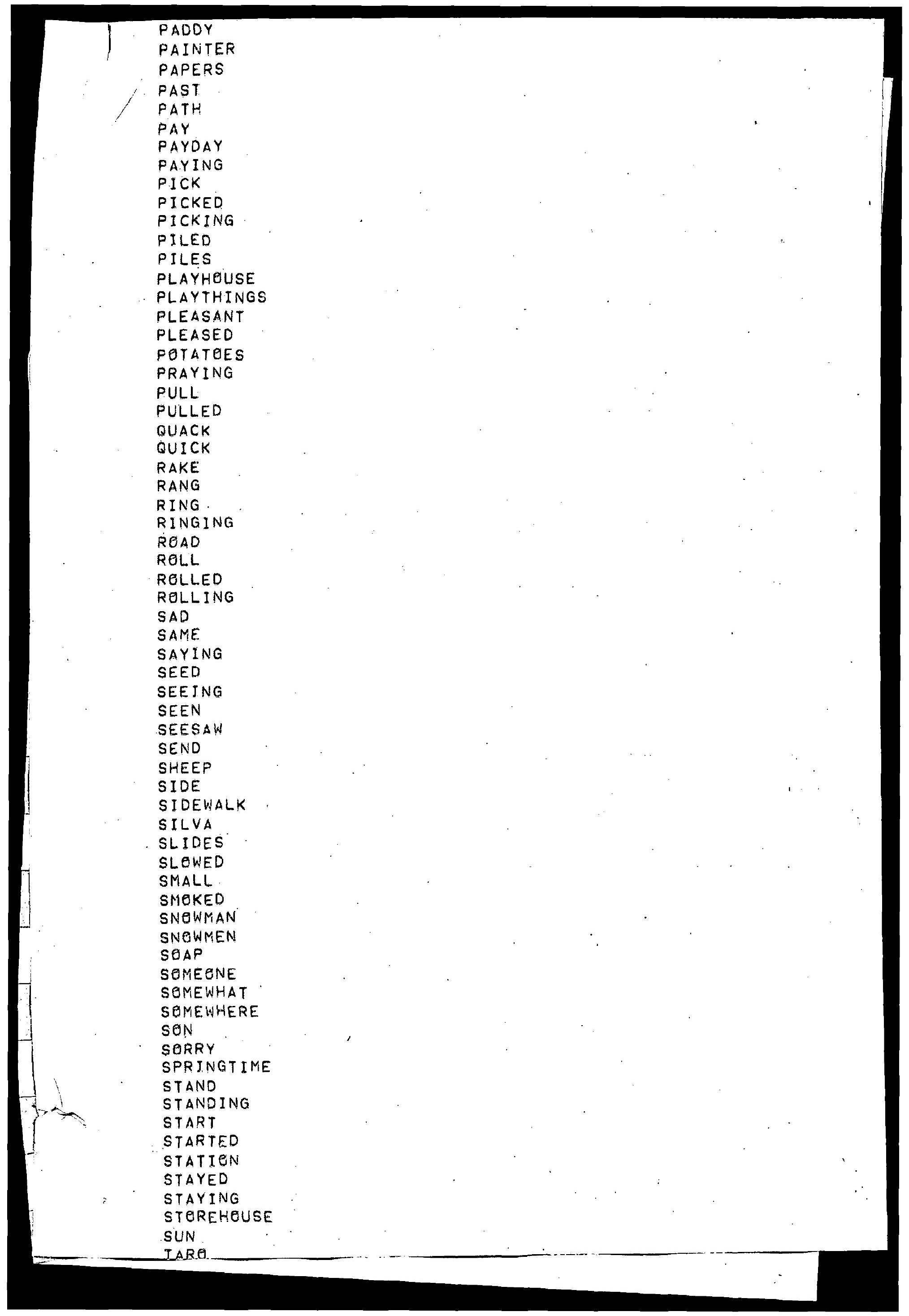


TEN

THERE'S

THEREBY

TIE

TITHE

TITHED

TOGETHER

TOLD

TONY

TEULHOUSE

TOOLS

TEP

TREETEPS

TRICK

TRIED

TURN

TURNED

UNDERCLOTHES

UNDERSTAND

USED

WAITED

WAITING

WANT ING

WATCH

WATCHED

WATCHING

WATER

WENDY

$W H-E E=E$

WHATEVER

WHEAT

WHEELED

WHEELS

WHENEVER

WHE!S

WILLING

WINDOW

WISE

WORKMEN

THERE ARE 371 WURDS IN LEVEL 5 OF SERIES E. IN COMPARING SERIES E WITH SERIES $C$, EACH AT LEVEL, 5 THERE IS A $7.28 \%$ MATCH,

THERE ALSO IS A $19.41 \%$ MATCH (GUTSIDE OF LEVEL 5 ) FANGING FROM, S-114 TO $4=102$. 
THE BACK-UP WORDS FOR LEVEL 6 ARE:

$\triangle C T$

$\triangle H E A D$

ALIKE

ANYMQRE

APART

AREN $\cdot T$

ARMS

AUNT JANE

AWHILE

$B A=A=A$

BABIES

BARS

BASKETBALL

BEAMS

BECOME

BEDDING

BEDTIME

BELOW

BIDDY

BIKES

BIRDIES

BITE

BITING

BITS

BLOCK

BLUEBELL

BLUEBERRIES

BLUEBERRY

BOASTED

BODIES

BODY

BEOY-HOUSE

BEXES

BRANCH

BRANCHES

RRIDGES

BRIGHT

BRIGHT-EYED

BROWN-AND-WHITE

BUILDER

BURNING

EUS

BUSH

BUSHES

BUTTER

BUTTERCUP

BUTTERNUT

BUZZ

CAGE

CAREFULLY

CARLES

CART

CERTAINLY

CHANCED

CHANGE

CHASE

CHASING

CHIN

CLEANER.

CLEVER

ClOCK 
CLOSE

CLESER

COAX

COOK

COOL

COVERED

COWBOY

CRACKING

CRUWDED

CREWFEOT

CRYING

CUPBOARD

CUPCAKES

CURLS

CURLY

CURLY $=$ HAIRED

DADDY

DASHED

DAYTIME

DIRT

DIRTY

DOCTORFFATHER

DOCTERS

DEESN T T

DOWNSTAIRS

DRA'W

DRESS-UP

DRESSES

DRINK

DRIVE

DRIVER

DRIVING

UREP

DREPPED

EARTHMOVERS

EARTHY

EASIER

EASILY

EGG-HOUSES

EIGHT-YEAR-OLD

EXCITED

EYE = HOLES

FARAWAY

FEELING

FIERCE

FILLED

FINALLY

FIREBELL

FIREHOUSE

FIREMAN'S

FIREMEN

FISHES

FIXED

FLAG

FLEW

FLYER

FOLLOWING

FUOL

FEOLISH

FOUTSTEPS

FRIDAY

FRIENDLY

FRUITS

FUNNIEST

FUNNY-LOGKING

GALLOPING 


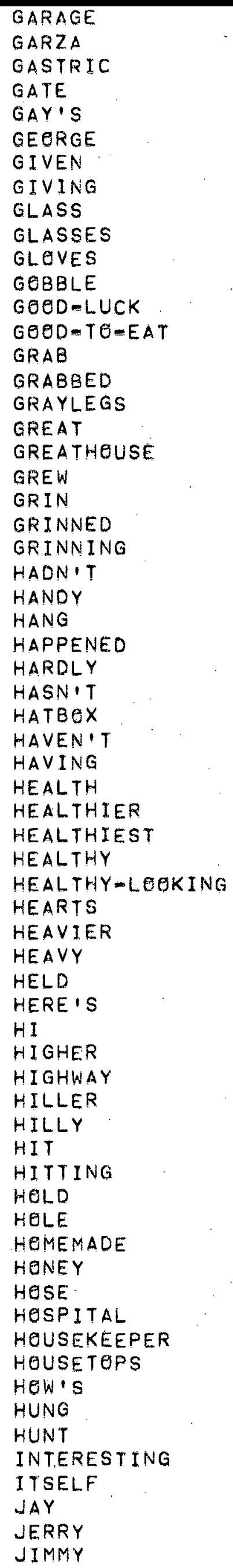


KATYDIDS

KEEPER

KICKED

KILLS

KING

KITTY

LADDER

LAKE

LANDED

LAN I

LARGER

LAY

LEAP

LEAS !

LEAVING

LED

LETTING

LIFE

LIONS

LIVING

LONGER

LONGHOUSE

LUNCHROEM

LUNCHTIME

$M=M-M$

MALEC

MARCHERS

MARY

MEAT

MEETING

MERRIEST

MERRY $=G O-R O U N D$

MOLLY

MOVING

MYSELF

NAILS

NEAREST

NECK

NEWEST

NEWSBOY

NO-WHEELED

NOD

NEDDED

NOISEMAKER

NOISEMAKING

NOISIEST

NOISY

NOSE

NOTE

EFFER

$\mathrm{OH}-\mathrm{H}-\mathrm{H}$

OIL

OIL-COVERED

OILY

OLDER

ONE-NHEELED

QNO

ONTE

EUGHT

OWNER

PABLO

PAIL

PALYTIME

PAN

PART

PARTIES 
PAT

PATTED

PAUL

PECKING

PETERSON'S

$P F T=F T$

PIE

PIGS

PINK

PIPER

PLAY - IN-THE-SUN

POINTING

POISONED

PUNIES

POEL

POOR

POP

POPPED

PORCH

POSTURE

POTATE

PUTTED

$P R=R \sim R$

PRETENDING

PRINCE

QUINN

QUIT

RACE

RACING

RAGS

RAINY

RAINY-DAY

RAKER!S

RAKING

RATS

RATTLE

REACH

REMEMBERED

ROCKY

RODE

ROSY

ROW

RUBBING

RUBS

RUNNY

RUSHING

SAM

SANDY

SANTOS

SATAN

SAVE

SAVING

SCARECROW

SCHOQLCHILDREN

SCHOOLTEACHER

SCREAMED

SECOND -FLOUR

SET

SHAKE

SHAKING

SHALL

SHIRT

SHOEK

SHOULDN'T

SHUT

SID 
SIDEWAYS

SINCE

SING

SITT ING

SLOW-MEVING

SLEWLY

SLY

SLYMAN

SMALLER

SMALLEST

SMARTEST

SMARTY

SMILE

SMOKING

SNIFF = SNIFF

SNIFFED

SNIFFY

SOAPBOX

SOMEDAY

SONNY

SERE

SPECKLE

SPEKE

SPETTED

STAIRWAYS

STARRY

STEPPED

STEVE

STOMACH=CLOCKS

STEMACHS

STOOD

STERYBOOK

STRAIGHT

STRANGE

STRANGER

STRAW

STRAW=PILE

STRINGER

SUDDEN

SUNNY-DAY

SURELY

SWIFTLY

SWIM

SWIMIING

TALL

TALLER

TALLEST

TASTE

TEACH

TEACHER

TEMPLES

THERE' LL

THESE

THREAT

THROWING

TOOTH

TOUCH

TRACK

TRAINER

TRUNKS

TWU - WHEELED

UNCLE BOB

UNDERHANDED

UPPER

UPSET 


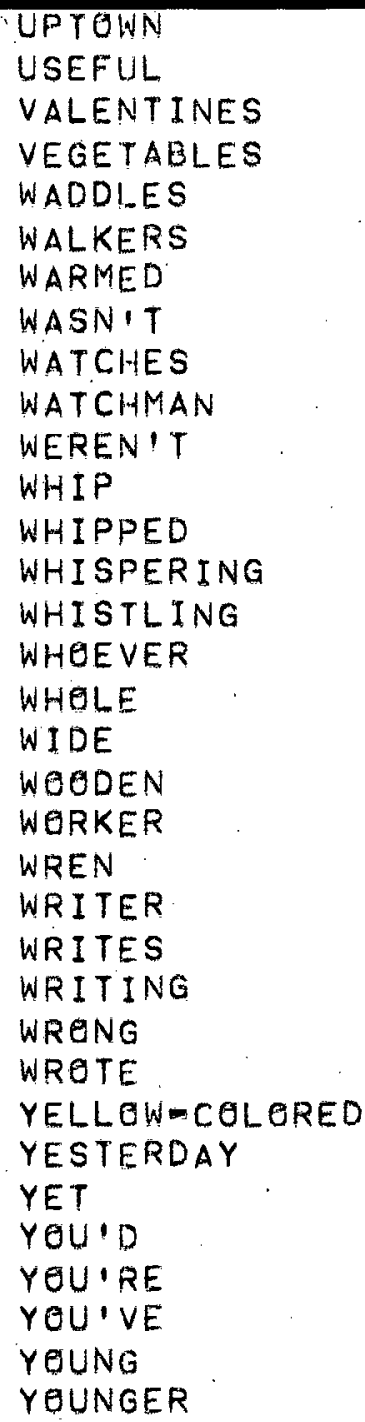

THERE ARE 609 WORDS IN LEVEL 6 OF SERIES E. IN COMPARING SERIES E WITH SERIES C , EACH AT LEVEL, 6. THERE IS A $7.55 \%$ MATCH. THERE ALSO IS A $15.60 \% \mathrm{MATCH}$ (EUTSIDE OF LEVEL 6) RANGING FROM, $g=214$ TO $4=146$. 
THE BACK-UP WORDS FOR LEVEL 7 ARE:

ACCIDENT

AERIAL

AGREE

AIMED

ALLERTON

ALVIN'S

AMATEUR

AMEN

AMERICA

AMY

ANKLE

ANYBGDY:D

APPROACHING

ATTACKED

$B A B C O C K$

BALANCE

BARTH

BASE

BATTED

BATTERY

BEACH

BEAUTY

BEGGED

BLADES

BLAZED

BLEW

BLOWN

BONNIE

$B O W$

BRADFORD

BRASS

BRAVE

BRIGHTEN

BRIGHTLY

BROKE

BREKEN

BUCKET

BUILT

BULB

BUNDLES

BUTTON

CABIN

CALM

CALMLY

CANDLES

CARDS

CAROLERS

CARVER

CASE

CHARLES

CHECK

CHEERFUL

CHICAGO

CHESE

CHOSEN

CHR ISTMAS

CHURN

CLEARING

CLIP

CLOSELY

CLOSET 
CLOTHESPIN

CLEWN

CECBA

CODE

CONNECT

CONSTANCE

CQPPER

CRAMER

CRASH

CREEK

CREPS

CRUELTY

CUPFUL

CURB

DAN

DANA

DANGER

DANGEREUS

DAPHNE

DARKNESS

DAYDREAMING

DECK

DEERSKIN

DEFROST

DELIVER

DIAL

DIED

DIPLOMACY

DIRECTIONS

DIRECTOR

DISAPPEARED

DISHES

DOUBT

DOUBTFULLY

DRAWN

DRIFTING

DUG

EDDIE

EDGE

ELDER

ELECTRIC

ENGINEER

ENVELOPE

EVENLY

EXPECTED

EXPLAINED

EXPLURING

FAINT

FEAST

FINGERS

FINISHED

FLAT

FLOODED

FOOLISHLY

FORT

GARDENIA

GATHER

GEESE

GILES

GINGERBREAD

GLOOMILY

GLEOMY

GOOSE

GOVERNOR

GRAIN 
GROWN-UPS

GRUNTED

GUESTS

HALL

HANDFUL

HANS

HEAVILY

HILDE'S

HONK

HEPKINS

HERN

HOWL

HEZAK

HUFFMAN

HUNGRILY

INGRID

INVENTIONS

INVENTOR

INVITED

JERK

$J A B$

JOKE

KATALIN

KATRINA

KILPATRICK

KINDLY

KNIFE

$K N O B$

LANES

LANTERN

LEATHER

LEONARD

LEVERS

LIST

LOCKED

LOGS

LUCKILY

MAGNET

MAILBOX

MASSACHUSETTS

MEOICINE

MELTS

MESSAGE

MILES

MILKMEN

MILLIEN

MODEL.

MESTLY

METIENS

METORBEAT

MUDDY

NANCY'D

NEARLY

NEMETH

NEWLY

NICKEL

NINE

NEISILY

NOTICED

BK

OLDMFASHIENED

ORDERS

OTTO

PAPPAS

PARROT'S

PARTLY 


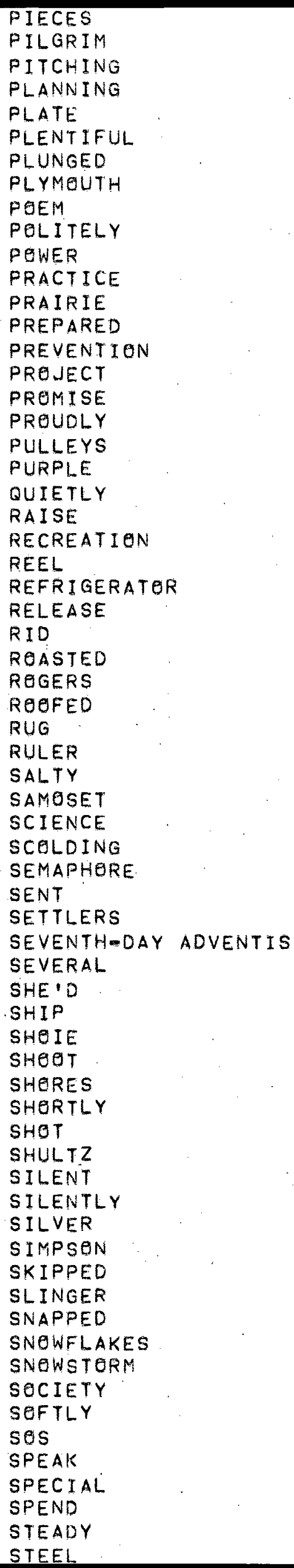




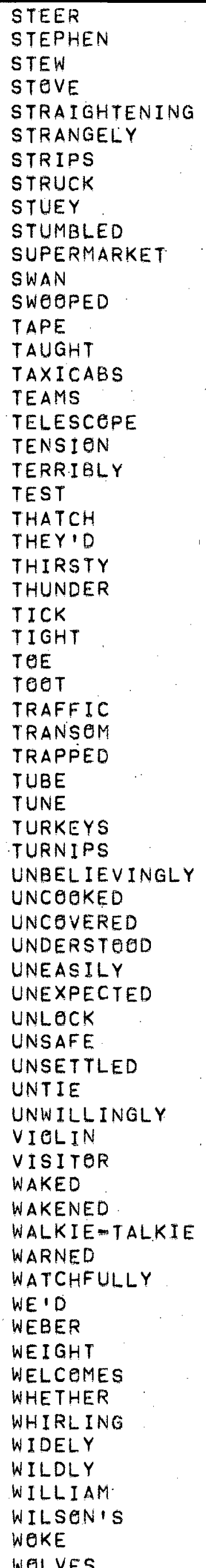



THERE ARE 446 WORDS IN LEVEL 7 OF SERIES E. IN COMPARING SERIES E
WITH SERIES $C$, EACH AT LEVEL, 7 THERE IS A $4.93 \%$ MATCH. THERE ALSO IS A $16.37 \%$ MATCH IOUTSIDE BF LEVEL 7 I RANGING FROM, $g=280$ TO $4=126$ 
THE BACK=UP WORDS FOR LEVEL 8 ARE:

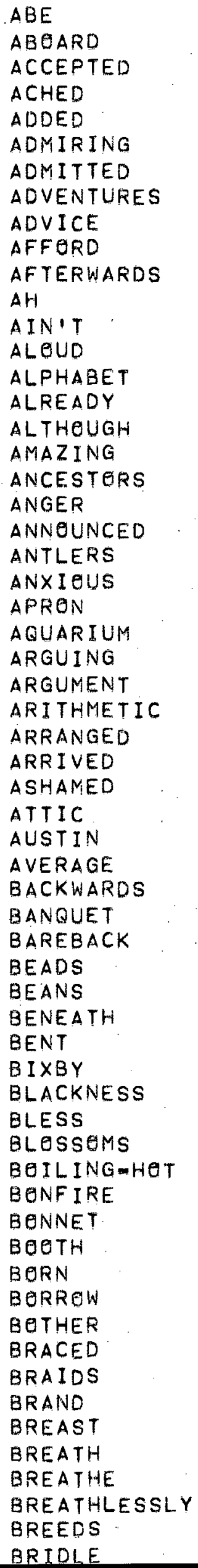


BRIEF

BRUSH

BUNK

BURST

BUSINESS

CALIFORNIA

CAPTURED

CARTWRIGHT

CAUTIOUSLY

CEILING

CELEBRATED

CELERY

CENTERED

CENTS

CHAIRBQUND

CHANTED

CHARLEY

CHARLIE

CHATTED

CHEEKS

CHERUKEE

CHINATOWN

CHINESE

CHOKED

CHOPS

CHORES

CHUCKLED

CLAIM

CLAPPED

CLASSES

CLEUDY

CLUBS

CEAL

COCONUT

COLLAR

COMFORT

COMMITTEE

COMPLAINED

CONFUCIUUS

CONSIDER

CONTEST

CONTINUED

CONVENIENT

CEPYCAT

CORRAL

COSTS

COSTUMES

COTTON

COUPLE

COURT

COUSIN

CRAWFORD

CRAWL

CRIPPLED

CUNNING

CUNNINGHAM

CURIUUS

CUSTEMERS

D.C.

DALE

DAMP

DANCES

DANDER

DARED

DEAD

DEAL 
DEBBIE

DEBT

DECEIVE

DECLARED

DECORATIUNS

DECOY

DELIGHTED

DESERT

DESERVES

DESK

DESPAIR

DESTROY

DETOUR

DEW

DIDOS

DIFFERENCE

DIFFICULTY

DIEN

DIPPED

D ISAPPOINTMENT

DISCEURAGED

DISCUVERED

DISEASE

DISSATISFIED

DISTANCE

DISTANT

DITCH

DIVED

DIVIDED

DOE

DRAGQN

DRAIN

DREW

DREVE

DROWNED

DULL

DUMB

DUST

EARTHQUAKE

EMPTINESS

ENVY

ERRAND

ESPECIALLY

EXAMINE

EXCUSE

EXERCISE

EXPERIMENTS

FACT

FAILIURE

FAITHFUL

FAULT

FELLOW

FERNWOOD

FEVER

FIFTEEN

FLAMES

FLIGHT

FOEL I SHNESS

FORENOON

FERTUNATELY

FERTY

FORWARD

FEURTEEN

FREEZE

FREST

FROWINED 
FUR

FURN I TURE

GERTRUDE

GRACEFUL

GRATEFULLY

GREUPS

GROWL

GRUMBLED

GUN

GUPPIES

HALFWAY

HAMILTON

HAMMER

HARE

HARM

HASTILY

HAVA

HEAVEO

HEIGHT

HERD

HEY

HID

HEE

HOGBACK

HENERS

HUG

HUNDREDS

HUT

ICEBERG

ILLS

IMAG INE

IMMEDIATELY

IMPATIENTLY

IMPOLITELY

INCHES

INSISTED

INSTANTLY

INTERRUPTED

INTRODUCING

INVESTMENT

ISABELLA

ISLAND

JACKET

$J A M$

JAMES

JOHNSTON

JOSEPH

JeY

JUDGING

KALLER

KANGAROO

KAYAK

KETTLE

KIMMY

KIRSTEN

LACOUR

LAD

LADY

LAID

LAME

LANKY

LARSEN

LATHERED

LAUGHTER

LAWN 
LEAKING

LEDGE

LEI

LESS

LESSON

LEVEE

LI

LICK

LID

LIE

LIN

LINCELN

LIPS

LOANED

LOHMANN

LONELY

LONESOME

LORD!S

LOSE.

LETTIE

LOUELLA

LEUIS

LUMP

LYING

MA

MAILE'S

MAIN

MAMMA

MANAGE

MANALUK'S

MAPLE

MARKED

MARRIAGE

MARRY

MASTER

MATE

MATHILDA

MATEAKA

MAYOR

MEADOW

MEAL

MEANT

MEASURED

MEASUREMENT

MEEKLY

MEISTER

MEMBERS

MEXICO

MID

MINE

MIRACLE

MISCHIEF

MISSISSIPPI

MISSOURI

MIST

MISTAKEN

MITE

MIX

MOM

MOMENT

MENTHS

MEON

MOWER

MUMPS

MURMUR

NATURALLY 
NEAT

NEEDLE

NEGRE

NEITHER

NETS

NOODLES

NERTH

NURSE

UARS

OBL IGE

UFFICER

OKLAHOMA

OUTFIT

OUTWARD

GVERAGE

QWE

$P A$

PACKED

PADDLE

PALE

PANT ING

PARDON

PASSENGER

PASTEUR

PATCH

PATIENT

PAUSED

PEACE

PEACHES

PEBBLES

PECULIAR

PEONY

PERSEN

PIGEON

PILLOW

PINES

PING

PITY

PLAINLY

PECAHENTAS

PQRTER

POSTMASTER

POUNO

POURED

POWHATAN

PREACHER

PRECIOUS

PRESIDENT

PRINTED

PRISONER

PRIZE

PROBLEM

PROTECTED

PROTESTED

PROVED

PUDDLE

PUNCH

QUESTIONED

QUUTATION

RADISH

RAM

REALIZED

REALTIONS

REAR

REASON

REBECCA 


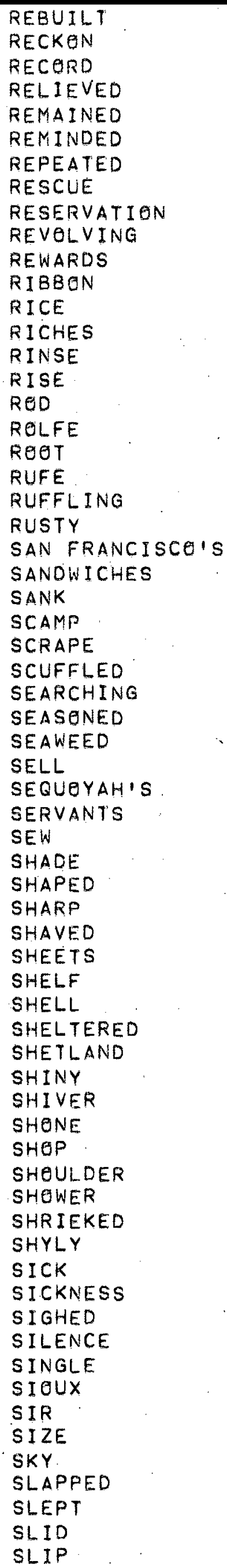


SNAILS

SNATCHED

SNUG

SUARED

SOBBING

SULES

SENG

SOO-PUNG

SOUTH

SPANKED

SPARED

SPARKLING

SPARKS

SPEECH

SPEECHLESS

SPELLING

SPENT

SPILLED

SPIN

SPINE

SPIRITS

SPREUTS

SPUTTERED

SQUISH

STAG

STERNLY

STIFFLY

STEOL

STRAY

STREAKS

STRETCH

STUBBERN

STUCK

STUDY

STUFF

STUPID

SUCCEEDED

SUFFER

SUGGESTED

SWALLOWED

SWITCHING

TAG

TAMEST

TASK

TEARS

TENDERLY

TENTS

TERRIFIC

TERROR

THEE

THICK

THOMAS

THREATENED

THUMBS

THUNDERBOLT

TIDE

TILL

TIPPING

TOASTY

TOSSED

TOWERING

TRADE

TRAILER

TRAVELWAYS

TREMBLED

TR IBF 
TRIM

TROPICAL

TROUGH

TROUSERS

TRUSTED

TRUTH

TUBS

TUTU

TWELVE

TWENTY

TWICE

TWINKLED

TWO-TONED

UNCAMFORTABLE

URGED

USUALLY

VICIUUS

VIRGINIA

VIRUS

WAGGING

WAILED

WAIST

WANDERED

WARRIORS

WASHINGTON

WASTING

WEAKNESS

WEDGES

WEI

WEST

WHINE

WHINNIED

WHISKERS

WHITTLING

WHOM

WICKED

WIN

WIPED

WON

WOELL.Y

THERE ARE 697 WURDS IN LEVEL 8 EF SERIES E. IN COMPARING SERIES E WITH SERIES C , EACH AT LEVEL, 8 THERE IS A $4.73 \%$ MATCH.

THERE ALSO IS A $8.75 \%$ MATCH 18 UTSIDE OF LEVEL 8 ) RANGING FROM, $9=279$ TU $4=146$. 
THE BACK $=$ UP WORDS FOR LEVEL 1 ARE:

$$
\begin{aligned}
& \text { DICK } \\
& \text { JANE } \\
& \text { MIKE } \\
& \text { PAM } \\
& \text { PENNY } \\
& \text { PUFF } \\
& \text { SALLY } \\
& \text { TIM }
\end{aligned}
$$

THERE ARE 21 WORDS IN LEVEL 1 GF SERIES E. IN COMPARING SERIES E WITH SERIES D. EACH AT LEVEL, 1 THERE IS A .00\% MATCH. THERE ALSE IS A $61.90 \%$ MATCH IOUTSIDE OF LEVEL 1 I RANGING FROM, $11=29$ TO $3=37$. 
THE BACK -UP WORDS FGR LEVEL 3 ARE:
DICK
DID
JANE
JESUS
MIKE
PAM
PENNY
PUFF
TIM

THERE ARE 85 WORDS IN LEVEL 3 OF SERIES E. IN COMPARING SERIES E WITH SERIES D, EACH AT LEVEL, 3 THERE IS A $7.06 \%$ MATCH. THERE ALSO IS A $78.82 \%$ MATCH IOUTSIDE OF LEVEL 31 RANGING FROM. $11=83$ TO $4=6$. 
THE BACK IUP WORDS FOR LEVEL 4 ARE:

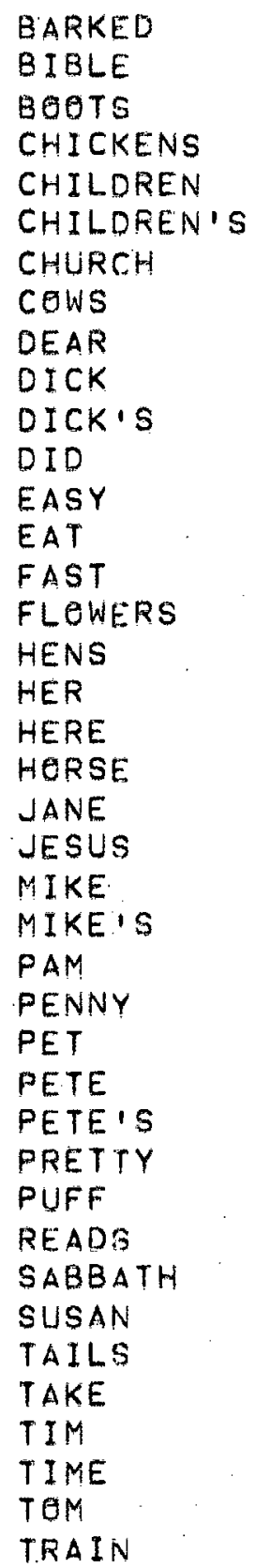

THERE ARE 186 WORDS IN LEVEL 4 GF SERIES E. IN COMPARING SERIES E WITH SERIES D ,EACH AT LEVEL, 4 THERE IS A $13.98 \%$ MATCH. THERE ALSO IS A $62.37 \%$ MATCH (OUTSIDE OF LEVEL 4 ) RANGING FROM, $11=98 \mathrm{TH} \quad 3=37$. 
THE BACK-UP WORDS FOR LEVEL 5 ARE:

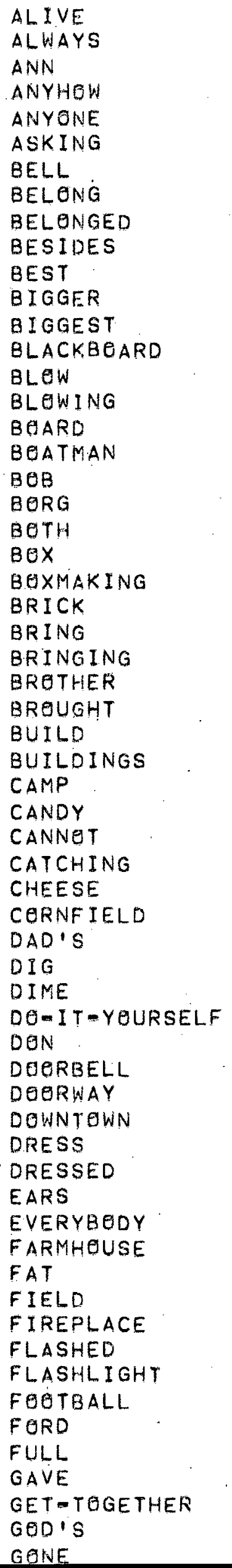


GRASSY

GREENHOUSE

HANDING

HARRIS

HAYDEN

HE'S

HEADING

HEAR

HEARD

HEARING

HEINZ

HELPFUL

HENRY'S

HEREAFTER

$\mathrm{HIGH}$

HILLSIDES

HOOK

HOOKED

HOWEVER

HUNGRY

HURRIED

HURT

I'VE

JULLY

KODA

KRAFT

LARGE

LETTER

LETTERING

LEW

LIGHT

LINDA

LINES

LISTEN

LISTENED

LISTENING

LIVED

LIVEL_ENG

LONG - TAILED

LONGING

LOTS

LOUGHBOROUGH

MARRIOTT

MET

MILTON

MINUTE

MISSION

NAMED

NEAR

NEARBY

NEARING

NEED

NEEDED

NEI GHBOR

NEI GHBORHOOD

NEVER

NEWSPAPER

NICE

NIGHTBIRDS

NOISE

' 'CLECK

ONLY

PAINTER

PAST

PATH

PAY 
PAYDAY

PAYING

PILED

PILES

PLACE

PLACED

PLANE

PLANTED

PLANTING

PLANTS

PLAYED

PLAYHUUSE

PLAYTHINGS

PLEASANT

PLEASED

PEND

PUTATOES

PRAYING

QUICK

RACCOONS

RAKE

RANG

RING

RINGING

ROAD

RELL

ROLLED

ROLLING

SEED

SEEING

SEEN

SEESAW

SEND

SHERT

SHOULD

SHEUT

SHEUTED

SHOUT ING

SHOVEL

SHOWING

SIDEWALK

SIT

SIX

SLIDES

SMALL

SMOKED

SNOWMAN

SNOWMEN

SOAP

SOFT

SURRY

STOREHOUSE

SUDDENLY

TARO

TELEPHONE

THERE'S

THEREBY

THIRD

TITHED

TEOLHOUSE

TOELS

TOP

TRUCK

UNDERCLOTHES

UNDERSTAND USE 


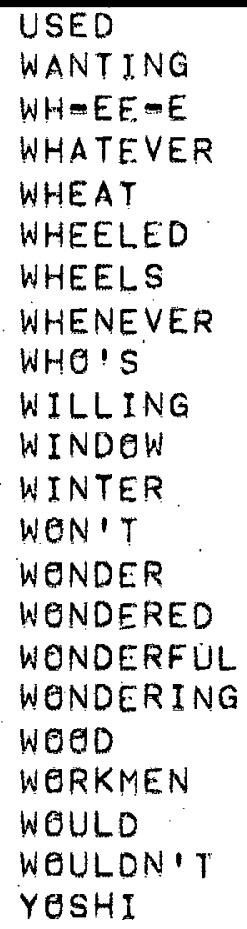

THERE ARE 371 WORDS IN LEVEL 5 OF SERIES E. IN COMPARING SERIES E WITH SERIES D. EACH AT LEVEL, 5 THERE IS A .00\% MATCH. THERE. ALSO IS A $27.22 \%$ MATCH (EUTSIDE OF LEVEL 5 ) RANGING FROM, $11=106$ TO $6-46$. 
THE BACK =UP WORDS FUR LEVEL 6 ARE:

$A C T$

AGAINST

$A G O$

AHEAD

AL.IKE

ALMEST

ALONE

ALSO

ANGRY

ANYMORE

APART

AREN I T

$\triangle R M S$

AUNT JANE

AWHILE

$B A-A-A$

BABIES

BAKE

BAKING

BANKER

BARS

BASKETBALL

BEAMS

BECOME

BEDDING

BEDTIME

BETTY

BIDDY

BIKES

BIRDIES

BITE

BITING

BITS

BLECK

BLUEBELL

BLUEBERRIES

BLUEBERRY

BOASTED

BODIES

BODY

BODY=HOUSE

BOTTOM

BOTTOMER

BOUGHT

BOXES

BRANCH

BRANCHES

BREAD

BREAK

BREAKFAST

BRIDGES

BRIGHT

BRIGHT =EYED

BREWN-AND-WHITE

BUILDER

BURNING

BUS

BUSH

BUSHES

BUTTER 
BUTTERNUT

BUZZ

CAREFUL

CAREFULLY

CARLOS

CARRIED

CARRY

CAUGHT

CERTAINLY

CHANCED

CHANGE

CHASE

CHASING

CHIN

CLEANER

CLEVER

CLOCK

CLOSE

CLOSER

COAX

CoOK

COOL

CORNER

COVERED

COWBOY

CRACKING

CREAM

CROSS

CROWFOOT

DASHED

DIRTY

DOCTOR FFATHER

DOCT GRS

DOESN'T

DONE

DOWNSTAIRS

DRAW

DRESS=UP

DRESSES

DRINK

DRIVE

DRIVER

DRIVING

DROP

DROPPED

EARTHMOVERS

EARTHY

EASIER

EASILY

EATEN

$E G G=$ HOUSES

EIGHT

$E I G H T-Y E A R=O L D$

ELEPHANT

EMPTY

EMPTY $-H E A D E D$

ENGINE

EVEN

EVENING

EXCITED

EXCITING

EYE-HOLES

FACE

FAIR

FELT

FEW 
F IERCE

FILLED

FINALLY

FIREBELL

FIREHOUSE

FIREMAN'S

FIREMEN

FISHES

FIXED

FLAG

FLEW

FLIES

FLOQR

FLYER

FELLOWING

FOOL

FOOLISH

FOOTSTEPS

FOREST

FURGETTING

FURGIVE

FORGQT

FERTH

FEURTH

FEX

FEXES

FEXY

FREE

FRIDAY

FRIENDLY

FRIGHTENED

FRUITS

FUNNIEST

FUNNY = LOOKING

GALLEPING

GARZA

GASTRIC

GATE

GAY'S

GEERGE

GIVEN

GIVING

GLASS

GLASSES

GLOVES

GOBBLE

GOES

GOOD = LUCK

GOOD-TO-EAT

GRAB

GRABBED

GRAYLEGS

GREAT

GREATHOLSE

GREW

GRIN

GRINNED

GRINNING

HADN'T.

HANDKERCHIEF

HANDY

HANG

HAPPENED

HASNIT

HATBEX

HAVEN'T 
HAVING

HE'LL

HEALTH

HEALTHIER

HEALTHIEST

HEALTHY

HEALTHY $=$ LOOKING

HEARTS

HEAVIER

HEAVY

HELD

HERE!S

HIGHER

HIGHWAY

HILLER

HILLY

HIT

HITTING

HOLD

HOLE

HOMEMADE

HONEY

HOUSETOPS

HOW'S

HUGE.

HUMMINGBIRD

HUNG

HUNT

HUSBAND

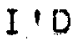

ICE

INSTEAD

INTERESTING

IRON

JAY

JERRY

JIMMY

JEHNNY

JUICE

KATYDIDS

KEEPER

KEPT

KICKED

KILLS

KING

KITCHEN

KITTY

KNOCK

LADDER

LAY

LEAP

LEAST

LEAVING

LED

LETTING

LIFE

LIFTED

LIONS

LIVING

LOUDER

LOUDEST

LOVE

LOW

LOWER

LUCKY

LUNCHROAM 
LUNCHTIME

$M-M=M$

MALEC

MARCHERS

MATTER

MEAN

MEAT

MEETING

MERRIEST

MERRY -GO-ROUND

MIDDLE

MIGHT

MIND

MELLY

MOST

MEUTH

NAILS

NEAREST

NECK

NEWEST

NEWSBOY

NU-WHEELED

NOD

NODDED

NOISEMAKER

NOISEMAKING

NOISIEST

NOISY

NOSE

NOTE

NUMBER

NUTMEAT

EAK

OBEY

BFFER

UFTEN

$\mathrm{BH}=\mathrm{H}=\mathrm{H}$

OIL

OIL-CBVERED

OILY

QLDER

ONE-WHEELED

ONO

ENTE

QUGHT

UVEN

PABLE

PAIL

PALYTIME

PAN

PARADE

PART

PARTIES

PAUL

PAW

PEANUT

PECKING

PETERSEN'S

PETTED

PFT $=F T$

PIE

PIGS

PINK

PIPER

$P L A Y=I N=T H E=S U N$

PLENTY. 
PeEl

PUOR

$P E P$

PGPPED

PORCH

PESTURE

PUTATO

PUTTED

$P R-R-R$

PRESENTS

PRETENDING

PRINCE

PUMPKINS

PUP

QUARREL

QUEER

QUICKLY

QUIET

QUINN

QUIT

RACE

RACING

RAGS

RAINY

RAINY=DAY

RAKER'S

RAKING

RATS

RATTLE

REACH

REAL

REALLY

REMEMBERED

REPL IED

REPLY

REST

RETURNED

ROARED

RECK

ROCKY

RODE

REPE

RESY

ROW

RUBBED

RUBB ING

RUBS

RUNNY

RUSHING

SAM

SANDY

SANTOS

SATAN

SAVE

SAVING

SCARECROW

SCARY

SCHOOLCHILDREN

SCHOOLTEACHER

SCREAMED

SECOND-FLOOR

SET

SEVEN

SHAKE

SHAKING

SHALI 
SHOULDNIT

SID

SILLY

SINCE

SING

SITTING

SLOW-MEVING

SLOWLY

SLY

SLYMAN

SMALLER

SMALLEST

SMARTEST

SMARTY

SMILE

SMOKING

SNIFF-SNIFF

SNIFFED

SNIFFY

SOAPBOX

SENNY

SORE

SOUND

SPECKLE

SPUKE

SPOTTED

SQUEAKY

SQUEALING

STAIRS

STAIRWAYS

STAR

STARRY

STEPPED

STOMACH $=$ CLOCKS

STOMACHS

STEOD

STERYBOEK

STRA I GHT

STRANGE

STRANGER

STRAW

STRAW-PILE

STRING

STRINGER

STRUNG

STRONGER

SUCH

SUDDEN

SUMMER

SUNNY-DAY

SUPPER

SUPPERTIME

SUPPESED

SURE

SURELY

SWIFTLY

SWIM

SWIMMING

TABLE

TAKEN

TAKING

TALL

TALLER

TALLEST

TASTE

TEACH 
TEACHER

TEETH

TEMPLES

TERR I BLE

THERE'LL

THESE

THEY 'LL

THEYIRE

THREE - WHEELED

THROAT

THROWING

TIMMY

TINY

TIRED

TOOTH

TOUCH

TOWARD

TRACK

TRAINER

TROTTED

TROUBLE

TRUNKS

UGLY

UNCLE BOB

UNDERHANDED

UNHAPPY

UPPER

UPSET

UPSTAIRS

UPTOWN

USEFUL

VALENTINES

VEGETABLES

VILLAGE

VINE

WADDLE - WADDLE

WADDLES

WALKERS

WARMED

WATCHES

WATCHMAN

WE'LL

WE!RE

WEAR

WEATHER

WEEK

WEEKEND

WELL-TO-DO

WEREN IT

WET

WHAT'S

WHILE

WHIP

WHIPPED

WHISPER ING

WHISTLING

WHOEVER

WHOLE

WIDE

WIFE

WILD

WILDCATS

WOU-FF

WERKER

WREN

WR ITER 


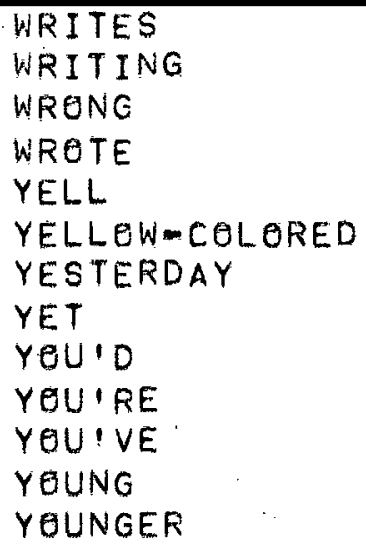

THERE ARE 609 WORDS IN LEVEL 6 OF SERIES E. IN COMPARING SERIES E WITH SERIES D. EACH AT LEVEL, 6 THERE IS A .00\% MATCH. THERE ALSO IS A. $8.37 \% \mathrm{MATCH}$ IOUTSIDE OF LEVEL 6) RANGING FROM, 11-111 TO $3=4$. 


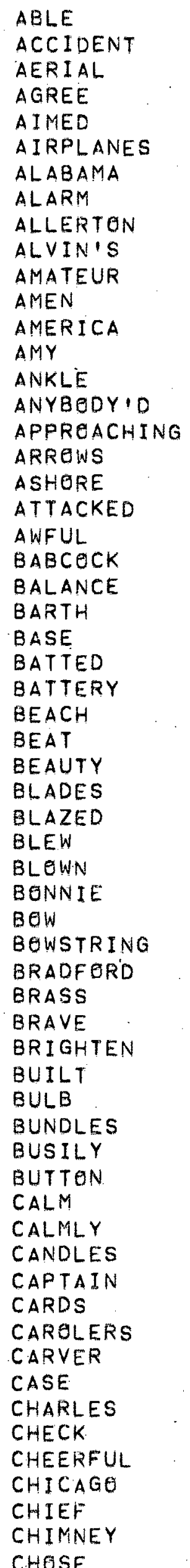


CHESEN

CHR I STMAS

CHURN

CIRCLE

CLEARING

CLIP

CLESELY

CLOSET

CLOTHESPIN

CLOWN

COCEA

CODE

CEMMEN

COMPANY

CONNECT

CONSTANCE

COPPER

CRAMER

CRASH

CREEK

CRUPS

CRUELTY

CUPFUL

CURB

DAN

DANA

DANGER

DANGEREUS

DAPHNE

DARKNESS

DAYDREAMING

DECK

DEERSKIN

DEFROST

DELIVER

DIAL

DIED

DIFFERENT

DIPLEMACY

DIRECTIONS

DIRECTOR

DISAPPEARED

DISHES

DOUBT

DEUBTFULLY

DRAWN

DRIFTING

DUG

DURING

EDDIE

EDGE

E. I THER

ELDER

ELECTRIC

ELECTRICITY

ENGINEER

ENVELOPE

EVENLY

EXACTLY

EXPLORING

FAINT

FALLEN

FANCY

FARTHER

FEAST

FIERCELY 
FINGERS

FINISHED

FLAT

FLEODED

FOOLISHLY

FERT

FRESH

FRESHLY

FRIGHT

GARDENIA

GEESE

GILES

GINGERBREAD

GLOOMILY

GLOOMY

GOOSE

GQVERNER

GRAIN

GREATLY

GROWN=UPS

GRUNTED

GUESTS

HALL

HANDFUL

HANDLE

HANS

HARBOR

HEAVILY

HELICOPTER

HILDE'S

HISTERY

He

HOBBLE

HONK

HOPKINS

HORN

HOURS

HOWL

HOZAK

HUFFMAN.

HUNGRILY

IMPORTANT

IMPOSSIBLE

INVENTIONS

INVENTOR

INVITED

JERK

JOINED

JUKE

KATALIN

KATRINA

KNIFE

KNOB

LANES

LANGUAGE

LANTERN

LEANED

LEATHER

LEONARD

LEVERS

LIBRARY

LIGHTNING

LIST

LOGS

LOESE

LOESELY 
LOOSEN

LOVELY

LUCKILY

MAGNET

MAILBOX

MASSACHUSETTS

MEDICINE

MELTS

MESSAGE

METAL

MILES

MILKMEN

MILLION

MODEL

MESTLY

MOTIENS

METORBEAT

MUDDY

MUSIC

NANCY 10

NEARLY

NEMETH

NINE

NOISILY

NETICED

OCEAN

$O K$

OLD-FASHI UNED

ORDERS

OTTO

PAPPAS

PARENTS

PARROT'S

PARTLY

PEDAL

PERFECT

PERFECTLY

PERFUME

PILGRIM

PILOT

PIONEERS

PITCHING

PLANNING

PLATE

PLENTIFUL

PLOWED

PLUNGED

PLYMEUTH

POEM

POLE

POLITELY

PESITIEN

POSSIBLE

POSSIBLY

PQWER

PRACTICE

PRAIRIE

PREPARED

PREVENTIUN

PROBABLY

PROJECT

PREMISE

PREUD

PROUDLY

PULLEYS

PURPLE 
QUIETLY

QUITE

RADIO

RAISE

RECREATION

REEL

REFRIGERATOR

RELEASE

RID

ROASTED

ROGERS

ROQFED

RUG

RULER

SADLY

SAFE

SAFELY

SAILING

SALE

SALTY

SAMOSET

SCATTERED

SCIENCE

SCELDING

SCRUBBED

SEMAPHORE

SENT

SERIEUS

SERIEUSLY

SETTLEMENT

SETTLERS

SEVENTH DAY ADVENTIS

SEVERAL

SHE'D

SHIP

SHOIE

SHEOT

SHBRES

SHORTLY

SHOT

SHOVED

SHULTZ

SIGHT

SILENT

SILENTLY

SILVER

SIMPSON

SKIPPED

SLINGER

SMELL

SNAPPED

SNOWFLAKES

SNOWSTORM

SOCIETY

Ses

SQUP

SPACE

SPEAK

SPECIAL

SPEND

SPREAD

SQUANTE

STAMPED

STANDISH

STEADILY

STEADY 
STEEL

STEER

STEPHEN

STEW

STOVE

STRAIGHTENING

STRANGELY

STRIPS

STRUCK

STUEY

STUMBLED

SUGAR

SUIT

SUPERMARKET

SWAN

SWOEPED

TAPE

TAUGHT

TAXICABS

TEAMS

TELESCOPE

TENSION

TERR IBLY

TEST

THANKFUL

THATCH

THEY'D

THIRSTY

THUNDER

TICK

TIGHT

TEE

TOOT

TRAFFIC

TRANSOM

TRAPFED

TURKEYS

TURNIPS

UNBELIEVINGLY

UNCEOKED

UNCEVERED

UNDERSTEOD

UNEASILY

UNEXPECTED

UNLOCK

UNSAFE

UNSET TLED

UNTIE

UNWILLINGLY

USELESS

VACATIEN

VACUUM

VAN VLIET

VIOLIN

VISITOR

VQICE

WAKED

WAKENED

WALKIE-TALKIE

WARNED

WATCHFULLY

WE'D

WEBER

WEIGHT

WELCOMES

WHE THER 
WHIRL ING

WIDELY

WILDLY

WILLI AM

WILSON'S

WIRE

WOKE

WOLVES

WOMEN

WONDERFULLY

WORRIED

WERRIEDLY

WORSE

WORSHIP

WOUND THERE ARE 445 WORDS IN. LEVEL 7 GF SERIES E. IN COMPARING SERIES E
WITH SERIES 0 , EACH AT LEVEL, 7 THERE IS A THERE ALSO IS A $5.84 \% \mathrm{MATCH}$ (BUTSIDE OF LEVEL 7 ) RANGING FROM, $11=115$ TO $8=170$. 
THE BACK-UP WORDS FOR LEVEL 8 ARE:

\section{$\triangle B E$}

$\triangle B Q A R D$

ACCEPTED

ACHED

$A D D E D$

$\triangle D M I R I N G$

ADMITTED

ADVENTURES

$A D V I C E$

AFFORD

AFTERWARDS

AH

AIN IT

ALASKA

ALFRED

ALLQWED

ALOUD

ALPHABET

ALREADY

ALTHOUGH

AMAZING

AMENG

ANCESTORS

ANGER

ANNEUNCED

ANTLERS

ANXIQUS

APARTMENT

APPEARED

APRON

AQUARIUM

ARGUING

ARGUMENT

AR I THMETIC

ARRANGED

ARR IVED

ASHAMED

ATTENTION

ATTIC

AUSTIN

AVERAGE

AWAKENED

BACKWARDS

BANQUET

BAREBACK

BEADS

BEANS

BENEATH

BENT

BIXBY

BLACKNESS

BLESS

BLESSOMS

BUILING-HOT

BENFIRE

BONNET

BOETH

BORN

BORREW

BOTHER

BRACED 
BRAIDS

BRAND

BREAST

BREATH

BREATHE

BREATHLESSLY

BREEOS

BRIDLE

BRIEF

BRUSH

BUBBLES

BUCK

BUD

BUNK

BURST

BUSINESS

CALIFORNIA

CAPTURED

CARRETS

CARTWRIGHT

CATTLE

CAUTIOUSLY

CEILING

CELEBRATED

CELERY

CENTERED

CENTS

CHAIRBOUND

CHANTED

CHARLEY

CHARLIE

CHATTED

CHEEKS

CHEROKEE

CHINATOWN

CHINESE

CHEKED

CHOPS

CHORES

CHUCKLED

CLAIM

CLAPPED

CLASSES

CLIFF

CLETH

CLUUDY

CLUBS

COAL

COCONUT

COLLAR

COMF ERT

CEMMITTEE

COMPLAINED

CENFUCIOUS

CONSIDER

CONTEST

CONTINUED

CENVENIENT

COPYCAT.

CORRAL

COSTS

COSTUMES

CETTEN

COUPLE

CEURSE

CEURT 
COUSIN

CRAWFORD

CRAWL

CRIFPLED

CUNNING

CUNNINGHAM

CURIOUS

CUSTEMERS

D.C.

DALE

DAMP

DANCES

DANDER

DARED

DAUGHTER

DEAD

DEAL

DEBBIE

DEBT

DECE IVE

DECLARED

DECORATIONS

DECOY

DEL I GHTED

DENMARK

DENNIS

DESERT

DESERVES

DESK

DESPAIR

DESTREY

DETUUR

DEW

DIDES

DIFFERENCE

DIFF I CULTY

DINING

DION

DIPPED

DISAPPEINTED

DISAPPOINTMENT

DISCUURAGED

OISCUVERED

DISEASE

DISSATISFIED

DISTANCE

DISTANT

DITCH

DIVED

DIVIDED

DOE

DOLLARS

DRAGEN

DRAIN

DREW

DREVE

DROWNED

DULL

DUMB

DUST

EAGER

EAGERNESS

EARNED

EARTHQUAKE

ELEVEN

EMPTINESS 
ENGLAND

ENGLISH

ENITH

ENJUYED

ENERMOUS

ENTER

ENVY

ERRAND

ESPECIALLY

EXAMINE

EXCEPT

EXCITEMENT

EXCUSE

EXERCISE

EXPERIMENTS

EXTRA

FACT

FAILURE

FAITHFUL

FAMILIAR

FAMEUS

FERNWEOD

FEVER

FIFTEEN

FIFTY

FIGHT

FIGURE

FIRMLY

FLAMES

FLIGHT

FLEATING

FLUCK

FLUFFY

FLUSHED

FOULISHNESS

FORENOEN

FERTUNATELY

FERTY

FURWARD

FOURTEEN

FREEZE

FROST

FROWNED

FUR

FURNITURE

GASPED

GAZED

GENTLE

GERTRUDE

GIANT

GIFT

GIGGLE

GLANCED

GLARE .

GOLD

GUODNESS

GESPEL

GOURDFUL

GRACEFUL

GRANNY

GRATEFULLY

GROUPS

GREWL.

GRUMELED

GUN

GUPPIES 
HALFWAY

HAMILTEN

HAMMER

HAPPINESS

HARE

HARM

HASTILY

HEAVED

HEIGHT

HERD

HEY

HID

HEE

HOGBACK

HOLIDAYS

HELLOW

HENORABLE

HONORS

HETEL

HUG

HUNDREDS

HUT

ICEBERG

ILLS

IMAG INE

IMMEDI ATELY

IMPATIENTLY

IMPOLITELY

INCHES

INSISTED

INSTANTLY

INTERRUP.TED

INTREDUCING

INVESTMENT

ISABELLA

ISLAND

JACKET

JAM

JAMES

JAPAN

JEALOUS

JEAN

JEROME

JOHNSTON

$J O S E P H$

JOY

JUDG ING

KALLER

KANGAROO

KAYAK

KETTLE

KIMMY

KIRSTEN

LACOUR

LAD

LADY

LAID

LAME

LANKY

LARSEN

LATHERED

LAUGHTER

LAWN

LEAFY

LEAKING

LFDGF 
LEI

LESS

LESSEN

LEVEE

LI

LICK

LID

LIE

LIN

LINCELN

LIPS

LOANED

LOFT

LOHMANN

LONELY

LONESOME

LORD'S

LOSE

LOTTIE

LOUELLA

LOUIS

LUMP

LYING

$M A$

$M A D$

MAGIC

MAILE'S

MAIN

MAMMA

MANAGE

MANALUK'S

MAPLE

MARKED

MARRIAGE

MARRY

MASTER

MATE

MATHILDA

MATOAKA

MAYGR

MEADEW

MEAL

MEANT

MEASURED

MEASUREMENT

MEEKLY

MEISTER

MEMBERS

MENTIUN

MEXICO

MIO

MINE

MIRACLE

MISCHIEF

MISSISSIPPI

MISSEURI

MIST

MISTAKEN

MITE

MIX

MOM

MOMENT

MENTHS

MOEN

MOWER

MUMPS 
MURMUR

MUTTERED

NARREW

NATURALLY

NEAT

NEEDLE

NEGRE

NEITHER

NETS

NONE

NOODLES

NURSE

QARS

UBLIGE

EFF ICER

EKLAHOMA

QUTFIT

OUTWARD

OVERAGE

OWE

PA

PACKED

PADDLE

PAID

PAIN

PAIRS

PALACE

PALE

PANTING

PARDEN

PARIS

PASSENGER

PASTEUR

PATCH

PATIENT

PAUSED

PEACE

PEACHES

PEBBLES

PECULIAR

PEERED

PEONY

PERSON

PILLEW

PINES

PING

PITY

PLAINLY

PECAHONTAS

POEM

PORTER

PESTMASTER

PUUND

PQURED

POWHATAN

PREACHER

PRECIUUS

PRESIDENT

PRINCESS

PRINTED

PRISENER

PRIZE

PREBLEM

PROTECTED

PROTESTED

PROVED 
PUDDI.E

PUNCH

QUESTIONED

QUETATION

RADISH

RAM

RANCH

REALIZED

REALTIONS

REAR

REASON

REBECCA

REBUILT

RECKON

RECORD

REFUSED

RELIEVED

REMAINED

REMINDED

REPEATED

RESCUE

RESERVATIUN

REVOLVING

REWARDS

RIBBON

RICE

RICHES

RINSE.

RISE

ROD

ROLFE

RUET

ROYAL

RUFE

RUFFLING

SACK

SADDLE

SADNESS

SAN FRANCISCO'S

SANDWICHES

SANK

SARAH

SATISFIED

SCAMP

SCRAPE

SCUFFLED

SEA

SEARCHING

SEASENED

SEAWEED

SELL

SEQUOYAH'S

SERVANTS

SEW

SHADE

SHAPED

SHARP

SHAVEO

SHEETS

SHELF

SHELL

SHELTERED

SHETLAND

SHINY

SHIVER

SHENE 
SHOP

SHOULDER

SHOWER

SHR IEKED

SHYLY

SICK

SICKNESS

SI GHED

SILENCE

SILK

SINGLE

SIOUX

SIR

SIZE

SLID

SLIP

SMITH

SMOOTH

SNAILS

SNATCHED

SNUG

SOARED

SOBBING

SOLES

SOUTH

SPANKED

SPARED

SPEECH

SPEECHLESS

SPELLING

SPENT

SPILLED

SPIN

SPINE

SPIRITS

SPROUTS

SPUTTERED

SQUISH

STAG

STARTLED

STATEMENT

STATES

STATUE

STEAMING

STERNLY

STIFFLY

STOLEN

STOEL

STRAY

STREAKS

STRETCH

STRIKE

STUBBERN

STUCK

STUDY

STUFF

STUPID

SUCCEEOED

SUFFER

SUGGESTED

SWALLOWED

SW IRLING

SWITCHING

SWUNG

SYLLABLES

SYRUP 
TAG

TAMEST

TASK

TEARS

TENDERLY

TENTS

TERRIF IC

TERRER

TEXAS

THEE

THEMSELVES

THICK

THEMAS

THEUGH

THEUSANDS

THREAD

THREATENED

THREW

THUMBS

THUNDERBOLT

TIDE

TILL

TIPPING

TOASTY

TONGUES.

TESSED

TOWERING

TRAILER

TRAVELWAYS

TREMBLED

TRIBE

TRIM

TROPICAL

TROUGH

TROUSERS

TRUSTED

TRUTH

TUBS

TUTU

TWELVE

TWENTY

TWICE

TWINKLED

TWISTED

TWO-TONED

UNCOMFORTABLE

UNITED

UNLESS

VIRG IN IA

VIRUS

WAILED

WAIST

WASHINGTON

WASTING

WEAKNESS

WEDGES

WE I

WEST

WHINE

WHINNIED

WHI SKERS

WHITTLING

WHOM

WHOSE

WICKED

WIN 
WIPED

WISDOM

WON

WOOLLY

WORE

THERE ARE 697 WORDS IN LEVEL 8 OF SERIES E. IN COMPARING SERIES E WITH SERIES D, EACH AT LEVEL, 8 THERE IS A $.29 \%$ MATCH.

THERE ALSO IS A $2.44 \% \mathrm{MATCH}$ (OUTSIDE OF LEVEL 8 ) RANGING FROM, $11-115$ TO $7=84$. 


\section{APPENDIX E}

This table $1 \mathrm{~s}$ an alphabetical word list of each vord in all rive series.

The series letter corresponds to the following basal readirig series:

Series A: The Bookmark Reading Propram, by Harcourt Brace Jovanorich, Inc.: New York, 1974.

Series B: The Houghton Mifflin Readers, by Houghton Miffiin Company, Boston, 1966.

Series C: $\frac{\text { Jidilian Basal Readers, by Mackjilan Company, New }}{\text { York, 1966. }}$

Sexies D: Harper and Row Design for Readingo by Harpex and Row, Fublishers, Inc., New York, 1972.

Series E: Seventh-day Adventist Basai Series, Pacific Press,

When a word appears in a series the level and page it is first in.r. troduced on appears as $05 / 034$ (levei 5, page 34). If the rord does not appear in a series it is shown as $/$. 
- - per

IRITING

$A$

ABBIE

ABBOTT

$\triangle B B Y$

$\triangle B E$

$A B L E$

$\triangle B Q A R D$

ABQUT

ABOVE

ACCENTED

ACCEPT

ACCEPTED

ACCIDENT

ACCERD

ACCOUNT

ACCUSE

$A C H$

ACHEO

$A C I D$

ACORN

ACERNS

ACROSS

$A C T$

ACTED

ACT ING

ACTIEN

ACTER

ACTERS

$A D A M$

ADAM'S

$A D D$

$A D D E D$

ADDING

ADDRESS

ADDRESSED

ADMIRATION

ADMIRE

ADMIRED

ADMIRING

ADMITTED

ADOBE

$\triangle D V E N T U R E$

ADVENTURES

ADVICE

$\triangle E R I A L$

AFFECTS

AFFERO

AFRAID

AFRICA

AFRICAN

$\triangle F T E R$

AFT.ERNEIEN

AFTERWARDS

AGAIN

AGAINST

$\triangle G E$

$\triangle G Q$

AGREE

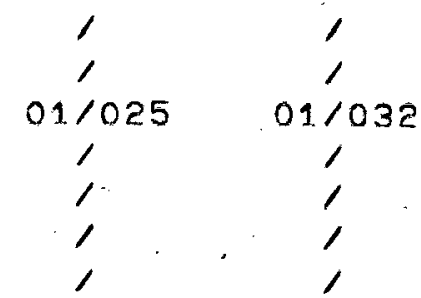

$07 / 140$

1

$05 / 052$

$07 / 172$

$09 / 061$

,

$07 / 194$
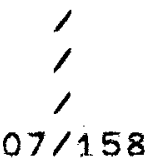

$09 / 279$

$08 / 221$

$08 / 154$

,

,

,

1

西

,

,

,

,

1

,

'

$09 / 272$

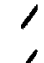

$07 / 046$

$09 / 111$

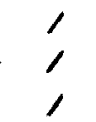

$09 / 157$

$07 / 014$

$08 / 255$

$09 / 098$

$01 / 014$

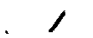

$07 / 170$

$07 / 169$

$08 / 149$

$06 / 116$

$06 / 1.75$

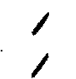

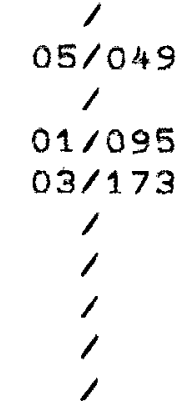

$06 / 195$

$06 / 069$

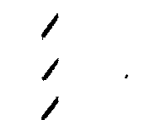

$06 / 256$

$03 / 035$

$06 / 285$

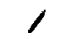

1

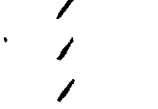

$03 / 221$
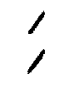

1

'

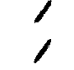

$06 / 189$

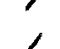

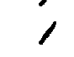

,

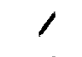

$03 / 036$

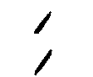

$01 / 138$

$05 / 163$

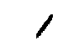

021066

$05 / 027$

$05 / 174$

$05 / 123$

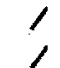

$07 / 175$

$09 / 032$

$05 / 071$

$09 / 233$

$08 / 245$

$07 / 221$

1

$06 / 129$

$04 / 087$

$07 / 107$

$09 / 212$

,

1

,

,

'
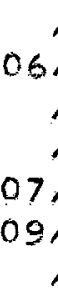

,

,

1

I

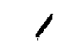

$06 / 114$

$07 / 139$

$09 / 104$

$09 / 097$

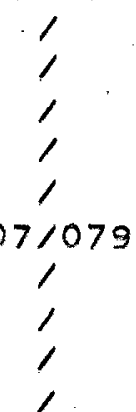

$06 / 126$
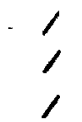

$07 / 072$

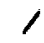

$04 / 054$

$08 / 088$

1

$06 / 083$

1

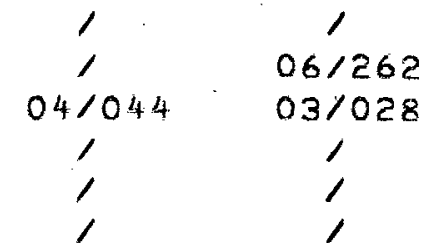

$08 / 140$

$07 / 060$

$08 / 086$

$08 / 074$

$11 / 094$
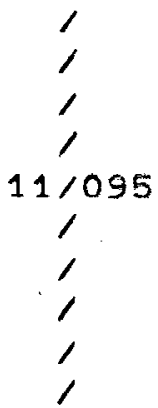

$08 / 138$

$08 / 138$

$08 / 045$

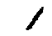

$11 / 050$

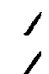

$09 / 073$

$09 / 072$

$04 / 021$

$08 / 072$

$11 / 115$

1

,

,

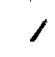

1

I.

,

,

,

1

1

1

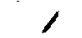

$08 / 127$

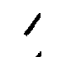

$08 / 085$

$09 / 061$

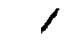

$08 / 129$

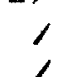

,

$08 / 205$
$06 / 149$

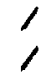

$08 / 235$

$07 / 174$

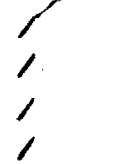

$08 / 159$

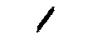

$05 / 144$

$06 / 166$

$08 / 024$
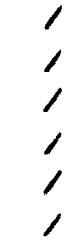

$08 / 177$

$08 / 116$

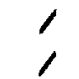

$08 / 125$

$08 / 015$

$07 / 018$

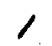

$08 / 175$

$05 / 179$

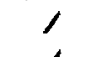

$04 / 115$

$06 / 061$

$08 / 014$

$06 / 150$

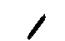

$06 / 057$

$07 / 117$

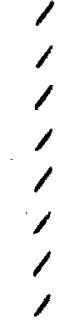




\section{AGREED \\ $A H$}

AHEAD

AHOY

A IM

A IMED

$A I N \cdot T$

$A I R$

A IRPLANE.

A IRPLANES

AIRPGRT

ALABAMA

ALARM

ALARM CLOCK

ALASKA

ALFRED

$A L I C E$

$A L I K E$

$A L I V E$

$A L L$

ALLEGI ANCE

ALLERTEN

ALLOSAURUS

$A L L O W$

ALLOWED

ALMOST

ALGNE

$A L O N G$

ALEUD

ALPHABET

ALREADY

ALSO

ALTHEUGH

ALVIN'S

ALWAYS

$A M$

AMATEUR

$\triangle M A Z E D$

$\triangle M A Z I N G$

AMEN

AMERICA

$\triangle M E R I C A N$

AMERICANS

$A M O N G$

AMES

AMOUNT

AMUSEMENTS

$\triangle M Y$

$A N$

ANCESTGR

ANCESTORS

ANCHORAGE

$\triangle N D$

ANDREA

ANDY

ANGELO

ANGELO!S

ANGER

ANGIE

$\triangle N G R Y$
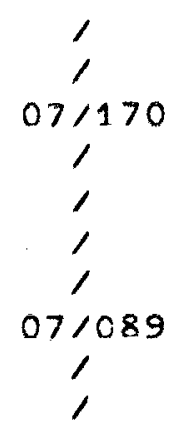

$04 / 127$

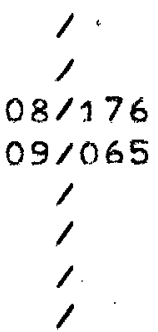

$05 / 010$

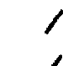

$08 / 237$

$07 / 097$

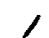

$07 / 083$

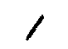

1

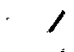

$08 / 195$

$07 / 083$

$09 / 020$

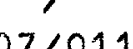

$04 / 048$
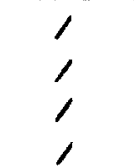

$07 / 212$

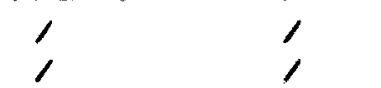

$09 / 106$

$01 / 011$

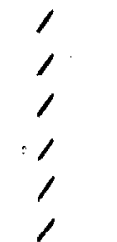

$07 / 156$
$05 / 221$

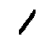

$06 / 123$

$06 / 163$

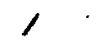

$02 / 094$
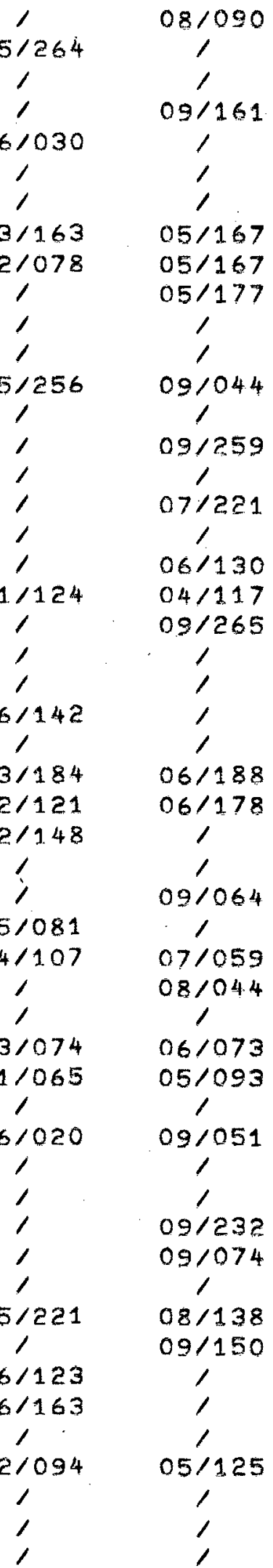

$05 / 167$

$05 / 167$

$05 / 177$

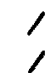

$09 / 044$

$09 / 259$

1

$07 / 221$

1

$06 / 130$

$04 / 117$

$09 / 265$
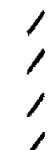

$06 / 188$

$06 / 178$

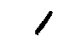

1

$09 / 064$

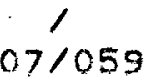

$08 / 044$

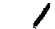

$06 / 073$

$05 / 093$

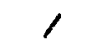

$09 / 051$
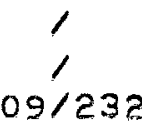

091074

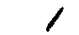

$08 / 138$

$09 / 150$
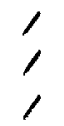

$05 / 125$

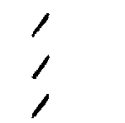

$01 / 006$

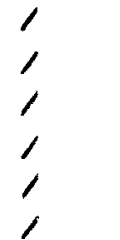

$03 / 169$

$06 / 207$

$06 / 012$
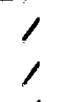

1

$08 / 097$

$06 / 170$

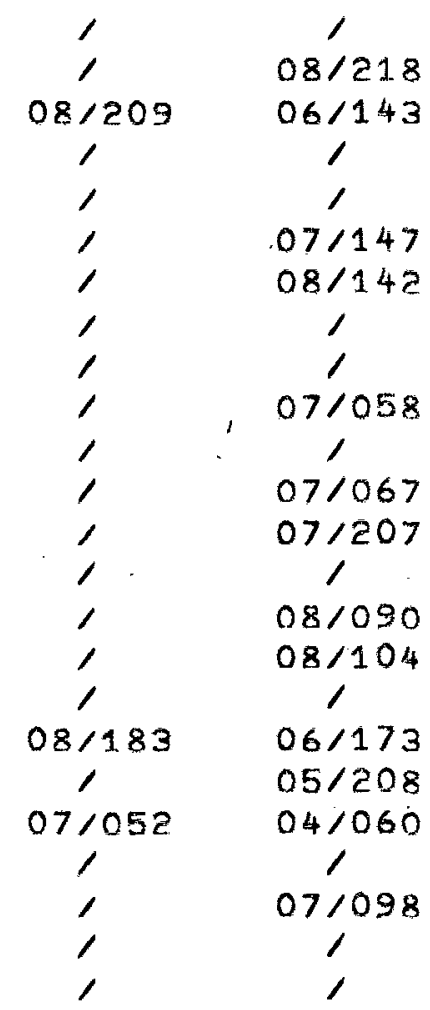

$08 / 054$

$06 / 068$

$06 / 155$

$11 / 025$
$08 / 191$

$11 / 023$

1

$11 / 025$

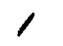

1

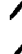

$07 / 095$

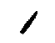

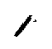

1

1

1

$11 / 026$

$11 / 024$
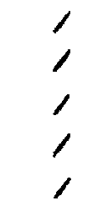

$08 / 025$

$11 / 027$

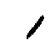

$03 / 008$

1

$08 / 045$

$08 / 133$

$06 / 197$

$08 / 064$

$07 / 231$

$05 / 163$

$04 / 151$

$07 / 211$

1

$08 / 136$

$07 / 050$

$07 / 097$

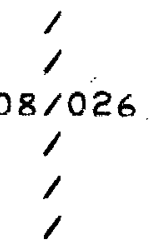

$07 / 193$

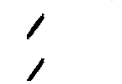

$08 / 066$

$02 / 013$

$07 / 153$

$11 / 036$

$.11 / 039$

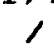

$\gamma$

$08 / 131$

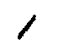

$06 / 211$
$08 / 134$ 
ANIMAL

ANIMALS

ANKLE

ANN

ANNACENDA

ANNEUNCED

ANNOUNCER

ANNEYS

ANETHER

ANSWER

ANSWERED

ANSWERING

ANSWERS

ANT

ANTARCTICA

ANTLERS

ANXIGUS

ANXI UUSLY

ANY

ANYBODY

ANYBOOY,D

ANYHOW

ANYMORE

ANYONE

ANYTHING

ANYWAY

ANYWHERE

APART

APARTMENT

APARTMENTS

APPARENTLY

APPEARED

APPLE

APPLES

APPROACHING APPREVE

APREN

$\triangle Q U A R I U M$

$\triangle R A B$

ARCTIC

$\triangle R E$

AREAS

$\triangle R E N \cdot T$

ARGUING

ARGUMENT

AR I THMET IC

$\triangle R I Z O N A$

$A R M$

ARMS

ARMY

ARNELO

ARGUND

ARRANGED

ARRIVE

ARRIVEO

ARREWS

ARTIST

AS

$\triangle S H A M E D$

ASHERE

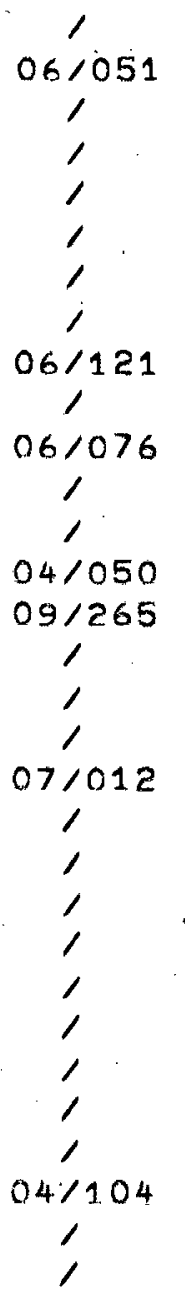

$08 / 133$

$06 / 176$

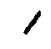

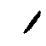
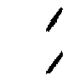

$08 / 122$

$08 / 255$

$09 / 265$

$04 / 025$

$08 / 246$

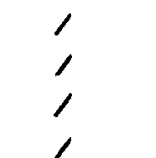

$08 / 228$

$07 / 136$

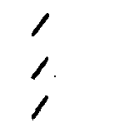

$04 / 064$

$09 / 085$

$$
\text { , }
$$

$08 / 109$

$04 / 02 ?$

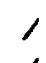

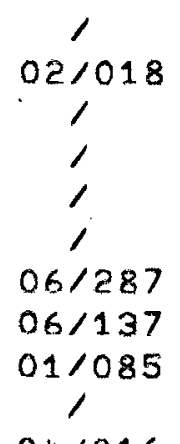

$04 / 016$

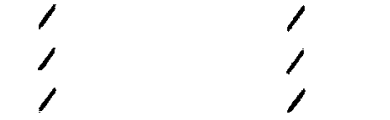

$08 / 273$

$09 / 049$

$05 / 161$

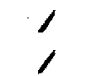

,

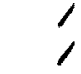

$02 / 141$

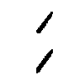

$03 / 203$

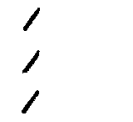

$05 / 151$

$04 / 052$

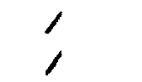

$03 / 058$
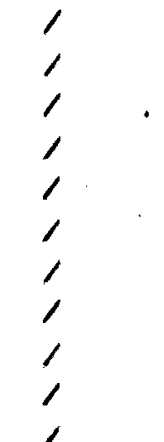

$03 / 052$

$06 / 010$

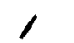

$01 / 157$

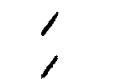

$05 / 116$

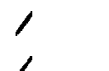

$01 / 133$

$05 / 225$

$07 / 125$

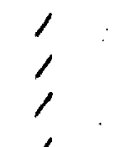

$05 / 184$
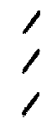

$05 / 125$

$05 / 126$

$09 / 275$

$08 / 021$

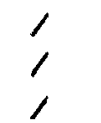

$09 / 023$
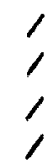

$05 / 022$
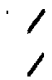

,
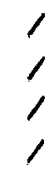

$07 / 032$

$06 / 125$

$09 / 022$

$04 / 140$
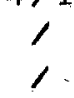

$08 / 139$

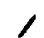

$05 / 084$

1

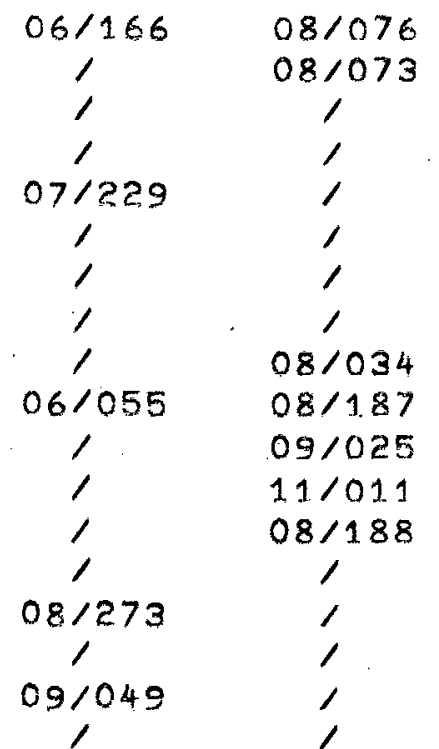

$08 / 023$

$08 / 028$

$08 / 186$

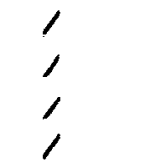

$09 / 016$

$08 / 192$

$08 / 192$

$09 / 078$

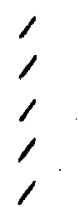

$08 / 174$

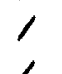

1

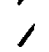

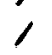

,

$05 / 014$

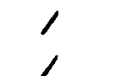

\section{1}

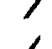

,

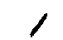

$11 / 033$

1
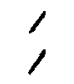

$08 / 017$

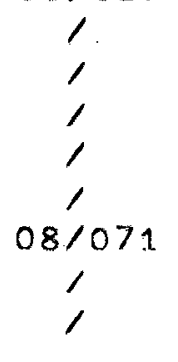

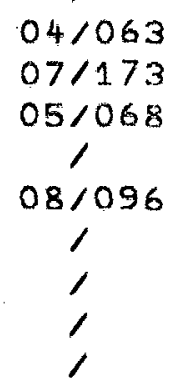

$05 / 024$

$05 / 218$

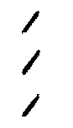

$08 / 069$

$08 / 016$

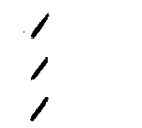

$07 / 081$

$05 / 220$

$06 / 193$

$05 / 032$

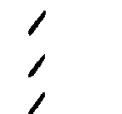

$06 / 202$

$08 / 192$

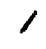

$08 / 051$

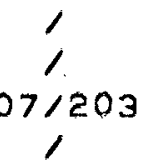

$08 / 149$

$08 / 246$

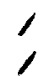

$04 / 025$

$06 / 040$

$08 / 167$

$08 / 167$

$08 / 193$

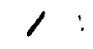

$06 / 072$

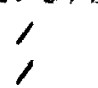

$05 / 045$

$08 / 134$

$08 / 010$

$07 / 146$

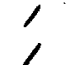

$08 / 182$

$07 / 107$ 
AS IDE

$A S K$

ASKED

$A S K I N G$

ASKS

ASLEEP

ASTONISHED

ASTONISHING

ASTONISHMENT

ASTRENAUT

$A T$

ATE

ATTACHED

AT TACKED

ATTENTION

ATTIC

ATTRACTED

AUDIENCE

AUGUST

AUNT

AUNT JANE

AUSTIN

AUTOMOBILES

AUTUMN

AVENUE

AVERAGE

AW AKE

AWAKENED

AWAY

AWE

AWFUL

AWHILE

AWL

$A Y E$

$B A=A-A$

BABCECK

BABIES

$B A B S$

$B A B Y$

BACK

BACKS

BACKWARD

BACKWARDS

BACKYARD

BACEN

BAD

BADGE

$B A G$

BAGPIPE

BAGPIPES

BAKE

BAKERY

BAKING

BALANCE

BALCONY

BALL

BALLGON

BANANA

BANANAS

BAND

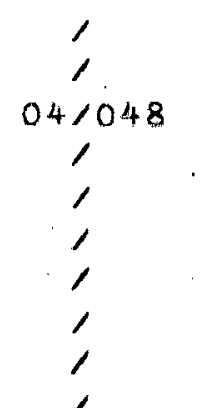

$02 / 030$

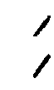

1

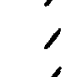

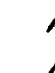

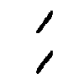

$09 / 111$

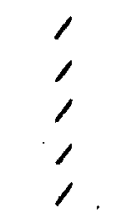

$09 / 162$

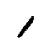

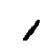

,

$01 / 019$

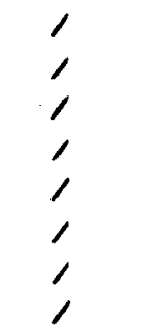

1

$05 / 135$

$04 / 040$

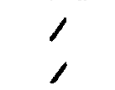

$08 / 268$
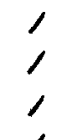

1

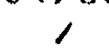

$07 / 018$

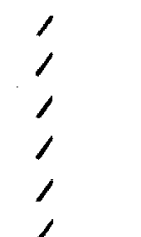

$06 / 161$

1

$07 / 024$

,
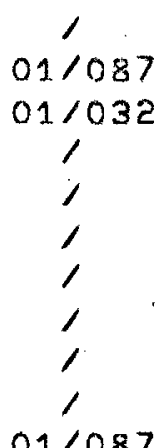

$04 / 058$

$06 / 076$

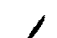

$05 / 182$

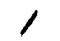

$06 / 165$

$06 / 022$

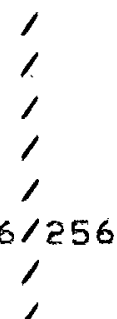

$06 / 080$

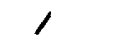

$01 / 094$

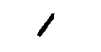

1

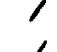

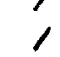

1

$03 / 045$

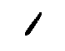

$01 / 119$

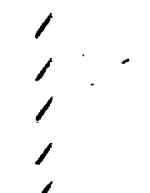

$01 / 056$

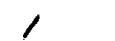

021088
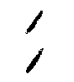

$04 / 046$

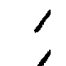

$06 / 007$

$01 / 019$

$03 / 128$
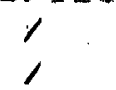

$02 / 122$

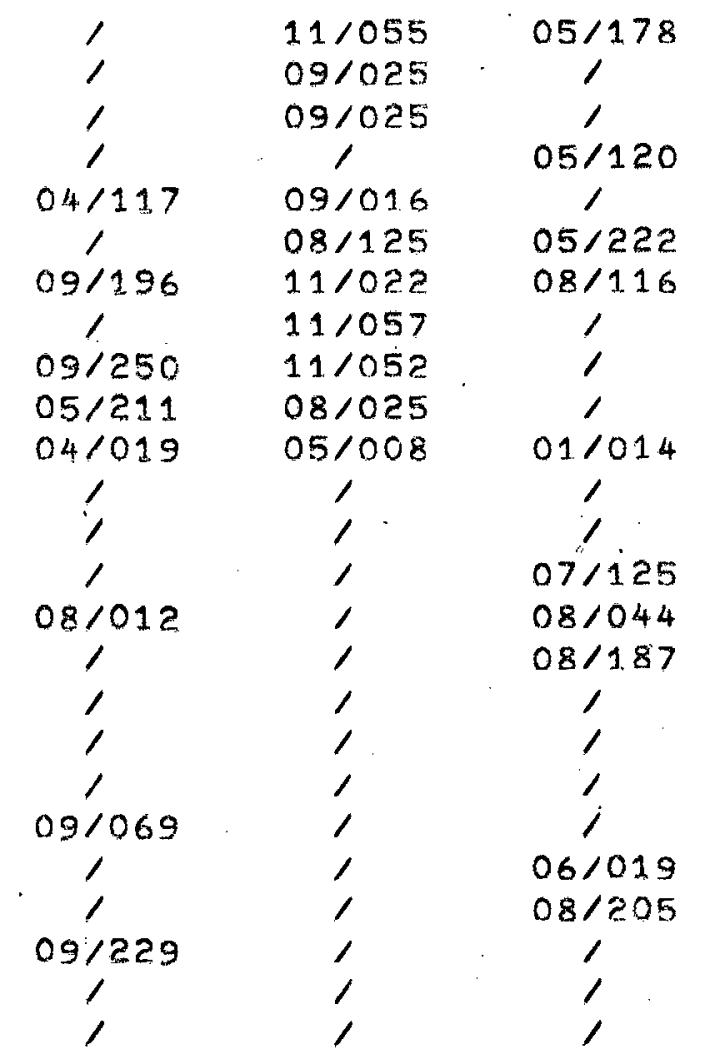

$08 / 018$

$07 / 151$

$09 / 048$

$04 / 091$

$08 / 022$

$07 / 147$

$09 / 060$

$09 / 235$
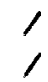

$08 / 220$

$05 / 125$

$04 / 114$

$04 / 040$

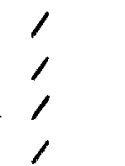

$06 / 186$

$04 / 173$

$09 / 257$

$07 / 020$
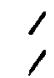

$04 / 146$

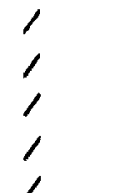

$05 / 190$

$08 / 041$
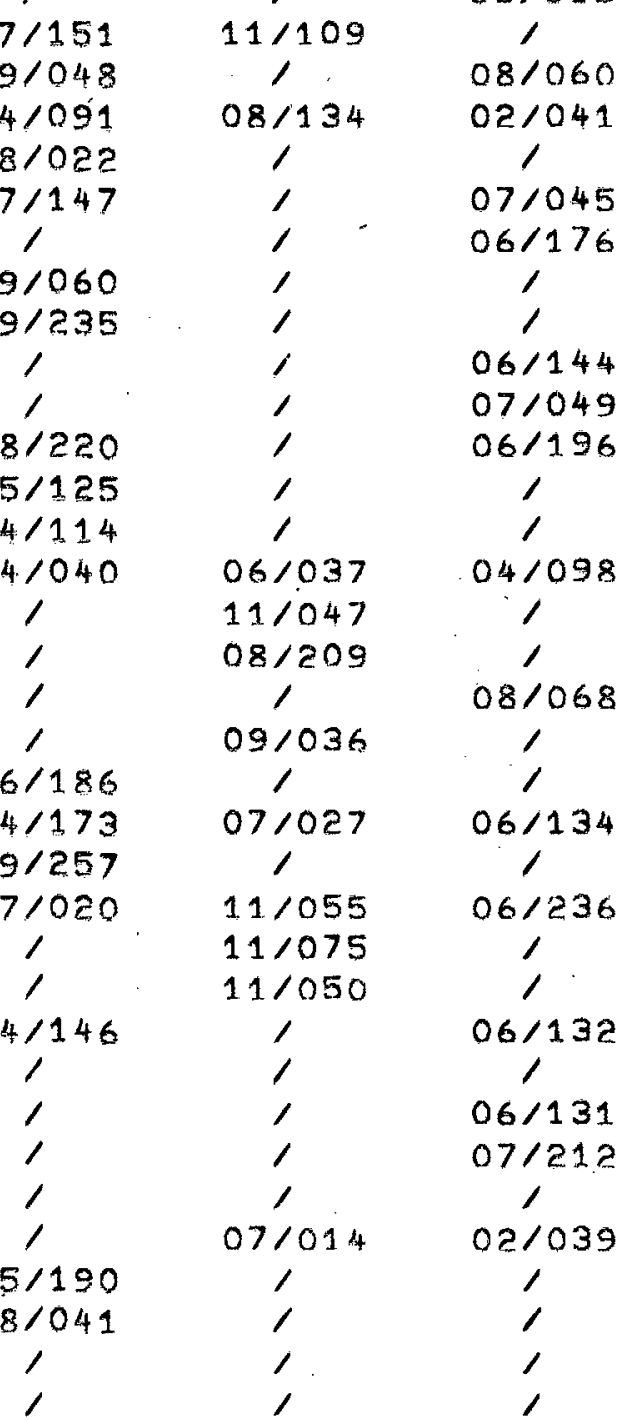
BANDANNA

BANGED

BANK

BANKER

BANKS

BANQUET

BARBECUES

BAREBACK

BARGAIN

BARK

BARKED

BARKING

BARN

BARREL

BARS

BARTH

BASE

BASEBALL

BASKET

BASKETBALL

BASKETS

BAT

BATES

BATHE

BATHREOM

BATHS

BATTED

BATTER

BATTERY

BATTLE

BE

BEACH

BEACHES

BEADS

BEAMED

BEAMS

BEANS

BEAR

BEARD

BEARS

BEAST

BEAT

BEAUTIFUL

BEAUTY

BECAME

BECAUSE

BECKIE

BECOME

BED

BEDDING

BEDROOM

BEDS

BEDTIME

BEE

BEEHIVE

BEEN

BEEZUS

BEFERE

BEGAN

BEGGAR

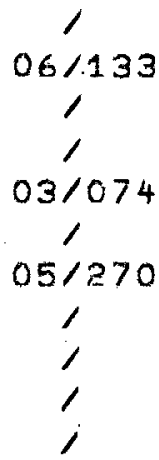

$05 / 254$

$04 / 119$

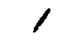

$06 / 113$

1
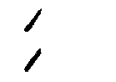

$06 / 040$

,

1

,

I

,

,

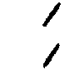

$05 / 149$

',

,

,

$05 / 102$

$03 / 145$

,

,

$08 / 008$

1

$07 / 149$

$06 / 105$

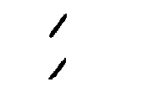

$04 / 094$

,

,

$1 / 029$

$05 / 108$

$07 / 058$

$$
1
$$

$05 / 108$

$05 / 073$

$05 / 051$

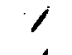

,

1

1

$06 / 280$

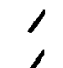

$04 / 048$

$06 / 246$
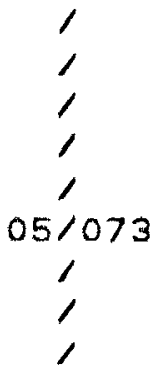

$04 / 199$

$02 / 018$

$06 / 184$

$06 / 191$

$05 / 051$
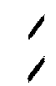

$02 / 037$
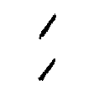

1

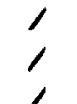

1

$06 / 159$

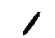

$03 / 090$

$$
1
$$

$02 / 063$

$02 / 069$
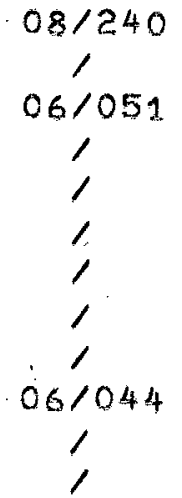

$05 / 099$

$08 / 171$
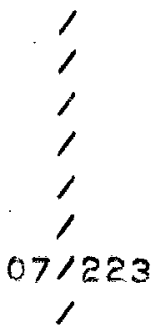

$09 / 233$

$07 / 249$

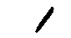

$07 / 2.36$
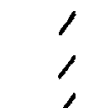

$09 / 165$

$05 / 072$

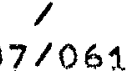

,
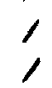

$07 / 049$

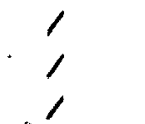

$06 / 077$

$06 / 1.64$

,

$06 / 047$

$09 / 233$

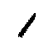

$05 / 143$

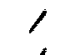

$05 / 142$

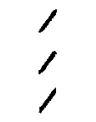

$06 / 149$

$08 / 013$

$05 / 209$

$05 / 019$

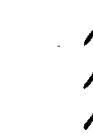

$06 / 178$

$08 / 202$

$08 / 017$

$08 / 067$

$08 / 012$

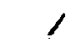

$09 / 044$

$08 / 154$

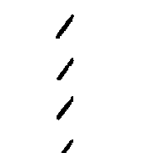

$07 / 138$

$08 / 196$

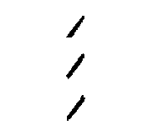

$09 / 065$

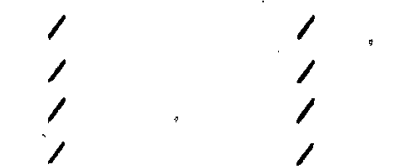

$07 / 029$

$07 / 197$

$05 / 065$

$06 / 128$

$07 / 100$

$07 / 026$

$06 / 050$
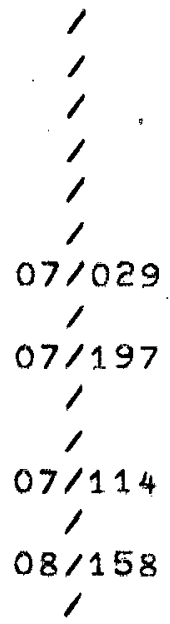

$06 / 180$

$08 / 06 ?$

$07 / 083$

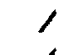

$07 / 091$

$05 / 095$

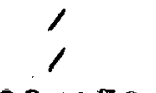

$07 / 128$

$08 / 150$

$05 / 164$

$07 / 217$

$09 / 041 \quad 05 / 153$

$05 / 157$
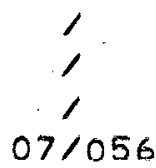

$06 / 219$

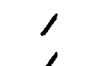

$06 / 219$

$05 / 107$

$06 / 063$

$05 / 198$

$05 / 178$

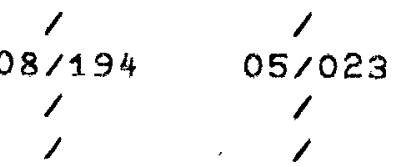


BEGGED

BEGIN

BEGINNING

BEGINS

EEGUN

BEHAVED

BEHIND

BEING

BELIEVE

BELIEVED

BELINDY

BELL

BELONG

BELONGED

BELONGS

BELEW

BEN

BEN'S

BEND

BENEATH

BENJAMIN

BENT

BERRIES

QERRY

QESIDE

BESIDES

BEST

BET

BETSY

BETSY'S

BETTER

BETTY

BETWEEN

BEYEND

BIBLE

BICYCLE

BIDDY

BIG

BIGGER

BIGGEST

BIKE

BIKES

BILL

BILLIONS

BILLY

BILLY!S

BING

BIRCHBARK

BIRD

B IRD=CAGE

BIRDIES

BIRDS

BIRDS '

BIRTHOAY

BIRTHDAYS

BISCUIT

BIT

BITE

BITING

BITS

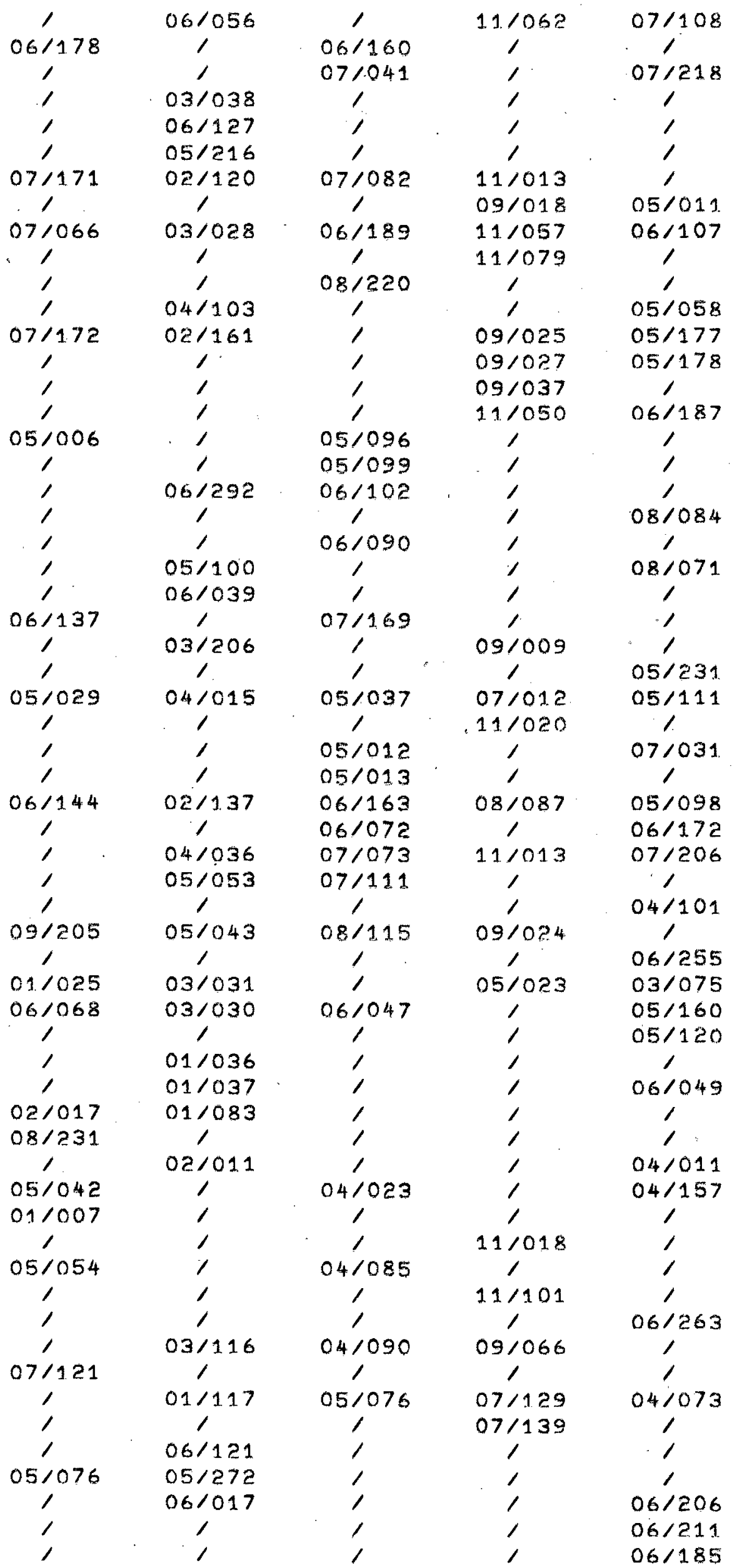


BIXBY

BLACK

BLACKBEARD

BLACKBOARD

BLACKNESS

BLACKTOP

BLADES

BLAME

BLAMED

BLANCHARD

BLANKET

BLANKETS

BLAST

BLAST $=$ OFF

BLAZED

BLESS

BLEW

BLINKED :

BLINKY

BLIZZARD

BLOCK

BLECKS

BLOED

BLOSSOMS

BLOW

BLOWHOLE

BLOWING

BLOWN

BLUE

BLUE-EYED

BLUEBELL

BLUEBERRIES

BLUEBERRY

BLUEBENNET

BEARD

BOARDS

BEASTED

BOAT

BOATMAN

BGATS

$B O B$

BOBBY

BODIES

BUDY

BODY=HOUSE

BOILED

BUILER

BOILING-HOT

BEL.D

BELE

BOLE'S

BONE

BENFIRE

BONNET

BONNIE

BOOIE

BOEK

BOEKLET

BOGKS

BEOMER

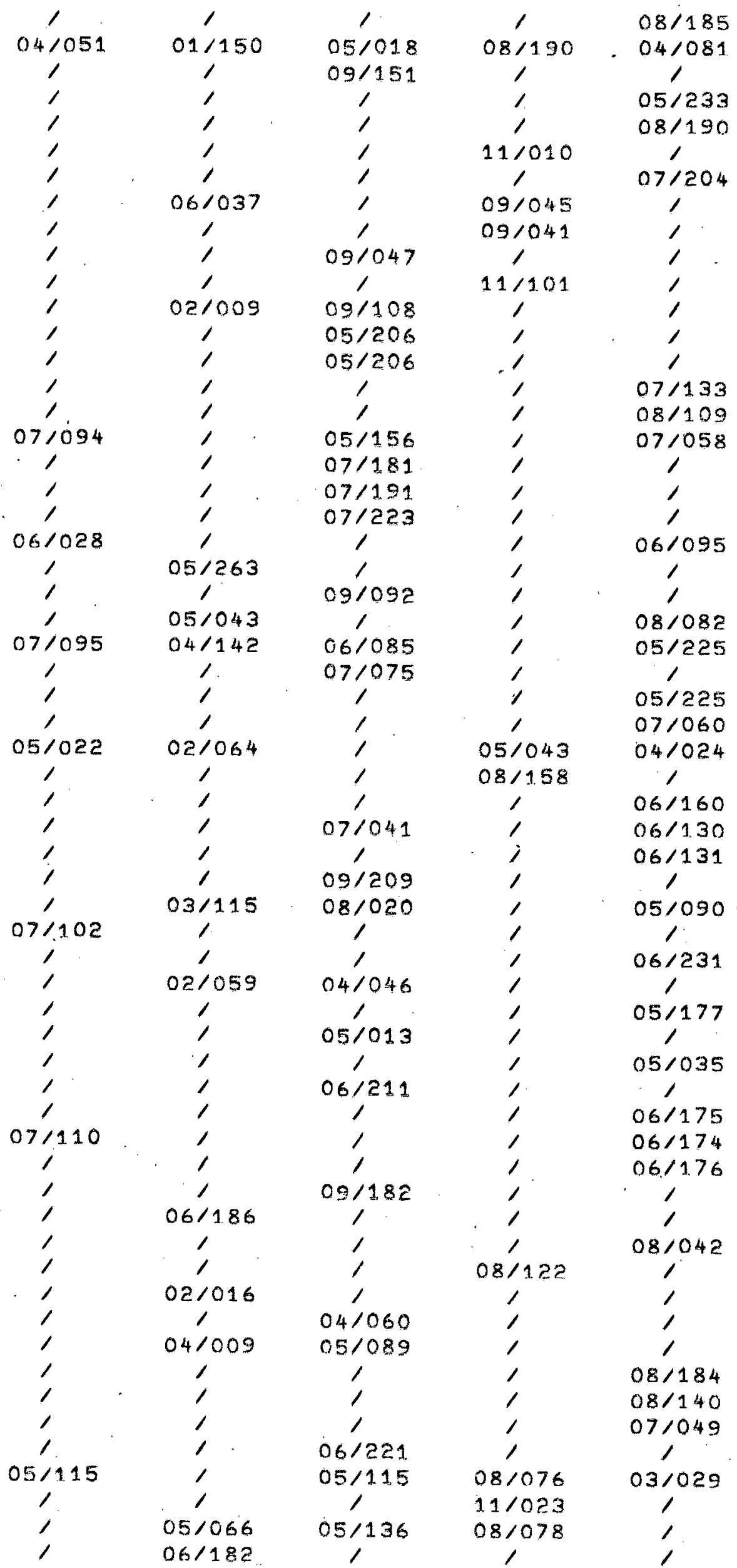




BOONESBEROUGH
BOOTH
BOETS
BORG
BORN
BORROW
BOTH
BOTHER
BOTHERED
BOTTLE
BOTTLES
BOTTEM
BOTTOMER
BOUGHT
BOUNCE
BOUNCED
BOUQUET
BOW
BOW-WEW
BOWED
BOWL.
BOWLS
BEWSPRIT
BOWSTRING
BOX

\section{BOXES}

BEXMAKING

BOY

BOY'S

BOYS

BQZES

BFACED

QRADFERD

ERAIDS

GRAILLE

BRANCH

BRANCHES

BRAND

BRASS

BRAVE

BRAVES

BRAVG

BRAY

BRAYED

BRAYING

BRAZIL

BREAD

BREAK

BREAKFAS $r$

BREAST

BFEATH

BRFATHE

RREATHLESSLY

BREATHS

BREEDS

BREMEN BAND

BRICK

BRIDGE

BRIDGES

BRIDLE
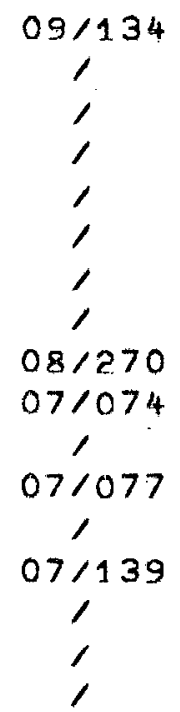

$09 / 044$

$05 / 096$

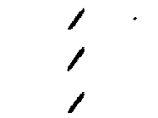

$09 / 083$
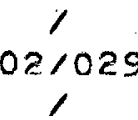

$$
1
$$

$04 / 074$

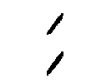

$05 / 169$

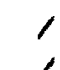

,

1

,

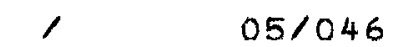

,
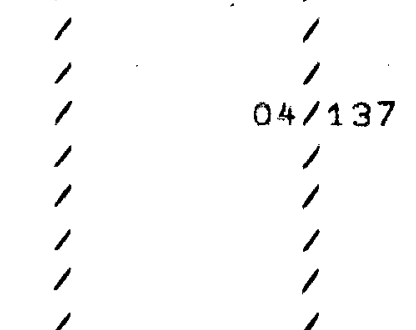

$08 / 257$

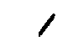

$08 / 162$

$07 / 175$

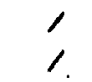

$08 / 9.53$

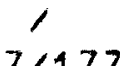

$07 / 177$

$05 / 089$

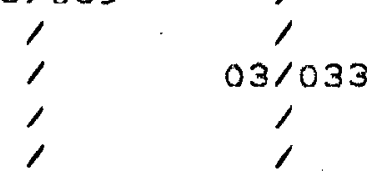

$04 / 137$
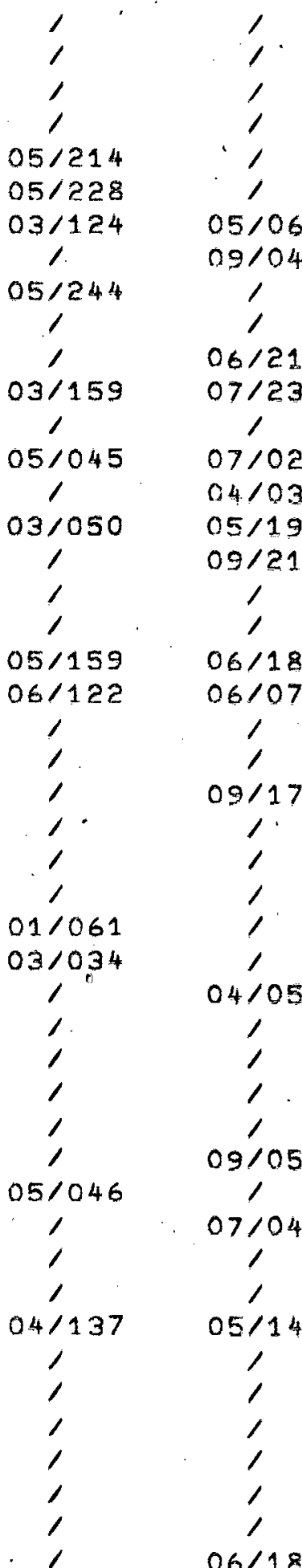

$05 / 065$

$09 / 044$

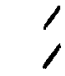

$06 / 212$

$07 / 238$

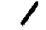

$07 / 023$

041030

$05 / 193$

$09 / 215$

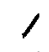

$05 / 159$

$06 / 122$

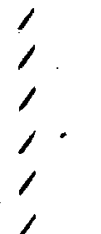

$01 / 061$

$03 / 034$

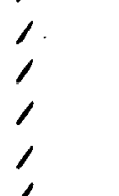

$5 / 046$

$06 / 183$

$04 / 029$

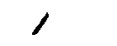

$06 / 043$

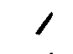

- 1

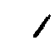

1

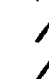

$3 / 033$

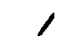

$06 / 183$

$06 / 077$

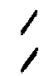

$09 / 171$

,

,

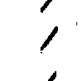

1

$04 / 052$

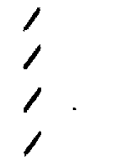

$09 / 059$

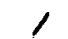

$07 / 044$
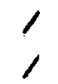

$05 / 149$

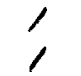

,

I

,

$06 / 186$

$07 / 098$

$07 / 245$

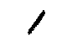

$07 / 100$

$07 / 101$

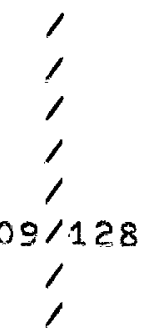

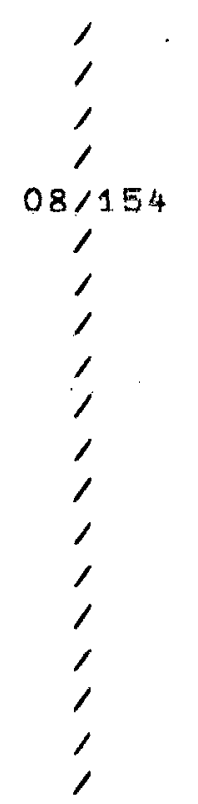

$11 / 090$

$11 / 089$

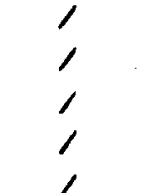

$07 / 139$

$11 / 010$

$07 / 120$

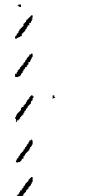

$08 / 124$

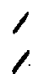

1

$y$

$1 / 021$

$11 / 060$

$11 / 070$

$11 / 062$

$11 / 070$

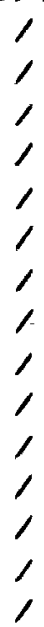

$08 / 021$

$04 / 123$

$05 / 051$.

$08 / 169$

$08 / 051$

$05 / 036$

$08 / 015$

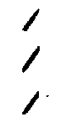

$06 / 184$

$06 / 189$

$06 / 176$

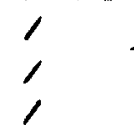

$07 / 146$

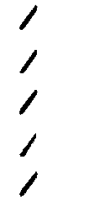

$07 / 147$

$05 / 035$

$06 / 212$

$05 / 188$

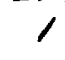

$04 / 051$

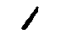

$08 / 239$

$07 / 117$

$08 / 193$

$06 / 098$

$06 / 098$

$08 / 194$

$07 / 165$

$07 / 081$

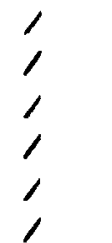

$06 / 209$

$06 / 202$

$06 / 079$

$08 / 254$

$08 / 032$

$08 / 113$

$08 / 115$

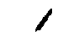

$08 / 018$

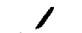

$05 / 191$

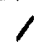

$06 / 226$

$08 / 011$ 


BRIEF
BRIGHT
BRIGHT-CELORED
BRIGHT-EYED
BRIGHTEN
BRIGHTER
BRIGHTLY
BRING
BRINGING
BRISKLY
BRITISH
BREAD
BROKE
BROKEN

BRONTESAURUS

BREOM

BROTH

BRETHER

BRETHER'S

BROTHERS

BREUGHT

BROWN

BREWN-AND-WHITE

BREWN-EYED

BREWNIS

BRIUSH

BRUSHES

BRUSHING

BUBBLES

BUCK

BUCKET

BUCKSKIN

BUCKY

BUD

BUDDIES

BUDDY

BUDGE

BUDS

BUG

BUGS

BUILD

BUILDER

BUILDINGS

BUILT

BULB

BULLDOG

BULLDOZER

BULLETS

BULLFINCH

BUMBLE

BUMP

BUMPED

BUMPER

BUNCH

BUNDLE

BUNDLES

BUNG

BUNK

BURIED

BURN

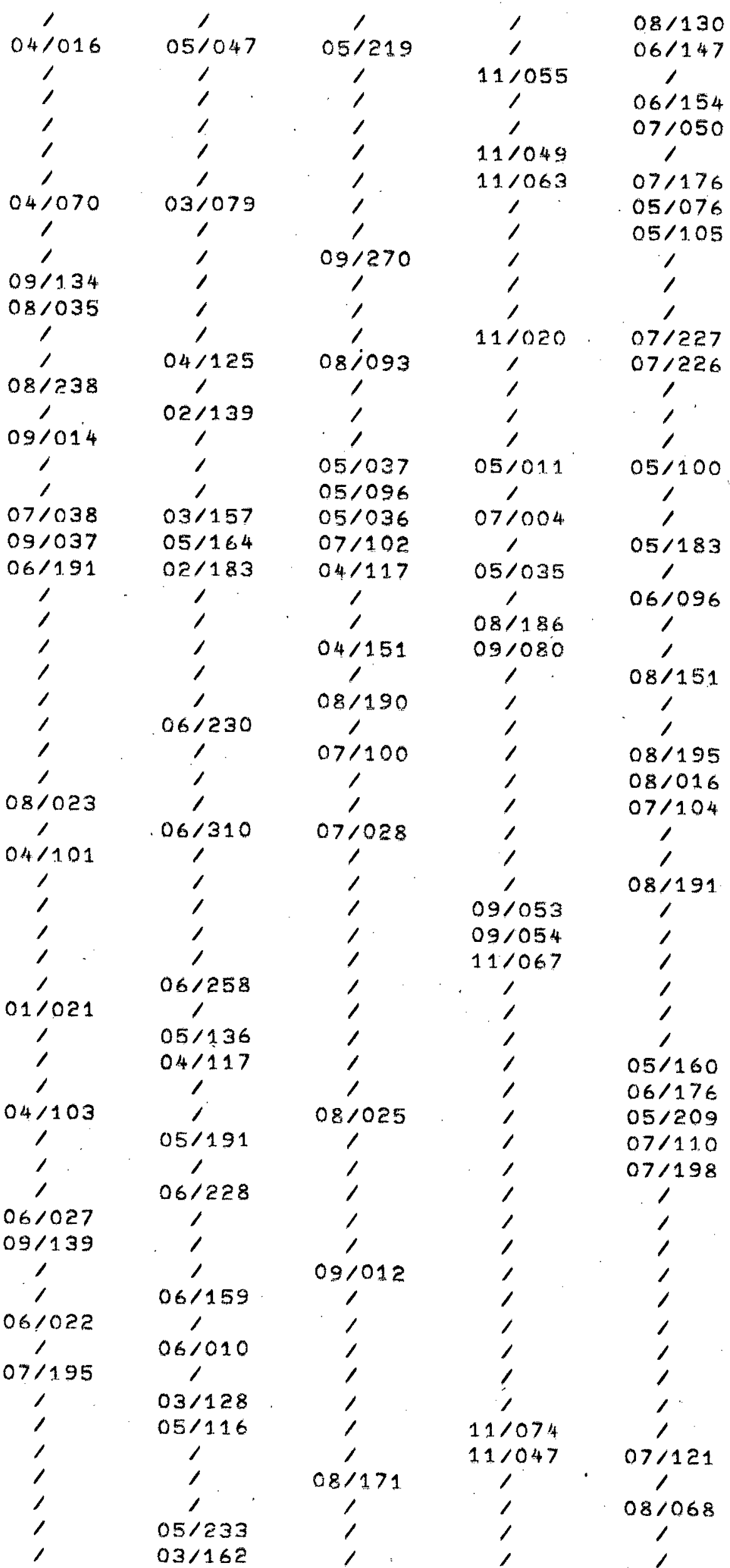


BURNING

BURRO

BURRE'S

BURROS

BURST

BUS

BUSH

BUSHES

BUSHONGO

EUSILY

BUSINESS

BUS INESSMAN

BUSMAN

BUSTLING

BUSY

BUT

BUTCH

BUTCHER

BUT T

BUTTER

BUTTERCUP

BUT TERNUT

BUTTGN

BUT.TENS

BUY

BUYER

BUYING

BUZZ

$B Y$

$C A B$

CABIN

CABINET

CABINS

CABOESE

CAGE

CAGES

CAKE

CAKES

CALF

CALIFORNIA

CALL

CALLED

CALL ING

CALLS

CALM

CALMLY

CAME

CAMERAS

CAMP

CAN

CAN'T

CANADA

CANADIAN

CANARIES

CANDLE

CANDLES

CANDY

CANNON

CANNET

CANOE

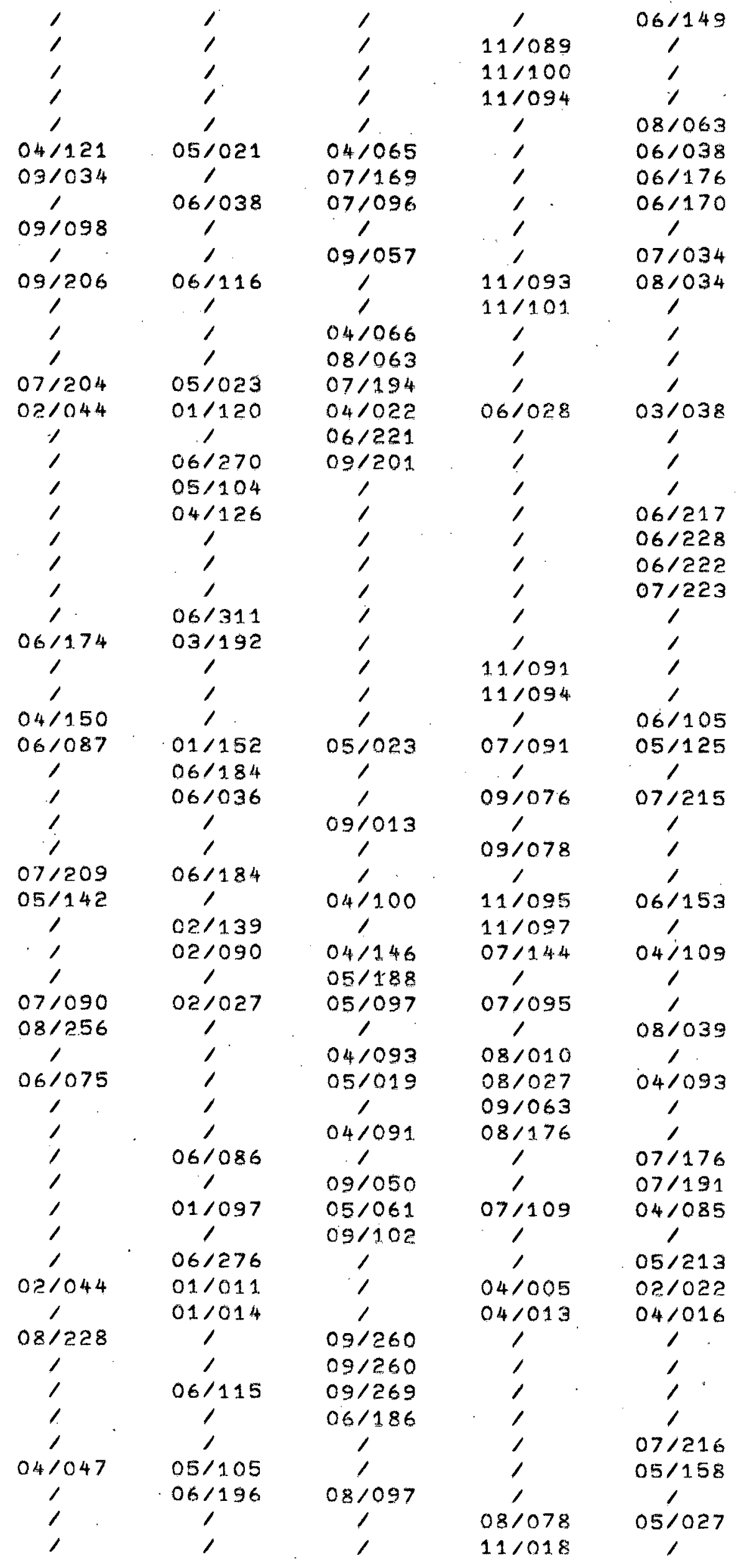


CANVAS

CANYEN

CAP

CAPE

CAPITAL

CAPPY

CAPPYIS

CAPS

CAPTAIN

CAPTURE

CAPTURED

CAR

CARD

CARDBEARD

CAROS

CARE

CARED

CAREFUL

CAREFULLY

CARLA

CARLOS

CARNATION

CAROLERS

CARPENTER

CARPET

CARR I AGE

CARRIED

CARRUTS

CARRY

CARRYING

CARS

CART

CARTONS

CARTWRIGHT

CARVER

CASE

CASH

CASK

CAST

CASTLE

CAT

CATBIRD

CATCH

CATCHING

CATHERINE

CATS

CATTLE

CAUGHT

CAUSING

CAUTIOUSLY

CAVES

CEILING

C.ELEBRATED

CELERY

CELLAR

CENTAVE

CENTAVOS

CENTERED

CENTS

CERTAIN

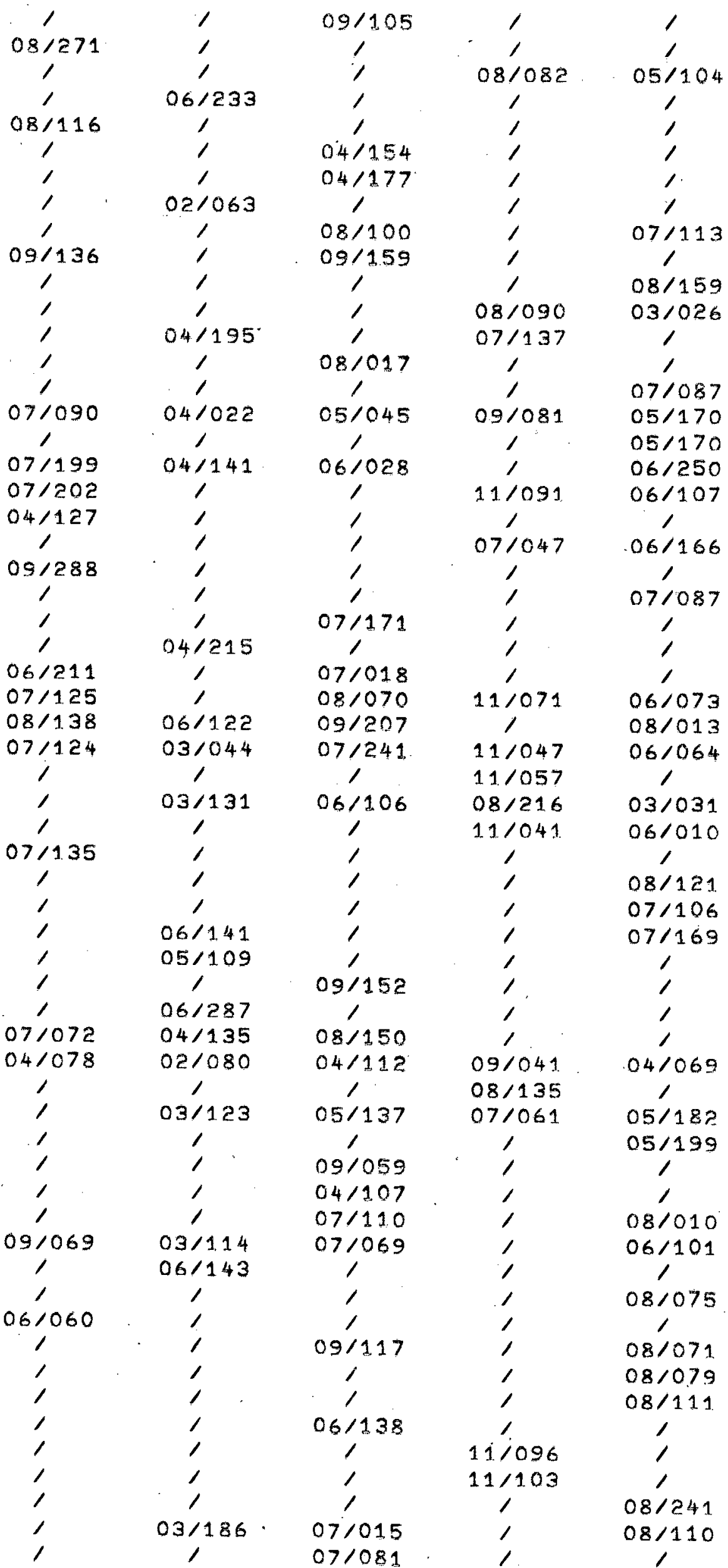


CERTAINLY

CHAIN

CHAIR

C.HATRBOUND

CHALET

CHALK

CHAMPIONSHIP

CHANCE

CHANCED

CHANGE

CHANTED

CHARGED

CHARLES

CHARLEY

CHARLIE

CHARMING

CHASE

CHASE'S

CHASED

CHASING

CHATTED

CHATTER

CHECK

CHECKERS

CHEEKS

CHEERFUL

CHEERFULLY

CHEERIEST

CHEESE

CHEROKEE

CHERRIES

CHERRY

CHEST

CHET

CHEWS

CHICAGO

CHICKEN

CHICKEN LITYLE

CHICKENS

CHIEF

CHILD

CHILDREN

CHILDREN'S

CHIMNEY

CHIMPANZEE

CHIN

CHINA

CHINATOWN

CHINESE

CHITTER = CHATTER

CHOKED

CHOESE

CHEOSES

CHOP

CHOPS

CHORES

CHOSE

CHOSEN

CHRISTMAS

CHUCKLED

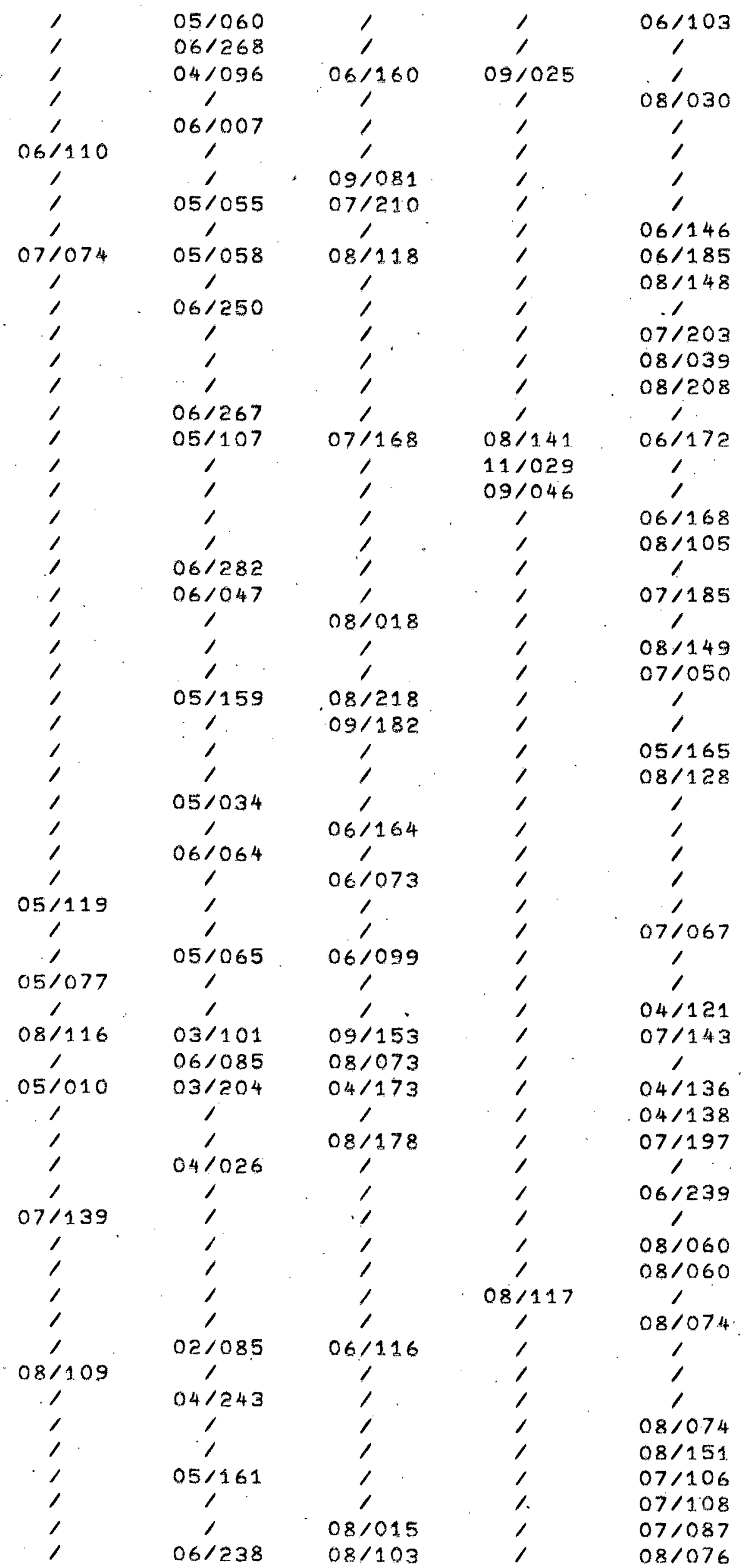




\section{CHURCH}

CHURN

CHUTE

CIBELA

CIDER

CINDER

CIRCLE

CIRCUS

CITY

CLAIM

CLAIMS

CLANCY

CLANG

CLAP

CLAPPED

CLAPPERS

CLASS

CLASSES

CLATTERED

CLAWS

CLAY

CLEAN

CLEANED

CLEANER

CLEANING

CLEAR

CLEARING

CLEVER

CLIFF

CLIMATES

CLIMB

CLIMBED

CLIMBING

CLIP.

CLIPPERS

CLIPPITY

CLECK

CLOSE

CLESELY

CLOSER

CLOSET

CLOTH

CLOTHES

CLOTHESL INE

CLOTHESPIN

CLETHING

CLOUD

CLOUDY

CLAVER

CLEWN

CLUBS

CLYDE

$\mathrm{COACH}$

COAL

COAT

COAX

$C \theta C K-A=D O \theta D L E-D O E$

COCOA

COCONUT

CODE
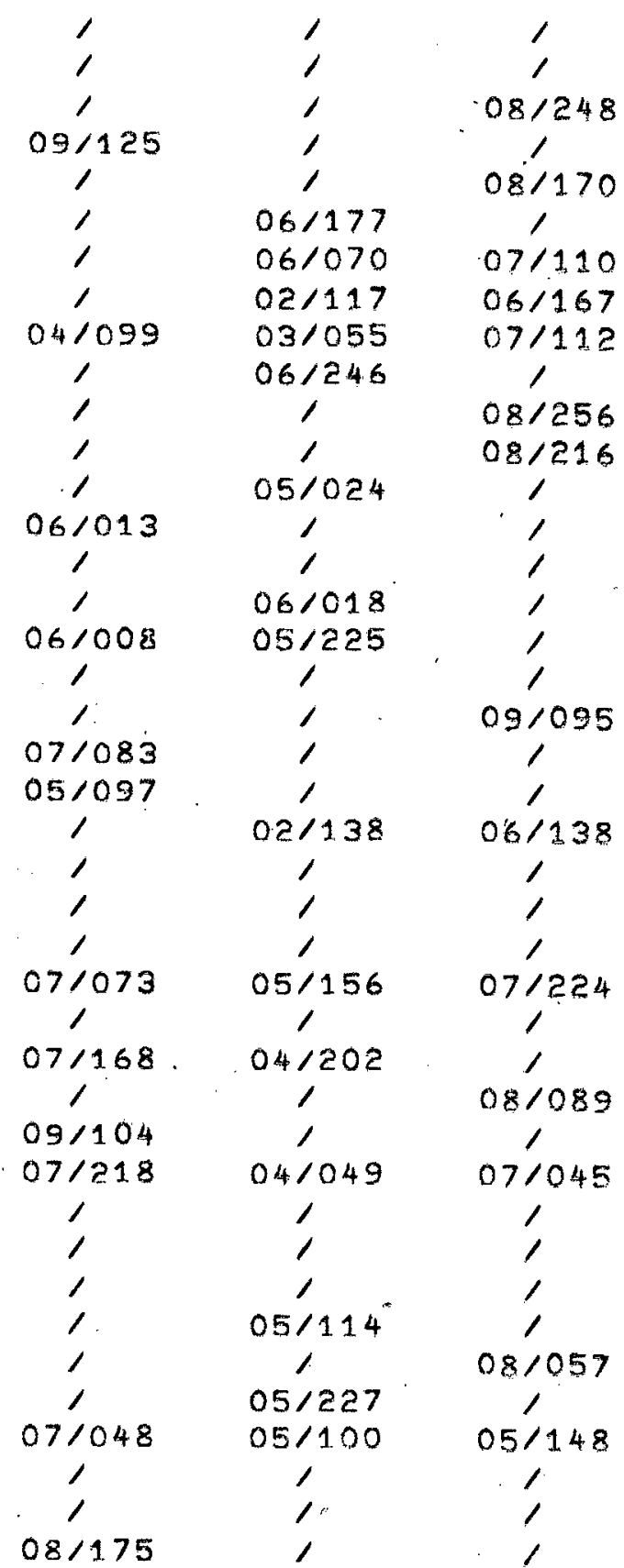

$09 / 017$

$08 / 188$

$08 / 190$

$04 / 225$

$02 / 133$

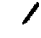

$04 / 023$

$04 / 0$

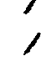

1

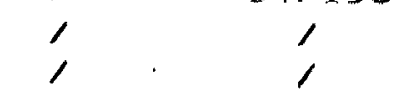

$04 / 054$

$06 / 182$

$05 / 025$

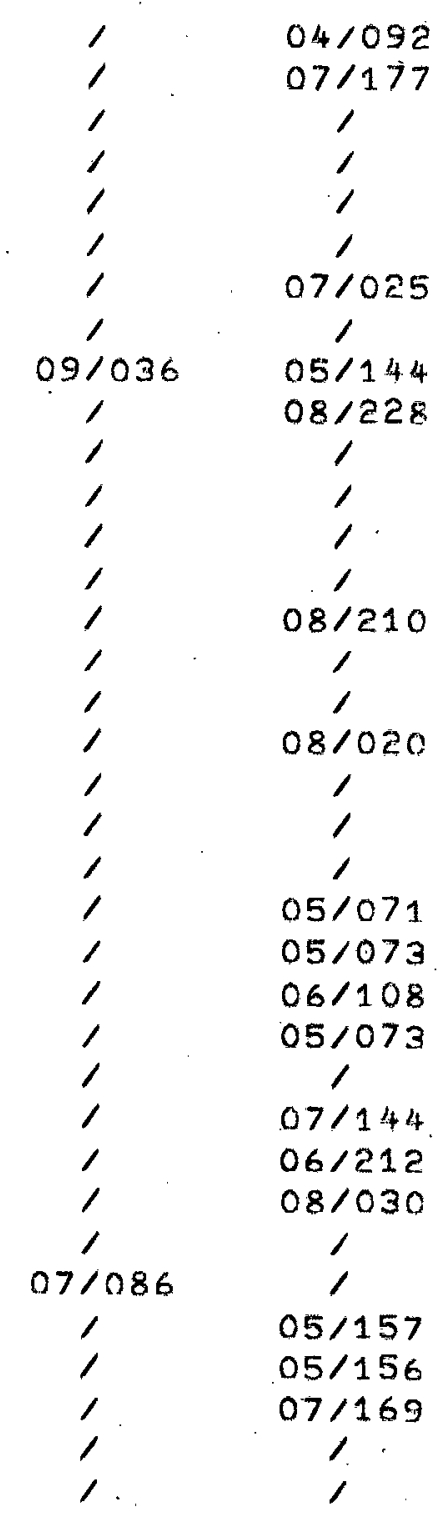

$06 / 071$

$06 / 109$

$07 / 037$

$06 / 111$

$07 / 194$

$08 / 045$.

$05 / 176$

$07 / 240$

,

$08 / 200$

$08 / 043$

$07 / 184$

$08 / 160$

$05 / 186$

$08 / 090$

$04 / 033$

$06 / 111$

$05 / 096$.

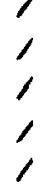


COFFEE

COILED

CEIN

COLD

COLGATE

COLLAR

COLLECTING

COLLECTION

COLLINS

CELER

CELORS

COLT

CELTS

COMB INED

COMBING

COME

COMES

COMFORT

COMFORTABLE

COMFERTABLY

CEMING

COMMANDER

COMMAS

COMMITTEE

COMMON

COMMUNICATE

COMPANY

COMPARED

COMPASS

COMPETE

COMPLAINED

COMPLETELY

COMPLEX

COMPUTE

COMPUTER

COMPUTERS

COMSAT

CONCERNED

CONCERT

CONCRETE

CONDITIEN

CENE

CONFESS

CONFUCIOUS

CONFUSED

CUNGRATULATIONS

CONGRESS

CONNECT

CONSIDER

CONSTANCE

CONTENTED

CONTENTMENT

CONTEST

CONTESTANTS

CONTESTS

CONTINUED

CONTRACTS

CONTRARY

CENTROL

CONVENIENT

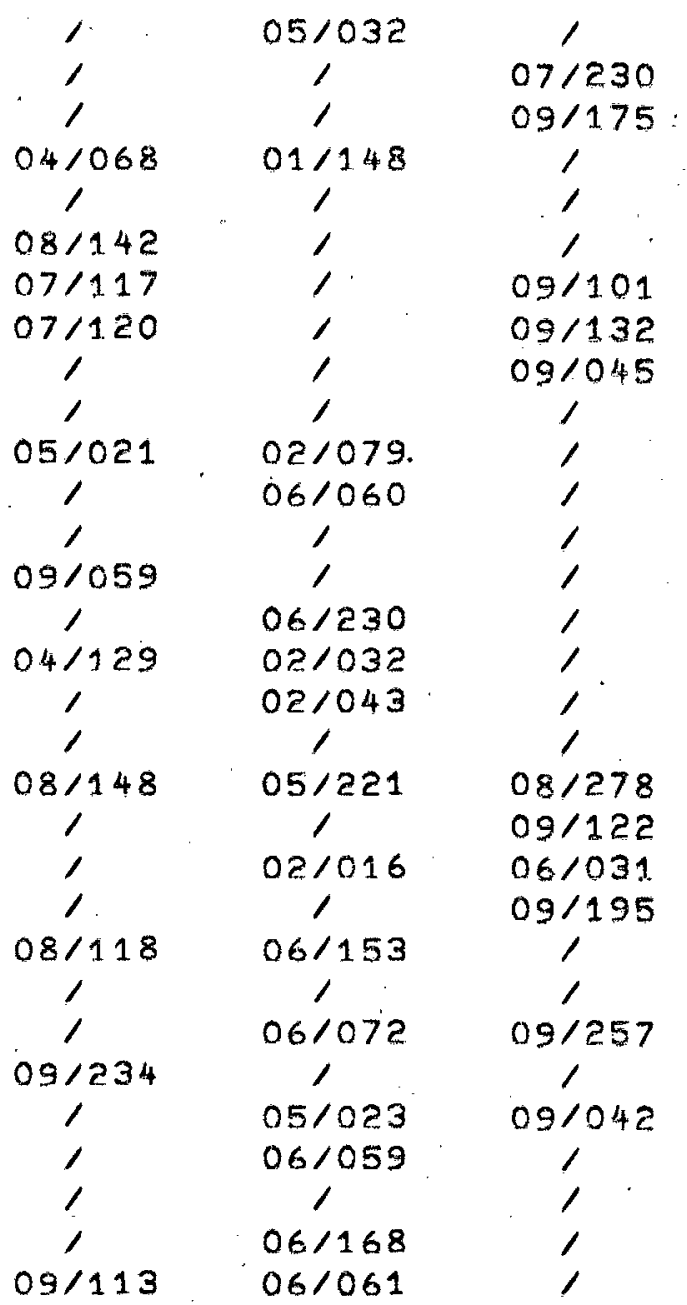

$09 / 113$

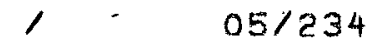

I

I

1

$09 / 219$

$09 / 239$

1

1

$08 / 220$

1

,

/

1

$09 / 236$

$09 / 143$

1

.

$09 / 271$

1

,

I

1

$08 / 220$

1

$09 / 226$

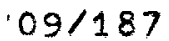

$09 / 035$

$.09 / 035$

$09 / 014$

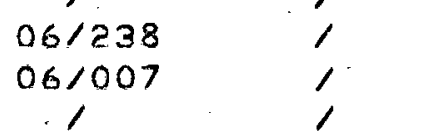

$08 / 199$

$06 / 170$

$06 / 073$

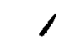

$06 / 140$

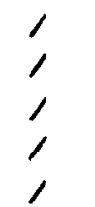

$06 / 295$

$06 / 291$

$06 / 019$

$06 / 022$

$05 / 246$

$06 / 268$
SERIES B SERIES C

SERIES

SERIES E

$11 / 066$

$11 / 049$

$07 / 098$

$08 / 160$

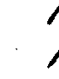

$03 / 052$

$04 / 041$

1

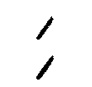

$09 / 041$
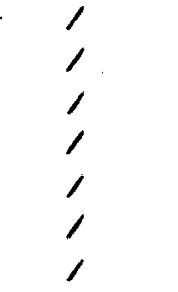

$08 / 084$

1

,

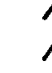
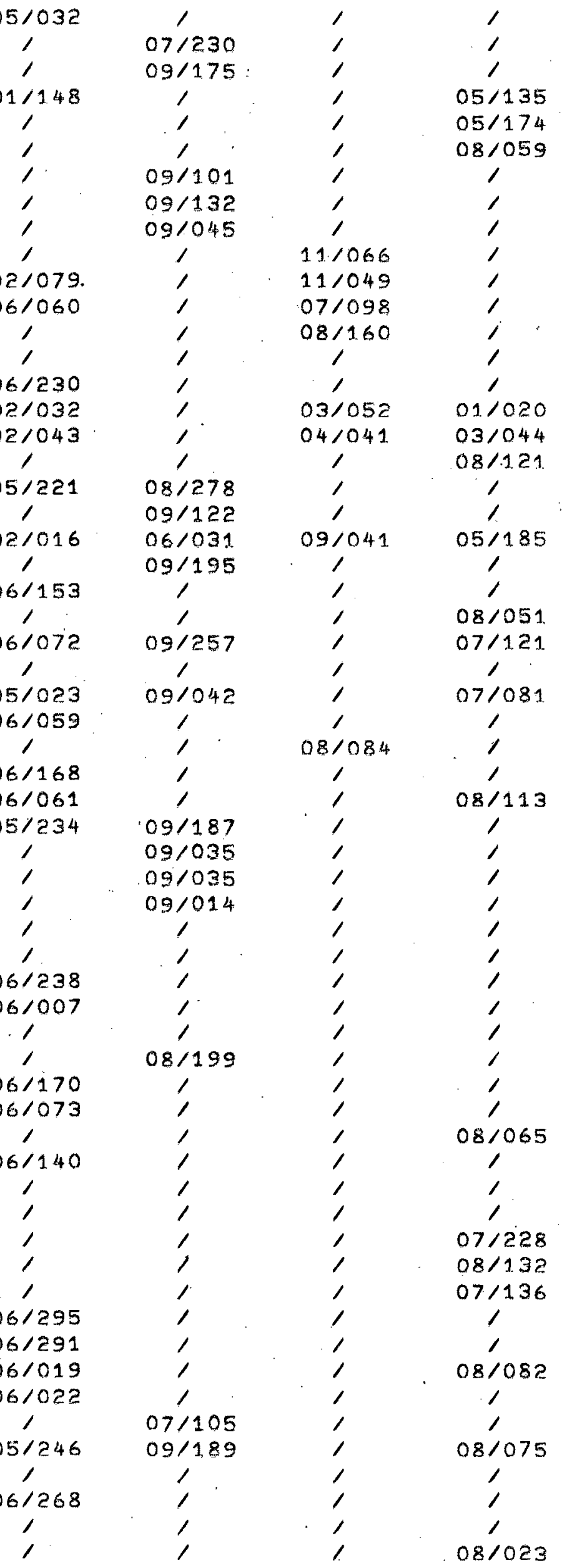


\section{coek \\ COOKBOQK \\ CUOKED \\ COOKIES \\ COOL \\ CeOP \\ COPPER \\ COPY \\ COPYCAT}

CORAL REEFS

CORN

CQRNER

CORNERS

CORNFIELD

CORRAL

CURRECT

COST

COSTS

COSTUME

COSTUMES

COTTAGE

COTTAGES

COTTEN

COULD

COULDNIT

COUNCIL

COUNCILER

CEUNT

COUNTDEWN

COUNTED

COUNTING

COUNTRIES

COUNTRY

COUNTRY'S

COUNTS

COUPLE

COURAGE

COURSE

COURT

COUSIN

COUSINS

COVE

COVER

COVERED

COVERING

COW

COWBOY

COWBOYS

COWS

COWSLIPS

CRACK

CRACKING

CRACKLING

CRAMER

CRAMMED

CRASH

CRATES

CRAWFERD

CRAWL

CRAWLED

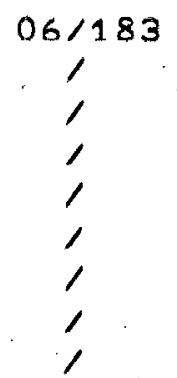

$09 / 083$

1

$07 / 130$



$07 / 053$

,

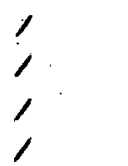

$08 / 092$

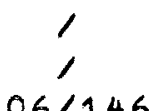

$06 / 146$

,

,

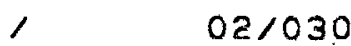

I

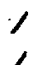

1

$06 / 177$

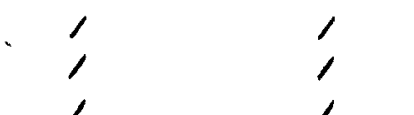

$09 / 142$

$07 / 169$

$09 / 025$

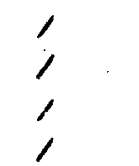

$08 / 232$

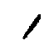

,

,

,

,

,

,

,

,

,

,

,

1

1

,

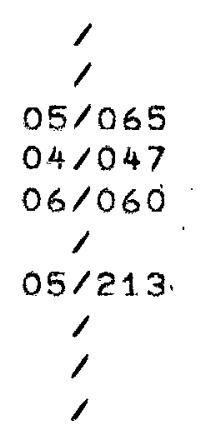

$05 / 271$

$05 / 033$

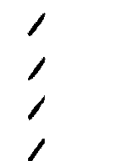

$05 / 045$

$06 / 053$

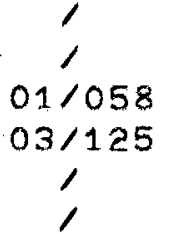

$02 / 030$
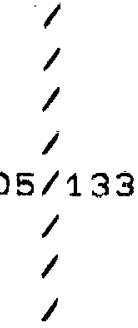

$06 / 086$

$05 / 140$

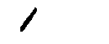

$05 / 147$
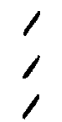

$04 / 051$

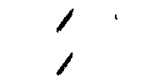

$01 / 028$

$01 / 027$

$02 / 011$

$05 / 218$

$05 / 133$

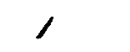

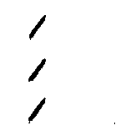

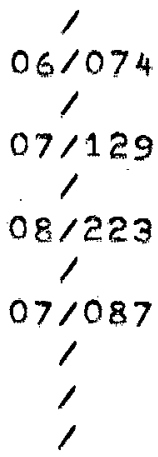

$07 / 178$
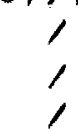

$09 / 033$

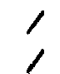

$09 / 278$
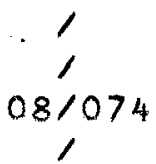

$05 / 063$

$05 / 085$

$09 / 210$

$08 / 154$

$05 / 023$

$05 / 212$

$05 / 070$

1

$08 / 049$

$07 / 094$

1

$08 / 141$

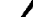

$03 / 281$

$08 / 033$

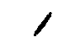

1

$06 / 206$

$09 / 243$

$06 / 101$

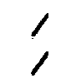

$05 / 097$

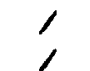

$05 / 099$

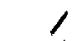

$06 / 099$

$09 / 101$

1

$09 / 186$

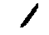

$08 / 043$

I

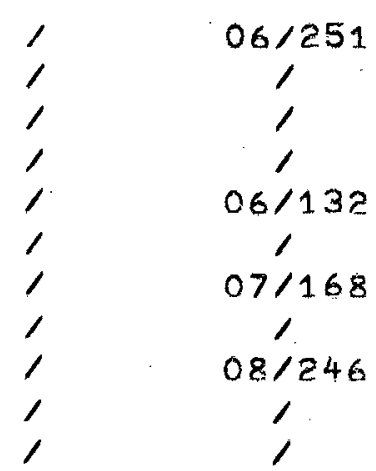

$08 / 061$

$11 / 01.8$
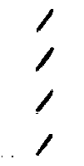

,

1

$11 / 046$

,

$08 / 024$

$09 / 047$

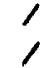

$09 / 026$

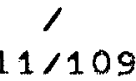

$09 / 017$

$11 / 036$

$11 / 025$

$11 / 037$

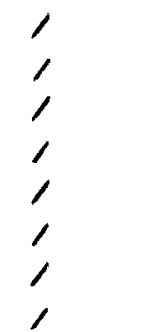

$08 / 192$

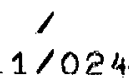

$11 / 024$
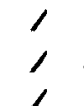

$06 / 039$

$05 / 220$

$08 / 010$

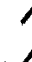

$08 / 030$

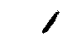

$08 / 025$

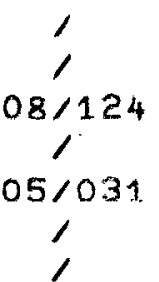

$06 / 191$

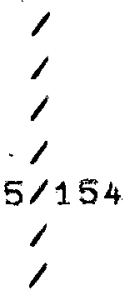

$08 / 213$

$08 / 023$

$08 / 227$

$08 / 105$

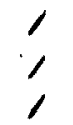

$06 / 104$

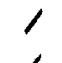

$06 / 035$

$04 / 128$

1

$06 / 202$

$07 / 225$

1

$07 / 234$

$08 / 140$

$08 / 206$ 
WERD LIST

CRAWL ING

CRAYONS

CRAZY

CREAKING

CREAKY

CREAM

CREATURE

CREDIT

CREEK

CREPT

CREW

CRIED

CRIPPLED

CRISPY

CRECEDILE

CREP

CROPS

CRESS

CROSSING

CRESSREADS

CROWD

CREWDED

CREWDS

CROWFGOT

CROWNED

CRUEL

CRUELTY

CRY

CRYING

CUBA

CUNNING

CUNNINGHAM

CUP

CUPBEARD

CUPCAKES

CUPFUL

CURB

CURIEUS

CURIEUSLY

CURLED

CURLS

CURLY

CURLY $=E A R E D$

CURLY $=H A I R E D$

CURRENTS

CURTAIN

CURVE

CUSHION

CUSTAMER

CUSTOMERS

CUT

CUTEST

CUTTING

CUTTINGS

D.C.

$D A D$

DAD'S

DADDY

DADOY'S

DADGER

SERIES

SERIES

SERIES $C$

SERIES D

SERIES E

$06 / 030$

$06 / 1.16$

,

,

'

,

,

,

$05 / 1.03$

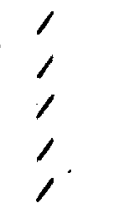

1

$06 / 214$

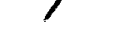

$07 / 097$

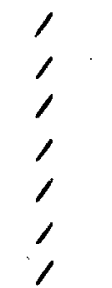

$05 / 140$

$09 / 120$

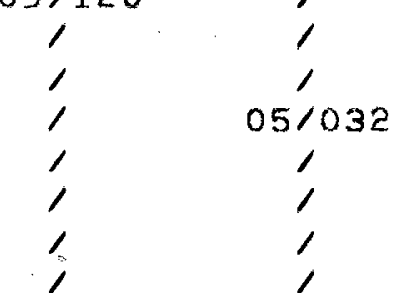

$08 / 077$

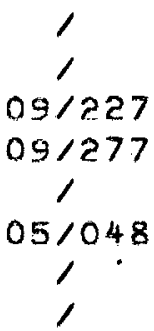

$08 / 256$

$04 / 056$

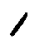

,

1
$03 / 112$
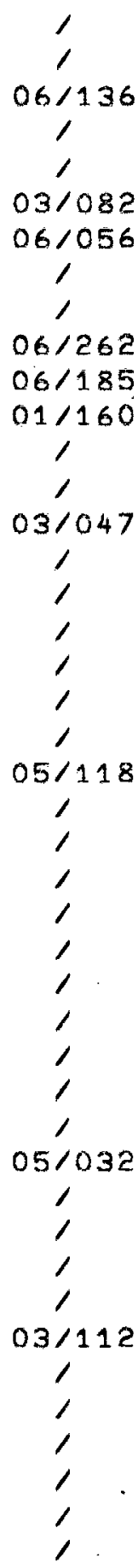

$06 / 262$

$06 / 185$

$01 / 160$

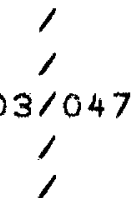

,

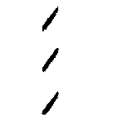

$05 / 118$
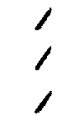

1

,

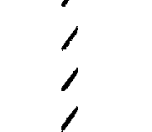

$05 / 032$
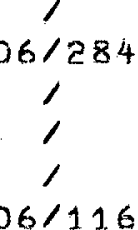

$03 / 170$

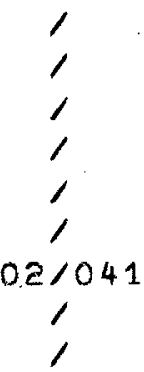

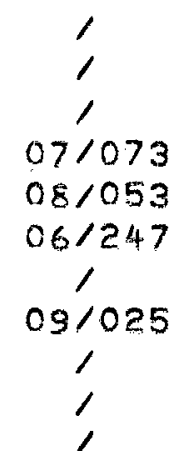

$06 / 080$

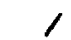

$08 / 019$

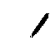

$08 / 027$

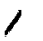

$07 / 144$

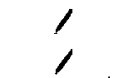

$08 / 063$

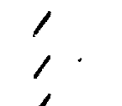

$09 / 215$

$09 / 168$

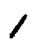

$05 / 020$
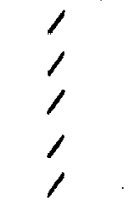

$09 / 114$
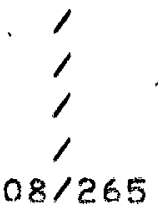

$07 / 037$

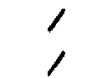

$07 / 188$

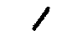

$08 / 116$

$09 / 179$

$08 / 112$
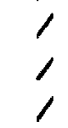

$05 / 202$

$05 / 044$

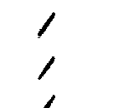

$05 / 108$
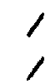

$05 / 122$

$08 / 071$

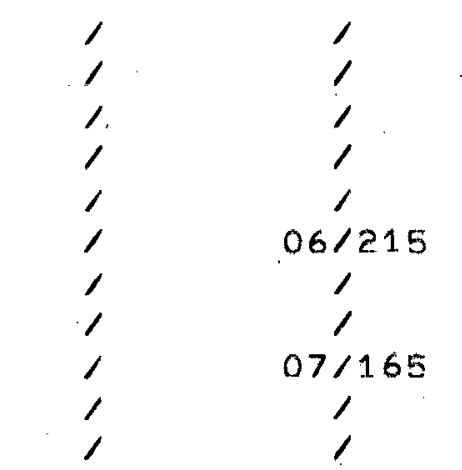

$11 / 022$
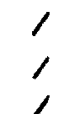

,
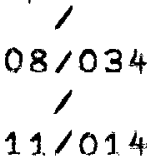

$11 / 053$

$11 / 054$

$11 / 094$
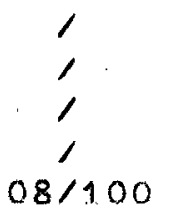

$09 / 047$
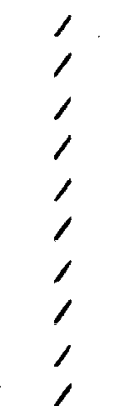
DADS

DAISY

DALE

DAMP

DAN

DANA

DANCE

DANCES

DANCING

DANDER

DANGER

DANGEREUS

DANGLING

DANNY

DANNY DRAKE

OAPHNE

DARED

DARING

DARK

DARKNESS

DASHED

DASHING

DAUGHTEF

DAVE

DAVID

DAVID'S

DAWSON

DAY

DAY:S

DAYDREAM

DAYDREAMING

DAYS

DAYTIME

DEAD

DEAL

DEAR

DEBBIE

DEBT

DECALRED

DECEIVE

DECEMBER

DECIDE

DECIDED

DECK

DECLARED

DECQDE

DECURATIENS

DECOY

DEEP

DEERSKIN

DEFEATED

DEFEND

DEFROST

DELICI UUS

DELIGHT

DEL I GHTED

DELIVER

DELIVERING

DELIVERY

DEMANDING

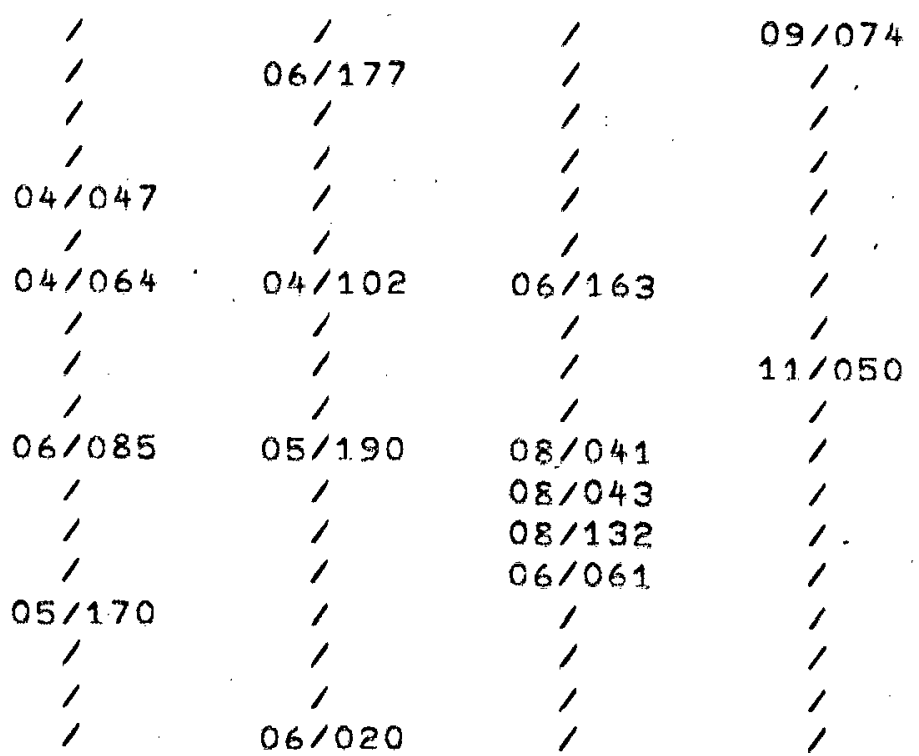

$04 / 005 \quad 01 / 150$

, $06 / 042$

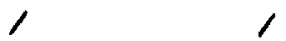

1. $\quad 06 / 134$

$08 / 179 \quad 05 / 059$

, 1

,

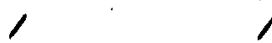

11

$04 / 095 \quad 02 / 036$

1

,

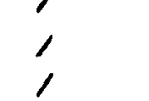

$06 / 089$

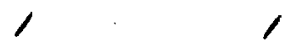

$07 / 073$

1

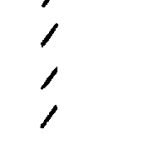

$09 / 121$

1

$07 / 148$

1

$09 \% 104$
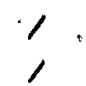

,

1

,

1

091020

1

$06 / 220$

1

$06 / 220$
$07 / 035$

1

I

1

$08 / 148$

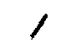

$06 / 095$

1

$04 / 113$

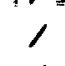

1

$04 / 115$

1

1

$08 / 101$

$04 / 206$

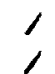

$06 / 063$

1
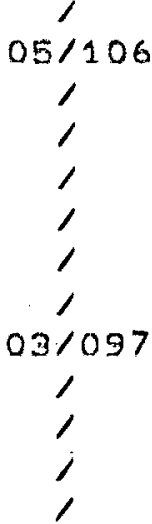

$06 / 130$

$05 / 221$
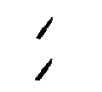

,

,

$06 / 143$
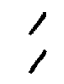

,
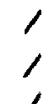

$08 / 045$
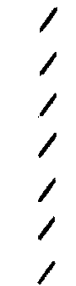

$09 / 172$

$09 / 258$

$09 / 203$

$09 / 065$

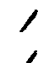

$08 / 016$

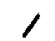

$08 / 011$

$11 / 010$
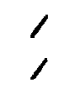

$09 / 059$

$03 / 004$

$05 / 011$

$08 / 078$

$11 / 051$

$11 / 040$

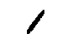

$08 / 159$

$08 / 190$

$09 / 116$

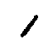

,
$08 / 169$

$08 / 144$

$07 / 215$

$07 / 058$

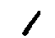

081080 1

$08 / 152$

$07 / 176$

$07 / 179$

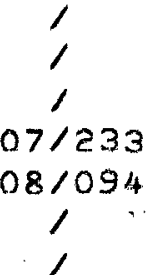

$07 / 078$

$06 / 194$

$08 / 132$

$06 / 007$

1

$04 / 082$

,

$07 / 226$

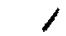

$06 / 263$

$08 / 083$

$08 / 213$

$04 / 076$

$08 / 021$

$08 / 262$

1

$08 / 255$

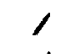

$06 / 021$

$07 / 098$

$08 / 056$

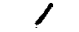

$08 / 052$

$08 / 255$

$05 / 195$

$07 / 141$

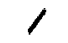

$07 / 228$

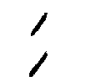

$08 / 068$

$07 / 206$ 
SERIES O SERIES'E

DENMARK

DENNIS

DEPENO

DEPENDS

DESCENDAMT

DESCENDANTS

OESCRI BED

DESERT'

DESERTS

DESERVE

DESERVES

DESIRE

DESK

DESPAIR

DESTREY

DETECTIVE

DETECTIVES

DETOUR

DEW

DIAGRAM

DIAL

DIALS

DIAMOND

DIARY

DICK

DICK'S

DID

DIDN'T

DIDES

DIED

DIFFERENCE

DIFF I CULT

DIFFICULTY

DIG

DIGNITY

DIME

DING

DINING

DINNER

DINOSAURS

DION

DIPLOMACY

DIPPED

DIRECT

DIRECTION

DIRECTIENS

DIRECTOF

DIRT

DIRTY

DISAPPEARED

DISAPPOINT

DISAPPOINTED

DISAPFOINTMENT

DISCQURAGED

DISCOVER
DIFFERENT

$\begin{array}{lccc}\text { DISCOVERED } & / & 05 / 231 & 07 / 234 \\ \text { DISEASE } & / & / & 1 \\ \text { DISH } & 05 / 164 & / & 06 / 043 \\ \text { DISHES } & / & \prime & \end{array}$

$\begin{array}{lccc}\text { DISCOVERED } & / & 05 / 231 & 07 / 234 \\ \text { DISEASE } & / & / & 1 \\ \text { DISH } & 05 / 164 & / & 06 / 043 \\ \text { DISHES } & / & \prime & \end{array}$

$\begin{array}{lccc}\text { DISCOVERED } & / & 05 / 231 & 07 / 234 \\ \text { DISEASE } & / & / & 1 \\ \text { DISH } & 05 / 164 & / & 06 / 043 \\ \text { DISHES } & / & \prime & \end{array}$

$\begin{array}{lccc}\text { DISCOVERED } & / & 05 / 231 & 07 / 234 \\ \text { DISEASE } & / & / & 1 \\ \text { DISH } & 05 / 164 & / & 06 / 043 \\ \text { DISHES } & / & \prime & \end{array}$

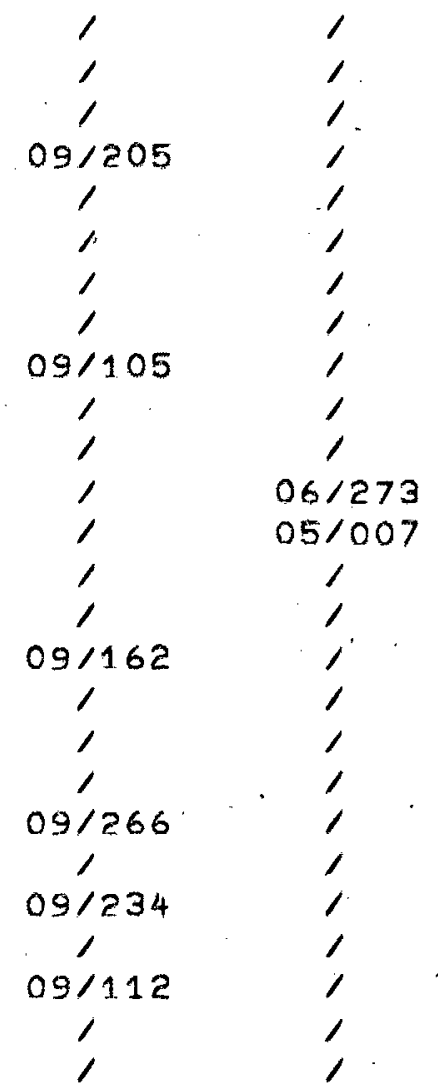

$02 / 013$

$07 / 104$

1

$06 / 095$

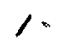

$07 / 007$

$09 / 192$
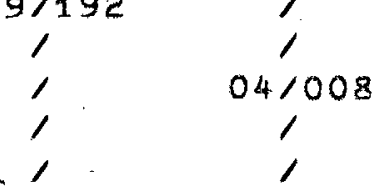

1

,

$07 / 082$

1

,

I

,

$08 / 079$

1

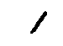

I $04 / 120$

, $05 / 236$

$09 / 065$

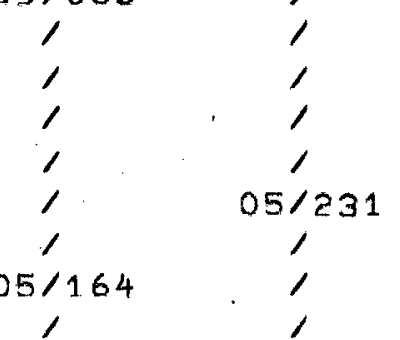

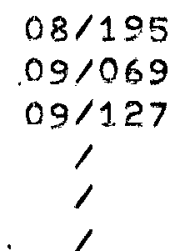

$09 / 062$

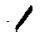

$09 / 069$

$09 / 194$

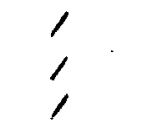

$09 / 170$

$09 / 110$
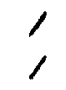

1

$09 / 215$

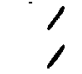

$09 / 153$

$06 / 050$

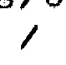

I

,

,

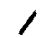

$08 / 075$

$07 / 121$

$09 / 064$

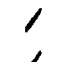

$09 / 279$

$05 / 247$

$04 / 018$

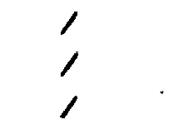

$06 / 297$

$06 / 275$

$05 / 232$
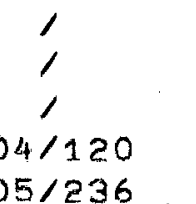

$08 / 048$
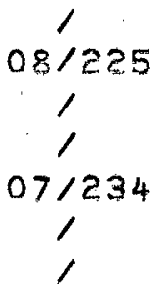

$\begin{array}{lccc}\text { DISCOVER } & / & / & 07 / 234 \\ \text { DISCOVERED } & / & 05 / 231 & / \\ \text { DISEASE } & / & / & 06 / 043 \\ \text { DISH } & 05 / 164 & / & /\end{array}$

$\begin{array}{lccc}\text { DISCOVERED } & / & 05 / 231 & 07 / 234 \\ \text { DISEASE } & / & / & 1 \\ \text { DISH } & 05 / 164 & / & 06 / 043 \\ \text { DISHES } & / & \prime & \end{array}$

081020

$08 / 141$

$11 / 026$

$11 / 046$

,

,

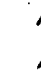

'

1

,

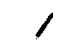

$09 / 005$

$09 / 014$

1

,

1

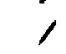

,

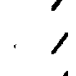

$05 / 032$

$08 / 095$
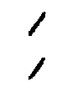

$07 / 036$

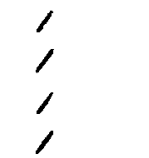

$07 / 029$

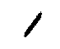

,

,

,

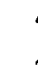
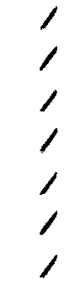

1

,

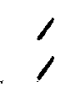

$11 / 061$

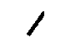




\section{DISLIKE \\ DISPLACE \\ DISSATISFIED \\ DISTANCE \\ DISTANT \\ DITCH \\ DIVED \\ DIVIDED \\ DIVIDING \\ DIZZY}

$D E$

DO-IT-YEURSELF

$D O=N O T H I N G$

DOCK

DOCTOR

DOCTER-FATHER

DECTORS

DOE

DUES

DOESN'T

$D \theta G$

DOGHOUSE

DEGS

DOING

DELL

DOLLARS

DOLPHIN

DEMES

DON

DON:T

DENE

DONG

DENKEY

DONKEYS

DENNA

DOODLE

DEER

DOERBELL

DOORWAY

DET

DOUBLED

DEUBT

DOUBTED

DOUBTFULLY

DEUGH

DOVEKIE

DOWN

DOWNSTAIRS

DOWNTEWN

DEZED

DEZEN

$D R$

DR CARVER

DR .

DRAGGING

DRAGON

DRAGBNS

DRAIN

DRAINBOARD

DRANK

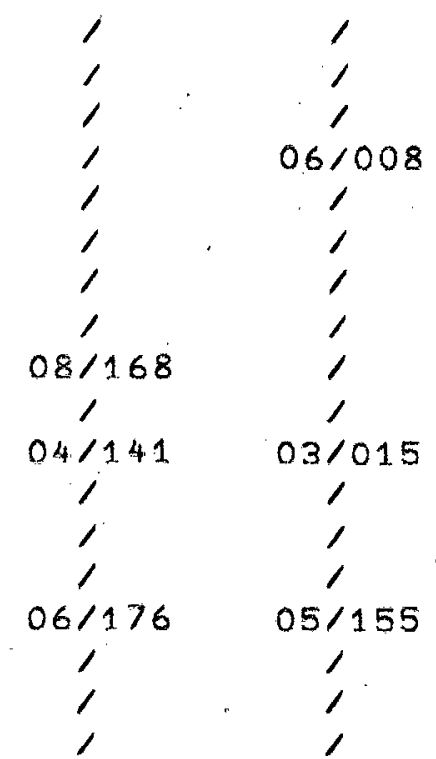

$07 / 011$

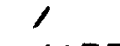

$04 / 077$

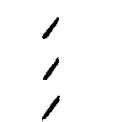

$06 / 029$

1

1

$09 / 258$

1

$05 / 042$

$08 / 075$

1

$05 / 084$

$06 / 026$

1

$05 / 139$

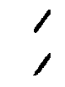

,

,
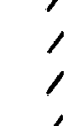

$09 / 176$

$01 / 039$

1

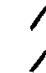

$09 / 145$

$06 / 1.95$

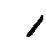

$07 / 065$

,
$01 / 075$

$04 / 117$

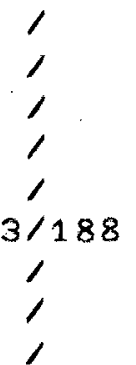

$02 / 021$

$02 / 137$

$05 / 247$
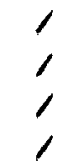

$03 / 122$

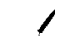

$01 \% 039$

$06 / 091$

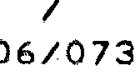

1

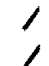

$02 / 013$

$04 / 035$

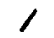

$06 / 007$

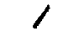

$05 / 223$

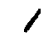

$04 / 135$

,

$03 / 214$

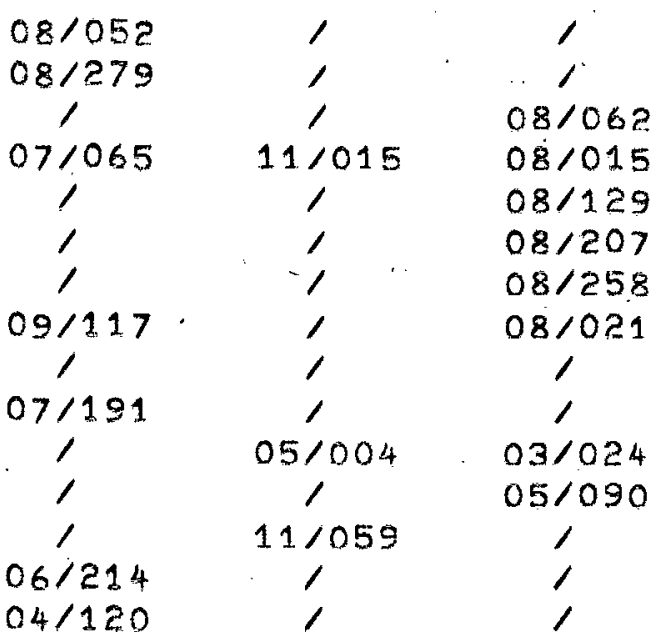

$06 / 192$

$06 / 189$

$08 / 069$

$05 / 179$

$06 / 109$

$03 / 045$

$04 / 062 \quad 09 / 023$

$09 / 022$

$09 / 052$

$09 / 025$

$11 / 019$

,

$06 / 061$

$07 / 063$

1

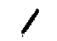

$04 / 038$

$06 / 169$

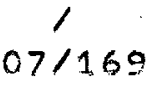

1

$09 / 237$

$06 / 013$

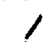

,

,

1

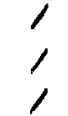

$07 / 159$

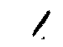

,

,

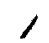

$08 / 162$

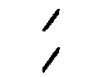

$06 / 226$

$08 / 103$

$07 / 247$

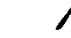

$03 / 045$

$05 / 010$

$08 / 030$

,

1

$04 / 018$

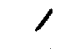

$11 / 041$

$11 / 063$

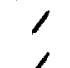

$07 / 113$

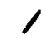

$08 / 121$
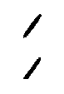

1

1

1

$06 / 034$

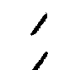

,

,

,

,

,
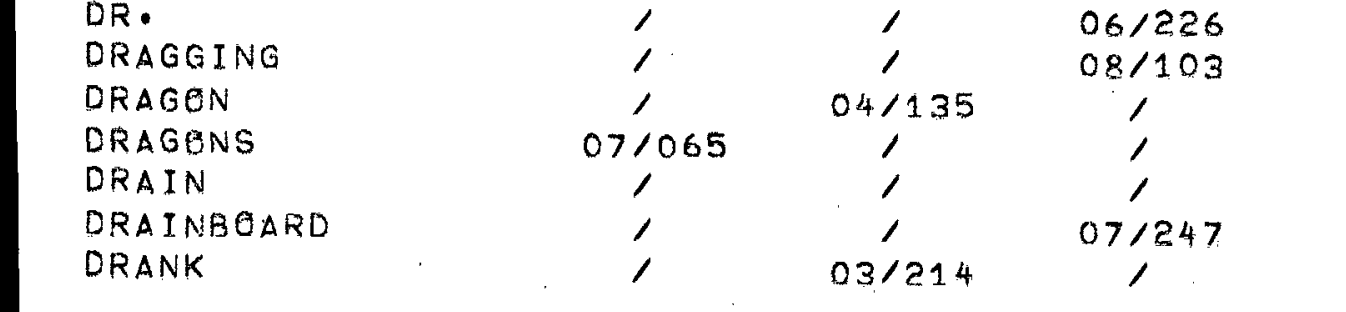
DRAW

DRAWIER

DRAWERS

DRAWN

DREAM

ORESS

DRESS UP

DRESSED

DRESSES

DREW

DRIED

DRIFTING

DRINK

DRIPPING

DRIVE

DRIVEN

DRIVER

DRIVER IS

DRIVES

DRIVING

DREOP

DROP

DREPPED

DREFPINE

DRGUGHT

DROVE

DREWN

DREWNED

DREWNING

DRY

DRYER

DUCHESS

DUCK

DUCKED

DUCKLING

DUCKS

DUCKY LUCKY

DUG

DUKE

DULL

DUMB

DUNES

DUNN

DURING

DUST

DUSTY

EACH

EAGER

EAGERLY

EAGERNESS

EAGLE

EAGLE FEATHER

EAR

EARLY

EARN

EARNED

EARRINGS

EARS

EARTH

EARTHMOVERS

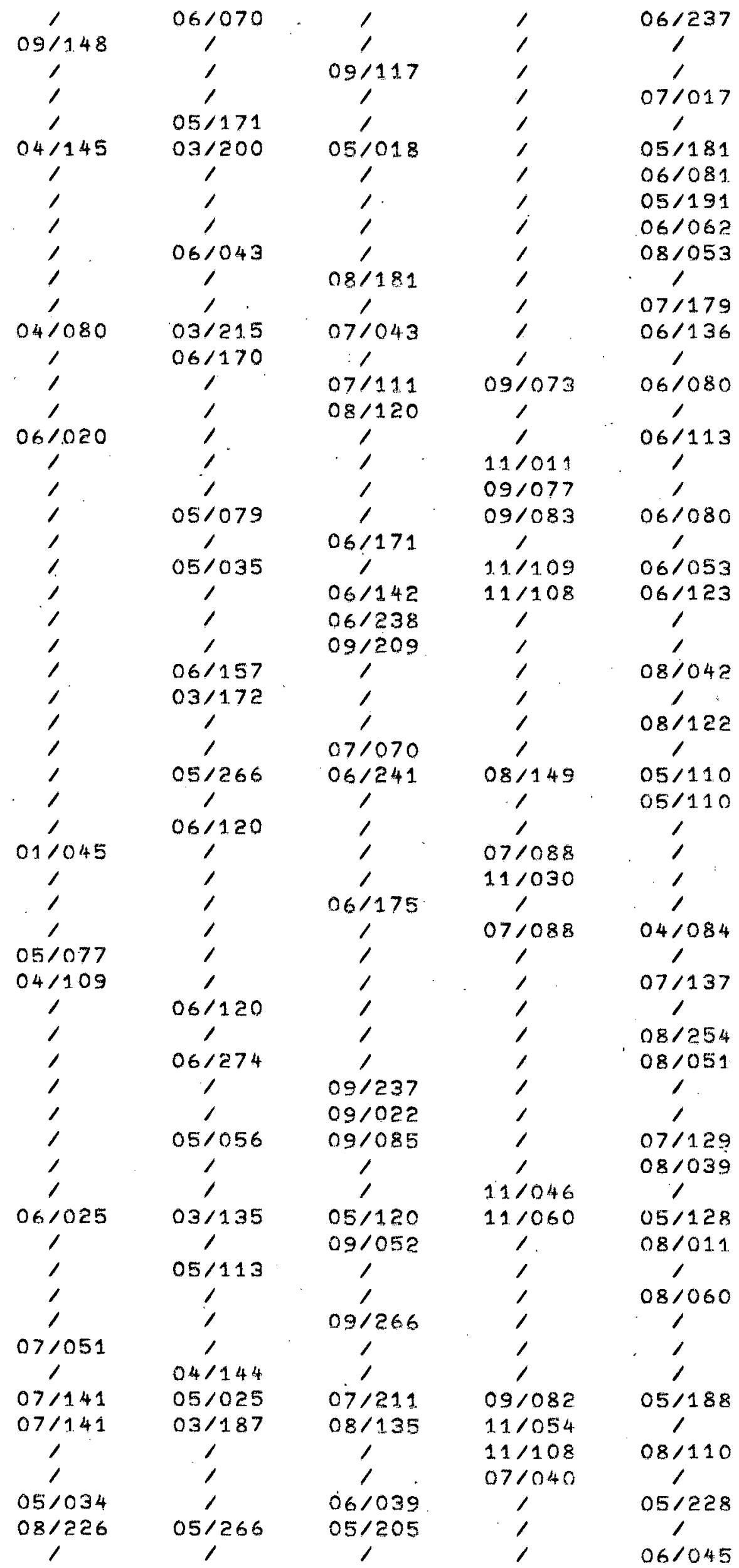


EARTHQUAKE

EARTHY

EASEL

EASIER

EASILY

EASY

EAT

EATEN

EATING

EATS

ECHO

EDDIE

EDGE

EDWIN

EGBERT

$E G G$

$E G G=H E U S E S$

EGGS

EGRET

EIGHT

EIGHT-YEAR $-O L D$

EITHER

ELDER

ELECTRIC

ELECTRICAL

ELECTRICITY

ELEPHANT

ELEPHANTS

ELEVATORS

ELEVEN

ELF

ELLEN

ELLEN'S

ELSA

ELSE

ELSE'S.

ELVAR

ELVES

EMBLEM

EMPT IED

EMPTINESS

EMPTY

EMPTY = HE ADED

END

ENDING

ENEMIES

ENEMY

ENGINE

ENGINEER

ENGLAND

ENGLISH

ENITH

ENJEY

ENJOYABLE

ENJOYED

ENJEYMENT

ENERMOUS

ENOUGH

ENTER

ENTERS

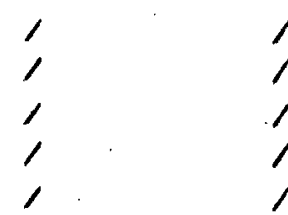

$08 / 049$

$04 / 063$

1

,

$09 / 239$

$08 / 092$

$06 / 033$

$06 / 136$

$05 / 015$

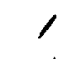

$09 / 034$

$08 / 035$

1

$07 / 170$

$09 / 217$

1

1

$06 / 033$

$07 / 218$

$1 \quad 06 / 085$

$-1 \quad 06 / 291$

,

$07 / 168$

$06 / 038$

1

$07 / 173$

1

,

I

,

,

,

1

$08 / 198$

1

$07 / 126$

$08 / 071$

$$
1
$$

$06 / 193$

$08 / 040$

$09 / 022$

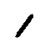

$07 / 164$

1
$05 / 048$

$01 / 055$

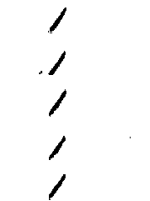

$05 / 220$

,

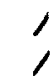

$04 / 148$

$06 / 044$

$05 / 188$

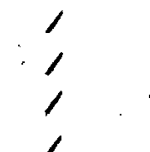

$02 / 123$
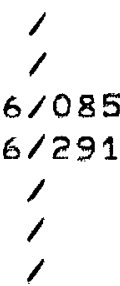

$03 / 208$

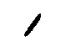

1

1

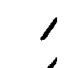

$05 / 148$

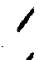

$03 / 066$

$06 / 260$

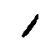

$06 / 184$

$05 / 021$

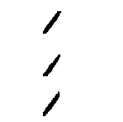

$05 / 157$
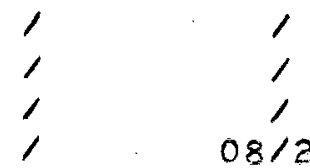

$03 / 187$

$06 / 020$

',

,
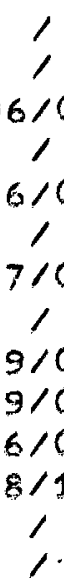

1
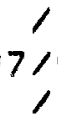

1

1
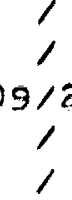

1

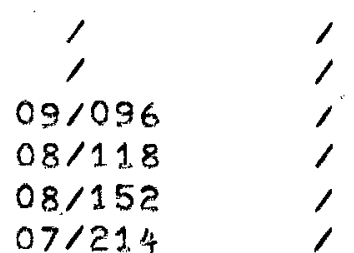

1.1/063

$07 / 049$

1

$04 / 124$

$07 / 248$

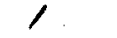

$06 / 077$

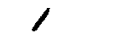

$06 / 063$

1

$07 / 046$

$09 / 013$

$09 / 020$

$06 / 090$

$08 / 185$

$07 / 154$

$05 / 017$

$05 / 017$

$07 / 066$

$07 / 088$

$09 / 257$

$07 / 244$

$07 / 192$

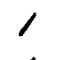

1

$09 / 238$

$08 / 195$

$09 / 019$

$08 / 223$

$05 / 098$

$08 / 190$

$11 / 075$

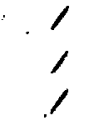

$08 / 139$

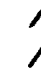

,

,

,

1

,

1

,

1

,

,

,

,

,

1

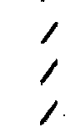

$08 / 079$

$11 / 027$

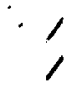

1

1

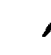

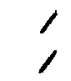

$08 / 041$

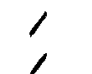

$07 / 018$

$08 / 090$

$06 / 193$

$06 / 249$

$06 / 114$

$04 / 028$

$04 / 019$

$06 / 127$

,

1

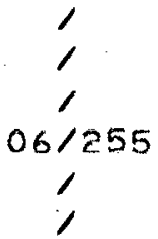

$06 / 091$

$06 / 172$

$07 / 219$

$07 / 053$

$07 / 218$

$07 / 216$

$06 / 035$

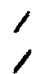

$08 / 047$
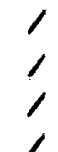

$05 / 176$
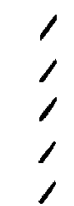

$08 / 231$

$06 / 183$

$06 / 184$

$05 / 085$

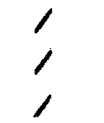

$06 / 067$

$07 / 187$

$08 / 157$

$08 / 046$

$08 / 105$

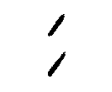

$08 / 009$

$08 / 074$

$05 / 150$

$08 / 054$

$\begin{array}{cc}07 / 092 & 05 / 150 \\ 1 & 08 / 054 \\ 1 & 1\end{array}$


ENTRANCE

ENTRY

ENVELEPE

ENVELOPES

ENVY

ERASER

ERRAND

ESCALATSR

ESCAPE

ESCAPED

ESCAPES

ESKIMO

ESPECIALLY

ESTHER

EUROPE

EVEN

EVENING

EVENLY

EVENTS

EVER

EVERY

EVERYBEOY

EVERYENE

EVERYTHING

EVERYWHERE

EXACTLY

EXAMINE

EXAMINING

EXAMPLES

EXCEPT

EXCHANGE

EXCITED

EXCITEMENT

EXCITING

EXCLAIMED

EXCUSE

EXERCISE

EXIT

EXPANDS

EXPECTED

EXPECTIEN

EXPENSIVE

EXPERIMENT

EXPERIMENTAL

EXPERIMENTS

EXPLAIN

EXPLAINED

EXPLODE

EXPLERE

EXPLORING

EXPRESS

EXTRA

EYE

EYE-HOLES

EYEBRGWS

EYEGLASSES

EYES

FABULGUS

FACE

FACES

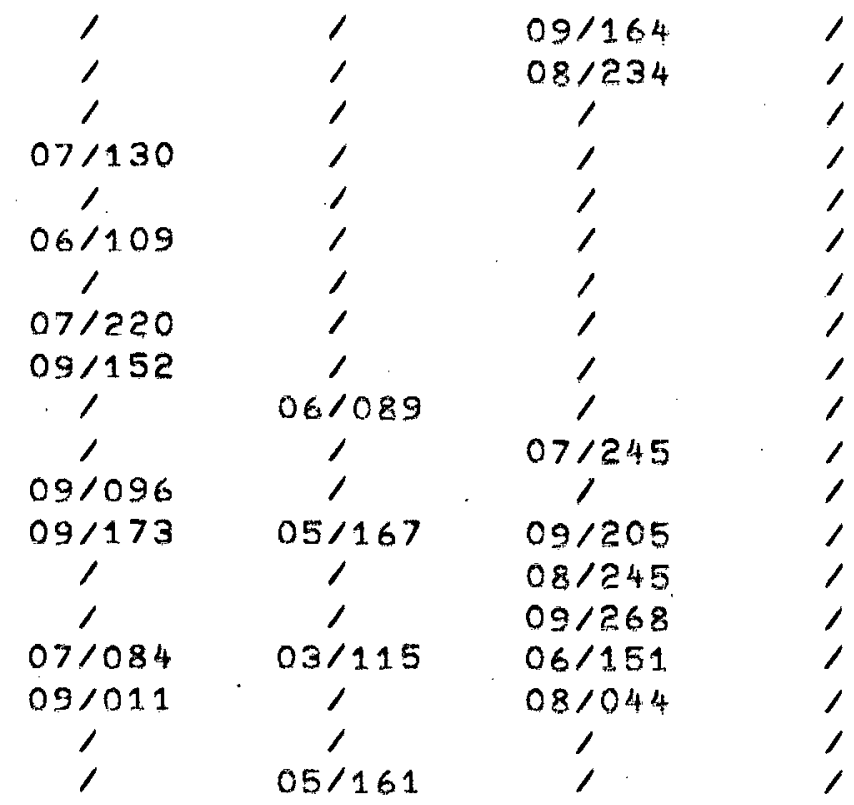

$05 / 041$

$06 / 041$

$07 / 034$

$02 / 089$

021090

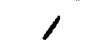

$06 / 070$

',

$09 / 210$

, $05 / 058$

$1 /$

,

1

$08 / 074$

$05 / 089$

1

o7/195

$08 / 220$

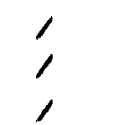

$09 / 264$

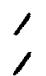

$07 / 164$

,

,

I

,

,

1

1

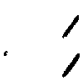

$06 / 091$

$09 / 126$

1

$07 / 007$

1

$05 / 031$

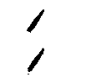

$05 / 164$
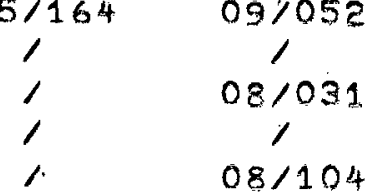

$05 / 120$

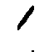

$06 / 144$
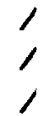

$05 / 165$

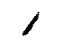

,

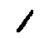

$06 / 196$

$05 / 062$

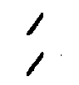

$06 / 292$

$04 / 048$

1

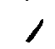

$03 / 202$

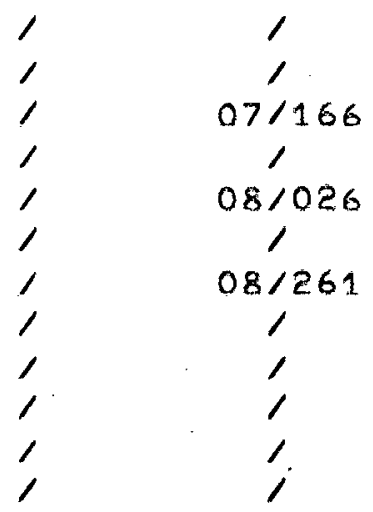

$08 / 193$

$06 / 085$

$06 / 213$

$07 / 190$

$09 / 074$

$09 / 040$

$08 / 185$

$09 / 041$

$09 / 040$
$09 / 018$

1

$08 / 239$

$09 / 025$

$08 / 076$

$09 / 183$

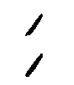

,

,

1

$11 / 087$

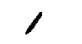

$11 / 087$

$11 / 057$

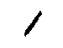

$08 / 031$

$\gamma$

$08 / 104$

1

$09 / 083$

$09 / 065$

$06 / 091$.

$09 / 124$

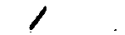

$07 / 149$

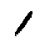

$08 / 100$

$07 / 230$
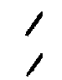

$08 / 263$

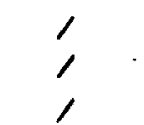

$06 / 227$

$0 G / 216$

$07 / 021$

$11 / 058$

$11 / 033$

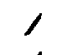

,

$08 / 126$

$08 / 126$
$05 / 119$

$05 / 131$

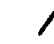

$07 / 091$

$08 / 029$

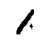

$08 / 014$

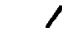

$06 / 098$

$08 / 060$

$06 / 103$

$07 / 119$

$08 / 025$

$08 / 042$
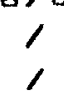

$07 / 187$
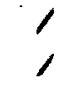$$
1
$$

$08 / 115$

$07 / 131$

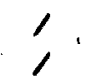

$07 / 113$

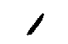

$08 / 023$

$06 / 253$
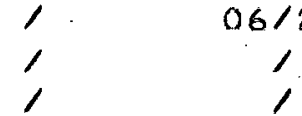

$05 / 162$

$06 / 015$ 
FACT

FACTORIES

FACTS

FAILED

FAILURE

FAINT

FAIR

FAIRY

FAITHFUL

FALL

FALLEN

FALLING

FAMILIAR

FAMILIES

FAMILY

FAMEUS

FANCY

FAR

FARAWAY

FARM

FARMER

FARMERS

FARMHEUSE

FARMS

FARTHER

FAST

FASTEN

FASTENED

FASTER

FAT

FATHER

FATHER 'S

FATHERS

FAUCET

FAUCETS

FAULT

FAVER

FAVORITE

FAVERITES

FEAR

FEARFULLY

FEARLESS

FEAST

FEATHER

FEATHERS

FED

FEED

FEEDING

FEEL

FEEL ING

FEET

FELL

FELLOW

FELT

FENCE

FERNWEOD

FEVER

FEW

FF OUND

FIELD

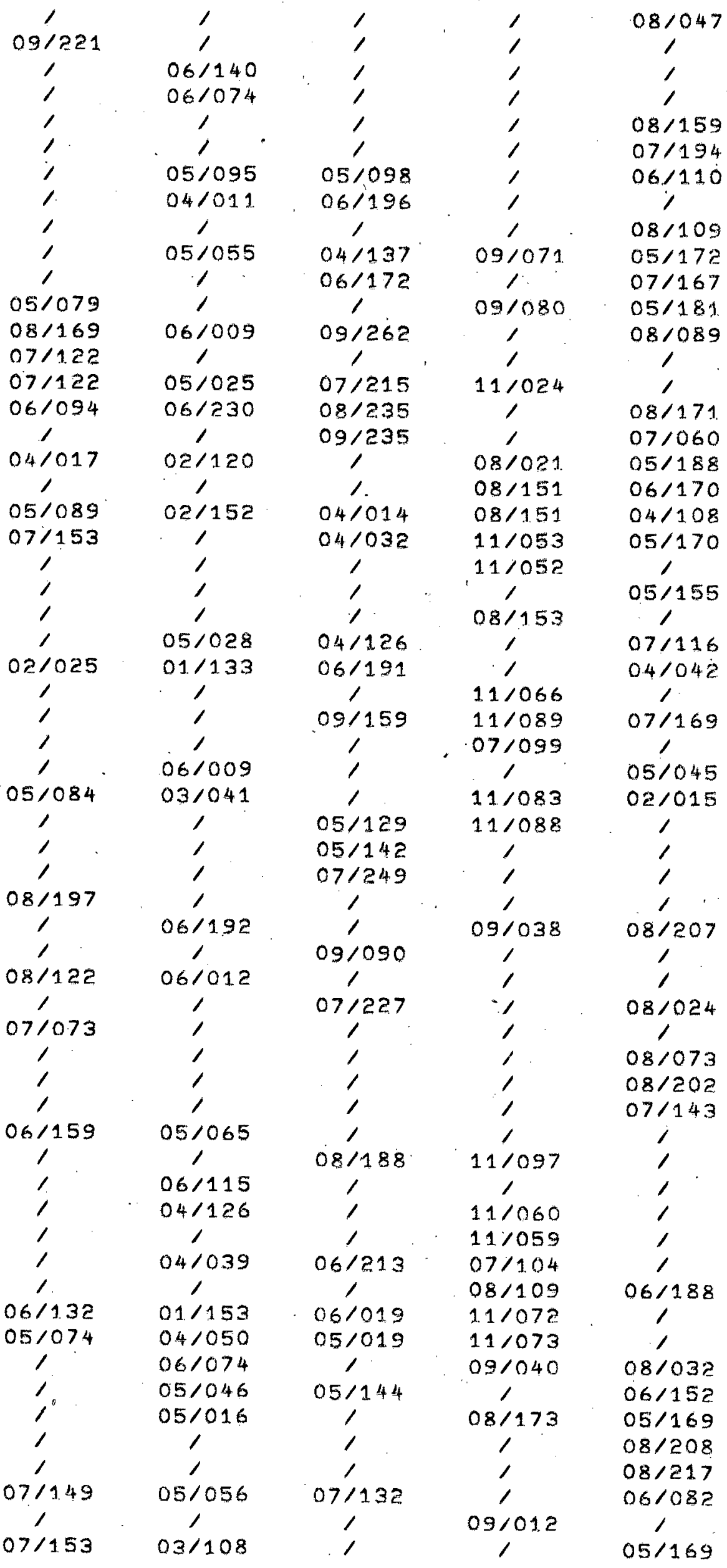


WERD LIST.

FIELDS

FIERCE

FIERCELY

FIFE

FIFTEEN

FIFTEEN-MAN

FIFTH

FIFTIETH

FIFTY

FIGHT

FI GURE

FIGURED

FIJI

FIJIANS

FILL

FILLED

FINALLY

FIND

FINDING

FINDS

FINE

FINEST

FINGERS

FINISH

FINISHED

FINNALLY

FIRE

FIREBELL

FIREHOUSE

FIREMAN'S

FIREMEN

FIREPLACE

FIRES

FIREWEOD

FIRMLY

FIRST

FISH

FISHES

FISHING

FITS

FIVE

FIX

FIXED

FLAG

FLAMES

FLASH

FLASHED

FLASHLIGHT

FLAT

FLAVGR

FLEA

FLEW

FLIERS

FLIES

FLIGHT

FLOAT

FLOATED

FLOATING

FLECK

FLOEDED
SERIES

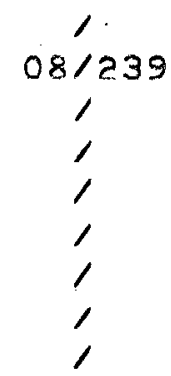

$06 / 085$

$08 / 069$

$09 / 076$

$09 / 082$

1

$04 / 067$

$07 / 163$

1

'

$06 / 062$

$07 / 031$

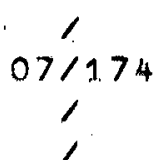

$07 / 019$

1
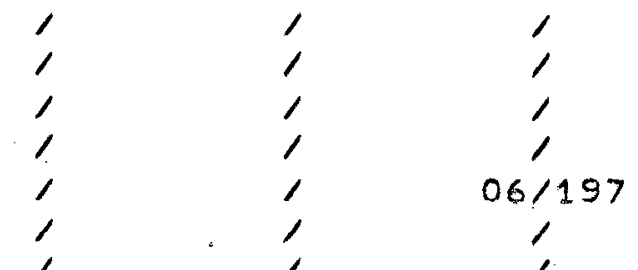

$05 / 060$
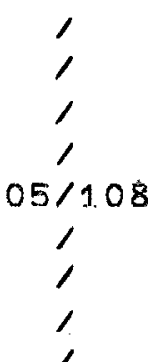

$04 / 051$

,

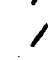

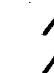

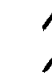

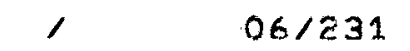

/ $\quad 04 / 158$

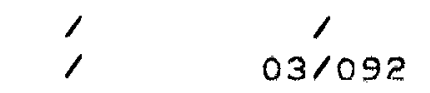

$06 / 1.80$

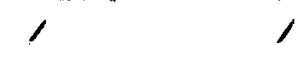

$08 / 024$

$05 / 079$
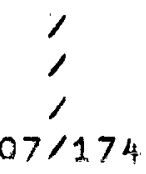

$04 / 223$

$04 / 030$

$05 / 135$

$01 / 148$

$06 / 197$

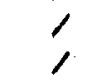

$04 / 130$

$05 / 013$

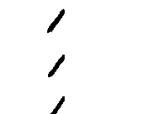

$05 / 024$

$06 / 017$

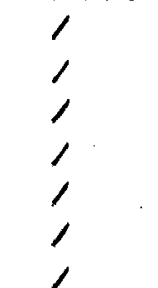

$09 / 204$

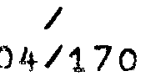

$08 / 123$

$08 / 052$

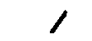

$05 / 195$

$05 / 197$

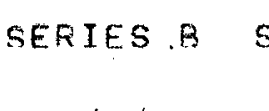

SERIES

SERIES D SERIES E

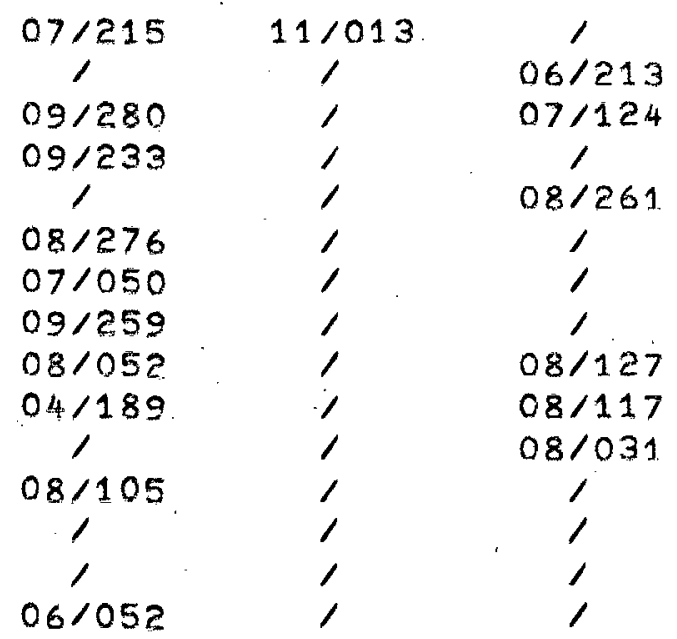

$06 / 103$

$05 / 200$

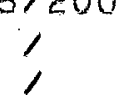

$06 / 109$

$02 / 035$

$08 / 048$

$09 / 072$

$09 / 016$

$11 / 098$

$11 / 111$

'

$11 / 046$

1

.1

,

,

1

$11 / 047$.

$07 / 133$

$07 / 060$

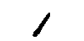

$07 / 06$ ?

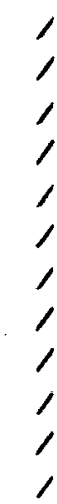

$08 / 017$
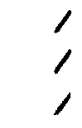

$06 / 179$

$07 / 200$

$07 / 132$

1,132

$04 / 130$

$06 / 147$

$06 / 067$

$06 / 065$

$06 / 067$

$05 / 186$

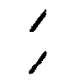

$08 / 203$

$05 / 082$

$06 / 120$

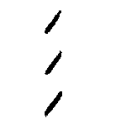

$06 / 202$

$06 / 086$

$08 / 190$<smiles>[TlH]</smiles>

$05 / 229$

$05 / 096$

$07 / 190$

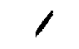

$06 / 155$

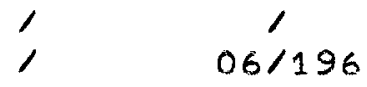

$08 / 201$

$08 / 250$

$08 / 238$

$07 / 018$ 
FLOODING

FLOGR

FLOPPED

FLORIDA

FLEUR

FLOWER

FLOWERS

FLUFFY

FLUSHED

FLUTTER

FLY

FLYER

FLYING

$F \in G$

FQLDED

FOLK

FOLKS

FELLOW

FOLLOWED

FOLLOWINO

FONDER

FOOD

FOOL

FOOLISH

FEUL ISHLY

FOOLISHNESS

FOeT

FEOTBALL

FEQTMEN

FOETSTEPS

FER

FORCE

FURD

FEREIGN

FOREMAN

FIRENEON

FOREST

FOREVER

FERGET

FERGETTING

FORGIVE

FORGET

FORGOTTEN

FORKS

FORMS

FORMULA

FORT

FERTH

FERTUNATELY

FERTUNES

FERTY

FERWARD

FESSIL

FOUGHT

FOUND

FOUNDRY

FOUNTAINS

FOUR

F QUR - LEAF

FOURTEEN

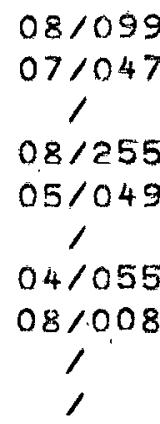

$06 / 085$

1

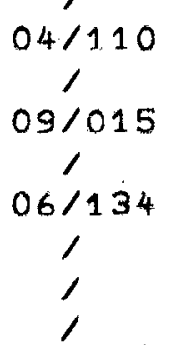

$06 / 062$

,

1

$07 / 099$

1

,

$02 / 052$

,

$08 / 024$

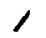

$07 / 067$

1

$04 / 138$

1

,

,

I

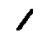

$08 / 139$

1

1

$09 / 042$

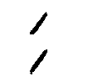

$08 / 242$
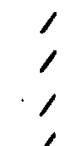

$05 / 108$

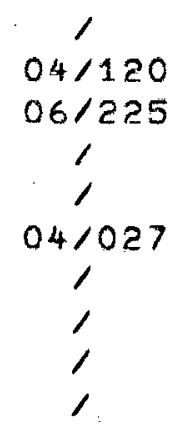

$01 / 127$

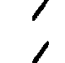

$05 / 194$
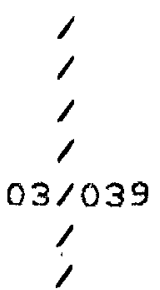

$02 / 142$

$06 / 074$
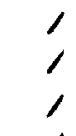

$04 / 055$

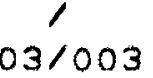

$06 / 227$
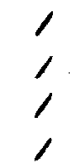

$06 / 030$
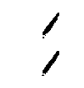

1

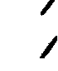

$05 / 273$

$06 / 090$

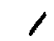

$06 / 081$

$05 / 272$

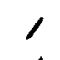

$06 / 082$

$06 / 019$

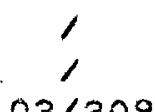

$03 / 209$

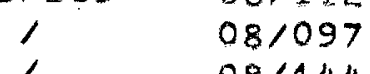

$02 / 061$

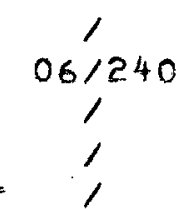

$07 / 222$

$09 / 279$

$07 / 246$

$06 / 138$
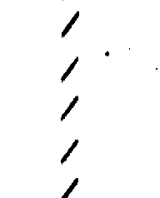

$09 / 054$ 1

$07 / 239$

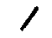

$08 / 034$

$07 / 087$

$08 / 117$

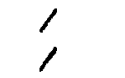

$06 / 029$

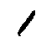

,

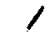

1

$07 / 101$

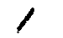

$09 / 271$

$08 / 098$

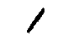

$07 / 167$

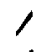

,

,

$$
\text { , }
$$

$06 / 139$

1
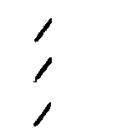

$08 / 221$
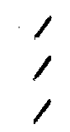

$08 / 026$

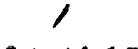

$09 / 165$

$06 / 112$

$08 / 144$

$05 / 024$

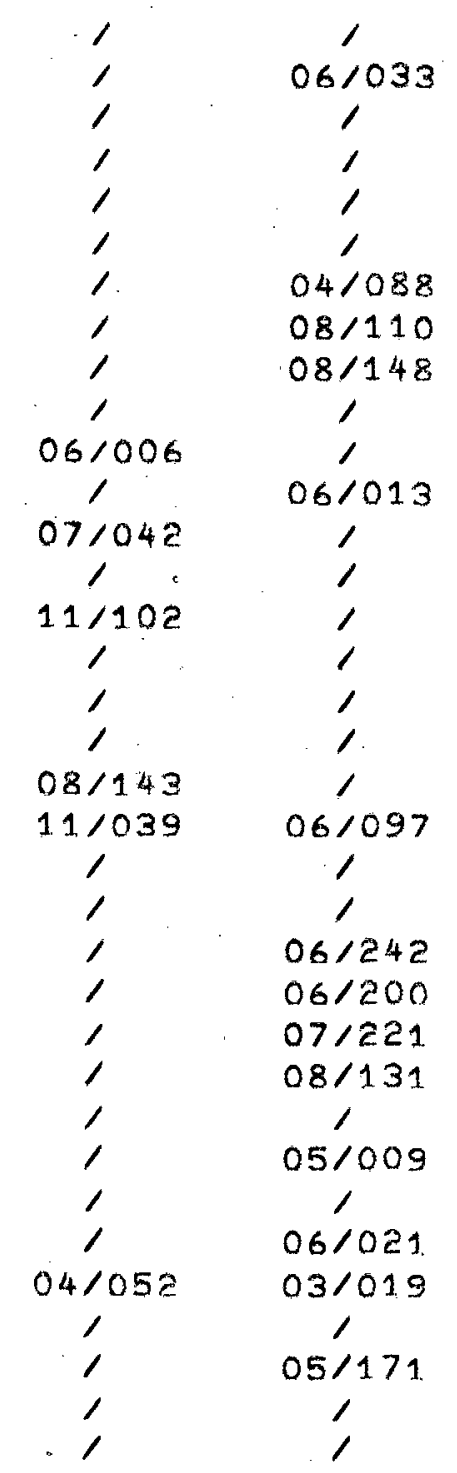

$08 / 085$

$06 / 116$

$09 / 080$

$08 / 170$

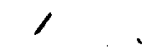

$09 / 082$

$09 / 060$

$11 / 031$
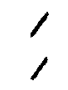

,

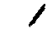

,
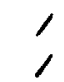

1

$11 / 011$
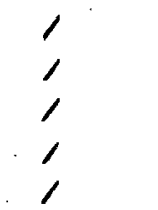

$09 / 016$

$08 / 043$

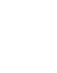

$06 / 061$

$06 / 203$

$06 / 059$

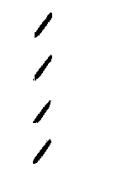

$07 / 217$

$06 / 122$

$08 / 117$

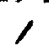

$08 / 031$

$08 / 035$

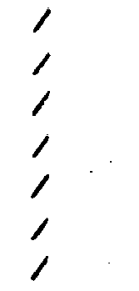

$08 / 019$ 
FOURTH
FEX

FOXES

FOXY

FEXY LEXY

FRAME

FRANCE

FRANK

FRANKLIN

FRECKLES

FREE

FREEDQM

FREEWAY

FREE ZE

FREEZING

FREIGHT

FRE IND

FRESH

FRESHLY

FRIDAY

FRIEND

FRIENDLY

FRIENDS

FRIGHT

FRIGHTEN

FRIGHTENED

FRIGHTENING

FRINGE

FRITZ

FRITZL

FROG

- FROGS

FROM

FRONT

FRONT-PAGE

FREST

FROWN

FROWNED

FRUIT

FRUITS

FULL

FUN

FUNNIEST

FUNNY

FUNNY-LOOKING

FUR

FURNITURE

FURTHER

FURTURE

$G$ WASHINGTON CARVER

GALE

GALLOPING

GAME

GAMES

GANG

GARAGE

GARAGES

GARDEN

GARDENIA

GARZA

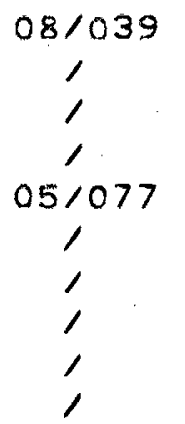

$06 / 133$

$07 / 195$

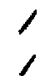

$07 / 203$

$04 / 112$

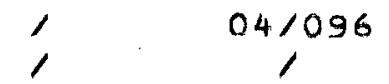

$06 / 087$

1

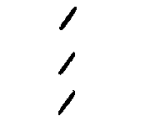

$05 / 031$

1

$04 / 016$

$06 / 148$

,

,

1

$08 / 159$

'

$04 / 033$

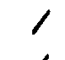

1

$07 / 056$

1

,

1

$06 / 195$

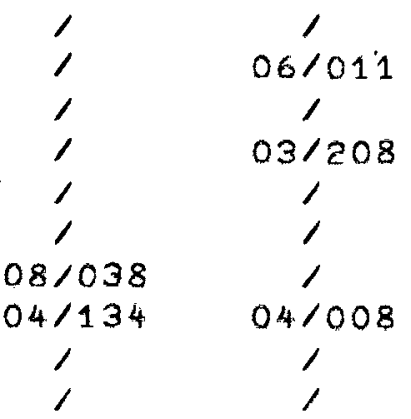

$06 / 017$

$04 / 034$

,

1

$04 / 038$

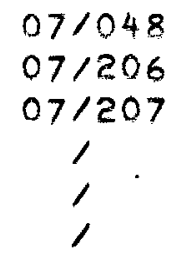

$09 / 095$

$06 / 064$

$06 / 083$

$09 / 166$

1

$09 / 172$

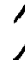

$08 / 099$

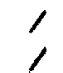

$08 / 131$

1

$05 / 075$

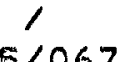

$05 / 067$

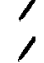

$07 / 095$

$07 / 137$

1

$09 / 268$

$08 / 167$

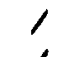

$04 / 127$

$07 / 135$

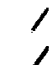

$08 / 134$

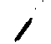

$08 / 233$

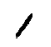

$07 / 077$

$04 / 126$

$01 / 066$

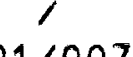

$01 / 007$

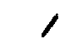

$05 / 010$
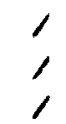

$8 / 120$

$08 / 139$

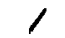

$09 / 153$

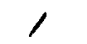

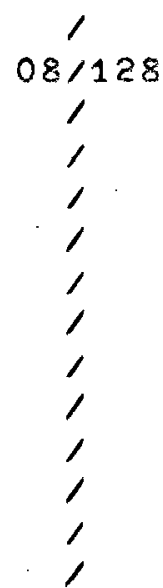

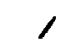

,

,

1

$08 / 185$

$09 / 060$

$08 / 161$
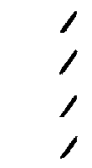

,

1

$07 / 103$

$08 / 102$

$08 / 091$

$08 / 213$

$09 / 058$

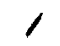

1

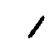

,

,

1

$06 / 017$

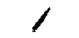

$09 / 010$
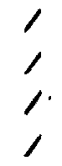

\section{,}
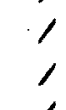

$07 / 035$

$07 / 057$

$09 / 074$

$09 / 024$

$09 / 038$

$11 / 053$
$06 / 259$

$06 / 165$

$06 / 170$

$06 / 167$
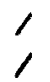

1

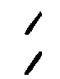

$06 / 115$

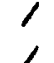

$08 / 247$

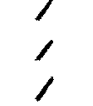

$07 / 135$

$07 / 138$

$06 / 025$

$04 / 110$

$06 / 048$

$07 / 098$

$06 / 197$
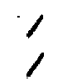

,

1

1

$05 / 124$

$/$

$08 / 111$

$08 / 023$ 1

$06 / 207$

$05 / 166$

$03 / 050$

$06 / 237$

$03 / 055$

$06 / 106$

$08 / 075$

$08 / 122$

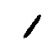

$$
\text { , }
$$$$
1
$$

$06 / 110$

$04 / 097$

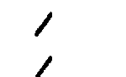

$06 / 157$

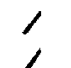

$07 / 047$

$06 / 126$ 
GAS

GASPED

GASTRIC

GATE

GATES

GATHER

GATHERED

GAVE

GAY

GAY'S

GAZE

GAZED

GEESE

GENEVIEVE

GENTLE

GENTLEMEN

GENTLENESS

GEORGE

GERALDINE

GERTRUDE

GESSLER

GET

GET - TEGETHER

GETS

GET TING

GHEST

GIANT

GIANTS

GIFT

GI GGLE

GILES

GINGERBREAD

GIRAFFE

GIRL

GIRL'S

GIRLS

GIVE

GIVEN

GIVES

GIVING

GLAD

GLADDENED

GLADLY

GLANCED

GLARE

GLASS

GLASSES

GLEN DEE

GLITTERING

GLOBE

GLEOMILY

GLEEMY

GLORY

GLOSSARY

GLUVE

GLOVES

GNOMES

GO

GEAT

GEATS

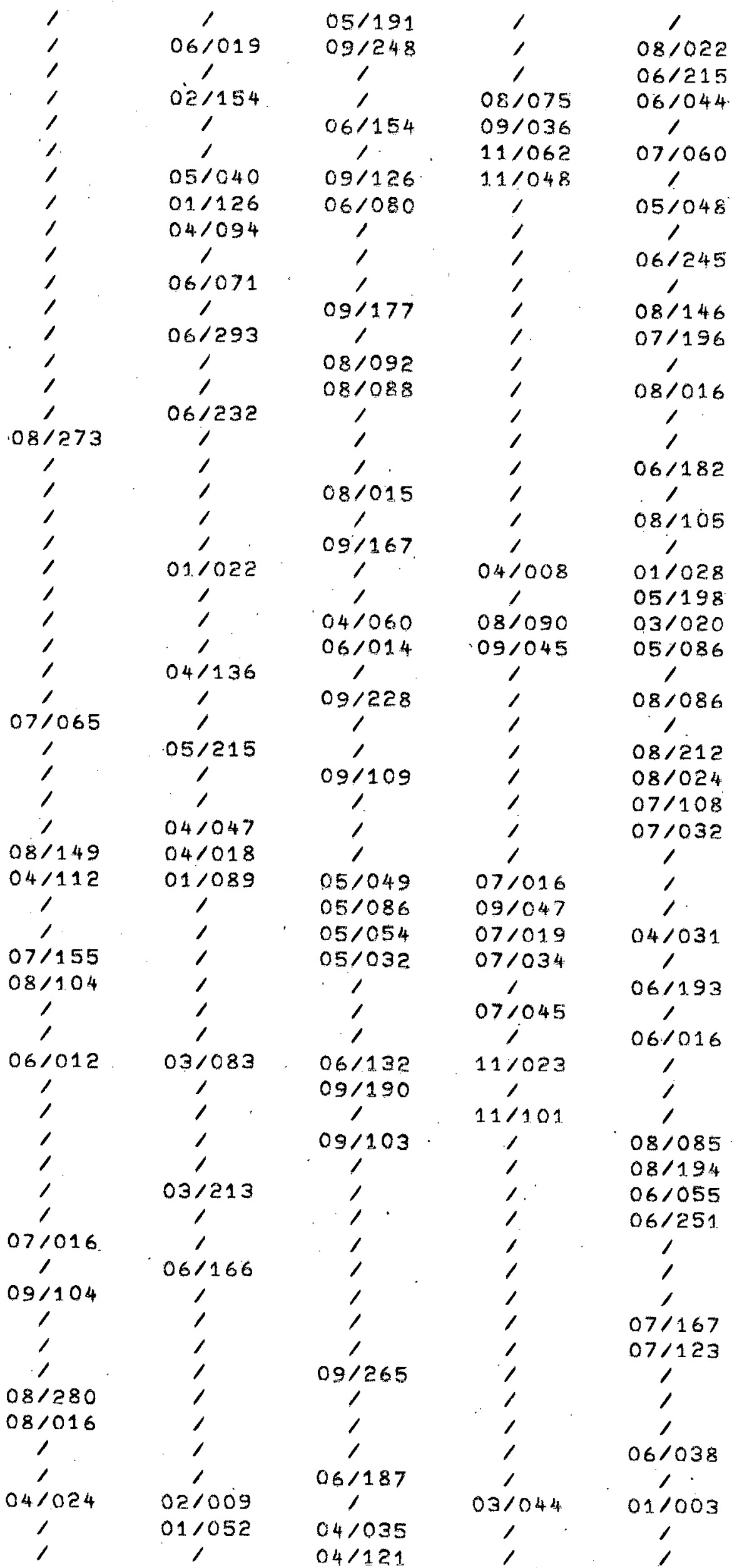


GOATSKIN

GABBLE

GOBLINS

GOD'S

GODMOTHER

GEES

GOING

GOLD

GQLDEN

GOMEZ

GONE

GOEBERS

GOQD

$G Q E D=B Y$

GOOD-BYE

GQED-LUCK

GOOD-TO-EAT

GOODNESS

GEODS

GOODY

GOOSE

GESPEL

GQT

GEURDFUL

GEVERNOR

GOWNS

GRAB

GRABBED

GRACEFUL

GRADUALLY

GRAIN

GRAND

GRANDER

GRANDFATHER

GRANDFATHER'S

GRANDFATHERS

GRANDMA

GRANDMETHER

GRANDMOTHER'S

GRANNY

GRANT

GRAPE

GRASP

GRASS

GRASSHGPPER

GRASSY

GRATEFUL

GRATEFULLY

GRAVES

GRAY

GRAYLEGS

GREAT

GREAT -GRANDFATHERS

GREAT-GREAT-GRANDFAT

GREATEST

GREATHOUSE

GREATLY

GREEN

GREENF IELD

GREENHOUSE

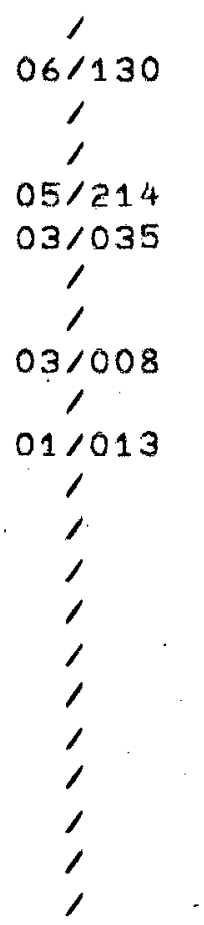

$08 / 016$

$06 / 188$

$01 / 009$

1

$06 / 209$

,

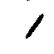

1

I

1

$02 / 026$

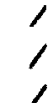

,

,

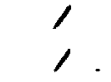

$09 / 059$

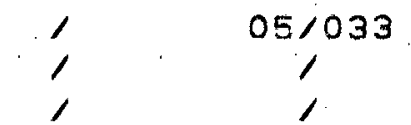

$04 / 109$

'

$06 / 056$

$05 / 112$

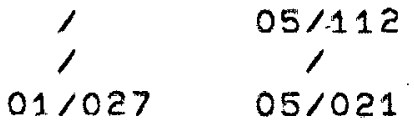

0.1037

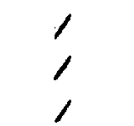

$09 / 142$

$05 / 0.98$

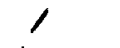

$08 / 077$
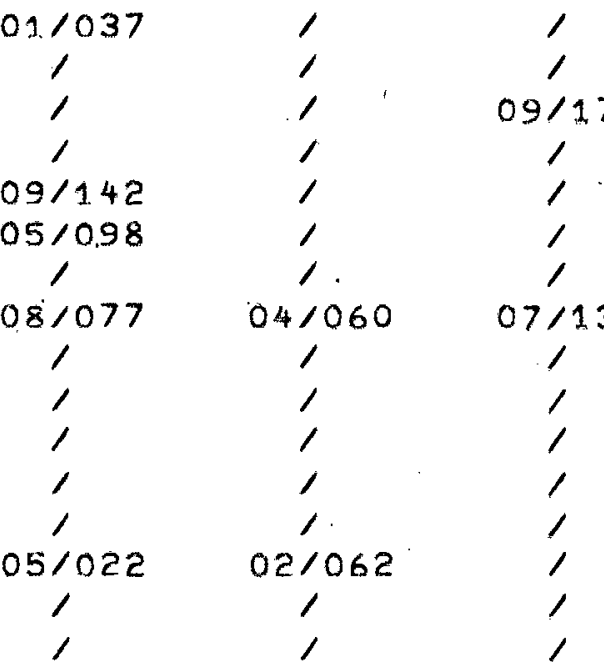

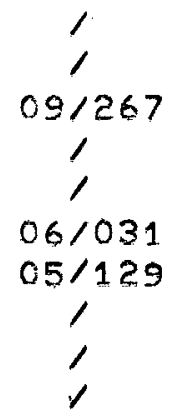

1

,

1

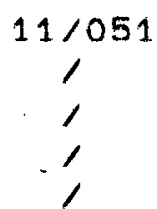

$09 / 075$

$05 / 013$

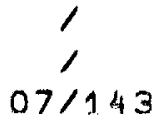

$06 / 132$

041025

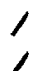

,

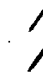

$06 / 147$

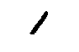

1

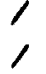

$05 / 026$

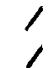

$08 / 208$
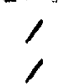

1

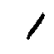

1

$06 / 155$

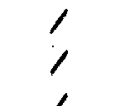

$0.6 / 211$

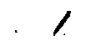

.1

$07 / 235$

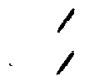

$08 / 231$

$05 / 154$

$09 / 172$

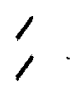

I

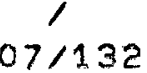

$07 / 132$

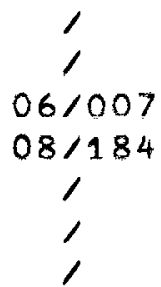

1

$11 / 093$

$09 / 074$

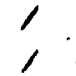

$09 / 081$
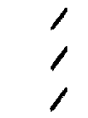

,

1

1

1

$09 / 080$

$09 / 029$

$11 / 046$

$11 / 025$

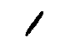

$07 / 021$

$09 / 048$
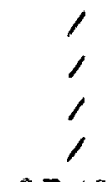

$07 / 089$
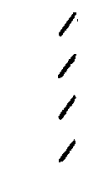

$11 / 065$

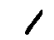

$09 / 027$

$11 / 025$

$11 / 025$

$11 / 092$

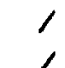

$06 / 020$

$11 / 028$
$06 / 211$

$05 / 147$

1

$06 / 008$

$08 / 157$

1

$05 / 183$

$04 / 021$.

1

$06 / 073$

$06 / 017$

$08 / 061$

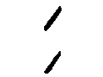

$07 / 221$

$08 / 106$

$04 / 095$

$08 / 142$

$07 / 106$

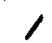

$06 / 120$

$06 / 117$

$08 / 170$

$07 / 116$

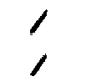

$04 / 103$
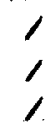

$04 / 112$

$08 / 045$

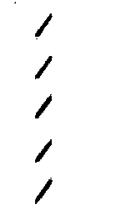

$05 / 181$

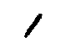

$08 / 150$
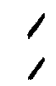

$06 / 130$

$06 / 189$

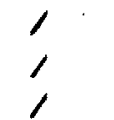

$06 / 195$

$07 / 125$

$05 / 051$ 
GREG

GREW

GRIMM

GRIN

GRIND

GR INNED

GRINNING

GRUANED

GROCERIES

GRECERY

GROUCH

GRUUND

GREUP

GROUPS

GREVES

GROW

GREWING

GREWL

GROWLING

GROWN

GRQWN-UPS

GROWS

GRUMBLED

GRUMPY

GRUNTED

GUARDS

CUES

GUESS

GUESTS

GUINEA PIGS

GUITAR

GUN

GUNPOWDER

GUNS

GUPPIES

HA

HABIT

HABITAT

HAD

HADN'T

HAIR

HAITI

HALF

HALFWAY

HALL

HAMBURGER

HAMEURGERS

HAMILTON

HAMMER

HAND

HANDED

HANDFUL

HANDFULS

HANDING

HANOKERCHIEF

HANOLE

HANDS

HANDSEME

HANDWERK

HANDY

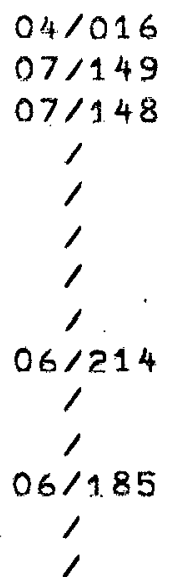

$08 / 259$

$06 / 172$

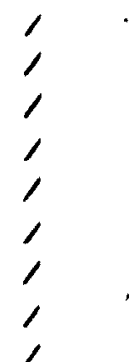

$07 / 133$

1

$07 / 167$

$09 / 086$

$08 / 153$

$09 / 198$

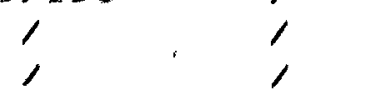

$06 / 077$

,

,

1

$09 / 247$

$02 / 017$

$$
1
$$

$07 / 033$

$09 / 121$

$09 / 125$

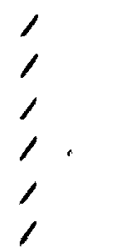

$05 / 033$

,

,

,

$08 / 059$

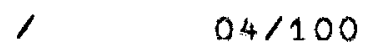

$05 / 182$

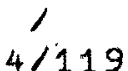

$02 / 134$

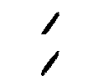

$05 / 216$

$01 / 143$

$04 / 049$

$05 / 148$

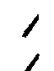

,

,

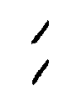

$03 / 119$

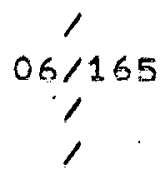

$06 / 188$

$07 / 099$

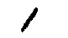

$09 / 186$

',

$09 / 114$

1

$07 / 114$

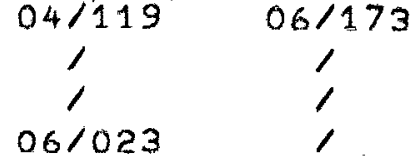

$06 / 181$

,

,

$09 / 026$

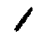

$09 / 195$

$08 / 230$

$08 / 024$

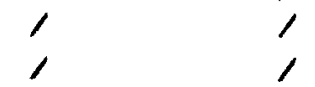

$08 / 017$

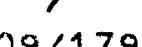

$07 / 154$
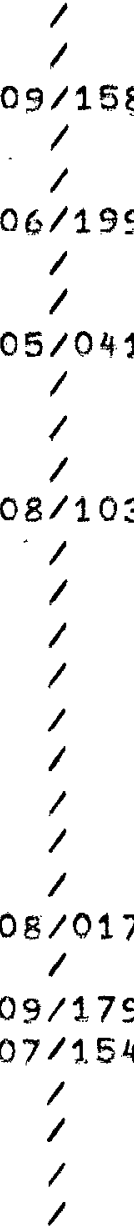

$09 / 158$

$06 / 199$

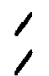

$05 / 041$

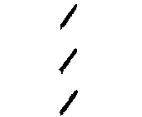

$08 / 103$
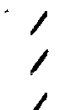

1
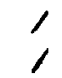

,

'

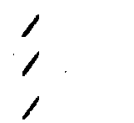

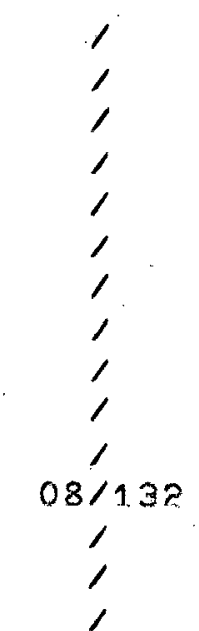

,
$08 / 159$

$09 / 066$

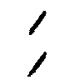

$11 / 040$

$11 / 060$

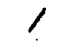

,

,

',

$11 / 026$

1

1

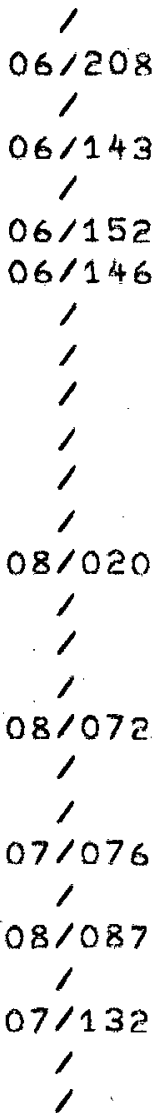

$03 / 049$

$07 / 144$
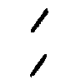

$08 / 074$

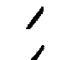

$08 / 246$

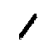

,

$04 / 106$

$06 / 176$

$07 / 011$

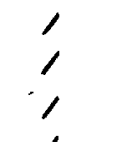

$07 / 071$

$07 / 144$

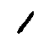

1

$08 / 075$

$09 / 057$

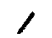

1
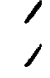

1

$08 / 098$

$11 / 101$

11/014

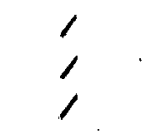

$08 / 032$

$07 / 173$

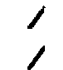

$08 / 105$

$08 / 228$

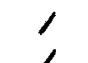

$07 / 036$

$05 / 115$

$06 / 060$

$07 / 012$

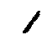


HANG

HANG ING

HANS

HAP

HAP'S

HAPPEN

HAPPENED

HAPPENING

HAPPILY

HAPPINESS

HAPPY

HARBOR

HARBERS

HARD

HARD = EARNED

HARDLY

HARDWGRKING

HARE

HARK

HARM

HARNESS

HARNESSED

HARRIS

HAS

HASN T T

HASTILY

HAT

HATBEX

HATCH

HATE

HATED

HAUS

HAVA

HAVE

HAVEN'T

HAVING

HAWAII

HAWK

HAY

HAYDEN

HE

HE $1 D$

HE'LL

HE'S

HEAD

HEADING

HEALTH

HEALTHIER

HEALTHIEST

HEALTHY

HEALTHY = LOEKING

HEAR

HEARD

HEARING

HEART

HEARTIES

HEARTS

HEAT

HEAVED

HEAVEN
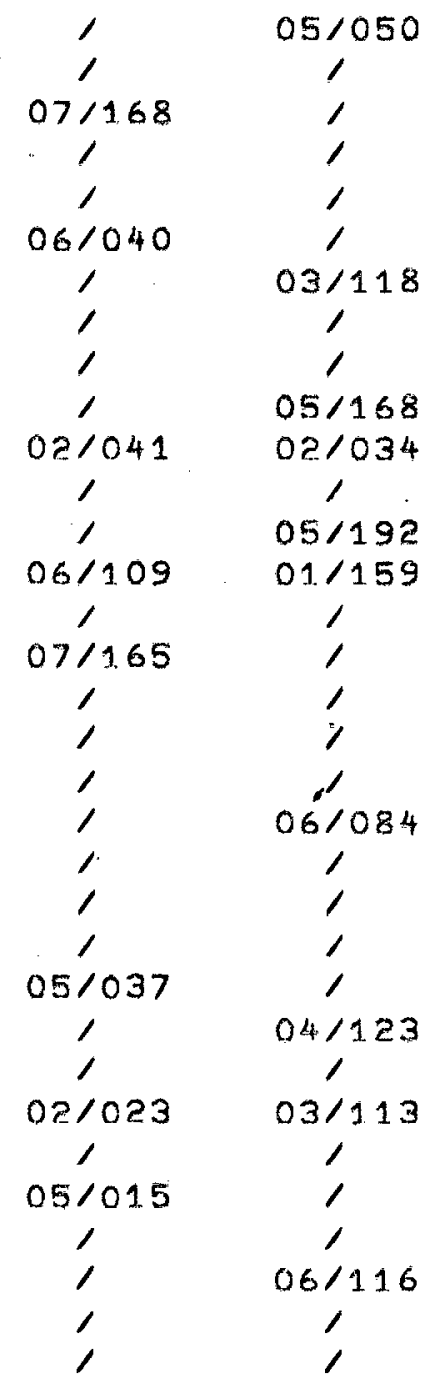

$05 / 117$

1

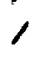

,

1

1

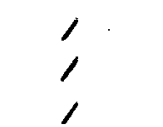

$07 / 104$

$06 / 122$

1

,

1

1

1

$07 / 075$

$07 / 138$

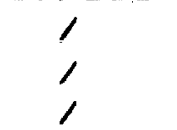

$08 / 153$

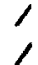

$09 / 052$

,

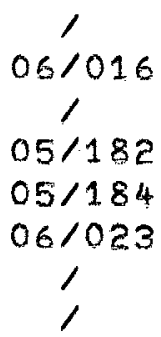

$08 / 184$

$06 / 184$

$05 / 066$

$09 / 234$

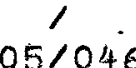

1046

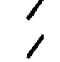

1
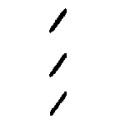

$08 / 236$

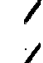

$04 / 022$

$04 / 123$

1

$03 / 113$

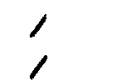

$06 / 116$

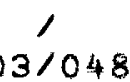

$03 / 048$

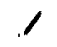

1

$05 / 152$

1

$01 / 053$

1

1

$01 / 008$
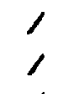

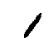
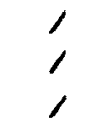

$01 / 068$

$02 / 121$
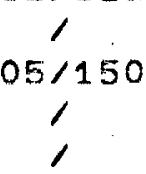

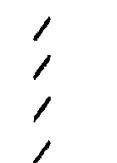

$06 / 176$

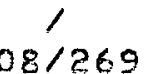

$08 / 135$

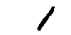

$04 / 128$

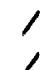

$09 / 259$
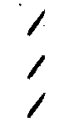

$07 / 217$

$07 / 095$

$06 / 065$

$06 / 183$
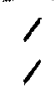

1

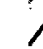

1
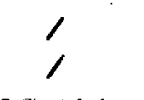

$05 / 143$

$08 / 061$

$03 / 234$

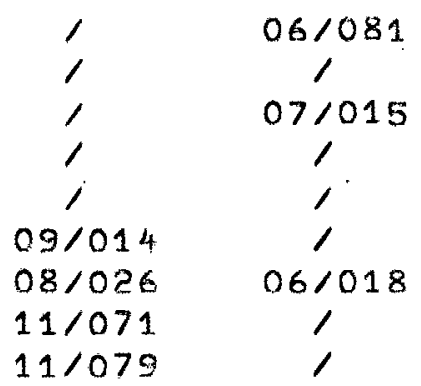

$08 / 135$

$04 / 074$

$07 / 106$

$05 / 079$

1

$06 / 181$

$08 / 030$

1

$08 / 013$

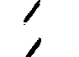

$05 / 186$

$\gamma$

$06 / 035$

$08 / 022$

$04 / 007$

$06 / 039$
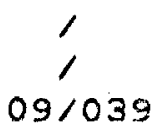

$09 / 039$

1

$04 / 016$

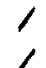

,

,

,

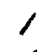

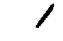

$05 / 030$

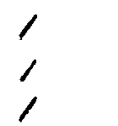

$08 / 190$
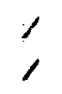

1

,

1

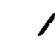

$08 / 008$

$11 / 067$

1

1

1

I

1

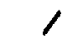

$08 / 051$

$08 / 1.05$

$03 / 047$

$06 / 186$

$06 / 106$

$08 / 079$

$08 / 201$

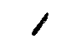

$05 / 167$

$04 / 062$

$06 / 089$

$05 / 120$

$05 / 157$

$06 / 245$

$06 / 207$

$06 / 192$

$06 / 192$

$06 / 256$

$05 / 088$

$05 / 098$

$05 / 093$

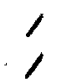

$06 / 186$

$08 / 159$

$08 / 097$ 
HEAVENS

HEAVIER

HEAVILY

HEAVY

HEE - HAW

HEELS

HEIGHT

HEINZ

HELD

HEL I CUPTER

HELIPERT

HELIUM

HELLO

HELP

HELPFUL

HELPING

HELPLESS

HELPLESSLY

HELPS

HEN

HENERY'S

HENNY PENNY

HENRI

HENRY:S

HENS

HER

HERD

HERDS

HERE

HERE'S

HEREAFTER

HERO

HEROES

HERR

HERR ING

HERSELF

HERSHEY

HES I TATED

HEY

$H I$

$H I=Y I-Y I I P P I-Y I$

HIBERNATE

HICCUPS

HID

HIDDEN

HIDE

HIGH

HIGHER

HIGHNESS

HIGHWAY

HILDE'S

HILL

HILLER

HILLS

HILLSIDE

HILLSIDES

HILLTEP

HILLY

HIM

HIMSELF

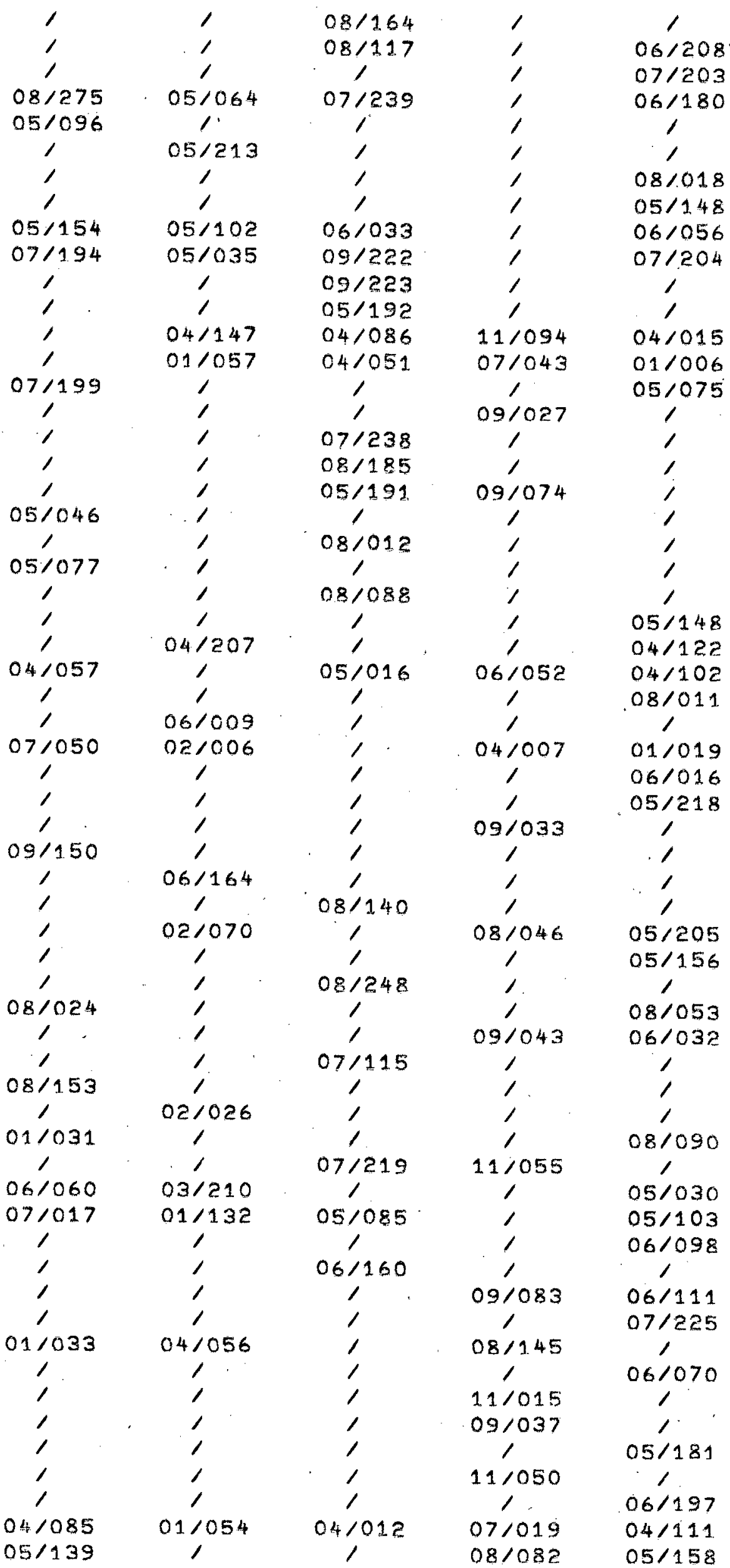


HIND

HIPPGPOTAMUS

HIS

HISPANIOLA

HISTARY

HIT

HITCHED

HITTING

HO

HOBBLE

HOBBY

HOE

HOGAN

HEGBACK

HELD

HOLDING

HOLE

HOLES

HOL IDAY

HOL IDAYS

HOLLOW

HOLLY

HOLMES

HOME

HOMEMADE

HOMEWARD

HONEY

HONK

HENGR

HONERABLE

HONORS

HOOFS

Heek

HOQKED

HOOP

HQP

HOPE

HOPED

HOPEFUL

HOPING

HOPKINS

HOPPED

HOPPING

HORN

HURNETS

HORNS

HORR IBLE

HORSE

HORSES

HESE

HOSPITAL

HET

HETEL

HOTTER

HOUND

HEUND-PUP

HQUR

HEURS

HOUSE

HOUSEKEEPER

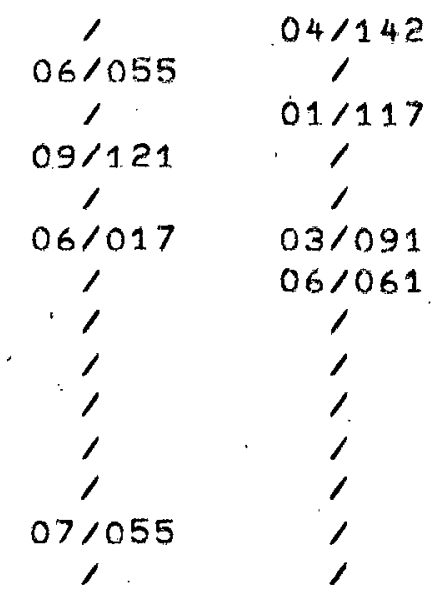

$07 / 025 \quad 01 / 132$

$01 / 137$

$04 / 010$.

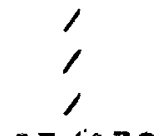

$07 / 079$

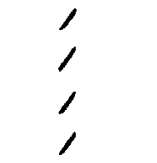

$05 / 135$
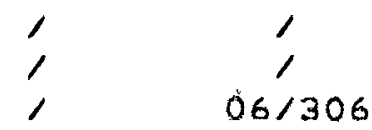

1

1

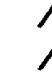

,

. 1

1

$01 / 038$

1

$06 / 054$

1
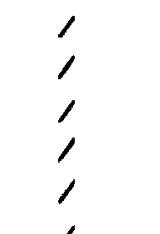

$07 / 070$

$07 / 098$

$07 / 054$

1

1

$05 / 068$
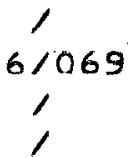

1

'

1

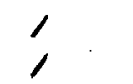

,

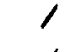

1

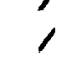

$06 / 306$

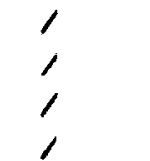

$06 / 287$

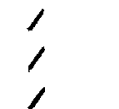

$01 / 067$

$03 / 046$

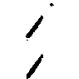

1

'

,

$06 / 040$

$05 / 194$

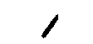

$02 / 038$

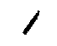

,

$02 / 008$

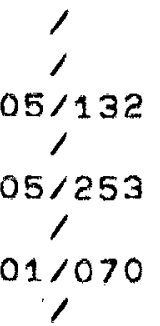

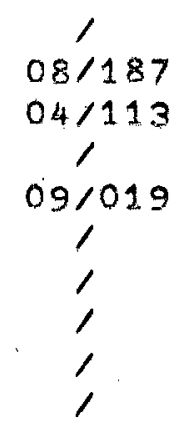

$06 / 096$
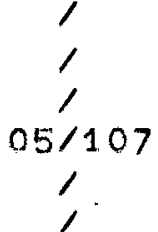

$06 / 018$

$08 / 069$

$05 / 208$

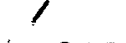

$08 / 217$

$08 / 100$

$05 / 129$

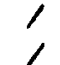

$07 / 029$

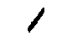

$09 / 226$

$06 / 166$

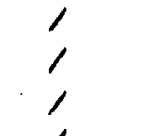

$07 / 072$

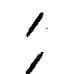

$07 / 097$

$08 / 020$

$08 / 031$
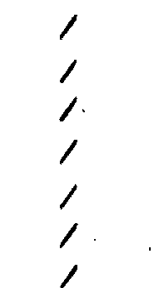

$05 / 134$

$05 / 138$
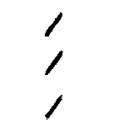

$07 / 063$

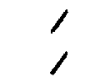

$06 / 039$

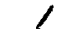

$07 / 224$

$04 / 151$

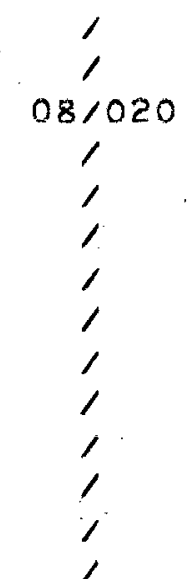

$11 / 073$

$11 / 017$

$08 / 149$

$11 / 090$

1

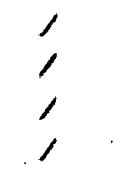

$05 / 024$

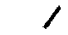

$11 / 024$

1

,

,

,

'

,

,

1

$07 / 085$

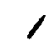

$11 / 099$
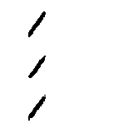

$11 / 022$

,

,

,

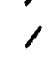

,

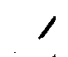

1

$08 / 189$
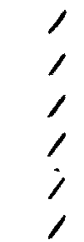

$08 / 045$

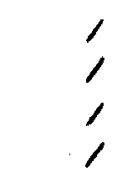

$07 / 154$

$06 / 141$

$06 / 219$

$07 / 105$

$07 / 142$

$08 / 110$

$08 / 029$

$06 / 108$

1

$06 / 046$

1

$08 / 025$

$08 / 255$

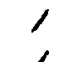

$04 / 052$

$06 / 230$

$06 / 228$

$07 / 061$

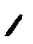

$08 / 087$

$08 / 135$

$05 / 064$

$05 / 067$

1

$04 / 144$ 1

$06 / 068$

$06 / 068$

$07 / 107$

$06 / 045$

$06 / 101$

$07 / 009$

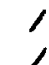

$04 / 046$

$06 / 052$

$06 / 188$

$05 / 175$

$08 / 052$

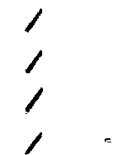

$07 / 101$

$03 / 022$

$06 / 199$ 
HOUSES

HOUSETOPS

HOVER

HOW

HOW'S

HEWEVER

HOWL

HEZAK

HUB

HUE

HUFFMAN

HUG

HUGE

HUGGED

HUGGINS

HUI

HULLA

HUMAN

HUMMING

HUMMINGB I RD

HUNDRED

HUNDREDS

HUNG

HUNGRILY

HUNGRY

HUNT

HUNTED

HUNT ING

HURDY - GURDY

HURRAH

HURRICANE

HURR IED

HURRY

HURT

HUSBAND

HUSTLING

HUT

HYACINTH

HYDREFOIL

$I$

I. D

$I \cdot L L$

$I, M$

I V VE

ICE

ICE CREAM

I CEBERG

IDEA

IF

I LLS

IMAGINATIEN

IMAGINE

IMMED I ATELY

IMPAT IENT

IMPATIENTLY

IMPELITELY

IMPERTANT

IMPESSIBLE

IN

INCH

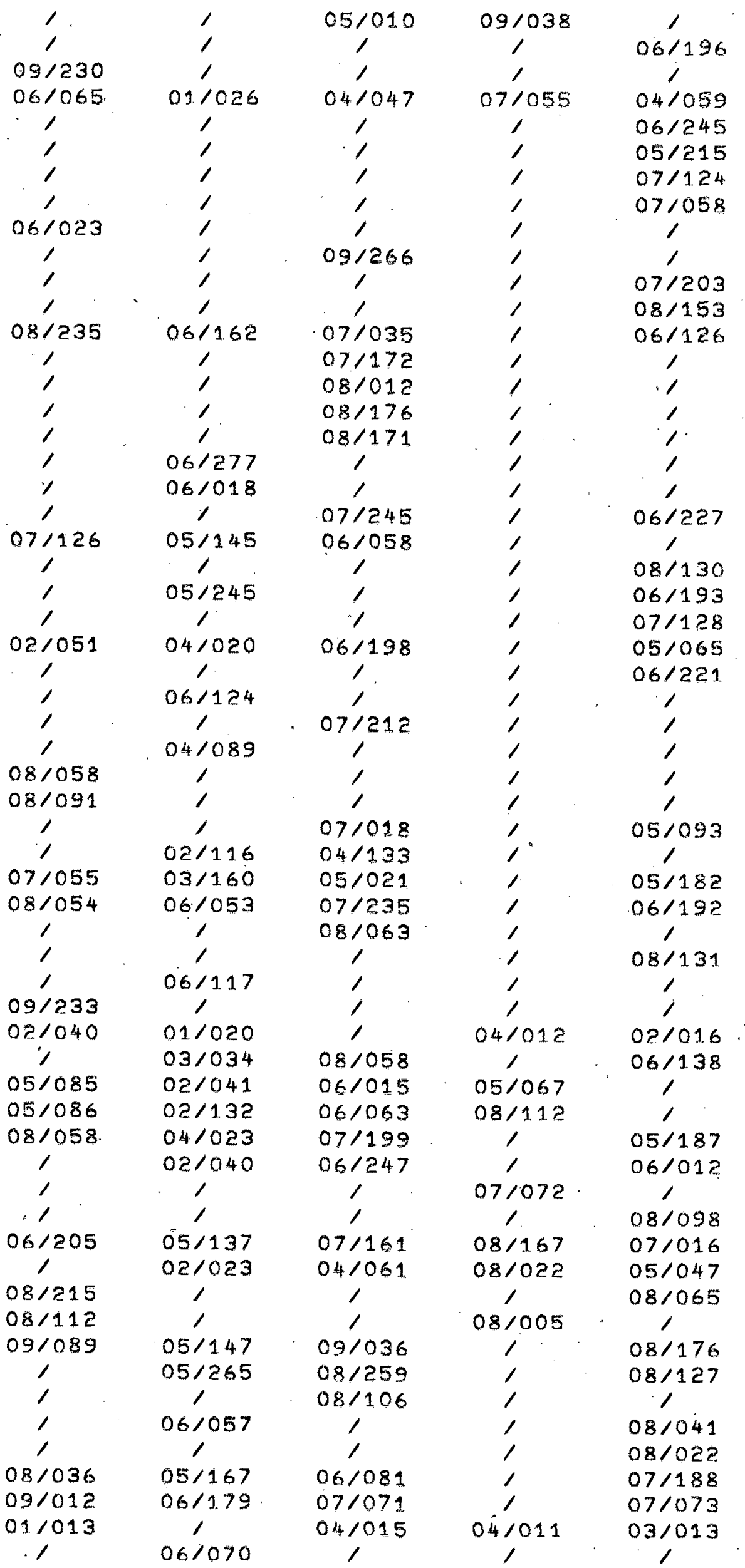


WORD LIST

INCHES

INDEED

INDEX

INDIA

INDIAN

INDIANS

INFERMATION

INGRID

INN

INNING

INOU IRED

INSECTS

INSIDE

INSISTED

INSTANTLY

INSTEAD

INTELLIGENCE

INTEND

INTEREST

INTERESTED

INTEREST ING

INTERNATI UNAL

INTERRUPTED

INTE

INTRODUCED

INTRODUCING

INVENTED

INVENTIEN

INVENTIONS

INVENTUR

INVESTMENT

INVISIBLE

INVITE

INVITED

INWARD

IRENE

IREN

IRONWOOD

IS

ISABEL

I.SABELLA

ISLAND

ISLANDS

ISN'T

IT

IT'S

ITALY

ITEMS

ITS

ITSELF

IVERGON IS

JACK

JACKDAW

JACKET

$J A C K S O N$

JAGGED

JAIL

JAM

JAMES

JAN
SERIES A SERIES B SERIES C SERIES D SERIES E

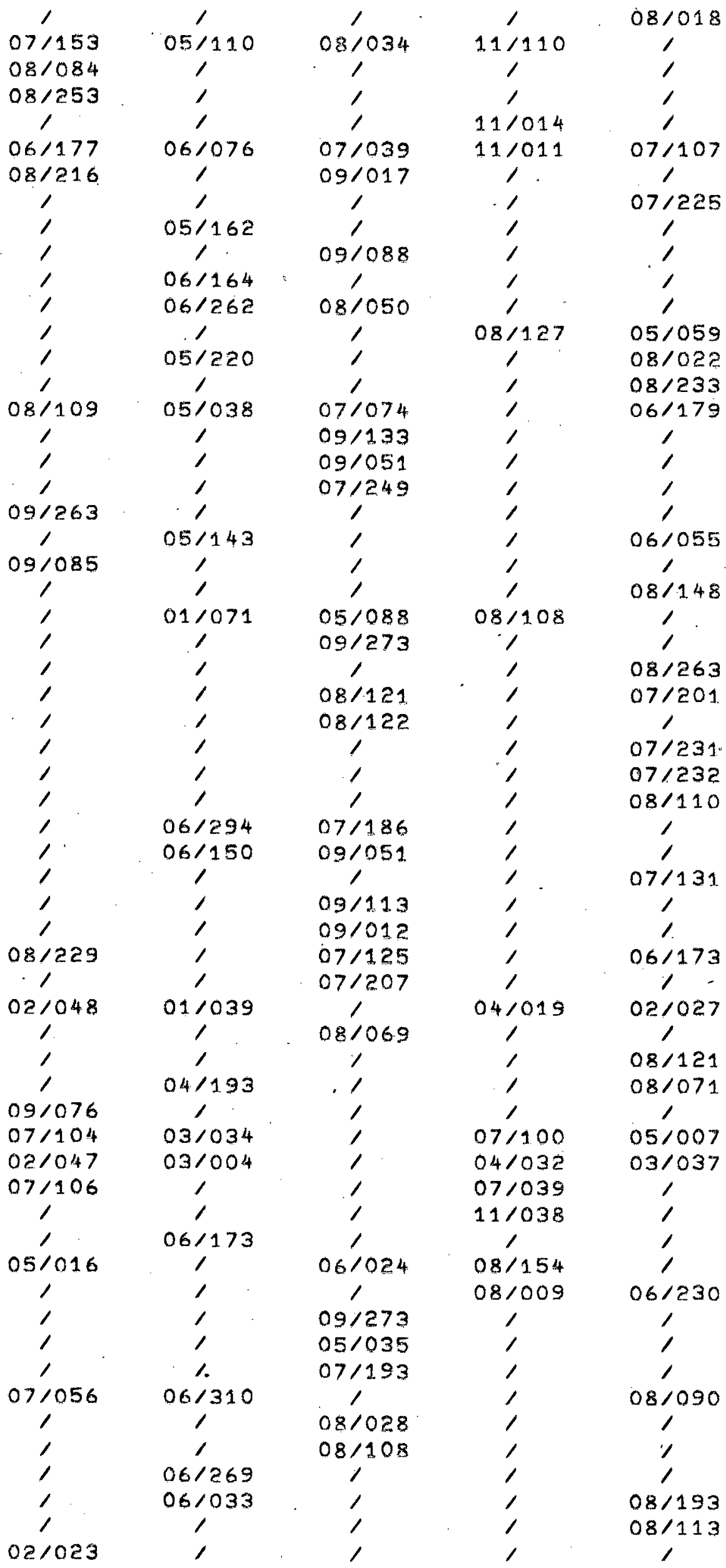


JANE

JANET

JANET'S

JANIE

JAPAN

JAR

JARS

JAWS

JAY

JEALOUS

JEAN

JEFF

JELLY

JENN IFER

JENNY

JERK

JEROME

JERRY

JESUS

JEWELS

$J I G$

JIGGLED

$J I L L$

JIM

JIMMY

JINGLED

$J O B$

JUE

JUHANN

$J O H N$

JUHN:S

JOHNNY

JOHNNY APPLESEED

JUHNSTON

JOINED

JUKE

JUKES

JOKING

JULLY

JONATHAN

JUNES

JESEPH

JOY

JOYFULLY

JUD

JUDGE

JUDGES

JUDGING

JUDGMENT

JUGGLE

JUICE

JUICIER

JUICY

JUL I ANA

JUMBO

JUMP

JUMPED

JUMPING

JUMPS

JUNE

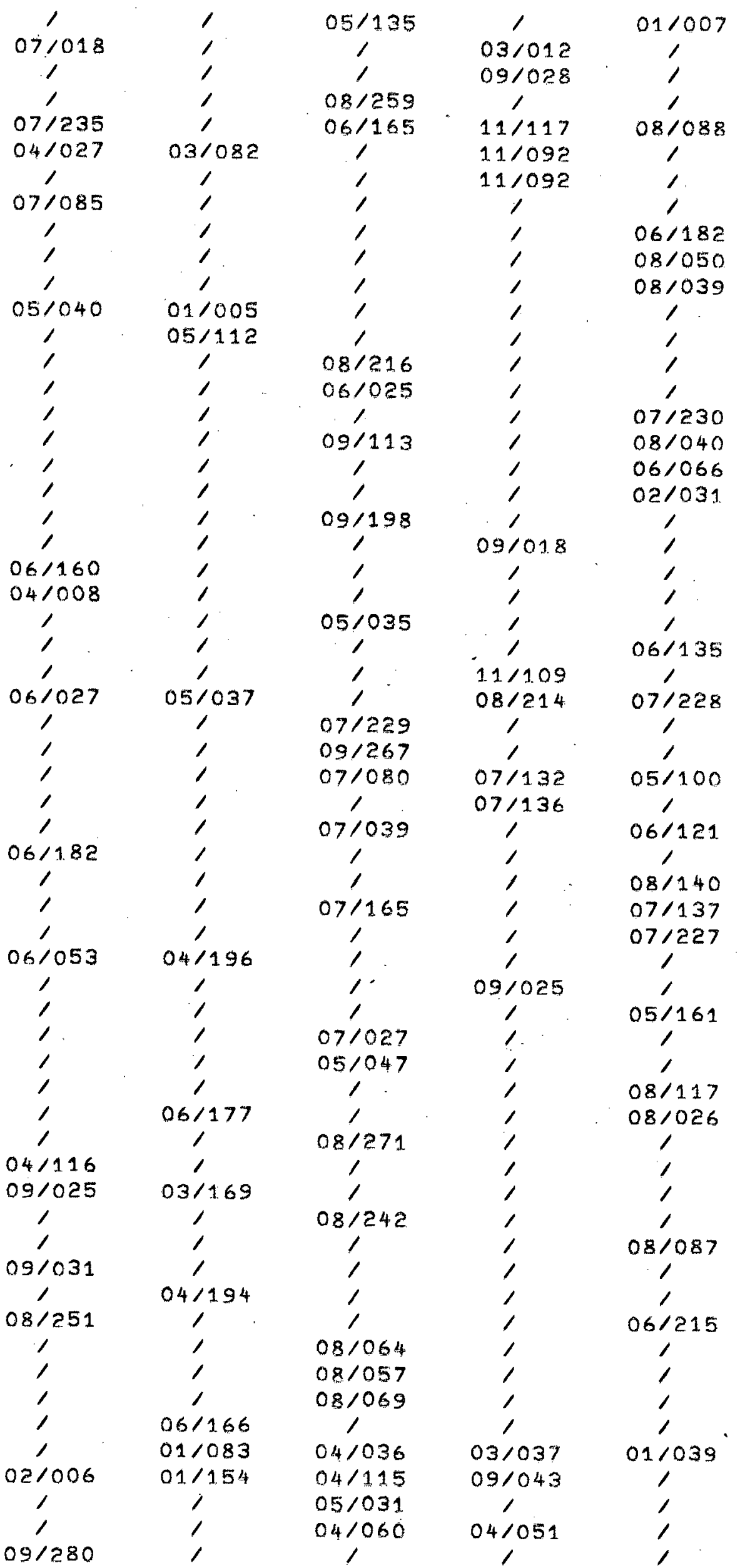


JUNEAU

JUNGLE

JUST

KAKAI

KALLER

KANGAROE

KATALIN

KATHY

KATRINA

KATYDIDS

KAYAK

KEEN

KEEP

KEEPER

KEEPING

KEEPS

KENNELS

KEPT

KETTLE

KEVIN

KEY

KICKED

KIDS

KILOEE

KILL

KILLER

KILLS

KILPATRICK

KIM

KIMIS

KIMMY

KIND

KINDLY

KINDNESS

KING

KINGDEM

KINNDLI

KIRSTEN

KITCHEN

KITE

KITES

KITTEN

KITTENS

KITTY

KNEES

KNEW

KNIFE

KNIGHT

KNITTING

KNGB

KNECK

KNOCKED

KNOW

KNOWING

KNOWINGLY

KNEWN

KNOWS

$\mathrm{KOBO}$

KEDA

KRAFT

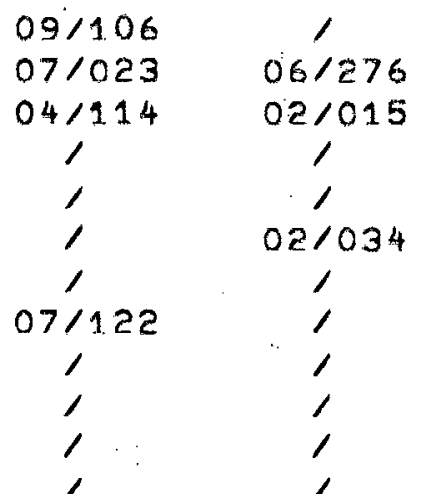

$06 / 132 \quad 03 / 167$

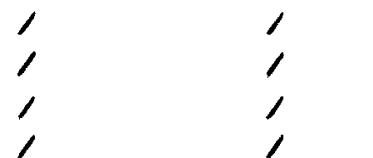

/ $02 / 092$

$02 / 009$

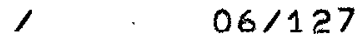

, $\quad 06 / 179$

$02 / 009$
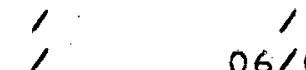

$07 / 092$

1

1

$04 / 112$

1

1

$06 / 120$

$07 / 165$

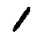

$05 / 074$

$09 / 048$

I

$5^{\prime} / 094$

1

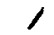

$06 / 076$

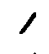

1

$07 / 067$

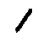

$07 / 071$

,
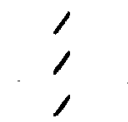

$07 / 066$

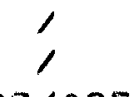

$07 / 095$
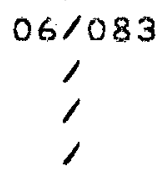

1

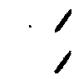

$03 / 195$

,

$05 / 246$

1

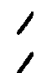

$05 / 078$

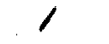

$01 / 121$

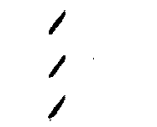

$06 / 136$

$03 / 168$

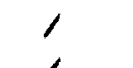

$06 / 053$

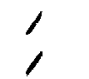

$04 / 121$

$01 / 072$

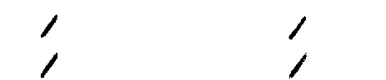

$06 / 210$

$04 / 060$
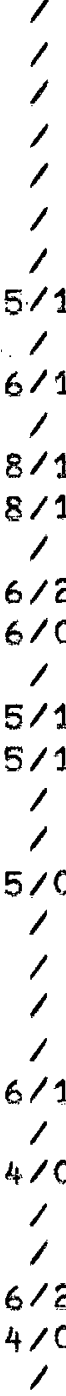

,

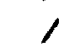

$05 / 186$

$06 / 197$

$08 / 154$

$08 / 167$

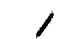

$06 / 238$

$06 / 089$

$05 / 165$

$05 / 166$

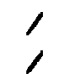

$05 / 074$

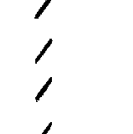

$06 / 144$

1

$04 / 028$

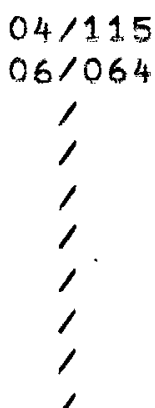

$06 / 031$

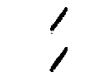

$07 / 185$

$06 / 118$

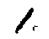

$08 / 217$

$06 / 092$.

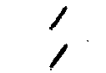

$09 / 113$

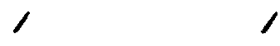

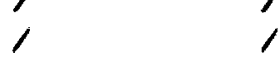

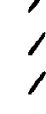

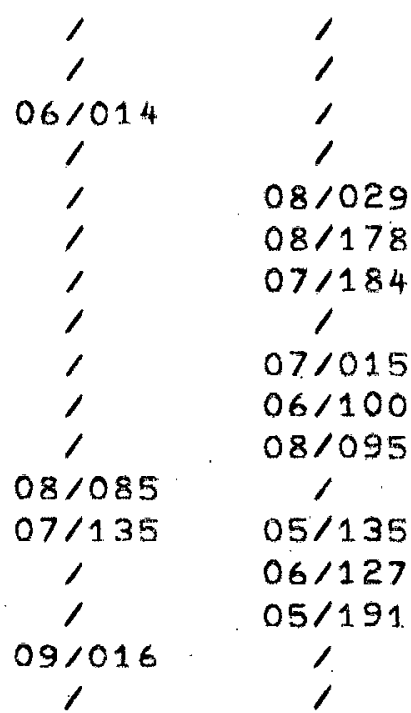

$06 / 112$

$08 / 144$

$07 / 196$

$06 / 075$

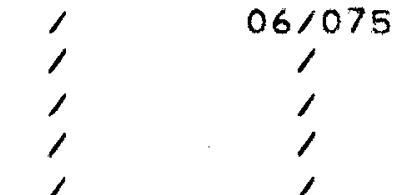

$06 / 225$

$07 / 042$

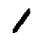

$07 / 132$

$08 / 180$

$08 / 191$

$05 / 076$

$07 / 035$

$11 / 115$

,

,

,

,

$07 / 134$

,

,

'

$08 / 205$
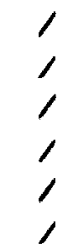

$07 / 151$

$11 / 058$

$11 / 070$

$09 / 075$

$07 / 149$

$11 / 117$

',

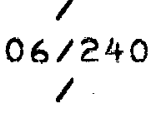

$08 / 020$

$06 / 014$

$05 / 082$

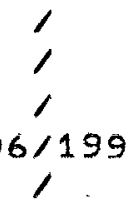

$05 / 178$

$07 / 147$

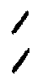

$07 / 186$

$06 / 195$

1

$04 / 055$

$05 / 157$

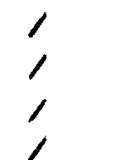

$05 / 081$

$05 / 163$ 
LABERATERY

LACEUR

LAD

LADDER

LADY

LAID

LAKE

LAKES

LAMB

LAMBS

LAME

LAMP

LAND

LANDED

LANDING

LANDLERD

LANES

LANGFORD

LANGUAGE

LANGUAGES

LAN I

LANKY

LANTERN

LARGE

LARGER

LARK

LARS

LARSEN

LAST

LATCHED

LATE

LATER

LATHERED

LATIN

LAUGH

LAUGHED

LAUGHING

LAUGHS

LAUGHTER

LAWN

LAY

LAZIER

LAZY

LEAD

LEADER

LEADING

LEADS

LEAFY

LEAKING

LEANED

LEANING

LEAP

LEAPED

LEARN

LEARNED

LEARNING

LEAST

LEATHER

LEAVE

LEAVES

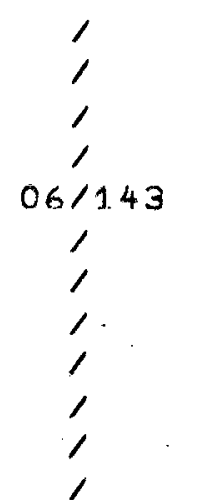

$05 / 017$
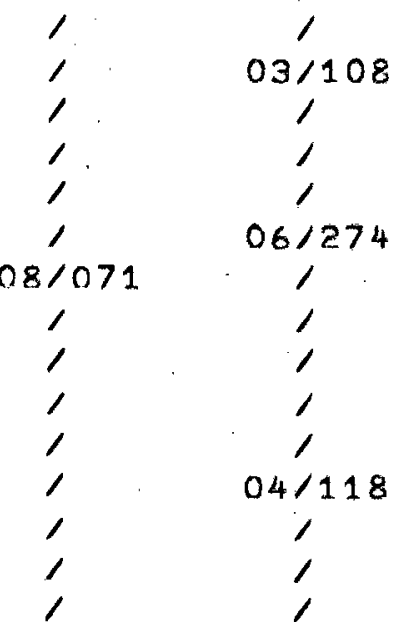

$04 / 039$

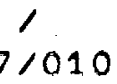

1

,

I

$6 / 147$

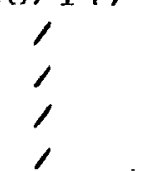

$05 / 038$

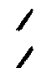

$06 / 106$
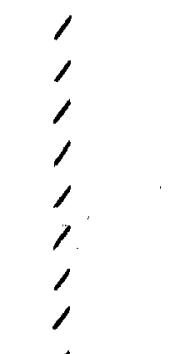

$06 / 065$

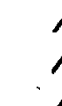

$07 / 174$

. 1

$06 / 068$

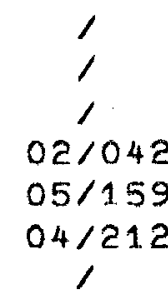

$05 / 191$

$03 / 089$

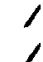

$05 / 192$

$06 / 274$

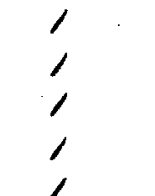

$04 / 118$

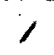

1

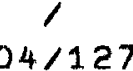

$06 / 117$

$04 / 205$

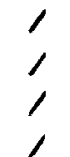

$02 / 024$

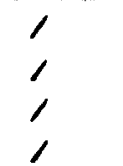

$05 / 152$

$06 / 240$

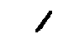

$05 / 232$
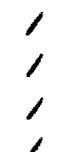

$05 / 144$

$05 / 107$

1

$03 / 066$

$05 / 145$

$05 / 215$

$04 / 099$

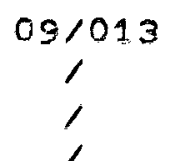

$08 / 014$

$08 / 051$
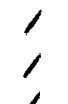

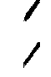

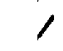

,
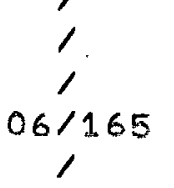

$07 / 109$

$07 / 083$

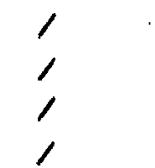

$07 / 202$
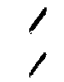

$09 / 222$

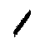

$05 / 071$

1

1

$06 / 247$

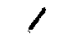

$09 / 266$

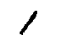

$04 / 035$

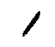

1

1

1

1

$07 / 127$

$08 / 029$

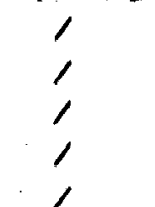

$07 / 241$

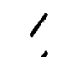

$07 / 182$

$06 / 110$
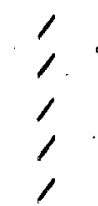

$07 / 058$

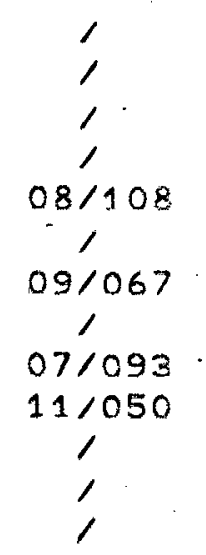

$08 / 146$

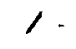

,

,

1

1

,

$$
\text { , }
$$

1

'

$08 / 015$

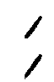

$07 / 154$

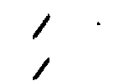

$11 / 012$

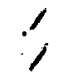

$08 / 170$

$08 / 071$

$11 / 077$

$11 / 027$

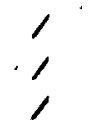

$11 / 058$

$11 / 058$

$11 / 038$

$11 / 087$

$11 / 064$
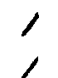

,

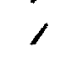

1

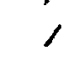

$08 / 167$

$09 / 049$
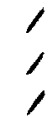

$09 / 082$

$09 / 080$
$.08 / 021$

$08 / 029$

$06 / 148$

$08 / 056$

$08 / 160$

$06 / 105$

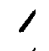

,

1

$08 / 128$

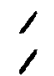

$06 / 122$

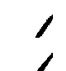

$07 / 059$

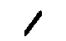

$07 / 130$

1

$06 / 058$

$08 / 148$

$07 / 092$

$05 / 189$

$06 / 118$

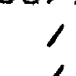

$08 / 021$

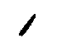

1

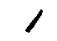

$06 / 070$

$08 / 142$

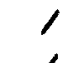

$04 / 133$

$05 / 113$

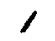

$08 / 185$

$08 / 043$

$06 / 203$

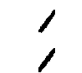

$07 / 026$

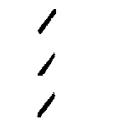

$08 / 030$

$08 / 131$

$07 / 061$.

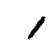

$06 / 121$

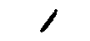

$05 / 184$

$05 / 200$

$05 / 185$

$06 / 225$

$07 / 141$

$05 / 125$ 
LEAVING

LECLERC

LED

LEDGE

LEFT

LEFTEVER

LEG

LEGEND

LEGS

LEI

LEMENADE

LEND

LENGTH

LEENARD

LESS

LESSON

LET

LET'S

LETS

LETTER

LETTERING

LETTERS

LETTING.

LETTUCE

LEVEE

LEVEL

LEVERS

LEW

LI

LIBERTY

LIBRARIAN

LIBRARY

LICHEN

LICK

LID

LIE

LIES I

LIEUTENANT

LIFE

LIFEGUARD

LIFTED

LIGHT

LIGHTNING

LIGHTS

LIKE

LIKED

LIKES

LILLY

LIN

LINCELN

LINDA

LINES

LIEN

LIENS

LIPS

LIST

LISTEN

LISTENED

LISTENING

LITTER :

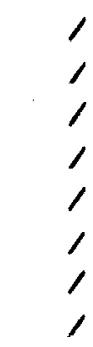

$05 / 016$

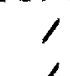

,

,

,

,

$05 / 168$

$04 / 028$

$06 / 205$

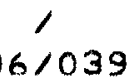

$06 / 039$
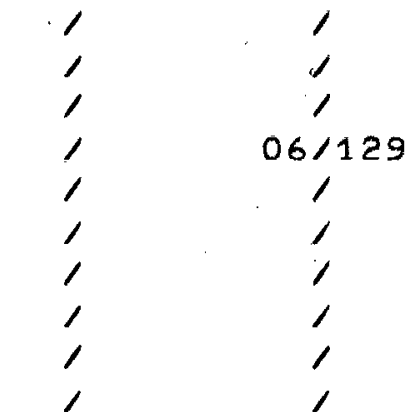

$07 / 136$

$07 / 135$

$08 / 221$

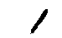

,

1

,

'

$04 / 009$

$04 / 026$

$04 / 042$

',

,

1

021029

$05 / 129$

$05 / 071$

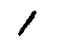

$06 / 015$

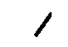

,

$06 / 040$

$06 / 129$

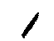

,

$y$

1

,

1

1

1

,

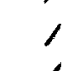

1

$05 / 162$

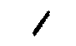

$05 / 157$

$02 / 013$

$05 / 022$

$01 / 074$

031009

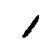

,

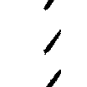

$03 / 065$

$06 / 039$

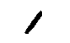

$06 / 173$

$03 / 040$

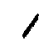

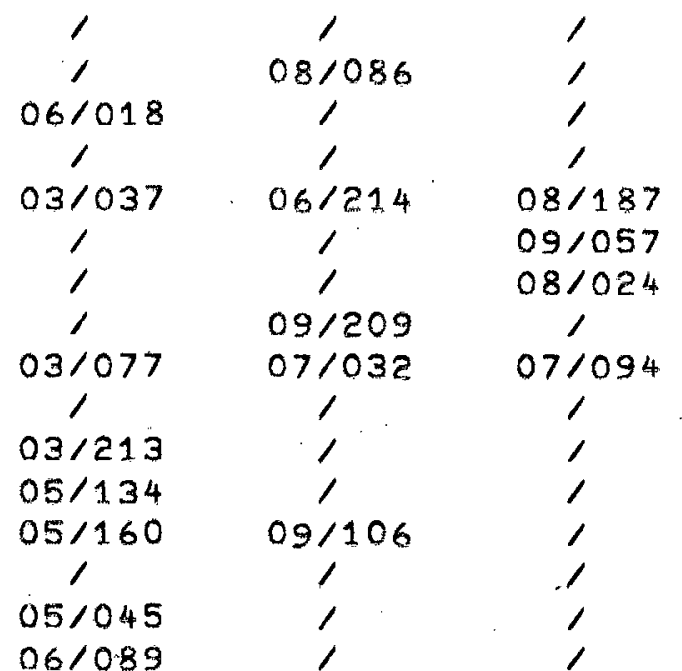

$04 / 066$

$04 / 044$

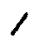

$05 / 040$
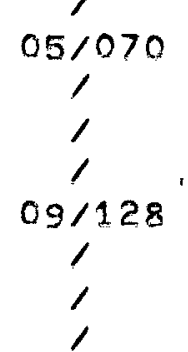

$09 / 222$

$06 / 054$

$06 / 054$

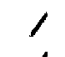

1

$08 / 167$

$09 / 161$

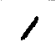

$07 / 061$

$07 / 102$

$04 / 103$

$06 / 092$

$04 / 105$

1

$05 / 015$

1

$07 / 080$
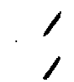

$05 / 017$

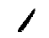

$07 / 201$
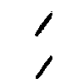

'

$06 / 196$

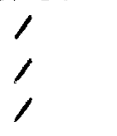

$07 / 064$

$11 / 01$ ह

$09 / 077$

$06 / 127$

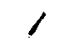

$06 / 144$

$08 / 012$

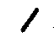

,

,

1

$08 / 079$

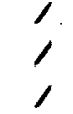

$07 / 025$

$08 / 018$

$08 / 106$

$04 / 087$

$04 / 057$

$05 / 108$

$05 / 113$

$06 / 078$

$08 / 122$

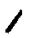

$07 / 233$

$05 / 161$

$08 / 06 ?$

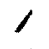

$07 / 185$

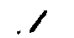

$08 / 074$

$08 / 200$

$08 / 146$

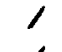

$06 / 175$

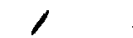

$06 / 112$

$05 / 096$

$07 / 018$

$08 / 213$

1

$05 / 005$

$08 / 080$

$05 / 038$

$03 / 030$

$03 / 047$

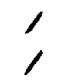

$08 / 063$

$08 / 140$

$05 / 077$

$05 / 223$

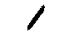

$06 / 210$

$08 / 192$

$07 / 032$

$05 / 152$

$08 / 144$

$09 / 060$

$05 / 152$

$05 / 221$ 
LITTLE

LIVE

LIVED

LIVELONG

LIVELY

LIVES

LIVING

LIZARD

LOAD

LOADING

LOAF

LOANED

LOCAL

LECK

LOCKED

LOCKET

LOCKS

LOFT

LOGGERS

LOGS

LOHMANN

LOLLIPOP

LONELY

LONESUME

LONG

LONG - LOST

LONG - TAILED

LENGER

LONGEST

LONGHUUSE

LONG ING

LOEK

LOEKED

LOBKING

LGEKBUT

LOOKS

LOOSE

LOESELY

LUOSEN

LOUSENED

LOP

LOPES

LORD

LORD!S

LOSE

LOST

LOT

LoTS

LOTTIE

LOUD

LOUDER

LQUDEST

LOUDLY

LOUDSPEAKER

LOUELLA

LOUGHBOROUGH

LOUIS

LOVE

LOVED

LOVELY

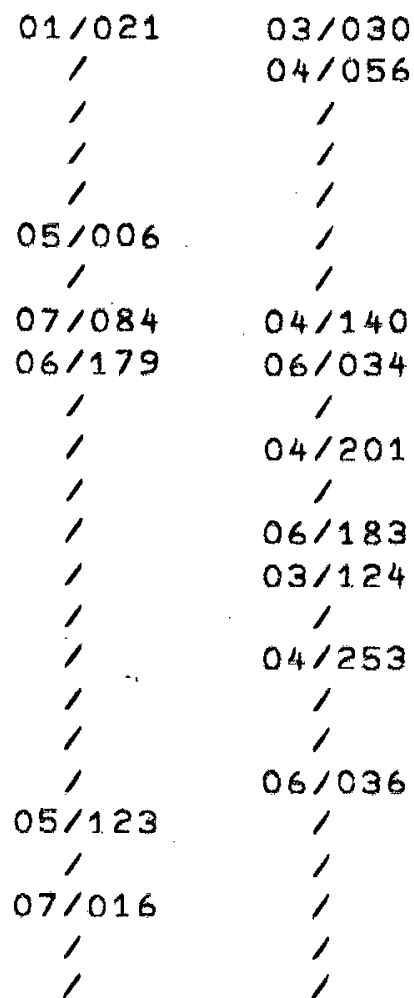

$05 / 121$

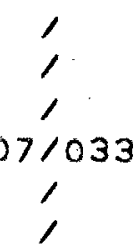

$02 / 030$

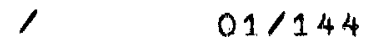

,

,

,

,

,

I

,

,

1

$08 / 139$

$01 / 033$

$06 / 026$

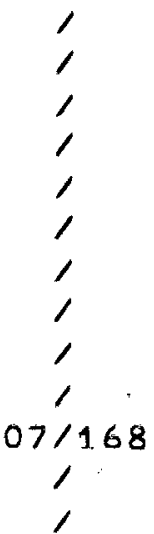

$02 / 157$

1

,

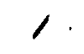

,

$01 / 006$

$01 / 144$

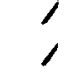

$03 / 028$

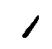

,

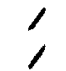

$06 / 181$

$06 / 185$

$06 / 153$

1

$05 / 226$

$05 / 232$

$05 / 046$

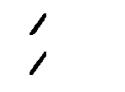

$04 / 032$

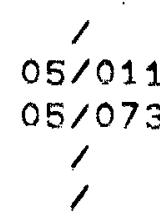

$05 / 080$

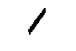

1

$08 / 041$

1

1

$06 / 139$

1

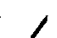

$08 / 110$

,

1

$08 / 070$
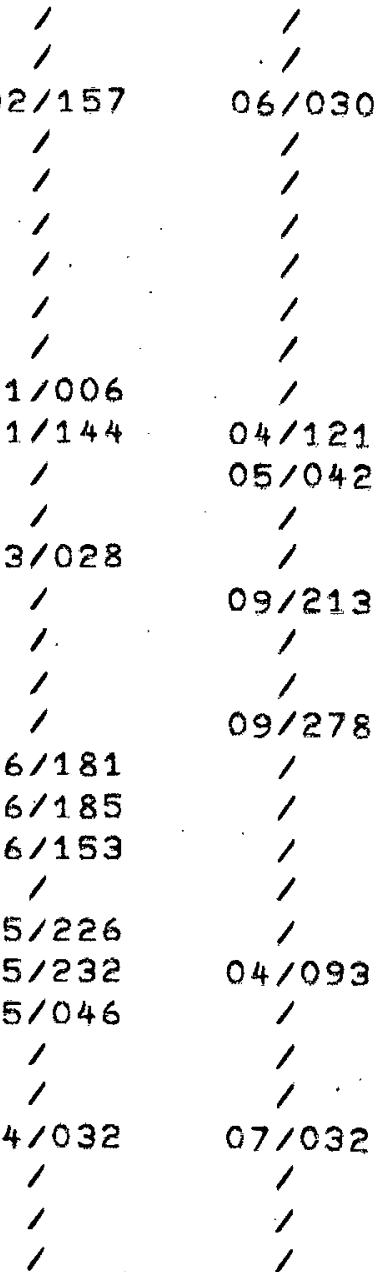

$07 / 060$

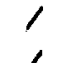

$09 / 059$

$05 / 045$

$05 / 100$

$06 / 182$

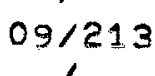

$09 / 278$

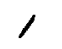

'

,

$04 / 093$

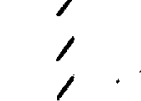

$07 / 032$

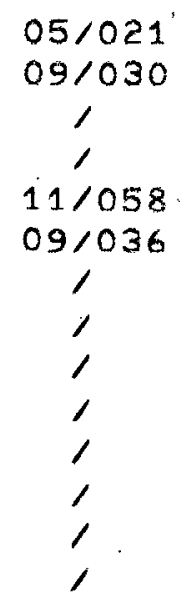

$09 / 076$

$09 / 076$

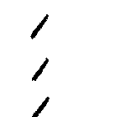

,

,

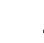

$07 / 094$

$11 / 020$

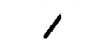

$09 / 072$

1

,

$05 / 003$.

$08 / 018$

$05 / 026$

$11 / 102$

$05 / 007$

$06 / 008$
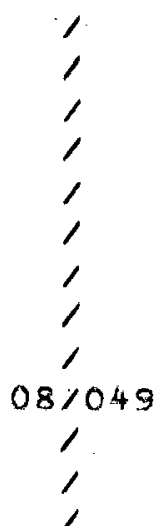

$11 / 057$

$11 / 051$

$03 / 044$

$05 / 146$

$05 / 109$

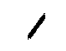

$06 / 018$

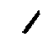

,

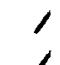

$08 / 058$

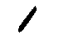

$07 / 194$

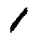

$08 / 141$

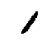

$07 / 121$

$08 / 104$

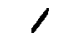

$08 / 014$

$08 / 175$

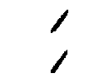

$05 / 226$

$06 / 150$

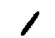

$06 / 196$

$05 / 158$

$01 / 008$

$04 / 099$
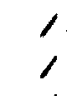

$07 / 138$

$07 / 226$

$07 / 238$
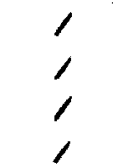

$08 / 107$

$08 / 072$

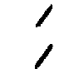

$05 / 077$

$08 / 104$

$06 / 145$

$06 / 149$

$06 / 187$

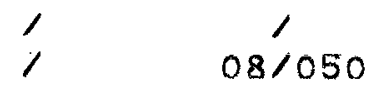

$05 / 192$

$08 / 113$

$06 / 085$

1

$07 / 093$ 
LOVINGLY

LOW

LOWER

LUBBER

LUCK

LUCKILY

LUCKY

LUCY

LUCY'S

LUGS

LUMBER

LUMP

LUNCH

LUNCHROSM

LUNCHTIME

LYING

$M=M-M$

$M A$

MA'AM

MABUNA'S

MACHINE

MACHINES

MACLEOD

$M A D$

MADE

MAGIC

MAGICIAN

MAGNET

MAGNIFYING

MAIOSERVENTS

MAIL

MAILBEX

MAILE!S

MAILED

MAILS

MAIN

MAJESTY

MAKE

MAKE $=B E L I E V E$

MAKES

MAKING

MALEC

MALLARD

MAMA

MAMMA

MAMMALS

MAMMY

MAN

MAN'S

MANAGE

MANAGED

MANALUK'S

MANE

MANY

MANY = COLDRED

MAP

MAPLE

MAPS.

MARBLE

MARCHERS

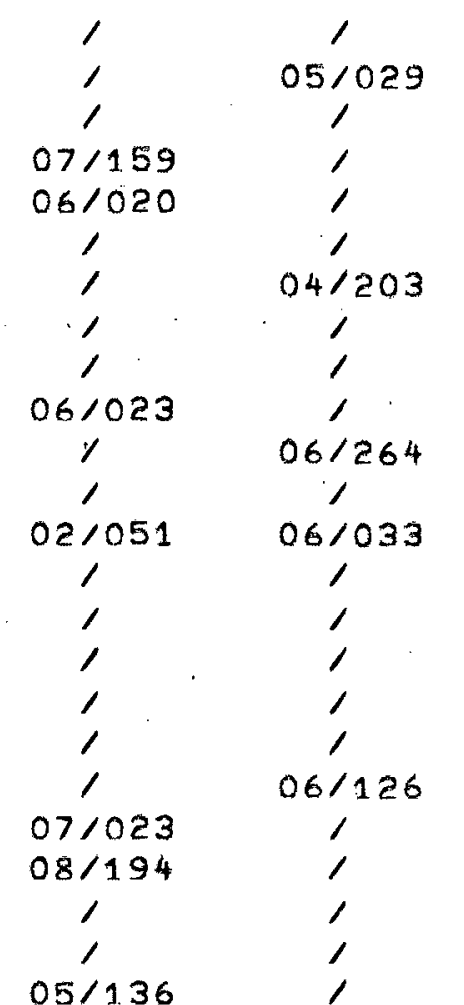

\section{$04 / 012$ \\ $02 / 140$}

$08 / 118$

1

$08 / 222$
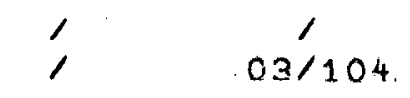

'

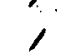

$06 / 039$

,

1

$04 / 027$

,

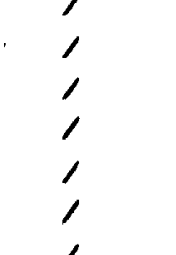

$07 / 087$

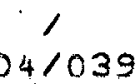

$04 / 039$

1

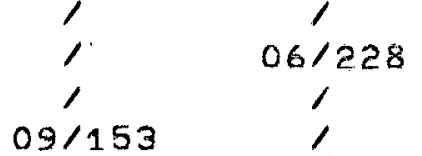

$05 / 114$

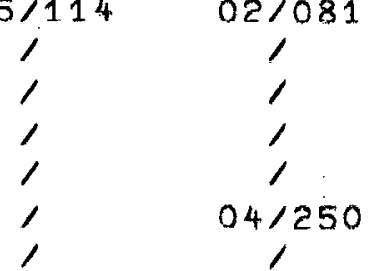

$04 / 133$

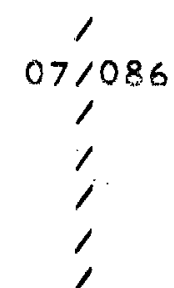

$05 / 073$

$05 / 079$
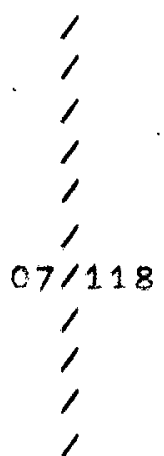

$08 / 117$

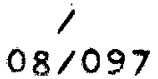

$05 / 105$

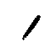

$06 / 090$
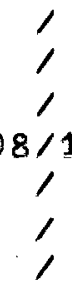

$07 / 144$

$05 / 229$

$05 / 266$

$01 / 069$

$03 / 006$
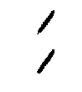

,
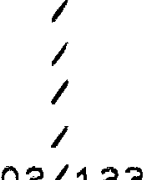

$02 / 123$

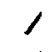

$07 / 103$

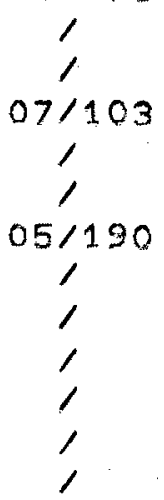

$05 / 190$

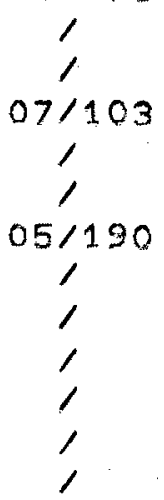

$05 / 138$
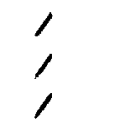

$09 / 042$

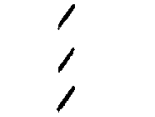

081054

$04 / 071$
$08 / 155$

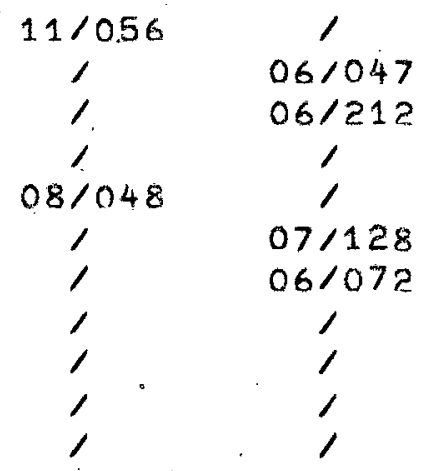

$08 / 071$

$05 / 185$

$06 / 059$

$06 / 059$

$08 / 072$

$06 / 017$

$08 / 141$
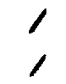

$05 / 173$

$08 / 039$

$08 / 087$

$08 / 022$

$09 / 008$

$08 / 128$

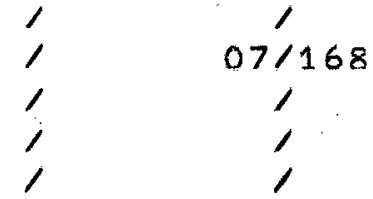

$07 / 205$

$08 / 082$

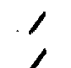

$08 / 124$

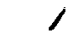

$05 / 027$

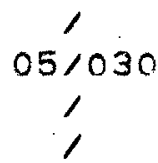

$11 / 093$

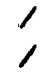

1

$08 / 028$

$11 / 100$

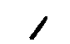

I

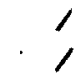

$07 / 143$

$11 / 089$

$11 / 012$

1

$11 / 031$
041061

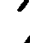

$05 / 091$

$06 / 116$

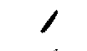

$08 / 144$

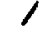

1

$04 / 104$

$08 / 023$

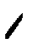

$08 / 090$
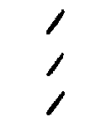

$08 / 185$

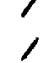

$06 / 032$ 
MARCO

MARCO'S

MARE

MARIA

MARIE

MARK

MARKED

MARKER

MARKET

MARKS

MARR I AGE

MARRIED

MARRIOTT

MARRY

MARSH

MARSHES

MARY

MARYGELD

MASK

MASSACHUSETTS

MASTER

MASTS

MATCHES

MATE

MATHILDA

MATUAKA

MATTER

MATTRESSES

MAY.

MAYBE

MAYNARD

MAYER

MAYS

$M E$

$M E=O W$

MEADOW

MEADOWS

MEAL

MEAN

MEANT

MEASURED

MEASUREMENT

MEAT

MECHANICAL

MEOICINE

MEECHAM

MEEKLY

MEET

MEETING

MEISTER

MELT

MELTED

MELTS

MELVIN

MEMBERS

MEMORIAL

MEMERY

MEN

MENTIEN

MEOW

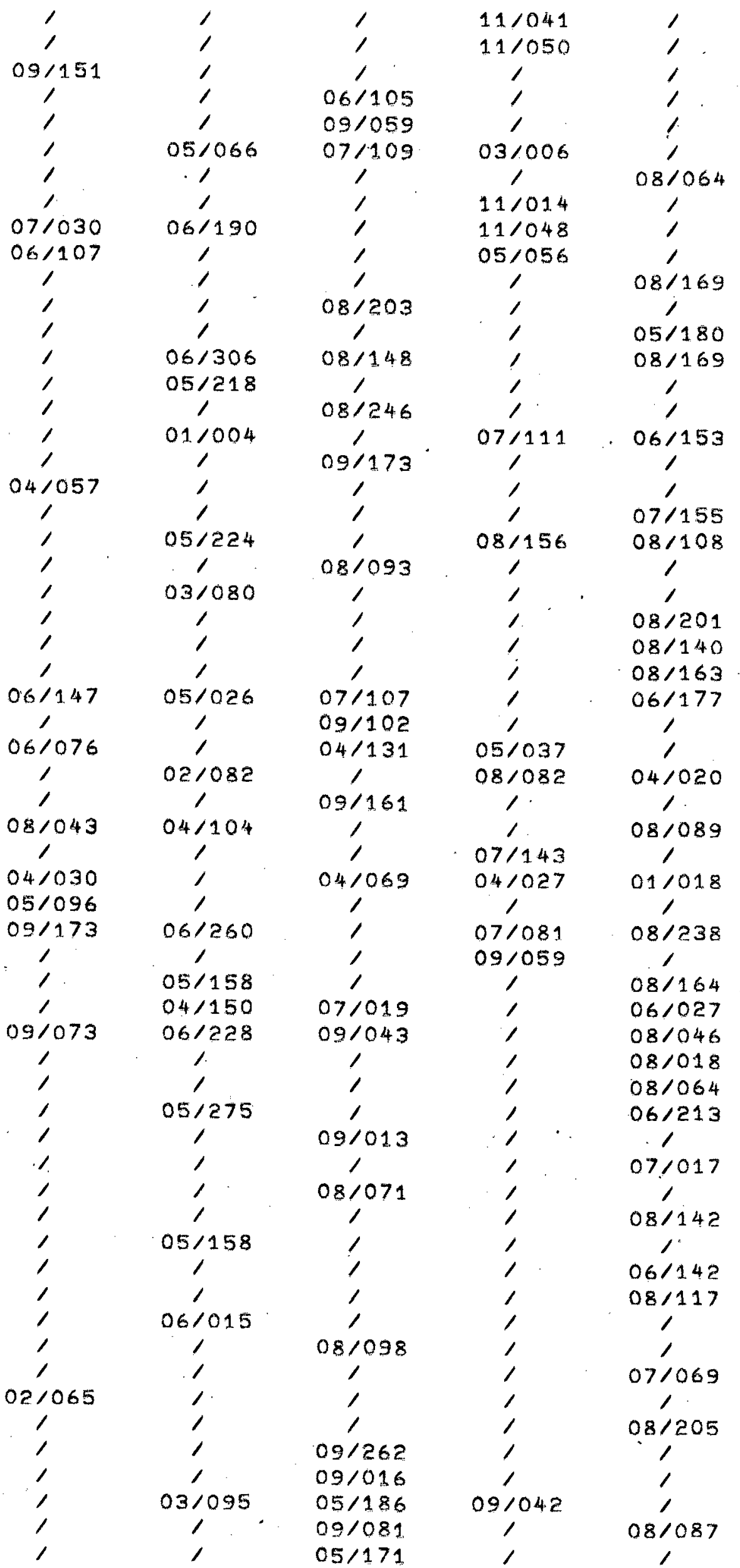


MERCHANT

MERRIEST

MERRILY

MERRY

MERRY = GQ-ROUND

MESA

MESSAGE

MESSAGES

MESSENGER

MET

METAL

METEOR

METEORITE

MEXICAN

MEXICO

MICHI

MICREPHONE

MICROSCOPE

MICREWAVE

MICROWAVES

MID

MIDAS

MIDDLE

MIDNIGHT

MIGHT

MIKE

MIKE'S

MILD

MILES

MILITARY

MILK

MILKMEN

MILL

MILLION

MILLIONS

MILTON

MIND

MINDY

MINE

MINERALS

MINI -

MINNY

MINUTE

MINUTES

MIRACLE

MIRRER

MIRRERS

MIRRES

MISCHIEF

MISERABLE

MISS

MISS HUCKLEBERRY

MISS LESTER'S

MISS PUPPY

MISSES

MISSION

MISSISSIPPÍ

MISSOURI

MIST

MISTAKE-

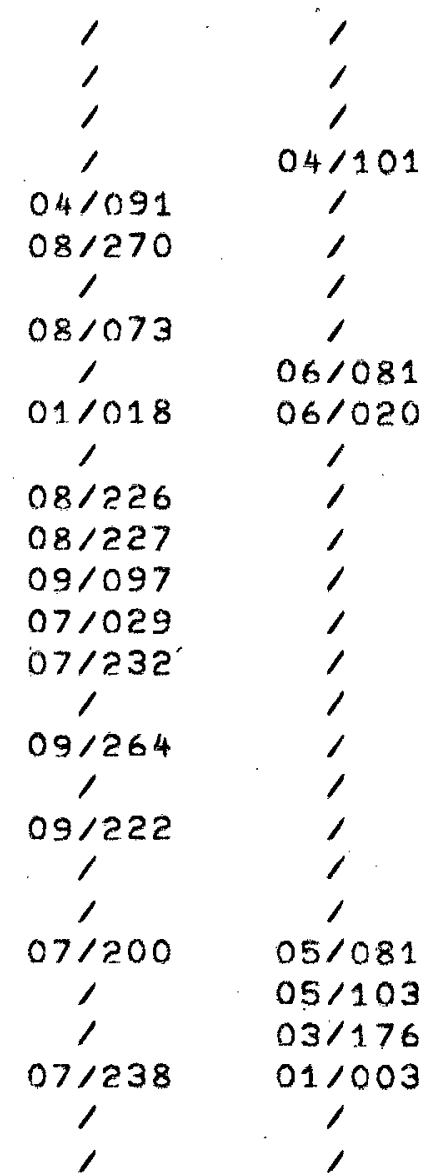

$06 / 059$

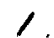

$04 / 081$

1

$05 / 124$

1

$07 / 082$

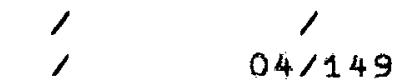

$09 / 250$

$09 / 257$

1

$08 / 091$

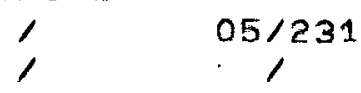

,

'

$7 / 163$

1.

$06 / 052$

$05 / 031$

$04 / 133$

,

,

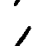

1

$09 / 167$
$04 / 149$

1

$01 / 014$

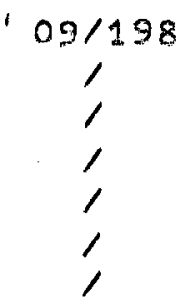

$09 / 066$

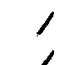

$09 / 029$

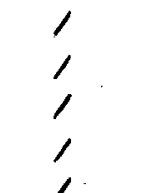

$09 / 014$

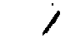

$09 / 071$

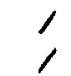

$09 / 173$

$07 / 180$

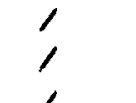

$04 / 100$

$07 / 223$

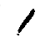

$09 / 262$

$07 / 131$
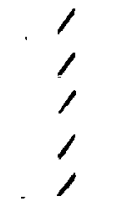

$07 / 213$

$07 / 131$
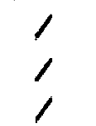

$09 / 012$

$08 / 029$

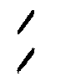

$08 / 190$

$06 / 166$

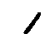

$06 / 137$

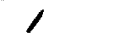

$04 / 017$

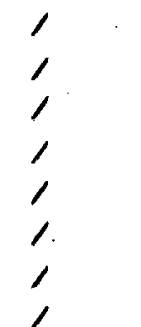

$05 / 249$

$09 / 100$

$06 / 123$
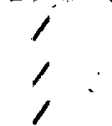

$$
\text { , }
$$

,
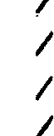

$09 / 214$

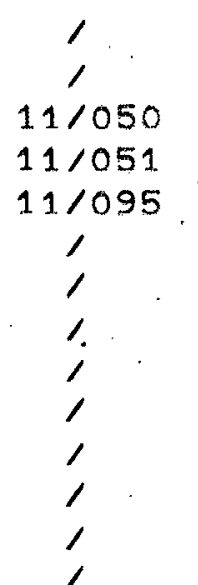

$11 / 083$

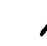

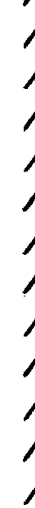

$11 / 014$

,

,

,

I

,

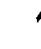

,

1

1

1

1

,

,

1

$11 / 081$

1

$11 / 063$

I

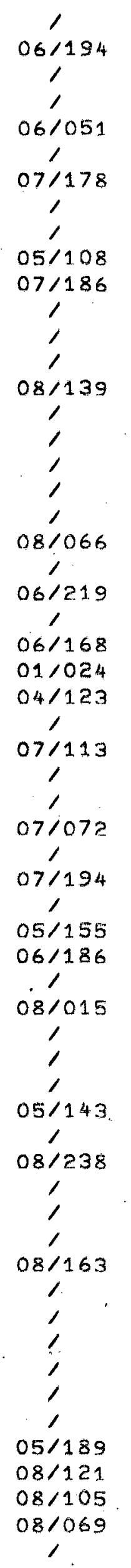


MISTAKEN

MISTS

MITE

MIX

MIXED

MODEL

MODELS

MOLDS

MOLLY

MOLTING

MOM

MOMENT

MONDAY

MONEY

MONGRELS

MONKEY

MONKEYS

MONKEYSHINES

MONSTERG

MONTH

MENTHS

MEQN

MOENLIGHT

MOQSE

MURE

MORNING

MERNINGS

MEST

MOSTLY

MOTHER

MOTHER'S

MOTHERS

METIEN

METIONS

MOTOR

MOTERBEAT

MEUNTAIN

MOUNTAINS

MOUNTAINTEP

MEUSE

MEUTH

MOVE

MUVED

MOVEMENT

MOVEMENTS

MEVING

MUWER

$M R$

$M R$ FIG

MR.

MR - GRANT

MR. MCGREGOR'S

MRS.

MRS - SNEW

$\mathrm{MUCH}$

MUD

MUDDY

MUFFLED

MULE

MULLAH

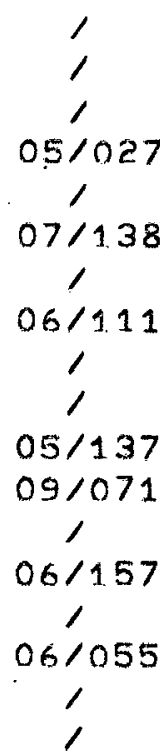

$06 / 030$

$08 / 079$

$04 / 011$

$04 / 011$

1

$06 / 158$

$01 / 009$

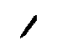

$07 / 026$

$04 / 010$

'

$08 / 070$

,

1

1

$07 / 029$

1

,

$03 / 159$
$1 \quad 04 / 025$

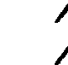

$09 / 199$

$04 / 116$
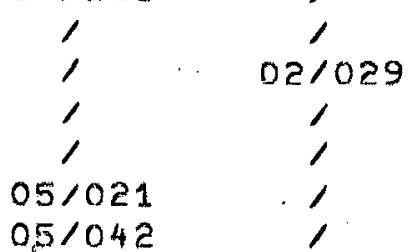

$02 / 032$

$06 / 075$

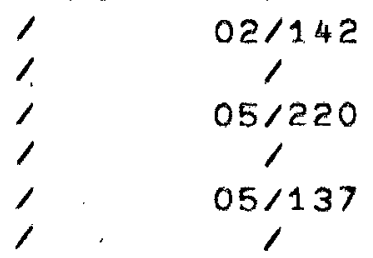

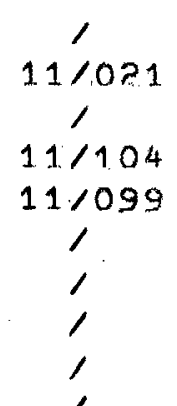

$09 / 015$

$07 / 146$

$07 / 009$

$08 / 073$

$09 / 083$

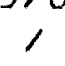

,

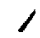

$08 / 024$

$09 / 032$

$04 / 125$

1

$09 / 080$

$05 / 106$

$05 / 038$

1

$06 / 095$

,

1

$05 / 018$

$05 / 016$

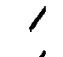

$08 / 120$

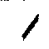

$05 / 155$
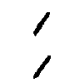

$07 / 176$

$07 / 064$

$07 / 068$

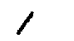

$08 / 251$

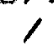

I

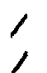

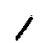

$04 / 150$

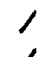

$06 / 012$

1

$06 / 086$

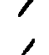

$09 / 101$

1

$07 / 169$

$11 / 046$

$06 / 004$

$09 / 053$

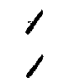

$03 / 02 ?$

$05 / 008$

$07 / 145$

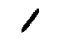

,

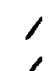

$11 / 087$

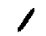

$11 / 116$

$09 / 015$

$$
1
$$

$09 / 028$

$09 / 015$

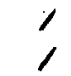

$09 / 019$

$07 / 03 ?$

$09 / 007$
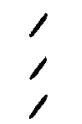

$07 / 134$

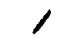

$08 / 107$

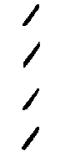

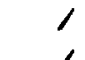

$08 / 042$

$08 / 149$

$08 / 085$

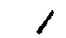

$07 / 193$

1

$06 / 182$

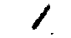

$08 / 175$

$08 / 013$

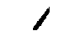

I

$06 / 144$

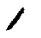

1

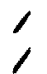

$08 / 031$

$08 / 036$

$08 / 029$
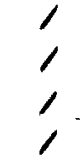

$06 / 139$

$07 / 035$

$02 / 019$
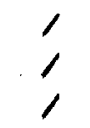

$07 / 140$

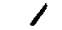

$07 / 176$

$08 / 029$

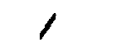

071090

$06 / 127$

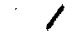

$05 / 040$

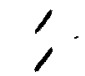

$06 / 115$

$08 / 043$

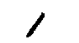

,

,

,

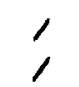

$05 / 089$

$07 / 016$ 
MUMPS

MURMUR

MUSEUM

MUSHRUSH

MUSIC

MUSICAL

MUSICIANS

MUST

MUSTACHE

MUSTARD.

MUTTERED

MUTTERING

MY

MYSELF

MYSTERIOUS

MYSTERY

NAILS

NAME

NAMED

NAMES

NANCY'D

NARRATOR

NARREW

NATION

NATIONAL.

NATIVES

NATURALLY

NAVAJO

NAVEL

NAVY

NEAR

NEARBY

NEAREST

NEARING

NEARLY

NEAT

NECESSARY

NECK

NEDDIE

NEED

NEEDED

NEEDLE

NEEDLES

NEEDS

NEGRO

NE I GHBOR

NEIGHBORHQOD

NEI GHBORS

NE I THER

NEMETH

NEST

NESTS

NET

NETS

NEVER

NEVERTHELESS

NEW

NEW YORK CITY

NEWBERN

NEWEST

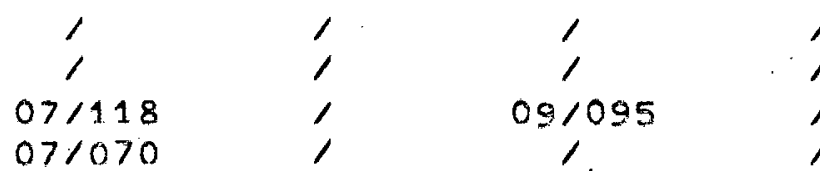

$\prime$
$08 / 111$
$04 / 113$
$/$
$09 / 190$
$/$

$08 / 188$

$04 / 049$

$05 / 047$
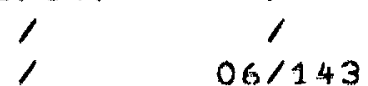

1

$05 / 128$

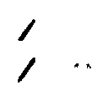

071032

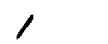

1

$09 / 111$

1

$07 / 051$

$08 / 258$

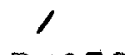

$07 / 078$

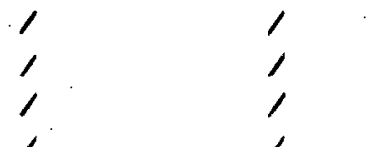

$08 / 015$

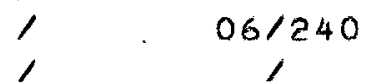

, 06/147

1

$05 / 054$

' 1058

1

$06 / 034$

$09 / 134$

$05 / 153$

$07 / 205$

,
$04 / 093$

$06 / 272$

$04 / 019$

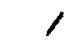

$03 / 058$

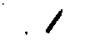

$05 / 160$

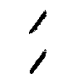

$11 / 051$

$09 / 233$

$08 / 016$

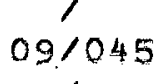

$08 / 245$

$06 / 125$

$06 / 104$

$06 / 175$

$04 / 211$

$06 / 243$

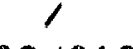

$02 / 019$

$01 / 122$

1

I
$04 / 097$

$4 / 093$

1

$09 / 109$

1

$05 / 049$

$05 / 052$

,

1

$08 / 091$

$09 / 258$

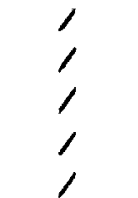

,

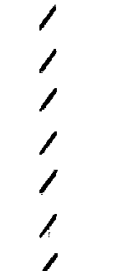

$06 / 236$
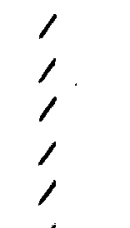

$09 / 114$

$07 / 227$

1
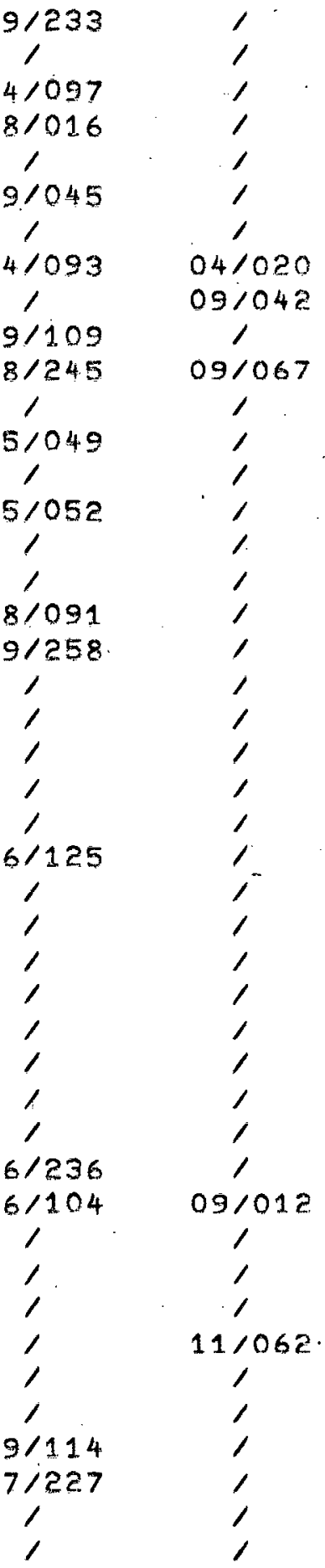

$04 / 020$

$09 / 042$

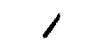

$09 / 067$

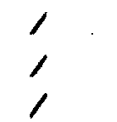

,

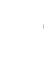

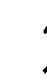

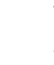

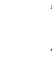

,

1

,

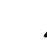

,

,

I

,

,

1

,

$09 / 012$

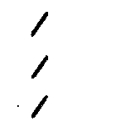

$11 / 062$.

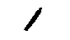

,

,

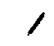

,
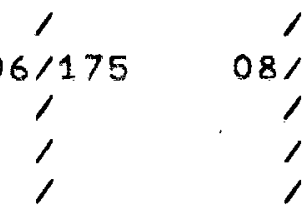

$08 / 121$

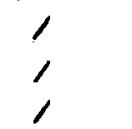

$09 / 010$

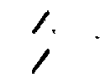

$04 / 043$

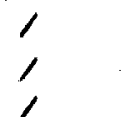

$07 / 098$

$08 / 155$

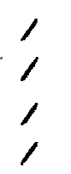

$08 / 235$

$08 / 189$

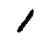

1

$07 / 088$
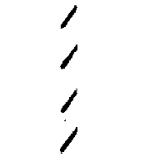

$08 / 095$

$03 / 036$

$06 / 176$

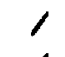

$06 / 007$

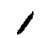

$05 / 040$

$07 / 079$

$08 / 206$

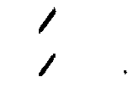

$08 / 057$

1

1

$05 / 061$

$05 / 174$

$06 / 125$

$05 / 156$

$07 / 039$

$08 / 105$

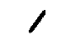

$06 / 120$

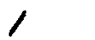

$05 / 070$

$05 / 093$

$08 / 086$

1

$08 / 124$

$05 / 025$

$05 / 005$

$08 / 094$

$07 / 187$
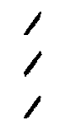

$08 / 083$

$05 / 173$

$04 / 094$

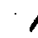

$06 / 126$ 
NEWLY

NEWS

NEWSBOY

NEWSPAPER

NEXT

NEXT =BEST

NIBBLE

NICE

NICEST

NICK

NICKEL

NICKELS

NIGHT

NIGHTBIRDS

NIGHTS

NIGHTTIME

NINA

NINE

NINETY

NINE TY $=F$ IVE

NIX

NO

NO-WHEELEQ

NOBGDY

NOD

NEDDED

NEISE

NOI SEMAKER

NOI SEMAKING

NUISIEST

NOISILY

NOISY

NONE

NONSENSE

NOODLE

NOODLES

NOON

NEONTIME

NOR

NERTH

NOSE

NOT

NET - TEO-BIG

NUTE

NOTES

NUTHING

NETHINGNESS

NOTICE

NOTICED

NOTICING

NETIEN

NOVEMBER

NEW

NUMBER

NUMBERS

NURSE

NUTMEAT

E.K.

O'CLOCK

UAK

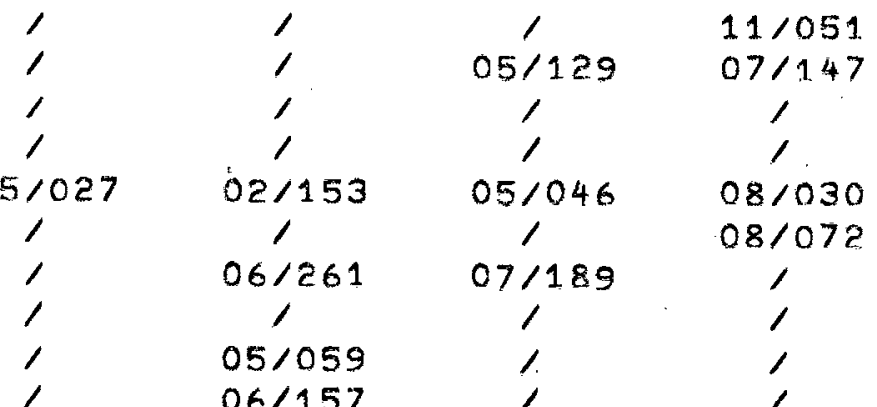

$06 / 157$

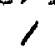

$03 / 185$

$06 / 069$

,

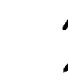

$09 / 111$

1

$08 / 037$

'

$04 / 021$

1

1

1

I
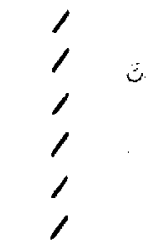

$09 / 257$

1

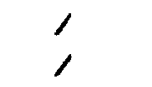

$06 / 103$

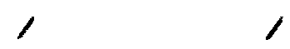

/ $\quad 06 / 147$

,

$02 / 014$

I

,

$08 / 206$

1

$07 / 203$

1

,

1

$04 / 058$
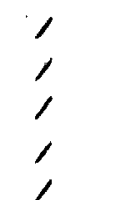

$08 / 035$

\section{$01 / 151$}

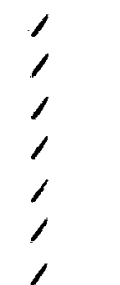

$06 / 052$

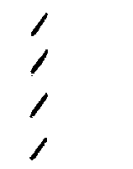

$05 / 145$

$04 / 031$

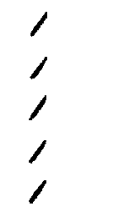

$03 / 167$

$04 / 007$

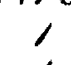

1

$04 / 122$

,

$06 / 012$

$03 / 099$

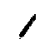

$05 / 174$

$06 / 115$

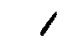

$01 / 009$
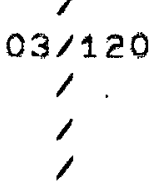

$06 / 227$
$01 / 038$
$07 / 031$

$07 / 031$

$07 / 051$

$04 / 189$
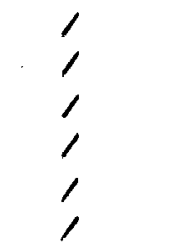

$06 / 246$

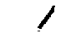

$04 / 018$

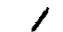

$07 / 182$

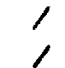

$05 / 144$
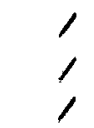

$07 / 209$

$08 / 169$
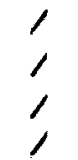

,

1

1

1

1

,

1

$07 / 181$

$07 / 194$

$08 / 077$

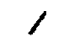

$08 / 027$

/ $9 / 136$

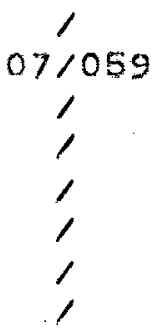

1

1

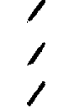

1

1

,

,

1

1

,

,

1

$08 / 058$

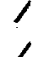

$03 / 061$

$09 / 056$

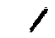

$11 / 007$

$08 / 139$

$04 / 024$

$09 / 024$

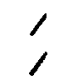

$09 / 072$
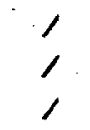

,

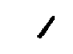

$05 / 029$

$07 / 02 ?$
$07 / 012$

1

$06 / 096$

$05 / 164$

,

,

$05 / 192$

!

$07 / 167$

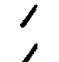

$05 / 226$

$09 / 044$

$11 / 030$

$05 / 015$

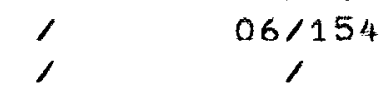

$08 / 040$

$06 / 236$ 1

$05 / 134$

$06 / 097$ 
EARS

GATS

BBEY

BBLIGE

BCEAN

QCEANEGRAFHERS

OCRACQKE

EF

EFF

UFFER

OFFERED

OFFICE

OFFICER

OFTEN

GH

$\mathrm{OH}=\mathrm{H}-\mathrm{H}$.

OIL

OIL-COVERED

UILY

OK

OKLAHOMA

OLAF

ELD

OLD=FASHIQNED

OLDER

OLDEST

OLDTEWN

ELIVE

ON

ONCE

UNE

ENE - WHEELED

$O N E=Y E A R=O L D$

ONES

ONIEN

ONLOEKERS

ONLY

ENO

ONTO

GPEN

QPENED

OPERATION

OPOSSUM

OPPOSITE

$O R$

ERANGE

ORANGE - CELURED

ERANGES

ORANGUTAN

ORBIT

QRBITED

QRBITS

ORDERED

ORDERS

GRDINARY

ORIULES

ORVILLE

OSTRICH

OTHER

OTHERS

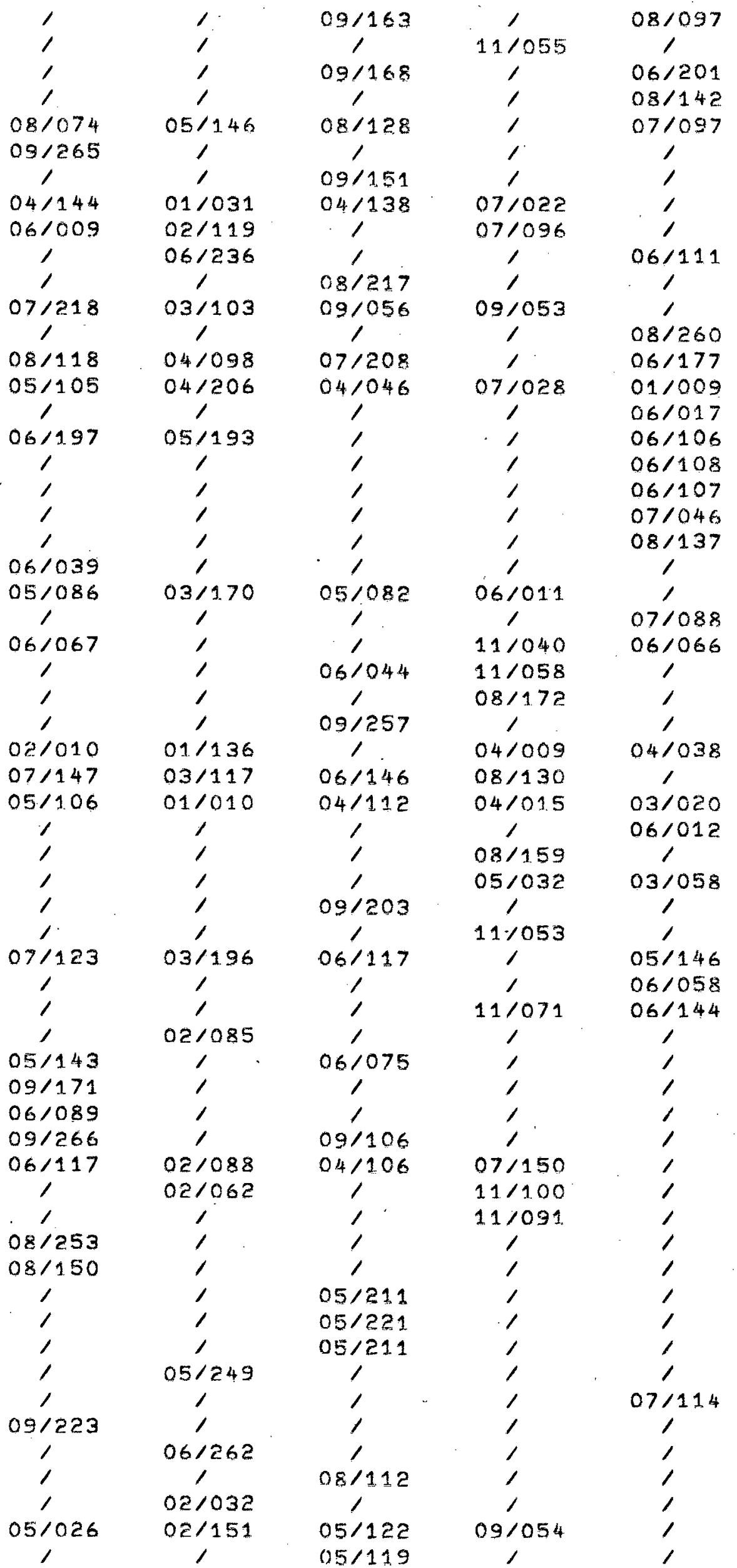


ETTE

EUCH

EUGHT

OUR

QURSELVES

EUT

QUT - QF - THE - WAY

OUTDGERS

QUTF IT

OUTNUMBERED

OUTSIDE.

UUTSMARTEO

BUTWARD

- EVEN

UVER

GVERAGE.

CVERJEYED

OVERLUOKING

EWE

OWL

OWLGLASS

OWN

OWNED

OWNER

OWNING

OWNS

OXEN.

BXYGEN

$P A$

PABLO

PACK

PACKAGE

PACKED

PACKET

PACKS

PADDLE

PADDY

PADRES

PAGE

PAGES

PAID

PAIL

PAIN

PAINFUL

PAINT

PAINTED

PAINTER

PAINTING

PAINTS

PAIR

PAIRS

PAJAMAS

PAL

PALACE

PALE

PALYTIME

PAM

PAN

PANCAKE

PANEL

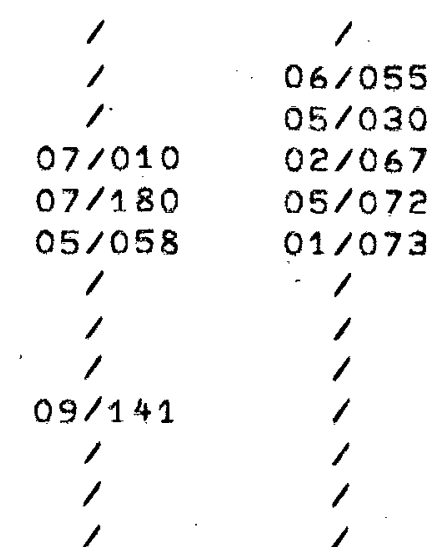

$06 / 112$

$02 / 043$

1

$07 / 096$
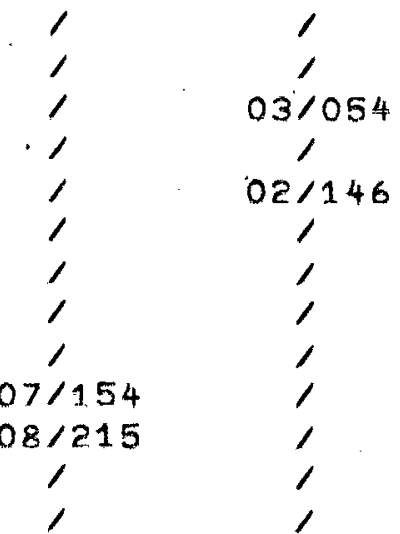

$05 / 140$

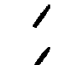

,

,

,

1

$08 / 256$

$06 / 118$

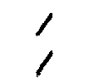

$07 / 037$
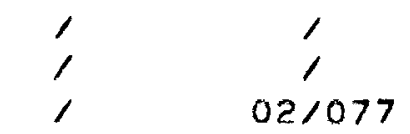

\%

$05 / 020$

1

$07 / 088$

1

1

$07 / 012$

'

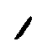

$07 / 160$

$06 / 067$

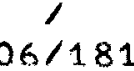

$06 / 181$

$02 / 136$

$02 / 077$
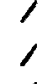

'

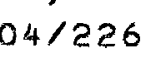

,

$02 / 135$

$05 / 264$

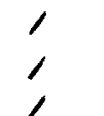

$04 / 029$

,

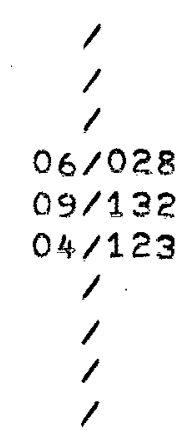

$06 / 025$

$09 / 251$

1

$06 / 241$

$05 / 045$

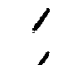

$07 / 221$

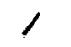

$07 / 043$

$07 / 158$

$05 / 100$

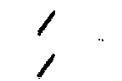

r

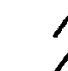

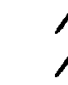

1

$05 / 191$

$05 / 075$

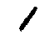

$09 / 242$

$05 / 192$
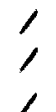

11097

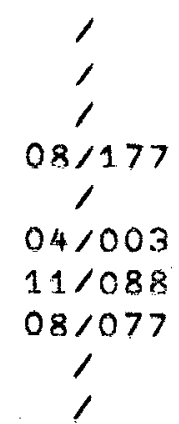

$07 / 163$

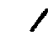

1

$08 / 049$

$04 / 180$
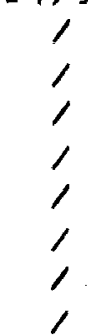

$08 / 201$
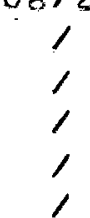

$09 / 013$
$09 / 050$

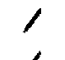

1

$08 / 098$

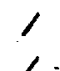

1

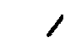

$08 / 019$

$08 / 036$

$11 / 059$

$11 / 115$

$09 / 076$

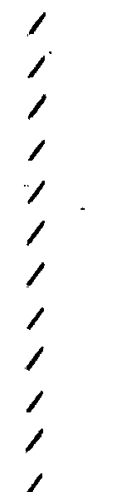

$08 / 133$

$11 / 039$

$07 / 070$

$06 / 167$

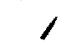

$04 / 018$

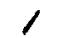

$05 / 126$

$08 / 011$

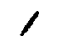

$05 / 155$

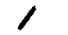

$08 / 057$

$06 / 131$

$08 / 201$

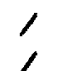

$08 / 214$

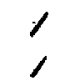

$05 / 166$

$05 / 178$

$06 / 067$
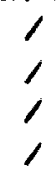

$08 / 141$

$06 / 165$

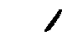

$08 / 220$

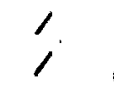

$08 / 095$

$05 / 165$
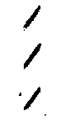

$08 / 053$

$06 / 117$

$08 / 117$

$08 / 103$

$09 / 023$

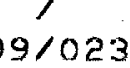

$08 / 102$

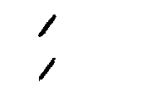

$08 / 026$

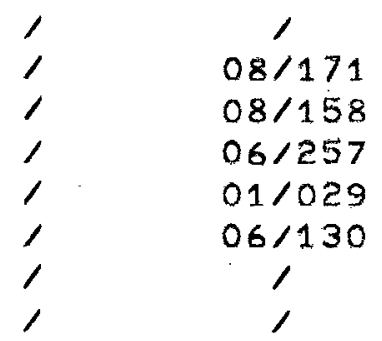


PANTING

PANTS

PAPA

PAPER

PAPERS

PAPPAS

PAPPY

PARADE

PARAGRAPHS

PARDON

PARENTHESES

PARENTS

PARIS

PARK

PARKED

PARNELL

PARROT

PARROT'S

PARSLEY

PART

PARTIES

PARTLY

PARTS

PARTY

PASS

PASSAGE

PASSED

PASSENGER

PAST

PASTEUR

PASTURE

PAT

PATCH

PATCHES

PATH

PATIENT

PATIENTLY

PATRICK

PATTED

PATTER

PATTERN

PATTY

PATTY'S

PAUL

PAULA

PAUSED

PAW

PAWS

PAY

PAYDAY

PAYING

PAYMENT

PEABEDY

PEACE

PEACH

PEACHES

PEANUT

PEANUTS

PEBBLES

PECKING

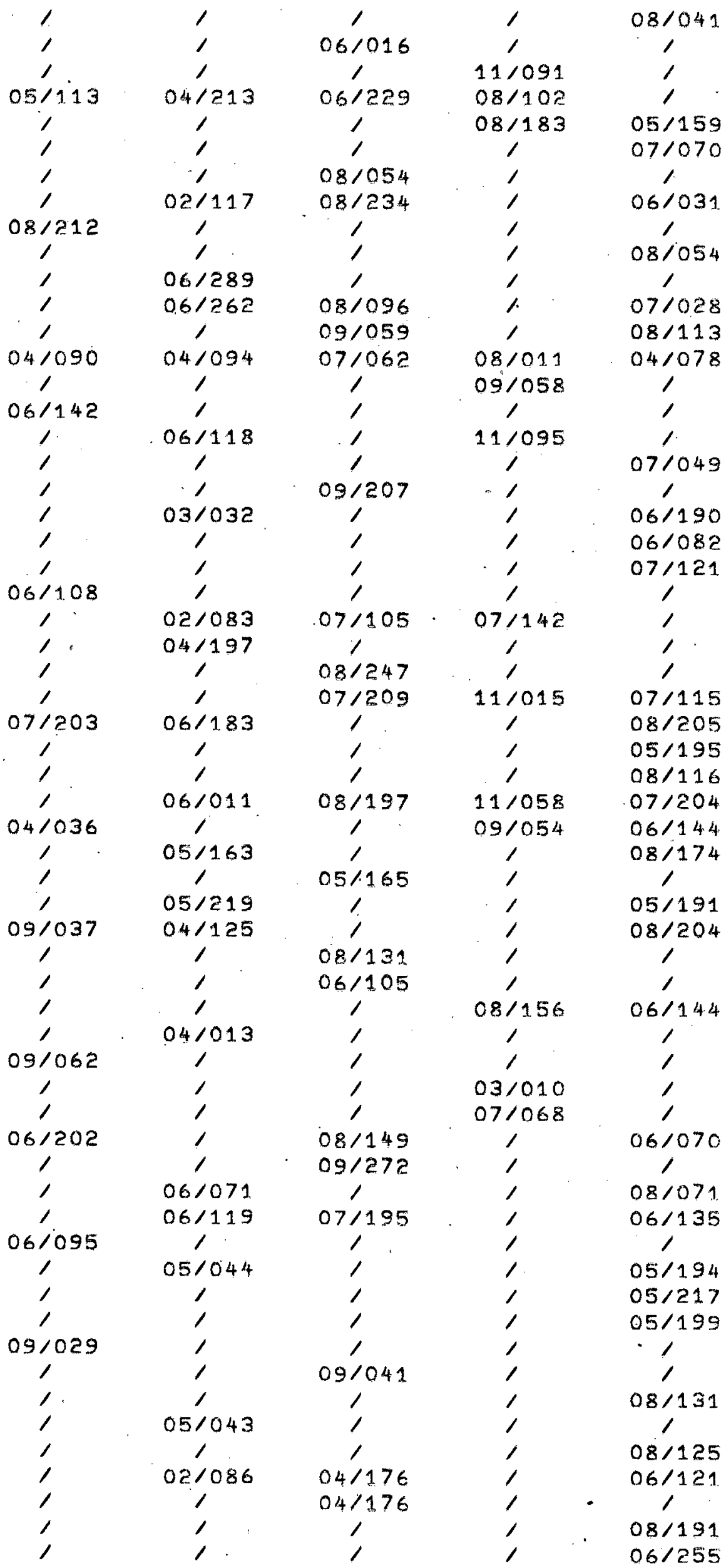


PECUL IAR

PEDAL

PEDDLER

PEDDLERS

PEDRE

PEEKING

PEEP

PEEPED

FEEPING

PEERED

PEGGY

PEN

PENCIL

PENCILS

PENELOPE

PENGUIN,

PENNIES

PENNY

PEONY

PEQPLE

PEPPERPET

PERCHED

PERFECT

PERFECTLY

PERFERMANCE

PERFUME

PERHAPS

PERIODS

PERMISSIEN

PERSEN

PESTERING

PET

PETE

PETE'S

PETER

PETER RABBIT

PETERSON'S

PETS

PETTEO

PFT-FT

PIANE

PICK

PICKED

PICKING

PICKLE

PICKLES

PICNIC

PICNICS

PICTURE

PICTURES

PIDGEON

PIE

PIECE

PIECES

PIER

PIERRE

PIES

PIG

PIGEON

PIGEENS
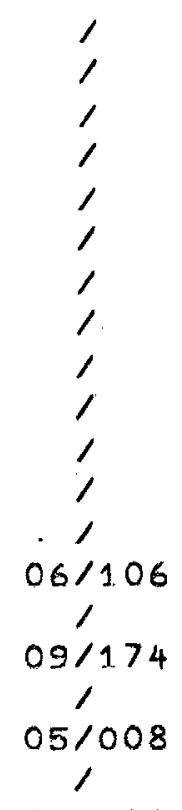

$04 / 102$

$08 / 185$

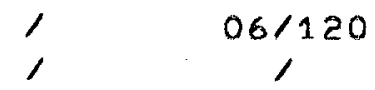

$08 / 116$

,

,

'́,

$05 / 1.58$

$05 / 040$

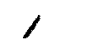

$05 / 014$

,

,

'

$04 / 088$

,
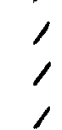

$05 / 021$

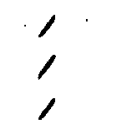

$06 / 206$

1

$08 / 096$

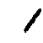

$01 / 063$

1
$06 / 022$

$06 / 235$

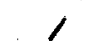

$05 / 054$

$05 / 100$

$02 / 077$

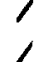

,

1

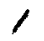

$03 / 097$

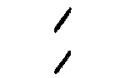

$05 / 284$

$04 / 034$

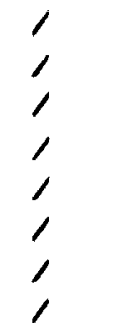

$06 / 008$

$05 / 044$
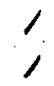

,

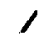

$04 / 111$

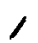

$02 / 078$
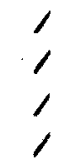

$04 / 046$

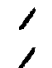

$05 / 096$

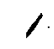

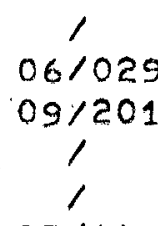

$07 / 146$

$06 / 175$

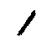

1

$08 / 224$

$08 / 025$

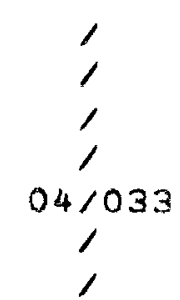

$08 / 128$

$11 / 089$

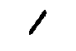

1

1

$09 / 233$

$08 / 257$

$06 / 049$

$06 / 059$

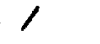

$05 / 117$

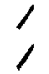

$09 / 193$

1

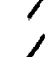

$08 / 122$

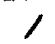

$09 / 096$

1

$08 / 013$

$04 / 090$

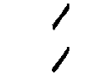

$05 / 135$

1

$05 / 073$

$06 / 022$

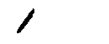

$09 / 066$

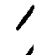

,

,

,

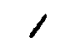

$04 / 017$

$04 / 053$

$07 / 064$

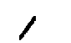

$08 / 084$

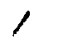

$07 / 174$

1

$08 / 084$

'́.

$05 / 093$

$07 / 153$

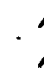

.

,

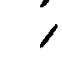

,

1

I

,

1

$07 / 033$

$07 / 033$

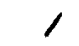

$07 / 155$

$07 / 155$

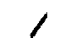

$$
1
$$

$11 / 106$

$11 / 109$

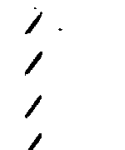

$08 / 072$

$07 / 212$
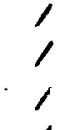

,

,

1

$08 / 075$

'

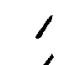

,

,

$01 / 030$

$08 / 264$

$05 / 154$

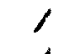

$07 / 210$

$07 / 208$

$07 / 047$

$05 / 193$

,

$08 / 067$

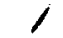

$04 / 135$

$04 / 010$

$04 / 073$

$07 / 073$

$07 / 073$

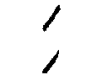

$06 / 215$

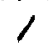

$06 / 142$

$06 / 223$

$05 / 153$

$05 / 154$

$05 / 223$

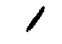

$$
\text { , }
$$
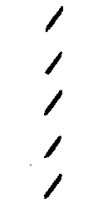

$06 / 206$

$07 / 102$

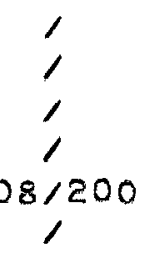




PIGGY=BACK
PIGS
PILE
PILED
PILES
PILGRIM
PILLOW
PILOT
PINCH
PINES
PING
PINK
PINS
PINT

FINTA

PIENEERS

PIPER

PIPES

PIRATES

PITCH

PITCHER

PITCHING

PITTER

PITY

PLACE

PLACED

PLAID

PLAIN

PLAINLY

PLAN

PLANE

PLANET

PLANKTEN

PLANNING

PLANT

PLANTED

PLANTING

PLANTS

PLATE

PLATES

PLATFERM

PLAY

PLAY - IN-THE-SUN

PLIAYED

PLAYFUL

PLAYGROUND

PLAYHOUSE

PLAYING

PLAYS

PLAYTHINGS

PLAYTIME

PLAZA

PLEASANT

PLEASE

PLEASED

PLEASURE

PLEDGE

PLENTIFUL

PLENTY

PLOW

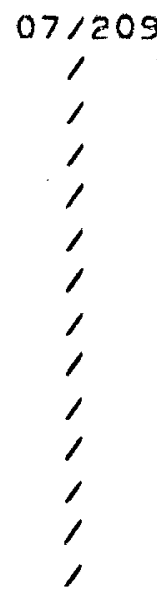

$09 / 111$

$09 / 136$

,

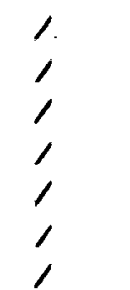

$06 / 119$

1

$09 / 059$

$06 / 155$

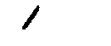

$06 / 008$

$04 / 023$

$06 / 010$

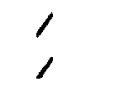

$05 / 047$

1

1

1

$08 / 239$

$04 / 020$

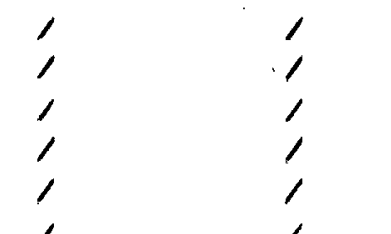

,

,

1

$07 / 049$

$09 / 224$

$06 / 138$

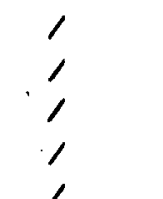

$06 / 189$

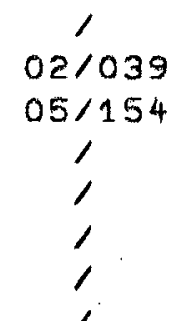

$05 / 217$

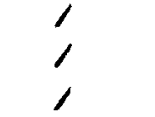

$04 / 216$

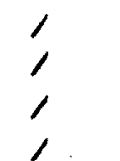

$03 / 106$

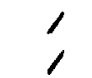

$06 / 084$

$04 / 013$

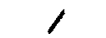

$03 / 171$

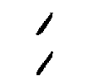

$04 / 225$
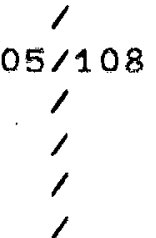

$.06 / 295$

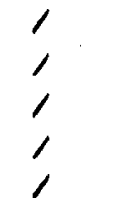

$06 / 227$

$01 / 017$

$01 / 021$

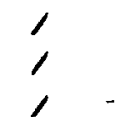

$06 / 059$

$03 / 055$
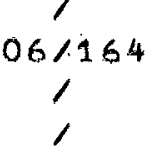

$05 / 030$

$03 / 098$

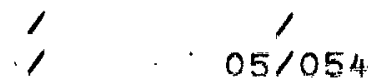

$07 / 070$

$06 / 223$

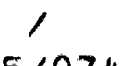

$05 / 074$

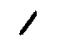

,

'

$08 / 163$

$04 / 105$

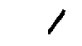

$09 / 265$

'́241

$07 / 241$

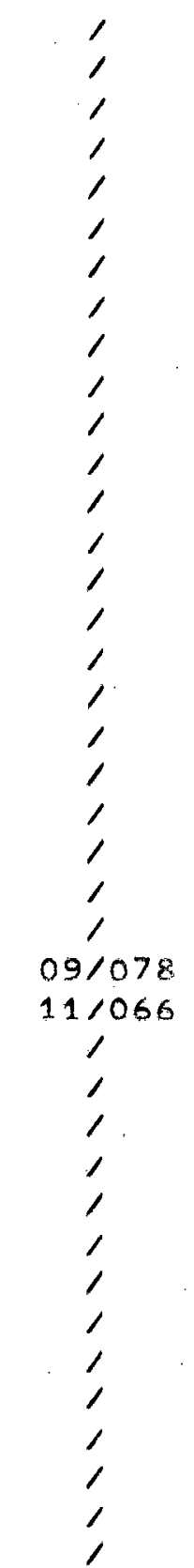

$11 / 096$

$05 / 052$

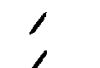

1

,

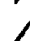

1.

$09 / 030$

$07 / 153$

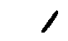

$08 / 167$

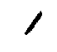

$07 / 015$

,

,

I

1

,

$06 / 196$

$05 / 155$

$05 / 151$

$07 / 154$

$08 / 240$

$07 / 211$

1

$08 / 033$

$08 / 063$

$06 / 062$
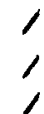

$07 / 215$

$06 / 071$

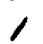

,

1

$07 / 098$

$08 / 022$

$05 / 030$

$05 / 122$

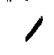

1

$08 / 069$

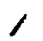

$05 / 140$

'

$07 / 140$

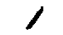

$05 / 168$

$05 / 170$

$05 / 168$

$07 / 103$

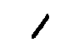

$02 / 025$

$06 / 258$

$05 / 050$

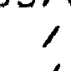

$05 / 089$

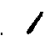

$05 / 009$

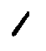

$05 / 197$

$04 / 041$

$05 / 163$

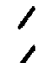

$07 / 117$

$06 / 194$ 
PLOWED

PLUCK

PLUNGE

PLUNGED

PLYMEUTH

FECAHONTAS

POCKET

POEM

POEMS

PUETRY

POINT

POINTED

POINTING

PUINTY $=$ EARED

POISON

POISENED

POISONOUS

POKED

POLE

POLICE

POLICEMAN

POLICEMEN

PELISH

POLISHED

PELITELY

POND

PONIES

PONY

PEOL

P'EOR

$P A P$

PUPCORN

POPPED

POPPER

POPPY

PUPSICLES

PURCH

PORCUPINE

PORRIDGE

PORTER

POSITIEN

POSSESSIEN

POSSIBLE

PASSIBLY

PUST

PUSTMAN

POSTMAN'S

PUSTMASTER

PESTMEN.

POSTURE

POTATE

POTATEES

PETTED

POTTER

POTTERS

PETTERY

PEUND

POUNDS

PQUR

PQURED

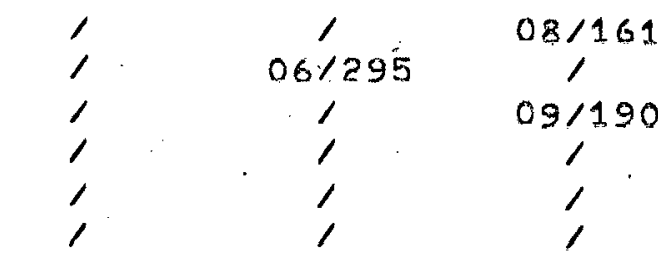

$04 / 100$

$07 / 222$

$06 / 071$

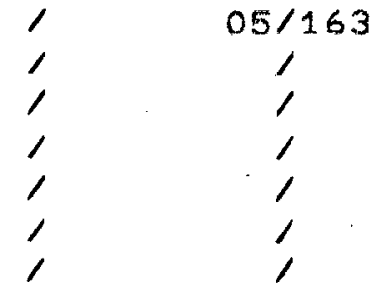

$05 / 150$

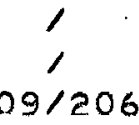

$09 / 206$

$1 / 045$

1

$04 / 080$

,

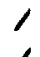

,

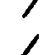

$08 / 203$

1

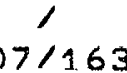

$07 / 163$
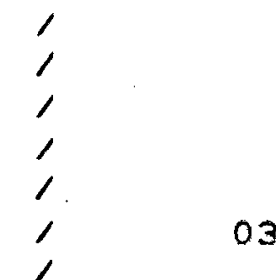

,

,

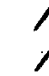

1

$09 / 222$

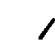

I

,
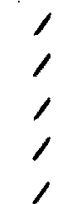

$08 / 200$
$06 / 121$

$06 / 179$

$03 / 102$

$02 / 035$

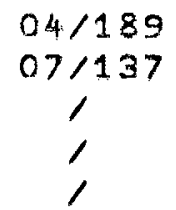

$07 / 183$

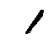

$07 / 188$

$08 / 050$

1

$08 / 042$

1

$05 / 202$

$09 / 047$

$05 / 076$

$01 / 013$

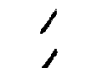

$04 / 091$

1
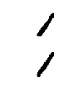

$03 / 131$
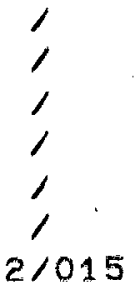

$02 / 015$.

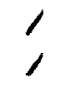

,

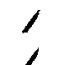

$03 / 103$

,

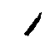

,
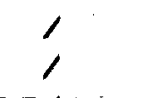

$05 / 136$

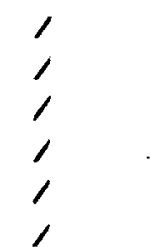

$06 / 187$

$05 / 255$

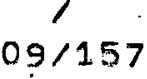

1

$07 / 237$

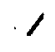

$05 / 013$

$06 / 180$

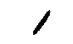

$06 / 237$

$06 / 172$

$06 / 237$

$07 / 191$

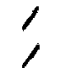

$08 / 187$

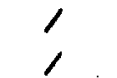

$09 / 083$

$09 / 210$

$09 / 067$

$09 / 047$

$07 / 183$

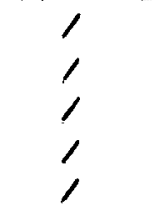

$08 / 019$

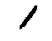

$07 / 171$

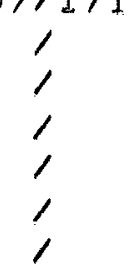

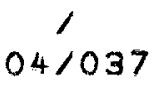

$07 / 025$

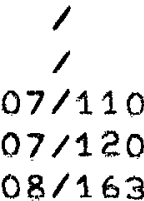

$08 / 083$

$07 / 024$

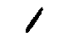

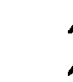

$11 / 019$

11/ $/ 018$

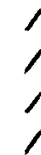

$08 / 138$

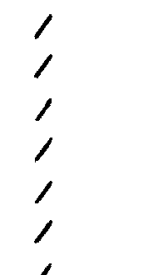

$07 / 081$

$11 / 095$

$08 / 153$

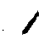

$11 / 103$

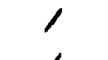

$09 / 080$

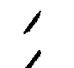

1

1

,

,

,

,

,

1

$09 / 053$

$09 / 051$

$09 / 057$

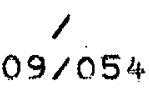

$08 / 202$

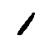

$06 / 237$

$06 / 258$

$05 / 149$

$06 / 228$

$11 / 092$

$11 / 092$

$11 / 098$

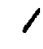

1

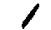

$08 / 062$

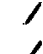

,

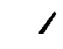

$07 / 080$

$07 / 044$

$05 / 197$

$6 / 110$

$06 / 070$

$06 / 178$

$06 / 216$

$06 / 223$

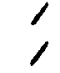

$06 / 084$

$08 / 106$

$07 / 181$

$07 / 073$

$07 / 076$

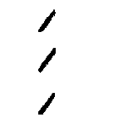


PQURING

PEWDER

PQWER

POWHATAN

$P R=R=R$

PRACTICE

PRACTTCED

PRAIRIE

PRAISE

PRAISED

PRAYER

PRAYING

PREACHER

PRECIUUS

PREFIX

PREPARE

PREPARED

PREPARES

PREPARING

PRESENT

PRESENTS

PRESIDENT

PRETEND

PRETENDED

PRETENDING

PRETTY

PRETZELS

PREVENTION

PRINCE

PRINCESS

PRINTED

PRISENER

PRIZE

PRIZES

PREBABLY

PREBLEM

PREBLEMS

PREFESSOR

PROGRAM

PROJECT

PROMISE

PROPELLER

PREPER

PREPERTY

PRETECT

PROTECTED

PRETECTS

PRETESTED

PROUD

PREUDEST

PREUDLY

PREVE

PROVED

PTERANADEN

PUDDLE

PUEBLOS

PUERTG RICE

PUFF

PULL

PULLED

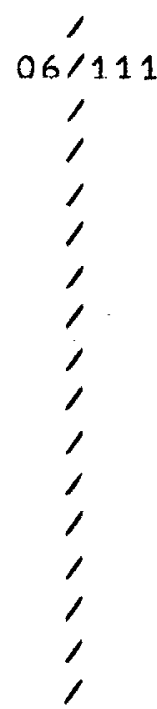

$09 / 081$

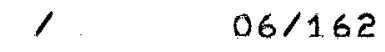

$1 \quad 05 / 060$

$09 / 236$

1

$09 / 138$

1

$07 / 160$

$08 / 204$

1

$07 / 186$

$07 / 186$

1

$09 / 135$

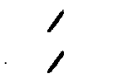

$08 / 068$

1

$08 / 045$

1

1

$07 / 107$

$09 / 228$

1

,

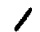

$06 / 078$

1

$06 / 187$

$07 / 034$

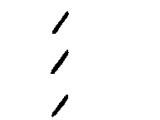

$08 / 242$

$09 / 130$

$09 / 085$

i

$04 / 074$

$06 / 008$

$06 / 165$

$06 / 094$

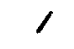

$06 / 085$

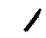

$04 / 057$

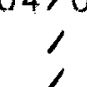

$05 / 265$

$04 / 055$

$0.5 / 092$

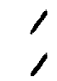

$02 / 083$

$05 / 099$

$05 / 029$

I

1

$04 / 118$

$06 / 137$

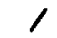

$06 / 040$

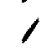

$05 / 111$

$06 / 177$
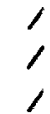

$04 / 143$

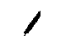

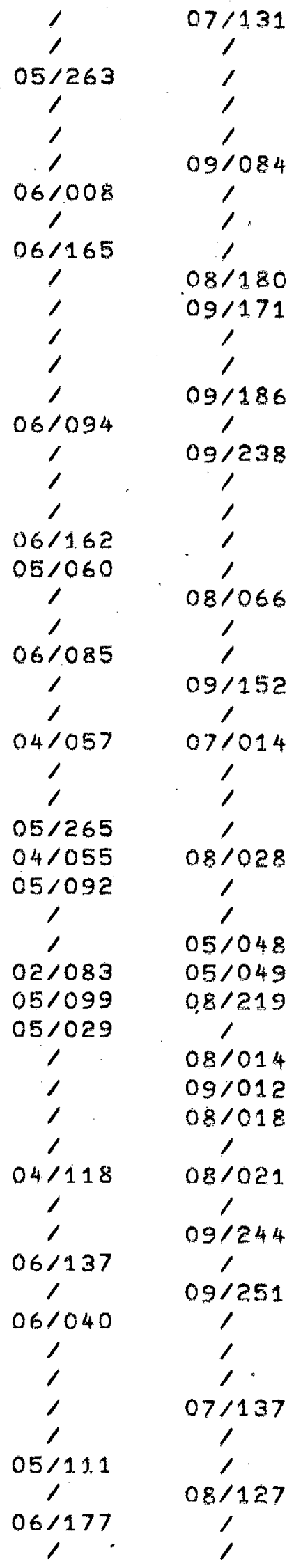
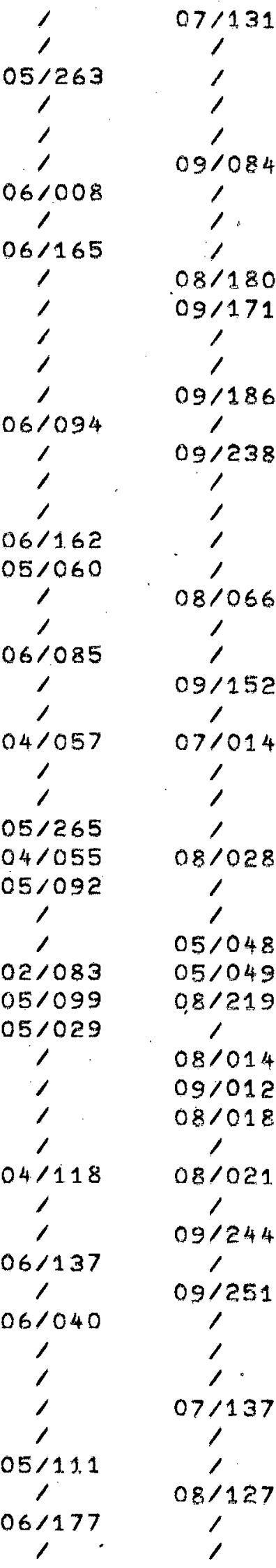

$01 / 159$

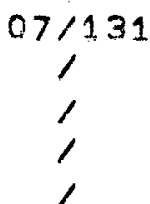

$09 / 084$

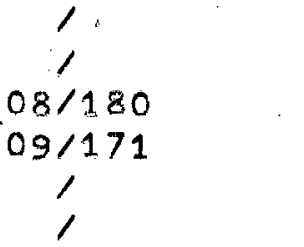

$9 / 186$

1238

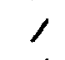

$08 / 066$

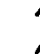

$09 / 152$

$07 / 014$
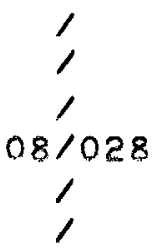

$05 / 048$

$05 / 049$

$08 / 219$

1

$08 / 014$

$09 / 012$

$08 / 018$

$08 / 021$

$09 / 244$

$09 / 251$

1

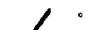

$07 / 137$

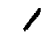

$08 / 127$

,

1
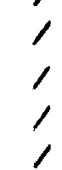

$06 / 131$

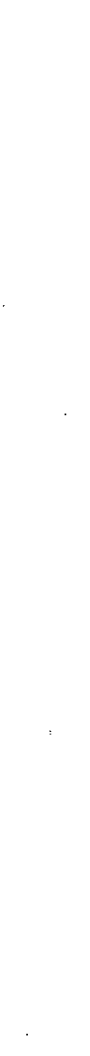

,

$07 / 081$

$08 / 159$

$06 / 222$

$07 / 079$

$07 / 027$

,

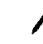

$05 / 203$

$08 / 106$

$08 / 032$

1

1

$07 / 132$

I

!

$06 / 200$

$08 / 127$

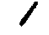

$06 / 032$

$04 / 083$

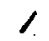

$07 / 043$

$06 / 220$

$08 / 170$

$08 / 135$

$08 / 159$

$08 / 083$

$07 / 040$

$08 / 130$

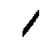

,

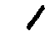

$07 / 069$

$07 / 027$

1

I

'

$08 / 158$

$08 / 149$

$07 / 201$

$11 / 026$

$11 / 047$

$07 / 141$

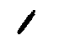

$08 / 115$

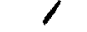

$08 / 191$

1 
PULLEYS

PULLING

PULP

PUMPKINS

PUNCH

PUNCTUATIEN

PUNISH

FUP

PUPILS

PUPPET

PUPPETS

PUPPIES

PUPPY

PUPPY'S

PURE

PURPLE

PURPESES

PUSH

PUSHED

PUSHER

PUSHERS

PUSHING

PUT

PUTS

PUTTER

PUTTING

PUZZLE

PUZZLED

QUACK

QUACKING

QUARREL

DUARRELING

QUARTER

QUARTERS

QUEEN

QUEER

QUESTIUN

QUESTI ONED

QUESTIONS

QUICK

QUICKLY

QUIET

QUIETLY

QUIMBY

QUINN

QUIT

QUITE

QUETATIEN

RABBIT

RABBITS

RACCOON

RACCEONS

RACE.

RACING

RACKET

RADIO

RADISH

RAFT

RAGE

RAGGED

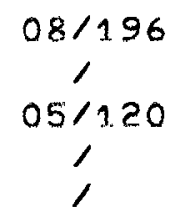

$08 / 117$

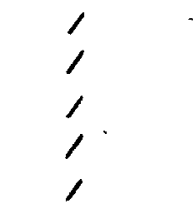

081009
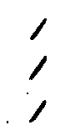

,

$09 / 104$

$08 / 126$

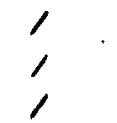

$04 / 135$

1

,

,

1

$05 / 109$

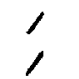

,

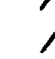

,

r

1

$06 / 075$

$07 / 165$

$08 / 055$
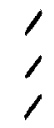

,

,

$01 / 017$

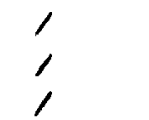

$05 / 066$

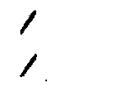

$07 / 194$

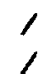

,

,

,

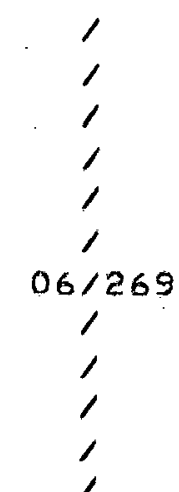

\section{$03 / 085$}

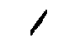

$06 / 292$

$03 / 031$

1

$03 / 096$

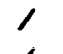

,

,

$01 / 027$

1

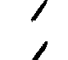

$06 / 067$

1

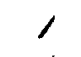

061060

$06 / 278$

$06 / 306$

$04 / 099$

$04 / 014$
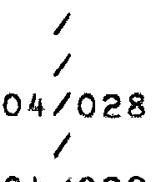

$04 / 032$

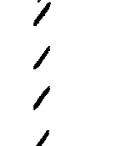

$05 / 134$

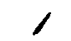

$01 / 064$

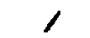

$02 / 017$

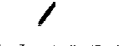

$02 / 084$

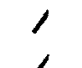

$06 / 286$
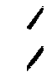

$06 / 057$

$04 / 090$

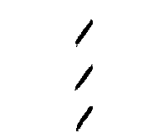

$09 / 214$
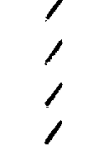

$09 / 066$

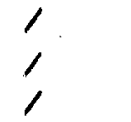

$05 / 043$

$05 / 055$

',

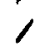

$06 / 034$

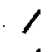

,

1

$04 / 067$
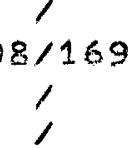

$08 / 226$

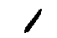

$07 / 089$

$05 / 057$

1

$08 / 073$

1

$08 / 245$

$08 / 078$
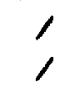

$06 / 233$

$07 / 029$

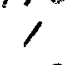

$08 / 014$
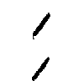

$06 / 249$

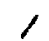

$05 / 154$
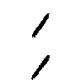

$09 / 114$

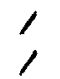

$09 / 047$

$08 / 140$

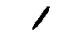

$07 / 105$

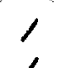

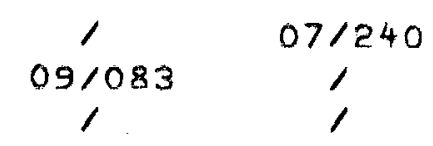

$06 / 248$

$08 / 026$

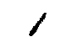

$06 / 097$

$07 / 123$

$07 / 122$

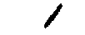

$07 / 025$
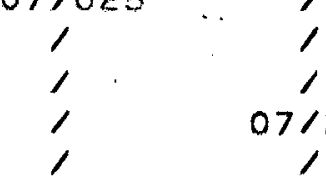

$07 / 231$

$11 / 106$ $11 / 069$

$11 / 113$

$11 / 113$

$11 / 096$

$09 / 026$

$09 / 076$

$09 / 060$

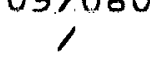

$11 / 011$

$08 / 142$
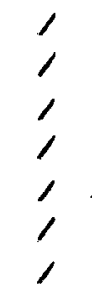

$09 / 021$

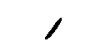

$09 / 024$
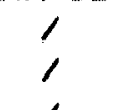

1

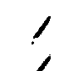

1
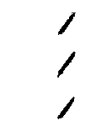

071026

$07 / 1.07$

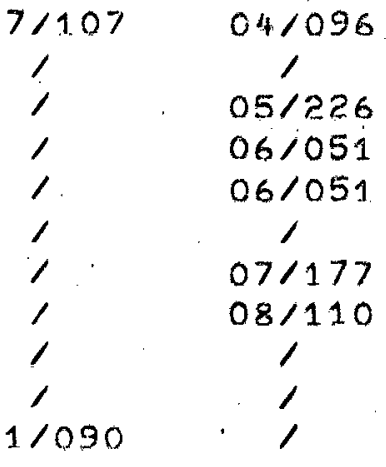


RAGS

RAILROAD

RAIN

- RAINCUAT

RAINED

RAINIEST

RAINY

RAINYEDAY

RAISE

RAISED

RAISING

RAISINS

RAKE

RAKER 'S

RAKING

RAM

RAMBLING

RAMON

RAMON'S

RAN

FANCH

RANCHERS

RANG

RANGER

RAP

RAPIDLY

RAPUNZEL

RARE

RASPBERRY

RATHER

FATS

RATTLE

REACH

REACHED

READ

READING

READS

READY

READY $=M I X$

REAL

REAL IZE

REALIZED

REALIZES

REALLY

REALTIUNS

REAPFEARED

REAR

REARED

REASUN

REBECCA

REBUILT

RECEIVE

RECEIVED

RECKON

RECEGNIZE

RECOGNIZED

RECERD

RECOVERED

RECREATION

RED

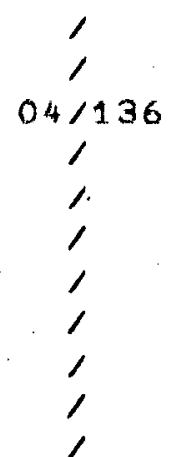

$06 / 170$

1

,

,

,

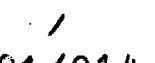

$01 / 014$

,

,

',

$05 / 099$

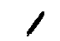

$07 / 187$

$07 / 090$

1

$08 / 156$

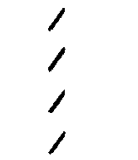

$06 / 041$

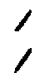

$07 / 050$

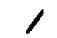

$06 / 034$

$08 / 068$

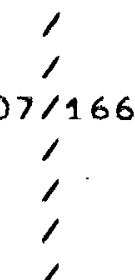

$08 / 179$

,

I

$09 / 241$

$08 / 169$

1

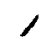

,

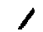

$05 / 022$

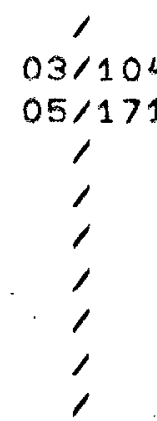

$05 / 172$
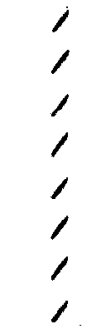

$01 / 153$

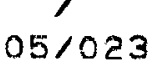

$05 / 245$

$05 / 132$

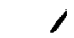

$06 / 035$

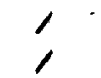

$06 \% 024$

$06 / 126$

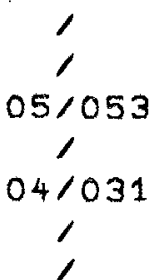

$03 / 202$
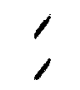

,

$05 / 169$

$03 / 032$
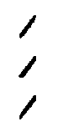

,
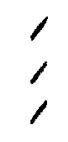

$05 / 223$

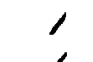

$06 / 242$

$06 / 012$

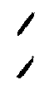

$01 / 059$

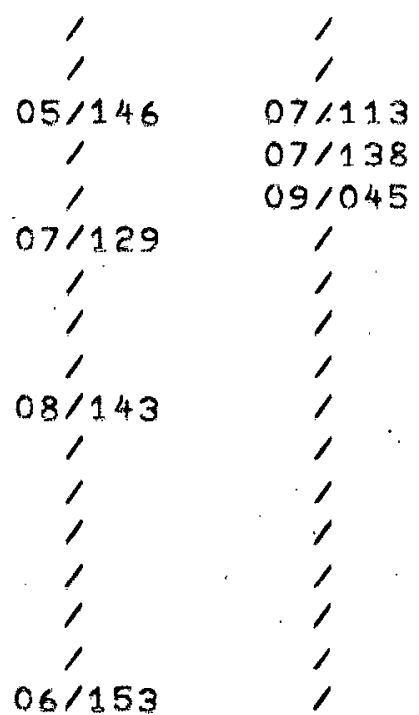

$07 / 109$

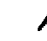

,
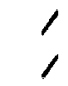

,

1

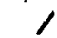

$09 / 185$

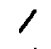

1

$07 / 104$

$06 / 076$

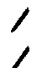

$05 / 111$

$06 / 217$

$06 / 083$

$09 / 049$

,

$06 / 097$

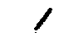

$09 / 119$

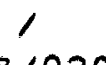

$08 / 030$
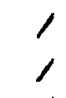

$07 / 106$

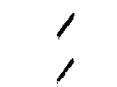

$09 / 125$

1

$08 / 110$

$09 / 091$

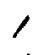

$11 / 083$

$11 / 083$

$07 / 090$

$06 / 108$

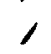

,

,

$06 / 025$

$06 / 029$

$07 / 100$

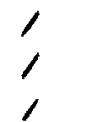

$05 / 196$

$06 / 205$

$06 / 233$

$08 / 238$
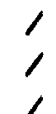

$04 / 066$

$08 / 009$

$05 / 061$

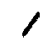

,

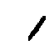

,

,

1

$06 / 205$

$06 / 183$

$06 / 045$

$08 / 078$

$11 / 037$

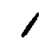

$08 / 183$

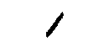

$11 / 017$

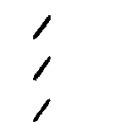

$11 / 038$
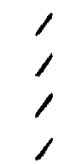

$11 / 082$
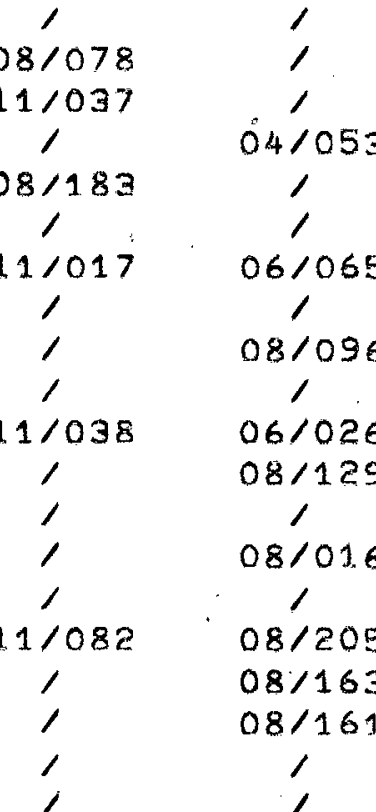

$04 / 053$

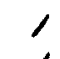

$06 / 065$

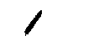

$08 / 096$

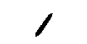

$06 / 026$

$08 / 129$

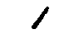

$08 / 016$

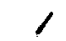

$08 / 205$

$08 / 163$

$08 / 161$

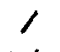

$08 / 148$

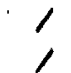

$08 / 201$

$07 / 074$

$06 / 018$ 
REDDISH

\section{REDS}

REDWOED

REEL

REFRIGERATQR

REFUSED

REFUSES

REINS

RELATED

RELAY

RELAYED

RELEASE

RELIEVED

REMA INED

REMARKABLE

REMEMEER

REMEMBERED

REMINDED

REPAIR

REPEAT

REPEATED

REPLIED

REPLY

REFERT

REPORTEP

REPRESENT

REPTILES

RESCUE

RESERVATIEN

RESERVOIRS

RESPECT

REST

RESTAURANT

RESTED

RETOLD

RETREAT

RETURN

RETURNED

REVELVING

REWARD

REWARDS

RHYMING

RIBBON

RIBBONS

RICE

RICH

RICHARD

RICHES

RID

RIDDLE

RIDDLES

RIDE

RIDES

RIDING

RIEHL

RIGHT

RIKKI

RING

RING ING

RINSE.

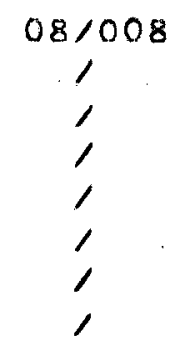

$09 / 208$

$09 / 238$

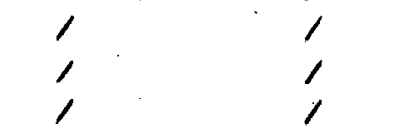

$06 / 071$

$06 / 118$

$07 / 119$

$03 / 077$

,

,

$06 / 091$

$05 / 155$

1

$04 / 195$

$07 / 196$

1

$08 / 241$

$09 / 171$

1

$08 / 249$

1

$06 / 036$

$07 / 219$

,

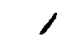

$08 / 158$

,

091040

$09 / 012$

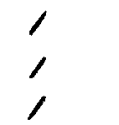

$04 / 081$

,

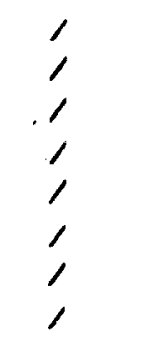

$05 / 157$

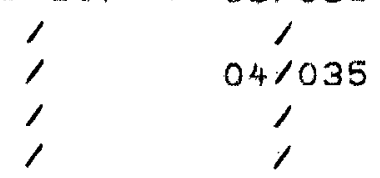

1

,

,

,

1

$04 / 129$

,
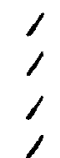

$04 / 130$

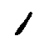

$06 / 147$

. 1

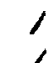

$05 / 095$

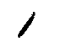

$05 / 262$
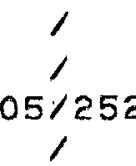

$06 / 067$

$01 / 011$

$01 / 007$

$02 / 124$

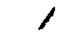

$03 / 038$
SERIES C

SERIES D

SERIES E

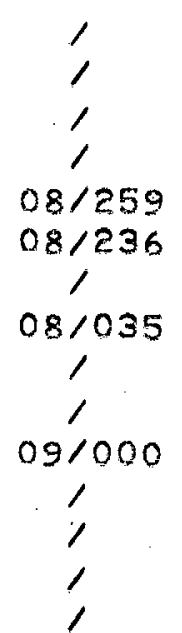

$07 / 153$

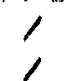

$08 / 261$

$07 / 148$

1

$08 / 249$

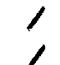

$05 / 252$

$09 / 267$

$04 / 100$

$08 / 125$
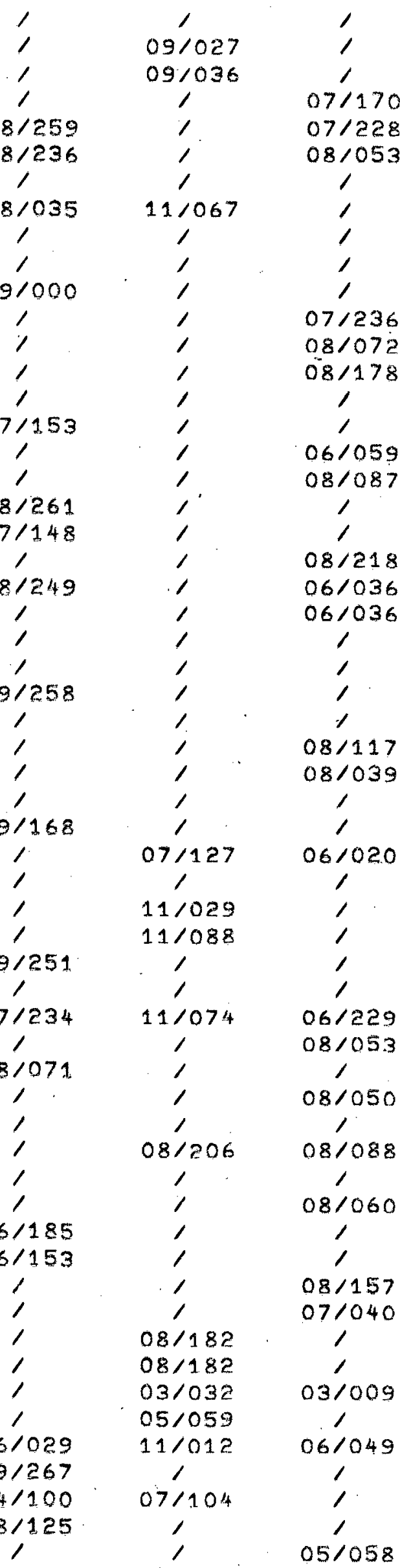

$07 / 236$

081072

$08 / 178$

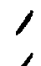

$06 / 059$

$08 / 087$

1

$08 / 218$

$06 / 036$

$06 / 036$
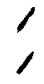

,

$8 / 117$

$08 / 039$

1

$06 / 020$

$07 / 127$

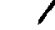

$11 / 029$

$11 / 088$

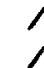

$11 / 074$
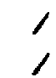

,

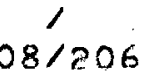

$08 / 206$

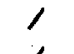

$06 / 185$

$06 / 153$
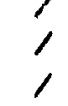

1

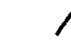

,

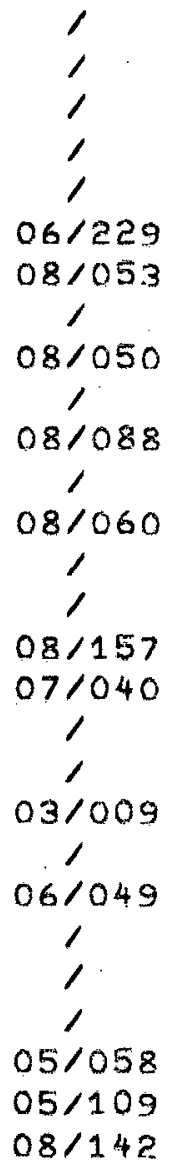


WORD LIST

RIPE

RISE

RIVER

RIVERS

RUAD

READS

REAN

REAR

ROARED

ROAST

REASTED

REBBED

FEBBERS

ROBE

RQBERT

ROBOT

REBOTS

ROCK

ROCKET

RECKETS

ROCKS

ROCKY

ROD

RODE

RODEE

RUGER

REGERS

ROLFE

RELL

RGLLED

RELLING

ROMONA

REOF

ROOFED

ROOM

REOSTER

ROQT

ROPE

ROSE RED

ROSE =PETALS

ROSY

RETER

ROTTEN

REUGH!

ROUND

REUNDED

ROUNDISH

ROUNDS

RUUSE

REUTE

ROW

REWS

ROYAL

RUBBED

RUBBING

RUBS

RUFE

RUFFLE

RUFFLING

RUG
SERIES A SERIES

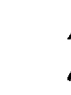

$06 / 149$

1

$04 / 061$

1

$07 / 073$

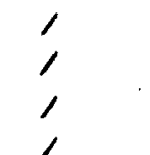

$05 / 094$

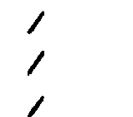

$09 / 214$

1

$04 / 024$

1

$05 / 071$

. 1

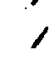

1

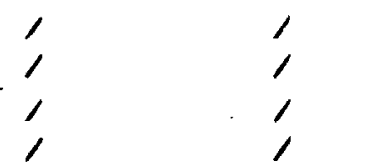

$06 / 159$

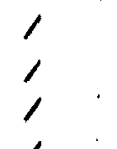

$05 / 012$

$05 / 092$

1

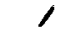

$07 / 1.87$

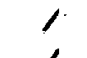

$07 / 198$

$08 / 097$

$08 / 218$

$06 / 1.85$

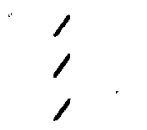

$08 / 064$

$09 / 107$
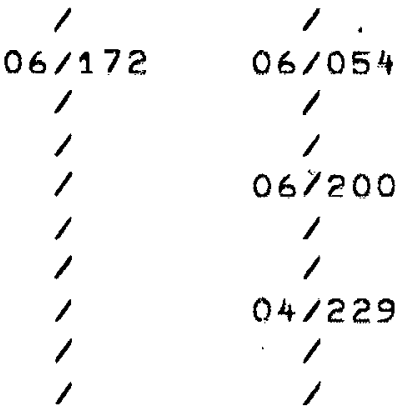

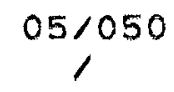

$05 / 267$

$03 / 033$

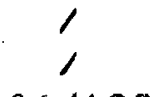

$06 / 199$

$05 / 271$

$06 / 138$

$04 / 139$

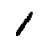

,

1

1

,

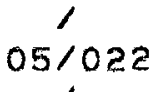

$04 / 060$

$04 / 211$

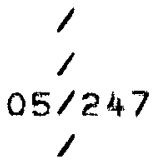

$03 / 121$

$05 / 080$

$06 / 090$

$02 / 089$

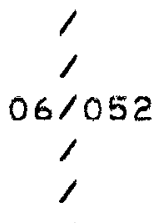

$03 / 014$
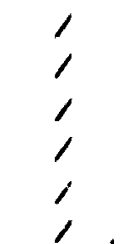

$06 / 054$

$06 \% 200$

041229

,

SERIES C SERIES D

SERIES E<smiles>C1CCCCC1</smiles>

$07 / 207$

$07 / 132$

1

$07 / 110$

1

$07 / 201$

$09 / 118$

1

,

$06 / 139$

$09 / 029$

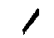

071047

$04 / 128$

$05 / 181$

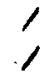

$07 / 111$

$07 / 123$

$07 / 206$

1

,

1

'

$08 / 012$

$07 / 178$

1

$06 / 110$

,

$05 / 195$

1

$09 / 175$

1

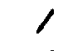

1

1

$07 / 186$

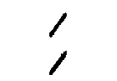

$08 / 013$

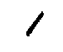

$08 / 196$

$06 / 091$

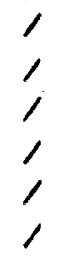

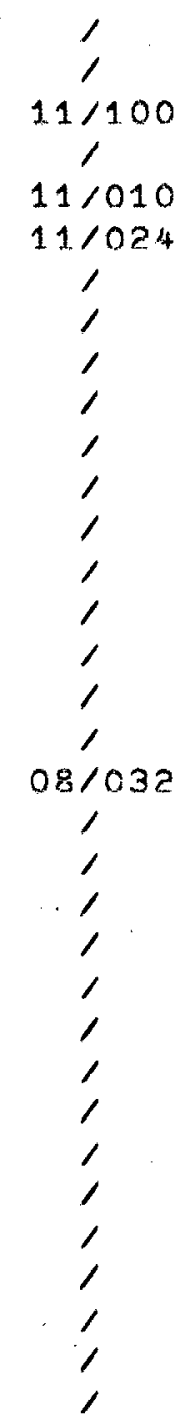

$08 / 099$

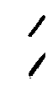

,

,

,

I
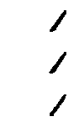

$08 / 125$

$11 / 013$

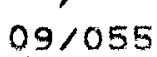

$9 / 055$

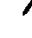

I

'
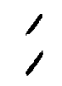

$07 / 015$

$08 / 169$

$05 / 118$

$05 / 119$

$5 / 11.8$

$07 / 120$

$08 / 141$

$06 / 223$

$6 / 197$

$06 / 204$

$11 / 013 \quad 06 / 208$

$06 / 236$

$08 / 029$

$08 / 035$

$07 / 226$ 


\begin{tabular}{|c|c|c|c|c|c|}
\hline RUIN & $07 / 158$ & 1 & I & I & I \\
\hline RULER & $09 / 106$ & ' & 1 & , & $07 / 197$ \\
\hline RULES & 1 & $06 / 047$ & I & I & ' \\
\hline RUN & $04 / 105$ & $01 / 131$ & $04 / 036$ & $07 / 099$ & $01 / 017$ \\
\hline RUNNING & ' & $02 / 086$ & I & $08 / 130$ & 1 \\
\hline RUNNY & 1 & ' & 1 & I & $06 / 242$ \\
\hline RUNS & 1 & ' & $04 / 124$ & ' & ' \\
\hline RUSHED & 1 & $-05 / 248$ & $09 / 043$ & ' & 1 \\
\hline RUSH ING & I & $y$ & 1 & 1 & $06 / 147$ \\
\hline $\begin{array}{l}\text { RUSTLE } \\
\text { RUSTLERS }\end{array}$ & ' & 1 & $\begin{array}{l}07 / 096 \\
07 / 121\end{array}$ & 1 & 1 \\
\hline $\begin{array}{l}\text { RUSTLERS } \\
\text { RUSTY }\end{array}$ & , & , & 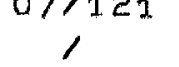 & $09 / 029$ & $08 / 189$ \\
\hline FUSTY:S & 1 & 1 & 1 & $09 / 029$ & 1 \\
\hline RYAN & 1 & , & $09 / 095$ & I & , \\
\hline SABBATH & 1 & 1 & 1 & ' & $04 / 105$ \\
\hline SACK & 1 & ' & $06 / 018$ & , & $08 / 191$ \\
\hline$S A D$ & 1 & $05 / 054$ & 1 & $09 / 035$ & $05 / 220$ \\
\hline SADDLE & ' & 1 & ' & I & $08 / 011$ \\
\hline SADLY & I & ' & $06 / 157$ & 1 & $07 / 140$ \\
\hline SADNESS & ' & 1 & I & ' & $08 / 233$ \\
\hline SAFE & 1 & ' & ' & $08 / 122$ & $07 / 206$ \\
\hline SAFELY & I & $05 / 099$ & 1 & $11 / 030$ & $07 / 214$ \\
\hline SAFETY & I & $06 / 243$ & $\prime$ & I & 1 \\
\hline SAID & $02 / 024$ & $01 / 030$ & 1 & $05 / 062$ & $02 / 008$ \\
\hline SAILING & ' & ' & 1 & ' & $07 / 101$ \\
\hline SAILOR & 1 & $03 / 118$ & ' & 1 & I \\
\hline SAILORS & , & , & $08 / 041$ & ' & ' \\
\hline SAINT & 1 & $06 / 162$ & ' & ' & $\prime$ \\
\hline SAKES & 1 & 1 & $08 / 057$ & ' & ' \\
\hline SALE & I & 1 & ' & I & $07 / 009$ \\
\hline SALES & 1 & $05 / 057$ & 1 & 1 & 1 \\
\hline SALLY & 1 & 1 & I & 1 & $01 / 012$ \\
\hline SALLY'S & 1 & 1 & 1 & ' & $04 / 043$ \\
\hline SALT & $08 / 122$ & $05 / 149$ & $06 / 081$ & / & 1 \\
\hline SALTY & 1 & I & I & I & $07 / 099$ \\
\hline SALUTE & I & 1 & $09 / 262$ & ' & / \\
\hline SAM & ' & 1 & I & 1 & $06 / 201$ \\
\hline SAME & $06 / 061$ & $03 / 189$ & / & $08 / 040$ & $05 / 196$ \\
\hline SAMOSET & / & ' & I & , & $07 / 130$ \\
\hline SAN FRANCISCE'S & / & 1 & I & / & $08 / 060$ \\
\hline SAN JUAN & $09 / 085$ & 1 & I & I & 1 \\
\hline SAN SALVADGR & $09 / 118$ & ' & I & I & ' \\
\hline SAND & I & $02 / 033$ & I & 1 & I \\
\hline SANDINO & 1 & ' & I & $11 / 092$ & I \\
\hline SANDINE'S & ' & ' & I & $11 / 092$ & / \\
\hline SANDWICH & 1 & ' & $08 / 070$ & , & / \\
\hline SANDWICHES & $09 / 190$ & $04 / 111$ & 1 & l & $.08 / 024$ \\
\hline SANDY & $01 / 006$ & ' & I & I & $06 / 188$ \\
\hline SANG & / & $06 / 010$ & $07 / 129$ & I & I \\
\hline SANK & / & I & I & 1 & $08 / 177$ \\
\hline SANTA CLAUS & ' & ' & $08 / 015$ & ' & I \\
\hline SANTA MAPIA & $09 / 111$ & ' & ' & , & , \\
\hline SANTES & I & ' & ' & ' & $06 / 165$ \\
\hline SARAH & , & ' & $08 / 233$ & ' & $08 / 142$ \\
\hline SAT & $02 / 005$ & $02 / 035$ & $05 / 143$ & $07 / 103$ & I \\
\hline SATAN & 1 & ' & I & , & $06 / 232$ \\
\hline SATELLITE & / & 1 & $09 / 073$ & 1 & ' \\
\hline SATELLITES & $09 / 239$ & ' & 1 & ' & ' \\
\hline SATISFIED & , & ' & $09 / 178$ & '. & $08104 ?$ \\
\hline SATURDAY & , & $03 / 190$ & $06 / 047$ & $08 / 091$ & $07 / 054$ \\
\hline
\end{tabular}


SAUCEPAN

SALCER

SAUSAGE

SAVE

SAVING

SAW

SAWMILL

SAY

SAYING

SAYS

SCAMP

SCAMPER

SCAMPERED.

SCARCELY

SCARECREW

SCARED

SCARING

SCARY

SCATTERED

SCENE

SCHEOL

SCHEOLCHILDREN

SCHOOLREOM

SCHOOLTEACHER

SCHWA

SCIENCE

SCIENTISTS

SCISSORS

SCITUATE

SCELD

SCOLDED

SCOLDING

SCOOTER

SCORED

SCOWLED

SCRAPBOOK

SCRAPE

SCRATCH

SCRATCHING

SCREAMED

SCREAMING

SCREECHED

SCREECHING

SCREEN

SCRUBBED

SCRUNCHED

SCUFFLED

SCURRIED

SEA

SEARCH

SEARCHED

SEARCHING

SEASON

SEASONED

SEASENS

SEAT

SEAWEED

SECEND

SECOND =FLOER

SECRET

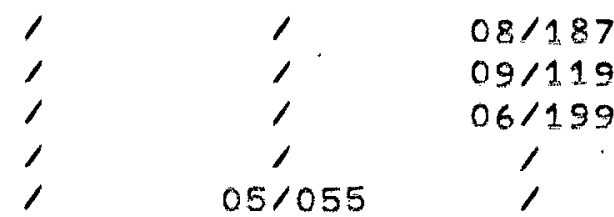

$05 / 149$

$06 / 079$

1

,

,

'

,

1

,

$\frac{1}{09 / 100}$

$05 / 005$

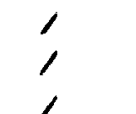

$09 / 061$

$09 / 264$

$09 / 265$

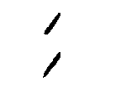

$07 / 028$

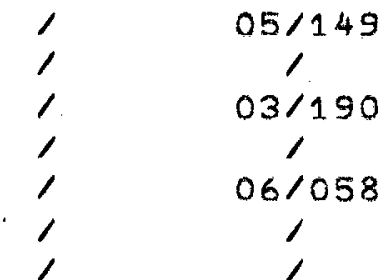

,

.

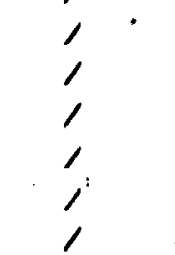

$06 / 159$

,

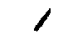

$07 / 035$

$09 / 145$

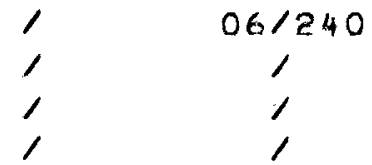

$06 / 260$

$05 / 027$

$03 / 160$

,
$04 / 088$

1

$04 / 154$

1

$05 / 100$

1

$07 / 208$

1

$09 / 048$

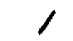

$05 / 132$

$08 / 047$

$09 / 014$

$09 / 129$

$0.6 / 245$

$05 / 208$
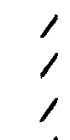

I

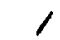

$07 / 079$

1

$09 / 234$
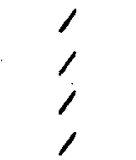

$09 / 089$

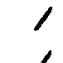

I

$/$
$08 / 153$
$/$
$08 / 241$
$/$

$05 / 047$

$09 / 269$

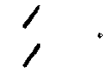

$09 / 120$

$06 / 185$

$07 / 170$

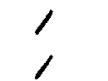

$09 / 134$

1

1

$05 / 170$

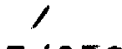

$07 / 053$

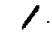

$06 / 045$
SERIES D

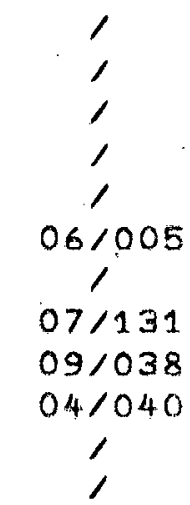

$08 / 132$

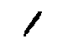

,

,

,

1

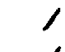

$09 / 050$

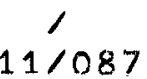

$11 / 087$

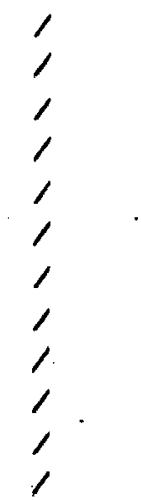

$07 / 155$

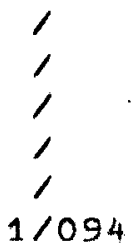

$11 / 094$

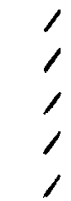

$.08 / 066$
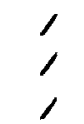

,

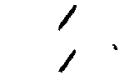

$11 / 011$

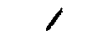

$11 / 030$

$07 / 174$
$06 / 027$

$06 / 194$

$04 / 117$

,

$05 / 093$

$08 / 076$
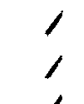

$06 / 035$

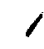

$06 / 142$

$07 / 139$

$04 / 091$

$06 / 208$

$06 / 206$

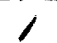

$07 / 168$

,

,

,

,

$07 / 166$

,

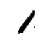

,

1

$08 / 164$

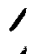

1

$06 / 203$
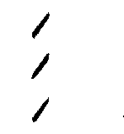

$07 / 110$

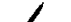

$08 / 096$

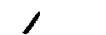

$08 / 031$

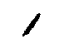

$08 / 209$

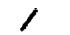

$08 / 218$

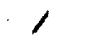

$07 / 01 ?$

$08 / 092$

$05 / 171$

$06 / 041$

$07 / 049$ 
SECTIONS

SEE

SEE-ABLE

SEED

SEEDS

SEE ING

SEEMED

SEEN

SEES

SEESAW

SEIZE

SELECTIENS

SELFISH

SELL

SEMAPHORE

SEND

SENSE

SENSOR

SENT

SENTENCE

SEPTEMBER

SEQUOYAH'S

SERAF INA

SERAF INA'S

SERAPE

SERAPES

SERGEANT

SERIQUS

SER.I OUSLY

SERRVANTS

SERVE

SERVICE

SET

SETH

SETTLED

SETTLEMENT

SETTLERS

SEVEN

SEVENTEEN

SEVENTH-DAY $\triangle D V E N T I S$

SEVERAL

SEW

SEWING

SHADE

SHADOW

SHADOWS

SHADOWY

SHAFTS

SHAG

SHAKE

SHAKES

SHAKING

SHALL

SHAPE

SHAPED

SHARED

SHARK

SHARP

SHARPLY

SHAVED
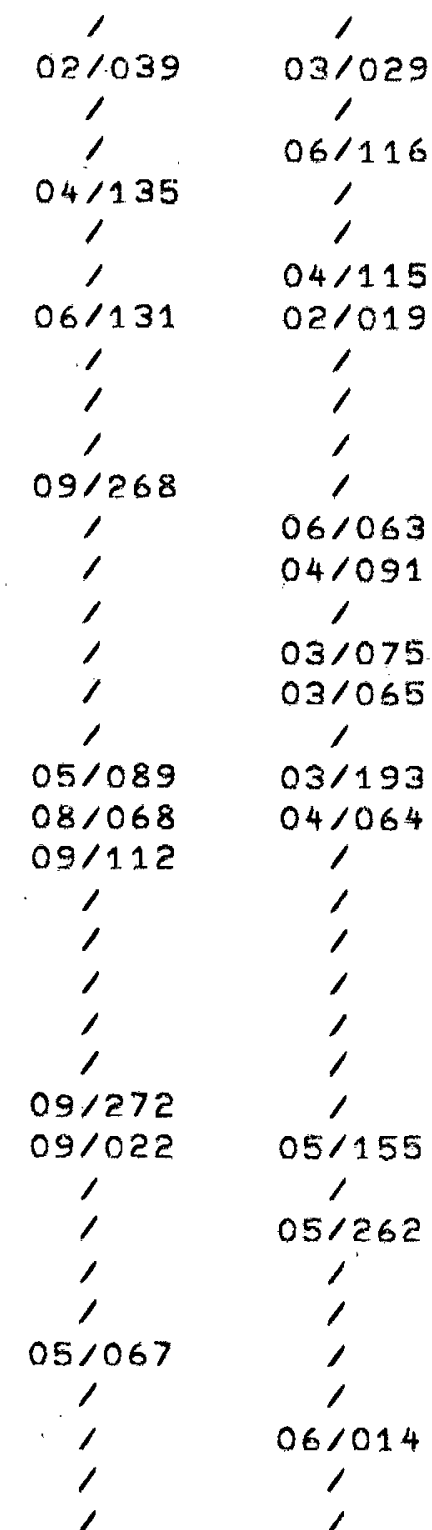

$08 / 054$

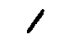

$09 / 165$

$09 / 046$

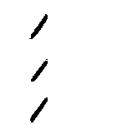

021017

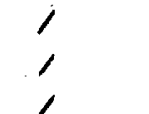

$04 / 096$

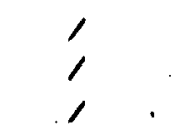

$.06 / 063$

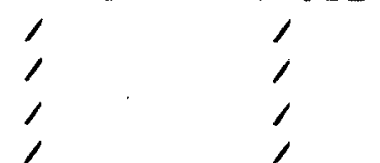

$06 / 010$

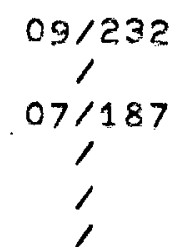

$06 / 230$

$05 / 044$

$04 / 092$

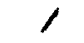

$09 / 172$

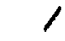

$09 / 180$

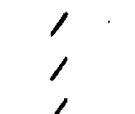

$09 / 049$

$09 / 032$
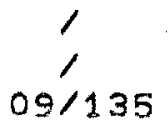

1
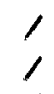

I

$09 / 092$

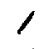

$09 / 181$

$08 / 263$

1

$07 / 207$

,

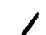

$06 / 064$

$08 / 156$

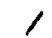

$09 / 015$

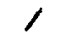

$09 / 106$

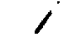

$09 / 176$

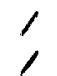

$08 / 239$

$07 / 110$
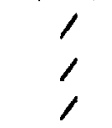

$07 / 108$

$07 / 162$

1

$08 / 111$

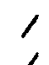

,

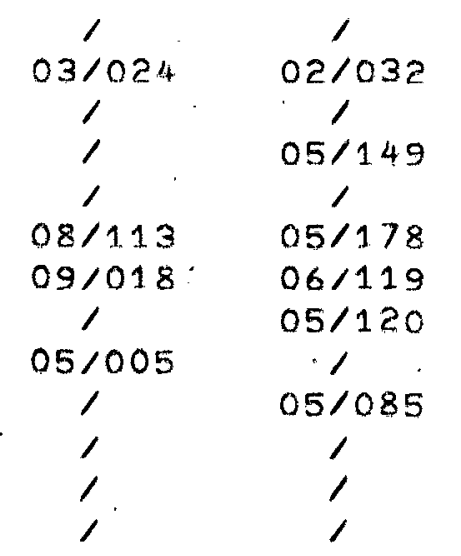

$08 / 022$

$07 / 178$

$05 / 142$

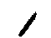

$07 / 110$

'

$08 / 128$

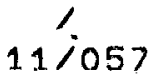

$11 / 059$

$11 / 090$

$11 / 101$

,

,

,

,

'

,

1

1

,

I

,

,

,

$11 / 029$

$07 / 008$

$07 / 008$

$11 / 013$

$11 / 049$

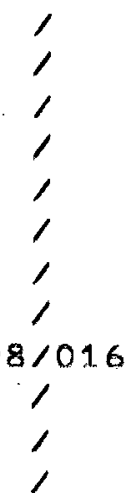

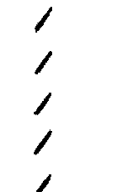

$07 / 196$

$07 / 192$

$08 / 108$

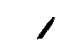

$06 / 215$

1

$07 / 122$

$07 / 105$

$06 / 079$

$07 / 053$

$07 / 050$

$08 / 045$

1

$08 / 013$

1

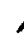

,

1 .

$06 / 026$

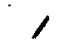

$06 / 136$

$06 / 222$

1

$08 / 065$

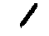

$08 / 031$

$08 / 084$ 
SHAW

SHAWNEE

\section{SHE}

SHEID

SHE'LL

SHEEP

SHEET

SHEETS

SHELF

SHELL

SHELTER

SHELTERED

SHEPHERO

SHER IFF

SHETLAND

SHINE

SHINING

SHINY

SHIP

SHIPS

SHIRT

SHISH

SHIVER

SHOCKED

SHOE

SHGES

SHOIE

SHOLDERS

SHENE

SHOOK

SHOCT

SHOP

SHOPS

SHORES

SHERT

SHERTLY

SHOT

SHOULD

SHEULDER

SHEULDERS

SHQULON'T

SHGUT

SHEUTED

SHEUTING

SHEVED

SHEVEL

SHEVELED

SHOW

SHOWED

SHOWER

SHOWING

SHOWS

SHRIEKED

SHRUNK

SHUFFL ING

SHULTZ

SHUT

SHUTTLE

SHYLY

SICK

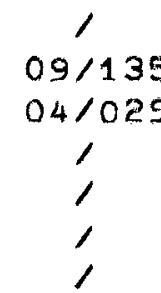

$05 / 127$

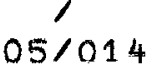

$09 / 158$

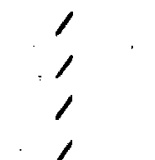

$07 / 017$

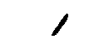

$07 / 040$
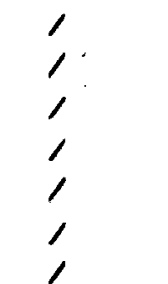

$07 / 161$
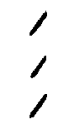

$07 / 099$

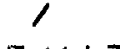

$05 / 147$

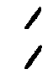

$07 / 131$
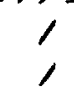

$07 / 110$

$08 / 086$

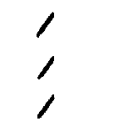

$06 / 187$

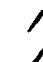

$08 / 022$

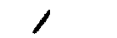

$06 / 015$

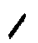

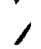

$$
1
$$$$
1
$$
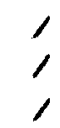

$$
\text { , }
$$

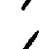
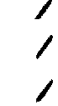

$06 / 018$

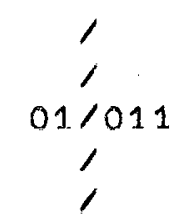

$04 / 124$

$06 / 013$

$05 / 096$

$06 / 257$

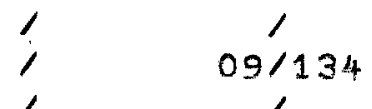

,

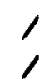

$05 / 111$

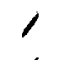

$05 / 190$

$06 / 232$

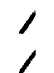

$06 / 236$

$02 / 119$

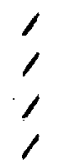

$05 / 169$

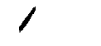

$05 / 164$

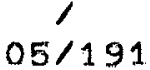

$03 / 046$

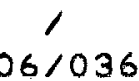

$02 / 010$ 1

$06 / 233$

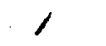

$04 / 037$
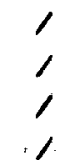

$06 / 182$

1

$06 / 280$
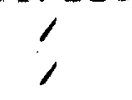

$06 / 311$

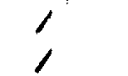

$04 / 137$

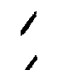

$05 / 272$
$06 / 138$

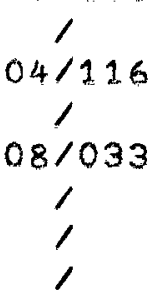

$07 / 022$

$09 / 134$

$08 / 016$

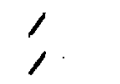

$07 / 160$

$06 / 044$

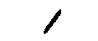

$06 / 130$

$06 / 101$

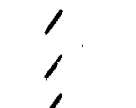

$08 / 053$

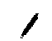

$09 / 017$
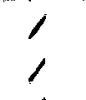

$06 / 118$

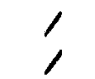

$07 / 134$

$07 / 045$

$06 / 066$
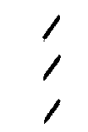

$06 / 159$

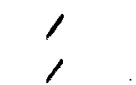

$09 / 047$

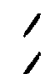

$04 / 026$

$05 / 049$
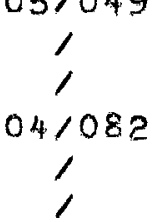

$09 / 101$

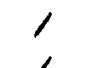

$08 / 201$

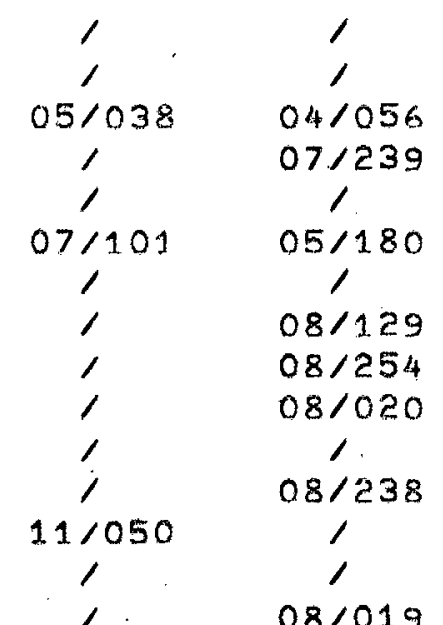

$08 / 019$

$08 / 064$

$07 / 097$

$08 / 221 \quad 06 / 123$

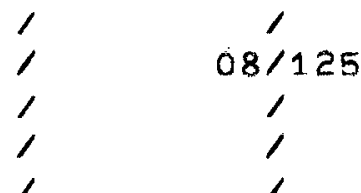

$07 / 234$

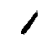

$08 / 069$

$06 / 067$

$07 / 148$

$08 / 035$

$11 / 016$

$11 / 014$
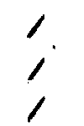

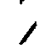

,

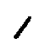

,

$9 / 045$

$08 / 084$

$09 / 044$
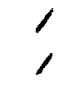

$08 / 010$

$08 / 079$

$$
1
$$

$09 / 063$
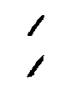

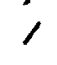

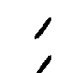

$08 / 216$
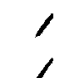

$11 / 012$
$07 / 105$

$05 / 184$

$07 / 176$

$07 / 232$

$05 / 157$

$08 / 125$

$06 / 109$

$05 / 092$

$05 / 092$

$05 / 093$

$07 / 234$

$05 / 124$

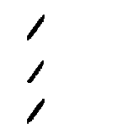

$08 / 190$

$05 / 054$

$08 / 052$

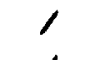

$07 / 070$

$06 / 099$

$08 / 021$

$08 / 115$ 
SICKNESS

SID

SIDE

SIDES

SIDEWALK

SIDEWAYS

SIGH

SIGHED

SIGHT

SIGN

SIGNAL

SILENCE

SILENT

SILENTLY

SILK

SILL

SILLY

SILVA

SILVER

SILVERSPOT

SIMEN

SIMPLE

SIMPLY

SIMPSEN

SINCE

SINES

SING

SINGLE

SINGS

SINK

SIOUX

SIR

SIR CHARLES

SIRREE

SISTER

SISTER'S

SISTERS

SIT

SITS

SITTING

SIX

SIXTEEN

SIXTY-NINE

SIZE

SK.ATE

SKATER

SKATES

SKATING

SKEIN

SKIED

SKIING

SKILLS

SKINKS

SKIP

SKIPPED

SKIRT

SKIRTS

SKIS

SKUNK

SKY

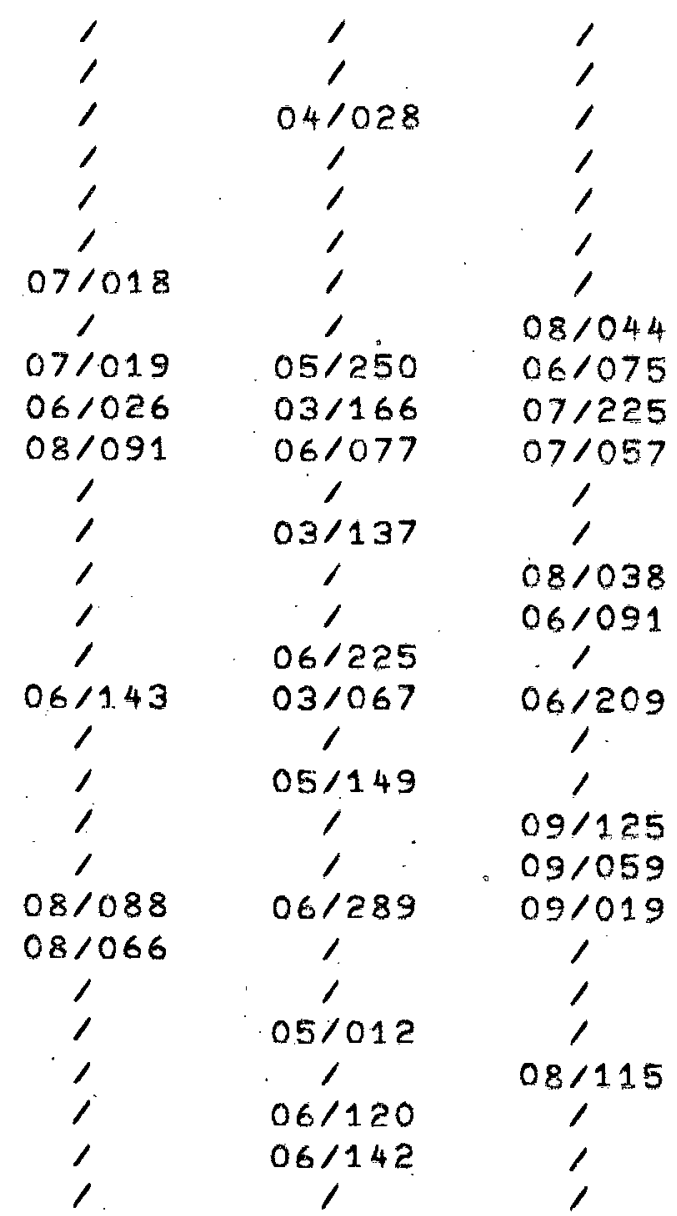

$05 / 160$

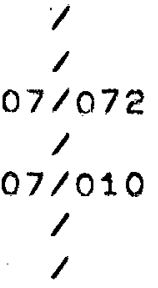

$04 / 063$

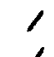

$05 / 109$

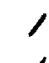

,

,

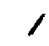

,

I

,
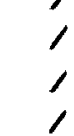

$05 / 167$

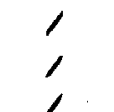

$07 / 056$

1

$09 / 090$

.1

$04 / 007$

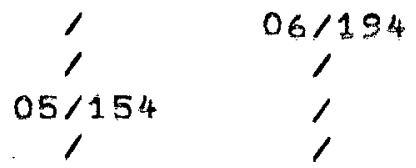

$08 / 019$

$05 / 012$

$05 / 044$

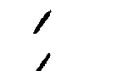

$04 / 097$

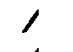

$06 / 085$
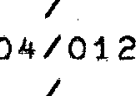

,

,

i

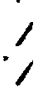

$\therefore$

$06 / 132$

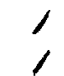

,

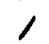

$06 / 117$

$06 / 042$

$05 / 073$

$04 / 114$

$05 / 024$

$06 / 060$

1

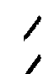

$06 / 027$

$06 / 031$

$08 / 200$

$07 / 216$

$07 / 216$

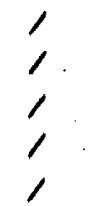

$09 / 249$

$07 / 215$

$07 / 206$
$03 / 185$

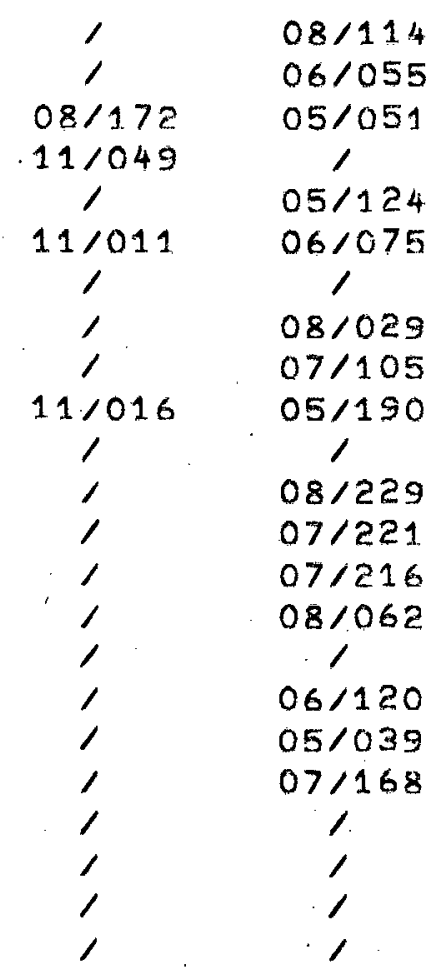

$07 / 053$

$06 / 138$.

$08 / 014$

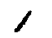

$08 / 015$

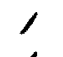

,

,

,

$05 / 016$

$07 / 039$

$07 / 004$

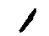

,

1

$06 / 195$

$08 / 144$

.

$08 / 041$

$08 / 228$

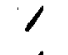

$05 / 068$

,

$05 / 045$

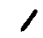

$06 / 021$

$05 / 134$

$1 / 099$

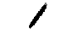

1

$07 / 042$

$09 / 010$

$07 / 041$

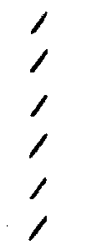

$08 / 045$
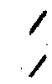

,

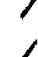

$07 / 084$
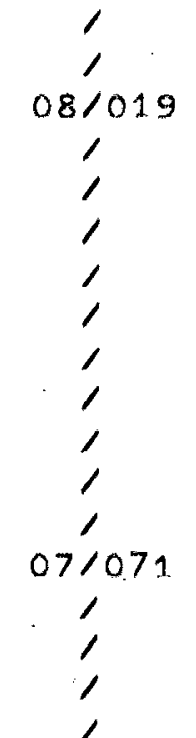

$08 / 094$ 


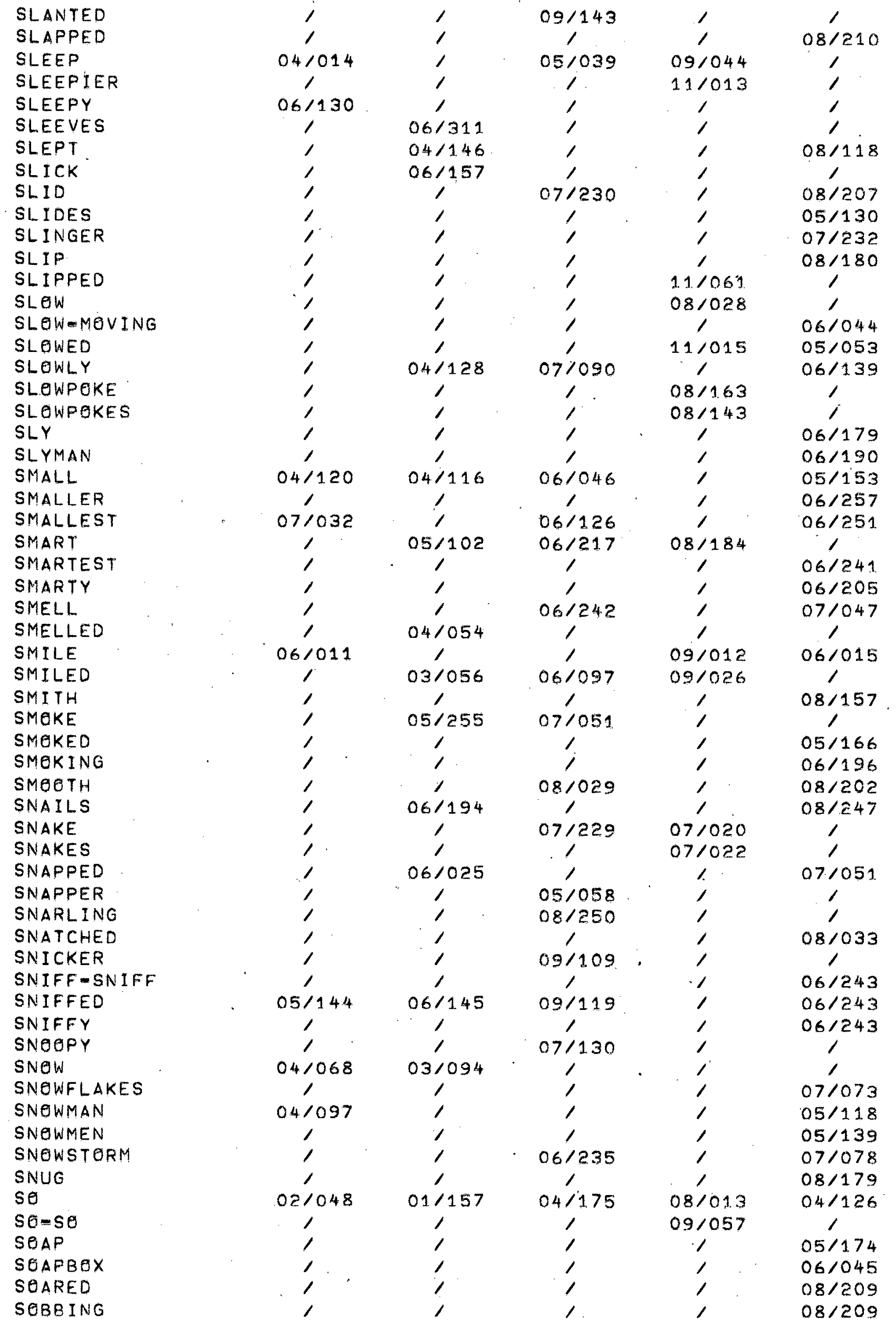




\section{SOBS}

SECIETY

SOCK

SOCKET

SOCKS

SEFT

SOFTLY

SOIL

SOLD

SOLDIERS

SOLEMN

SULES

SOLVE

SOLVING

SOME

SOMEBODY

SOMEDAY

SOMEHOW

SOMEONE

SEMERSAULT

SOMETHING

SOMETHINGNESS

SOMETIME

SOMET IMES

SOMEWHAT

SOMEWHERE

SON

SENG

SENNY

SENS

SOO-PUNG

SOON

SUONER

SORE

SORRY

SERT

SES

SUUND

SEUNDS

SEUP

SOUTH

SOW

SPACE

SPAIN

SPANIARDS

SPANISH

SPANKED

SPARED

SPARK

SPARKLED

SPARKLING

SPARKS

SPATTERED

SPEAK

SPEAKING

SPECIAL

SPECKLE

SPEECH

SPEECHLESS

SPEED

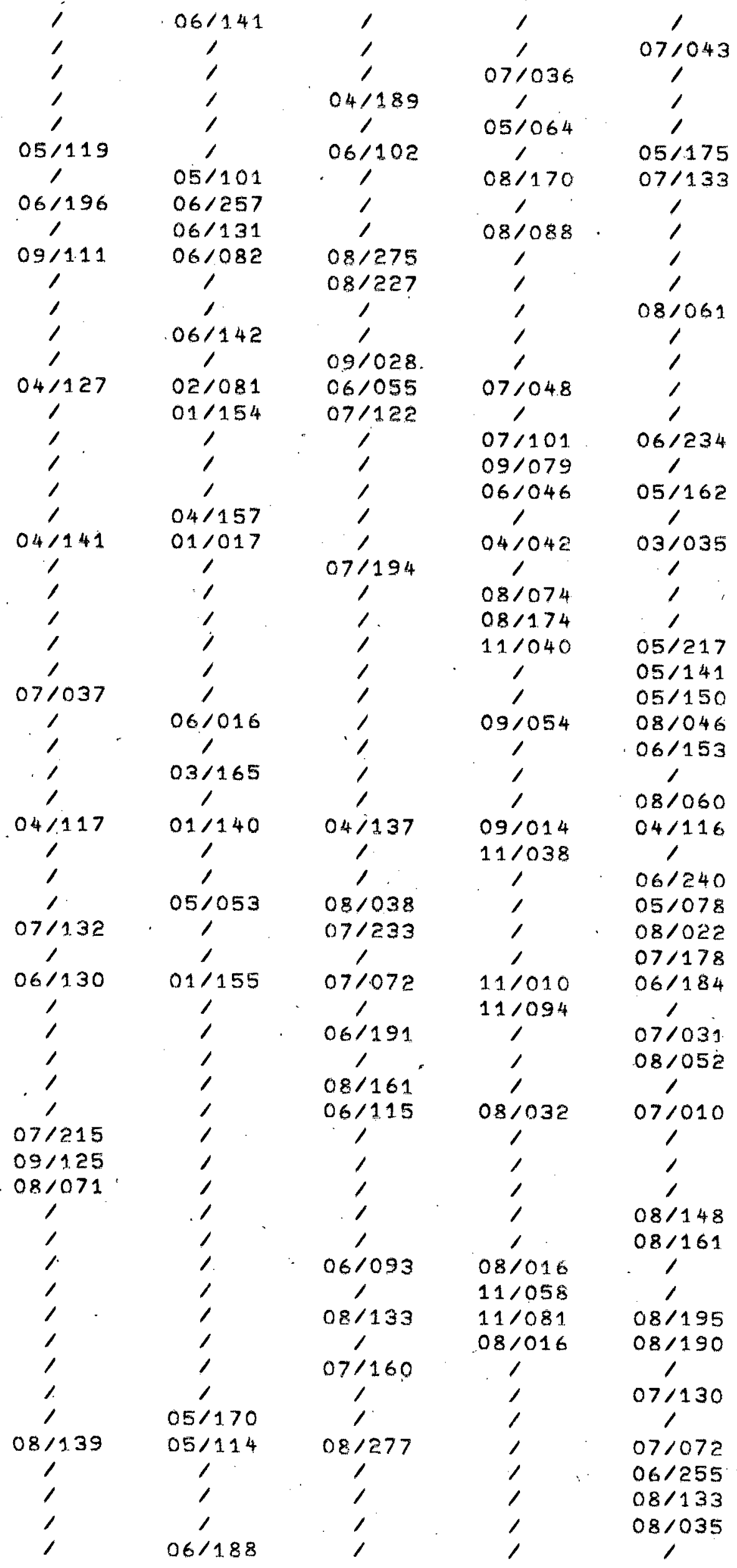


SPELL

SPELLER

SPELLING

SPELLS

SPEND

SPENT

SPICES

SPIDER

SPIED

SPILL

SPILLED

SPIN

SPINDLE

SPINE

SPINNERETS

SPIRITS

SPITZ

SPLASH

SPLASHED

SPLIT

SPLUTTERING

SPOIL

SPOILED

SPOKE

SPOEKIER

SPOOKY

SPEONS

SPET

SPETS

SPETTED

SPQUTS

SPRANG

SPRAYS

SPREAD

SPREADING

SPRIG

SPRING

SPRINGFIELD

SPRINGTIME

SPRINKLE

SPREUT

SPREUTS

SPUTTERED

SQUANTO

SQUARE

SQUAWKS

SQUEAKY

SQUEALING

SQUEEZE

SQUINTING

SQUIRREL

SQUIRRELS

SQUISH

STAG

STAGE

STAIRS

STAIRWAYS

STAKE

STALL

STAMPED
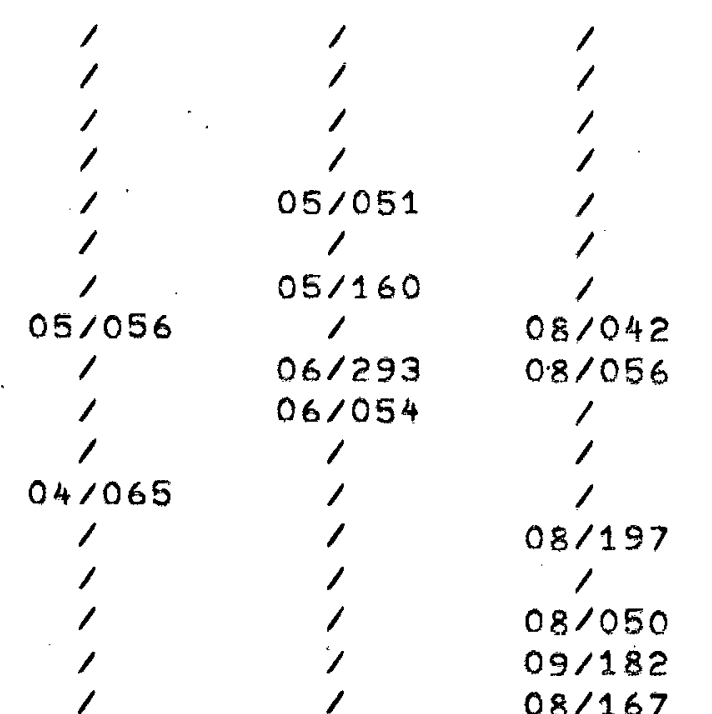

$02 / 033$

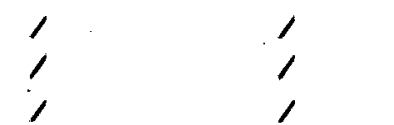

$06 / 185$

1

$05 / 135$

1,

1,1

$06 / 103, /$
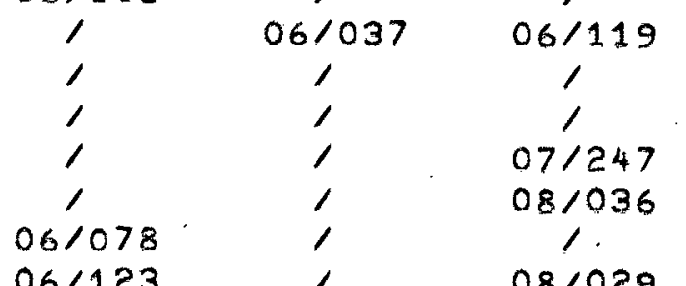

$06 / 123$

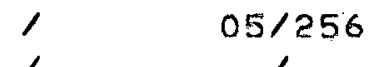

,

,

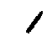

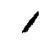

,

(

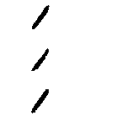

$09 / 098$

,

,

,

1

$06 / 1.51$

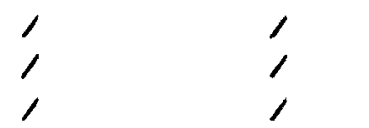

$06 / 286$

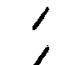

$06 / 268$

$05 / 041$
$08 / 181$

$08 / 199$

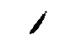

$08 / 196$

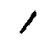

,

,
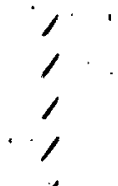

$08 / 197$

$08 / 050$

$09 / 182$

$08 / 167$

$07 / 100$

$08 / 108$

$08 / 182$
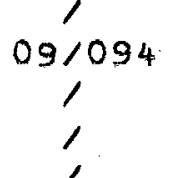

$06 / 119$

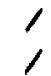

$07 / 247$

$08 / 036$

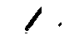

$08 / 029$

$09 / 207$

$06 / 181$
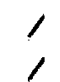

$09 / 190$

$08 / 129$

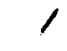

$07 / 053$

$08 / 061$

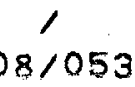

$08 / 053$

$07 / 231$

$08 / 031$

$07 / 097$

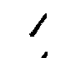

,
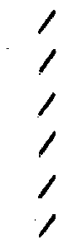

$09 / 118$

$11 / 029$

$11 / 010$

,

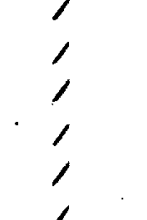

$08 / 153$

$11 / 012$

$08 / 159$

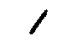

,

I

,

I

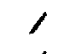

I

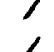
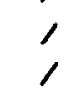

$07 / 086$

$07 / 124$
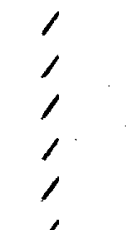

11. 1091

,

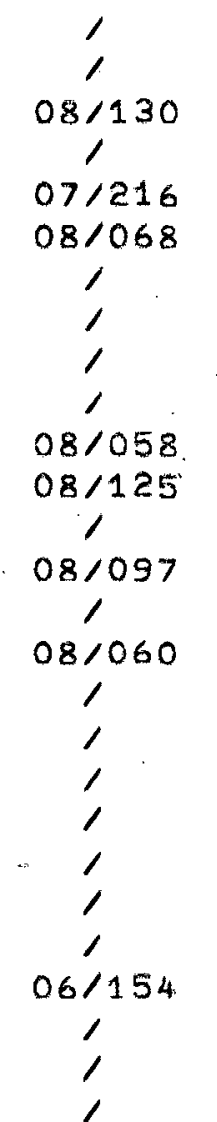

$01 / 016$

\section{$06 / 220$}

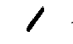

$07 / 184$
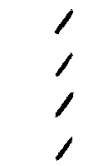

$05 / 148$

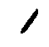

$08 / 062$

$08 / 056$

$07 / 135$

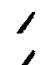

$06 / 205$

$06 / 118$
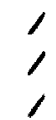

1

$08 / 143$

$08 / 069$

$06 / 241$

$06 / 245$

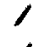

$07 / 124$ 
STAN

STAND

STANDING

STANDISH

STANDS

STAR

STAR-SPANGLED

STARE

STARED

STARING

STARRY

STARS

START

STARTED

STARTING

STARTLED

STARTS

STATE

STATEMENT

STATES

STATION

STATUE

STATUES

STATUTE

STAY

STAYED

STAYING

STAYS

STEADILY.

STEADY

STEAL

STEAL ING

STEAM

STEAMING

STEEL

STEEP

STEER

STEERED

STEGESAURUS

STEM

STEP

STEPHEN

STEPPED

STEPS

STERN

STERNLY

STEVE

STEW

STICK

STICKING

STICKS

STIFFLY

STILL

STILLNESS

STIRRING

STUCKADES

STUCKINGS

STELEN

STEMACH

STOMACH-CLOCKS

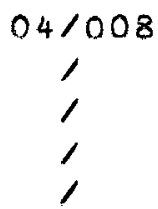

$04 / 009$

$06 / 166$

$04 / 036$

$07 / 133$

1

I

1

/ $05 / 109$

/.

,

',

$09 / 193$

$5 / 037$

,

,

l

$09 / 151$

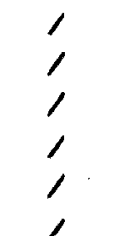

$06 / 223$

$08 / 239$

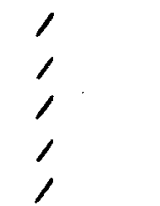

$07 / 165$

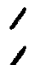

,

,

,

,

2'́13

(

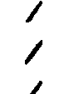

,

1

$08 / 123$

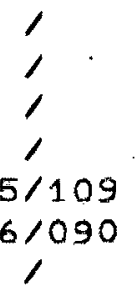

$03 / 101$
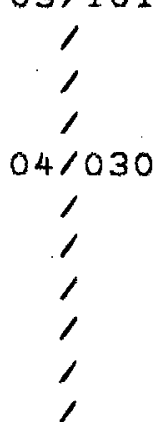

$06 / 074$

$06 / 186$

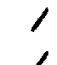

$05 / 219$

$06 / 187$
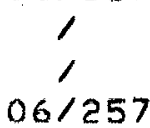

$04 / 146$

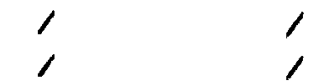

$07 / 102$
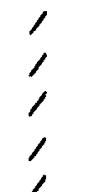

$07 / 146$

$05 / 049$

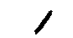

$01 / 155$

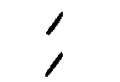

$06 / 076$

$05 / 222$

$06 / 069$
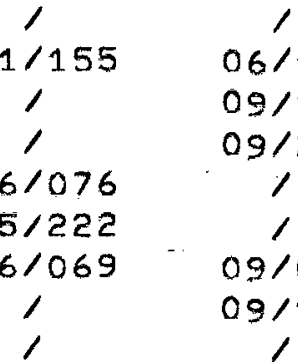

$06 / 173$

$09 / 171$

$09 / 203$

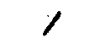

$09 / 095$

$09 / 122$
$09 / 265$

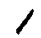

071097

081089

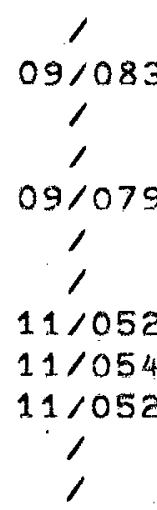

$08 / 023$

$08 / 133$

$09 / 042$

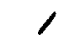

$08 / 187$

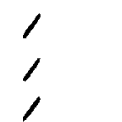

$09 / 075$

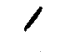

,

$07 / 024$

$09 / 018$

$07 / 068$

$09 / 076$
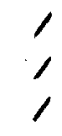

,

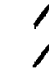

1

$11 / 089$

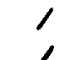

1

$09 / 009$

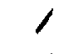

$09 / 045$

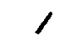

$07 / 010$

1

$08 / 08$

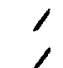

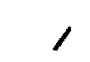

$05 / 165$

$05 / 159$

$07 / 113$

1

$06 / 246$

1

$08 / 035$

$06 / 263$

05/127

$05 / 139$

$08 / 034$

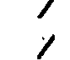

$08 / 202$

$08 / 079$

$05 / 192$

$08 / 137$

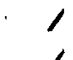

$05 / 059$

$05 / 062$

$05 / 073$

$07 / 149$

$07 / 148$

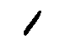

,

$08 / 215$

$07 / 165$

$07 / 212$

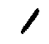

,

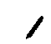

$07 / 116$

$06 / 042$

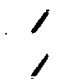

$08 / 145$

$06 / 043$

$07 / 219$

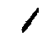

,

081072

$09 / 027 \quad 05 / 044$

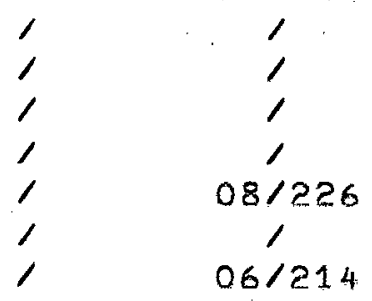


STBMACHS

STONE

STEED

STOEL

STOP

STEPPED

STOPS

ST GRE

STEREHUUSE

STERIES

STORMY

STERY

STORYBOEK

STORYTELLER

STORYTELLER'S

STOVE

STRAIGHT

STRAIGHTENING

STRANGE

STRANGELY

STRANGER

STRAP

STRAPS

STRAW

STRAW-PILE

STRAY

STREAK

STREAKS

STREAM

STREET

STREETS

STRENGTH

STRETCH

STRETCHED

STRIKE

STRING

STRINGER

STRIPES

STRIPS

STREDE

STREKE

STRENG

STRENGER

STRUCK

STRUGGLED

STRUGGLING

STUBBERN

STUCK

STUDIED

STUDY

STUDYING

STUEY

STUFF

STUMBLED

STUMP

STUPID

STURDY

SUBJECT

SUBJECTS

SUBMARINES

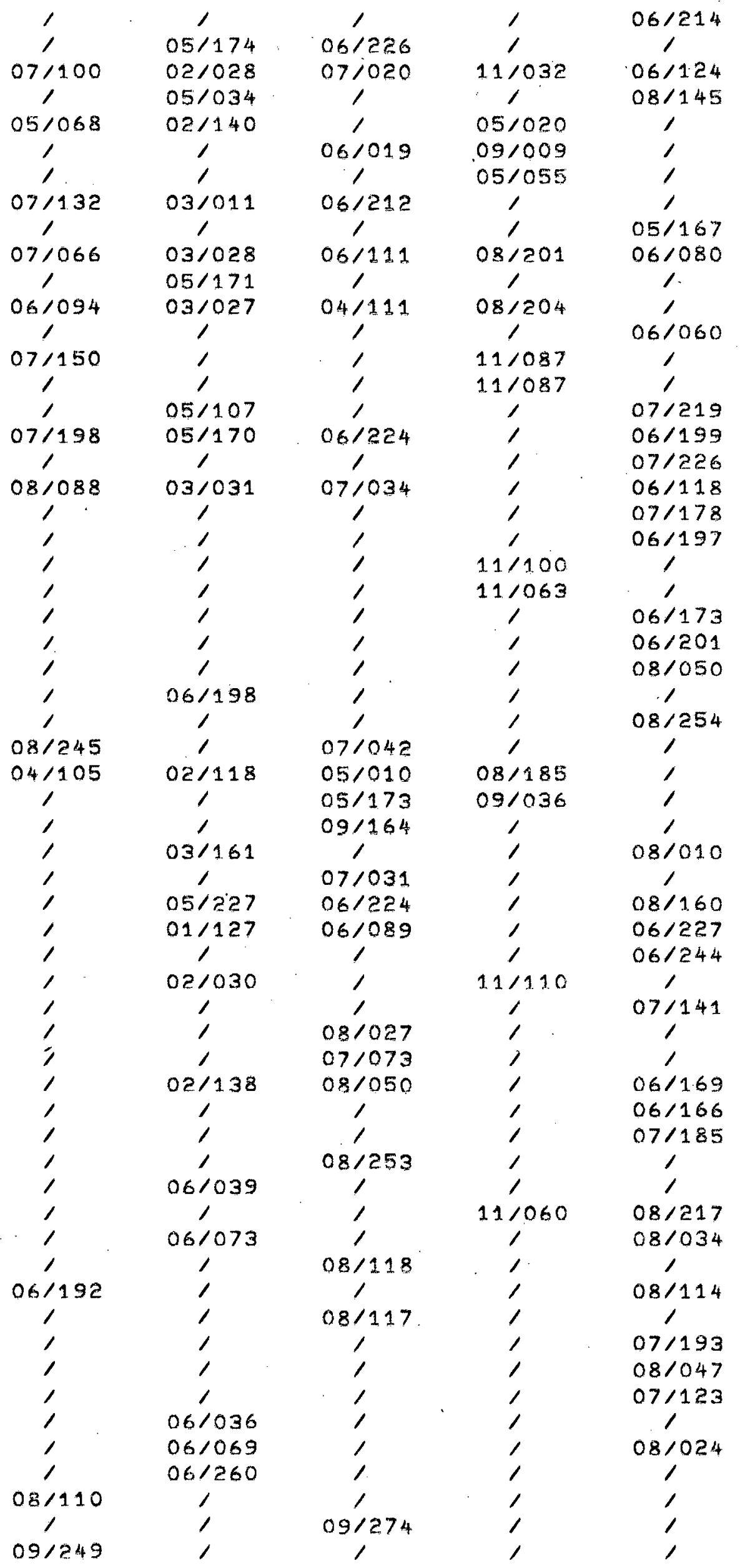


SUBWAY

SUCCEEDED

SUCCESS

SUCCESSFULI.Y

SUCH

SUCK

SUDDEN

SUDDENLY

SUE

SUFFER

SUFFIX

SUGAR

SUGGESTED

SUIT

SUITS

SULKED

SUMMER

SUN

$S U N=Y E E$

SUNDAY

SUNNY

SUNNY-DAY

SUNR I SE

SUNSET

SUPERMAN

SUPERMARKET

SUPERTOBL

SUPPER

SUPPERTIME

SUPPLIES

SUPPORTS

SUPPOSE

SUPPOSED

SURE

SURELY

SURFACE

SURPRISE

SURPR I SED

SURPRISES

SURPRISING

SURRENDER

SUSAN

SUSPECTED

SWALLOWED

SWAM

SWAN

SWANS

SWEATER

SWEEP

SWEET

SWELLED

SWENSON

SWEPT

SWIFTLY

SWIM

SWIMMING

SWIMS

SWINGING

SW IRLED

SWIRLING

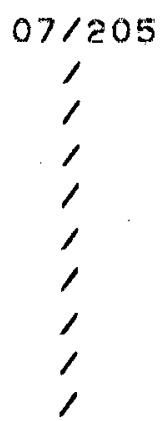

$09 / 208$

$09 / 155$

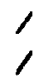

,

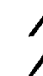

$04 / 066$

$01 / 005$

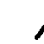

I

$\gamma$

,

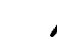
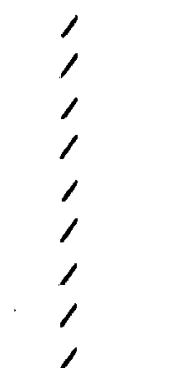

$07 / 159$

$07 / 1.66$

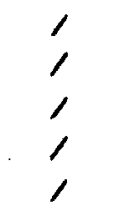

$09 / 136$

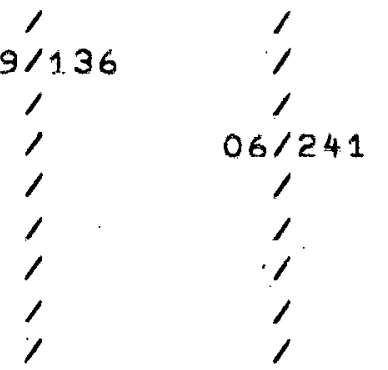

,

,

,

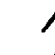

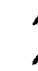

,

,

,

$03 / 095$

$03 / 012$
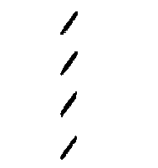

$03 / 013$

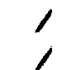

1

$05 / 148$

6/016

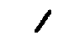

$05 / 137$

1

$03 / 186$

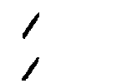

$03 / 194$
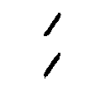

$06 / 123$

$05 / 112$

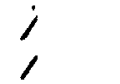

$06 / 131$
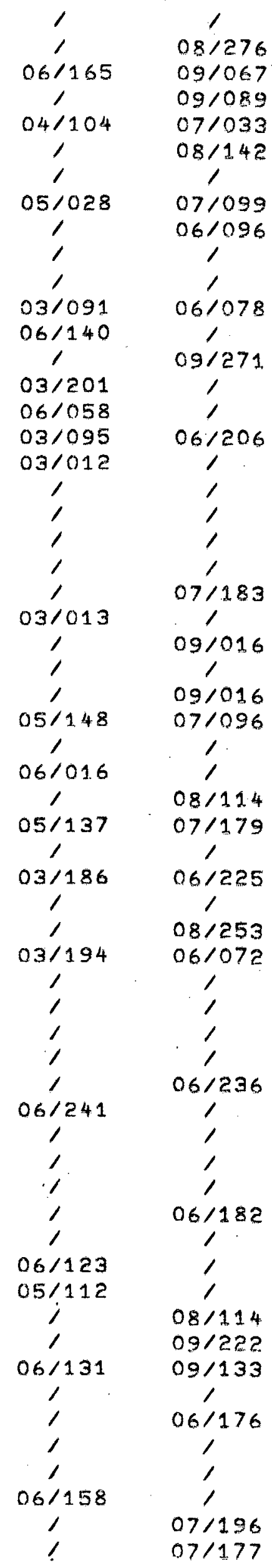

$08 / 114$

$07 / 179$

1

$06 / 225$

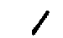

$08 / 253$

$06 / 072$

!

,

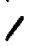

$06 / 236$

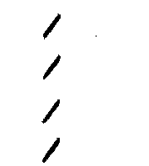

$06 / 182$
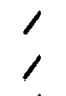

$08 / 114$

$09 / 222$

$09 / 133$

$06 / 176$
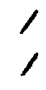

$07 / 196$

$07 / 177$

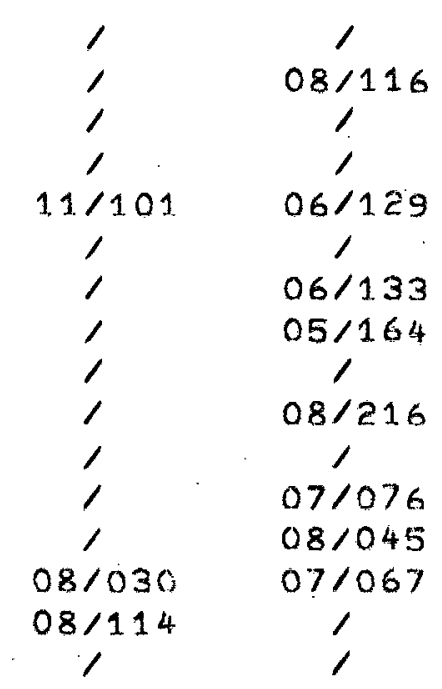

$06 / 069$

$.05 / 143$

$07 / 006$

$07 / 133$

$09 / 053$

$11 / 013$

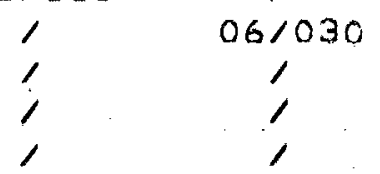

$07 / 031$.

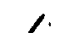

$06 / 209$

$06 / 212$

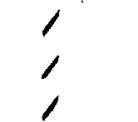

$06 / 134$

$06 / 028$

$06 / 198$

$07 / 017$

$09 / 053$

$07 / 049$

$09 / 016$
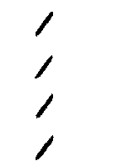

$09 / 030$

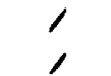

$07 / 013$
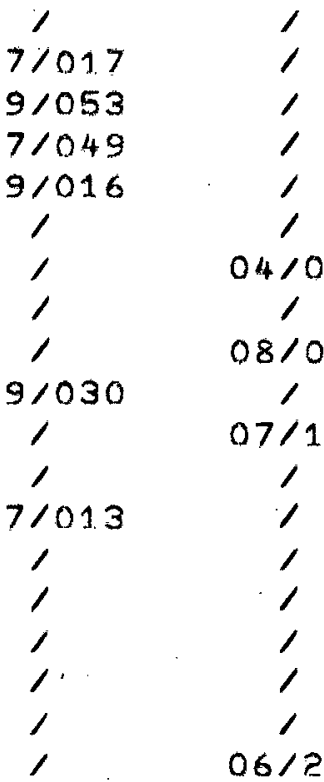

$04 / 036$

$08 / 036$
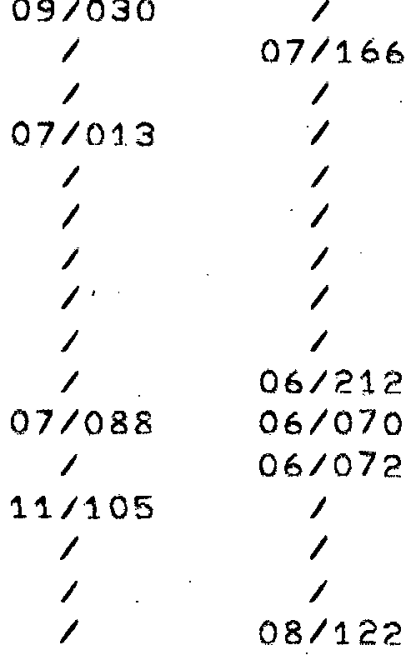

$06 / 212$

$06 / 070$

$06 / 072$

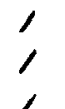

$08 / 122$ 
SWISS

SWITCHES

SWITCHING

SWITZERLAND

SWEBPED

SWORD

SWUNG

SYLLABLES

SYMBELS

SYNCOM

SYRUP

$T=S H I R T$

T-SHIRTS

TABLE

TADPOLES

TAG

TAIL

TAILS

TAKE

TAKEN

TAKES

TAKING

TALE

TALES

TALK

TALKED

TALKING

TALKS

TALL

TALLER

TALLEST

TAMEST

TANGLED

TAP

TAPE

TAPES

TARANTULA

TARE

TASK

TASTE

TASTED

TASTIEST

TAUGHT

TAXI

TAXICABS

TEACH

TEACHER

TEAMMATES

TEAMS

TEARING

TEARS

TEASE

TEASED

TEASING

TEASING BOY

TED

TEDOY

TEDDY'S

TEENY

TEETH

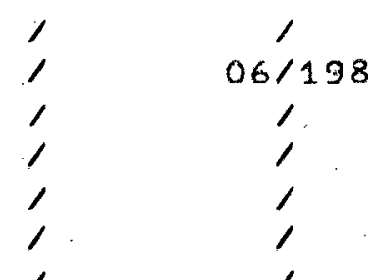

$08 / 166$

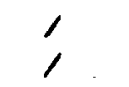

$08 / 139$

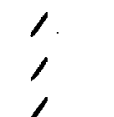

$05 / 038$

$06 / 155$

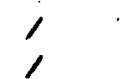

1

$06 / 063$

$06 / 112$

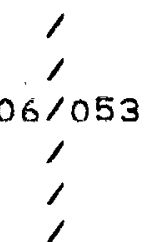

$04 / 103$

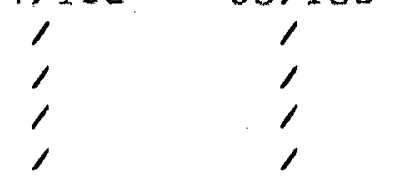

$05 / 269$

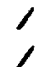

1

$07 / 232$

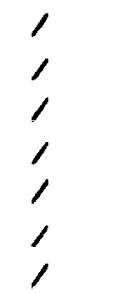

$06 / 137$

$05 / 011$

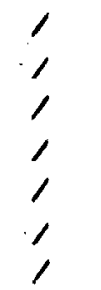

$07 / 053$

$05 / 008$

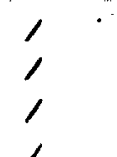

$01 / 086$

$03 / 171$

$06 / 079$

$05 / 038$

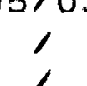

1

'

1

$04 / 103$
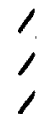

$2 / 037$

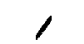

$05 / 223$
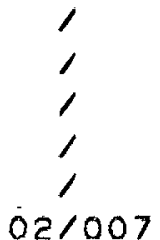

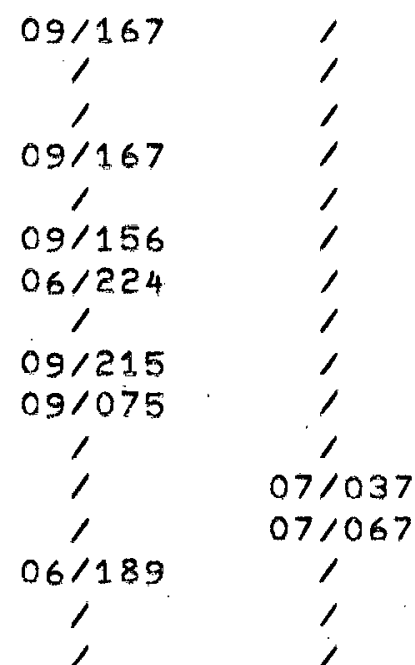

$06 / 117$

l $04 / 086$

$1 \quad 05 / 142$

$05 / 246$

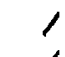

$06 / 100$

$04 / 080$

$06 / 063$

$05 / 116$

$04 / 089$

1
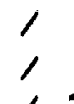

$09 / 073$

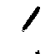

$07 / 089$

$08 / 042$
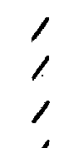

$07 / 096$

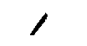

$09 / 061$

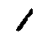

$09 / 078$

$1 / 115$

$11 / 087$

$07 / 122$

$08 / 195$

$08 / 074$

,

1

1

1

1

1

,

1

,

,

,

1

$09 / 204$

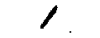

$09 / 223$
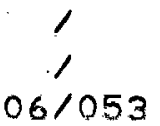

$09 / 092$

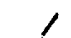

$08 / 030$
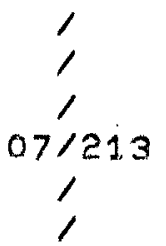

$05 / 125$

$05 / 132$

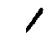

$07 / 065$

$08 / 133$

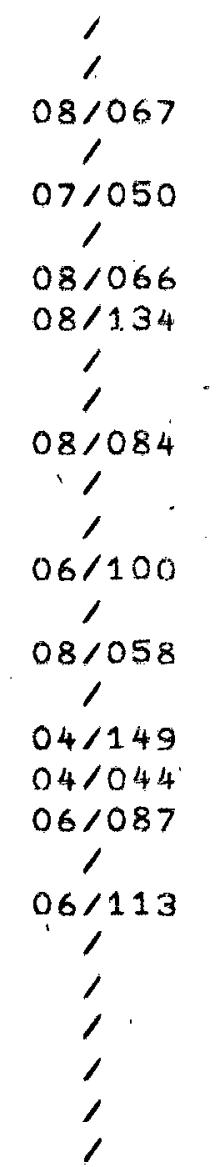

$06 / 187$

$06 / 235$

$06 / 212$

$08 / 178$

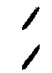

$07 / 197$

,

$05 / 081$

$08 / 124$

$06 / 243$

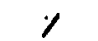

$07 / 052$

$07 / 059$

$06 / 154$

$06 / 056$

$07 / 026$

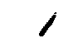

$08 / 036$

$\begin{array}{cc}\prime & 1 \\ \prime & 1 \\ \prime & 1 \\ \prime & 1 \\ \prime & 1 \\ \prime & 06 / 204\end{array}$


WORD LIST

TELEPWONE

TELESCOPE

TELEVISIEN

TELL

TELLING

TELLS

TELSTAR

TEMPERAMENTAL

TEMPERATURE

TEMPLES

TEN

TENDERLY

TENNIS

TENSION

TENT

TENTS

TEPEE

TEPEES

TERRIBLE

TERRIBLY

TERRIFIC

TERRER

TERRY

TERRY'S

TEST

TEXAS

THAN

THANK

THANKFUI

THANKS

THAT

THAT'S

THATCH

THE

THEE

THEIR

THEM

THEMSELVES

THEN

THERE

THERE 'LL

THERE'S

THEREBY

THESE

THEY

THEY D

THEY'LL

THEY'RE

THICK

THIEF

THING

THINGS

THINK

THINKING

THINKS

THIRD

THIRSTY

THIRTEEN

THIRTY

THIS
SERIES

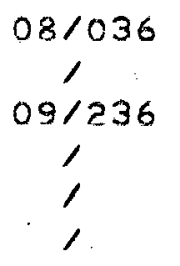

$09 / 239$

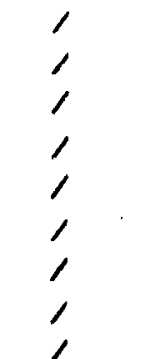

$08 / 088$
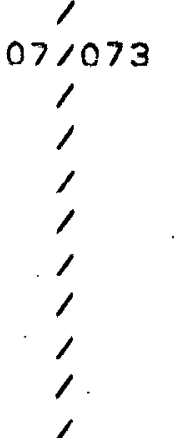

$07 / 202$

$05 / 166$

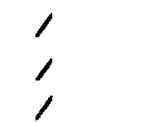

$01 / 005$

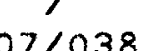

$04 / 129$

$07 / 179$

$02 / 011$

$07 / 039$

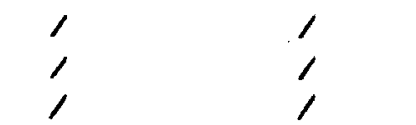

$07 / 104$

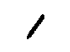

,

1

$04 / 041$

$04 / 088$

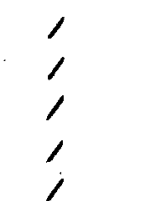

$08 / 035$

$04 / 082$
$01 / 092$

$01 / 123$

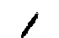

$05 / 050$

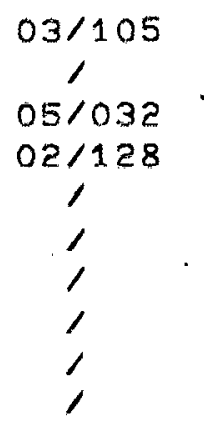

$04 / 199$

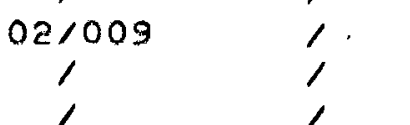

$09 / 211$

$07 / 179$

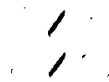

$$
1
$$

$05 / 076$

$05 / 076$

$04 / 1.56$

$02 / 141$

$01 / 125$

1

1

1

$01 / 015$

$03 / 045$

$01 / 136$

1

$01 / 134$

$01 / 024$

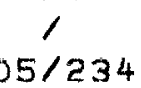

$06 / 069$

$02 / 068$

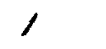

$02 / 010$

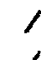

$03 / 161$

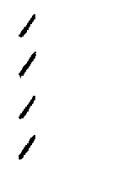

$09 / 071$

1

$04 / 072$

$07 / 133$

$04 / 099$

$04 / 068$

$04 / 061$

1

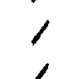

$05 / 052$

$05 / 115$

$08 / 113$

$04 / 060$

$05 / 082$

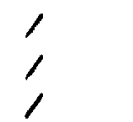

$07 / 053$

$04 / 150$

1

$0.7 / 190$

1

$5 / 137$

$05 / 089$

$05 / 089$

$06 / 062$

$07 / 048$

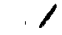

$09 / 059$

$05 / 025$
SERIES D

SERIES E

$05 / 109$

$07 / 174$

$07 / 007$

$09 / 023$

$09 / 052$

1

$11 / 062$

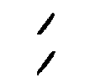

$11 / 023$

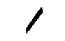

1

1

,

,

,

1

,

1

1

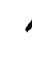

,

$08 / 088$

$05 / 041$

1

1

$05 / 010$

$07 / 084$

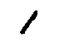

$04 / 010$

\section{1}

$09 / 046$

$08 / 021$

1

$08 / 018$

$08 / 031$
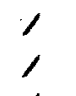

1

$08 / 014$
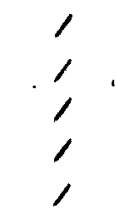

$08 / 082$

$08 / 081$

$08 / 039$

$09 / 010$

$08 / 210$

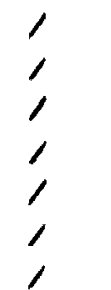

$06 / 172$

$05 / 151$

$08 / 240$

$07 / 238$

$8 / 157$

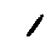

1

$06 / 218$

$07 / 214$

$08 / 159$

$08 / 158$

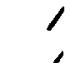

$07 / 233$

$08 / 051$

$03 / 069$

$07 / 102$

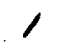

$02 / 024$

$05 / 055$

$07 / 121$

$02 / 040$

$08 / 108$

$05 / 088$

$04 / 090$

$08 / 051$

$04 / 070$

$04 / 026$

$06 / 120$

$05 / 102$

$05 / 223$

$06 / 073$

$04 / 065$

$07 / 040$

$06 / 112$

$06 / 042$

$08 / 065$

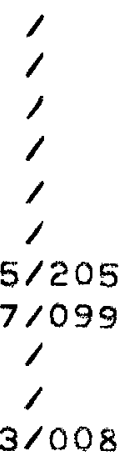


THOMAS

THOSE

THEUGH

THOUGHT

THOUGHTFULLY

THOUGHTLESS

THOUSAND=PQUND

THOUSANDS

THREAD

THREATENED

THREE

THREE - WHEELED

THREE-YEAR $=$ OLD

THREW

THREAT

THREUGH

THREW

THFEWING

THUMB

THUMBS

THUNDER

THUNDERBELT

THURSDAY

TICK

TICKETS

TICKLED

TIDE

TIDY

TIE

TIED

TIGER

TIGHT

TILL

TIM

TIMBER

TIME

TIMES

TIMMY

TIMETHY TURTLE

TINA

TINA'S

TING $=A=L$ ING

TINKLED

TINY

TIPPING

TIRE

TIRED

T.IRED-OUT

TIRES

TITHE

TI THED

TITLE

TITO'S

TO

TUASTY

TOBEGGAN

TODAY

TEE

TEES

TOGETHER

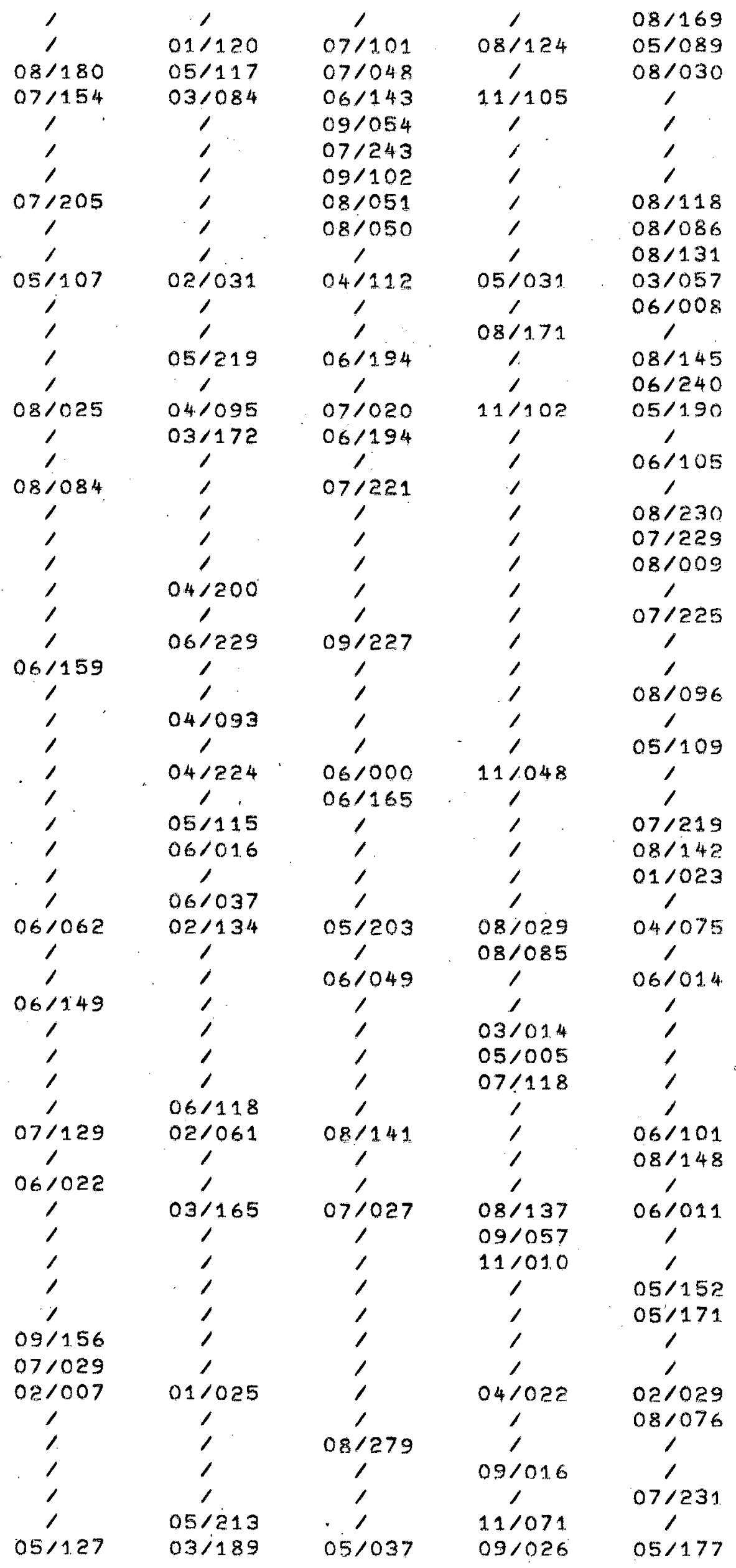


TELD

TOLL

TOM

TEMMY

TOMMY:S

TOMERROW

TONGUE

TONGUES

TONIGHT

TONIO

TENS

TONY

TOO

TOEK

TOOLHEUSE

TOELS

TOQT

TEOTH

TOETHPASTE

TOOTING

TOP

TOPIC

TOPICS

TUSSED

TOTING

$\mathrm{TOUCH}$

TOUCHED

TEUCHING

TOWARD

TUWER

TOWER ING

TOWN

TOY

TRACK

TRACKS

TRACTOR

TRADE

TRADING

TRAFFIC

TRAILER

TRAIN

TRAINED

TRAINER

TRAIT

TRANSOM

TRAP

TRAPPED

TRAVEL

TRAVELWAYS

TREASURE

TREAT

TREATED

TREE

TREE-SHADED

TREES

TREETOPS

TREMBLED

TREMENDEUS

TRIAL

TRIBE

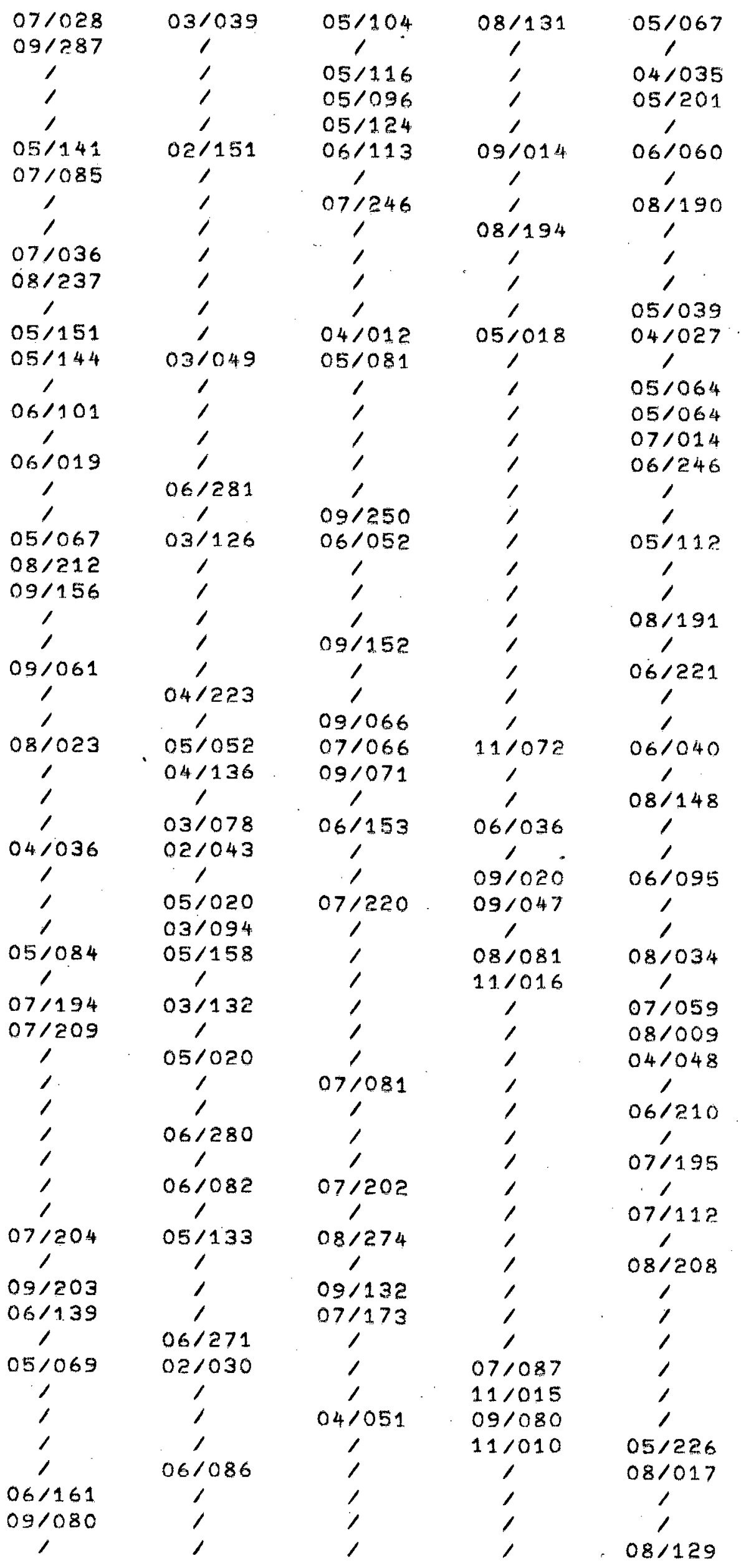


TR ICK

TRICKS

TRIED

TRIES

TRIM

TRIP

TRIPS

TREJAN

TREPICAL

TRET

TRETTED

TROUBLE

TREUBLES

TREUGH

TROUSERS

TROUT

TRQIL

TRUCK

TRUCKS

TRUDGED

TRUE

TRUILY

TRUNKS

TRUST

TRUSTED

TRUTH

TRY

TRYING

TUBE

TUBS

TUESDAY

TUGGED

TUMBLING

TUNE

TUNES

TUNNELS

TURKEY LURKEY

TURKEYS

TURN

TURNED

TURNIP

TURNIP-SEED

TURNIPS

TURNEUT

TURNS

TURTLE

TUTU

TV

TWANG

TWELVE

TWENTY

TWENTY-ENE

TWICE

TWIG

TWIN

TWINKLED

TWINS

TWIRLING

TWISTED

TWIT
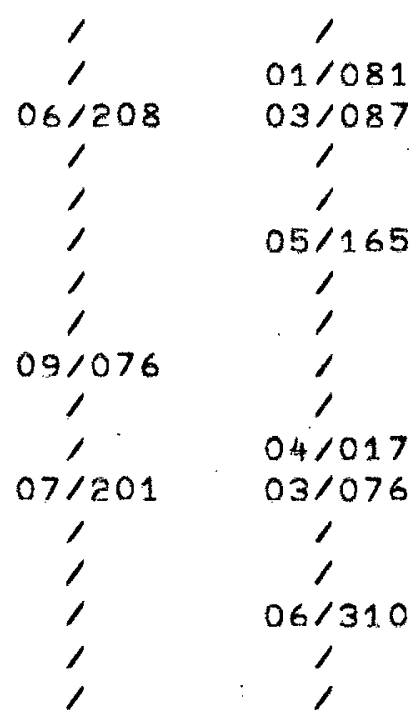

$04 / 109$

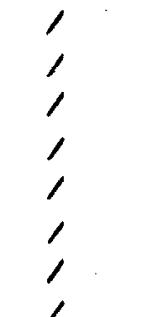

$06 / 087$

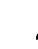

$\gamma$
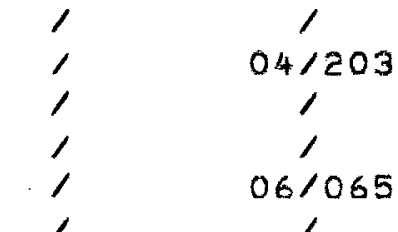

$08 / 037$

$05 / 077$
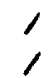

$06 / 145$

$04 / 074$

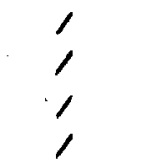

$01 / 053$
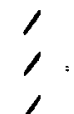

,

,

$08 / 140$

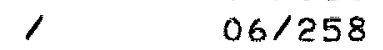

, . $03 / 183$

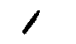

I

,

,

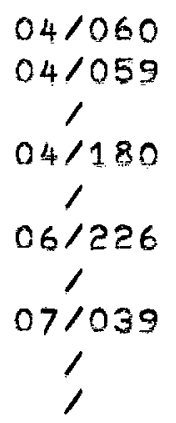

$08 / 059$

$07 / 233$

1

,

$09 / 182$

071029

$05 / 113$

$05 / 183$

$07 / 125$

$07 / 177$
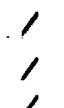

$07 / 150$

$05 / 043$

$05 / 167$

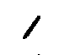

,

1

$08 / 089$

$07 / 126$

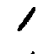

$08 / 277$
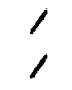

I

$08 / 055$

1

$08 / 236$

$06 / 100$

$07 / 237$

1

$05 / 042$

$09 / 171$

1

$07 / 108$

1

$06 / 155$

1

$05 / 036$

$05 / 038$

$07 / 195$

081080

$09 / 211$

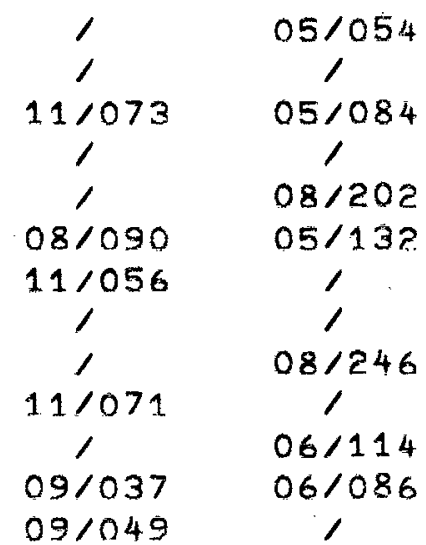

$08 / 141$

$08 / 063$

,

$05 / 102$

$11 / 115$

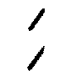

I

1

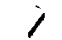

$11 / 071$

$11 / 095$

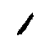

,

'

$08 / 194$

$1.1 / 051$

$11 / 076$
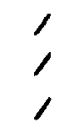

$08 / 072$
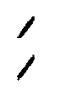

,

1

1

$08 / 146$

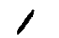

$11 / 017$

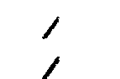

$11 / 103$

$11 / 109$

'
1

$07 / 228$

$07 / 224$

$06 / 073$

$$
1
$$

$08 / 017$

$08 / 145$

$04 / 086$

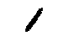

$07 / 233$

$08 / 124$

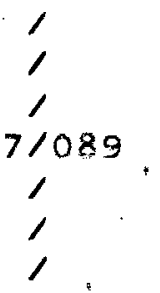

$07 / 072$

$05 / 098$

$05 / 097$

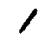

$07 / 146$

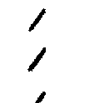

$08 / 083$

$05 / 021$.

$08 / 018$

$08 / 019$

1

$08 / 012$

$08 / 076$

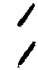

$08 / 264$ 
TWe

TWO $=$ TONED

TWO-WHEELED

TWO-WHEELER

TWO-YEAR - OLD

TYRANNOSAURUS

TYRANT

UGLY

UH

UMBRELLA

UMBRELLAS

UMLOADING

UMPIRE

UNABLE

UNBEL IEVINGLY

UNCLE

UNCLE BOB

UNCOMFERTABLE

UNCEOKED

UNCEVERED

UNDER

UNDERCLETHES

UNDERHANDED

UNDERNEATH.

UNDERST AND

UNDERSTANDS

UNDERSTEOD

UNEARTHED

UNEASILY

UNEXPECTED

UNHAPPILY

UNHAPPINESS

UNHAPPY

UNIFERM

UNIT

UNITED

UNITED STATES

UNLESS

UNLECK

UNPACK

UNRELL

UNSAFE

UNSEE-ABLE

UNSETTLED

UNTIE

UNTIED

UNT IL

UNWILLINGLY

UP.

UPON

UPPER

UPSET

UPSTAIRS

UPTEWN

UPTURNED

UPWARD

URGED

US

USABLE

USE

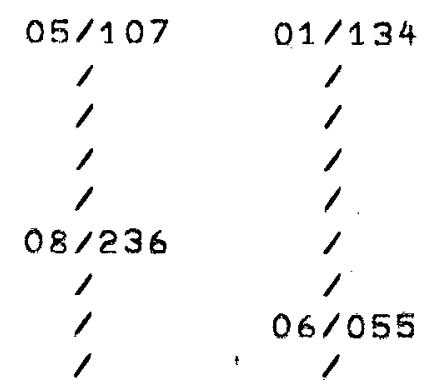

$04 / 141$

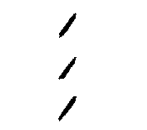

$07 / 213$

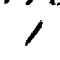

,

'

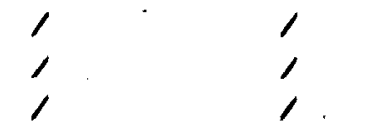

$05 / 059$

,

$09 / 181$

,

,

,

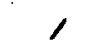

$07 / 232$

$07 / 211$

1

$06 / 072$

1/127

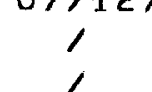

$07 / 210$

$06 / 160$

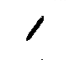

,

I

,

$06 / 024$

$01 / 005$

$01 / 005$

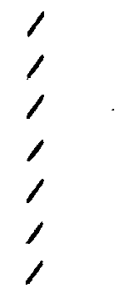

$09 / 035$

$05 / 061$

1

$06 / 102$

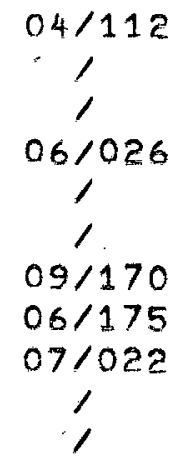

$08 / 041$

$09 / 088$

$08 / 253$

$09 / 266$

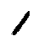

1

,

$03 / 209$

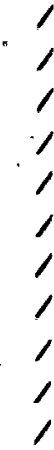

$04 / 124$
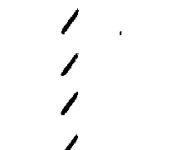

$05 / 250$

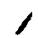

,
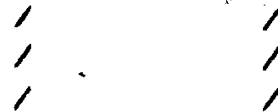

$07 / 187$

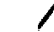

$08 / 028$

$07 / 177$

$06 / 055$

$03 / 174$

$01 / 131$
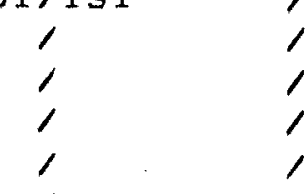

'

$06 / 258$

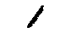

$01 / 019$

'

$04 / 026$

$09 / 180$

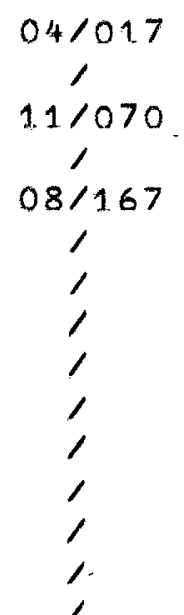

$06 / 010$

$06 / 084$
$07 / 030$

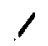

,

1

$8 / 142$

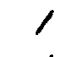

1

$11 / 026$

$11 / 062$

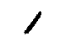

,

,

,

1

1

1

,

,

,

,

,

,

,

,

,

,

,

$8 / 137$

$11 / 074$

$08 / 153$

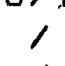

,

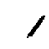

1

$11 / 017$

$11 / 010$

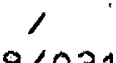

$03 / 054$

$08 / 083$

$06 / 010$
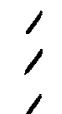

$$
1
$$

$06 / 222$

$07 / 131$

$06 / 019$

$08 / 090$

$07 / 051$

$07 / 139$

$05 / 205$

$06 / 179$

$05 / 210$

1

$07 / 134$

$07 / 124$

$07 / 188$

,

$06 / 061$

1

$08 / 079$

$08 / 016$

$07 / 196$

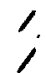

$07 / 214$

$07 / 155$

$07 / 142$

$05 / 113$

$07 / 028$

$03 / 034$

$05 / 221$

$06 / 247$.

$06 / 214$

$06 / 244$

$06 / 070$ 1

$08 / 141$

$.08 / 158$

$09 / 031$

$03 / 046$

$05 / 085$ 
WORD LIST

USED

USEFUL

USELESS

USUAL.

USUALLY

VACATION

VACUUM

VALENTINES

VALLEY

VALUE

VAN VLIET

VASES

VEGETABLE

VEGETABLES

VEICLE

VELVET

VELVET'S

VENTURE

VERY

VICIOUS

VIEW

VILLAGE

VILLAGERS

VINE

VINEGAR

VIULET

VIOLIN

VIRGINIA

VIRUS

VISIBLE

VISIT

VISITED

VISITING

VISITOR

VISITERS

VUICE

VOICES

VOLCANO

VUTE.

VOWEL

VOYAGE

WADDLE-WADDLE

WADDLED

WADDLES

WAFERS

WAGGED

WAGGER

WAGGING

WAGGLES

WAGEN

WAILED

WAIST

WAIT

WAITED

WAITING

WAKED

WAKENED

WALK

WALKED

WALKER
SERIES $B$

SERIES

SERIES D

SERIES E

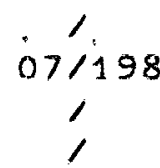

$08 / 0.71$

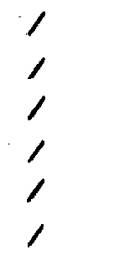

$08 / 176$

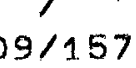

,

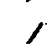

1

021034

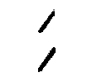

$07 / 023$

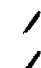

,

,

,

,

,

,

.

$08 / 155$

$8 / 135$

1

$09 / 112$

$06 / 054$

$08 / 167$

$09 / 11.2$

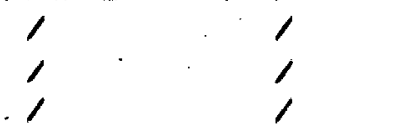

$06 / 163$

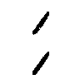

$04 / 011$

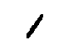

$02 / 116$

$06 / 220$

1

$06 / 153$

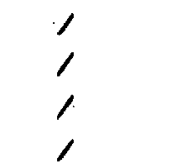

$05 / 009$

$02 / 115$

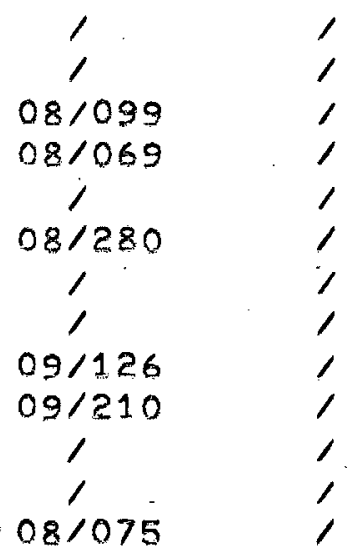

$08 / 239$

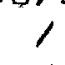

$09 / 281$

$05 / 103$

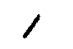

$09 / 244$

$07 / 067$

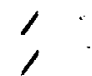

$06 / 099$

,

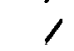

1

$07 / 197$

$07 / 158$

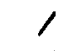

1

$08 / 085$

$07 / 060$

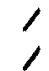

$06 / 098$

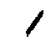

$09 / 268$

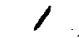

$08 / 260$

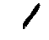

1

$06 / 120$

$06 / 117$

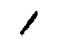

$07 / 147$

$06 / 206$

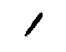

1

$06 / 214$

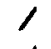

,

1

$05 / 185$

$05 / 189$
$11 / 053$

$05 / 086$

$06 / 012$

$07 / 125$

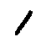

$08 / 013$

$07 / 173$

$07 / 226$

$06 / 014$

$08 / 040$

$07 / 015$

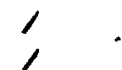

$06 / 207$

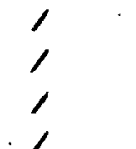

$11 / 011$

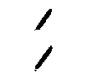

$11 / 015$

1. $1 / 069$

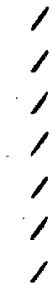

$11 / 088$
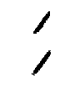

,

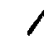

$11 / 054$

$11 /$

,

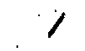

,
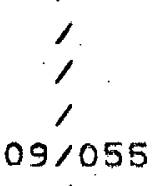

091042

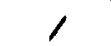

091075
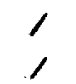

$09 / 054$

$09 / 055$

$09 / 060$

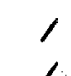

$03 / 037$

$08 / 013$

$09 / 073$

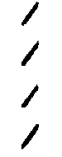

1

$08 / 074$

$06 / 196$

$06 / 256$

,

$07 / 088$

$08 / 115$

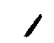

$05 / 111$

$05 / 194$

1

$07 / 129$

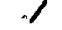

$07 / 079$

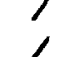

1

1

$06 / 244$

1

$06 / 238$
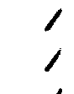

081059

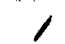

$04 / 049$

$08 / 057$

$08 / 153$

$05 / 137$

$05 / 194$

$05 / 139$

$07 / 224$

$07 / 230$

$04 / 119$
$08 / 157$

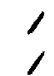


WALKEFS

WALKIE = TALKIE

WALKING

WALKS

WALL

WALLS

WALRUS

WANDER

WANDERED

WANT

WANTED

WANTING

WANTS

WAR

WARD

WARDEN

WARM

WARMED

WARMER

WARN

WARNED

WARR I OR

WARR IORS

WARSHIPS

WAS

WASH

WASHED

WASHINGTON

WASN!T

WASPS

WASTE

WASTING

WATCH

WATCHED

WATCHES

WATCHFULLY

WATCHING

WATCHMAN

WATER

WATERIS

WATERMELON

WATERS

WATERSHED

WAVE

WAVED

WAVING

WAWA

WAY

WAYSIDE

WE

WE'D

WE'LL

WE!RE

WE IVE

WEAK

WEAKNESS

WEALTH

WEAR

WEATHER

WEAVER

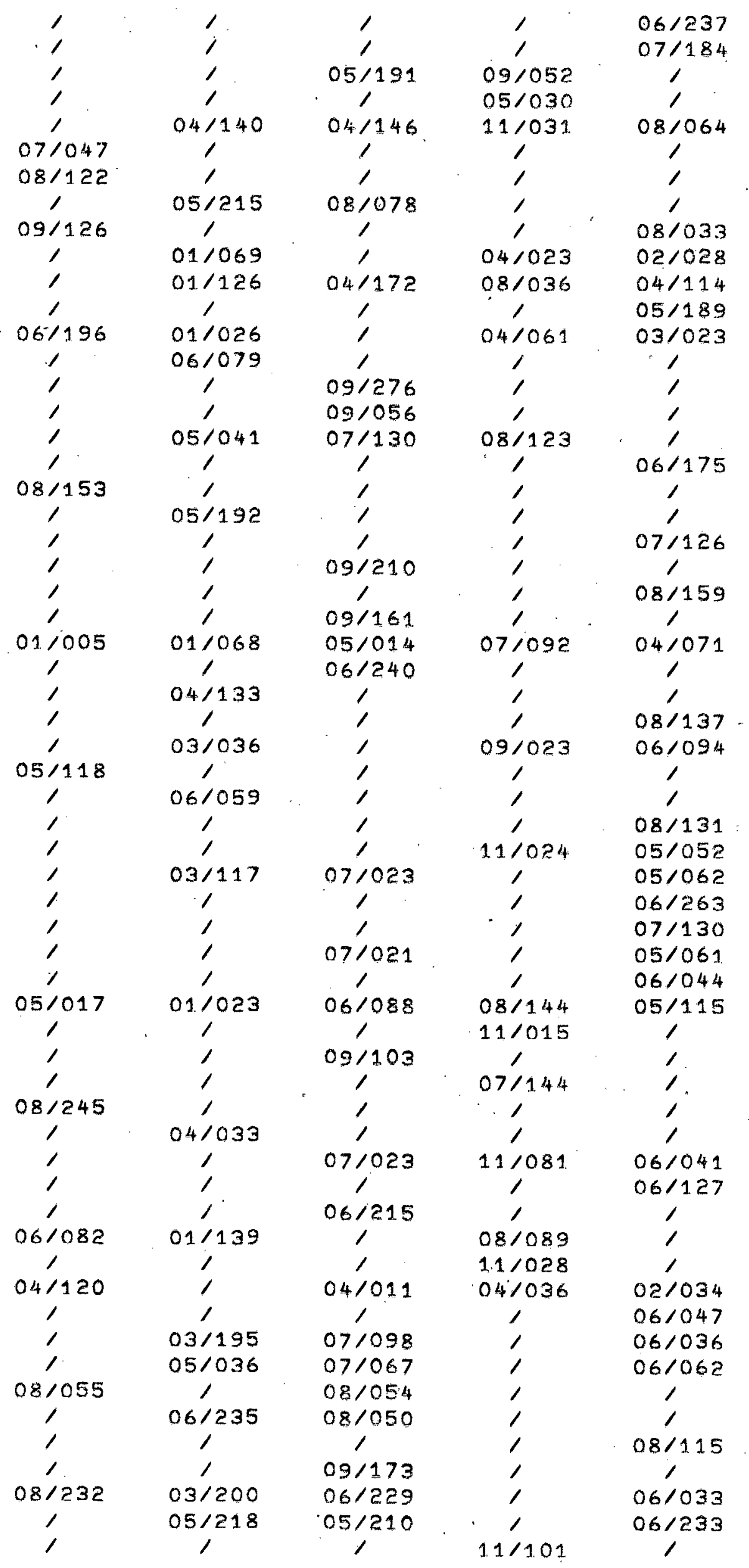


WEB

WEBER

WEDDING

WEDGES

WEDNESDAY

WEEK

WEEKEND

WEEP

WEI

WEIGH

WE I GHED

WE I GHT

WELCOME

WELCOMES

WELL

WELL - MADE

$W E L L=T O=D G$

WELLS

WENOY

WENT

WERE

WEREN 'T

WEST

WESTERN

WET

$W H=E E=E$

WHALES

WHARF

WHAT

WHAT'S

WHATEVER

WHEAT

WHEEL

WHEELED

WHEELS

WHEN

WHENEVER

WHERE

WHETHER

WHICH

WHILE

WHIMPERED

WHINE

WHINNIED

WHIP

WHIPPED

WHIRL

WHIRLED

WHIRL ING

WHIRLWIND

WHIRLY

WHISKERS

WHISPER

WHISPERED

WHISPERING

WHISPERS

WHISTLE

WHISTLED

WHISTLING

WHITE

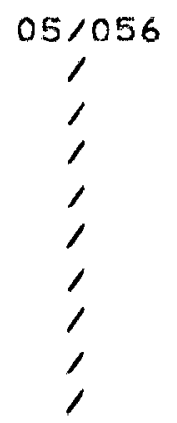

$08 / 133$

,

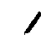

$06 / 034$

,

,

1

$05 / 011$

$01 / 030$

$05 / 141$

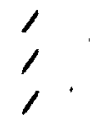

$02 / 034$

1

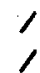

$04 / 025$

$07 / 106$

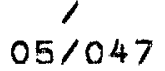

$06 / 022$

1

$04 / 012$

1

$05 / 075$

$09 / 242$

$07 / 101$
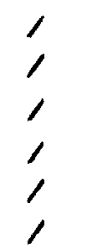

$07 / 198$
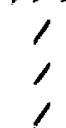

$07 / 198$

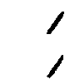

1

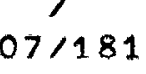

$07 / 210$

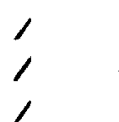

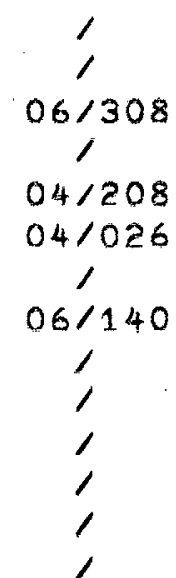

$03 / 054$

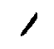

,

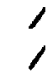

$01 / 130$

$02 / 039$
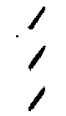

$01 / 149$

1

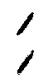

$01 / 012$
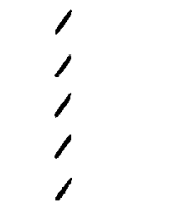

$03 / 033$

$02 / 014$
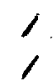

$05 / 156$

$02 / 091$

$03 / 075$

$06 / 226$

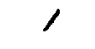

$06 / 242$
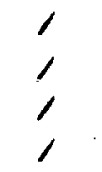

,

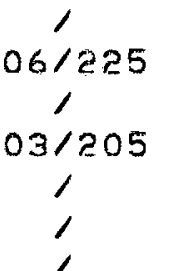

$03 / 084$

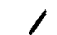

$02 / 038$

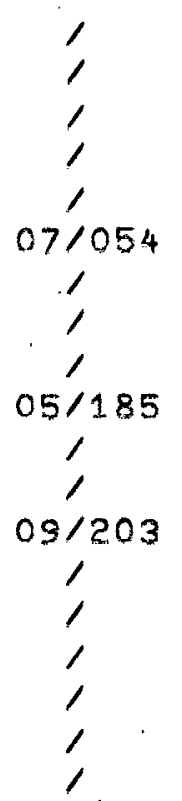

$04 / 118$

$05 / 032$

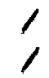

$09 / 209$

$05 / 020$

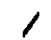

$08 / 141$

$08 / 125$

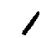

$06 / 062$

'

$04 / 158$

,

$05 / 101$

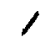

$05 / 077$

1

$06 / 109$

$06 / 241$
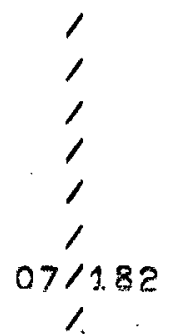

$08 / 118$
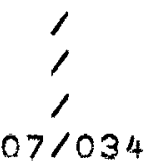

$07 / 034$

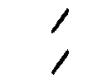

$05 / 064$

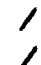

$05 / 015$

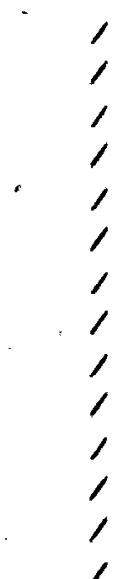

$07 / 039$

$08 / 066$

1

$06 / 071$

$06 / 116$

$08 / 061$

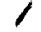

$07 / 189$

$07 / 130$

$08 / 077$

$11 / 103$

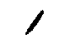

$09 / 015$

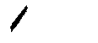

$08 / 086$

$08 / 140$
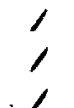

$08 / 1.47$
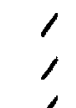

2́,

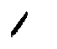

1

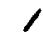

$11 / 099$

$11 / 049$

$08 / 077$

$11 / 082$

$09 / 037$

1

$11 / 016$

1

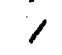

I

1

1

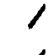

,

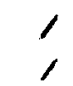

,

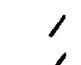

$09 / 026$

$09 / 020$

$09 / 081$.
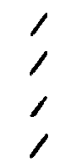

$07 / 023$

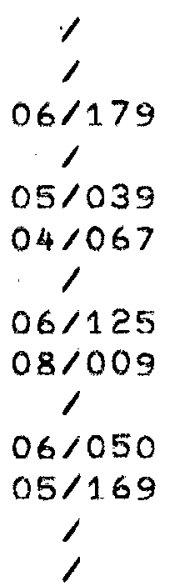

$03 / 031$

$06 / 018$

$05 / 153$

$05 / 167$

$05 / 153$

$05 / 121$

$05 / 153$

$04 / 009$

$07 / 041$

$06 / 019$

$08 / 225$

$08 / 012$

$06 / 210$

$06 / 217$

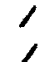

$07 / 204$

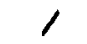

$08 / 165$

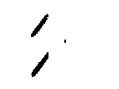

$06 / 021$

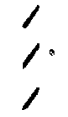

$06 / 090$

$04 / 080$ 
WHITE $=$ HAIRED

WHITENESS

WHITES!

WHITTLING

WHIZZED

WHIZZING

WHO

WHOIS

WHOA

WHOEVER

WHOLE

WHOM

WHOC-OO-OO

WHOOOO-OO

WHOSE

WHY

WICK

WICKED

WIDE

WIDELY

WIDOW

WIFE

WI GGLED

WILBUR

WILD

WILDCATS

WILDLY

WILL

WILL I AM

WILLING

WILLINGLY

WILSEN'S

WIN

WINO

WINDEW

WINDOWS

WINGS

WINK

WINTER

WINTERS

WINTERS !

WIPE

WIPED

WIRE

WIRES

WISDOM

WISE

WISEST

WISH

WI SHES

WI SH ING

WITCH

WITH

WI THOUT

WI ZARD

WOBBLY

WOKE

WELF

WOLVES

WOMAN

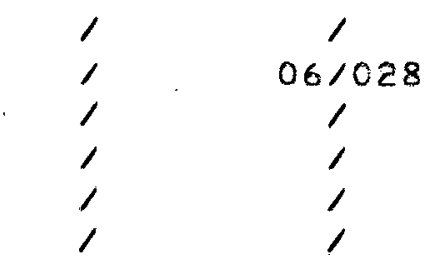

$05 / 0.98 \quad 01 / 004$
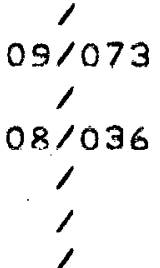

$09 / 125$

$06 / 015$
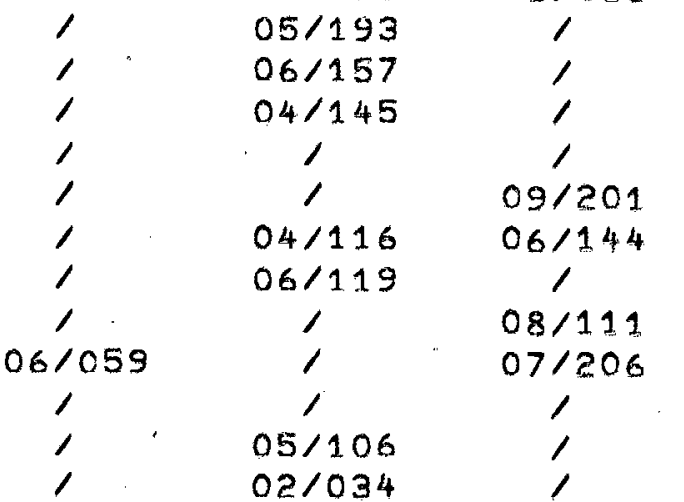

$07 / 029$

'́1012

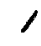

1

$04 / 067$

,

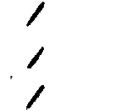

$06 / 139$

,

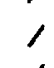

1

$04 / 018$

1

1

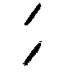

$07 / 074$

1

$07 / 027$

$08 / 088$

1

$04 / 094$
$04 / 116$

$06 / 119$

/ $\quad 09 / 167$

$02 / 084$

$05 / 028$

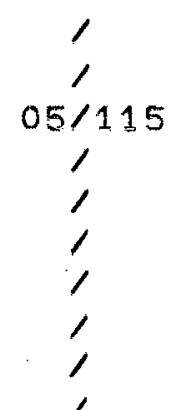

$03 / 123$

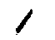

$04 / 115$

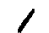

$02 / 147$

$02 / 156$

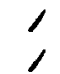

$01 / 012$
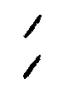

1

$05 / 086$

$05 / 270$

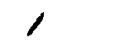

$04 / 090$

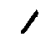

$08 / 029$

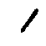

$08 / 087$

$09 / 122$

$09 / 122$

$09 / 051$

$05 / 016$

1

,

$09 / 201$

$06 / 144$

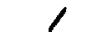

$8 / 111$

1

$05 / 156$

$06 / 192$

,

$06 / 181$

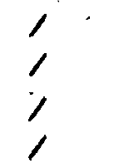

$06 / 093$

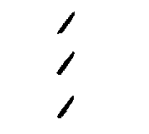

$08 / 027$

$11 / 010$

$07 / 151$
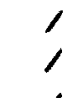

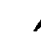

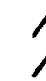

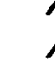

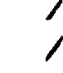

$07 / 069$

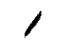

,

1

,

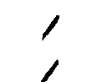

,
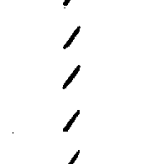

$05 / 022$

1

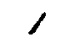

$11 / 079$

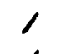

1

$08 / 123$

1

$1.1 / 080$
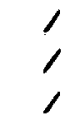

$11 / 032$

$11 / 087$

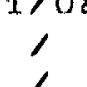

1

'

1

$08 / 156$

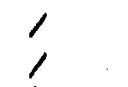

$06 / 095$

$08 / 019$

$11 / 058$

$08 / 100$

,

$11 / 117$

$09 / 061$

$04 / 014$

$08 / 082$

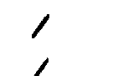

$06 / 025$

$07 / 040$

1

$07 / 015$

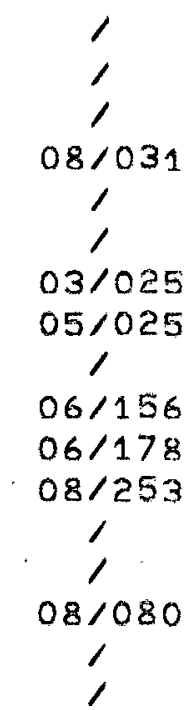

$08 / 146$

$06 / 229$

$07 / 149$

$06 / 181$

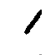

$06 / 169$

$06 / 211$

$07 / 098$

$03 / 017$

$07 / 117$

$05 / 233$

$07 / 039$

$08 / 083$

$05 / 102$

1

1

$05 / 168$

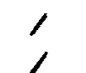

$08 / 143$

$07 / 080$

$08 / 066$

$05 / 193$

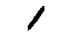

'
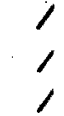

$02 / 037$

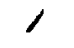

$07 / 098$

$07 / 126$ 
WOMAN'S

WOMEN

WON

WON'T

WENDER

WONDERED

WONDERFUL

WONDERFULLY

WONDERING

WOE-FF

WeOD

WOODCHUCK

WOEDCUTTER

WOODEN

WOUDLAND

WOODS

WOODSHED

WoUF

WOEL

WOOLLY

WERO

WORDS

WORE

WORK

WORKED

WORKER

WORK ING

WORKMAN

WURKMEN

WORKOUT

WORKS

WORLD

WORR IED

WORR IEDLY

WORRIES

WURRY

WORRYING

WORSE

WORSHIP

WORTH

WERTHY

WOULD

WOULDN'T

WOUND

WRAPPED

WRECKED

WREN

WRIGHT

WRINKLED

WR ITE

WR I TER

WRITES

WRITING

WRITTEN

WRENG

WRETE

YANKEE

YARD

YARDS

YEAR

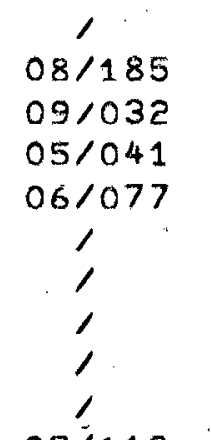

$05 / 118$

$06 / 152$

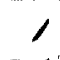

$08 / 097$

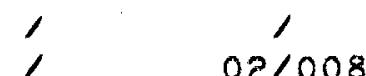

,

,

,

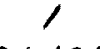

$06 / 041$

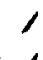

\section{$05 / 142$}

1

,

,

,

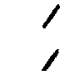

$04 / 085$

$07 / 126$

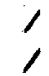

$07 / 125$

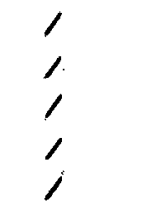

$06 / 146$

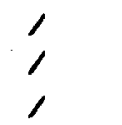

$09 / 125$
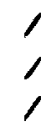

$05 / 129$

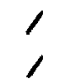

$06 / 110$

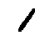

1

$07 / 149$

$04 / 018$

1

$06 / 091$

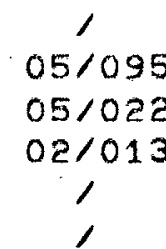

$05 / 104$

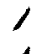

,
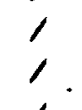

,

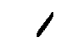

$02 / 008$

$06 / 227$

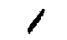

$05 / 114$
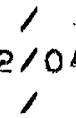

$05 / 216$

$02 / 133$

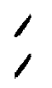

I

,

,

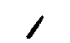

$04 / 198$

1

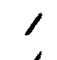

$05 / 033$

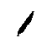

$05 / 235$

1

$06 / 236$

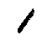

$02 / 011$

$05 / 027$

1

$06 / 018$
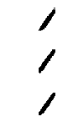

$06 / 055$

$05 / 066$
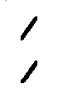

1

$04 / 027$
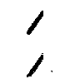

$05 / 152$

1

$03 / 184$
$02 / 043$

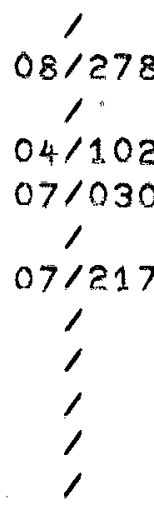

$06 / 195$

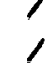

$06 / 180$

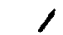

$05 / 091$

1

$04 / 146$

$04 / 189$

$06 / 229$

$05 / 129$

$05 / 205$

1

$05 / 202$

1

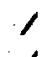

$05 / 170$

$07 / 187$

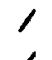

$08 / 074$

$07 / 159$

$07 / 174$

$08 / 205$

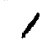

$09 / 076$

$09 / 194$

$06 / 054$

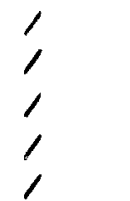

$08 / 111$

$09 / 151$

$05 / 040$

$05 / 043$

$08 / 017$

$07 / 163$

$06 / 233$

$09 / 237$

$07 / 22 ?$

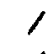

$11 / 060$

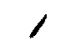

$07 / 037$

$08 / 176$

$05 / 136$

$08 / 150 \quad 05 / 192$

$05 / 187$

$05 / 17 ?$

$07 / 011$

$\begin{array}{cc}09 / 079 & 05 / 191 \\ 1 & 06 / 188\end{array}$

$08 / 142 \quad 05 / 198$

1

$11 / 014$

$08 / 172$

$08 / 121$.

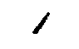

1

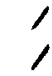

$07 / 157$

$07 / 131$

1

$07 / 114$

$09 / 021$

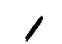

$09 / 072$

$11 / 052$

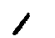

$08 / 168$

$08 / 085$

$11 / 031$
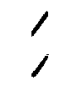

I
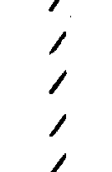

$11 / 115$
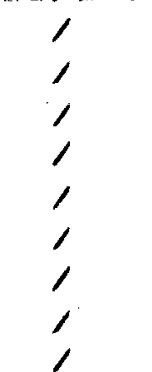

$08 / 181$

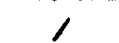

$08 / 203$

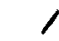

$11 / 039$

$09 / 043$

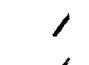

$09 / 039$

$09 / 046$

$08 / 060$

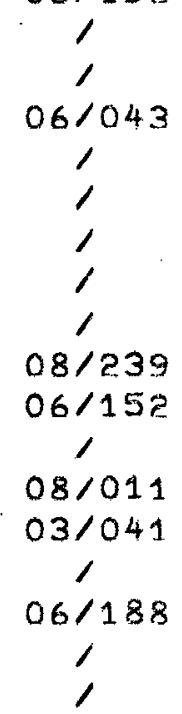

$.05 / 093$

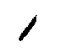

$08 / 019$

$07 / 079$

$07 / 190$

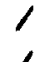

$07 / 080$

$07 / 155$

$08 / 035$

$05 / 026$

$05 / 214$

$07 / 171$

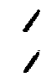

$06 / 201$

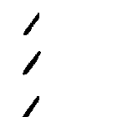

$06 / 240$

$06 / 102$

$06 / 141$

$08 / 058$

$06 / 124$

$06 / 019$

'


WERD LIST

YEARS

YELL

YELLED

YELLING

YELLOW

YELLEW-COLGRED

YELLEWING

YES

YESTERDAY

YET

YEDER

YOSHI

YOU

YOU'D

YOU'LL

YOU'RE

YOU.VE

YOUNG

YOUNGER

YOUR

YOURS

YUURSELF

YOURSELVES

YUGOSLAVIA

ZEBRA

ZERE

ZI PEPPI

2 I PEPPI'S

ZIP

ZIPPEP

200
SERIES A SERIES B SERIES

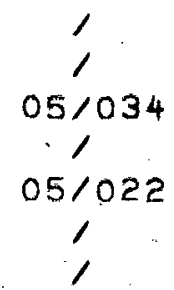

$02 / 052$

$07 / 118$

$06 / 033$

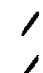

$04 / 029$

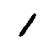

1

$07 / 103$

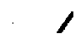

$08 / 149$

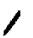

$05 / 114$

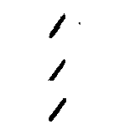

$09 / 100$

1

,

/

I

1

$06 / 052$

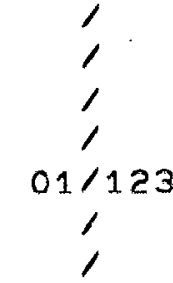

$01 / 025$

1

$05 / 048$

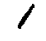

1

$02 / 033$

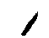

$04 / 038$

$05 / 029$

$05 / 035$

$04 / 205$

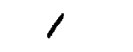

I. $04 / 129$

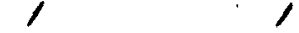

$06 / 043$

$09 / 028$

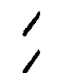

$04 / 139$

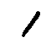

1

$05 / 058$

$05 / 071$

$07 / 229$

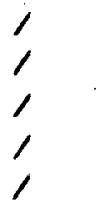

$03 / 119$

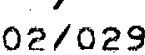

SERIES D

SERIES E

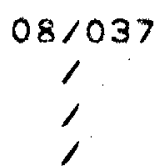

$11 / 029$

$$
1
$$

$11 / 013$

$05 / 019$

,

,

,

$04 / 006$

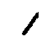

$09 / 013$

$09 / 011$

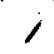

$08 / 106$

1

$05 / 007$

$05 / 039$

$07 / 1.06$

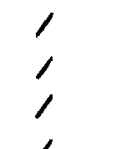

$11 / 054$

$11 / 058$

1

1

$08 / 072$

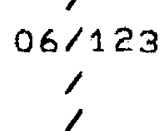

$04 / 089$

$06 / 097$

1

$04 / 012$

$06 / 224$

$06 / 034$

$05 / 087$

$02 / 021$

$06 / 095$

$05 / 040$

$06 / 035$

$06 / 140$

$06 / 041$

$06 / 066$

$04 / 107$

1

$05 / 087$

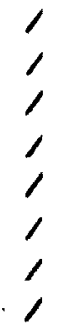

$04 / 138$ 


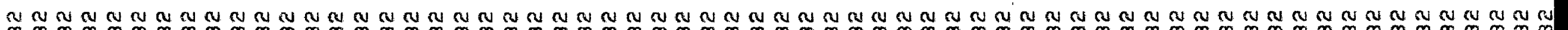

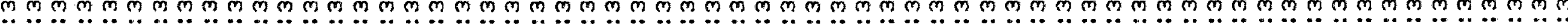

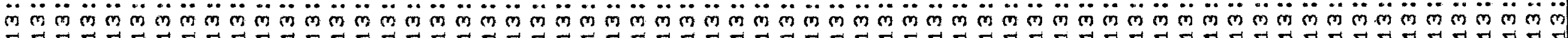

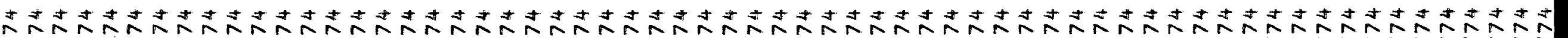
فn

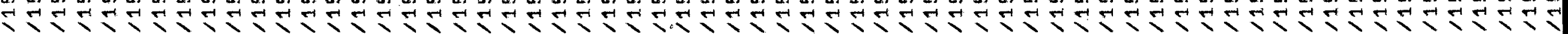

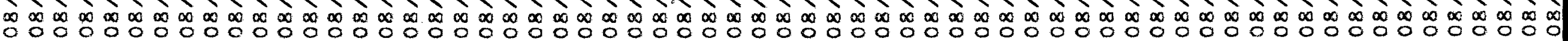

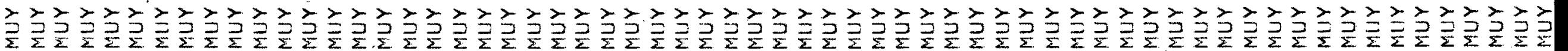

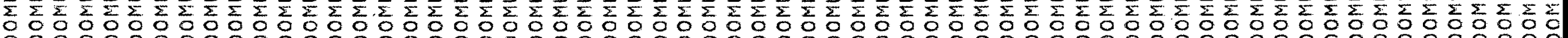

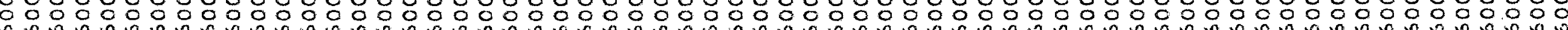

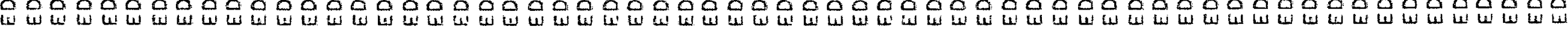

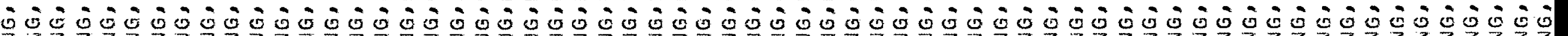

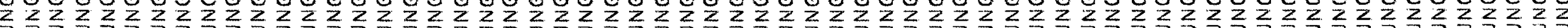

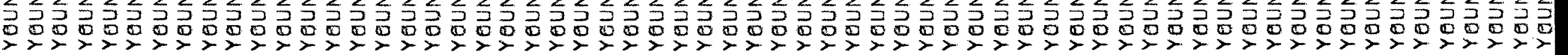

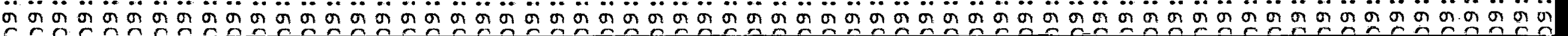




\section{BIBIJIOGRAPHY}

Anderson, Iwing $H_{*}$ "The Psychology of Learning to Read," Universjey of Michigan Sciool of gucation Bulletin, Decenber, 1.947.

3lack, IIIlel. The Amerjcan Schoolbook. New York: Willian Morrcow \& Company, Inc., 1967 .

Bond, Guy I, Eax Miles A. Tinker, Reading Diffloulties: Their Diagrosis and Corsection. New York: Appleton-Century-Crof's, 1967.

Buckingham, B. and E. W. Dolch. A Combined Word List. New Yorir: Ginn \& Company, 1936.

Carpenter, Chaxles, History of American Schoolbooks. Philadelphia: University of Pennsy?vania Press, 1963.

Dechant, Enerald $V$. Inproving the Teaching of Reading. Englewood Cliffs, New Jersey: Prentice-Hall, Inc., 1964.

Irurkin, Dolores, Teaching Them to Read. Boston: Allyn and Bacon, Ine. , 1970 .

Durr, Villian K., ed. Reading Instruction. Boston: Houghton Mirfin Company, 1967.

Flesch, Rudolf: Why Johnny Can't Read. New Yoxk: Harper and Row, 1965.

Hafner, Iawrence E. end Haylen B. Jolly. Datterns of Teaching Reading in the: Elementaxy School. New York: The facmilian Company, 1972.

Kamis, Albext J. How To Increase Reading Ability. Longmans, Green and Co., Inc., 1956.

Hamis, Albert J, Mae Knight Clark and Others. Teacher's Annoted Edition axd Guide to Accompany Better Than Gold. New York: The Macmilian Company, 1966.

Hester, Kathleen B. Teaching Every Child to Read, New York: Harper and Row. 1964 .

HiIdreth, Gertrudes Teaching Reading. New Yorks Holt, Finehart and Hinston, Inc., 1958

Jomsin, Ida Ha, Alfonso R, Ramirez, Mildred Beatty Smith and Others, Teachers adition for use with Seking Adventure. Glenview, ITInots: Scott, Foresinan \& Co., 1973. 
McCullough, Constance H. "Lingistics, Psychology, and the Teaching of Reading". Readines on Reading. Scranton, Pennsylvania: International Tex60ok 0.969.

Morrjon, Ida E. Teaching Reading in the Elementary School. New York: The Ronald Press Company, 1968.

Sebesta, Sam Leaton and Carl J. Vallen. The First $R$, Readings on Teaching Reajing. Chicago: Science Research Associates, Inc., 1972.

Spache, George D. and Evelyn B. Spache. Reading in the Elementary School. Boston: Allyn and Bacon, Inc., 1973.

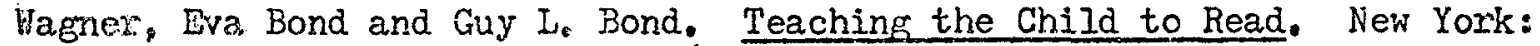
The Mackillan Company, 1960.

Hallen, Carl J. Competency in Teaching Reading. Chicago: Science Research Associates, Inc., 1972. 